\title{
Tables of products of tensor operators and Stevens operators
}

\author{
Lindgård, Per-Anker
}

Publication date:

1974

Document Version

Publisher's PDF, also known as Version of record

Link back to DTU Orbit

Citation $(A P A)$ :

Lindgård, P-A. (1974). Tables of products of tensor operators and Stevens operators. Risø National Laboratory. Denmark. Forskningscenter Risoe. Risoe-R No. 313

\section{General rights}

Copyright and moral rights for the publications made accessible in the public portal are retained by the authors and/or other copyright owners and it is a condition of accessing publications that users recognise and abide by the legal requirements associated with these rights.

- Users may download and print one copy of any publication from the public portal for the purpose of private study or research.

- You may not further distribute the material or use it for any profit-making activity or commercial gain

- You may freely distribute the URL identifying the publication in the public portal

If you believe that this document breaches copyright please contact us providing details, and we will remove access to the work immediately and investigate your claim 


\title{
Danish Atomic Energy Commission
}

\author{
Research Establishment Risø
}

\section{Tables of Products of Tensor Operators and Stevens Operators}

by Per-Anker Lindgård

July 1974 


\title{
Tables of Products of Tensor Operators and Stevens Operators
}

\author{
by \\ Per-Anker Lindgård \\ Danish Atomic Energy Commission \\ Research Establishment Ris $\emptyset$ \\ Physics Department
}

\begin{abstract}
Numerical tables of products of tensor (Racah) operators, $R_{\ell, m}(J)$, and Stevens operators, $O_{\ell}^{\mathbf{m}}(\mathrm{J})$, working within a $\mathrm{J}$-multiplet are given as a function of $X=J(J+1)$. Examples of the use of the tables, such as the calculation of commutation relations and thermal averages, are given. All values of $\ell$ and $m$ up to six are included.
\end{abstract}


ISBN 8755002811 


\section{CONTENTS}

Page

1. Introduction $\ldots \ldots \ldots \ldots \ldots \ldots \ldots \ldots \ldots \ldots \ldots \ldots \ldots \ldots \ldots, v$

2. Definitions $\ldots \ldots \ldots \ldots \ldots \ldots \ldots \ldots \ldots \ldots \ldots \ldots \ldots \ldots \ldots \ldots$

2.1. Tensor Operators $\ldots \ldots \ldots \ldots \ldots \ldots \ldots \ldots \ldots \ldots \ldots$ VI

2. 2. Symmetry Properties $\ldots \ldots \ldots \ldots \ldots \ldots \ldots \ldots \ldots \ldots \ldots$ VII

2.3. Stevens Operators $\ldots \ldots \ldots \ldots \ldots \ldots \ldots \ldots \ldots \ldots \ldots \ldots$ VIII

3. Applications and Examples $\ldots \ldots \ldots \ldots \ldots \ldots \ldots \ldots \ldots \ldots \ldots$ VIII

3.1. Calculation of Commutation Relations and Equations of Motion $\ldots \ldots \ldots \ldots \ldots \ldots \ldots \ldots \ldots$ VIII

3. 2. Thermodynamic Averages $\ldots \ldots \ldots \ldots \ldots \ldots \ldots \ldots \ldots$ XI

References $\quad \ldots \ldots \ldots \ldots \ldots \ldots \ldots \ldots \ldots \ldots \ldots \ldots \ldots \ldots \ldots \ldots \ldots$ XII

Table 1. Products of Tensor operators $\mathbf{R}_{\ell m} \mathbf{R}_{\ell^{\prime} \mathbf{m}^{\prime}} \ldots \ldots \ldots \ldots \ldots, \quad$

Table 2. Products of Stevens operators $o_{\ell}^{m}(c) o_{\ell}^{m^{p}}(c) \ldots \ldots \ldots \ldots . \quad 92$

Products of Stevens operators $o_{\ell}^{m}(s) O_{\ell !}^{m^{\prime}}(s) \ldots \ldots \ldots 134$

Table 3. Coefficients relating Stevens operators to tensor operators .......................... 167 



\section{INTRODUCTION}

The use of spin Hamiltonians ${ }^{1)}$ is well established in the theory of solid state physics. Most directly of course in the theory of magnetic phenomena - but also in other problems, the statistics of which can be eimulated by a spin system. An example is structural phase change phenomena $^{2}$ ).

In work of this nature it is most convenient - except in the simplest cases - to use tensor operators having general transformation properties, rather that to use the spin component operators. Tensor (Racah) operators, $\mathbf{R}_{\ell, m}$, transform under rotation of the frame of coordinators as

$$
\sqrt{\frac{4 \pi}{2 \ell+1}} Y_{\ell m}
$$

where $\mathrm{Y}_{\ell \mathrm{m}}$ is a spherical harmonic ${ }^{3)}$. The Stevens operators, $\mathrm{O}_{\ell}^{\mathrm{m}}$, are related to the tesseral harmonics ').

The rather tedious and lengthy calculation of products of these operators frequently occurs and for this reason we have compiled numerical tables of the most commonly met products. The products may occur in the calculation of time derivatives of tensor operators in systems in which the Hamiltonian is a sum of tensor operators $H=\underset{\ell^{\prime}, m^{\prime}}{\Sigma} a_{\ell^{\prime} m^{\prime}} R_{\ell^{\prime} m^{\prime}}$,

$$
\dot{\mathrm{R}}_{\ell \mathrm{m}}=\frac{1}{\mathrm{i} \mathrm{L}}\left[\mathrm{R}_{\ell \mathrm{m}} \cdot \sum_{\ell^{\prime} \mathrm{m}^{\prime}} \mathbf{a}_{\ell^{\prime} \mathrm{m}^{\prime}} \mathbf{R}_{\ell^{\prime} \mathrm{m}^{\prime}}\right]
$$

The products may also appear in the evaluation of thermal averages

$$
\operatorname{Tr}\left\{R_{\ell, m} \exp \left(-\beta \sum_{\ell^{\prime} m^{\prime}} a_{\ell^{\prime} m^{\prime}} R_{\ell^{\prime} m^{\prime}}\right)\right\}
$$

A number of properties of the tensor operators (rotations, expansions in angular momentum components and Bose operators and the relation between Racah and Stevens operators) was recently discussed in detail by Danielsen and Lindgard ${ }^{4}$ ), and we shall refer to these reports for details.

The present report gives the basic definitions in section 2 and examples on how and when to use the tables in section 3. Finally are presented the tables of the products, $R_{\ell m} R_{\ell^{\prime} m^{\prime}}$ and $O_{\ell}^{m}(c) O_{\ell^{\prime}}^{m^{\prime}}(c)$ and $O_{\ell}^{m}(s) O_{\ell^{\prime}}^{m^{\prime}(s)}$ for $\ell$ up to 6 , and the results for $\ell$ up 6 only. 


\section{DEFINITIONS}

\subsection{Tensor Operators}

The tensor (Racah) operators are defined by their transformation properties. Only non-commuting operators working within a J-multiplet are considered.

$R_{\ell m}$ transforms as $\sqrt{\frac{4 \pi}{2 l+1}} Y_{\ell m}$.

The tensor uperators $R_{\ell m}$ are often denoted $\tilde{\mathbf{O}}_{\ell m}$.

Tables in terms of angular momentum components are given in ref. 4. The simplest are for example

$$
\begin{aligned}
& R_{0,0}=1 \\
& R_{1,1}=-\frac{1}{\sqrt{2}} J^{+} \\
& R_{1,0}=J_{2} \\
& R_{1,-1}=+\frac{1}{\sqrt{2}} J^{-} .
\end{aligned}
$$

where

$$
\mathbf{J}^{ \pm} \quad=\mathbf{J}_{\mathbf{x}} \pm \mathbf{i} \mathbf{J}_{\mathbf{y}}
$$

The formula for the product of two non-commuting tensor operators was calculated by Danielsen and Lindgård ${ }^{4}$. In terms of $6-j$ and $3-j 8 y m-$ bols it is given as

$$
\begin{aligned}
& R_{\ell_{1} m_{1}} R_{\ell_{2} m_{2}}=\sum_{M=-L}^{L} \sum_{L=\left|l_{1}-l_{2}\right|}^{\ell_{1}+l_{2}}(-1)^{\ell_{1}+\ell_{2}+L} \\
& \cdot(2 L+1) \cdot f\left(l_{1} \ell_{2} L, J\right)\left(\sum_{m_{1} m_{2}-M}^{l_{1} l_{2} L}\right)(-1)^{M} R_{L M} \text {. }
\end{aligned}
$$

where

$$
f\left(\ell_{1} \ell_{2} L, J\right)=\left\{\begin{array}{l}
\ell_{1} \ell_{2} L \\
J
\end{array}\right\} \frac{\left\langle J|| R_{\ell_{1}}|| J\right\rangle\left\langle J|| R_{\ell_{2}} \| J\right\rangle}{\left\langle J\left\|R_{L}\right\| J\right\rangle} .
$$


The dependence of $\mathrm{J}$ is contained in $f\left(\ell_{1} \ell_{2} \mathrm{~L}, J\right) i$. e. in the $6-j$ symbol and the reduced matrix elements

$$
\left\langle J\left\|R_{\ell}\right\| J\right\rangle=\frac{1}{2^{\ell}} \sqrt{\frac{(2 J+\ell+1) !}{(2 J-\ell) !}} .
$$

Since all tensor operators can be expressed in terms of the length, $\sqrt{J(J+1)}$, and the components, $J_{2}$, of the angular momentum, $\vec{J}$, we can express $f\left(\ell_{1} \ell_{2} L, J\right)$ as a polynomial in $X=J(J+1)$. The coefficients to this polynomial are found numerically (double precision) by solving a system of linear equations for a number of values of $\mathrm{J}$. Having the polynomial expression for $f\left(\ell_{1} \ell_{2} L, J\right)$ it is simple to calculate the product. In order to reduce the volume of the tables we have only listed a number of products from which others can be obtained by using the following symmetry properties.

\section{2. Symmetry Properties}

Because of the invariance of the 6-j symbol with respect to interchange of columns we have

$$
f\left(\ell_{1} \ell_{2} L J\right)=f\left(\ell_{2} \ell_{1} L J\right)
$$

The 3-j symbol has the following symmetry properties ${ }^{4)}$.

$$
\left(\begin{array}{cc}
l_{1} & l_{2} L \\
m_{1} m_{2}-M
\end{array}\right)=(-1)^{l_{1}+l_{2}+L}\left(\begin{array}{c}
l_{2} l_{1} L \\
m_{2} m_{1}-M
\end{array}\right)=\left(\begin{array}{cc}
l_{2} l_{1} L \\
-m_{2}-m_{1} M
\end{array}\right) .
$$

Using these we can obtain from $R_{\ell_{1}} m_{1} R_{\ell_{2}} m_{2}$

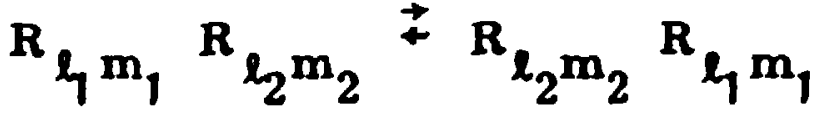

$$
\begin{aligned}
& \rightarrow R_{\ell_{1}},-m_{1}, R_{\ell_{2},}-m_{2}
\end{aligned}
$$

The commutator between two tensor operators is given by 


$$
\begin{aligned}
& \left.\left[R_{\ell_{1} m_{1}}, R_{\ell_{2} m_{2}}\right]=\sum_{M=-L}^{L} \sum_{\left|l_{1}-\ell_{2}\right|}^{L} i(-1)^{\ell_{1}+\ell_{2}+L}-1\right\} \\
& -(2 L+1) f\left(l_{1} \ell_{2} L, J\right)\left(_{m_{1} m_{2}-M}^{l_{1} \ell_{2}} L(-1)^{M} R_{L M} \cdot\right.
\end{aligned}
$$

The commutator is zero if $\ell_{1}+\ell_{2}+L$ is even.

\subsection{Stevens Operators}

The Stevens operators are reiated to the tensor operator's as follows

$$
\begin{aligned}
& O_{\ell}^{m}(c)=\frac{1}{K_{\ell}^{m}} \sqrt{\frac{2 \ell+1}{4 \pi}} \frac{1}{\sqrt{2}}\left(R_{\ell_{1}-m}+(-1)^{m} R_{\ell m}\right) \\
& O_{\ell}^{m}(s)=\frac{1}{K_{\ell}^{m}} \sqrt{\frac{2 \ell+1}{4 \pi}} \frac{1}{\sqrt{2}}\left(R_{\ell_{1}-m}-(-1)^{m} R_{\ell m}\right. \\
& O_{\ell}^{o}(c)=\frac{1}{K_{\ell}^{o}} \sqrt{\frac{2 \ell+1}{4 \pi}} R_{\ell O}, \quad O_{\ell}^{o}(s)=0,
\end{aligned}
$$

where $\mathrm{K}_{\ell}^{\mathrm{m}}$ are the normalization coefficients of the tesseral harmonies, which are specified in table 3, last page. The Stevens operators are labelled by $c$ or $s$ according to the cosine and sine character. The products of the Stevens operators can be obtained from those of the tensor operators. However, for convenience a number of Stevens operator products are given in table 2.

\section{APPLICATIONS AND EXAMPLES}

\subsection{Calculation of Commutation Relations and Equations of Motion}

Let us consider the simplest example, table 1:

$$
R_{11} R_{1,-1}-R_{1,-1} R_{11}=-R_{10}
$$

which, according to section 2 eq. (4), is the same as

$$
-\frac{1}{2}\left[\mathrm{~J}^{+}, \mathrm{J}^{-}\right]=-\mathrm{J}_{\mathrm{z}} \text {. }
$$


From table 2 we have

$$
o_{1}^{1}(c) o_{1}^{o}(c)-o_{1}^{o}(c) o_{1}^{1}(c)=-i o_{1}^{1}(s)
$$

which in angular momentum components is

$$
\left[J_{x}, J_{z}\right]=-i J_{y}
$$

From these, the commutation relation between higher order operators can be derived in a straight-forward, but tedious way. By using the tables and the symmetry relations (8), (9) and (10) this is greatly faciliated. In table $2 \mathrm{i}$ is denoted by $a$ ";".

Simply the commutator is twice the terms with odd $\ell$-sums.

Let us as an example consider a magnetic crystal with planar (xz) anisotropy described by the Hamiltonian ( 2 is the quantization axis)

$$
\begin{aligned}
H= & -\sum_{j, r} 2 \tilde{J}_{r} J_{j} \cdot J_{j+r}+D \sum_{j}\left(3 J_{y}^{2}-J(J+1)\right)_{j} \\
= & -\sum_{j, r} 2 \tilde{J}_{r}\left\{R_{10}^{j} R_{10}^{j+r}-R_{11}^{j} R_{1,-1}^{j+r}-R_{1,-1}^{j} R_{11}^{j+r}\right\} \\
& -\sum_{j} D\left\{R_{20}^{j}+\sqrt{\frac{3}{2}}\left(R_{22}^{j}+R_{2,-2}^{j}\right)\right\},
\end{aligned}
$$

where $\tilde{J}_{r}$ is the Heisenberg exchange interaction to the $r^{\prime}$ th neighbour and $D$ is the single ion anisotropy constant.

We shall use the tables to derive the equations of motion for $R_{11}$ and $R_{1,-1}$, which may be used in a Green's function theory for the excitation spectrum ${ }^{5)}$. For simplicity we note that $\sqrt{3} / 2=0.86603$ and $\sqrt{6} / 5=0.48990$ and find

$$
\text { is } \begin{aligned}
\dot{R}_{11}^{j}= & \sum_{r} 2 \tilde{J}_{r}\left\{R_{11}^{j} R_{10}^{j+r}-R_{10}^{j} R_{11}^{j+r}\right\} \\
& +D \sqrt{3} / 2\left\{R_{21}^{j}+R_{2,-1}^{j}\right\}
\end{aligned}
$$




$$
\begin{aligned}
\text { i h } \dot{R}_{1,-1}^{j}= & -\sum_{r} 2 \tilde{J}_{r}\left\{R_{1,-1}^{j} R_{10}^{j+r}-R_{10}^{j} R_{1,-1}^{j+r}\right\} \\
& -D \sqrt{3} / 2\left\{R_{21}^{j}+R_{2,-1}^{j}\right\}
\end{aligned}
$$

We notice that new tensor operators appear, i. e. $R_{10}$ and $R_{2,} \pm_{-1}$. The equations are usually solved by decoupling the higher order terms. We can use the tables to arrive at suitable forms to decouple. Consider the single ion anisotropy terms: using the multiplication table for $R_{11} R_{10}$ and $R_{10} R_{1,-1}$ we find the exact relation

\section{case 1:}

$$
\sqrt{3} / 2\left(R_{21}+R_{2,-1}\right)=\frac{3}{2}\left\{R_{11}\left(R_{10}-\frac{1}{2}\right)+\left(R_{10}-\frac{1}{2}\right) R_{1,-1}\right\} .
$$

Using this form we can decouple (21) and (22) by taking the average $\left\langle R_{10}\right\rangle=\left\langle J_{z}\right\rangle$. This corresponds to the random phase approximation. However, it is easy to find other exact relations. Using $R_{20} R_{11}$ and $R_{22} R_{1,-1}$ we find by eliminating $R_{31}$

\section{case 2:}

$$
\begin{gathered}
\sqrt{3 / 2}\left(R_{21}+R_{2,-1}\right)=\left\{R_{11}\left[R_{20}-\sqrt{6} R_{22}-(X-3 / 4)\right]\right. \\
\left.+\left[R_{20}-\sqrt{6} R_{2,-2}-(X-3 / 4)\right] R_{1,-1}\right\} .
\end{gathered}
$$

In this form we can decouple (21) and (22) in terms of $\left\langle R_{10}\right\rangle,\left\langle R_{20}\right\rangle$ and $\operatorname{Re}\left(R_{2, \pm 2}\right)$, where

$$
\left\langle R_{20}\right\rangle=\frac{1}{2}\left(3\left\langle J_{z}^{2}\right\rangle-X\right) \text { and } \sqrt{6} \operatorname{Re}\left\langle R_{2, \pm_{2}}\right\rangle=\frac{3}{2}\left\langle J^{+2}+J^{-2}\right\rangle / 2 \text {. }
$$

It is clear that in both forms the influence of the anisotropy vanishes, as it should for $\mathrm{J}=1 / 2$.

Using the standard technique for diagonalizing (21), (22) by Fourier transformation we find the excitation spectrum in the two approximations (one atom per unit cell, ferromagnet with planar anisotropy):

$$
b \omega_{q}=2\left\langle J_{z}\right\rangle \sqrt{\left(\tilde{J}_{o}-\tilde{J}_{q}\right)\left(\tilde{J}_{0}-\tilde{J}_{q}+\frac{3}{2} D f(T)\right)}
$$


where in the random phase approximation (23)

case 1:

$$
f(T)=\left\{\left\langle J_{z}\right\rangle-1 / 2\right\} /\left\langle J_{z}\right\rangle .
$$

and in the second order approximation (24) and (25)

case 2:

$$
f(T)=\left\{\left\langle J_{z}^{2}\right\rangle-\left\langle J^{+2}+J^{-2}\right\rangle / 2-\frac{4}{3} x+\frac{3}{4}\right\} /\left\langle J_{z}\right\rangle .
$$

This completes our example. Other cases can be treated similarly.

\subsection{Thermodynamic Averages}

Dalton and Rimmer ${ }^{6)}$ compiled tables of traces of spin operators and listed a number of examples for which they could be used. The present work has very much the same area of applicability - in fact in most cases it is preferable to use tensor operators rather than spin operators.

In general, we have the partition function for a system described by the Hamiltonian $\mathbf{H}^{7}$ )

$$
\begin{aligned}
& H=\sum_{\ell, m} a_{\ell m}{ }^{R} l m \\
& Z=\operatorname{Tr}\left\{e^{-\beta H}\right\}, \quad \beta=\frac{1}{k_{B} T} .
\end{aligned}
$$

Any thermodynamic function can be obtained from $\mathrm{Z}$ by differentiation. The thermodynamic average of an operator $R_{\ell m}$ is then

$$
\left\langle R_{\ell m}\right\rangle=\operatorname{Tr}\left\{R_{\ell m} e^{-\beta H}\right\} / Z \text {. }
$$

which may be evaluated at high temperatures by expanding expanding exp(- $\beta \mathrm{H})$. The tables can then be used to reduce the products, of operators. Another example is the calculation of correlation functions

$$
\left\langle R_{\ell m} R_{\ell ' m^{\prime}}\right\rangle \text {. }
$$

which can be reduced to single operator averages. These often occur in the derivatives of $Z$, say $d / d a m^{Z}$. 
In general the expectation value of a tensor operator can be evaluated using the expression for the matrix element within the $\mathbf{J}$-multiplet:

$$
\left\langle J, M_{1}\left|R_{\ell m}\right| J, m_{2}\right\rangle=(-1)^{J-m_{1}}\left(\begin{array}{ccc}
J & J \\
-m_{1} & m_{m_{2}}
\end{array}\right)\left\langle J|| R_{\ell}|| J\right\rangle .
$$

\section{REFERENCES}

1) K. W. H. Stevens, in: Magnetism, Edited by G. T. Rado and H. Suhl, Vol. 1 (Academic Press, New York, 1973) 1-23.

2) K. A. Maller, In: Structural Phase Transitions and Soft Modes, Proceedings of the NATO Advanced Study Institute at Geilo, 13-20 April 1971. Edited by E.J. Samuelsen, Eigil Andersen and Jens Feder (Universitetsforlaget, Oslo, 1971 ) 61 -96.

3) A. R. Edmonds, Angular Momentum in Quantum Mechanics (Princeton University Press, Princeton, N. J. 1957) 146 pp.

4) O. Danielsen and P. -A. Lindgărd, Risø Report No. 259 (1 972) 93 pp. and P. - A. Lindgård and $O$. Danielsen, J. Phys. C. I (1 974) 1523-35 and O. Danielsen, Risø Report No. 295 (1974) 228 pp.

5) D. N. Zubarev, Sov. Phys. Uspekhi, 3, (1 960) 320-345.

6) N. W. Dalton and D. E. Rimmer, AERE-R-5958 (1 968) 248 pp.

7) T. L. Hill, Statistical Mechanics (McGraw-Hill, New York), 1956,432 pp. 


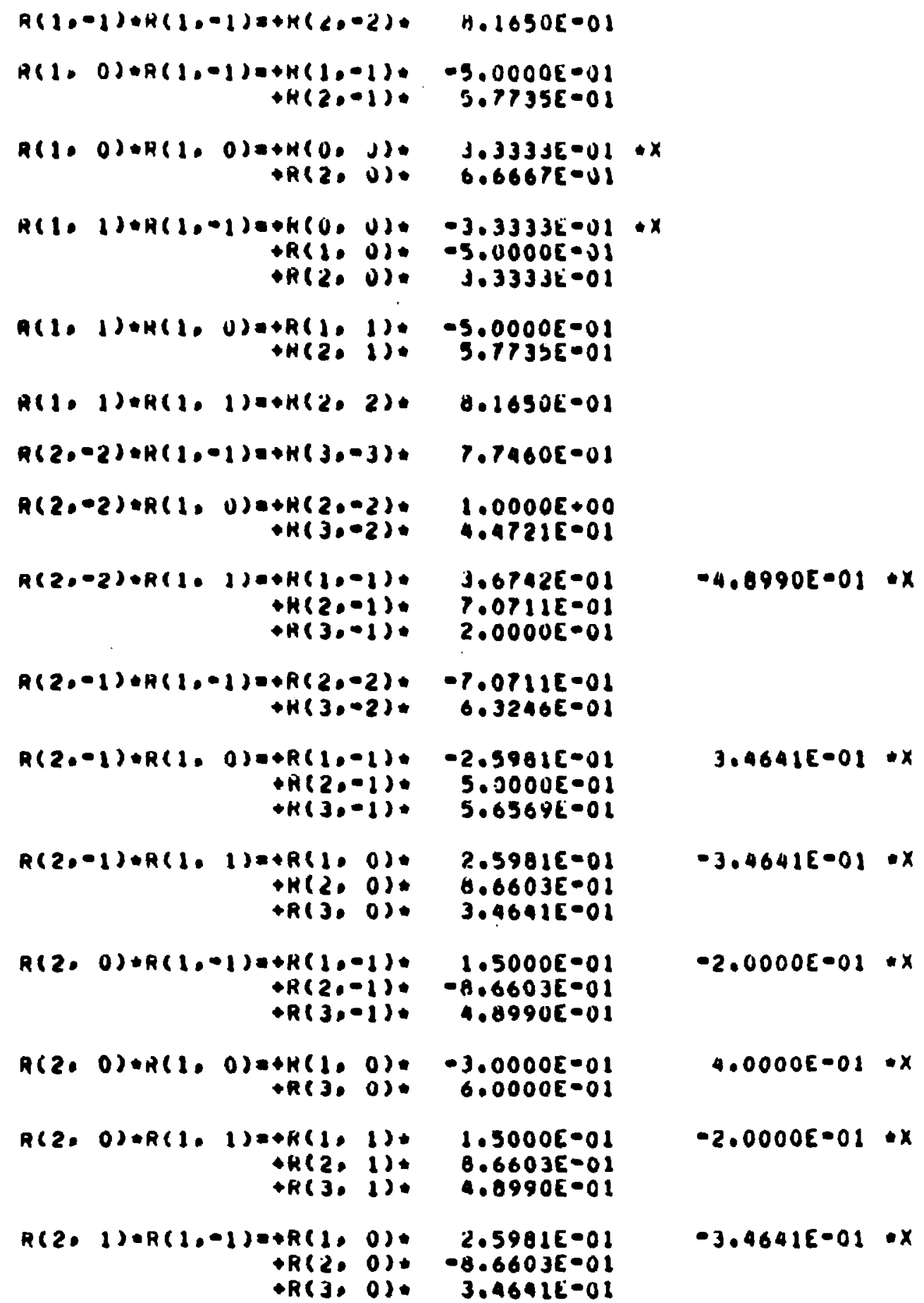

$-4.8990 E-01 \cdot x$

$3.46418-01+x$

$-3.4641 E-01 \cdot x$

$-2.0000 E-01 * x$

$4.0000 E-01 \cdot x$

$-2.0000 E=01 \cdot x$

$-3.4641 E-01 \cdot x$ 


\begin{tabular}{|c|c|c|c|c|c|c|c|}
\hline $4<2$ & $2 J \cdot R(10$ & $\begin{aligned}0)= & +H(1) \\
& +R(20 \\
& +R(3)\end{aligned}$ & $\begin{array}{l}\text { 1): } \\
\text { 1): } \\
\text { 1): }\end{array}$ & $\begin{array}{r}-2.5901 E=01 \\
-5.0000 E-01 \\
5.656 y E-01\end{array}$ & & $3.4641 E-01$ & $\cdot x$ \\
\hline$R<2$. & $1) \cdot R(1$. & $\begin{array}{r}1)=+R(20 \\
\\
P R C 3\end{array}$ & 2): & $\begin{array}{l}7.07115=01 \\
6.3246 E-01\end{array}$ & & & \\
\hline A 2 . & $2)+R(1$, & $\begin{aligned}-1)= & +H(1) \\
& +R(20 \\
& +R(10\end{aligned}$ & $\begin{array}{l}\text { 1): } \\
\text { 1): } \\
\text { i): }\end{array}$ & $\begin{array}{r}3.6742 E-01 \\
-7.0711 E=01 \\
2.000 E=01\end{array}$ & & $-4.0990 E-01$ & $\bullet x$ \\
\hline R(2. & $2) * R(1$ & $\begin{aligned}0)= & +R(2, \\
& \mapsto R(3)\end{aligned}$ & 2): & $\begin{array}{r}-1.0000 E+00 \\
4.4721 E=01\end{array}$ & & & \\
\hline$R(2)$ & $2)=R(1$. & $1)=+R(3$ & $3)$ & $1.7460 E-01$ & & & \\
\hline$R(2)$. & $-2) \cdot R(2$. & $-2) \cdot H(4 .-$ & $-4)$ & $7.1714 E-01$ & & & \\
\hline R120 & $-1) \cdot R(2$, & $\begin{aligned}-2)= & +R(j, 0 \\
& +R(4,-\end{aligned}$ & -3): & $\begin{array}{r}-1.3416 E+00 \\
5.0704 E-01\end{array}$ & & & \\
\hline A620. & $-1) \cdot R(20$ & $\begin{aligned}-1)= & +R(2, P \\
& +K(40-\theta\end{aligned}$ & $\begin{array}{l}-2) \\
-2)\end{array}$ & $\begin{array}{r}-1.3122 E+00 \\
5.4210 E-01\end{array}$ & & $3.49935-01$ & $\cdot x$ \\
\hline Ac2. & $0) \cdot R(2$. & $\begin{aligned}-7)= & +R(20- \\
& +R(3)= \\
& +R(40-\end{aligned}$ & $\begin{array}{l}-2) \\
-22 \\
-23\end{array}$ & $\begin{array}{r}1.0714 E+00 \\
-1.3416 E+00 \\
3.3197 E=01\end{array}$ & & $-2.0571 E-01$ & $\cdot x$ \\
\hline R 2 (2. & $O) \circ A(20$ & $\begin{aligned}-1)= & +R(1.0 \\
& +H(2,0 \\
& +H(30-0 \\
& +H(4.0\end{aligned}$ & $\begin{array}{l}\text { 1): } \\
\text { 1): } \\
\text { 1): } \\
\text { 1) }\end{array}$ & $\begin{array}{r}3.8971 E=01 \\
-5.3571 E=01 \\
-6.4053 E=01 \\
4.6948 t=01\end{array}$ & & $\begin{array}{r}-5.1962 E-01 \\
1.4286 E-01\end{array}$ & $: x$ \\
\hline Q12० & $O) \cdot R(2$. & $\begin{aligned}O)= & +R\left(0_{0}\right. \\
& +H\left(22^{\circ}\right. \\
& +F(4)^{\circ}\end{aligned}$ & $\begin{array}{l}0): \\
0): \\
01:\end{array}$ & $\begin{array}{r}-1.5000 E=01 \\
-1.0714 E+00 \\
5.1124 E-01\end{array}$ & $\cdot x$ & $\begin{array}{l}2.0000 E-01 \\
2.85 T 1 E-01\end{array}$ & $\cdot x 2$ \\
\hline A<2. & $1) \cdot A(20$ & $\begin{aligned}-2)= & +H(1.0 \\
& +H(2,0 \\
& +H(3)- \\
& +R(4 .-\end{aligned}$ & $\begin{array}{l}-11): \\
-1) \\
-1) \\
-1)\end{array}$ & $\begin{array}{r}-3.1020 c-01 \\
1.31222+00 \\
-1.03922+00 \\
1.91602-01\end{array}$ & & $\begin{array}{r}1.2120 E=01 \\
-3.4993 E-01\end{array}$ & $: x$ \\
\hline A 120 & 1) $0 R(20$ & 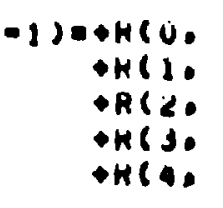 & 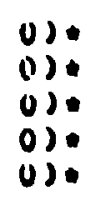 & $\begin{array}{r}1.5000 E-01 \\
2.2500 E-01 \\
5.35716-01 \\
-1.20006+00 \\
3.0200 k=01\end{array}$ & $\bullet x$ & $\begin{array}{l}-2,0000 E-01 \\
-3.0000 E=01 \\
-1.4286 E=01\end{array}$ & $\begin{array}{l}: x 2 \\
: x \\
: x\end{array}$ \\
\hline$R<2$ & H $H(2$. & 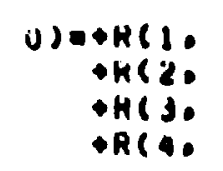 & $\begin{array}{l}\text { 1): } \\
\text { 1): } \\
\text { 1): } \\
\text { 1): }\end{array}$ & $\begin{array}{r}3.09716-01 \\
-5.35716-01 \\
-4.04536-01 \\
4.69406-01\end{array}$ & & $\begin{array}{r}-5.1962 E-01 \\
1.42 n 6 E-01\end{array}$ & $: x$ \\
\hline
\end{tabular}




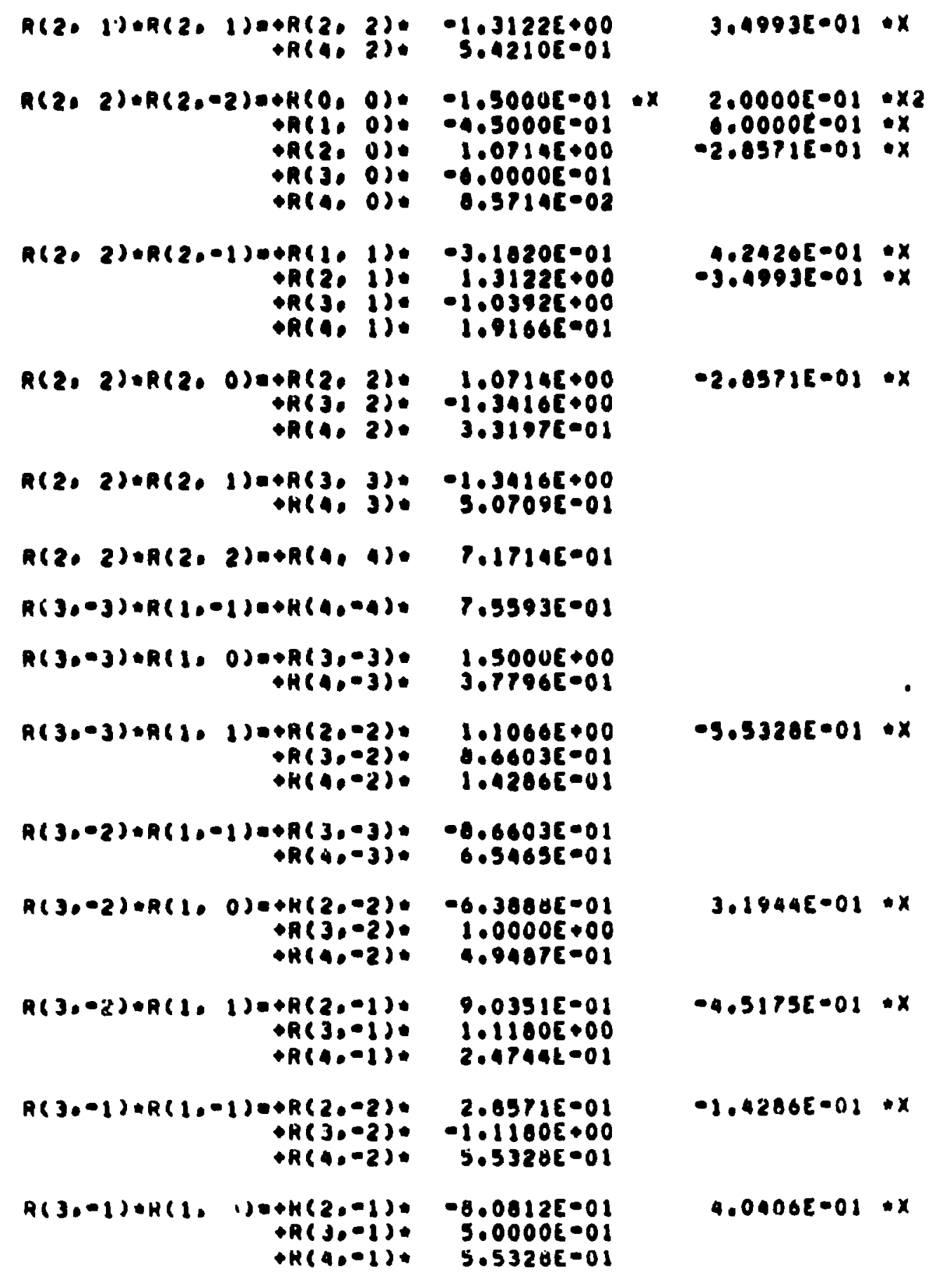




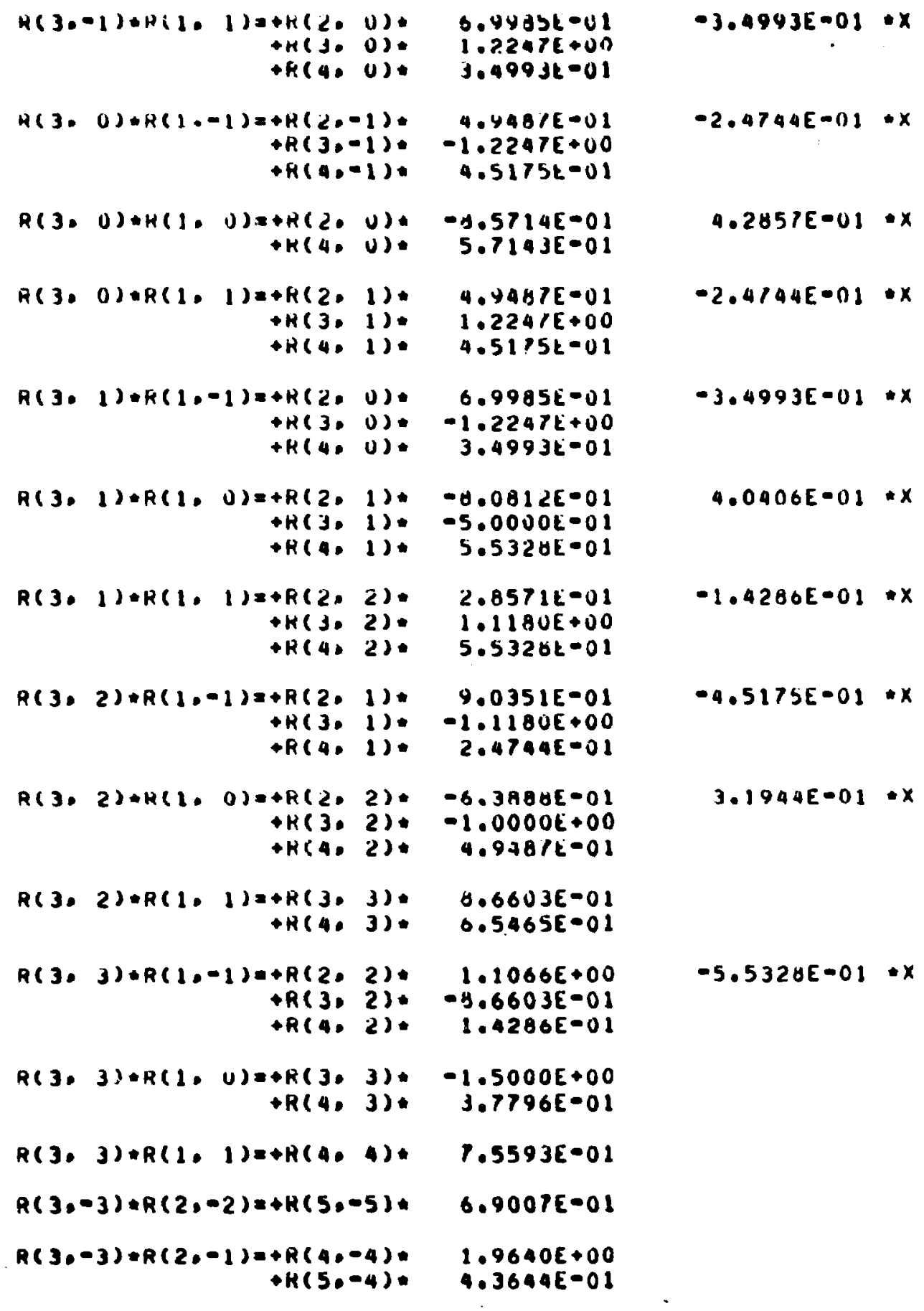




\begin{tabular}{|c|c|c|c|c|c|c|}
\hline$R(3,-3) * R(2$, & $\begin{aligned} 02= & +R(3,-3) \\
& +R(4,-3) \\
& +R(5,-3)\end{aligned}$ & $\begin{array}{l}2.7500 E+00 \\
1.7008 E+00 \\
2.5198 E=01\end{array}$ & $-3.3333 E-01$ & $x$ & & \\
\hline$R(3,-3) \cdot R(2$, & (1)= $\begin{aligned} &+R(2,-2)= \\
&+R(3,-2) \\
&+R(4,-2) \\
&+R(5,-2) *\end{aligned}$ & $\begin{array}{l}1.9166 E+00 \\
2.7500 E+00 \\
1.1135 E+00 \\
1.2599 E-01\end{array}$ & $\begin{array}{l}-9.5831 E=01 \\
-3.3333 E-01\end{array}$ & $\begin{array}{l}* x \\
x\end{array}$ & & \\
\hline$R(3,-3) \backsim R(2$, & (2)= $\begin{aligned} & +R(1,-1): \\
& +R(2,-1): \\
& +R(3,-1) \\
& +R(4,-1): \\
& +R(5,-1):\end{aligned}$ & $\begin{array}{l}1.9796 E-01 \\
1.9166 E+00 \\
1.7393 E+00 \\
5.2489 E-01 \\
4.7619 E-02\end{array}$ & $\begin{array}{l}-9.1292 E-01 \\
-9.5831 E-01 \\
-2.1082 E-01\end{array}$ & $\begin{array}{l}x \\
: x \\
: x\end{array}$ & $3.3197 E-01$ & $\star \times 2$ \\
\hline$R(3,-2)=R(2$ & $\begin{aligned}-2)= & +R(4,-4) \\
& +R(5,-4)\end{aligned}$ & $\begin{array}{r}-1.6036 E+00 \\
5.3452 E-01\end{array}$ & & & & \\
\hline$R(3 .-2)=R(2)$ & $\begin{aligned}-1)= & +R(3,-3) \\
& +R(4,-3): \\
& +R(5,-3)\end{aligned}$ & $\begin{array}{r}-2.7500 E+00 \\
5.6695 E-01 \\
5.0395 E-01\end{array}$ & $3.3333 E-01$ & $\bullet x$ & & \\
\hline$R(3,-2) \bullet R(2$, & (0)= $\begin{aligned} & +R(2,-2) \\
& +R(4,-2) \\
& +R(5,-2)\end{aligned}$ & $\begin{array}{r}-1.9166 E+00 \\
1.4846 E+00 \\
3.7796 E-01\end{array}$ & $9.5831 E=01$ & $x$ & & \\
\hline$R(3,-2)=R(2$, & $\begin{aligned}1)= & +R(1,-1): \\
& +R(3,-1) \\
& +R(4,-1) \\
& +R(5,-1)\end{aligned}$ & $\begin{array}{r}-4.0650 E-01 \\
2.1301 E+00 \\
1.5000 E+00 \\
2.3320 E-01\end{array}$ & $\begin{array}{r}7 \cdot 4539 E=01 \\
-2.5820 E=01\end{array}$ & $: x$ & $-2.7105 E-01$ & $\pm \times 2$ \\
\hline$R(3,-2) * R(2$, & $\begin{aligned}2)= & +R(1,0): \\
& +R(2,0): \\
& +R(3 \circ 0): \\
& +R(1,0): \\
& +R(5,0):\end{aligned}$ & $\begin{array}{l}2.0749 E-01 \\
1.9166 E+00 \\
2.4597 E+00 \\
9.5831 E-01 \\
1.0648 E-01\end{array}$ & $\begin{array}{l}-5.2707 E-01 \\
-9.5031 E-01 \\
-2.9014 E-01\end{array}$ & $\begin{array}{l}: x \\
: x \\
x\end{array}$ & $1.9166 E \cdot 01$ & $1 \times 2$ \\
\hline$R(3,-1) * R(2$ & $\begin{aligned}-2)= & +R(3,-3) \\
& +R(4,-3) \\
& +R(5,-3)\end{aligned}$ & $\begin{array}{r}1.7393 E+00 \\
-1.7928 E+00 \\
3.90415-01\end{array}$ & $-2.1082 E-01$ & $\cdot x$ & & \\
\hline$R(3,-1) \bullet R(2$, & $\begin{aligned}-1)= & +R(2,-2) \\
& +R(3,-2) \\
& +R(4,-2) \\
& +R(5,-2)\end{aligned}$ & $\begin{array}{r}1.4046 E+00 \\
-2.1301 E+00 \\
-4.7916 E=01 \\
4.8795 E-01\end{array}$ & $\begin{array}{r}-7 \cdot 4231 E-01 \\
2.5820 E-01\end{array}$ & $: x$ & & \\
\hline$R(3,-1) \cup R(2$. & $\begin{aligned}0)= & +R(1,-1): \\
& +R(2,-1): \\
& +R(3,-1): \\
& +R(1,-1) \\
& +R(5,-1)\end{aligned}$ & $\begin{array}{r}3.1493 E-01 \\
-1.2122 E+00 \\
-1.6500 E+00 \\
0.2993 E-01 \\
0.5175 E=01\end{array}$ & $\begin{array}{r}-5.77380=01 \\
6.0609 E-01 \\
2.0000 E-01\end{array}$ & $x$ & $2.0996 E-01$ &.$\times 2$ \\
\hline
\end{tabular}




\begin{tabular}{|c|c|c|c|c|c|c|c|}
\hline$R(3,-1) \bullet R(2$. & $\begin{aligned}1)= & +R(1): \\
& +R(20) \\
& +R(30) \\
& +R(40) \\
& +R(5)\end{aligned}$ & $\begin{array}{l}0): \\
\text { 0): } \\
0): \\
0): \\
03:\end{array}$ & $\begin{array}{r}-3.6365 E-01 \\
-1.2122 E+00 \\
7.77022-01 \\
1.3152 t+00 \\
3.3672 t-01\end{array}$ & $\begin{array}{r}6.6670 E-01 \\
6.0609 E-01 \\
-9.4281 E-02\end{array}$ & $\begin{array}{l}x \\
: x \\
x\end{array}$ & $-2.4244 E-01$ & $1 \times 2$ \\
\hline$R(3 .-1) \cdot R(2$. & $\begin{aligned}2)= & +H(10) \\
& +R(20) \\
& +H(30) \\
& +R(4) \\
& +R(5)\end{aligned}$ & $\begin{array}{l}\text { (1): } \\
\text { b): } \\
\text { i): } \\
\text { 1): } \\
\text { 1): }\end{array}$ & $\begin{array}{l}1.2857 t-01 \\
1.4840 E+00 \\
2.6944 t+00 \\
1.3553 t+00 \\
1.0443 t-01\end{array}$ & $\begin{array}{l}-2,3571 E-01 \\
-7.4231 E-01 \\
-3.2660 E-01\end{array}$ & $\begin{array}{l}: x \\
* x \\
* x\end{array}$ & $8.5714 E=02$ & $-\times 2$ \\
\hline$R(3,0)=R(2,-$ & $\begin{aligned}-2)= & +R(2,-= \\
& +H(3,- \\
& +R(4,- \\
& +R(5,-\infty\end{aligned}$ & $\begin{array}{l}-2): \\
-23: \\
-21: \\
-23 .\end{array}$ & $\begin{array}{r}-0.5714 E-01 \\
2.45976+00 \\
-1.6599 t+00 \\
2.8172 t-01\end{array}$ & $\begin{array}{r}4.2657 E-01 \\
-2.9014 E-01\end{array}$ & $: x$ & & \\
\hline$R(3,0) * R(2$, & $\begin{aligned}-1)= & +R(1,1,- \\
& +R(2,=- \\
& +R(3,-= \\
& +R(4,- \\
& +K(5,-=\end{aligned}$ & $\begin{array}{l}\text { 1) } \\
\text { (1): } \\
\text { 1): } \\
\text { 1): } \\
\text { 1) } \\
\text { (1): }\end{array}$ & $\begin{array}{r}-2.2264 E-01 \\
1.71411+06 \\
-7.7702 E-01 \\
-1.1737 E+00 \\
4.2592 E-01\end{array}$ & $\begin{array}{r}4.0827 E-01 \\
-8.5714 E-01 \\
9.4201 E-02\end{array}$ & $\begin{array}{l}* x \\
* x \\
x\end{array}$ & $-1.4846 E=01$ &.$\times 2$ \\
\hline$H(3, O) * H(2$, & $\begin{aligned} \text { O) } & +R(1) \\
& +R(3: \\
& +K(S,\end{aligned}$ & $\begin{array}{l}\text { u): } \\
\text { u): } \\
\text { u): }\end{array}$ & $\begin{array}{r}3.8571 E-U 1 \\
-2.200 U E+U 0 \\
4.1019 E=01\end{array}$ & $\begin{array}{r}-7.0714 E=01 \\
2.6667 E-01\end{array}$ & $: x$ & $2.5714 E-01$ & $-\times 2$ \\
\hline$R(3,0) \cdot H(2$. & 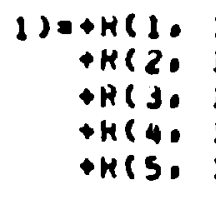 & $\begin{array}{l}\text { 1): } \\
\text { 1): } \\
\text { 1): } \\
\text { 1): } \\
\text { 1): }\end{array}$ & $\begin{array}{r}-2.2269 E-J 1 \\
-1.71436+00 \\
-7.77826-U 1 \\
1.1737 t+00 \\
4.25922-U 1\end{array}$ & $\begin{array}{l}4.0827 E-01 \\
0.5714 E-01 \\
Y .4281 E-02\end{array}$ & $\begin{array}{l}: x \\
: x \\
: x\end{array}$ & $-1.4846 E-01$ & $+\times 2$ \\
\hline$Q 13.0) \cdot R(2$. & $\begin{aligned}2) & +R(20: \\
& +H(30 \\
& +R(40 \\
& +R(5)\end{aligned}$ & $\begin{array}{l}25: \\
23: \\
25: \\
25=\end{array}$ & $\begin{array}{l}0.5714 E-01 \\
2.05976+00 \\
1.65946+00 \\
2.81726-01\end{array}$ & $\begin{array}{l}-4.2857 E-01 \\
-2.9814 E-01\end{array}$ & $\because x$ & & \\
\hline$R(3,1) \cdot R(2$. & 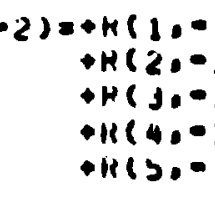 & $\begin{array}{l}\text { (1): } \\
\text { (1): } \\
\text { 1): } \\
\text { 1): } \\
\text { 1): }\end{array}$ & $\begin{array}{r}1.2 A 51 E-01 \\
-1.4646 E+00 \\
2.0444 E+00 \\
-1.3553 L+00 \\
1.044 J E-U 1\end{array}$ & $\begin{array}{r}-2.3571 E-01 \\
7.4231 E-01 \\
-3.2660 E-01\end{array}$ & $\begin{array}{l}: x \\
: x \\
: x\end{array}$ & $0.5714 E-02$ &.$\times 2$ \\
\hline$A(3,1) \cdot H(2$ & $\begin{array}{r}1)=+H(10) \\
+H(20) \\
+H(30 \\
+H(4) \\
+K(5)\end{array}$ & $\begin{array}{l}\text { u): } \\
\text { u): } \\
\text { u): } \\
\text { u): } \\
\text { u): }\end{array}$ & $\begin{array}{r}-3.6365 E=01 \\
1.2122 t+00 \\
7.7792 t-01 \\
-1.3152 t=00 \\
3.3672 t=01\end{array}$ & $\begin{array}{l}6.667 \cup E=01 \\
-6.060 \text { YE-UI } \\
-9.42 A 1 E-02\end{array}$ & $\begin{array}{l}* x \\
\bullet x \\
: x\end{array}$ & $-2.4244 E-01$ & $-\times 2$ \\
\hline
\end{tabular}




\begin{tabular}{|c|c|c|c|c|c|c|c|c|}
\hline Reso & $1) \cdot R(20$ & 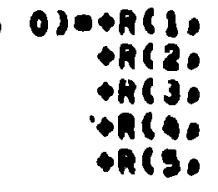 & $\begin{array}{l}\text { j): } \\
\text { ij: } \\
\text { j): } \\
\text { ij: }\end{array}$ & $\begin{array}{r}3.1493 E-01 \\
1.21222+00 \\
-1.05006+00 \\
-8.29935-01 \\
1.5175 E=01\end{array}$ & $\begin{array}{c}-5.17300-01 \\
-6.06006-01 \\
2.00006-01 \\
.\end{array}$ & $\begin{array}{ll}\bullet x \\
0 x \\
0 x\end{array}$ & $2.0996 t-01$ & $\cdot \times 2$ \\
\hline neso & 13012 & 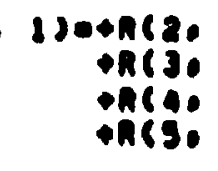 & $\begin{array}{l}25: \\
83: \\
83: \\
850\end{array}$ & $\begin{array}{r}-1.40405+00 \\
-2.13015+00 \\
4.89166001 \\
4.07955001\end{array}$ & $\begin{array}{l}7.42316=01 \\
2.5820[-01\end{array}$ & $\ddot{0 x}$ & & \\
\hline ne3o & $12+128$ & 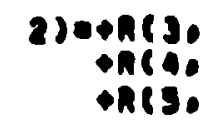 & 3): & $\begin{array}{l}1.73936+00 \\
1.79886+00 \\
3.90416-01\end{array}$ & $-2.10025 \cdot 01$ & $\cdot x$ & & \\
\hline acso & $2 d \bullet A<2$ & 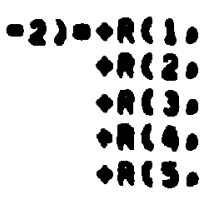 & $\begin{array}{l}0 \text { 8): } \\
\text { oj: } \\
\text { 03: } \\
\text { 0j: } \\
\text { 0): }\end{array}$ & 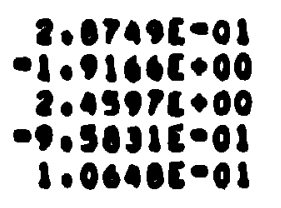 & $\begin{array}{r}-5.2707 c-01 \\
9.90316001 \\
-2.90146-02\end{array}$ & $\begin{array}{l}: x \\
: x \\
: x\end{array}$ & $1.01005-01$ & $\cdot \times 2$ \\
\hline acose & $\begin{array}{c}\text { R) } \\
.\end{array}$ & 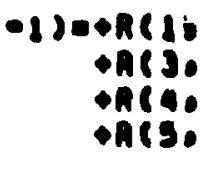 & $\begin{array}{l}\text { 1): } \\
\text { ij: } \\
\text { ij: } \\
\text { ij: }\end{array}$ & $\begin{array}{r}-1.06508-01 \\
2.13016 \bullet 00 \\
-1.50008+00 \\
2.33206-01\end{array}$ & $\begin{array}{r}7.45385-01 \\
-2.5820[-01\end{array}$ & $\because x$ & $-2.7105 t \cdot 01$ & $\cdot \times 2$ \\
\hline ac3. & $2) \cdot n(20$ & $\begin{array}{r}0)++R(2, \\
+R(4) \\
+R(s,\end{array}$ & $\begin{array}{l}2) \\
23:\end{array}$ & $\begin{array}{r}1.91606+00 \\
-1.40465+00 \\
3.77965-01\end{array}$ & $-9.50312-01$ & $\bullet x$ & & \\
\hline neso & $28 \cdot n(2$. & 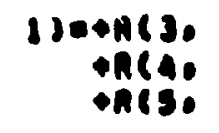 & 3:0 & $\begin{array}{l}-2.7300 c+00 \\
-5.86955-01 \\
5.0395 t-01\end{array}$ & $3,33336-01$ & $\bullet x$ & & \\
\hline Ac3o & $2) \cdot n(20$ & $2)=+h(4)$ & 30 & $\begin{array}{l}1.00306+00 \\
5.3452 c-01\end{array}$ & & & & \\
\hline 2630 & $3) \cdot A(2$ & 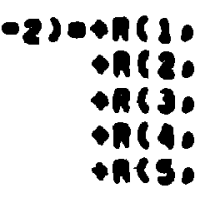 & $\begin{array}{l}130 \\
130 \\
i j \\
i j \\
130\end{array}$ & 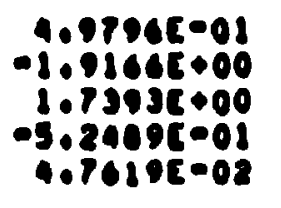 & $\begin{array}{r}-9.12925-01 \\
9.59318-01 \\
-2.10026-01\end{array}$ & $\begin{array}{l}: x \\
: x \\
* x\end{array}$ & $3.31876-01$ & $-\times 2$ \\
\hline arose & $330 R 62$ & 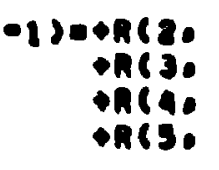 & $\begin{array}{l}250 \\
2 j: \\
230 \\
250\end{array}$ & $\begin{array}{r}-1.91006+00 \\
2.75005+00 \\
-1.1135 E+00 \\
1.2590+01\end{array}$ & $\begin{array}{r}9.50316-08 \\
-3.33936-08\end{array}$ & $: x$ & & \\
\hline nes. & $3) \cdot A 120$ & 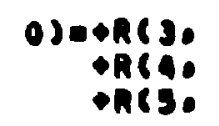 & $\begin{array}{l}\text { 3): } \\
\text { 3:० }\end{array}$ & $\begin{array}{r}2,75005 \bullet 00 \\
-1.70005+00 \\
2.51905-01\end{array}$ & $-3.33336 \cdot 01$ & $\cdot x$ & & \\
\hline
\end{tabular}




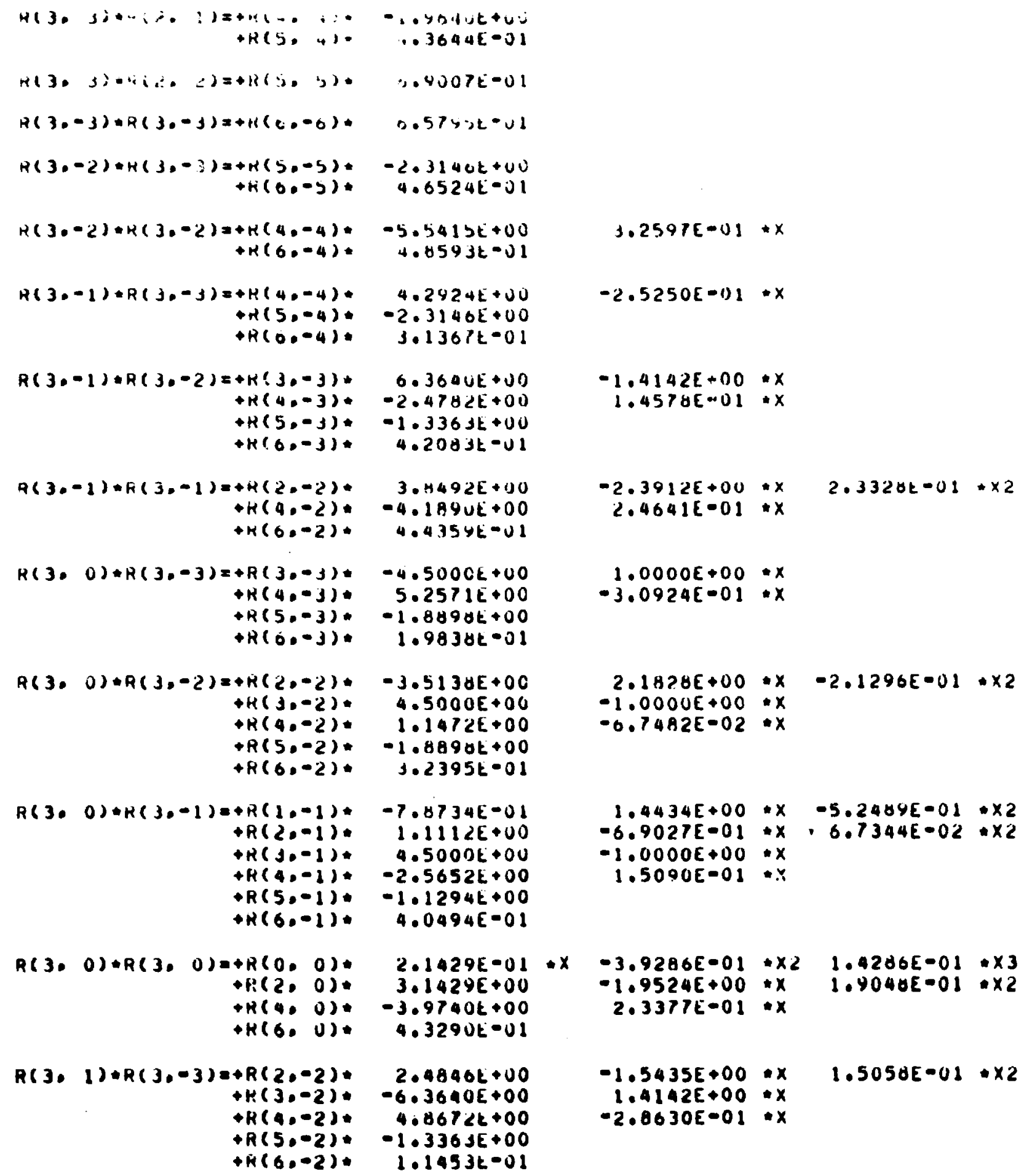

$x-3.9286 E-01 * \times 2 \quad 1.4206 E-01 * \times 3$ $-1.9524 E+00: x \quad 1.4040 E-01: \times 2$ $2.3377 E-01: x$

$-1.5435 E+00 * x \quad 1.5058 E-01 * \times 2$ $1.41+2 E+00 \div x$ $-2.0630 E-01 \cdot x$ 


\begin{tabular}{|c|c|c|c|c|c|c|c|c|c|}
\hline N13. & $1)+\cdots 1,0$ & $\begin{aligned}A)= & +11(1) \\
& +R(20) \\
& +H(4)= \\
& +H(50)= \\
& +\pi(6)=\end{aligned}$ & $\begin{array}{l}-1) \\
\text {-1): } \\
\text {-1): } \\
\text {-1): } \\
\text {-1): }\end{array}$ & $\begin{array}{r}1.1+7+k-U 1 \\
-3.0431 E+0 U \\
3.7467 L+00 \\
-1.14550 E+00 \\
2.2100 E=01\end{array}$ & & $\begin{array}{r}-1.3171 E+U U \\
1.8904 E+U U \\
-2.2040 E-01\end{array}$ & $\begin{array}{l}: x \\
: x \\
: x\end{array}$ & $\begin{array}{r}4.1910 E-01 \\
-1.0443 E-01\end{array}$ & $\begin{array}{l}: \times 2 \\
\times 2\end{array}$ \\
\hline 4130 & $1)+7(30-$ & 1) $\begin{aligned} & +H(0) \\
& +K(1) \\
& +R C 20 \\
& +H(3) \\
& +H(4) \\
& +R(5) \\
& +R<0 .\end{aligned}$ & $\begin{array}{l}0 \text { ): } \\
0 \text { ): } \\
\text { 0): } \\
0 \text { ): } \\
\text { 0): } \\
0 \text { ): } \\
\text { 0): }\end{array}$ & $\begin{array}{r}-2.142 Y E-01 \\
-3.2143 E-U 1 \\
-2.3511 E+0 U \\
4.3000 E+00 \\
0.6234 E-01 \\
-1.7857 E+00 \\
1.2468 E-01\end{array}$ & $\pitchfork x$ & $\begin{array}{r}3.9266 E=01 \\
5.8929 E-01 \\
1.9643 E+00 \\
-1.0000 E+00 \\
-3.8961 E=02\end{array}$ & $\begin{array}{l}: x< \\
: x \\
: x \\
: x \\
x\end{array}$ & $\begin{array}{l}-1.4206 E=01 \\
-2.1424 E=01 \\
-1.4206 \mathrm{E}=01\end{array}$ & $\begin{array}{l}1 \times 3 \\
\times 12 \\
02\end{array}$ \\
\hline R०3. & $1)=H(3$, & $\begin{aligned}0)= & +R(1) \\
& +R(2) \\
& +R(3) \\
& +R(4) \\
& +H(5) \\
& +K(0 .\end{aligned}$ & $\begin{array}{l}\text { 1): } \\
\text { 1): } \\
\text { ()) } \\
\text { 1): } \\
\text { 1): } \\
\text { 1): }\end{array}$ & $\begin{array}{r}-7.8734 E-01 \\
1.1112 E+00 \\
4.5000 E+00 \\
-2.5652 E+00 \\
-1.1294 E+00 \\
4.0494 E-01\end{array}$ & & $\begin{array}{r}1.4434 E+00 \\
-6.9027 E-01 \\
-1.0000 E+00 \\
1.5090 E=01\end{array}$ & $\begin{array}{l}x \\
: x \\
: x \\
: x\end{array}$ & $\begin{array}{r}-5.248 Y E=01 \\
6.7344 E=02\end{array}$ & $\begin{array}{l}: \times 2 \\
\times 2\end{array}$ \\
\hline$R<3$. & $1) \cdot R(3$. & $\begin{aligned}l)= & +R(2 .) \\
& +K(4 .) \\
& +H(6 .)\end{aligned}$ & $\begin{array}{l}2): \\
23: \\
23:\end{array}$ & $\begin{array}{r}3.8492 E+00 \\
-4.1890 E+00 \\
4.435 \times E-01\end{array}$ & & $\begin{array}{r}-2.3912 E+00 \\
2.4641 E-01\end{array}$ & $: x$ & $2.3320 E=01$ & $1 \times 2$ \\
\hline$R<3$. & $2) \div R(3$. & $\begin{aligned}3)= & +R(1 .: \\
& +H(2: 0 \\
& +R(3)= \\
& +R(4)= \\
& +R(5.5 \\
& +R(6 .)\end{aligned}$ & 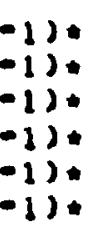 & $\begin{array}{r}-5.5673 E=01 \\
3.9286 E+00 \\
-6.3640 E+00 \\
3.6278 E+00 \\
-7.9869 E=01 \\
5.7267 E=02\end{array}$ & & $\begin{array}{r}1.0207 E+00 \\
-2.4405 E+00 \\
1.4142 E+00 \\
-2.1340 E-01\end{array}$ & $\begin{array}{l}x \\
: x \\
: x \\
: x\end{array}$ & $\begin{array}{r}-3.7115 E=01 \\
2.3820 E=01\end{array}$ & $\begin{array}{l}: \times 2 \\
: \times 2\end{array}$ \\
\hline AC 3. & & $\begin{aligned}= & +R C O 0^{\circ} \\
& +R\left(10^{\circ}\right. \\
& +H\left(30^{\circ}\right. \\
& +R(4)^{\circ} \\
& +H\left(50^{\circ}\right. \\
& +R C 60^{\circ}\end{aligned}$ & $\begin{array}{l}03: \\
03: \\
0): \\
0\}: \\
03: \\
03:\end{array}$ & $\begin{array}{r}2.1429 E=01 \\
6.4286 E=01 \\
-4.5000 E+00 \\
1.6364 E+00 \\
-1.4286 E+00 \\
1.2987 E=01\end{array}$ & $x$ & $\begin{array}{r}-3.9286 E-01 \\
-1.1786 E+00 \\
1.0000 E+00 \\
-2.7273 E-01\end{array}$ & $\begin{array}{l}x 2 \\
: x \\
: x \\
x \\
x\end{array}$ & $\begin{array}{l}1.4286 E=01 \\
4.2857 E=01\end{array}$ & $\begin{array}{l}+\times 3 \\
1 \times 2\end{array}$ \\
\hline AC 3 . & $2)=R(3$ & $\begin{aligned}1)= & +H(1) \\
& +H(2) \\
& +R(4) \\
& +R(5) \\
& +H(6)\end{aligned}$ & $\begin{array}{l}\text { 1): } \\
\text { 1): } \\
\text { 1): } \\
\text { 1): } \\
\text { 1): }\end{array}$ & $\begin{array}{r}7.1874 E-01 \\
-3.0431 E+00 \\
3.7467 E+00 \\
-1.8558 E+00 \\
2.2180 E-01\end{array}$ & & $\begin{array}{r}-1.3177 E+00 \\
1.8904 E+00 \\
-2.2040 E-01\end{array}$ & $\begin{array}{l}: x \\
-x \\
* x\end{array}$ & $\begin{array}{r}4.7910 E=01 \\
-1.0443 E=01\end{array}$ & $\begin{array}{l}\times 2 \\
\times 2\end{array}$ \\
\hline$R<3$. & $2)=R(3)$ & $\begin{aligned}0)= & +R(20 \\
& +R(3) \\
& +R(4) \\
& +R(5) \\
& +H(6)\end{aligned}$ & $\begin{array}{l}2): \\
2): \\
2): \\
2):\end{array}$ & $\begin{array}{r}-3.5138 E+00 \\
4.5000 E+00 \\
1.1472 E+00 \\
-1.8890 E+00 \\
3.2395 E=01\end{array}$ & & $\begin{array}{r}2.1828 E+00 \\
-1.0000 E+00 \\
-0.7482 E-02\end{array}$ & $\begin{array}{l}: x \\
: x \\
: x\end{array}$ & $-2.1296 E=01$ & $+\times 2$ \\
\hline
\end{tabular}


H(3, 2).A(3, 1) $+H(3,3), 0.3640 E+00$ $+H(4,3),-2.4782 E+00$ $\rightarrow R(5,3)=-1.3363 E+00$

$R(3,2) \oplus R(3,2)=+R(4,4), \quad-5.5415 E+00$

$R(3,3)+R(3,-3)=+R(0,0):-2,1424 E=0$ $R(2,0)$ $R(3.0)=-4.5000 k+0$ H(4, 0). 1.9470E+00 R(5., O). - -3.5714E-0 $\rightarrow R(6,0)$. $2.1645 k-02$

$R(3,3)+H(3,-2)=+A(1,1),-5.5673 t-0$ $+R(2,1) .3 .9286 E+00$ $+R(3 ., 1)=0.36902+00$ $+K(4 ., 1) .3 .6275 t+00$

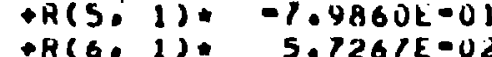

$R(3,3) \cdot H(3,-1)=+H(2,2), 2.4846 E+0 O$ $+H(3,2)=-6.3 A 40 E+00$ $+K(4.2) .408672 E+00$ $+H(5,2) *-1.3363 E+00$
$+H(6,2) * \quad 1.1453 E=01$

$R(3,3)+R(3, J)=+H(3,3) *-4.500 u k+00$ $\rightarrow R(4,3)=5.25 P 1 k+0$ $\rightarrow H(b, 3) .-1.0 A 9 b E+0$ $903 \forall t-01$

$R(3,3) * R(3,1)=+K(4,4), 4.2424 t+00$ $+R(5.4)=-2.3146 E+00$ $+F(6,4)$ 3.136/k-0)

$A(3,3)+H(3,2)=+K(5,5),-2,3146 E+00$ $+R(6.5)$. $4.6524 E-01$

$R(3,3)+H(1,3)=+R(0,6) * 0.5795 E-1) 1$ $R(4,-4) \cdot 4(1,-1)=+H(5,-5), \quad$ R.4536E-01

$R(4,-4) \cdot M(1,0)=+R(4,-4), \quad 2,0000 E+00$ $+R(5 .-4)$. $3.33 j 3 t=01$

$R(4,-4)+H(1,1)=+H(3,-9)=2.2040 E+00$ $+K(4,-3)=1.0000 E+00$ $+!^{2}(3,-3)$. I. IIIL-O
$-1.41426+00: x$
$1.4578 E-01: x$

$3.2597 E-111 \cdot x$

$3.9286 \varepsilon-01: x 2-1.4286 E-01 * x 3$ $1.7679 E+00 * x=6.4286 E-01: \times 2$ $-2.4405 E+00 * x \quad 2.3610 E-01 * \times 2$ $1.0000 E+00: x$ $-1.168 B E-01$ * $x$

$1.0207 E+00=x-3.7115 E=01 * \times 2$ $-2.4405 E+00 * x \quad 2.3810 E-01 * x 2$ $1.4142 E+00 \cdot x$

$-2.1340 E-01 * x$

$-1.5435 E+00 * x \quad 1.5058 E-01 * 2$

$1.41426+00=x$

$-2.8630 E-01 \cdot x$

$1.0000 E+00=x$

$-3.09045-01: x$

$-2.5250 E-01 \cdot x$

$-5.8794 k-01 \cdot x$ 


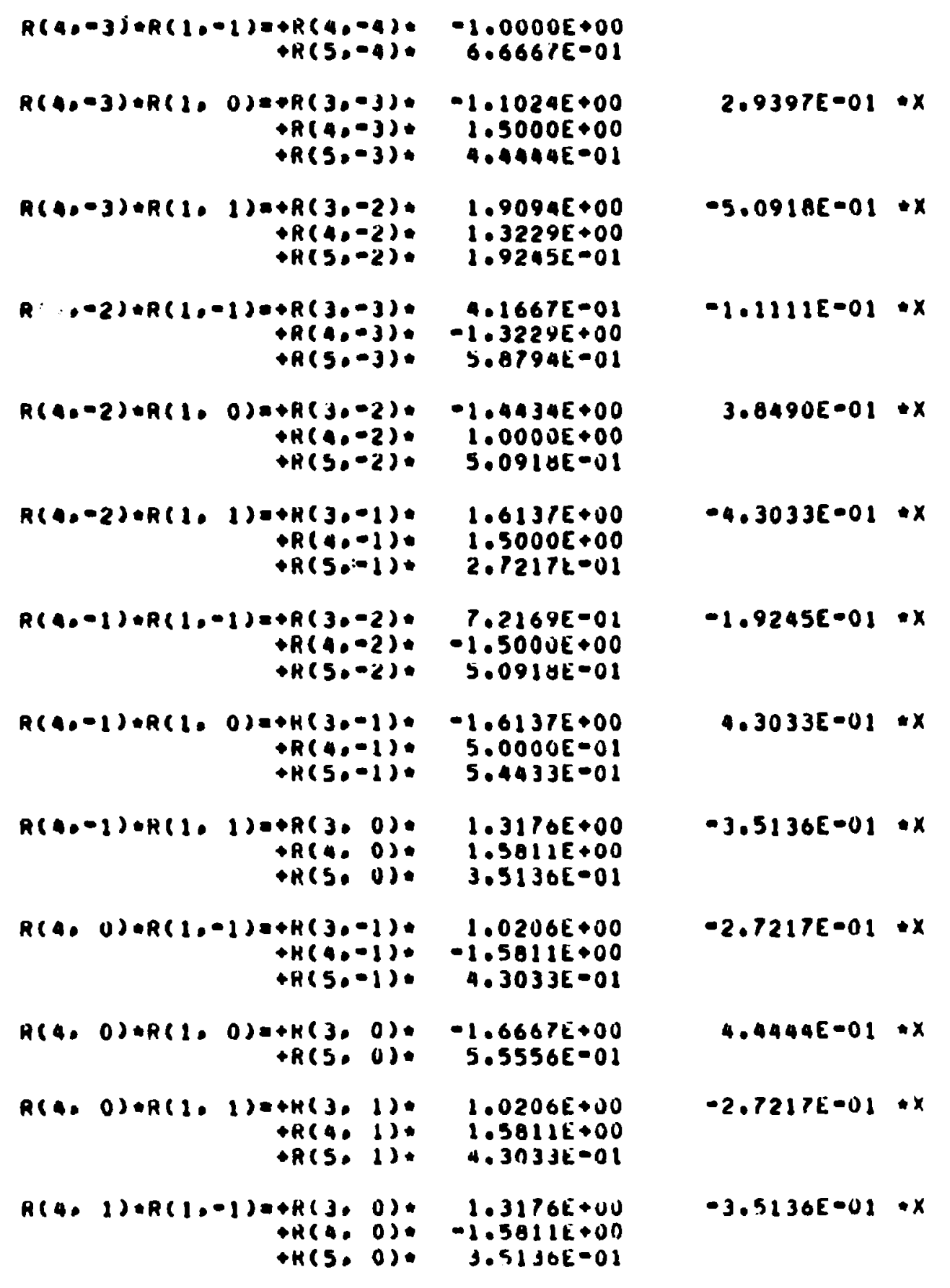




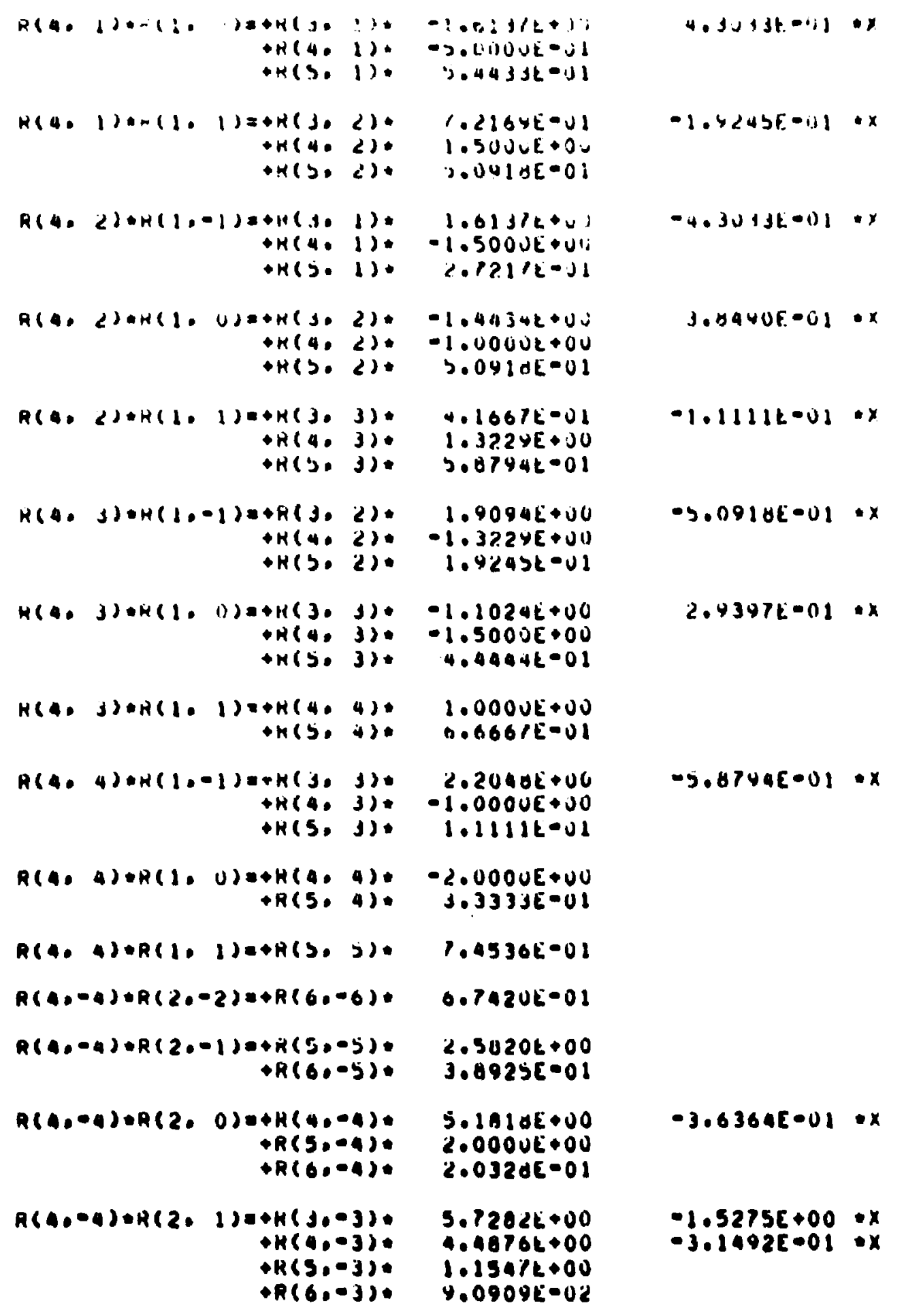




\begin{tabular}{|c|c|c|c|c|c|c|}
\hline$R(4,-4) \oplus R(2$. & $\begin{aligned} 21= & +K(2,-2): \\
& +K(3,-2) \\
& +K(4,-2) \\
& +K(3,-2) \\
& +H(0 .-2)\end{aligned}$ & 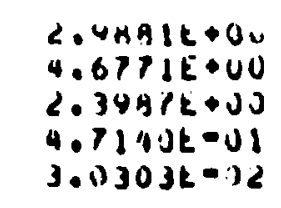 & $\begin{array}{l}-2.2904[+00 \\
-1.2472 E+011 \\
-1.6833 L=01\end{array}$ & $\begin{array}{l}: x \\
: x \\
: x\end{array}$ & $3.48412=01$ & $=x_{2}$ \\
\hline$A(A,-3) \cdot A(2$, & $\begin{aligned}-21 & +H(5,-5) \\
& +H(6 .-5)\end{aligned}$ & $\begin{array}{r}-1.0251 E+00 \\
5.5000 E-01\end{array}$ & & & & \\
\hline$R(4,-3) \cdot R(2$, & $\begin{aligned}-11, & +H(4,-4) \\
& +R(5,-4) \\
& +H(6,-4)\end{aligned}$ & $\begin{array}{r}-4.4876 E+J 0 \\
1.154 / E+00 \\
4.6445 E-U 1\end{array}$ & $3.1492 E=01$ & $\cdot x$ & & \\
\hline$R(4,-Z) \cup A(2$, & $\begin{aligned}U)= & +R(3,-3) \\
& +K(4,-3) \\
& +K(3,-3) \\
& +K(6,-3)\end{aligned}$ & $\begin{array}{r}-4.9600 E+00 \\
1.2953 E+00 \\
2.000 U E+00 \\
3.1492 E-01\end{array}$ & $\begin{array}{r}1.3229 E+00 \\
-4.0409 E-0 z\end{array}$ & $: x$ & & \\
\hline$A(4,-3)=R(2$, & $\begin{aligned} 11= & +H(2,-2): \\
& +R(3,-2): \\
& +R(4,-2) \\
& +R(5,-2) \\
& +R(6,-2)\end{aligned}$ & $\begin{array}{r}-2.112 Y E+U 0 \\
1.6536 E+00 \\
4.2404 E+00 \\
1.6667 E+00 \\
1.7192 E-01\end{array}$ & $\begin{array}{r}1.6194 E+00 \\
-4.4046 E=01 \\
-2.9757 E=01\end{array}$ & $\begin{array}{l}: x \\
: x \\
: x\end{array}$ & $-2.81726=01$ & $* x \bar{c}$ \\
\hline$A(4,-3) \cdot A(2$, & $\begin{aligned}2)= & +K(2,-1): \\
& +R(3,-1): \\
& +R(4,-1): \\
& +K\left(b_{0}-1\right): \\
& +K\left(0_{0}-1\right)\end{aligned}$ & $\begin{array}{l}2.1129 E+00 \\
5.2291 E+00 \\
3.5981 E+00 \\
0.0192 E-01 \\
6.7760 E=02\end{array}$ & $\begin{array}{l}-1.6194 E+00 \\
-1.344 A E+00 \\
-2.5250 E-01\end{array}$ & $\begin{array}{l}\cdot x \\
: x \\
\cdot x\end{array}$ & $2.0172 E-01$ &.$\times 2$ \\
\hline$R(4,-2) \bullet A(2$. & $\begin{aligned}-2)= & +H(4,-4) \\
& +R(5,-4) \\
& +R(6,-4)\end{aligned}$ & $\begin{array}{r}2.3997 E+00 \\
-2.1602 E+00 \\
4.391 J E=01\end{array}$ & $-1.6833 E-01$ & $\cdot x$ & & \\
\hline$R(4,-2) * R(2$, & $\begin{aligned}-1)= & +K(3,-3) \\
& +R(4,-3) \\
& +R(6,-3)\end{aligned}$ & $\begin{array}{r}3.2476 E+00 \\
-4.2404 E+00 \\
4.8105 E-01\end{array}$ & $\begin{array}{r}-8.6603 E-01 \\
2.9757 E-01\end{array}$ & $\begin{array}{l}* x \\
* x\end{array}$ & & \\
\hline$A(4,-2) * k<2$. & $\begin{aligned}(0)= & +H(2,-2): \\
& +K(3,-2) \\
& +K(4,-2) \\
& +H(5,-2) \\
& +K(6,-2)\end{aligned}$ & $\begin{array}{r}1.3832 E+00 \\
-1.3301 E+00 \\
-1.4805 E+00 \\
1.5275 E+00 \\
3.9271 E=01\end{array}$ & $\begin{array}{r}-1.0605 E+00 \\
1.1547 E+00 \\
1.0390 E-01\end{array}$ & $\begin{array}{l}: x \\
: x \\
: x\end{array}$ & $1.0443 E=01$ & $\cdot \times 2$ \\
\hline$R(4,-2) \cdot R(2$. & 1)= $\begin{aligned} & +R(2,-1): \\
& +R(3,-1): \\
& +R(4,-1): \\
& +R(5,-1): \\
& +R(6,-1):\end{aligned}$ & $\begin{array}{r}-2.2588 E+00 \\
-1.3975 E+00 \\
2.8849 E+00 \\
1.88506+00 \\
2.5353 E-01\end{array}$ & $\begin{array}{r}1.7317 E+00 \\
3.7268 E-01 \\
-2.0245 E-01\end{array}$ & $\begin{array}{l}: x \\
: x \\
: x\end{array}$ & $-3.0117 E-01$ &.$\times 2$ \\
\hline
\end{tabular}




\begin{tabular}{|c|c|c|c|c|c|c|}
\hline$A(4,-2) \cdot R(2)$ & $\begin{aligned}2)= & +R(2,0) \\
& +R(30 \\
& +R(400) \\
& +R(500) \\
& +R(600)\end{aligned}$ & $\begin{array}{l}1.30326+00 \\
1.8412 E+00 \\
1.3005 E+00 \\
1.2910 E+00 \\
1.1736 E-01\end{array}$ & $\begin{array}{l}-1.0605 E+00 \\
-1.29106+00 \\
-3.01796-01\end{array}$ & $\begin{array}{l}: x \\
: x \\
: x\end{array}$ & $1.8443 E-01$ & $1 \times 2$ \\
\hline$R(4,-1) \cdot R(20$ & $\begin{aligned}-2)= & +R(3,-3) \\
& +R(4,-3) \\
& +R(5,-3) \\
& +R(6,-3)\end{aligned}$ & $\begin{array}{r}-1.53045+00 \\
1.59615+00 \\
-2.16025+00 \\
3.4015 E=01\end{array}$ & $\begin{array}{r}4.0825 E-01 \\
-2.5250 E-01\end{array}$ & $\bullet x$ & & \\
\hline$R(a,-1) \cup R(2$, & $\begin{aligned}-1) & =+R(2,-2): \\
& +K(3,-2): \\
& +R(4,-2): \\
& +H(5,-2): \\
& +P(6,-2)\end{aligned}$ & $\begin{array}{r}-7.9860 E-01 \\
4.3750 E+00 \\
-2.8049 E+00 \\
-6.8192 E-01 \\
4.53536-01\end{array}$ & $\begin{array}{r}6.1226 E-01 \\
-1.1667 E+00 \\
2.0245 E-01\end{array}$ & $\begin{array}{l}: x \\
: x \\
: x\end{array}$ & $-1.0640 E-01$ & $\cdot \times 2$ \\
\hline$R(4,-1) * A(2$, & $\begin{aligned} O S= & +K(2,-1): \\
& +K(3,-1): \\
& +R(40-1): \\
& +R(3,-1): \\
& +R(0,-1):\end{aligned}$ & $\begin{array}{r}1.9562 E+00 \\
-2.4200 t+00 \\
-3.1461 E+00 \\
0.1650 t=01 \\
1.3913 t-01\end{array}$ & $\begin{array}{r}-1.4947 E+00 \\
6.4550 E-01 \\
2.2078 E-01\end{array}$ & $\begin{array}{l}: x \\
: x \\
x\end{array}$ & $2.6002 E-01$ & $\cdot \times 2$ \\
\hline$A(4,-1) \neq A(2$, & 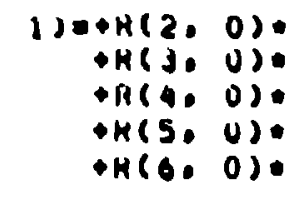 & $\begin{array}{r}-1.9562 E+00 \\
-3.9233 E+00 \\
1.0136 E+00 \\
1.0257 E+00 \\
3.3193 E-01\end{array}$ & $\begin{array}{r}1.4997 E+00 \\
9.1247 E-01 \\
-7.1133 E-02\end{array}$ & $\begin{array}{l}\cdot x \\
: x \\
0 x \\
\cdot x\end{array}$ & $-2.0082 E-01$ & $\cdot \times 2$ \\
\hline$R(4,-1) \cdot R(2$, & 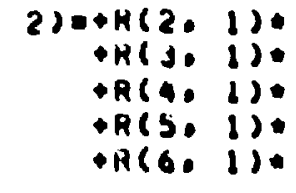 & $\begin{array}{l}7.9960 E-08 \\
3.9528 E+00 \\
4.5331 E+00 \\
1.6667 E+03 \\
1.7924 E-01\end{array}$ & $\begin{array}{l}-6.1226 E=01 \\
-1.0541 E+00 \\
-1.1012 E=01\end{array}$ & $\begin{array}{l}: x \\
: x \\
: x\end{array}$ & $1.0040 E-01$ & $\cdot \times 2$ \\
\hline 41.0100120 & $\begin{aligned}-2)= & +H(2,-2): \\
& +H(3,-2): \\
& +R(4,-2) \\
& +H(3,-2): \\
& +R(0,-2)\end{aligned}$ & $\begin{array}{r}3.5714 E=01 \\
-2.7951 E+00 \\
9.3005 E+00 \\
-1.9720 \mathrm{O}+00 \\
2.535 \mathrm{JE}=01\end{array}$ & $\begin{array}{r}-2.7381 E=01 \\
1.4536 E-01 \\
-3.0179 E=01\end{array}$ & $\begin{array}{l}\cdot x \\
: x \\
: x\end{array}$ & $4.7619 E=02$ & $1 \times 2$ \\
\hline$R(a, \infty)+(2,0$ & $\begin{aligned}-1)= & +H(2,-1) \\
& +K(3,-1) \\
& * H(4,-1) \\
& \leftrightarrow H(3,-1) \\
& \leftrightarrow R(6,-1)\end{aligned}$ & $\begin{array}{r}-1.4206 E+00 \\
1.0194 E+U 0 \\
-1.0136 E+D 0 \\
-1.4907 E+00 \\
+.0081 E-01\end{array}$ & $\begin{array}{r}1.0452 E+00 \\
-1.1783 E+00 \\
1.1133 E-02\end{array}$ & $\begin{array}{l}: x \\
: x \\
: x\end{array}$ & $-1.4040 k-01$ &.$\times 2$ \\
\hline m(40 $010+120$ & 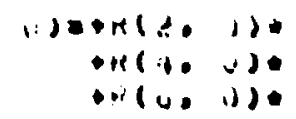 & $\begin{array}{r}2.1424 E+00 \\
-3.701 \text { } \\
4.9455-W 1\end{array}$ & $\begin{array}{r}-1.6429 E+00 \\
2.5914 E-01\end{array}$ & $\because x$ & $2.05 / 12=01$ & $0 \times 2$ \\
\hline
\end{tabular}




\begin{tabular}{|c|c|c|c|c|c|c|c|c|}
\hline$R(4)$ & $0)=R(2$. & $\begin{aligned} 110 & +R C 20 \\
& +H(30 \\
& +R(40 \\
& +H C 30 \\
& +K C 60\end{aligned}$ & $\begin{array}{l}1 \\
1) \\
1) \\
110 \\
1\end{array}$ & $\begin{array}{r}-1.72 .06 E+00 \\
-4.1194 E+00 \\
-1.0136 E+00 \\
1.0907 E+00 \\
1.0007 E-01\end{array}$ & $\begin{array}{l}1.0952 E+00 \\
1.1785 E \gg 00 \\
7.1133 E-02\end{array}$ & $\begin{array}{l}x \\
0 x \\
x\end{array}$ & $-1.9040 E-01$ &.$\times 2$ \\
\hline ACA. & U) $) \cdot A(2$. & $\begin{aligned}2)= & +H(2, \\
& +H(3) \\
& +R(4) \\
& +H(5) \\
& +R C 6 .\end{aligned}$ & $\begin{array}{l}2): \\
2): \\
2): \\
2) \\
2)\end{array}$ & $\begin{array}{l}3.571 \text { पE }-U 1 \\
2.7951 E+00 \\
1.3005 E+00 \\
1.9720 E+00 \\
2.5353 E-01\end{array}$ & $\begin{array}{l}-2.7301 E=01 \\
-7.4336 E=01 \\
-3.0179 E-01\end{array}$ & $\begin{array}{l}: x \\
: x \\
: x\end{array}$ & 4.761 YE.02 &.$\times 2$ \\
\hline RCA. & $1) * R(2$ & 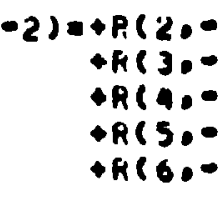 & 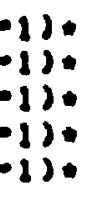 & $\begin{array}{r}7.9860 E-01 \\
-3.952 \mathrm{dE}+00 \\
4.533 \mathrm{~L}+00 \\
-1.6667 t+00 \\
1.7920 E-01\end{array}$ & $\begin{array}{r}-0.1226 E=01 \\
1.0541 E+00 \\
-3.1812 E=01\end{array}$ & $\begin{array}{l}x \\
\cdot x \\
x \\
x\end{array}$ & 1.06406 .01 & $\bullet \times 2$ \\
\hline$R\left(A_{0}\right.$ & l) & $\begin{aligned}-1)= & +R(20: \\
& +H(3 \circ: \\
& +R(40: \\
& +H(50 \\
& +R(60\end{aligned}$ & $\begin{array}{l}0): \\
0): 0 \\
0): \\
0 \\
0\end{array}$ & $\begin{array}{r}-1.9562 E+00 \\
3.923 \mathrm{JE}+00 \\
1.0136 \mathrm{0}+00 \\
-1.0257 t+00 \\
3.3145 E-01\end{array}$ & $\begin{array}{r}1.4997 E+00 \\
-9.1287 E-01 \\
-7.1133 E-02\end{array}$ & $\begin{array}{l}: x \\
: x \\
: x\end{array}$ & $-2.6082 E-01$ &.$\times 2$ \\
\hline$R(4)$ & $1) * R(2$. & 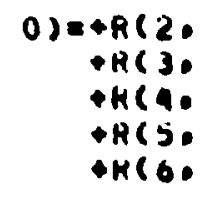 & $\begin{array}{l}\text { 1): } \\
\text { i): } \\
\text { 1): } \\
\text { 1): } \\
\text { 1). }\end{array}$ & $\begin{array}{r}1.9562 E+00 \\
2.4200 E+00 \\
-3.1961 E+00 \\
-1.1630 t=01 \\
4.391 \mathrm{JE}-01\end{array}$ & $\begin{array}{r}-1.4997 E+00 \\
-6.4550 E=01 \\
2.207 \text { UE }=01\end{array}$ & $\begin{array}{l}\cdot x \\
: x \\
: x\end{array}$ & $2.6002 E-01$ &.$\times 2$ \\
\hline$R C A_{0}$ & $1)+P(2$. & $\begin{aligned}1)= & +R(2 D \\
& +H(3 D \\
& +H(4 D \\
& +H(5)^{\circ} \\
& +R(6)\end{aligned}$ & $\begin{array}{l}2): \\
2) \\
2) \\
2) \\
2)\end{array}$ & $\begin{array}{r}-7.9360 E=U 1 \\
-4.3750 E+0 U \\
-2.084 Y E+U 0 \\
0.8192 .01 \\
4.5353 E=01\end{array}$ & $\begin{array}{l}6.1226 E \bullet 01 \\
1.1667 E \uparrow 00 \\
2.0245 E-01\end{array}$ & $\begin{array}{l}: x \\
: x \\
: x\end{array}$ & $-1.0648 E-01$ &.$\times 2$ \\
\hline Ro: & $1)=1+2$ & $\begin{aligned}2)= & +R(30 \\
& +4(4 \circ) \\
& +R(5 \circ \\
& +H(60\end{aligned}$ & $\begin{array}{l}3): \\
320 \\
30=\end{array}$ & $\begin{array}{l}1.5309 E+00 \\
3.5991 E+00 \\
2.1602 E+00 \\
3.4015 E-01\end{array}$ & $\begin{array}{l}-4.0825 E-01 \\
-2.5250 E=01\end{array}$ & $: x$ & & \\
\hline$R(4)$ & $=1.12$ & $\begin{aligned}-2)= & +H(2 . \\
& +H C j \circ \\
& +H(4) \\
& +H(50 \\
& +H(6)\end{aligned}$ & 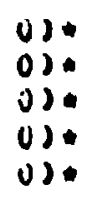 & $\begin{array}{r}1.3832 E+00 \\
-4.1412 E+00 \\
4.3005 E+00 \\
-1.2410 E+00 \\
1.1736 E=01\end{array}$ & $\begin{array}{r}-1.0605 E+00 \\
1.2910 E+00 \\
-1.01791=01\end{array}$ & $\begin{array}{l}* x \\
: x \\
* x\end{array}$ & $1.0443 E=01$ &.$\times 2$ \\
\hline$R(4)$ & $2) * R(2$. & $\begin{aligned}-,= & +K(2) \\
& +K(3) \\
& +r(4) \\
& +r(3) \\
& +r(3)\end{aligned}$ & $\begin{array}{l}\text { 1): } \\
\text { 1): } \\
\text { 1): } \\
\text { 1): } \\
\text { ile }\end{array}$ & $\begin{array}{r}-2.25 A O L+U 0 \\
1.3 Y 7 J E+00 \\
2.8 B A Y E+U U \\
-1 . H 856 E+J U \\
2.5353 E-01\end{array}$ & $\begin{array}{r}1.7317 E+00 \\
-3.7268 E=01 \\
-2.024 S E-01\end{array}$ & $\begin{array}{l}* x \\
* x \\
* x\end{array}$ & $-3.0117 E-01$ & $=x$ \\
\hline
\end{tabular}




\begin{tabular}{|c|c|c|c|c|c|c|c|c|}
\hline$R(4)$ & C? $\# R(2$. & $\begin{aligned}1)= & +R(2) \\
& +K(3) \\
& +R(4) \\
& +R(5) \\
& +R(6 .\end{aligned}$ & $\begin{array}{l}2): \\
2): \\
2): \\
2): \\
2):\end{array}$ & $\begin{array}{r}1.3 B 32 E+00 \\
4.3301 E+00 \\
-1.9305 E+00 \\
-1.5275 E+00 \\
3.9271 \mathrm{~L}=01\end{array}$ & $\begin{array}{r}-1.0605 E+00 \\
-1.1547 E+00 \\
1.0390 E-01\end{array}$ & $\begin{array}{l}x \\
: x \\
x\end{array}$ & $1.0443 E-01$ & $+\times 2$ \\
\hline$H(4$. & $2) * A(Z$ & $\begin{aligned}1)= & +H(3, \\
& +K(4) \\
& +K(6)\end{aligned}$ & $\begin{array}{l}\text { 3): } \\
\text { 3): } \\
\text { 3): }\end{array}$ & $\begin{array}{r}-3.2476 E+0 U \\
-4.2404 E+00 \\
4.8105 E-U 1\end{array}$ & $\begin{array}{l}0.6603 E-01 \\
2.9757 E=01\end{array}$ & $\begin{array}{l}* x \\
x\end{array}$ & & \\
\hline$R<4$. & $2) \cdot R(2$. & $\begin{aligned}2)= & +R(4, \\
& +R(5)^{\circ} \\
& +R 16 .\end{aligned}$ & $\begin{array}{l}\text { 4): } \\
\text { 4): } \\
\text { 4). }\end{array}$ & $\begin{array}{l}2.3987 E+U U \\
2.160 \angle E+U 0 \\
4.3913 E-O 1\end{array}$ & $-1.6833 E-01$ & $\cdot x$ & & \\
\hline$R(4)$ & $3) \leadsto P(2$. & $\begin{aligned}-2)= & +R(2) \\
& +K(30 \\
& +K(4) \\
& +R(5) \\
& +H(6)\end{aligned}$ & $\begin{array}{l}\text { 1): } \\
\text { 1): } \\
\text { 1): } \\
\text { (1): } \\
\text { 1): }\end{array}$ & $\begin{array}{r}2.112 y E+00 \\
-5.2291 E+00 \\
3.3981 t+00 \\
-0.8192 E-01 \\
6.7760 E-02\end{array}$ & $\begin{array}{r}-1.6199 E+00 \\
1.3944 E+00 \\
-2.525 U E-01\end{array}$ & $\begin{array}{l}* x \\
: x \\
: x\end{array}$ & $2.0172 E-01$ & $\times 2$ \\
\hline RiA. & J) $\# R(2$. & $\begin{aligned}-1)= & +R(2, \\
& +R(3) \\
& +R(4) \\
& +K(5) \\
& +R(6)\end{aligned}$ & $\begin{array}{l}2) \\
2) \\
2) \\
2 J \\
2)\end{array}$ & $\begin{array}{r}-2.112 Y E+00 \\
-1.6530 E+00 \\
4.2404 E+00 \\
-1.6667 E+00 \\
1.7142 E-01\end{array}$ & $\begin{array}{r}1.6199 E+00 \\
4.4096 E-01 \\
-2.9757 E-01\end{array}$ & $\begin{array}{l}* x \\
* x \\
* x\end{array}$ & $-2.0172 E=01$ & $+\times 2$ \\
\hline$R(4)$ & $3) \neq R(2$ & $\begin{aligned}0)= & +K(3) \\
& +K(4) \\
& +R(5) \\
& +R<0\end{aligned}$ & $\begin{array}{l}\text { 3): } \\
3): \\
3): \\
3):\end{array}$ & $\begin{array}{r}4.9600 E+00 \\
1.2955 E+00 \\
-2.0000 E+00 \\
3.1492 E-01\end{array}$ & $\begin{array}{l}-1.3229 E+00 \\
-4.0909 E-02\end{array}$ & $\begin{array}{l}* x \\
-x\end{array}$ & & \\
\hline$R(4)$ & $3)=12(2$ & $\begin{aligned}1)= & +K(4) \\
& +K(5) \\
& +R(6)\end{aligned}$ & $\begin{array}{l}\text { 4): } \\
4):\end{array}$ & $\begin{array}{r}-4.4876 E+00 \\
-1.1547 E+00 \\
4.6945 E-01\end{array}$ & $3.1492 E-01$ & $-x$ & & \\
\hline$R(4)$ & $3)=R(2$ & $\begin{aligned}2)= & +K(5) \\
& +K(6)\end{aligned}$ & 5): & $\begin{array}{l}1.825 / E+00 \\
5.5048 E-01\end{array}$ & & & & \\
\hline$R(4)$ & 4) $=R(2$. & $\begin{aligned}-2)= & +R(2) \\
& +R(30 \\
& +R(4) \\
& +R(5) \\
& +R(6)\end{aligned}$ & $\begin{array}{l}2): \\
2): \\
2): \\
2) \\
2)\end{array}$ & $\begin{array}{r}2.9881 E+U 0 \\
-4.6771 E+00 \\
2.3987 E+00 \\
-4.7140 E=01 \\
3.0303 E-02\end{array}$ & $\begin{array}{r}-2.2909 E+00 \\
1.2472 E+00 \\
-1.6833 E=01\end{array}$ & $\begin{array}{l}: x \\
: x \\
: x\end{array}$ & $3.9841 E-01$ & $+\times 2$ \\
\hline$R(A)$ & 4) $* R(2$ & $\begin{aligned}-1)= & +R(3) \\
& +R(4) \\
& +R(5) \\
& +R(6 .\end{aligned}$ & $\begin{array}{l}\text { 3): } \\
\text { 3): } \\
\text { 3): } \\
\text { 3): }\end{array}$ & $\begin{array}{r}-5.7282 E+00 \\
4.4876 E+00 \\
-1.1547 E+00 \\
9.0909 t=02\end{array}$ & $\begin{array}{r}1.5275 E+00 \\
-3.1492 E-01\end{array}$ & $\Rightarrow x$ & & \\
\hline$R(4)$ & 4) $\because R(2$. & $\begin{aligned}0)= & +R(4 .) \\
& +R(5) \\
& +R(6)\end{aligned}$ & 4): & $\begin{array}{r}5.181 \theta E+00 \\
-2.0000 E+00 \\
2.0320 E-01\end{array}$ & $-3.6364 E-01$ & $\cdot x$ & & \\
\hline
\end{tabular}




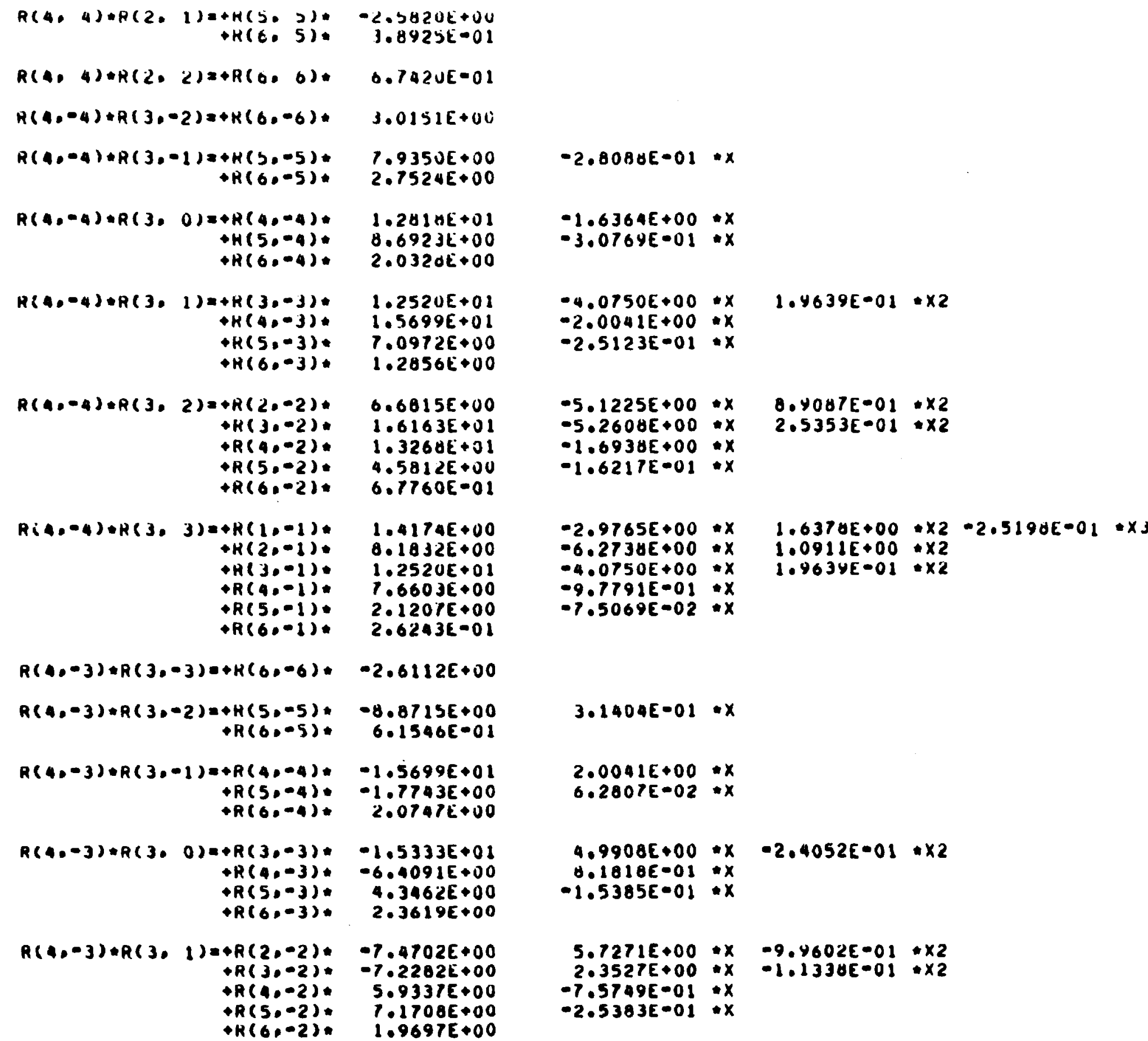

$R(4,-3) * R(3,1)=+R(2,-2):-7.4702 E+00$ $+R(3,-2),-7.2202 E+00$ $R(4,-2) .50 .0337 E+00$ $\rightarrow R(5,-2)$. $7.17005+00$ $+R(5,-2): \quad 1.1708 E+00$

$-2.8088 E-01 \cdot x$

$-1.6364 E+00 \cdot x$ $-3.0769 E=01: x$

$-4.0750 E+00 * x$ $-2.0041 E+00 \div$ $-2.5123 E-01 \cdot x$

$-5.1225 E+00 \cdot x$ $-5.2608 E+00$. $-1.6938 E+00$ : $X$ $-1.6217 E-01 * x$

$-2.9765 E+00 * x \quad 1.6374 E+00 \div \times 2-2.5190 E-01=x$ $-6.2738 E+00: \times \quad 1.0911 E+00 * \times 2$ $-4.0750 E+00: x \quad 1.963$ YE-01:x2

$-9.7791 E-01: x$

$0.9087 E-01+\times 2$ $2.5353 E-01 * \times 2$

$3.1404 E-01 \cdot x$

$2.0041 E+00 * x$ $6.2807 E-02: x$

$4.9908 E+00 \times x \quad-2.4052 E-01 \times 2$ $0.1818 E-01: x$ $-1.5385 E \cdot 01 \cdot x$

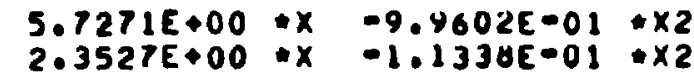
$-7.5749 E-01: x$

\section{$1.4639 E-01 \cdot \times 2$}

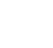




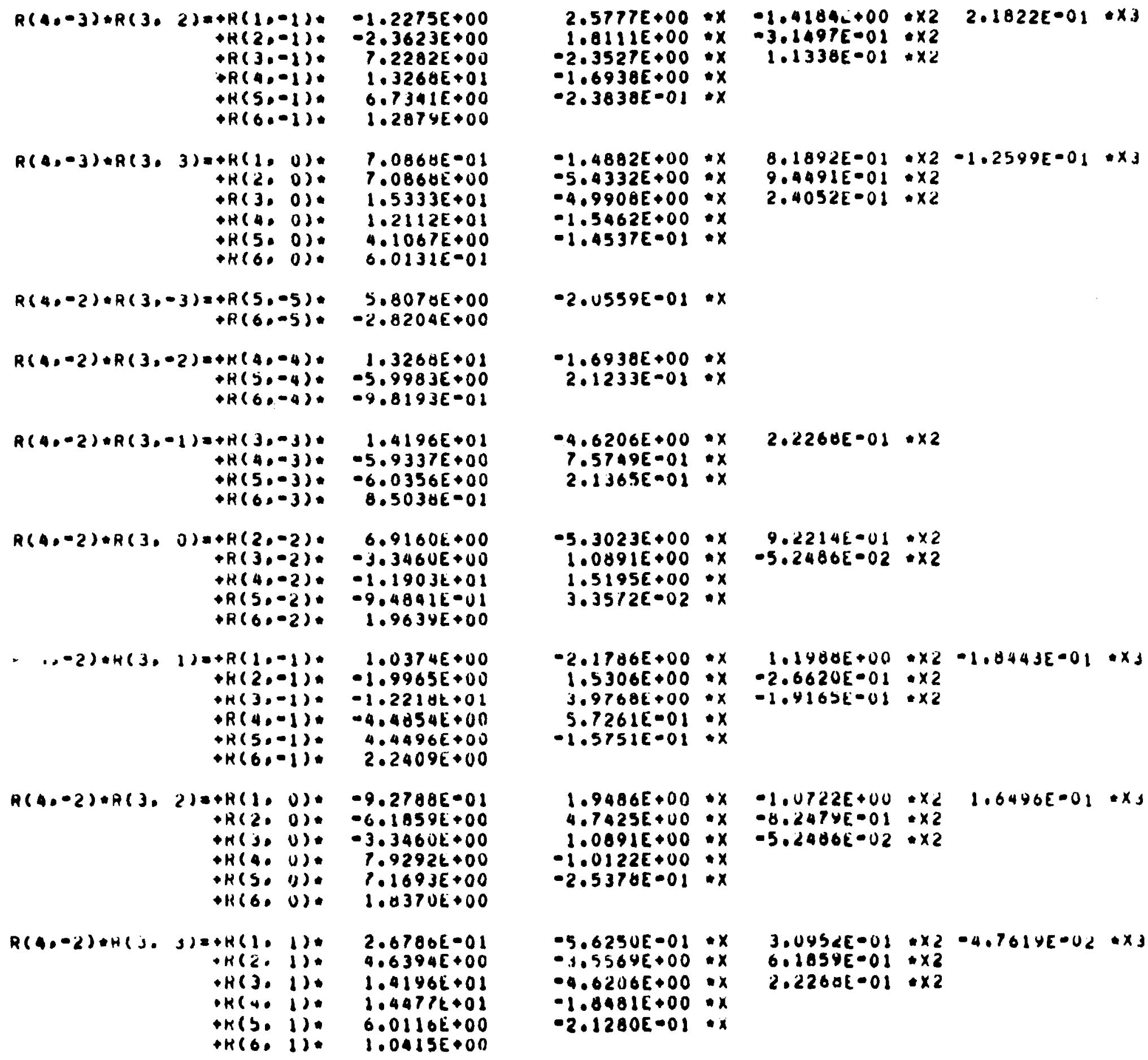

$6.9160 E+00$ $-3.3460 E+00$ $-1.1903 k+01$ $\begin{array}{ll}+R(4,-2): & -1.1903 E+01 \\ \rightarrow R(5,-2): & -9.4841 E-U 1 \\ \rightarrow R(6,-2) & 1.9634 E+00\end{array}$ $+R(3,-2)$

$, \ldots-2) \cdot H(3,1)=+R(1,-1)$

$1.0374 E+00$ $-1.9965 \mathrm{E}+00$ $R(3,-1)=-1.221 \forall t+01$ $\rightarrow(4 .-1),-4.4854 E+00$ $\begin{array}{ll}+R(5,-1): & 4.4496 E+00 \\ +K(6,-1) * & 2.2409 E+00\end{array}$

$R(4,02)+R(3,2)=+R(1,0)$

$-9.2700 E-01$ $+R(2,0) *-6.3959 E+00$ $+H(3, U)=-3.3460 E+00$ $+H(40, U) .7 .9292 E+00$ $1.16936+00$ $1.03706+00$

$R(4,-2)+H(j, J)=+H(1,1) *$ $0.67802=0$ in(3)

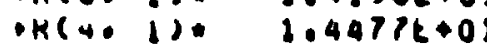
$+K(b .1) .6 .0116 E+00$ $+H(6$.$) 1) 1.0413 E+00$

$2.5777 E+00 \times x-1.4184 \div+00 \times 2$ $1.8111 E+00 * x-3.1497 E-01 * \times 2$

$-1.6938 E+00$ * $x$

$-1.4882 E+00 * x$ $-5.4332 E+00: x$ $-4.9908 E+00 * x$ $-1.5462 E+00 \cdot x$ $-1.5462 E+00: x$
$-1.4537 E-01 * x$

$8.1892 E-01 * x_{2}-1.2599 E-01 * x_{3}$ $9.4491 E=01: \times 2$

$2.40525-01 \times x$

$-2.0559 E-01 \cdot x$

$.000305+00, x$

$-1 \cdot 6938 E+00 * x$
$2.1233 E-01 * x$

$-4.6206 E+00 * x$ $7.57496-01: x$ $7.5749 E-01 * x$
$2.1365 E-01 * x$ $-5.3023 E+00 * x \quad 9.2214 E-01 * \times 2$

$1.0891 E+00: x \quad-5.2486 E-02 \times x 2$ $1.51956+00: x$
$3.3512 E-02: x$

$-2.1706 E+00 \cdot x$

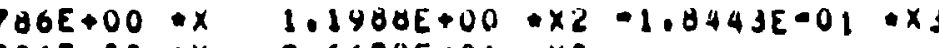
$1.5306 E+00 * x-2.6620 E=01 * \times 2$ $3.9760 E+00 * x=1.4165 E-01 * x 2$

$5.72616001 * x$

$1.9486 E+00 * x$

$1.9486 E+00 * x-1.0722 E+00 * x_{2} \quad 1.6496 E-01 * x_{3}$ $4.7425 E+00$ : $x \quad-8.247$ YE-01:x2

$1.0891 E+00 * x-5.2080 E-02 * x 2$

$-1.0122 E+00: x$

$-5.6250 E-01: x \quad 3.0452 E-01 * x 2-4.761 y E-02 \times x 3$ $0.10 .1054 E=01 \times 2$

$-9.6206 E+00 \div x$

$-1.8481 E+00$ : $x$

$-2.1280 E-01 . x$ 
$x \cdot 20-30169 \cdot 6=$

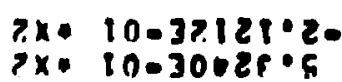

$x+00+32210^{\circ} 1$.

$x=00+3 s^{x}$

$2 \times 20-34060 \cdot 6$

$x \cdot 10-30206^{\circ} 2$.

$x+00+329+5 \cdot t$

x. $00+30000 \cdot 1$.

$3 x \cdot 10-39559 \cdot 1$

$x \cdot 10-31050^{\circ} 20$

$x=00+31860 \cdot 1$.

$3 x \cdot 10-3191608$

$x: 00+30060 \cdot 6$

$2 \times 10.38+11 \cdot 1 \cdot$

$x \cdot 10-3<140 \cdot 2=$

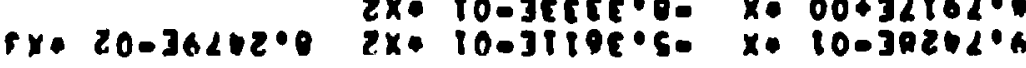

\section{$2 x+100398<1 \cdot 10$}

$6 x \cdot 10-36060 \cdot 1.2 x+10-3201901$

$x \cdot 20-32010 \cdot 10$

$x=00+31050^{\circ} t$

$x \cdot 00+3 e \operatorname{cec} s$

$x=00+30018 \cdot 8 \cdot$

$x * 10-30004^{\circ} 1$

$x+00+36150^{\circ} 8$

$2 \times \cdot 10-39618 \cdot 1$

ecene

seciose

$8 x \cdot 10 \cdot 30505 \cdot 1$

$2 x+10-30008^{\circ} 60$

$x \cdot 00+3002 d^{\circ} i$

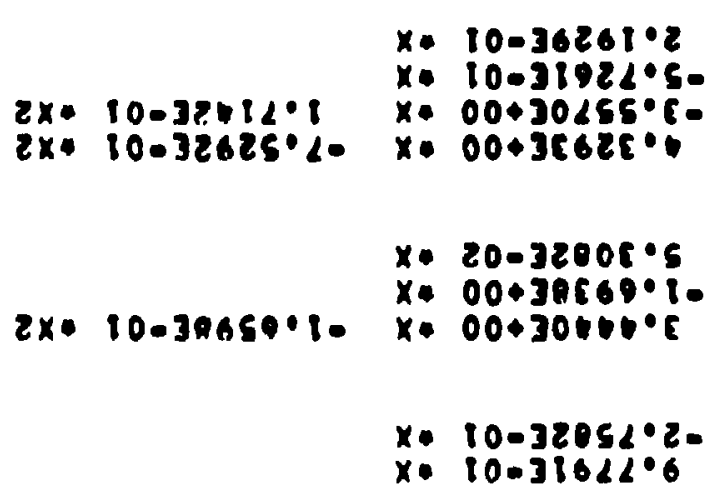

$x * 10-36261 \cdot 2$

x. $10.31922^{\circ} \mathrm{g}-$

$2 x * 10-32 \times 12 \cdot 1 \quad x * 00+30255: 6-$

$x \cdot 20-32008^{\circ} \mathrm{s}$

$x .10038021^{\circ} .6$

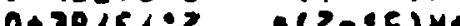

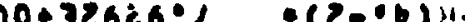

1003585C 9

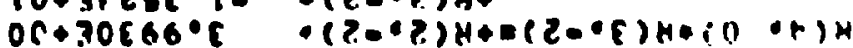

$00+70580 \cdot 2 \cdot 0(8-0.9) 40$

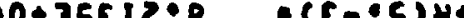

(0)

no+35568.

$00+30255 \cdot 1 \cdot(2 \cdot 0) 4+$

$00+3016605$ is is int

$10+3<100.1 \quad 18$ in)

$10+31050.1$ is $01,4+4$

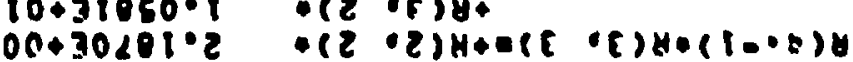

$00+30402 \cdot 2 \quad(1 \cdot 0) \mathrm{H}+$

$00+3060<.2$ - $(1$ is)

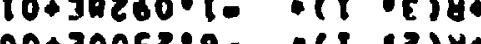

o-3nocona a

$00+36550.8 \quad 0.03)$

$10-3 B<000$ " $(0.5) \mathrm{H}$

$10039690.1-0(0.16) 4 \%$

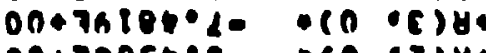

$00+30056 \cdot$ : $(0 \cdot 2) 4$.

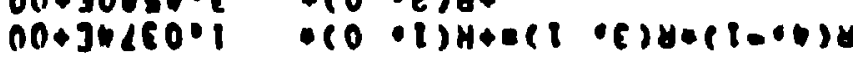

$00+30260 \cdot 1+(1-09) 40$

$00+3091004-0(10.5) 4+$

$00036062.00 \quad(1-0.6) 4+$

$00+36100.4$ (10.6) 84

$00+36060.4(1-02) 48$

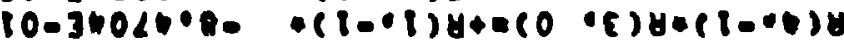

$10.32000 .00-18-00) 4$

$00+30568^{\circ} \cdot 0=(2-05) 4$

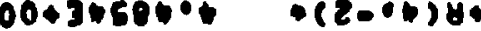

$10+39260^{\circ} 1 \quad(2-.6) y_{4}$

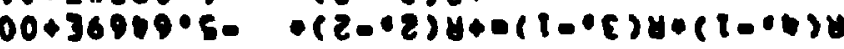

$00+35106^{\circ} 10 \cdot(6-09) 4+$

$00+39660^{\circ} 10-(E-0.5) H+$

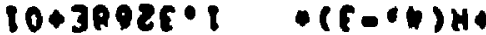

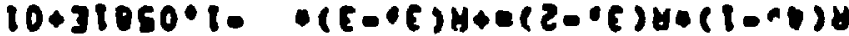

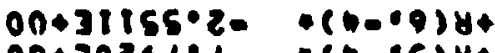

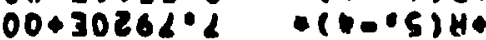

$00+36000^{\circ}<0$ o(th- 


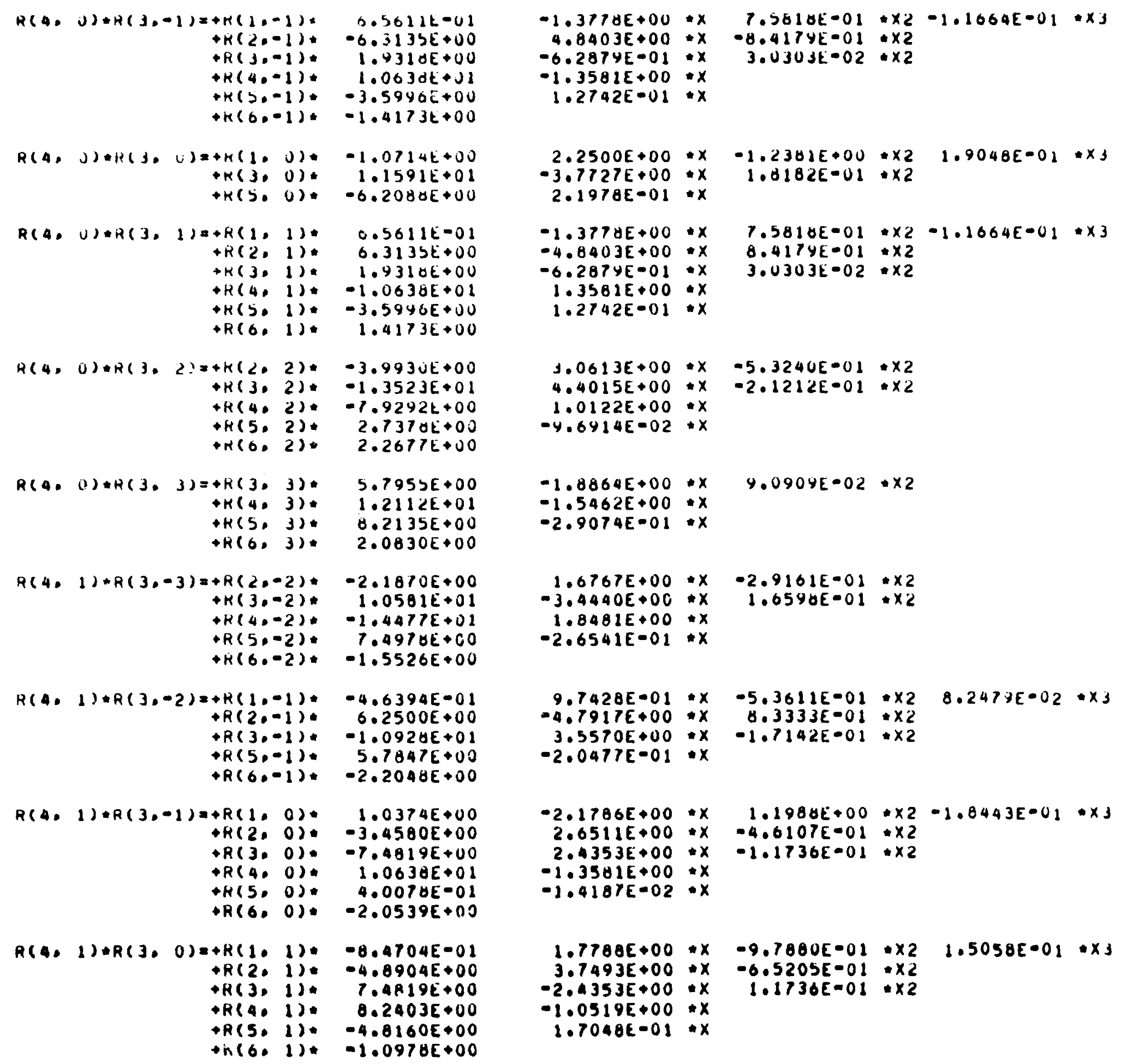




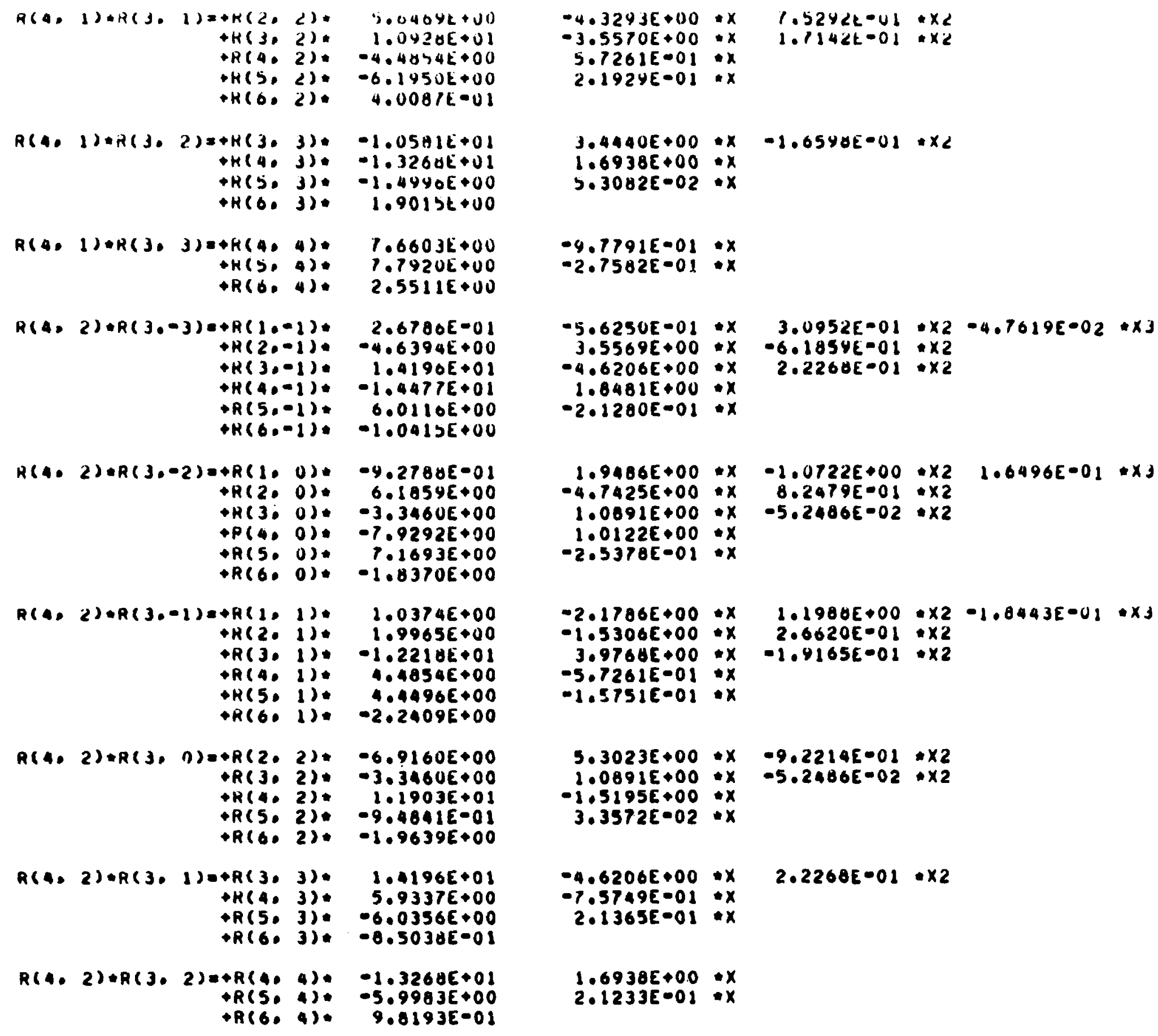

$R(4,1)+R(3,1)=+K(2,2), 300404 t+J 0)$ $+K(3,2): 1.0920 E+0)$ H(A. 2). H(6. 2). 4.008/E-U1

$R(4,1)+R(3,2)=+K(3,3)=-1.05 A 1 E+01$ $+H(4.3)=-1.3260 E+01$ $+R(5,3)=-1.4990 E+00$ $+H(6,3)=1.9015 t+00$

$R(4,1)+R(3,3)=+R(4,4): \quad T .6603 E+00$ $\rightarrow K(5,4)=1.7920 E+00$ $+R(6,4) * 2.5511 E+00$

$R(4,2)+R(3,-3)=+R(1,-2) * 2.6700 E-01$ $+R(2,-1) *-4.6394 E+00$ $+R(3,-1) \cdot 1.4190 E+01$ $+H(4,-1) \cdot-1.4477 E+01$ $+R(5 .-1)=0.0110 E+00$ $\rightarrow R(6,-1):-1,0415 E+00$

$R(4,2) \bullet R(3,-2)=+R(1,0) *$ $\rightarrow R(2,0)$ $6.1059 E+00$ (t) $0(50$ 0) $+R(6,0):-1.8370 E+00$

$R(4,2) * R(3,-1)=+R(1,1), 2.0374 E+00$ R(2. 1). 1.9965E*00 $R(3.1) *-1.2218 E+01$ $\rightarrow(40,1)=4.4854 E+00$ $+R(5,1)$
$+R(6,1)=-2.2409 E+00$

$R(4,2)+R(3, n)=+R(2,2)+-6.9160 E+00$ $\rightarrow R(3,2)+-3.3460 E+00$ $\rightarrow R(4.2)=1.1903 E+01$ $\rightarrow R(5,2):-9.4641 E-01$

$R(4,2) \cdot R(3,1)=+R(3,3), 1.4196 E+0)$ H(4, 3). 5.9337E+0O $+R(6,3) .0 .3038 E-01$

$R(4,2)+R(3,2)=+R(4,4):$ $+R(5,+)-5.9983 E+00$ $+R(6,4)$. $9.8193 E-01$

$-4 \cdot 3293 E+100 * x$ $-3.5570 E+00 * x$ $5.7261 E \bullet 01: x$ $2 \cdot 1924 E=01 \cdot x$

$3.4440 E+00 * x-1.6548 E=01 \div \times 2$ $1.6938 E+00: x$ $3.3082 E-02 * x$

$-9.7791 E-01: x$
$-2.75828-01: x$

$-5.6250 E-01 * x \quad 3.0952 E-01 * \times 2-4.7619 E-02+x 3$ $3.5569 E+00 \times x \quad-6.18546-01 \times x 2$ $-4.6206 E+00 * x \quad 2.2268 E-01 * x 2$

$1.8481 E+00 \cdot x$

$-2.1280 E-01 * x$

$1.9406 E+00 * x-2.0722 E+00 * \times 2$ $-4.7425 E+00 \times x \quad 8.2479 E-01 \times x 2$

$1.0891 E+00 * x$

$1.01226000 \div x$

$-2.1706 E+00 * x \quad 1.1980 E+00 * \times 2-1.0443 E-01 * x 3$

$20.20620 E=01$ :x2

$3.97602+00=x$

-5.72615001 ax

$5.3023 E+00 * x \quad-9.2214 E-01 * \times 2$

$2.0891 E+00 * x-5.2486 E-02 \times \times 2$

$1.5195 E+00: x$
$3.3572 E-02: x$

$-4.6206 E+00 * x \quad 2.2268 E-01 * x 2$

$-7.5749 E-01 * x$

$2.1365 E-01 \cdot x$

$1.6938 E+0.0+x$

$2.1233 E-01: x$ 


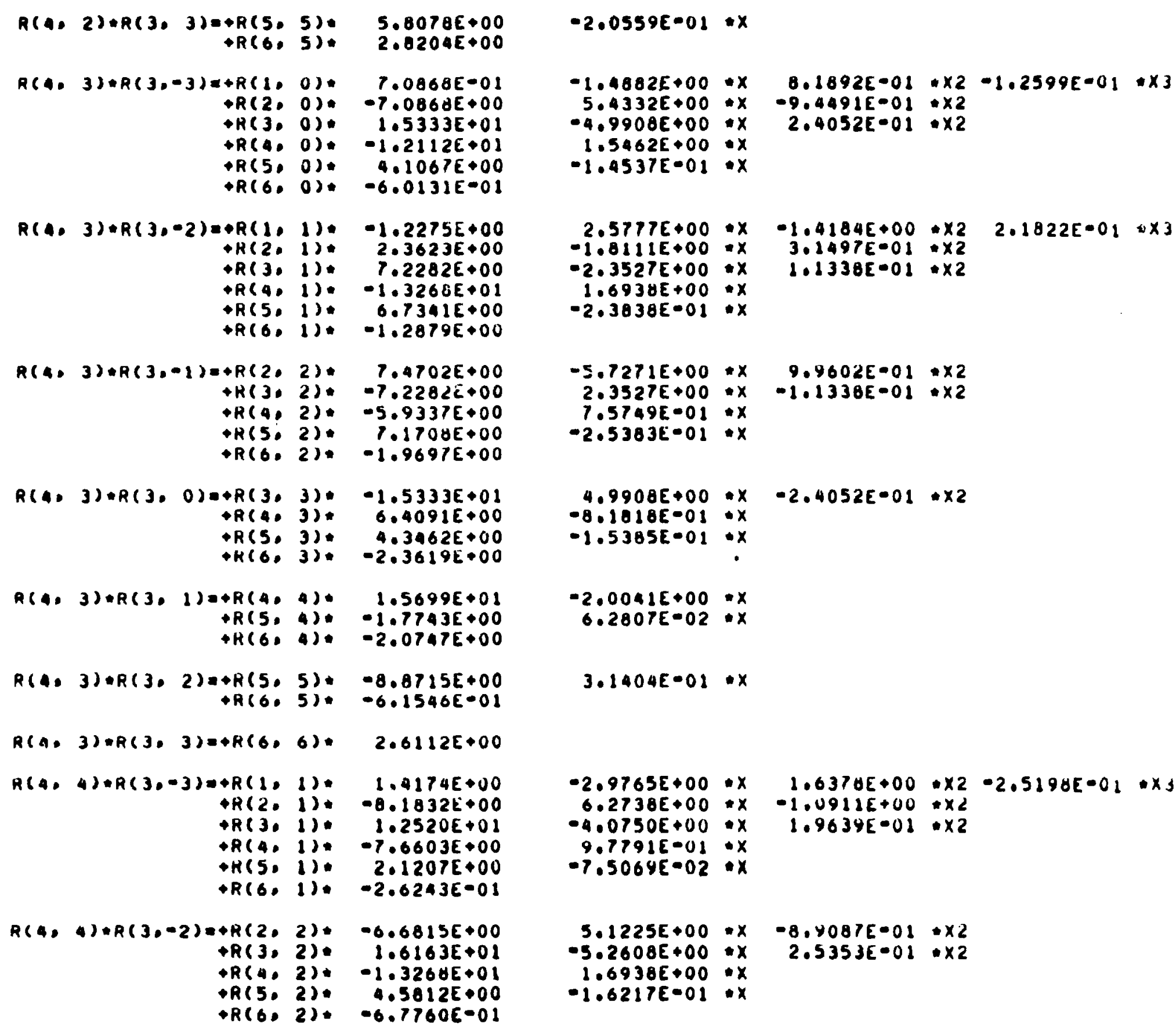

$R(4,2) \bullet R(3,3)=+R(5,5): \quad 5.807 \theta E+00$ $\rightarrow R(6,5), \quad 2.0204 E+00$

$R(4,3) \cdot R(3,-3)=+R(1,0), \quad 7.0868 E-01$ $\rightarrow R(2,0):-7.0868 E+00$ H(3. O) D. D.5333E+01 $R(4,0) .0102112 c+01$ $\begin{aligned} \rightarrow R(5, & 0): \quad 4.1067 E+00 \\ & -6.0131 E=01\end{aligned}$

$R(4,3) \otimes R(3,-2)=+R(1,1),-1.2275 E+00$ R(2. 1). 2.3623E+00 R(30 1) P.2202E+00 R(4. 1): $\rightarrow R(5,1): 0.7341 E+00$
$+R(6,1):-1.2879 E+00$

$R(4,3) \oplus R(3,-1)=+R(2,2), \quad T .4702 E+00$ $+R(3,2) .=7.22826+00$ पR( $+R(5,2): \quad 7.1708 E+00$
$+R(6,2) *-1.9697 E+00$

$R(4,3) * R(3,0)=+R(3,3) *-1.5333 E+01$ $\rightarrow R(4,3) * 6.4091 E+00$ $\rightarrow R(5,3): \quad 4.3462 E+00$
$+K(6,3)=-2.3619 E+00$

$A(4,3) \cap R(3,1)=+R(4,4) * 1.5699 E+0$ $+R(5,4):-1.7743 E+00$
$+R(6,4):-2.0747 E+00$

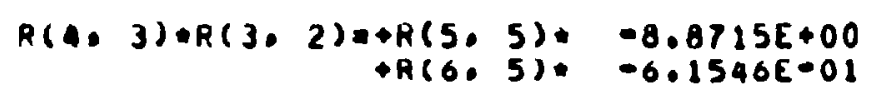
$R(A, 3) * R(3,3)=+R(6,6), \quad 2.6112 E+00$

$R(4,4)+R(3,-3)=+R(1,1), \quad 1,4174 E+00$ $4 R(2,1):-0.1832 E+00$ $+R(3.1) .1 .2520 E+0$ $+R(4,1)=-7.650$ $\rightarrow R(S) 1.) \quad 2.1207 E+00$ $\rightarrow R(6,1),-2.6243 E=0$

$R(4,4) \bullet R(3,-2)=+R(2,2) *-6.6815 E+00$ $\rightarrow R(3,2) . \quad 1.6163 E+0$ $\rightarrow R(4,2)+-1.3268 E+0$ $\rightarrow R(5,2)=9.5812 E+00$ $\rightarrow R(6,2) \cdot-6.7760 E=0$

$-2.0559 E-01 * x$

$-1.4682 E+00 * x \quad 8.1892 E-01 \times 2-1.2599 E-01 * \times 3$ $5.4332 E+00 * x \quad-9.4491 E-01: x 2$

$-4.9900 \varepsilon+00 \times x \quad 2.4052 E 001 \times x 2$

$-1.54625+00 \cdot x$

$2.57776+00 * x$ $-1.8111 E+00=x$
$-2.3527 E+00: x$ $-2.3527 E+00: x$
$1.6938 E+00: x$

$1.6938 E+00: x$
$-2.3838 E-01: x$

\section{$1.4184 E+00 * 2 \quad 2.1822 E=01 \approx \times 3$} $3.2497 E-01 * x 2$ $1.1338 E \cdot 01: \times 2$

$-5.7271 E+00 * x \quad 9.9602 E-01 * x 2$ $2.3527 E+00: x-1.1338 E-01: \times 2$ $7.5749 E-01: x$

$-2.5383 E-01 \cdot x$

$4.9908 E+00 * x-2.4052 E-01 * x 2$

$-8.1818 E-01 \cdot x$

$-1.5385 E-01 * x$

$-2.0041 E+00: x$

$6.2807 E=02 * x$

$3.1404 E-01 \cdot x$

$-2.9765 E+00 * x \quad 1.6370 E+00 * \times 2-2.5198 E-01 * x J$ $6.2738 E+00 \div x-1.0911 E+00 * x<$ $-4.0750 E+00 * x \quad 1.9639 E-01 * x 2$

$9.77916-01: x$
$-7.50695-02: x$

$5.1225 E+00 * x \quad-8.4087 E-01 * x 2$

$-5.2608 E+00 \times x \quad 2.5353 E-01 * x 2$

$1.6938 E+00$
$-1.6217 E-01: x$ 


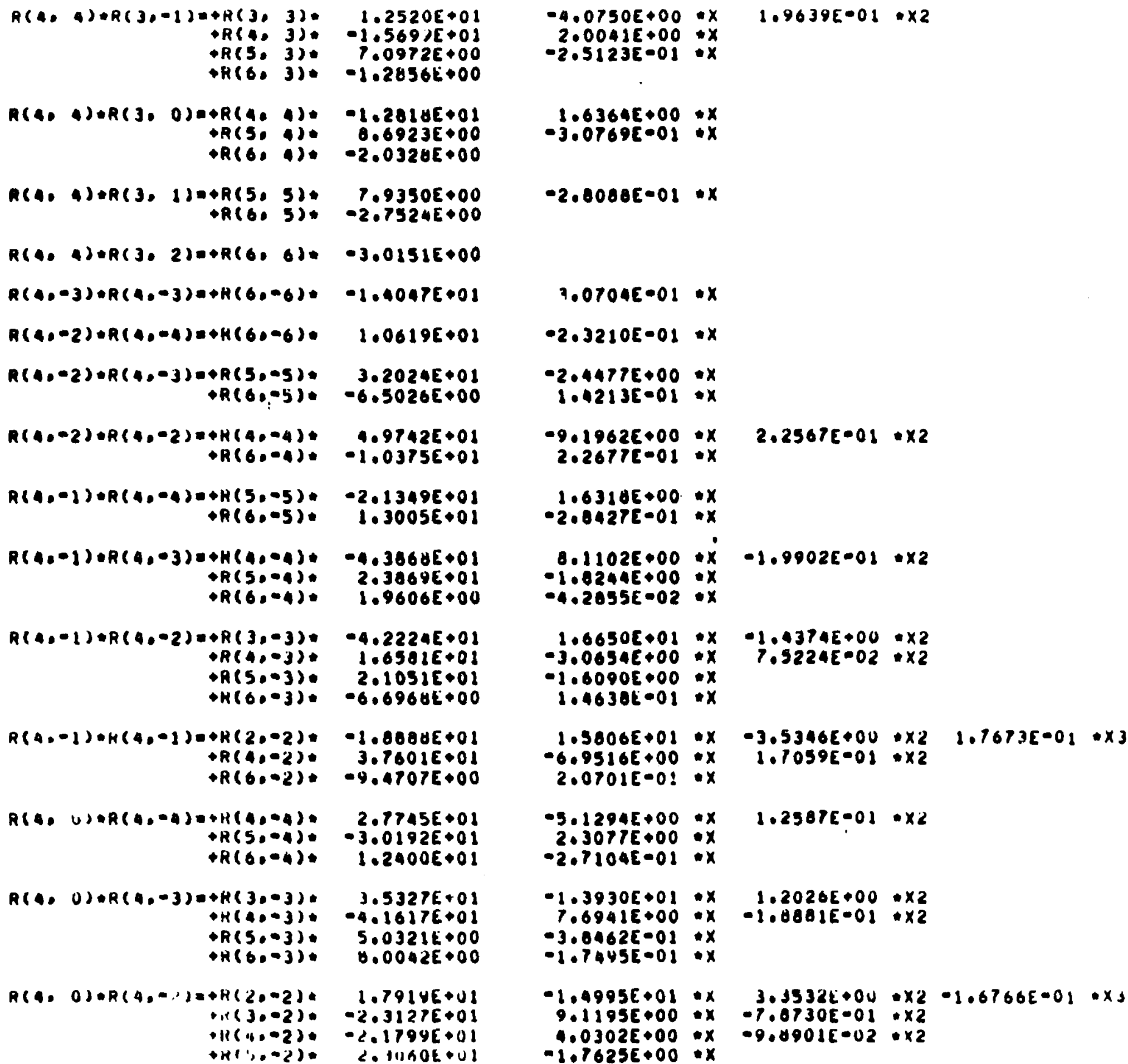

$R(4,0) \cdot R(4,-)=,+H(2,-2), \quad 1.7914 E+U)$ $\rightarrow(3 .-2),-2.31276+01$ $-11(16-2) \cdot-2.1799 E+01$

$-4.0750 E+00 \cdot x$ $2.00436+00 * x$

$1.63646+00 * x$ $1.63646+00 * x$
$-3.0769 E-01: x$

$-2.0088 E-01 * x$

$9.0704 E \cdot 01 * x$

$-2.3210 E-01 \cdot x$ $-2.44776+00 * x$ $1.42135-01$ * $x$

$-9.19626+00 \cdot x$ $2.26776-01+x$

$1.63105+00 * x$ $-2.0427 E-01: x$

$0.1102 E+00+x-1.9902 E-01+\times 2$

$-1.0244 E+00 * x$ $-4.2055 E-02$ : $x$

$1.6650 E+01 * x-1.4374 E+00 * \times 2$ $-3.06546+00 * x \quad 7.5224 E-02 * x 2$ $-1.6090 E+00 * x$ $1.46306-01: x$

$1.5006 E+01 * x-3.5346 E+00 * \times 2 \quad 1.7673 E-01 * \times 3$ $-6.9516 E+00 * x \quad 1.7059 E-01 * x 2$ $2.07015-0: * x$

$-5.1294 E+00 \cdot x \quad 1.2587 E-01 \cdot \times 2$ $2.3077 E+00$ \& $x$ $-2.7104 E-01 \cdot x$

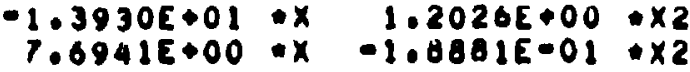
$-3.0462 E-01 * x$ $-1.74455001 \cdot x$

$-1.4995 E+01 * x \quad 3.4532 E+04+x 2-1.6766 E-01 * x s$ $9.1195 E+00 * x-7.0730 E-01: \times 2$

$4.0302 E+00 * x \quad-9.8901 E-02 * x 2$

$-1.7625 E+00: x$ 


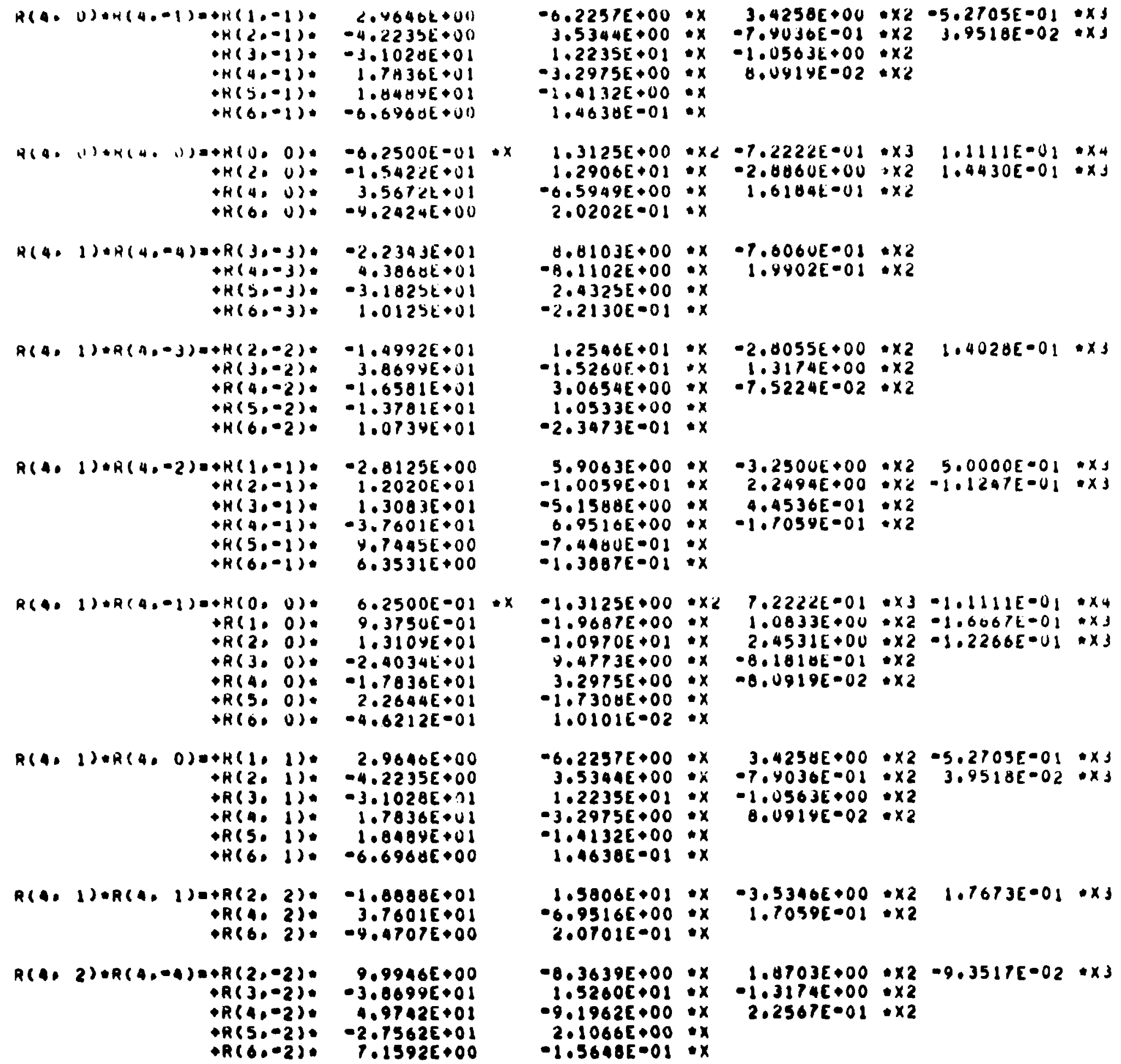

$1.4992 E+01$ $\rightarrow R(4,-2)=-1.6581 E+01$ $+R(5,-2),-1.3781 E+02$ $+H(6,-2) \cdot$

$1.0739 E+01$

$R(4,1)+R(4,-2)=+H(1,-1)$ $H(2 .-1)=1.2020 E+01$ $H(30-1)=1.3003 E+01$ H. $4.7445 E+00$

$6.2237 E+00 * x$ $3.5344 E+00: x$ $1.2235 E+01: x$ $=3.2975 E+00: x$ $-1+0132 E+00: x$
$1.4638 E-01: x$

$1.3125 E+00 * x<-7.2222 E-01 * x 3 \quad 1.1111 E=01 * x_{4}$ $1.2906 E+01+x-2.0060 E+00 \geqslant x 2$ $-6.5949 E+00 * x \quad 1.6184 k=01 * x$

$2.0202 E \cdot 01 \cdot x$

$0.8103 E+00 * x-7.6060 E=01: x 2$

$-8.1102 E+00: x \quad 1.9402 E=01: x$

$2.4325 E+00: x$

$-2.2130 E=01 \cdot x$

$1.2540 E+01 * x-2.8055 E+00 \times 2 \quad 1.4028 E-01 * 3$ $1.5260 F+01 * x$ $3.0654 E+00 * x$ $1.0533 E+00: x$ $-2.34735-01: x$

$5.9063 E+00 * x-3.2500 E+00 \times 2 \quad 5.0000 E-01 * \times 1$ $-1.0059 E+01: x \quad 2.2494 E+00: x^{2}-1.1247 E-01 * x 3$ - 5015805000 : $x$ $0.9510 E+00: x$ $-7.448 U E-01 \cdot x$ $-1.30875001: x$

$4.4536 E-01 \cdot x$

$6.3531 E+00$

$R(4,1) \otimes R(4,-1)=+H(0,0)$. $+R(1,0):$
$+R(2,0)$ - $R(3,0)$. $\rightarrow R(4,0)$. $\rightarrow R(5,0)$. $6.2500 E-01 . x$ $9.3750 E-01$

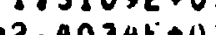
$1.70305+01$ $1.7036 E+01$ $-4.62125=01$ $+R(0,0)$.

$R(4,1)+R(4,0)=+R(1,1)$. $H(2,1)=-4.2235 E+00$ R(3. 1): -3.1028E+31 R(5. 1).

$R(0,1) \cdot R(4,1)=+R(2,2),-1.0888 E+0)$

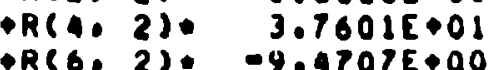

$R(4,2) \cdot R(4,-4)=+R(2,-2)$.

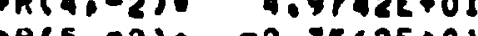
$\because R(5,-2),-2.7562 E+01$

$0.1 .31255000 \cdot x$ $-1.9687 E+00 * x$ $0.1 .09705+01: x$ $9.47735+00 * x$ $3.29755+00 * x$ $1.73005000 \times x$ $1.01016=02 * x$

$-6.2257 E+00 \cdot x$ $3.5344 E+00: x$ $1.2235 E+01 * x$ $-3.2975 E+00 * x$ $-1.4132 E+00 \cdot x$ $1.4638 E-01 * x$

$1.58065001 \cdot x$ $-6.9516 E+00: x$ $2.07016-01: x$
$2.01 * x$

$-0.3639 E+00 * x \quad 1.4703 E+00 * x-9.3517 E-02 * x 3$ $0.36306000 x$ $0.19625+00$ :x $2.10665+00$ *x $-1.5648 E-01: x$

$1.2222 E-01 * 33=1.11112 E-01 * x_{4}$ $1.0833 E+00=\times 2-1.6067 \mathrm{k}=01 \times \times 3$ $2.4531 E+0 U+\times 2-1.2266 E-U 1 \times 1$ $0.1810 E-01$ *x $-0.0919 E=02 * x 2$

$3.4258 E+00 \times 2-5.2703 E=01 \times 3$ $-7.4030[-01 \cdot \times 2 \quad 3.9518 E-02 \times x 3$ $-1.0563 E+00 \cdot \times 2$ $0.0914 E-02 \cdot \times 2$

$-3.5340 E+00 \div \times 2 \quad 1.7673 E \cdot 01 * \times 3$ 1.7059500

$1.31746000 \div \times 2$

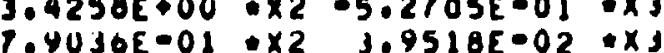

$1.3174 E+00: \times 2$ 


\begin{tabular}{|c|c|c|c|c|c|c|c|c|c|c|}
\hline 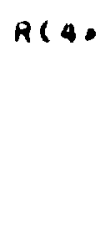 & $R(4 .-3)=$ & $\begin{array}{l}+R(1 .-1): \\
+R(20-1): \\
+R(30-1): \\
+R(4 .-1) \\
+H(5,-1) \\
+R(6 .-1):\end{array}$ & $\begin{array}{r}2.4804 E+00 \\
-1.7668 E+01 \\
1.7307 E+01 \\
1.6581 E+01 \\
-2.57826+01 \\
1.0405 E+01\end{array}$ & & $\begin{array}{r}-5.2088 E+00 \\
1.4786 E+01 \\
-6.02446+00 \\
-3.0654 E+00 \\
1.9706 E+00 \\
-2.2744 E-01\end{array}$ & $\begin{array}{l}: x \\
: x \\
: x \\
* x \\
* x \\
* x\end{array}$ & $\begin{array}{r}2.0662 t+00 \\
-3.3063 E+00 \\
3.0910 E=01 \\
7.5224 E-02\end{array}$ & $\begin{array}{l}\times 2 \\
\times 2 \\
\times 2 \\
\times 2 \\
\times 2\end{array}$ & $\begin{array}{r}-4.4090 E=01 \\
1.6532 E-01\end{array}$ & $\begin{array}{l}x y \\
x y \\
x y\end{array}$ \\
\hline$R<40$ & $2) \cdot R(4,-2)=$ & 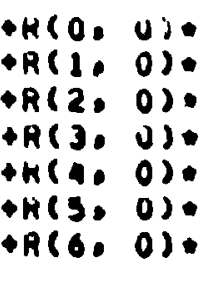 & $\begin{array}{r}-6.2500 E-01 \\
-1.8750 E+00 \\
-6.1680 E+00 \\
3.4716 E+01 \\
-2.17994 E+01 \\
-1.0064 E+01 \\
1.0167 E+01\end{array}$ & $x$ & $\begin{array}{r}1.3125 E+00 \\
3.9375 E+00 \\
5.1623 E+00 \\
-1.3689 E+01 \\
4.0302 E+00 \\
7.6923 E-01 \\
-2.2222 E-01\end{array}$ & $\begin{array}{l}: x z \\
: x \\
: x \\
: x \\
: x \\
: x \\
x \\
x\end{array}$ & $\begin{array}{r}-7.2222 E-01 \\
-2.1607 E+00 \\
-1.1344 E+00 \\
1.1010 E+00 \\
-9.0901 E-02\end{array}$ & $\begin{array}{l}* \times 3 \\
\times \times 2 \\
0 \times 2 \\
\times 2 \\
\times 2\end{array}$ & $\begin{array}{l}1.1111 E=01 \\
3.3333 E=01 \\
3.7720 E=02\end{array}$ & $\begin{array}{l}0 \times 4 \\
x y \\
x y \\
x y\end{array}$ \\
\hline$R<4$. & $2) \cdot R(4,-1)=$ & $\begin{array}{l}+R(1,1) \\
+R(2,1) \\
+R(30,1) \\
+R(A \circ \\
+R(5,1) \\
+R(6,1):\end{array}$ & $\begin{array}{r}-2.8125 E+00 \\
1.2020 E+01 \\
1.303 E+01 \\
-3.7601 E+01 \\
9.7495 E+00 \\
6.3531 E+00\end{array}$ & & $\begin{array}{r}5.9003 E+00 \\
-1.00396+01 \\
-3.1500 E+00 \\
6.9516 E+00 \\
-7.44006-01 \\
-1.3087 E-01\end{array}$ & $\begin{array}{l}: x \\
0 x \\
0 x \\
0 x \\
: x \\
0 x\end{array}$ & $\begin{array}{r}-3.2500 E \bullet 00 \\
2.2494 E+00 \\
-.45368-01 \\
-1.1054 E-01\end{array}$ & $\begin{array}{l}: \times 2 \\
\times \times 2 \\
\times \times 2 \\
\times \times 2\end{array}$ & $\begin{array}{l}0 E=01 \\
P E=01\end{array}$ & $\begin{array}{l}x_{x} \\
03 \\
x 3\end{array}$ \\
\hline RCA. & $2) \cdot R(4,0)=$ & $\begin{array}{l}+R(2,2) \\
+R(302) \\
+R(40,2) \\
+R(5,2)\end{array}$ & $\begin{array}{r}1.7919 E+01 \\
-2.3127 E+01 \\
-2.1799 E+01 \\
2.3060 E+01\end{array}$ & & $\begin{array}{r}-1.4995 E \diamond 01 \\
9.11956+00 \\
4.03025 \ngtr 00 \\
-1.7025 E \$ 00\end{array}$ & $\begin{array}{l}: x \\
: x \\
0 x \\
0 x\end{array}$ & $\begin{array}{r}3.3532 E+00 \\
-7.6730 E-01 \\
-9.8901 E-02\end{array}$ & $\begin{array}{l}\times 2 \\
\times 2 \\
\times 2 \\
\times 2\end{array}$ & $-1.6766 E=01$ & $\times 3$ \\
\hline ACA. & $2) \times R(4,1)=$ & $\begin{array}{l}+R(3.3) \\
+R(4.3) \\
+R(5.3) \\
+R(6.3)\end{array}$ & $\begin{array}{r}-1.2224 E+01 \\
1.6581 E+01 \\
2.1051 E+01 \\
-6.6968 E+00\end{array}$ & & $\begin{array}{r}1.6650 E+01 \\
-3.0654 E+00 \\
-1.6090 E+00 \\
1.0638 E-01\end{array}$ & $\begin{array}{l}\cdot x \\
0 x \\
0 x \\
0 x\end{array}$ & $\begin{array}{l}A E \bullet 00 \\
A E-02\end{array}$ & $\begin{array}{l}\times 2 \\
\times 2\end{array}$ & & \\
\hline c & $2) \cdot R(4,2)=$ & $\begin{array}{l}+R(4,4)= \\
\rightarrow R(6,4)=\end{array}$ & $\begin{array}{r}1.97 A 2 E \bullet 01 \\
-1.0375 E \bullet 01\end{array}$ & & $\begin{array}{r}-9.1962 E+00 \\
2.2677 E=01\end{array}$ & $\cdot x$ & $2.2567 E-01$ &.$\times 2$ & & \\
\hline 14. & $3) \times R(4,-4)=$ & $\begin{array}{l}+R(1,-1) \\
+R(2,-1) \\
+R(3,-1) \\
+R(4,-1) \\
+R(5,-1) \\
+R(6,-1)\end{array}$ & $\begin{array}{r}-1.0730 E \bullet 00 \\
1.0698 E \bullet 01 \\
-4.5709 E \bullet 01 \\
4.3060 E \bullet 01 \\
-1.9409 E \bullet 01 \\
-.235 \backsim E \bullet 00\end{array}$ & & $\begin{array}{r}3.9375 E+00 \\
-1.5648 E+01 \\
1.8056 E+01 \\
-8.1102 E+00 \\
1.0096 E+00 \\
-9.2577 E-02\end{array}$ & $\begin{array}{l}: x \\
: x \\
: x \\
: x \\
0 x \\
: x\end{array}$ & $\begin{array}{r}-2.1667 E \bullet 00 \\
3.4991 E \bullet 00 \\
-1.3580 E \bullet 00 \\
1.9902 E 001\end{array}$ & $\begin{array}{l}\times 2 \\
\times 2 \\
\times 2 \\
\times 2 \\
\times 2\end{array}$ & $\begin{array}{r}3.33 \\
-1.74\end{array}$ & $\begin{array}{l}0 \times 3 \\
0 \times 3\end{array}$ \\
\hline$(A)$ & $3)+A(4,-3)=$ & 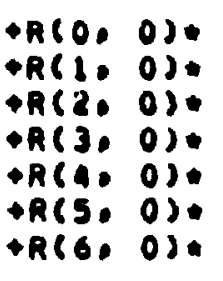 & $\begin{array}{r}6.2300 E-01 \\
2.8125 E+00 \\
-5.3971 E+00 \\
-1.8693 E+01 \\
1.1617 E+01 \\
-2.7676 E+01 \\
7.8561 E+00\end{array}$ & - & $\begin{array}{r}-1.3125 E+00 \\
-5.9063 E+00 \\
1.5170 E+00 \\
7.3712 E+00 \\
-7.69416+00 \\
2.11546+00 \\
-1.7172 E-01\end{array}$ & $\begin{array}{l}: x 2 \\
: x \\
: x \\
: x \\
: x \\
: x \\
: x \\
: x\end{array}$ & $\begin{array}{r}P .2222 E \bullet 01 \\
3.2500 E \bullet 00 \\
-1.0101 E \bullet 00 \\
-6.3636 E=01 \\
1.0001 E-01\end{array}$ & $\begin{array}{r}\times 3 \\
\times 2 \\
\times 2 \\
\times 2 \\
\times 2 \\
\times 2\end{array}$ & $\begin{array}{r}1.11111 E-01 \\
-5.0000 E-01 \\
5.0505 E-02\end{array}$ & $\begin{array}{l}: \times 4 \\
0 \times 3 \\
0 \times 3 \\
\times 1 \times 3\end{array}$ \\
\hline
\end{tabular}




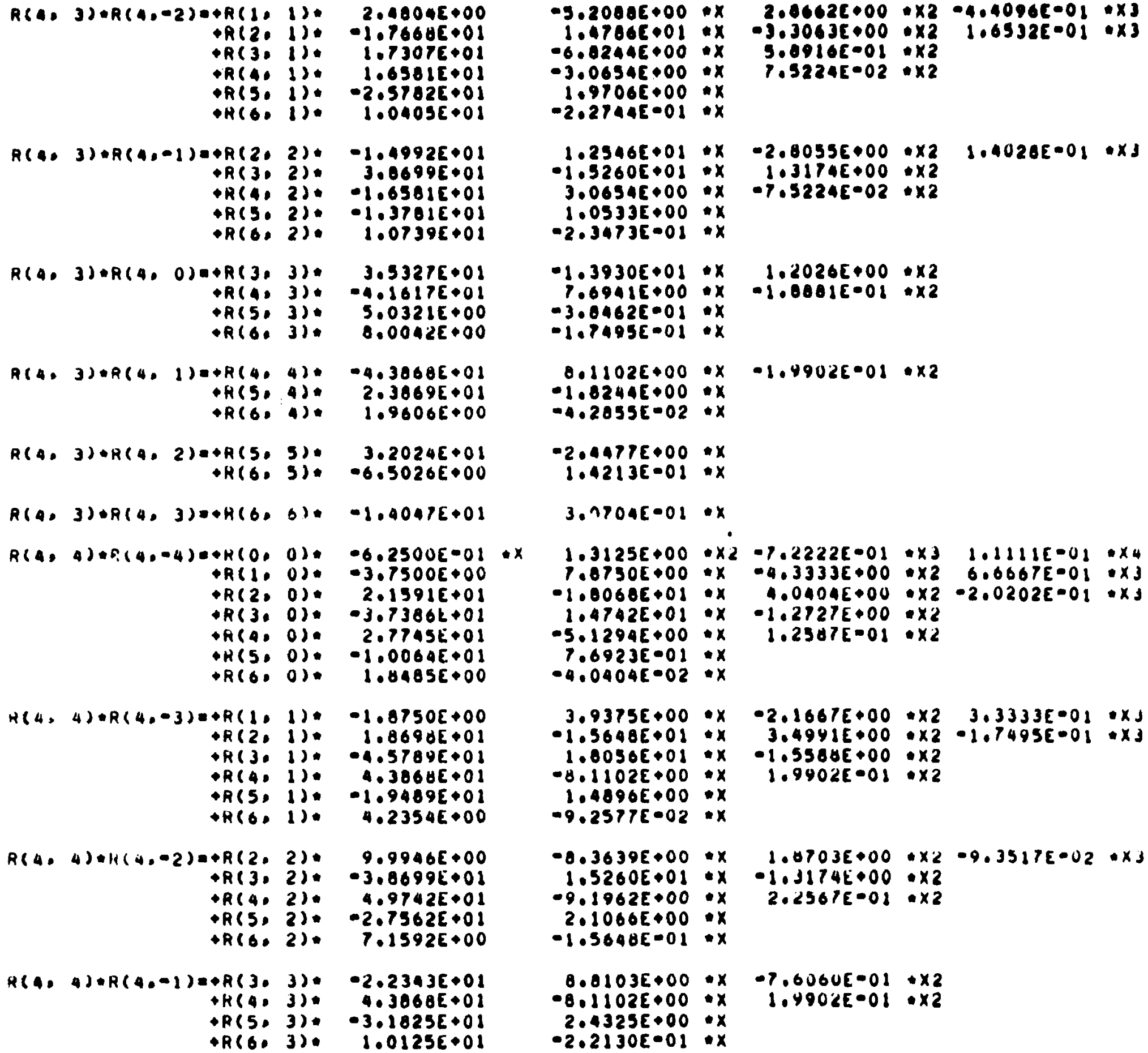

$R(4,3)+R(4,-2)=+R(1,1), 2.4004 E+00$ $+R(2,1)=-1.7668[+0)$ $+A(3,1) \quad 1.7307 E+0)$ $\rightarrow R(4)$ 1) $1.6581 E+0)$ $+A(5,1)$. $-2.57025+0$ QH(G. 1). 1.040SE+0)

$R(4,3) \cap R(4,-1)=+R(2,2)$ $+R(3,2)=3.0699 E+01$ PR(41. 2) - $1.65815+0$ $+R(5,2):-1.37015+01$ $\rightarrow R(6,2)$. $1.0739 E+0$

$R(4,3) \div R(4,0) 0+R(3,3), 3.53275+02$ $\rightarrow R(4,3)=-4,1617 E+01$ $\rightarrow R(5,3)=5.03215+00$ $\because R(6,3): 0.0042 E+00$

$R(4,3)+R(4,1)=+R(4,4):-4,3068 E+0$ $\begin{array}{ll}+H(5,4): & 2.3869 E+0) \\ +R(6, & 1.96065+00\end{array}$

$\begin{array}{rlr}R(4,3) * R(4,2)= & +A(5,5): \quad 3.2024 E+01 \\ & +H(6,3) \cdot-6.5026 E+00\end{array}$ $+A(6,3)=-6.50262000$

$R(4,3) \circ R(4,3)=+H(6,6),-1,404) E+0)$

$R(4,4)+R(4,-4)=+H(0,0),-6.2500 E-01$ *X $\rightarrow R(1,0)=-3.7500 E+00$ $\rightarrow R(2,0): 2.15916+0)$ -R(3. O). -3.7386k+0 $\rightarrow$ $(A, 0)$. $2.7745 E+01$ $\rightarrow H(5,0)=-1.0064 E+01$ $\rightarrow R(6,0) \% \quad 1.0485 E+00$

$H(4,4)+R(4,-3)=+R(1,1),-1.8750 E+00$ $+R(2,1)=1.8690 E+01$ $R(301) .0 .57005+0$ $\rightarrow R(A, 1)$ a. $3864 E+0$ $R(5,1) .1 .9489 E+0$ $\rightarrow R(6,1)=4.23545+00$

$R(4,4)+1((4,-2)=+R(2,2) * 9.9946 E+00$ $+R(3,2)=-3.8699 E+0$

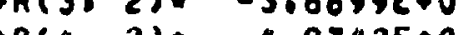
$R(5,2)=0.75625001$ $+R(6,2)$. $7.1592 E+00$

Q(4) 4$)+R(4,-1)=+R(3.3) *-2.2343 E+01$ $+K(4,3)=4.3068 E+0$ $+R(5,3)=-3.1825 E+0$ $\rightarrow R(6,3): 1.0125 E+01$

$-5.20006+00$ ox $1.97065+01: x$ $-6.02446+00 * x$ $-3.06545+00$ :x

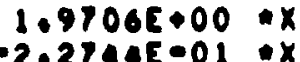
$-1.3260 E+01$. $x$ $3.06546+00 * x$ $1.0533 E+00: x$ $-2.3473 E-01$ : $x$ $-1.3930 E+01: x \quad 1.20265+00 * \times 2$
$\$ .6941 E+00 * x \quad-1.0001 E=01 * \times 2$ $-3.04626 \cdot 01 \cdot x$ $-1.74955=01 \cdot x$

$0.1102 E+00 \cdot x \cdot 1.4902 E-01 \cdot \times 2$ $-1.0244 E+00 * x$ $-4.2055 E-02 * x$

$-2.447 T E \bullet 00$
$1.4213 E=01 * x$ $3.17045 \cdot 01 \cdot x$

\section{$-2.80556+00 \times 2 \quad 1.4026 E-01 * x]$}

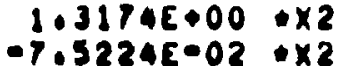

$1.20265+00 \times 2$

源 $1.0730 E+00 * x-4.3333 E+00 * \times 2 \quad 6.6067 E-01 * x J$ $-1.0060 E+01: x \quad 4.0404 E+00 * x 2-2.0202 E=01 * x J$ $1.4742 E+01$ : $\quad-1.2727 E+00: \times 2$

$7.6923 E \cdot 01 * x$

$0.0404 E-02 . x$

$3.9375 E+00$ * $\quad-2.1667 E+00 \quad \times 2 \quad 3.3333 E-01 * x J$ $-1.5648 E+01 * x \quad 3.4941 E+00 * x 2 \cdot 1.7495 E \bullet 01 * x 3$ $1.0056 E+01: x-1.5588 E+00 * x 2$ $-8.1102 E+00 * \times \quad 1.9402 E-01 \cdot \times 2$

$1.4896 E \bullet 00: x$

$-9.2577 E \cdot 02: x$

$-0.3639 E+00 * x \quad 1.4703 E+00 * \times 2-9.3517 E-02 * x 3$ $1.5260 E+01 * x-1.3174 E+00 \cdot x 2$ $-9.1962 E+00 * x \quad 2.2567 E=01: \times 2$

$2.10665+00: x$

$-1.50085001 \cdot x$

$0.0103 E+00 * x-7.6060 E-01+\times 2$ $-8.1102 E \bullet 00$ * $1.4902 E 001 \cdot x 2$ $2.4325 E+00: x$

$0.21305001: x$ 


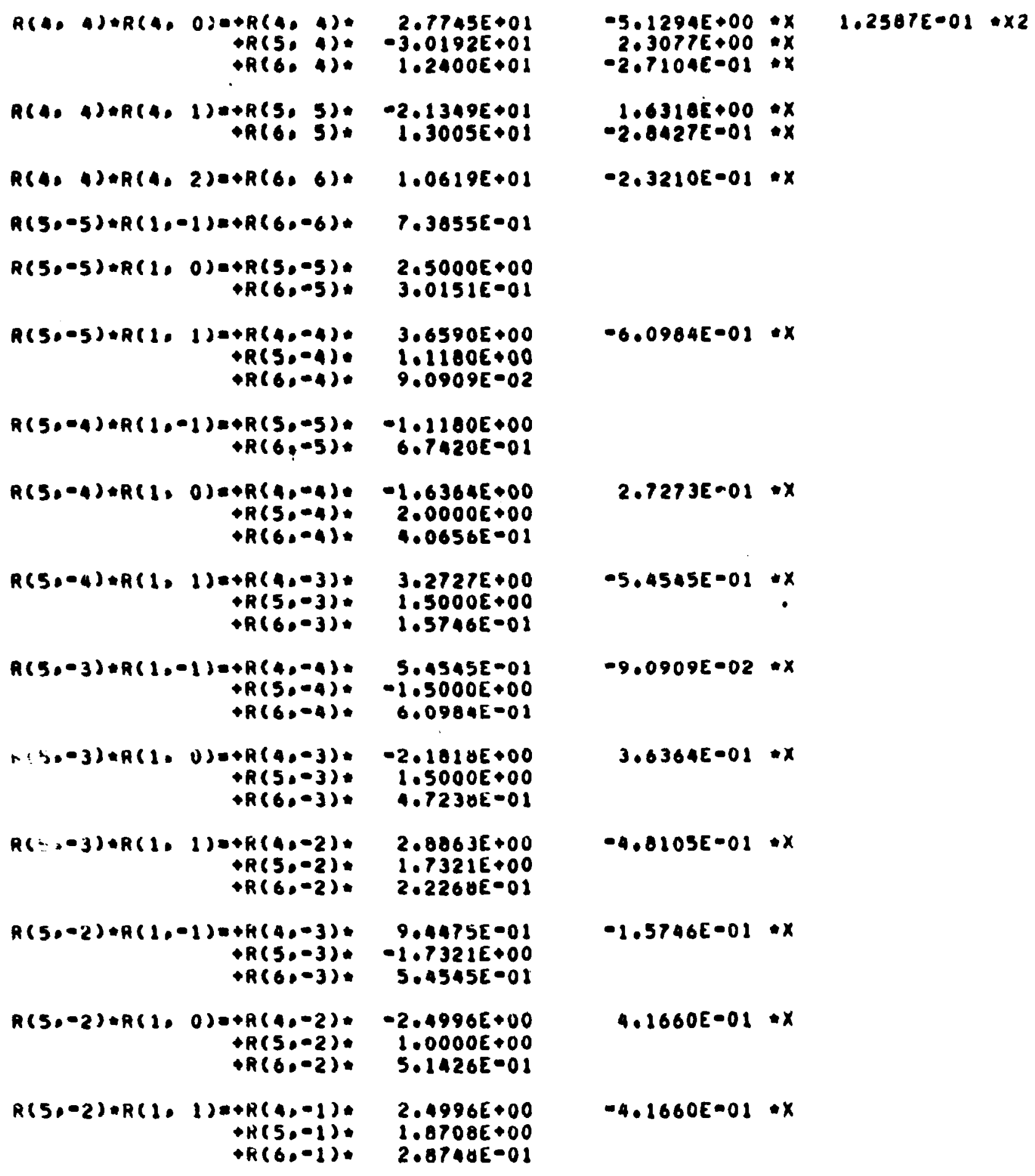

$-5.1294 E+00 * x$

$2.30776+00 \cdot x$

$-2.71046-01 \cdot x$

$1.63186+00$
$-2.8427 E-01: x$

$-2.3210 \varepsilon-01 \cdot x$

$-6.0984 E-01 * x$

$2.72735001 * x$

$-5.4545 E-01 \cdot x$

$1.2507 E-01 * 22$

.

$-9.0909 E-02 * x$

$3.6364 E-01 * x$

$-4.0205 E-01 * x$

$-1.5746 E-01 * x$

$4.1660 E-01 \cdot x$

$-4.1660 E=01 \cdot x$ 


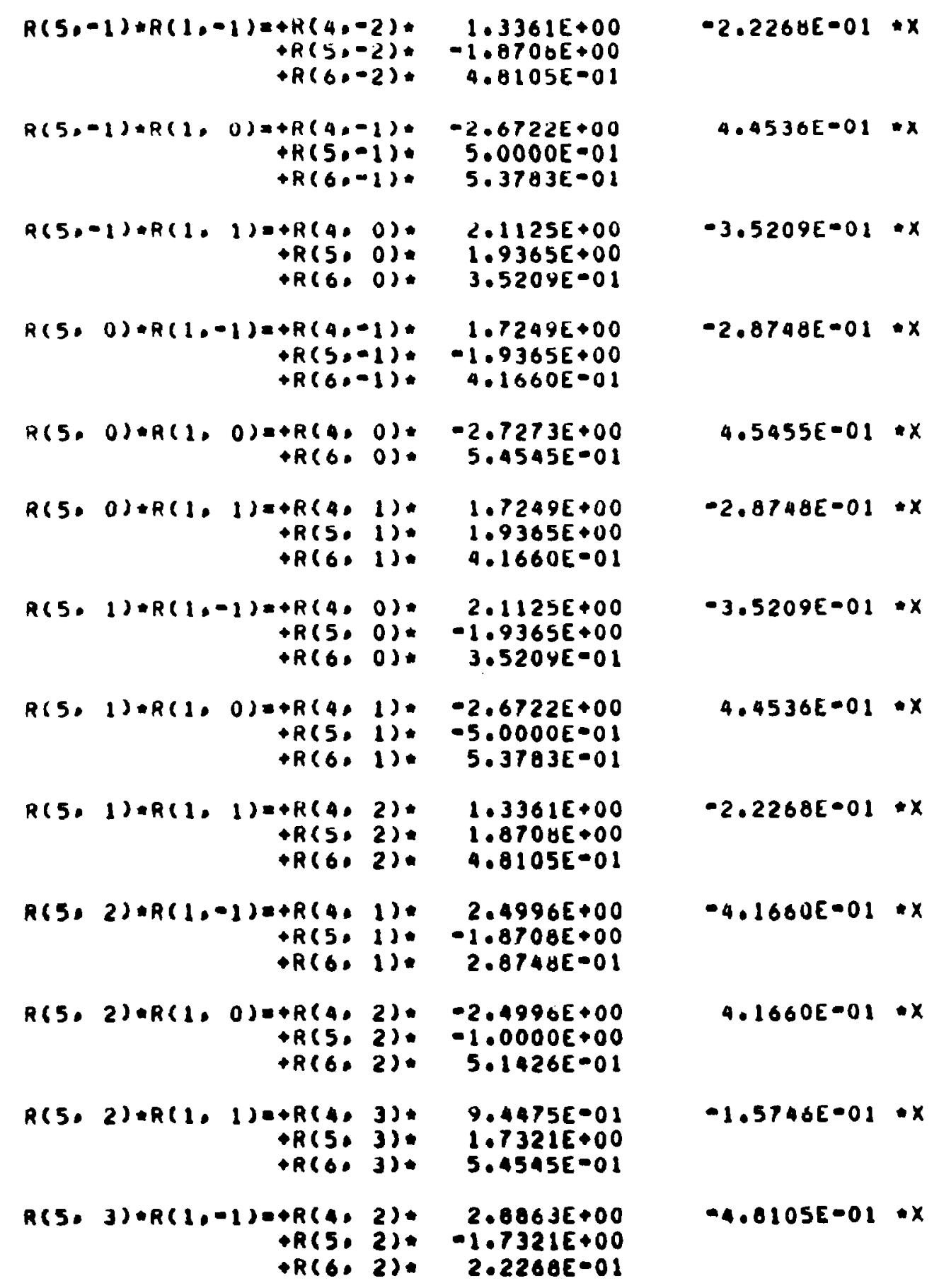




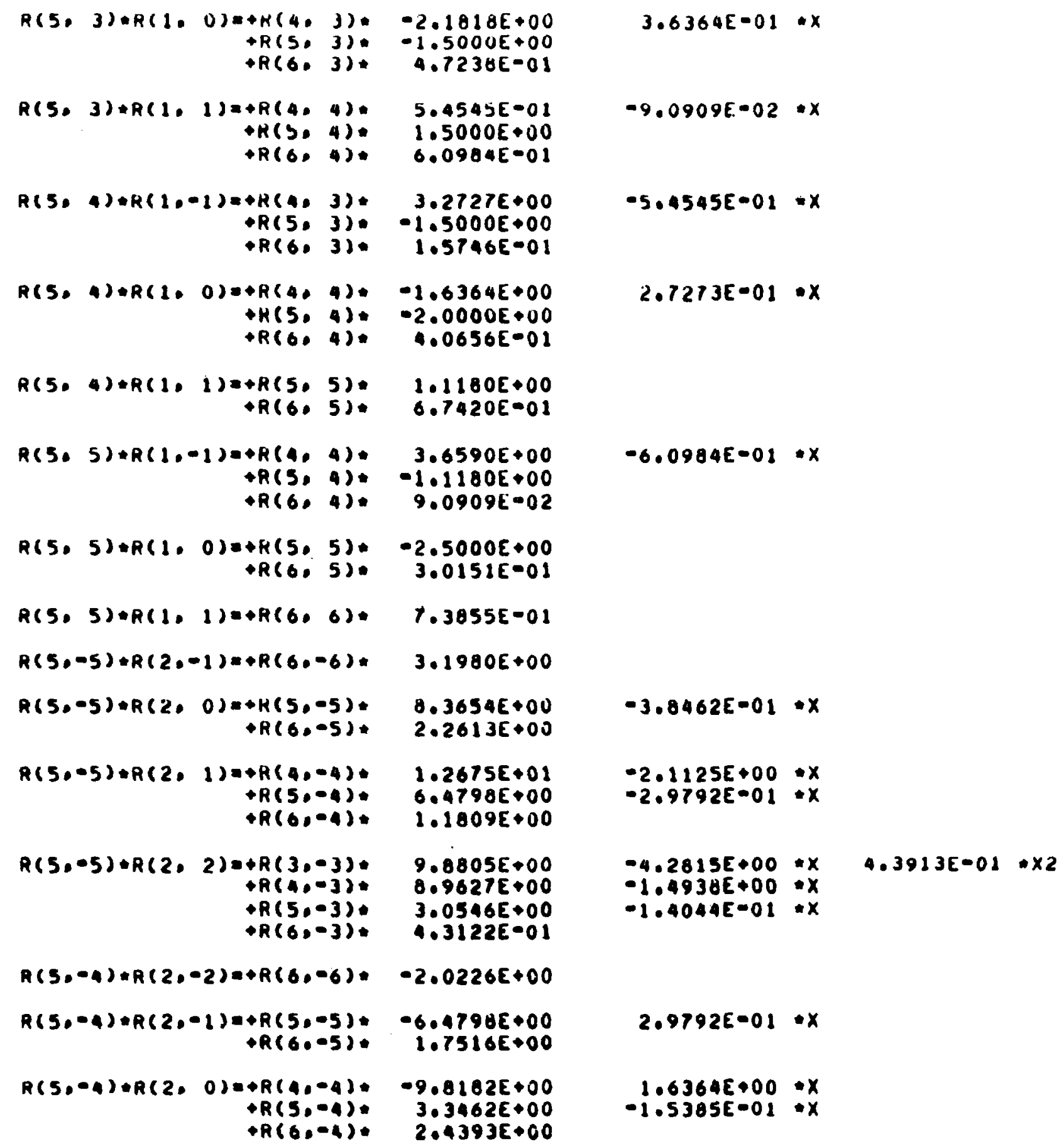

$3.6364 E-01 * x$

$-9.0909 E .02 \cdot x$

$-5.4545 E-01-x$

$2.7273 E-01 \cdot x$

$-6.0984 E-02 \cdot x$

$-3.8462 E-01 * x$

$-2.1125 E+00: x$

$-4.2815 E+00 * x \quad 4.3913 E=01 * \times 2$

$-1.4938 E+00 \cdot x$

$-1.4044 E=01: x$

$2.9792 E-01 \cdot x$

$1.6364 E+00 * x$
$-1.5385 E-01 * x$ 


\begin{tabular}{|c|c|c|c|c|c|c|}
\hline$R(5,-4) \bullet R(2$, & $\begin{aligned}1)= & +R(3,-3)= \\
& +R(4,-3): \\
& +R(5,-3)= \\
& +H(6,-3)=\end{aligned}$ & $\begin{array}{r}-6.2490 E+00 \\
5.6685 E+00 \\
6.7617 E+00 \\
1.7727 E+00\end{array}$ & $\begin{array}{r}2.7079 E+00 \\
-9.4475 E=01 \\
-3.1080 E-01\end{array}$ & $\begin{array}{l}x \\
i x \\
x\end{array}$ & $-2.1773 E-02$ & $+\times 2$ \\
\hline$A(5,-4) \cdot R(2)$ & $\begin{aligned}2)= & +A(3,-2) \\
& +R(1,-2) \\
& +R(5,-2) \\
& +R(6,-2)\end{aligned}$ & $\begin{array}{l}7.6534 E+00 \\
1.0605 E+01 \\
1.7322 E+00 \\
0.1018 E-01\end{array}$ & $\begin{array}{l}-3.3165 E+00 \\
-1.7675 E+00 \\
-2.1757 E-01\end{array}$ & $\begin{array}{l}x \\
: x \\
: x\end{array}$ & $3.4015 E-01$ & $\times 2$ \\
\hline$A(5 \cdot-3) \cdot R(2$ & $\begin{aligned}-2)= & +R(5,-5) \\
& +R(6,-5)\end{aligned}$ & $\begin{array}{r}3.0546 E+00 \\
-2.4772 E+00\end{array}$ & $-1.4044 E=01$ & $\cdot x$ & & \\
\hline$R(5,-3) \cdot R(2$ & $\begin{aligned}1)= & +R(4,-1) \\
& +R(5,-4): \\
& +R(6,-4):\end{aligned}$ & $\begin{array}{r}5.6605 E+00 \\
-6.7617 E+00 \\
3.2813 E-01\end{array}$ & $\begin{array}{r}-9.4475 E-01 \\
3.1008 E-01\end{array}$ & $: x$ & & \\
\hline$R(5,-3) \bullet R(2$, & $\begin{aligned}0)= & +R(3,-3) \\
& +R(4,-3) \\
& +R(5,-3) \\
& +R(6,-3)\end{aligned}$ & $\begin{array}{r}3.6076 E+00 \\
-9.8102 E+00 \\
-5.5769 E-01 \\
2.1257 E+00\end{array}$ & $\begin{array}{r}-1.5634 E+00 \\
1.6364 E+00 \\
2.5641 E-02\end{array}$ & $\begin{array}{l}: x \\
: x \\
x\end{array}$ & $1.6035 E-01$ & $\cdot \times 2$ \\
\hline$R(5,-3) \cdot R(2)$ & (1) $\begin{aligned} & +R(3,-2) \\
& +R(5,-2) \\
& +A(6,-2)\end{aligned}$ & $\begin{array}{r}-7.2157 E+00 \\
5.5769 E+C 0 \\
2.1213 E+00\end{array}$ & $\begin{array}{r}3.1268 E+00 \\
-2.5641 E-01\end{array}$ & $: x$ & $-3.2070 E-01$ & $\cdot \times 2$ \\
\hline$R(5,-3) \cdot R(2$, & $\begin{aligned}2)= & +R(3,-1) \\
& +R(4,-1) \\
& \mapsto R(5,-1) \\
& \mapsto R(6,-1)\end{aligned}$ & $\begin{array}{l}5.7045 E+00 \\
1.0605 E+01 \\
5.90216+00 \\
1.2197 E+00\end{array}$ & $\begin{array}{l}-2.4720 E+00 \\
-1.7675 E+00 \\
-2.7136 E-01\end{array}$ & $\begin{array}{l}\cdot x \\
: x \\
0 x\end{array}$ & $2.5353 E=01$ & $1 \times 2$ \\
\hline$R(5,-2) \cdot R(2$, & $\begin{aligned}-2) & +R(1,-1) \\
& +R(5,-4): \\
& +R(6,-4):\end{aligned}$ & $\begin{array}{r}-2.3142 E+00 \\
4.7322 E+00 \\
-2.3873 E+00\end{array}$ & $\begin{array}{r}3.8569 E-01 \\
-2.1757 E-01\end{array}$ & $: x$ & & \\
\hline$R(5,-2) \cdot R(2,-C$ & $\begin{aligned}-110 & +R(3,-3) \\
& +R(1,-3) \\
& +R(5,-3) \\
& +R(6 .-3)\end{aligned}$ & $\begin{array}{r}-1.8039 E+00 \\
6.1810 E+00 \\
-1.3769 E+00 \\
-4.7238 E-01\end{array}$ & $\begin{array}{r}7.0170 E-01 \\
-1.3636 E+00 \\
2.5641 E-01\end{array}$ & $\begin{array}{l}\cdot x \\
: x \\
: x\end{array}$ & $-0.0174 E-02$ & $\cdot \times 2$ \\
\hline$R(5,-2) \cdot R(2$, & $\begin{aligned}0)= & +R(3,-2) \\
& +R(4,-2) \\
& \leftrightarrow R(5,-2) \\
& \leftrightarrow R(6,-2)\end{aligned}$ & $\begin{array}{r}5.4118 E+00 \\
-7.4968 E+00 \\
-3.3462 E+00 \\
1.5428 E+00\end{array}$ & $\begin{array}{r}-2.3431 E+00 \\
1.2496 E+00 \\
1.5385 E-01\end{array}$ & $: x$ & $2.4052 E-01$ & $\cdot \times 2$ \\
\hline$A(5,-2) \cup A(2)$ & $\begin{aligned} J= & +R(3,-1) \\
& +R(A,-1) \\
& +R(5,-1) \\
& +H(6,-1)\end{aligned}$ & $\begin{array}{r}-6.9866 E+00 \\
-4.3294 E+00 \\
3.6143 E+00 \\
2.2407 E+00\end{array}$ & $\begin{array}{r}3.02756+00 \\
7.2157 E-01 \\
-1.6617 E-01\end{array}$ & $\begin{array}{l}\cdot x \\
: x \\
: x\end{array}$ & $-3.1051 E=01$ &.$\times 2$ \\
\hline
\end{tabular}




\begin{tabular}{|c|c|c|c|c|c|c|}
\hline$R(S,-2) \cdot R(2)$ & $\begin{array}{r}2) 0+R(3,0) \\
+R(1,0): \\
+R(5,0) \\
+R(6.05)\end{array}$ & $\begin{array}{l}1.03376+00 \\
9.60096+00 \\
6.39076+00 \\
1.61335+00\end{array}$ & $\begin{array}{l}-1.7+79 E \bullet 00 \\
-1.6135 E \bullet 00 \\
-1.0339 E-01\end{array}$ & $: x$ & $1.79206-01$ &.$\times 2$ \\
\hline$A(3,-1) \cdot R(2,0$ & 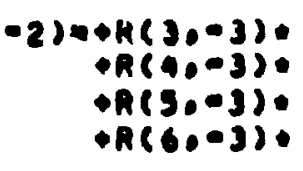 & $\begin{array}{r}6.01925 \cdot 01 \\
-4.32846+00 \\
5.90216+00 \\
-2.49961000\end{array}$ & $\begin{array}{r}-2.95958-01 \\
7.21576001 \\
-2.71366-01\end{array}$ & $: x$ & 3.03035002 & $1 \times 2$ \\
\hline$A(3,-1) \cdot R(20$ & 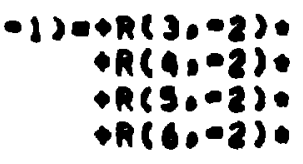 & $\begin{array}{r}-3.34025 \bullet 00 \\
9.25675: 00 \\
-3.6143 E \bullet 00 \\
-1.2498<\bullet 00\end{array}$ & $\begin{array}{r}1.14746+00 \\
-1.54286000 \\
1.66178-01\end{array}$ & $\begin{array}{l}: x \\
: x \\
0 x\end{array}$ & $-1.40456-01$ & $\cdot \times 2$ \\
\hline$A(3,-1) \cdot A(2$, & 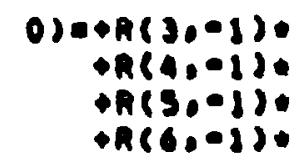 & $\begin{array}{r}6.16836 \mapsto 00 \\
-4.00036 \mapsto 00 \\
-5.01925+00 \\
6.0674 E-01\end{array}$ & $\begin{array}{r}-2.00296+00 \\
6.60046001 \\
2.30776-01\end{array}$ & $: x$ & $2.07446-01$ & $-1 \times 2$ \\
\hline$R(5,-1) \cdot R(20$ & 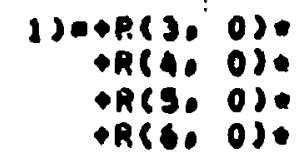 & 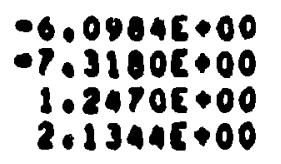 & $\begin{array}{r}2.64206+00 \\
1.21916+00 \\
-5.733 g c-02\end{array}$ & $\begin{array}{l}\cdot x \\
0 x \\
0 x\end{array}$ & $-2.7100[-01$ & $\cdot \times 2$ \\
\hline A $(5,0) 1) \cdot A(20$ & 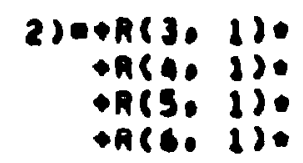 & $\begin{array}{l}2.64015 * 00 \\
6.1818 E \bullet 00 \\
6.83035 \% 00 \\
1.9761 E \bullet 00\end{array}$ & $\begin{array}{l}-1.14436+00 \\
-1.3636 E+00 \\
-3.14040=01\end{array}$ & $\begin{array}{c}x \\
0 x \\
0 x\end{array}$ & 1.17365001 &.$\times 2$ \\
\hline$R(s, 0) \cdot p(2$ & $\begin{aligned}-2)= & +R(3,-2): \\
& +R(4,-2) \\
& +A(5,-2)= \\
& \rightarrow R(6,-2)\end{aligned}$ & $\begin{array}{r}1.52465+00 \\
-6.33765+00 \\
6.59878000 \\
-2.2814 E+00\end{array}$ & $\begin{array}{r}-6.60605-01 \\
1.05636+00 \\
-3.03395-01\end{array}$ & $\begin{array}{l}x \\
0 x \\
0 x\end{array}$ & $0.77606-02$ & $1 \times 2$ \\
\hline$R(5,0) \bullet R$ & 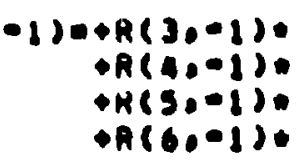 & $\begin{array}{r}-4.8212 E+00 \\
-.9627 E+00 \\
-1.2470 E+00 \\
-1.8039 E+00\end{array}$ & $\begin{array}{r}2.00826+00 \\
-1.19386+00 \\
5.733 s c-02\end{array}$ & $: x$ & $-2.1427 E-01$ & $9 \times 2$ \\
\hline$R(3,0)+R(2$. & $\begin{array}{r}O P R(3,0) \\
\rightarrow R(3, O)\end{array}$ & $\begin{array}{r}6.8182 E \bullet 00 \\
-5.5764 E \bullet 00\end{array}$ & $\begin{array}{r}-2.9545 E+00 \\
2.5641 E-01\end{array}$ & $\because x$ & $3.03036-01$ & $\cdot \times 2$ \\
\hline$R(5,0)=R(2$, & 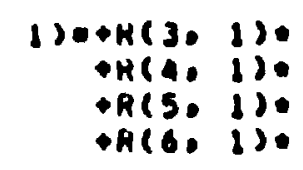 & $\begin{array}{r}-1.82126 \bullet 00 \\
-8.9627 E \bullet 00 \\
-1.2470 E \bullet 00 \\
1.8039 E+00\end{array}$ & $\begin{array}{l}2.00926 .00 \\
1.49385 \bullet 00 \\
5.73356-02\end{array}$ & $\ddot{: x}$ & $-2.1427 E-01$ & $-1 \times 2$ \\
\hline Hos. & 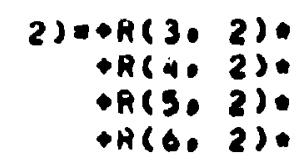 & $\begin{array}{l}1.3246 E+00 \\
6.3376 E+00 \\
6.5987 E+00 \\
2.2010 E+00\end{array}$ & $\begin{array}{l}-6.00065=01 \\
-1.05635+00 \\
-3.03396-01\end{array}$ & $: x$ & $6.7760 E-02$ & $\cdot \times 2$ \\
\hline
\end{tabular}




\begin{tabular}{|c|c|c|c|c|c|c|c|}
\hline$R(5$. & 1) $\begin{aligned} 1 . R(2,-2)= & +R(3) \\
& +R(4) \\
& +R(5) \\
& +R(6 .\end{aligned}$ & $\begin{array}{l}-11) \\
-1): \\
-1) \\
-1) \\
-11\end{array}$ & $\begin{array}{r}2.6407 E+00 \\
-8.1810 E+00 \\
6.8303 E+00 \\
-1.9761 E+00\end{array}$ & $\begin{array}{r}-1.1443 E+00 \\
1.3636 E+00 \\
-3.1404 E-01\end{array}$ & $\begin{array}{l}* x \\
* x \\
* x\end{array}$ & $1.1730 E-01$ & $\times 2$ \\
\hline$R(5)$ & $\begin{aligned} 12 * R(2,-1)= & +R(3) \\
& +R(4) \\
& +R(5) \\
& +R(6)\end{aligned}$ & $\begin{array}{l}0): \\
0): \\
0): \\
0)\end{array}$ & $\begin{array}{r}-6.0984 E+00 \\
7.3180 E+00 \\
1.2470 E+00 \\
-2.1344 E+00\end{array}$ & $\begin{array}{r}2.6426 E+00 \\
-1.2197 E+00 \\
-5.7335 E-02\end{array}$ & $\begin{array}{l}: x \\
: x \\
* x\end{array}$ & $-2.71 .04 E-01$ & $-\times 2$ \\
\hline$R<50^{\circ}$ & $\begin{aligned}1)=R(2,0)= & +R(3, \\
& +K(4) \\
& +R(5) \\
& +R(6)\end{aligned}$ & $\begin{array}{l}\text { 1): } \\
\text { 1): } \\
\text { 1): } \\
\text { 1): }\end{array}$ & $\begin{array}{r}0.4683 E+00 \\
4.0083 E+00 \\
-5.0192 E+00 \\
-8.0674 E=01\end{array}$ & $\begin{array}{r}-2.8029 E+00 \\
-6.6804 E-01 \\
2.3077 E-01\end{array}$ & $\begin{array}{l}* x \\
* x \\
* x\end{array}$ & $2.0748 E-01$ & $\times 2$ \\
\hline A 65 , & $\begin{aligned}1) * R(2,1)= & +R(3 . \\
& +R(4) \\
& +H(5) \\
& +R(6 .\end{aligned}$ & $\begin{array}{l}2): \\
21: \\
2): \\
21\end{array}$ & $\begin{array}{r}-3.3402 E+00 \\
-9.2567 E+00 \\
-3.6143 E+00 \\
1.2498 E+00\end{array}$ & $\begin{array}{l}1.4474 E+00 \\
1.5428 E+00 \\
1.6617 E=01\end{array}$ & $\begin{array}{l}: x \\
: x \\
* x\end{array}$ & $-1.4845 E-01$ & $\times 2$ \\
\hline$R(5$, & 1) $\begin{aligned} * R(2,2)= & +R(3) \\
& +R(4) \\
& +R(5) \\
& +R(6 .\end{aligned}$ & $\begin{array}{l}\text { 3): } \\
\text { 3): } \\
\text { 3): } \\
\text { 3): }\end{array}$ & $\begin{array}{l}0.0182 E-01 \\
4.3294 E+00 \\
5.9021 E+00 \\
2.4996 E+00\end{array}$ & $\begin{array}{l}-2.9545 E-01 \\
-7.2157 E-01 \\
-2.7136 E-01\end{array}$ & $\begin{array}{l}x \\
x \\
x \\
x\end{array}$ & $3.0303 E-02$ & $\times 2$ \\
\hline R65, & $\begin{aligned}2)+R(2,-2)= & +R(3) \\
& +R(4) \\
& +R(5) \\
& +R(6)\end{aligned}$ & $\begin{array}{l}01: \\
01: \\
01: \\
01\end{array}$ & $\begin{array}{r}4.0337 E+00 \\
-9.6809 E+00 \\
6.5987 E+00 \\
-1.6135 E+00\end{array}$ & $\begin{array}{r}-1.7479 E+00 \\
1.6135 E+00 \\
-3.0339 E=01\end{array}$ & $\begin{array}{l}x \\
: x \\
: x\end{array}$ & $1.7928 E=01$ & $\times 2$ \\
\hline$R 65$. & $\begin{aligned}2) * R(2,-1)= & +R(3) \\
& +R(4) \\
& +R(5) \\
& +R(6)\end{aligned}$ & $\begin{array}{l}\text { 1): } \\
\text { 1): } \\
\text { 1): } \\
\text { 1): }\end{array}$ & $\begin{array}{r}-6.9866 E+00 \\
4.3294 E+00 \\
3.6143 E+00 \\
.2 .2407 E+00\end{array}$ & $\begin{array}{r}3.0275 E+00 \\
-7.2157 E-01 \\
-1.6617 E-01\end{array}$ & $\begin{array}{l}: x \\
: x \\
: x\end{array}$ & $-3.1051 E-01$ &.$\times 2$ \\
\hline$R(5)$ & $\begin{aligned}2) \star R(2,0)= & +R(3, \\
& +F(4) \\
& +R(5) \\
& +R(6)\end{aligned}$ & $\begin{array}{l}2 \%: \\
2 \%: \\
2 \%\end{array}$ & $\begin{array}{r}5.4118 E+00 \\
7.498 B E+00 \\
-3.3462 E+00 \\
-1.5428 E+00\end{array}$ & $\begin{array}{r}-2.3451 E+00 \\
-1.2498 E+00 \\
1.5385 E-01\end{array}$ & $\begin{array}{l}-x \\
-x \\
* x\end{array}$ & $2.4052 E-01$ & $1 \times 2$ \\
\hline$R<5$. & $\begin{aligned}2) * R(2,1)= & +R(3) \\
& +H(4) \\
& +R(5) \\
& +R(6)\end{aligned}$ & $\begin{array}{l}31: \\
31: \\
31:\end{array}$ & $\begin{array}{r}-1.8039 E+00 \\
-8.1818 E+00 \\
-5.5769 E+00 \\
4.7238 E-01\end{array}$ & $\begin{array}{l}7.8170 E=01 \\
1.3636 E+00 \\
2.5641 E=01\end{array}$ & $\begin{array}{l}: x \\
: x \\
: x\end{array}$ & $-8.0174 E-02$ &.$\times 2$ \\
\hline A(s) & $\begin{aligned}2): R(2,2)= & +R(4) \\
& +R(5) \\
& +R(6)\end{aligned}$ & 4): & $\begin{array}{l}2.3142 E+00 \\
4.7322 E+00 \\
2.5873 E+00\end{array}$ & $\begin{array}{l}-3.8569 E-01 \\
-2.1757 E-01\end{array}$ & $\begin{array}{l}\cdot x \\
: x\end{array}$ & & \\
\hline A 5 . & $\begin{aligned}3) * R(2 .-2)= & +R(3) \\
& +R(40 \\
& +R(5) \\
& +R(6)\end{aligned}$ & $\begin{array}{l}\text { 1): } \\
\text { 1): } \\
\text { 1): } \\
\text { i): }\end{array}$ & $\begin{array}{r}5.7045 E+00 \\
-1.0605 E+01 \\
5.9021 E+00 \\
-1.2197 E+00\end{array}$ & $\begin{array}{r}-2.4720 E+00 \\
1.7675 E+00 \\
-2.7136 E-01\end{array}$ & $\begin{array}{l}x \\
: x \\
: x\end{array}$ & $2.5353 E-01$ & $1 \times 2$ \\
\hline
\end{tabular}




\begin{tabular}{|c|c|c|c|c|c|c|c|c|}
\hline$R(5$. & $3) * R(2$. & $\begin{aligned}-1)= & +R(3) \\
& +R(5) \\
& +R(6)\end{aligned}$ & $\begin{array}{l}23: \\
23: \\
23:\end{array}$ & $\begin{array}{r}-7.2157 E+00 \\
5.5769 E+00 \\
-2.1213 E+00\end{array}$ & $\begin{array}{r}3.1268 E+00 \\
-2.5641 E-01\end{array}$ & $\dot{x}$ & $-3.2070 E-01$ & $\times 2$ \\
\hline$R(5)$ & $3)=R(2$. & $\begin{aligned}0)= & +R(3: \\
& +R(4) \\
& +R(5) \\
& +R(6)\end{aligned}$ & $\begin{array}{l}\text { 3): } \\
\text { 3): } \\
\text { 3): } \\
\text { 3): }\end{array}$ & $\begin{array}{r}3.6078 E+00 \\
9.8102 E+00 \\
-5.5769 E=01 \\
-2.1257 E+00\end{array}$ & $\begin{array}{r}-1.3634 E+00 \\
-1.6364 E+00 \\
2.5641 E-02\end{array}$ & $\begin{array}{l}* x \\
: x \\
* x\end{array}$ & $1.6035 E-01$ &.$\times 2$ \\
\hline$R<50$ & $3) \div R(2$, & $\begin{aligned}1)= & +R(4) \\
& +R(5) \\
& +R(6)\end{aligned}$ & a): & $\begin{array}{l}-5.6605 E+00 \\
-6.7617 E+00 \\
-5.2813 E-01\end{array}$ & $\begin{array}{l}9.4475 E-01 \\
3.1088 E-01\end{array}$ & $\cdot x$ & & \\
\hline R(s. & $3) \circ R(2$. & $\begin{aligned}2) & =+R(50 \\
& \mapsto R(60\end{aligned}$ & $\begin{array}{l}52 \% \\
520\end{array}$ & $\begin{array}{l}3.0546 E \bullet 00 \\
2.4772 E \bullet 00\end{array}$ & $-1.4044 E-01$ & $\cdot x$ & & \\
\hline A6s. & 4) $* R(2$ & $\begin{aligned}-2) & =+R(30 \\
& +R(40) \\
& +R(50 \\
& +R(6)\end{aligned}$ & $\begin{array}{l}2): \\
23: \\
2) \\
23\end{array}$ & $\begin{array}{r}7.6534 E+00 \\
-1.0605 E+01 \\
4.7322 E+00 \\
-0.1818 E-01\end{array}$ & $\begin{array}{r}-3.3165 E+0 C \\
1.7673 E+00 \\
-2.1757 E-01\end{array}$ & $\begin{array}{l}: x \\
: x \\
: x\end{array}$ & $3.4015 E-01$ &.$\times 2$ \\
\hline R65. & $4) \cdot R(2$ & $\begin{aligned}-1)= & +R(3) \\
& +R(4) \\
& \mapsto R(5) \\
& \leftrightarrow R(6)\end{aligned}$ & $\begin{array}{l}\text { 3): } \\
\text { 3): } \\
\text { 3): } \\
\text { 3): }\end{array}$ & $\begin{array}{r}-6.2490 E+00 \\
-5.6685 E+00 \\
6.7617 E+00 \\
-1.7727 E+00\end{array}$ & $\begin{array}{r}2.7079 E+00 \\
9.4475 E=01 \\
-3.1088 E=01\end{array}$ & $\begin{array}{l}: x \\
: x \\
* x\end{array}$ & $-2.77736-01$ & $\times 2$ \\
\hline R65. & 4) $\bullet R(2$. & $\begin{aligned}0)= & +R(4, \\
& +R(5) \\
& +R 660\end{aligned}$ & 4): & $\begin{array}{r}9.8182 E \div 00 \\
3.34625+00 \\
-2.4393 E+00\end{array}$ & $\begin{array}{l}-1.6364 E+00 \\
-1.5385 E=01\end{array}$ & $: x$ & & \\
\hline$R 650^{\circ}$ & $1)+R(2$. & $\begin{array}{r}1)=+R(5) \\
+R(6,\end{array}$ & 5): & $\begin{array}{l}-6.4798 E+00 \\
-1.7516 E+00\end{array}$ & $2.9792 E=01$ & $\rightarrow x$ & & \\
\hline AC5. & $4) \cdot R(20$ & 2) $=+R(6$, & $6)$. & $2.0226 E \uparrow 00$ & & & & \\
\hline$R 650^{\circ}$ & $5) \cdot R(2$ & $\begin{aligned}-2)= & +R(30 \\
& +R(40 \\
& +R(5) \\
& +R(6)\end{aligned}$ & $\begin{array}{l}\text { 3): } \\
\text { 3): } \\
\text { 3): } \\
\text { 3): }\end{array}$ & $\begin{array}{r}9.0003 E+62 \\
-8.9627 E+00 \\
3.0546 E+00 \\
-4.3122 E-01\end{array}$ & $\begin{array}{r}-1.2815 E+00 \\
1.4938 E+00 \\
-1.40 A 4 E-01\end{array}$ & $\begin{array}{l}: x \\
: x \\
: x\end{array}$ & $4.3913 E=02$ &.$\times 2$ \\
\hline RC5. & $5)=F(2$ & $\begin{aligned}-1)= & +R(4.0 \\
& +R(50 \\
& +R(6)\end{aligned}$ & 1): & $\begin{array}{r}-1.2675 E+01 \\
6.4798 E+00 \\
-1.1809 E+00\end{array}$ & $\begin{array}{r}2.1125 E+00 \\
-2.9792 E-01\end{array}$ & $: x$ & & \\
\hline Res. & $5) \otimes R(2$. & $\begin{aligned}0)= & +R(5) \\
& +R(6)\end{aligned}$ & $\begin{array}{l}53 . \\
530\end{array}$ & $\begin{array}{r}0.3654 E+00 \\
-2.2613 E+00\end{array}$ & $-3.0462 E-01$ & $\bullet x$ & & \\
\hline $13{ }^{\circ}$ & $5) \oplus R($ & - 1$)=+R(60$ & 62. & $-3.1980 E+00$ & & & & \\
\hline$R 65$ & $-5 J * R$ & $-1)=+R(6$ & $-6)$. & $1.2663 E+01$ & $-3.0151 E-01$ & $* x$ & & \\
\hline
\end{tabular}




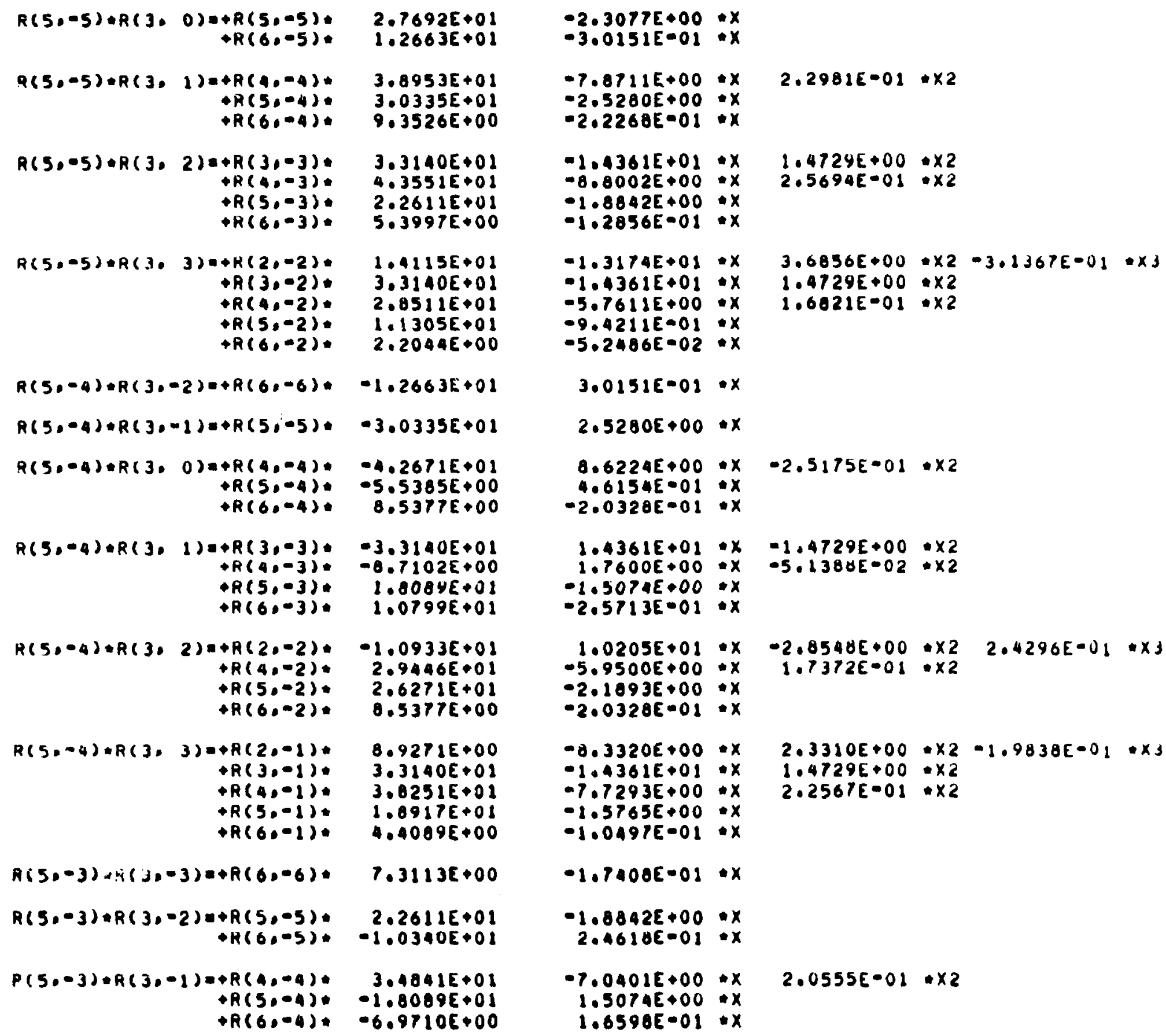

$3.6856 E+00 \div \times 2-3.1367 E-01 \div \times 3$

$-1.4361 E+01: x \quad 1.4729 E+00 \div \times 2$

$2.2981 E-01 * \times 2$

$1.4729 E+00: \times 2$
$2.5694 E-01 \div \times 2$

$2.42965=01 . x$

$1.7372 E-01 \cdot \times 2$

$2.3310 E+00=x 2-1.9838 E=01 \cdot x 3$ $1.4729 E+00=x$

$2.2567 E-01: \times 2$ 


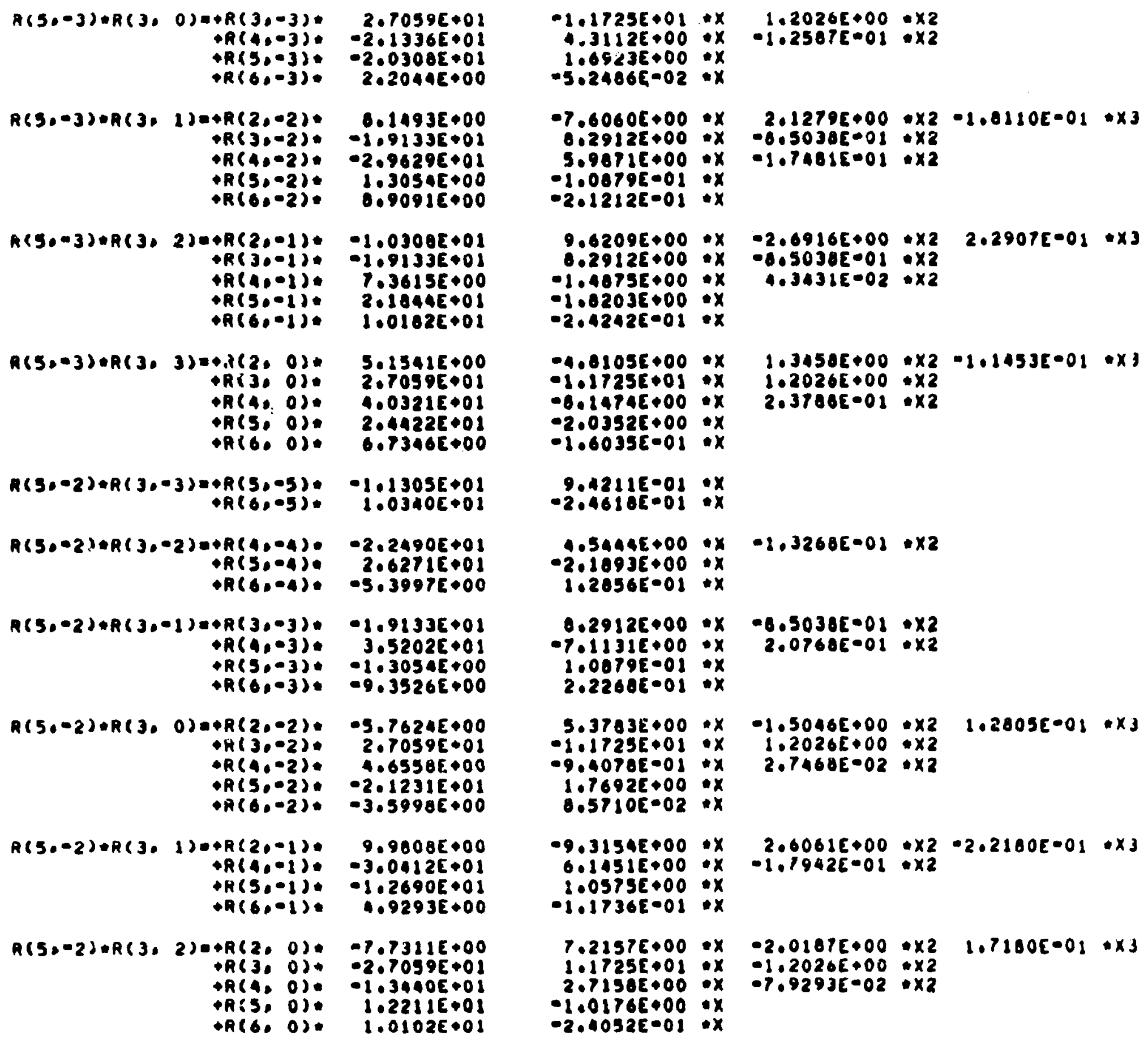




\begin{tabular}{|c|c|c|c|c|c|c|c|}
\hline 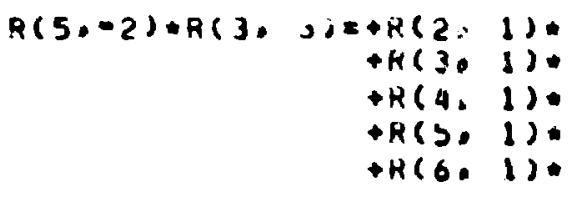 & $\begin{array}{l}2.5770 E+00 \\
1.9133 E+02 \\
3.6808 E+01 \\
2.7305 E+01 \\
0.9091 E+00\end{array}$ & $\begin{array}{l}-2.4052 E+00 \\
-4.2912 E+00 \\
-7.4375 E+00 \\
-2.2754 E+00 \\
-2.1212 E-01\end{array}$ & $\begin{array}{l}: x \\
: x \\
: x \\
: x \\
: x\end{array}$ & $\begin{array}{l}6.7289 E-01 \\
6.5030 E-01 \\
2.1715 E-01\end{array}$ & $\begin{array}{l}: \times 2 \\
: \times 2 \\
\times 2\end{array}$ & $-5.7267 E-02$ & $\times 5$ \\
\hline $\begin{aligned} R(5,-1)=R(3,-3) & +K(4,-4) \\
& +K(5,-=1) \\
& +K(6,-4)\end{aligned}$ & $\begin{array}{r}1.0411 E+01 \\
-1.0917 E+01 \\
1.1665 E+01\end{array}$ & $\begin{array}{r}-2.1036 E+00 \\
1.5765 E+00 \\
-2.7773 E=01\end{array}$ & $: x$ & $0.1420 E-02$ & $1 \times 2$ & & \\
\hline $\begin{aligned} R(3,-1)+H(3,-2)= & +R(3,-3) \\
& +R(4,-3) \\
& +R(5,-3)\end{aligned}$ & $\begin{array}{r}1.1434 E+01 \\
-3.305 Y E+01 \\
2.1644 E+01\end{array}$ & $\begin{array}{r}-4.9549 E+00 \\
6.600 E+00 \\
-1.0203 E+00\end{array}$ & $: x$ & $\begin{array}{r}5.0820 E-01 \\
-1.9504 E=01\end{array}$ & $\begin{array}{l}1 \times 2 \\
\div \times 2\end{array}$ & & \\
\hline $\begin{aligned} E(5,-1) \cup R(3,-1)= & +R(2,-2) \\
& +H(3,-2) \\
& +R(4,-2) \\
& +R(5,-2) \\
& +R(6,-2)\end{aligned}$ & $\begin{array}{r}3.7724 E+00 \\
-2.65 Y 15+01 \\
2.1844 E+01 \\
1.2690 E+01 \\
-8.24926+00\end{array}$ & $\begin{array}{r}-3.5209 E+00 \\
1.1514 E+01 \\
-4.9138 E+00 \\
-1.0575 E+00 \\
1.9639 E-01\end{array}$ & $\begin{array}{l}: x \\
: x \\
: x \\
: x \\
: x\end{array}$ & $\begin{array}{r}9.8501 E-01 \\
-1.1809 E+00 \\
1.2807 E=01\end{array}$ & $\begin{array}{l}: \times 2 \\
: \times 2 \\
\times 22\end{array}$ & $-8,3832 E=02$ & $\cdot 0 \times 3$ \\
\hline $\begin{aligned} R(5,-1)+R(3,0) & +R(2,-1) \\
& +H(3,-1) \\
& +H(4,-1) \\
& +R(5,-1) \\
& +R(0,-1)\end{aligned}$ & $\begin{array}{r}-8.7120 E+00 \\
1.6171 E+01 \\
2.3642 E+01 \\
-1.2923 E+01 \\
-7.5296 E+00\end{array}$ & $\begin{array}{r}8.1312 E+00 \\
-7.0073 E+00 \\
-4.7772 E+00 \\
1.0769 E+00 \\
1.7928 E-01\end{array}$ & $\begin{array}{l}: x \\
: x \\
: x \\
: x \\
x\end{array}$ & $\begin{array}{l}-2.2748 E+00 \\
7.1870 E-01 \\
1.3940 E-01\end{array}$ & $\begin{array}{l}\times 2 \\
\times 2 \\
\times 2\end{array}$ & $1.9360 E=01$ & $\cdot x_{4}$ \\
\hline $\begin{aligned} A(5,-1) \bullet R(3,1)= & +R(2,0) \\
& +R(3,0) \\
& +R(4,0) \\
& +R(5,0)\end{aligned}$ & $\begin{array}{r}P .2404 E+00 \\
1.6171 E+01 \\
-1.7671 E+01 \\
-2.0433 E+01\end{array}$ & $\begin{array}{r}-8.6244 E+00 \\
-7.0073 E+00 \\
3.5706 E+00 \\
1.7028 E+00\end{array}$ & $\begin{array}{l}x \\
: x \\
x \\
x\end{array}$ & $\begin{array}{r}2.4128 E+00 \\
7.1870 E-01 \\
-1.0425 E-01\end{array}$ & $\begin{array}{l}\times 2 \\
1 \times 2 \\
: \times 2\end{array}$ & $-2.0534 E=01$ & $x_{3}$ \\
\hline $\begin{aligned} R(5,-1)+R(3,2) & +R(2,1) \\
& +R(30,1) \\
& +R(4:\{1) \\
& +R(6,1)\end{aligned}$ & $\begin{array}{l}-4.7717 E+00 \\
-2.6571 E+01 \\
-2.8398 E+01 \\
0.2482 E+00\end{array}$ & $\begin{array}{r}4.4536 E+00 \\
1.1514 E+01 \\
5.7382 E+00 \\
-1.9639 E-01\end{array}$ & $\begin{array}{l}x \\
: x \\
x \\
x\end{array}$ & $\begin{array}{l}=1.2460 E+00 \\
-1.1009 E+00 \\
-1.6754 E=01\end{array}$ & $\begin{array}{r}\times 2 \\
\times 2 \\
\times 2\end{array}$ & $1.0604 E=01$ & $\times 3$ \\
\hline $\begin{aligned} R(5,-1), R(3,3) & +R(2,2) \\
& +R(3,2) \\
& +R(4,2) \\
& +R(5,2) \\
& +R(6,2)\end{aligned}$ & $\begin{array}{l}9.7403 E-01 \\
1.1434 E+01 \\
2.9512 E+01 \\
2.7305 E+01 \\
1.0648 E+01\end{array}$ & $\begin{array}{l}-9.09096-01 \\
-4.9549 E+00 \\
-5.9633 E+00 \\
-2.2754 E+00 \\
-2.5353 E-01\end{array}$ & $\begin{array}{l}: x \\
: x \\
: x \\
: x \\
: x\end{array}$ & $\begin{array}{l}2.3433 E=01 \\
5.0820 E=01 \\
1.7411 E-01\end{array}$ & $\begin{array}{l}: \times 2 \\
\div \times 2 \\
\times 2\end{array}$ & $-2.2645 E-02$ & $\cdot x_{3}$ \\
\hline $\begin{aligned} R(5,0) \div R(3,-3) & +R(3,-3) \\
& +R(4,-3) \\
& +R(5,-3) \\
& +R(6,-3)\end{aligned}$ & $\begin{array}{r}-5 \cdot 1136 E+00 \\
2.0160 E+01 \\
-2.1422 E+01 \\
1.1065 E+01\end{array}$ & $\begin{array}{r}2.2159 E+00 \\
-4.0737 E+00 \\
2.0352 E+00 \\
-2.7773 E-01\end{array}$ & $\begin{array}{l}: x \\
: x \\
: x \\
x\end{array}$ & $\begin{array}{r}-2.2727 E-01 \\
1.1094 E-01\end{array}$ & $\begin{array}{l}: \times 2 \\
\times \times 2\end{array}$ & & \\
\hline $\begin{aligned}-2) & = \\
& +R(2,-2) \\
& +R(3,-2) \\
& +R(4,-2): \\
& +R(5,-2): \\
& +R(6,-2)\end{aligned}$ & $\begin{array}{r}-2.1780 E+00 \\
2.0455 E+01 \\
-3.5195 E+01 \\
1.2211 E+01 \\
-.7621 E+00\end{array}$ & $\begin{array}{r}2.03246+00 \\
-8.0636 E+00 \\
7.1116 E+00 \\
-1.0176 E+00 \\
-1: 13386=01\end{array}$ & $\begin{array}{l}: x \\
: x \\
: x \\
: x \\
: x\end{array}$ & $\begin{array}{r}-5.6870 E-01 \\
9.0909 E-01 \\
-2.07645-01\end{array}$ & $\begin{array}{l}\times 2 \\
\times 2 \\
\times 2 \\
\times 2\end{array}$ & $4.0400 E=02$ &.$x d$ \\
\hline
\end{tabular}




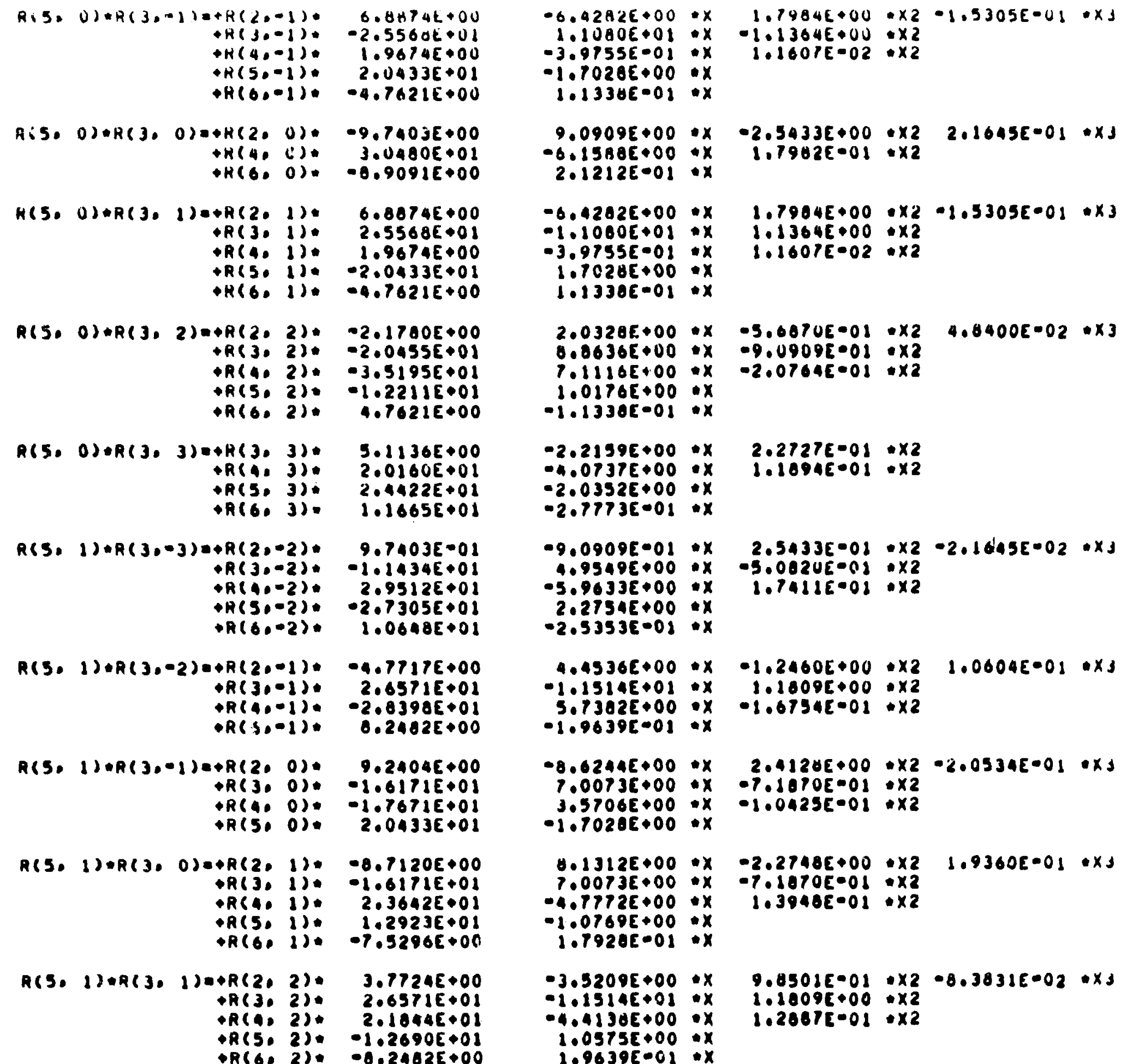

$R(3,1) \times R(3,1)=+R(2,2) * 3.7724 E+00$ $\rightarrow R(3,2) \cdot 2.6571 E+01$ PR(A, 2): $2.1844 E+0$ $\rightarrow R(5,2)=-1.2690 E \bullet 0)$

$-6.4282 E+00 \times x \quad 1.79846+00 \times \times 2-1.5305 E=01 \cdot x 1$ $1.1080 E+01: x-1.1364 E+00 \div \times 2$

$1.7026 E+00$ : $x$

$9.09095+00 \times x-2.5433 E+00 \cdot \times 2 \quad 2.1645 E-01 * x J$

9.09098000 ox

$0.15805+00$ : $x$

$-6.4202 E+00 * x$

$-1.1000 E+01$ : $x$

$\begin{array}{rl}-3.9755 E-01 & : x \\ 1.7028 E+00 & * x\end{array}$

$1.7028 E+00: x$
$1.1330 E-01: x$

$2.0328 E+00 * x-5.0070 E=01 \cdot \times 2 \quad 4.8400 E-02 \cdot \times 3$ $0.0636 E+00 * x \quad-9.0909 E \cdot 01 \cdot x 2$

$7.1116 E+00 \div x-2.0704 E=01: \times 2$

$1765+00: x$

$-1.13300-01: x$

$-2.21596+00$ *x $-4.0737 E+00$ : $x$ $-2.0332 E+00$ : $x$
$-2.7773 E-01: x$

$-9.0909 E-01 * x \quad 2.5433 E-01 * \times 2-2.1645 E-02 * x_{3}$ $4.9549 E+00 * x-5.0820 E-01 * \times 2$

$0.74115001: \times 2$
1.740

$2.2734 E+00$ : $x$

$-2.5353 E-02 \cdot x$

$4.4536 E+00 * x-1.2460 E+00 * \times 2 \quad 1.0004 E-01 * \times 3$ $-1.1514 E+01 * x \quad 1.1809 E+00 * \times 2$

$5.7302 E+00 * x-1.6754 E-01+x 2$

$-1.9639[001 \cdot x$

$-8.6244 E+00 * x \quad 2.4124 E+00 * \times 2-2.0534 E-01 \cdot X J$

$7.00735000: x=7.1870 E-01: \times 2$

$3.57065+00 * x-1.0425 E-01 \times x 2$

$-1.7020 E+00: x$

$.13125+00 * x-2.2748 E+00 * \times 2 \quad 1.9360 E-01 * x s$

$7.00735000 \div x \quad-7.10705=010 x 2$

$-4.7772 E+00 * x \quad 1.3940 E-01 * x 2$

$-1.07605+00$ : $x$

$1.70200-01$. $x$

$-3.52095+00$ :

$-1.15142+01$ : $x$

$-4.41305+00$ : $x$

$1.05756+00$ : $x$

$9.0501 E-01 * \times 2-8.3831 E=02 \cdot \times 3$

$1.10096+00 * \times 2$

$1.2887 E-01+\times 2$ 
A(5, 1).R(3, 2) $=+R(3,3),-1.1434 E+01$ $\rightarrow R(4,0):-3.3059 E+01$

$R(5,1)+R(3,3)=+R(4,4), 1.0411 E+0)$ $\rightarrow R(5,4)$. 1.0917E+0:

$A(5,2) \cap R(3,-3)=+R(2,-1)$ R(3.-1): -1.9133E+01 $R(4,-1): 3.6808 E+01$ $\rightarrow R(5,-1):-2.7305 E+01$

$R(5,2) \bullet R(3,-2)=+R(2,0)$ $+R(3,0)$ $-7.7311 E+00$ $2.7059 E+0$ R(5, 0) - $1.3440 E+01$ $+R(6,0)=1.0102 E+0$

$A(5,2) * R(3,-1)=+R(20 ;)$ $R(4,1)-9.900 \partial E+00$ $R(5,1)$. $+R(6,1) .4 .9293 E+00$

$R(5,2)+R(3,0)=+H(2,2) *-5.7624 E+O O$ $+H(3,2),-2.7059 E+01$ -R(4. 2). 4.6558E+00 $\rightarrow H(5,2): 2.1231 E+01$ $+R(6,2)$.

$-3.599 \forall E+00$

$R(5,2) \cdot A(3,1)=+R(3,3)$. $+R(4,3)$. $R(5,3) \quad 1.3054 E+00$

$R(5,2) \because R(3,2)=+K(4,4)$ $-K(5,4)$. $+K(6,4)$.

$-9.3520 E+00$

$-2.2490 E+01$ $-2.6271 E+01$ $-5.3997 E+00$

$R(5,2)+R(3,3)=+R(5,5)$ $+R(6,5)$.

$1.1305 E+01$ $1.0340 E+01$

$R(5,3) \because A(3,-3)=+R(2,0)$. $\rightarrow H(3,0)$. $+R(4,0)$ $+R(5,0)$.

$5.1541 E+00$ $-2.7059 E+01$ $4.0321 E+01$ $-2.4422 E+0$ $+K(6,0)$. $0.7346 E+00$

$R(5,3) \backsim R(3,-2)=+R(2,1) *-1.0300 E+01$ H(3. 1). $1.9133 E+0$ H H (40 1): rojolse+00 $\rightarrow H(O, 1): \quad 2.01020001$

\section{$4.9549 E+00 * x \quad-5.0820 E-01 * x 2$}

$6.6000 E+00: x$
$1.0203 E+00: x$

$-2.1036 E^{2}+00 \quad x$ $-1.5765 E+00: x$
$-2.7773 E-01: x$

$-2.4052 E+00 * x$ $0.2912 E+00 * x$ $-7.4375 E+00 * x$ $2.2754 E+00+x$
$-2.1212 E-01: x$

$7.2157 E+00: x$
$1.1725 E+01: x$

$2.7158 E+00 * x=1.2026 E+00 * x 2$

$1.0176 E+00: x$

$-2.4052 E-01 \cdot x$

$-9.3154 E+00 * x \quad 2.6061 E+00 \cdot \times 2 \cdot-2.2180 E-01 * x$

$6.1451 E+00 * x-1.7942 E-01 * \times 2$

$1.0575 E+00 \cdot x$

$-1.1736 E-01 \cdot x$

$5.3793 E+00 * x-1.5046 E+00 * \times 2 \quad 1.2805 E-01 * x$ $1.1725 E+01 * x-1.2020 E+00 * x 2$

$-9.4078 E-01 * x \quad 2.7468 E-02 * x 2$

$-0.2912 E+00 \cdot x$

$0.5036=01 \times 2$

$1.0879 E-01: x$
$2.22685-01: x$

\section{$4.5444 E+00 * x-1.3268 E=01 * \times 2$}

$2,1093 E+00 \div x$

$0.0211 E \cdot 01 \cdot x$

$-2.4610 E-01: x$

$-4.8105 E+00 * x \quad 1.3458 E+00 . \times 2-1.145 J E-01 * x$

$1.1725 E+01 * x-1.2026 E+00 * x 2$

$-8.1474 E+00: x \quad 2.3786 E-01 \cdot x$

$2.0352 E+00$ : $x$

$9.6204 E+00 * x$ $-8.2912 E+00: x$ 0.2912500

$-1.40756+00$ : $x$

$-2.4242 E-01 * x$

$2.6916 E+00 \div \times 2 \quad 2.2907 E-01 * x$ $0.5034 E-01$ : $x$

$4.34315002 \times x 2$ 


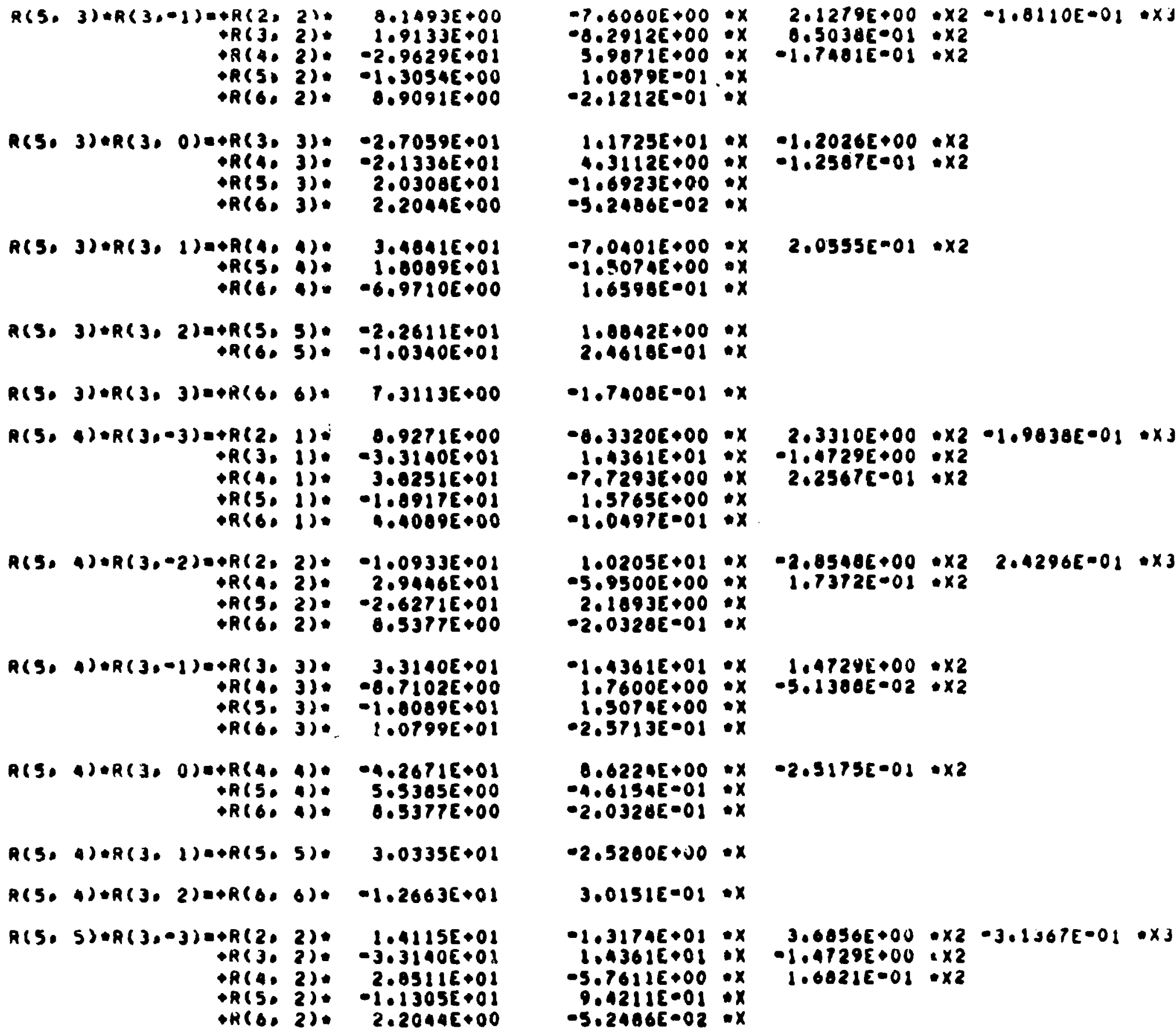

R(5, 3) $R R(3,0)=+R(3,3)=-2.7059 E \bullet 0$ $\rightarrow R(4 ., 3) \cdot-2.1336 E+0$ $\rightarrow R(6,3)$. $2.20448+00$

$R(5,3) \bullet R(3,1) \times+R(4,4), 3.40416+0)$ $\rightarrow R(5.4)$ 1.80098०0

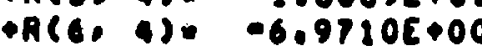

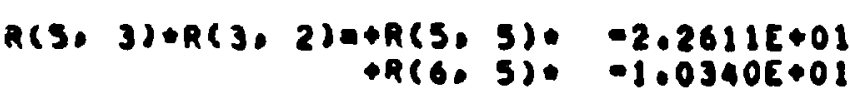

$R(5,3)+R(3,3)=+R(6,6) *$ T.3113E+00

$R(5,4) \cdot R(3,-3)=+R(2,1): 0.9271 E+00$ $+R(3,1)=3.3140 E+0$ R(4. 1). 3.0251E+0 $\rightarrow R(5,1) .-1.0917 E+01$ -R(6. 1). Q.4009E+00

$R(5,4) * R(3,-2)+4 R(2,2) *-1.0933 E+0$ $+R(A, 2) \cdot 2.9446 E+0$ $\rightarrow R(5,2)=-2.6271 E+0$ $+R(6,2)$. 0.537PE+00

$R(3,4) \bullet R(3,-1) 0+R(3,3): \quad 3.3140 E+0)$ A(4. 3). $-0.71025+00$ $\rightarrow R(5,3)=-1.0089 E+0$ $\rightarrow R(6.3) \% \quad 2.0799 E+01$

$R(5,4) \bullet R(3,0)=+R(4,4) *-4.2671 E+01$ $+R(5,4): 5.3385 E+00$

$R(5,4) \bullet R(3,1) 0+R(5,5) \cdot 3.0335 E+01$ $R(5,4) * R(3,2)=+R(6,6) \cdot-1.2663 E+0)$ $R(5,5) \cdot R(3, \cdots 3)=+R(2,2):-1 \cdot 4115 E+01$ R( $\rightarrow R(5,2) \cdot-1.1305 E+0$ $\rightarrow H(6,2)$. $2.20445+00$

$-7.6060 E+00 * x \quad 2.1279 E+00 \cdot \times 2-1.8110 E-01 \cdot X$ $-8.29126+00 * x \quad 0.5038 E 001 * x 2$

$-2.12125001 \cdot x$

$1.1725 E+01 * x-1.2026 E+00 * x 2$ $0.3112 \varepsilon+00$ : $x$-1.2567E-01 $\times 2$ $-1.6923 E+00$ * $x$ $-5.2486 E-02$ *x

$-7.04016+00 * x$ $-1.5074 E+00$ ०x $1.6598 \varepsilon 001 \cdot x$

$1.0042 E+00 * x$ $2.4618 E-01 \cdot x$

$1.7000 E-01: x$

$-8.3320 E+00 * x \quad 2.3310 E+00 * x 2-1.9838 E-01 * x 3$ $1 .+361 E+01 * x-1.4729 E+00+x 2$ $-7.7293 E+00 * x \quad 2.2567 E-01 * \times 2$ $1.57656+00$
$-1.04975-01: x$

$1.0205 E+01 * x-2.0540 E+00 * \times 2 \quad 2.4296 E=02 * x 3$ $-5.9500 E+00 * x \quad 1.7372 E-01 * \times 2$ $2.10935+00$ ix $-2.0328 E \cdot 01 \cdot x$ $-1.4361 E+01 * x \quad 1.47291+00 * \times 2$
$1.7600 E+00 * x-5.1300 E-02 * \times 2$ $1.50745+00$ : $x$ $-2.5713 E \cdot 01 \cdot x$

$0.6224 E+00 * x-2.3175 E-01 * x 2$ -4.61545001 : $X$ $-2.0328 E-01 \cdot x$

$-2.5200 E+00 \cdot x$ $3.0151 E-02 \cdot x$

$-1.3174 E+01 * x \quad 3.0056 E+00 * x 2-3.1367 E-01 * x 3$ $1.4361 E+01 \times x-1.4729 E+00=x 2$ $-5.7611 E+00$ * $\quad 1.6021 E=01 \times x^{2}$

$0.4211 E \cdot 01$ : $x$

$-5.2486 E 002 \cdot x$ 


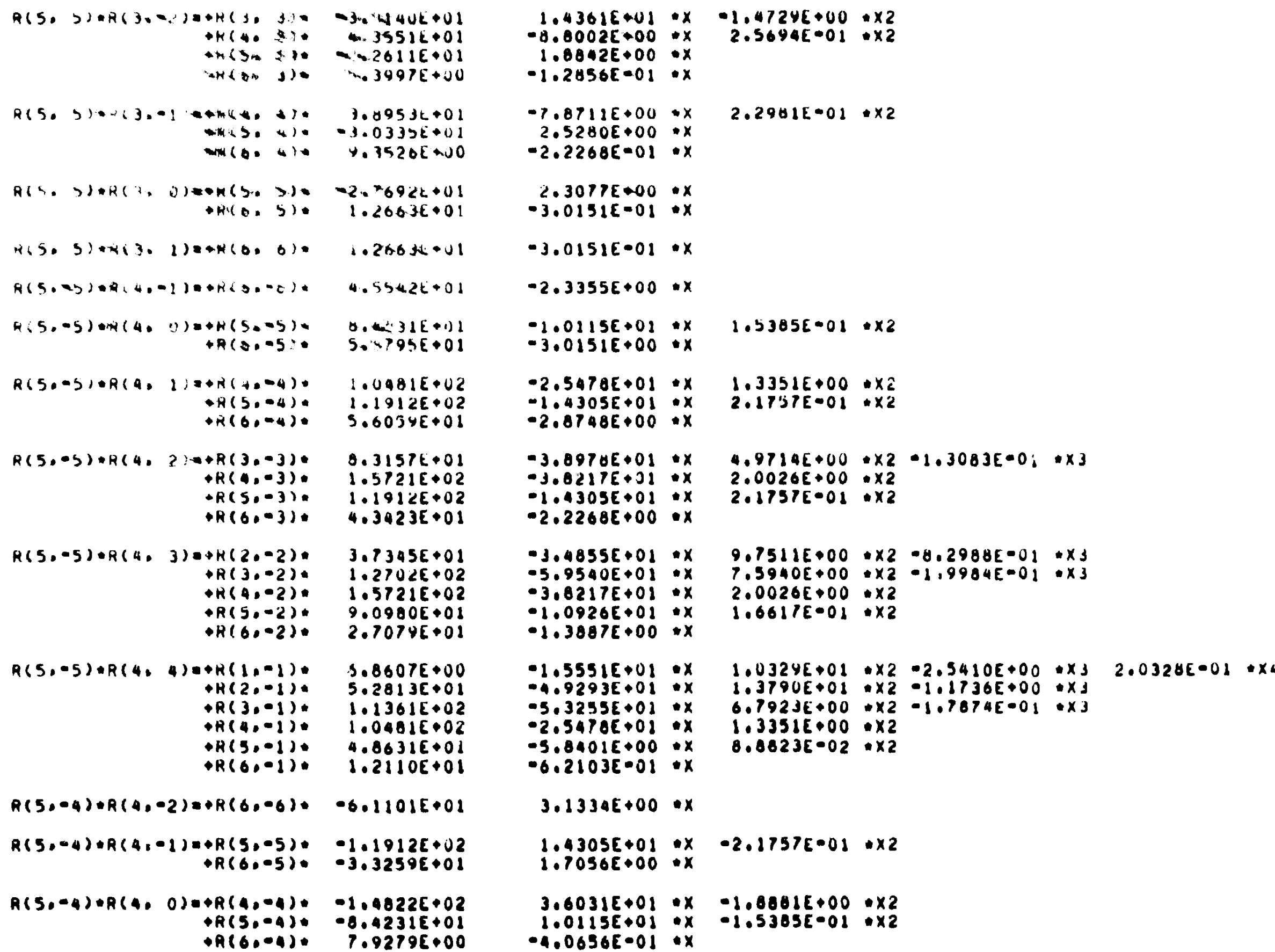

$1.43615 * 01: x$ $0.0002 E \div 00 * x$ $-1.00505000 . x$

$-7.87116+00 * x$ $2.5280 E+00: x$

$2.3077 E * 00 \cdot x$ $-3.0151 E-01: x$ $-3.01516001 \cdot x$ $-2.3355 E+00 * x$ $-1.01156+02: x$
$-3.01516+00: x$ $-2.54705+01 * x$ $-1.4305 E+01 \cdot x$ $-2.8748 E+00 * x$

$-3.09706001 \cdot x$ $-3.0217 E+31 * x$ $-1.030 s e+01$ : $x$ $-2.2268 E+00 \cdot x$

$-3.4855 E+01 * x$ $-5.9540 E+01 * x$ $-3.0217 E+01$ \& $x$ $-1.0926 E+01$ : $x$ $1.3887 E+00 * x$

$-1.5551 E+01 \cdot x$ $-1.9293 E+01$ *x $-3.3255 E+01: x$ $-2.3470 E+02$ : $x$ $-3.84016+00$ :x $-6.2103 E-01+x$ $3.1334 E+00 \cdot x$

$1.4305 E+01 * x-2.1757 E-01 * \times 2$ $1.7056 E+00 * x$

\section{$3.60318+01+x-1.00012+00 \div x 2$ \\ $1.0861 E+00 \times 2$}

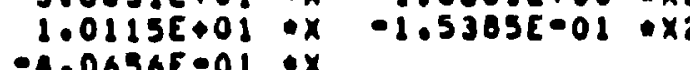

1.472 Y $+00 \times 2$

$2.2981 E-01 \cdot \times 2$

$1.3385 E-01 \div \times 2$

$1.33516+00 * x$

. I7ste-01 $x$

$4.9719 E+00 \cdot \times 2 \cdot 1.3083 E-0 i * \times 3$ $2.00265+00 \div \times 2$ $2.17576 \cdot 01: \times 2$

$9.7511 E+00 \div \times 2=0.2988 E-01 \div x$ $7.5940 E+00 \div \times 2-1,9984 E-01 \cdot x 3$ $2.0026 E+00: \times 2$
$1.6617 E \cdot 01: \times 2$

$1.032 y E+01 * \times 2-2.5410 E+00 * x 3 \quad 2.0328 E=01 * x$ lo3rque $6.792 J E+00 * \times 2-1.7874 E-01 \cdot x$

$1.33515+00 \div \times 2$

$8.0823 E-02 \cdot \times 2$ 


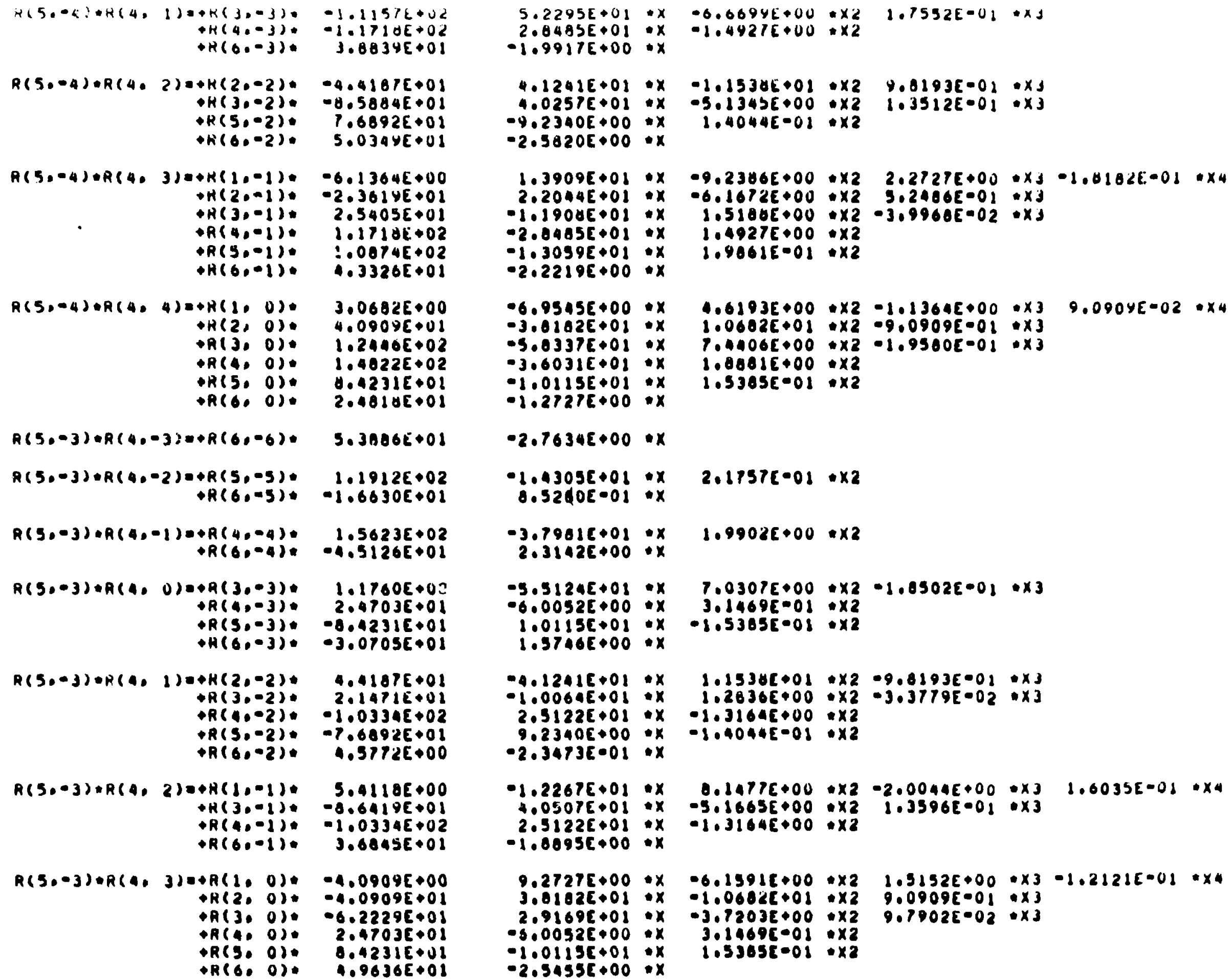

$-4.09095+00$
$-4.0909 E+01$

0.022296001

$2.4703 E+01$

$0.42316+01$

$1.9636 E+01$

$5.2295 E+01 * x$

$2.0485 E+01: x$

$4.1291 E+01 \cdot x$ $4.0257 E+01$ :x $-4.2340 E+00 \cdots x$ $-2.5820 E \cdot 00 \cdot x$

$1.3909 E+01 \cdot x$ $2.2004 E+01: x$ $-1.1900 E+01$ : $x$ $-2.8405 E+01$. $x$ $-1.30998+01$ : $x$ $-2,2219 E+00$ : $x$

$-6.9545 E+00 * x$ $-3.0102 E+01 \cdot x$

$-3.03376+01$ ix

$-3.6031 E+02$ :x

$-1.0115 E+01: x$

$-2.7634 E+00 \cdot x$

$-1 \cdot 4305 E+01$ : $x$ $0.5260 E-01 \cdot x$

$-3.79016+01: x$

$-5.5124 E+03 * x \quad 7.0301 E+00+\times 2-1.0502 E=01 * x 3$

$-6.00525+00 * x$ $1.0115 E+01: x$
$1.3746 E+00$

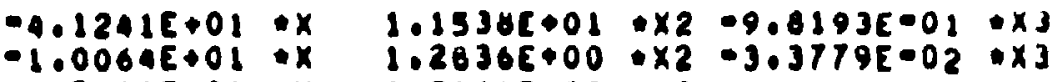
$2.3122 E+01 \quad x \quad-1.3164 E+00 \quad x 2$

$-2.3473 E=01$ *x

$-1.2267 E+01 * x \quad 0.1477 E+00 \times \times 2-2.0044 E+00 * \times 3 \quad 1.0035 E-01 * \times 4$ $4.0507 E+01: x-5,1665 E+00 \times x 2 \quad 1.3396 E=01 * \times 3$ $2.5122 E+01 * x-1,3164 E+00 \times x$

$-1.00956+00 \cdot x$

$9.27275+00 * x-6.15911+00 * x 2 \quad 1.5152 E+00 * x^{3}-1.2121 E=01 * x 4$ $3.01025+01 * x$ $2.91692+01 * x$ $-3,0052 E+00$ *x $-1.01155+01$ : $x$ $-2.54555+00 \cdot x$

$-1.15385001 * \times 2 \quad 9.81935-01 * x 3$ (1.3512E-01*x3

$-9.2366 E+00 * 2 \quad 2.2727 E+00 * x_{3}=1.81026-01 * x_{4}$ $6.1672 E+00 * x 2 \quad 3.2406 E-01 * x 3$

$1.4927 E+00 * x^{2}$

$\$ .6193 E+00 * \times 2-1.1364 E+00 * \times 3 \quad 9.0904 E-02 * \times 4$ $1.0602 E+01 \div \times 2=9.0909 E-01 \div \times 3$

$1.00015+00 \times x^{2}$

$.5385 E-01 \cdot \times 2$

\section{$2.17576-01 * \times 2$}

$1.9902 E+00 * 2$

$3.146980010 x 2$

$1.06026+01 \times 2 \quad 9.09095-01 * \times 3$

$-3.7203 E+00 * \times 2 \quad 9.7902 E-02 * x 3$

$3.1069501: \times 2$ 


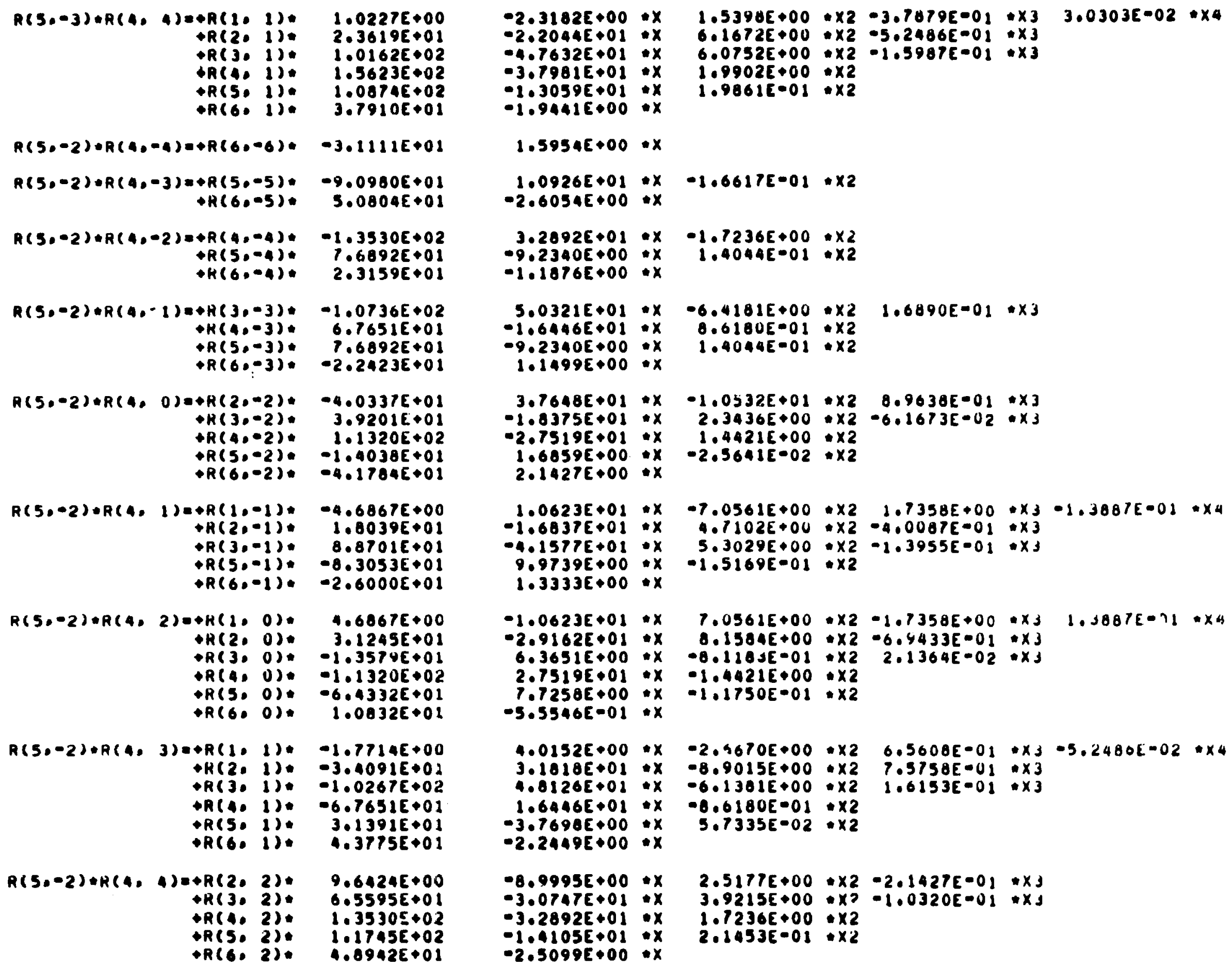

$-2.3182 E+00 \cdot x$ $2.2044 E+01$ : $X$ $0.76325+01: x$ $0.7012+01$ : $x$ $-1.30596+01$. $x$

$1.59546+00 \cdot x$

$1.0926 E+01 * x-1.6617 E-01 * \times 2$ $-2.6054 E+00 * x$

$3.2892 E+01 * x-1.7236 E+00 * x 2$ $-9.2340 E+00: x \quad 1.4044 E-01 * x 2$ $6 E+00: x$

$5.0321 E+01 * x-6.4181 E+00 * \times 2 \quad 1.6890 E=01 * x 3$ $-1.6446 E+01 * X \quad 8.6180 E=01 * X 2$

$9.2340 E+00$ :X 1.404

$3.7648 E+01 * x-1.0532 E+01 * \times 2 \quad 0.9638 E-01 * x 3$ $-1.8375 E+01 \cdot x \quad 2.3436 E+00 * x 2-6.1673 E-02 * x 3$

$-2.7519 E+01: x$ $1.68596+00: x$
$2.1427 E+00: x$

$1.44215+00 \times 2$

$1.0623 E+01 * x \quad-7.0561 E+00 * \times 2 \quad 1.7358 E+00 * x_{3}-1.3087 E-01 * \times 4$ $4.7102 E+00 \times 2-4.0087 E=01 \times x$

$0.1577 E+01$ \& $x \quad 5.3029 E+00$ : $x 2-1.3955 E-01$ : $x$

9.07350

$-1.002350010 x$

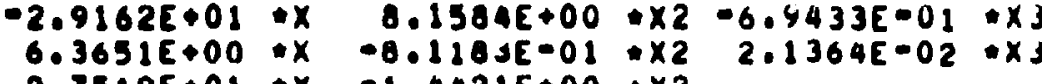

$2.7519 E+01 * x-1.4421 E+00 * x 2$

$-5.7250 E+00 * x-1.1750 E-01 * x 2$

$-5.5546 E-01 \cdot x$

$4.0152 E+00 * x \quad-2.9670 E+00 * x_{2} \quad 6.5600 E-01 * x_{3}-5.2480 E-02 * x_{4}$

$3.1010 E+01 * x \quad-8.9015 E+00 * x 2 \quad 7.5758 E-01 * x 3$

$4.8126 E+01 * x \quad-6.1301 E+00 * \times 2 \quad 1.6153 E-01: \times 3$

$1.6446 E+01 * x-0.6180 E-01 * x 2$

$-3.7690 E+00 * x \quad 5.7335 E-02 * x 2$

$-2.2449 E+00 * x$

$-8.9995 E+00 \cdot x$ $-3.0747 E+01$ : $x$ $-3.28925+01$ : $x$ $-1 \cdot 4105 E+01: x$
$-2.30995+00: x$

$2.5177 E+00 * \times 2-2.1427 E-01 * \times 3$

$3.9215 E+00 * x>-1.0320 E-01 * x y$

$\begin{array}{lll}1.7236 E+00 & : \times 2 \\ 2.1453 E-01 & : \times 2\end{array}$ 


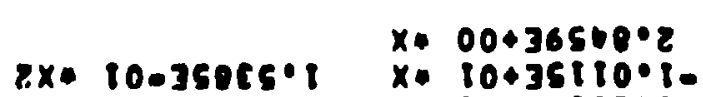
$8 x+10-31662 \cdot 9 \cdot x * 10+30102 \cdot 1$

$2 \times 410-31986^{\circ !}$

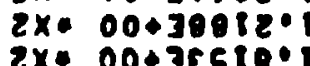

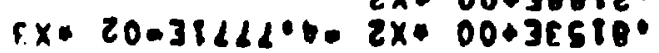

Ex. 200-35ee $8.2-$

$2 x+00+36219 \cdot 10$

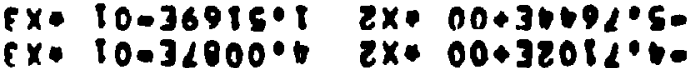

$2 x+00+32018.60$

$2 x+10032186 \cdot 1 \%$ $10-36260^{\circ}$.

$E x \cdot 10-36200 \cdot 1-2 x \cdot 00+36000^{\circ} \varepsilon$

$E x=10-3012509-2 x+10+31<000$

$x * 00+36560 \cdot 2 \cdot$ $x \cdot 10+36508 \cdot 10$

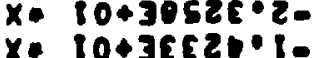

$x \cdot 00+39106 \cdot 1$. $x=00+30692^{\circ} E$ $x+10+309<0^{\circ} \mathrm{E}$ $x \cdot 10+3 \angle 609.8$

$x+10032020 \cdot 6$ $x * 10+36990.1$ $x+10+3629101$ $x=10+36986^{\circ} z-$ $x \cdot 10+396650.60$

$0 x \cdot 20031220 \cdot 1$ 8x* 20-31092090 $2 x+00+32951 \cdot 8$

ex. $10-3640001-2 x \cdot 0003628$ Ex 10-3EItL.E $2 x+00+38096 \cdot 0$.

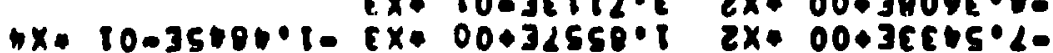

$x=00+30010.2$ $x+00+30681$. x. $10435902 \cdot 20$ $x \cdot 10+32262 \cdot 6=$ $x: 10039055 \cdot 1$

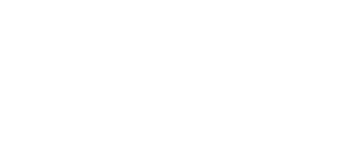

$5 x+20-3+801^{\circ} 8$

$2 x+10-3962001$
$2 x+00+32951 \cdot 1$

Ex. $00+32100 \cdot 6 \cdot$

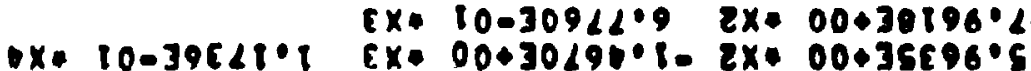

ex* 10-369ts.:

$2 x+100369150$
$2 x+100366009$

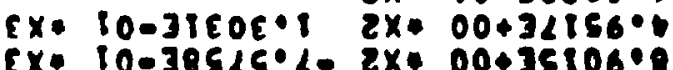

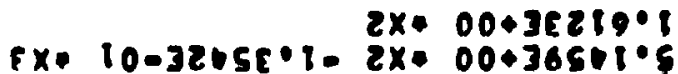
$5 x \cdot 10-31906 \cdot 1-$ $2 x \cdot 00030$

$2 x+20-36280 \cdot 0$ $x+00+32056 \cdot 1$ $x+10+35902 \cdot 8-$ $x+00436026 \cdot 8$.

$x=10-310<0.5-$ $x * 00+36826 \% 60$
$x * 10+36291+1$ $x=10+36208 \cdot 6$ $x_{0} \cdot 10+301010$.

$x * 00+36 t 5 t \cdot 2=$ $x \cdot 10+30920.6$ $x \cdot 00+39690 \cdot 1$. $x=10+36506 \cdot 1$
$x=10+30528.2$ $x+00+31<20: 2$ $x+00+3964<0.90$ $x=1003050008$
$10+35605.50 \quad-(0.09) 84$ $10+31626.0$ (t)

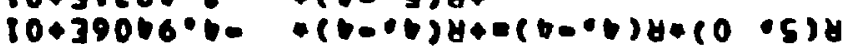

$10+35645.5$ - $(E$. $914+$ $20+36200 \cdot 1$ if 15$) 4+$ $10+36295 \cdot 6$ (c it)

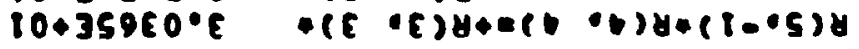

$10+31919 \cdot 2 *(2 \cdot 9) \mathrm{H}$

$10+31651.60$. $(5,02) 4+$

$20+39595 \cdot 10 \cdot(2 \cdot 1) 4+$

$10+31569.6-:(2 \cdot 6) \forall+$

$10+36200 \cdot 1-\quad(2 \cdot 2) y+a(6 \cdot b) \forall *(10-5) y$

$10+30091^{\circ} 10-(1 \cdot 9) 4+$

$10+32020.00$ : (1 . 65$) 4+$

$10+326020.00$ i 11 (1)

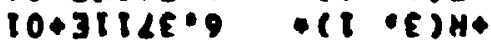

$10+36950^{\circ} \cdot(1 \cdot 2),+$

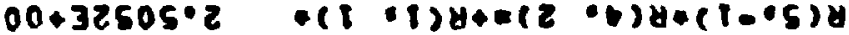

$10+30250 \cdot 10 *+(0 \cdot 9) 84$

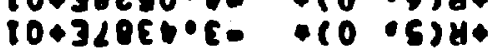

$10+30920 \cdot 6 \quad(0 \cdot 1) 4$

$10+39569.9$ :10 ०E)

$10+310<9 \cdot 10.10 .2) 4+$

$00+36010 \cdot 20$ e(0 it)

$10+32962 \cdot 8-(1-\cdot 9) 40$

$10+3+619.5 \quad(1-.6) y_{0}$

$10+340<0.6 \quad(10.1) 4+$

$10+36551050$ (10.5)

$10+3260000$ (

$00430196^{\circ} \mathrm{s}$

- (10.1)y+act

$00+30<00 \cdot 6$

$10+36508^{\circ} 0$

$10+3<60<.60$

$10+39292 \cdot 8 \cdot$

$-(2-0.9) 4$

$-(5-2) 4+$

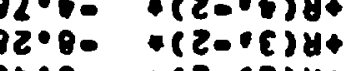

$10+30561 \cdot 1$ $-(2-0.2) 4+0(10 \cdot 0) y+(10 \cdot 5) y$

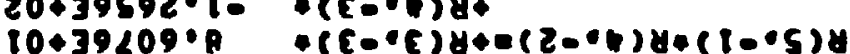

$10+38590^{\circ}=(10-0) 84$

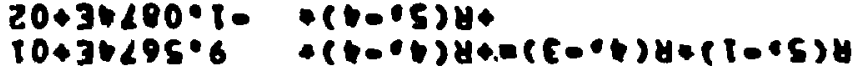

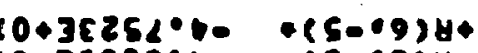

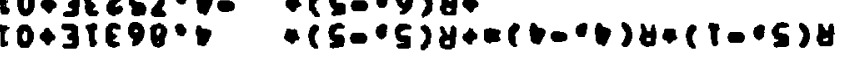




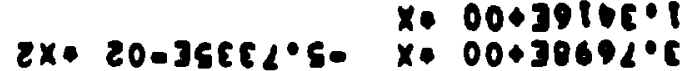

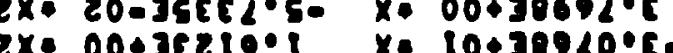

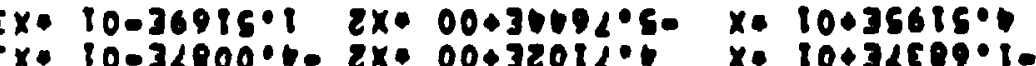

$2 x+10-31906^{\circ} 1$

$2 x \cdot 00+30912 \cdot 1$

$x+00+36540.8$

$x=10+36505 \cdot 10$

$x \cdot 10+3052 c^{\circ} 2$

$E x \cdot 20-31<2<\cdot 6 \cdot 2 x+00+3 E 510 \cdot 1$

$8 x \cdot 10-35065: 1$

$x \cdot 10+35110010$

$2 x \cdot 10-35065^{\circ} 1$.

$8 \times 0.00+30061 \cdot 10$

$\varepsilon x \cdot 29-36052 \cdot 6$

$x \cdot 10+35110 \cdot 1$

$x \cdot 10+3620 E^{\circ}:$

$2 x \cdot 10-30521 \cdot 10$

$2 x \cdot 10.32662 \cdot 9$

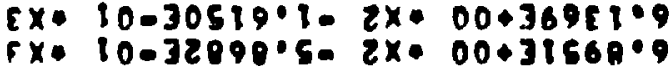

2x* $20-31008^{\circ} 9$

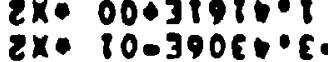

$E x+10.36<20^{\circ} 6$

$x \times 10-38962 \cdot 0$

$2 x \cdot 00+31152.60$

$\rightarrow x+20-3<205 \cdot 60+x \cdot 00+30<610$

$2 x \cdot 10-350 E 5^{\circ} t$

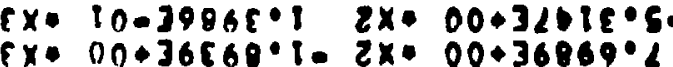

$n x \cdot 10-37515 \cdot 1$ sxo

$2 x+20-34002 \cdot 9$

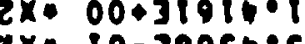

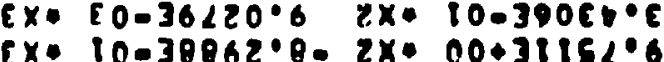

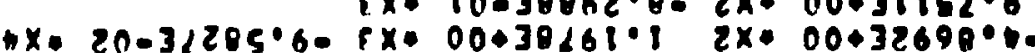

$2 x+10-30521 \cdot 10$

$2 x+10.31662^{\circ} \cdot 9$

Ex. $10-3051901-2 x+00+36961 \cdot 9$
$5 x \cdot 10-32890.5 \quad 2 x \cdot 00+31568 \cdot 9$

2x+10-350es:1

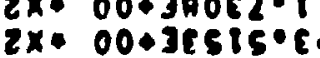

$6 \times 20-36052 \cdot 6$ $x \cdot 00+36596^{\circ} t$

$x+00+30522^{\circ}, 2$

$x+10+30102 \cdot 1$

$x=10+391180.0$

$x=00+35952 \cdot ?$

$x=00+39627^{\circ} \cdot 0$

$x \cdot 10+30202^{\circ} z$

$x+00+39609.2$

$x \cdot 10+35500.8$

$x=10+35110 \cdot 1$

$x, 10+302010$.

$x \cdot 00+35952 \cdot 1$

$x \cdot 00+39621000$

$x+10+3420<^{\circ} 2$

$x=10+3550 \cdot 0$

$x .00+3<05 c^{\circ}$

$x \cdot 00+36696 \cdot 10$

$x \cdot 00 \cdot 36 c 96 \cdot 1$

$x+00+38521^{\circ} 2$

$x=10+391100.0$

x. $10+39406 \cdot 2$

$x \cdot 10+3511001$

$x \cdot 10+36205 \cdot 8$
$10+31919.80 *(2009) 4$

100316etice -(20.5)4.

$20+39592^{\circ} 1 \quad(20 \cdot 0) 4$

$10+31200^{\circ} 60$ (2)- (2)

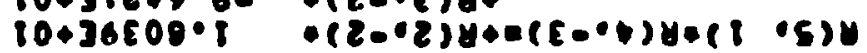

$10+35605 \cdot 5-\quad(60.9) 4$

$20+3010001$ (c-0.

$10+301050$ a

10+3ceso.

\section{$10+35605.5=(10.918$}

$10+97060^{\circ}$
$10+916500$

$10+31624 \cdot 80-(6 \cdot 5) 8+$

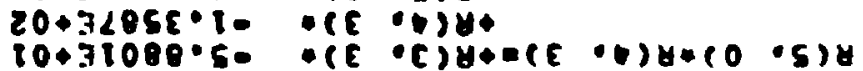

$10+3562908-*(299) 4+$

$10+32650.00 .(2.5) 4$

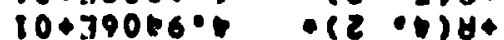

$20+3592001$ (c)

$10+3<009 \cdot 5$ a

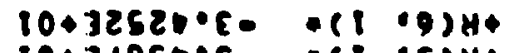

10+32060.

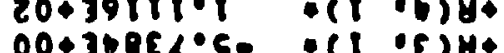

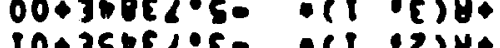

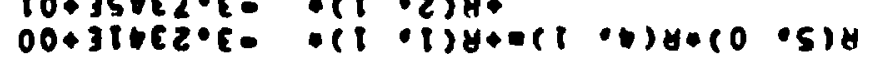

$10+31620 \cdot 0 \quad(0.5) 8+$

$10+36680.60$. $(0.6) y^{\circ}$

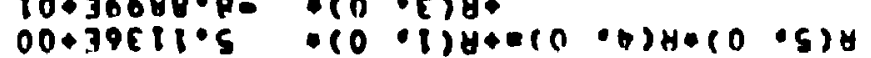

$10+32520.5 \quad(1.09) y+$

$10+32860.0+(1-0,5) 4+$

50.30111010 - (10.0)

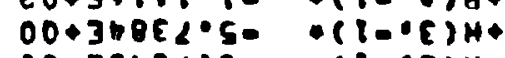

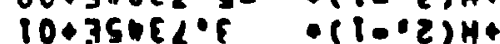

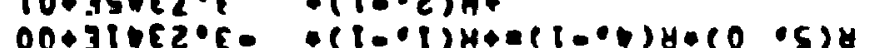

$10+35620 \cdot 6 \quad 0(20.0) 4+$

$10+35680.00 .(50.2) 4+$

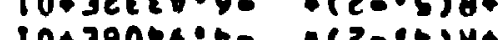

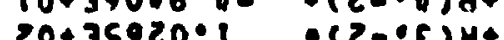

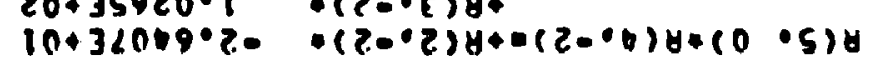

$10+31620.00-(60.5) 44$

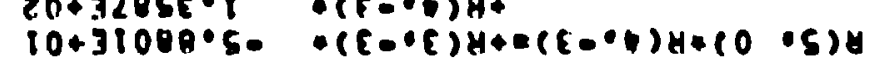




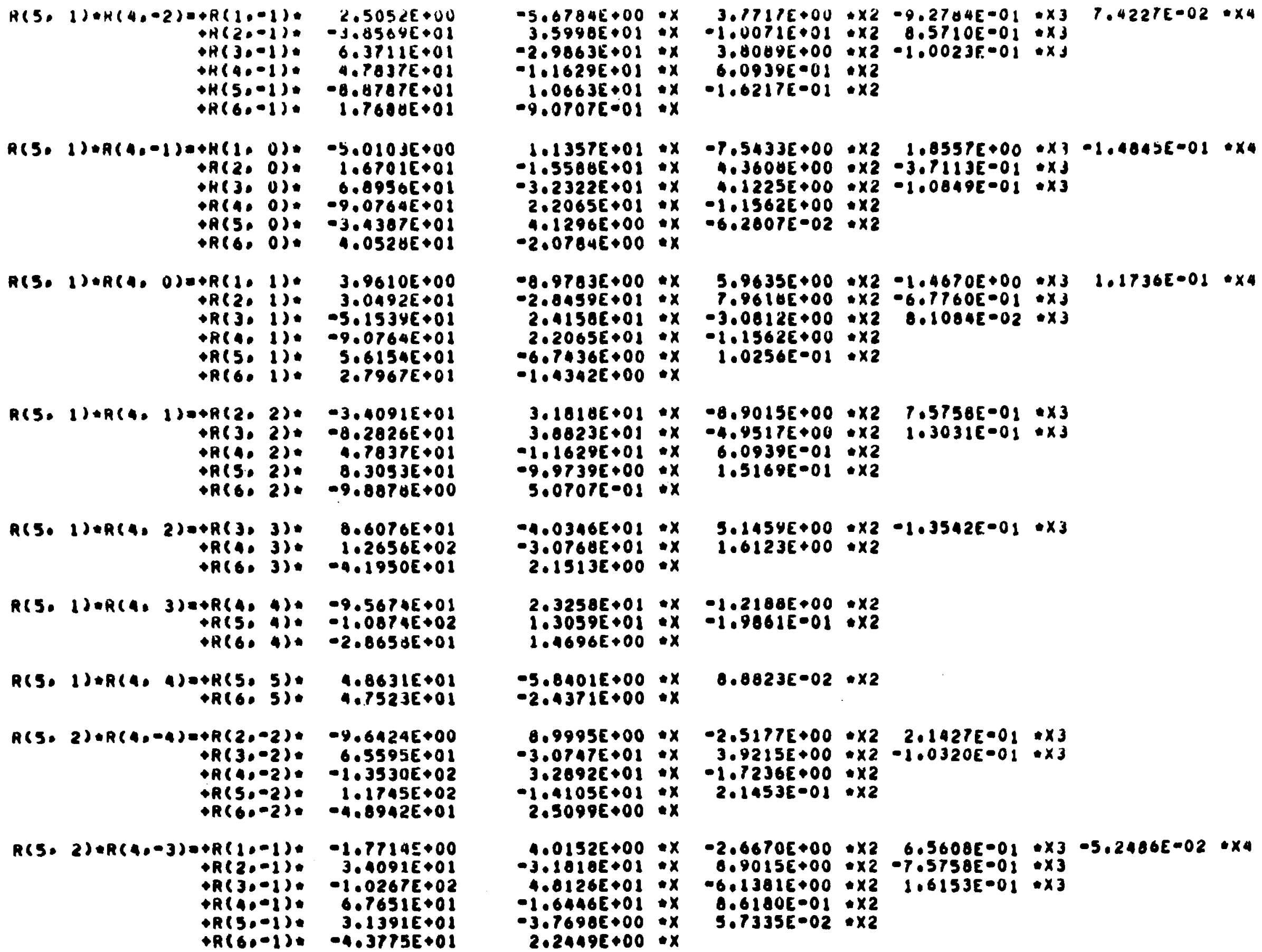

$H(5,1)+H(4,-2)=+R(1,-1) \cdot \quad 2.5052 E+00$ $-1.8569 E+0$ $6.3711 E+01$ H(50-1). H(60-1)

R(5. 1) $+A(4,-1)=+H(1,0)=-5.0103 E \bullet 00$ PA(2. 0) $1.6701 E+02$ - H(3. 0) 0.0956E+01 H AR(5. O) $4.05285+01$

$R(5,1) \bullet R(A, 0)=+R(1,1) \cdot 3.9610 E+00$ R R(2. 1). 3.0492E+01 TR(3. 1) -S.153YE+O

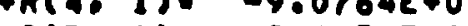
QR(5. 1) S.6154E+0 $+R(6,1)$ 2.7967E+01

$R(5,1) * R(4,1)=+R(2,2) *-3.4091 E+01$ $+R(3,2) *-8.2826 E+01$ -R(AD 2). A. 2037e+01 -R(5. 2$)$ O. 0.305JE+0 $\rightarrow R(6,2)$. $-9.8078 E+00$

$A(5,1) * R(4,2)=+R(3,3) * 8.6076 E+0)$ $\rightarrow R(A, 3): \quad 1.2656 E+02$

$R(5,1)+R(4,3)=+R(4,4) *-9.5674 E+0$ $+R(5,4):-1.0874 E+02$

R(5, 1)+R(4, 4)=+R(5, 5): $4.8631 E+01$

$R(5,2)+R(4,-4)=+R(2,-2),-9,6424 E+00$ $\rightarrow R(3,-2)=6.5595 E+0)$ $+R(4,-2),-1,3530 E+02$ $+R(5,-2): \quad 1.1745 E+02$
$+R(6,-2):-4.8942 E+01$

$R(5,2)+R(4,-3)=+R(1,-1):-1.77145+00$ $\rightarrow R(2,-1): 3.4091 E+01$ $\rightarrow R(30-1):-1.0267 E+02$ $+R(A+-1): 6.7651 E+01$ $+R(30-1): 3.1391 E+01$
$+R(60-1)=-4.3775 E+01$

$-5.6784 E+00 \cdot x$ $3.5998 E+01 \cdot x$ $-2.9863 E+01 * x$ 1.00635001 . $x$ $1.06638+01 * x$
$-9.07075-01 * x$

$1.1357 E+01 * x$ $-1.5508 E+01 * x$ $-3.2322 E+01: x$ $2.2065 E+01$
$-.1290 E+00: x$ $-2.0784 E+00: x$

$-0.9703 E+00 * x$ $-2.0459 E+01+x$ $2.4158 E+01: x$ $2.2065 E+01: x$
$-6.7436 E+00: x$ $\begin{array}{ll}-6 \cdot 7436 E+00 & : x \\ -1.4342 E \$ 00 & * x\end{array}$

$3.18106+01 \cdot x$ $3.0823 E+01: x$ $1.1629 E+01: x$ $-9.97395+00 * x$
$5.07075-01 * x$

$-4.03465+01 \cdot x$ $-3.07685+01 * x$ 2.1513E॰00 $\bullet x$

\section{$2.3258 E+01: x$} $1.9696 E+00$ : $x$

$\begin{array}{ll}-5.81016+00 \\ -2.43715+00 & : x\end{array}$

$8.9995 E+00 * x-2.5177 E+00 * \times 2 \quad 2.1427 E \cdot 01 * \times 3$ $-3.0747 E+01 * x \quad 3.9215 E+00 * x 2-1.0320 E=01 * x 3$ $3.2892 E+01 * x-1.7236 E+00 * x 2$

$-1.4105 E+01: x \quad 2.1453 E-01 * \times 2$ $2.5099 E \bullet 00 \bullet x$

$0.01525+00 \cdot x$ $-3.1010 E+01 \cdot x$ $4.0126 E+01: x$ $-1.6446 E+01$ ix $-3.7698 E+00: x$

$3.1717 E+00 * \times 2-9.27 \Delta 4 E-01 \times \times 3 \quad 7.4227 E-02 \times \times 4$ $-1.0071 E+01 \times \times 2 \quad 0.5710 E-01 \times x 3$ $3.0009 E+00 \times \times 2-1.00236-01 * x s$

$6.0939 E-01: \times 2$

$-1.6217 E-01 \cdot \times 2$

$-7.5433 E+00 \cdot \times 2 \quad 1.8557 E+00 * x 3-1.4845 E-01 \cdot x 4$ $4.3608 E+00 \quad \times 2-3.7113 E-01 \quad x 3$

$-1.1562 E+00 \div \times 2$

$0.20075-02 \div \times 2$

$5.9635 E+00 \cdot \times 2-1.4670 E+00 * \times 3 \quad 1.1736 E \cdot 01 \cdot \times 4$

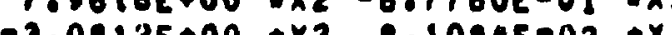

$-1.1562 E+00 \div \times 2$

$1.0256 E-01 * \times 2$

$-8.9015 E+00 * \times 2 \quad 7.5758 E-01 * \times 3$ $-4.9517 E+00 * \times 2 \quad 1.3031 E-01 * \times 3$ $6.0939 E-01 \div \times 2$

$1.5169 E-01 \cdot \times 2$

$3.1454 E+00 \cdot \times 2-1.3542 E-01 \cdot \times 3$

$1.6123 E+00 \times 2$

$-1.2100 E+00 \cdot \times 2$

$0.8823 E-02 * \times 2$

$-2.6670 E+00 * \times 2 \quad 6.5608 E-01 * x 3-5.2486 E-02 * x 4$

$0.9015 E+00 * x 2-7.5758 E-01: \times 3$

$-6.1381 E+00 \times x 21.6153 E-01 . \times 3$

$0.61005-01+\times 2$

$5.7335 E-02 \times \times 2$ 


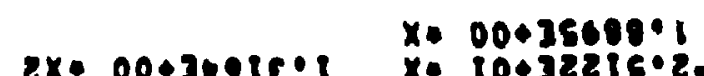

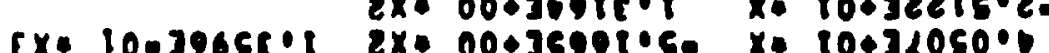

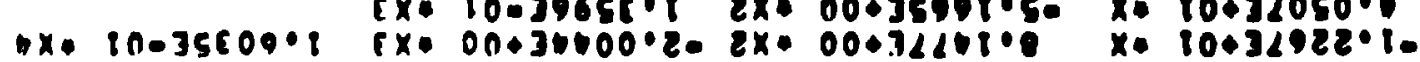
2x: 10035ecs? $x+10+3511001$

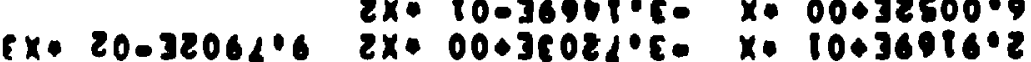

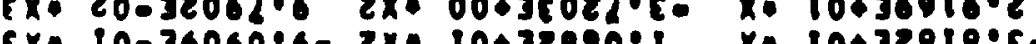

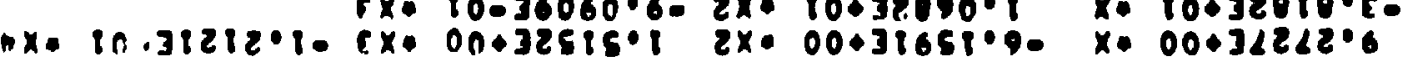

$2 x=10-31990^{\circ} t \quad x=00+31066^{\circ} !$ i. $x+10+31062 \cdot 6$ Ex. $10032065 \cdot 1-2 x+00+325100^{\circ} x+10+32696^{\circ} .6$ $8 x+00+32281 \cdot 9 \cdot x_{*}+10+30602 \cdot 8$

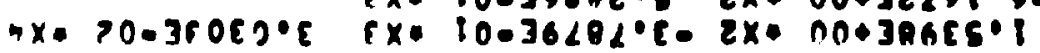

$2 x+10-35566 \cdot 1-2 x+10-36915 \cdot 80$
$2 x+00+36208 \cdot 5$ $2 x \cdot 10.36190 \cdot 8$ $x \cdot 00+30565 \cdot 1$. $x=00+3050908$ $2 x \cdot 10-30000 \cdot 8$
$2 x \cdot 00+39620.8$

$2 x+10-306000$ $2 x \cdot 16-300190$

$8 x \cdot 10=30689 \cdot$ ex. 00+31016.0

$2 x \cdot 20-31005 \cdot 2$ $2 x+00+312+6 \cdot 10$ $6 x+20-36<9109-2 x+00+39646.8$

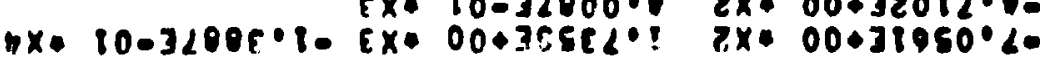

$2 x+10-30521010$ $5 x+00+31560 \cdot 8$ $E x \cdot 20-30981 \cdot 2$

$2 x \cdot 10-36811 \cdot 0$ $b x=10-3100 E \cdot 1$ Ex. $00+305 E 2 \cdot 1-2 x+00+31950 \cdot d$

\begin{tabular}{|c|c|c|c|}
\hline 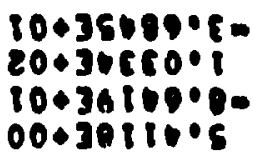 & $\begin{array}{l}-1 \\
: 11 \\
: 11 \\
01\end{array}$ & 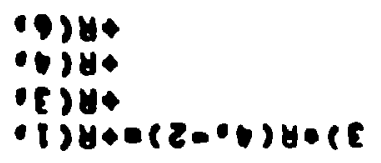 & - 534 \\
\hline 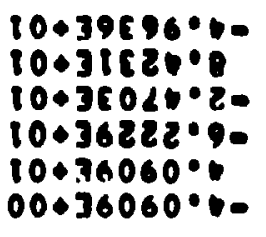 & $\begin{array}{l}: 10 \\
110 \\
110 \\
110 \\
110 \\
110\end{array}$ & 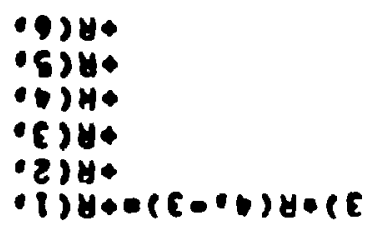 & $\cdot 5) y$ \\
\hline 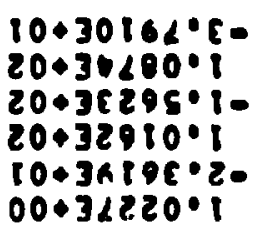 & 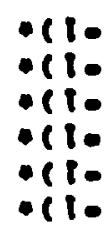 & 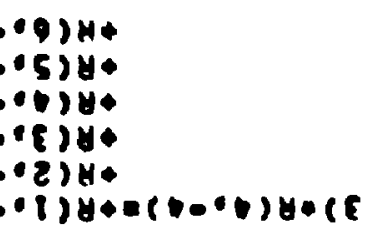 & - 5 in \\
\hline $10+31111 \cdot 0$ & $=10$ & $-9) y+a(0,0) H=12$ & - 5 siy \\
\hline $\begin{array}{l}10+30000 \cdot 50 \\
10+30860.60\end{array}$ & $\begin{array}{l}: 15 \\
-15\end{array}$ & 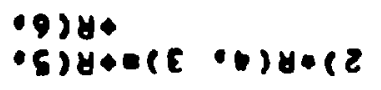 & - \\
\hline $\begin{array}{l}10+36516 \cdot 20 \\
10+32689 \cdot 2 \\
80+30656 \cdot 1\end{array}$ & $: 16$ & 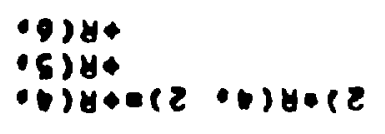 & - 518 \\
\hline $\begin{array}{l}10+362+2 \cdot 2 \\
10+32609 \cdot 2 \\
10+3159290 \\
20+39620 \cdot 10\end{array}$ & 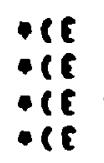 & 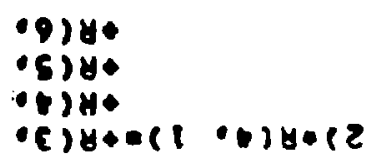 & -siy \\
\hline 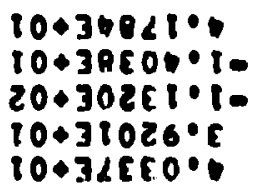 & $\begin{array}{l}-12 \\
: 12 \\
: 12 \\
: 12 \\
: 12\end{array}$ & 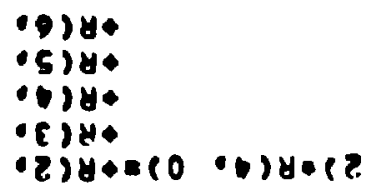 & (5) \\
\hline 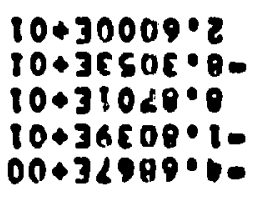 & 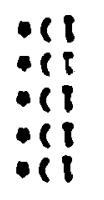 & 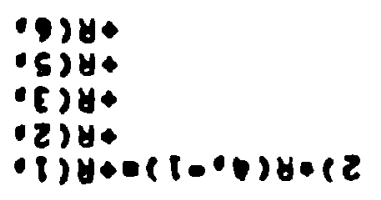 & - (s)y \\
\hline $\begin{array}{l}10+32690 \cdot 1- \\
10+326 E 990 \\
20+30261 \cdot 1 \\
10+36 \angle 56 \cdot 10 \\
10+35621 \cdot 6- \\
00+32909 \cdot 9\end{array}$ & $\begin{array}{l}\bullet 10 \\
-10 \\
: 10 \\
: 10 \\
: 10 \\
-10\end{array}$ & 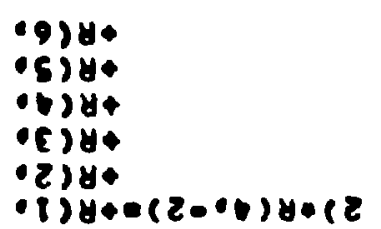 & \\
\hline
\end{tabular}




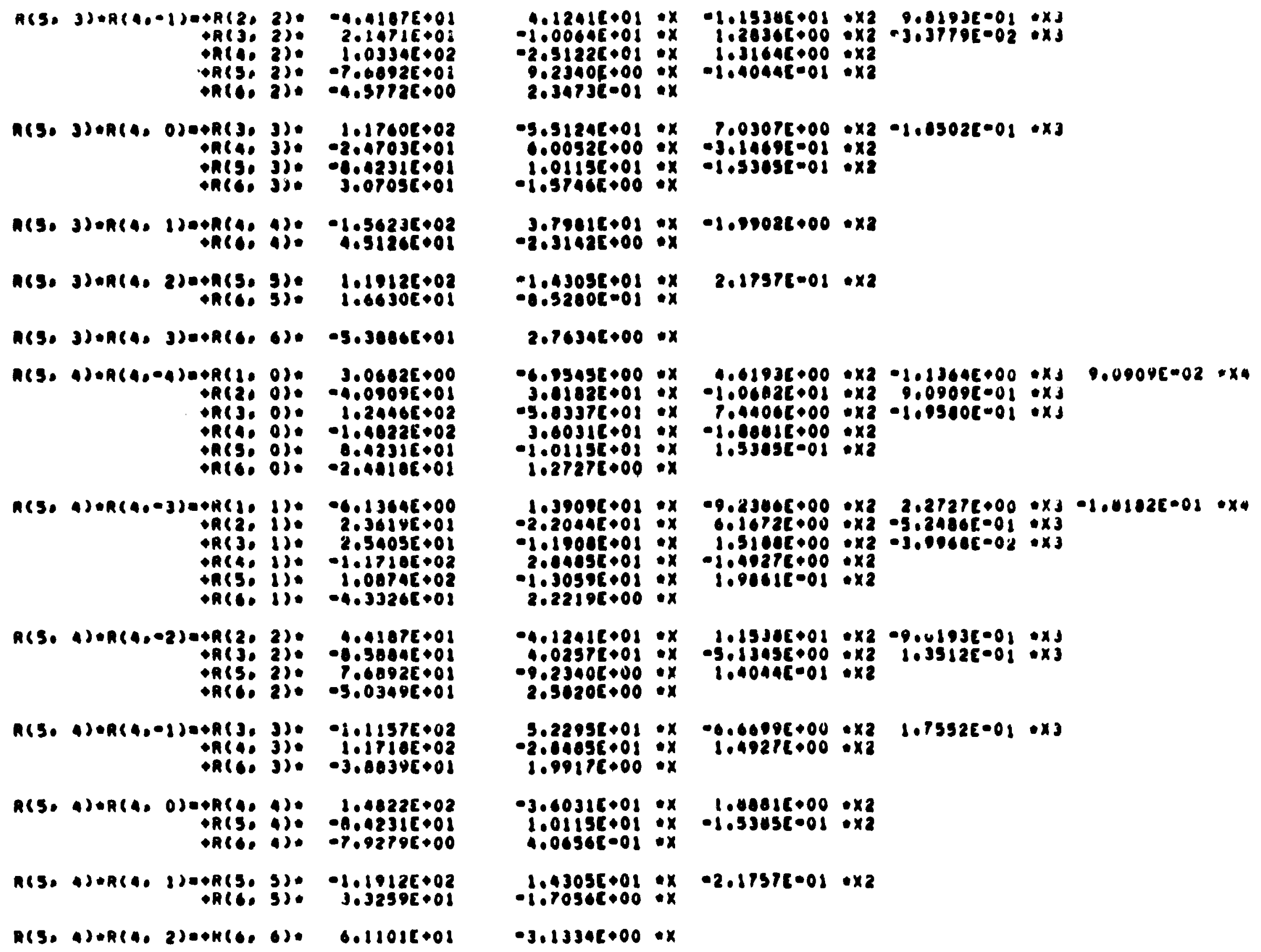

$-1 \cdot 11575 \cdot 02$ i. itiot +02 AR(6. 3). -3.80395०01

$R(5, A) \cup R(4,0)=Q R(A, A) \cdot$ R(S. H): $-A \cdot 4231 C 001$

A(5. A)+R(A. 1)=\$R(S, S): -1.1012E+02 PR(6. 5). 3.3239E०01

R(s. A)+R(A. 2)

$6.11016+01$

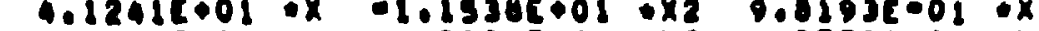

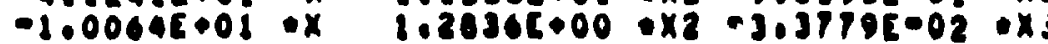
$-2.51226+01 * x \quad 1.31646000$ *xz $\begin{aligned} & 0.23406+00 \\ & 2.3473600\end{aligned}: x$

$-5.5124 c+01 * x \quad 7.03075+00 * x 2 \cdot 1.05026 \cdot 01 \cdot x^{2}$ $6.00526+00$ : $+3.14602=01$ *xz

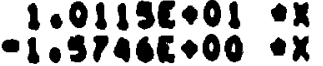

$\begin{array}{r}3.19616+01 \\ -2.31425000\end{array}: x$

$-1.4305[001 \cdot x$ $0.3200[=01$ :x

$-1,01028+00 \cdot \times 2$

$2.1795601 \cdot 02$

$2.76348+00 * x$

$-6.8545 E+00 * x^{2} \quad 4.61936+00 * x_{2}-1.13646+00 * x_{J} \quad 0.09045002 * x_{4}$

$3.01825001+x-1.06026+01 \times 28,9.0909601 * x 3$

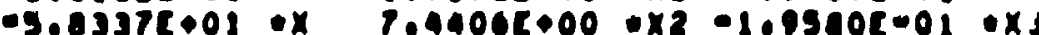

$3.0031<+01 \cdot x$

0.0115 to $x$

1.27275000 : $x$

$1.9846000 \times 2$

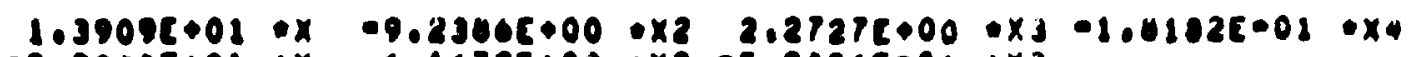
$-2.2040 \mathrm{Col}: x$ -1.19005001 . 200455001 : $x$ -1.3059601 : $x$

$2.2219<000$ :x

$6.10736+00 \div \times 2 \quad-5.24806-01 \div \times 3$

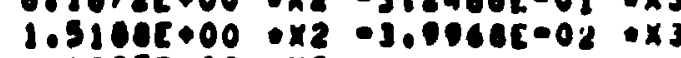

$1.90276+00 \times x$

$-4.12416001 \cdot x$ $0.02570+02: x$ $-9.2340[+00: x$ $2.50201+00 \cdot x$

$3.22995+01 \cdot x-0.00090+00 \times x \quad 1.7552 E-01 \cdot \times 3$

$-2.09058+01: x \quad 1.49218+00: x 2$ $1.99176 \circ 00 \cdot x$

$-3 \cdot 60316 \cdot 01 \cdot x$

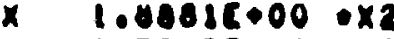

$1.01155001 \cdot x-1.53456001 \cdot x 2$ $4.00565-01 * x$

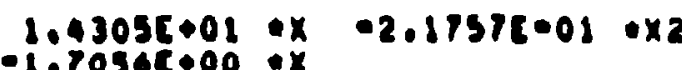

$-3.13346000 \cdot x$

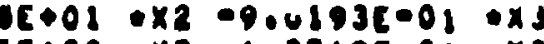

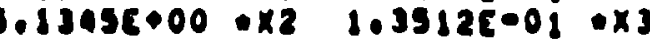

$.40445001 \times 2$ 


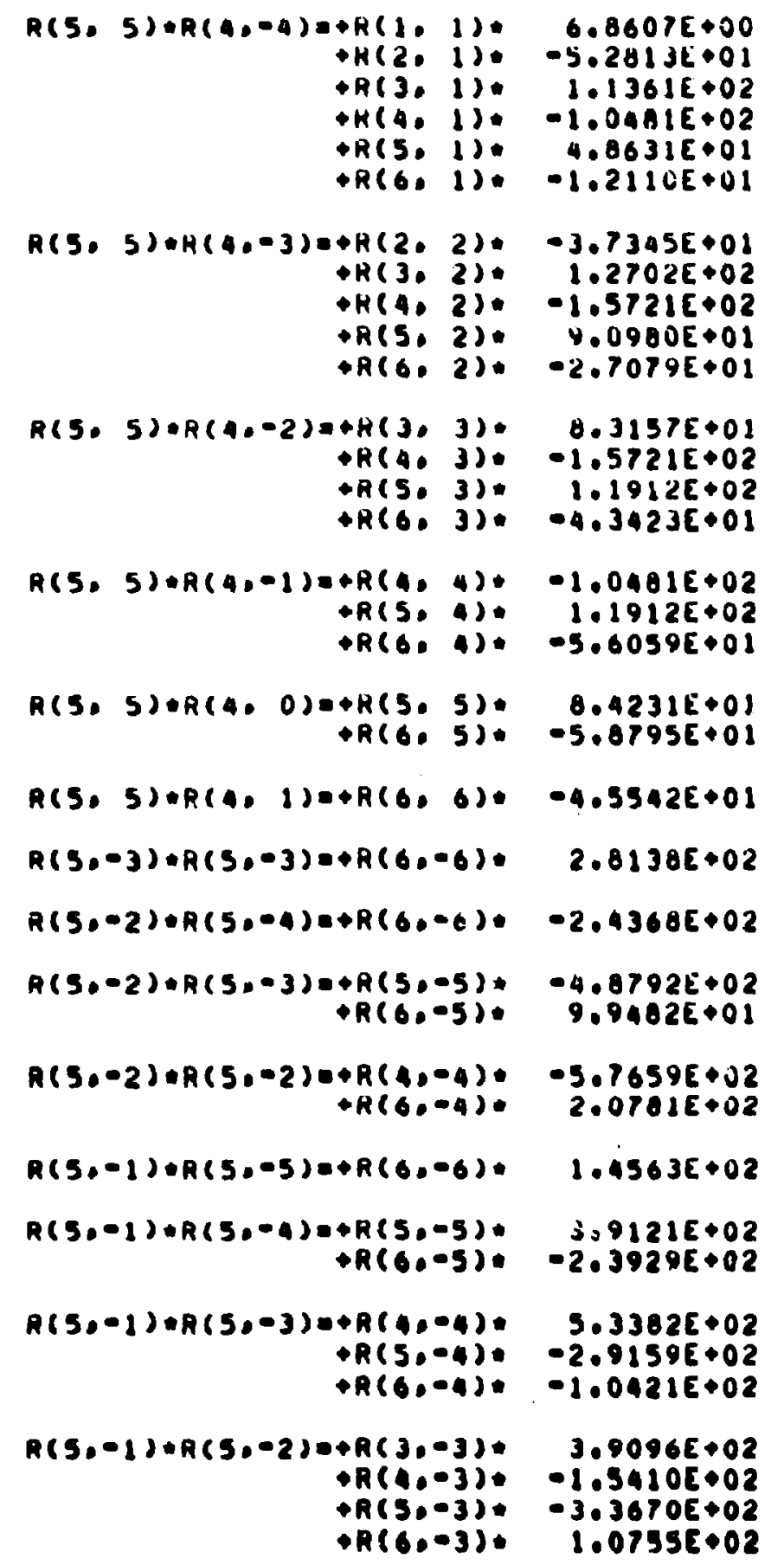
$9.9482 E+01$

$-5.7659 E+02$
$2.0701 E+02$ $1.4563 E+02$ $309121 E+02$ $-2.3929 E+02$

$-1.5551 E+01 \cdot x$ 40.028 $2.54705+010 x$ $-3.8401 E+00 \cdot x$ $6.2103 E-01 \cdot x$ $3.48556+01: x$ $3.0217 E+01$ : $x$ $-1.0026 \varepsilon+01$ *x I.3807E $+00 * x$

$-3.0978 E+01 * x$

$4.9714 E+00+x 2-1.3003 E=01$ * $x$ $3.0217 E+01 * x-2.0026 E+00 * x 2$

2.22685000 : $x$

$2.5478 E+01 \times x-1.3351 E+00 * x 2$ $-1.4305 E+01 \cdot x \quad 2.1757 E-01 \times x$ $2.8748 E+00: x$

$-1.0113 E+01 * x \quad 1.5385 E-01 * \times 2$ $3.01516+00 \cdot x$

$2.33556+00 * x$

$-2.2595 E+01 * x \quad 2.167 A E \cdot 01 \cdot \times 2$

$1.9560 E+01$ \& $-1.0770 E-01 \times 2$

$7.0647 E+01 \times x \quad-2.4920 E+00 \times 2$

$1.6534 E+02 * x \quad-1.2594 E+01 * \times 2$
$-1.6687 E+01 * x \quad 1.6007 E-01 * \times 2$

$-1.1694 \varepsilon+01 \cdot x \quad 1.1217 E-01$ *x2

$-6.1454 E+01 * x \quad 1.9985 E+00 * x 2$ $1.9215 E+01 \cdot x-1.0432 E-01 \cdot \times 2$

$-1.5300 E+02 * x \quad 1.1660 E+01 * \times 2-1.0250 E-01 * x_{3}$ $1,5005 E+01: x \quad-1.4090 E+00 \times \times 2$ $0.3685 E+00: x-0.0273 E-02 * \times 2$

$-2.01995+02 * x \quad 3.14945+01+x 2-1.44005+00 * x^{3}$ $4.4190[+01$ *x $-3.3650[+00 * \times 2 \quad 4.69 i 1[-02 * x 3$ $5.2092 E+01 * x-1.7201 E+00 * x 2$ $-0.6365 E+00 * x \quad 0.2045 E-02 * x 2$

\section{$0.29805-010 x\rfloor$}

$2.0320[\cdot 01 \cdot x 4$

$1.75525-01 * x]$ 
$2 x \cdot 10032<68 \cdot 1-x * 10+39954 \cdot 1$

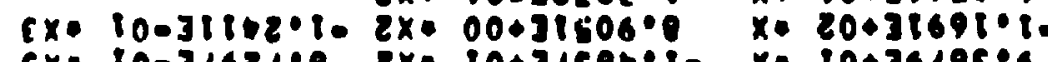

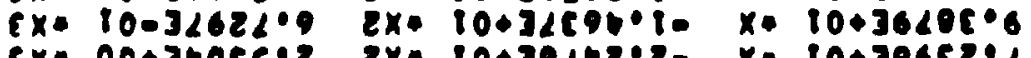

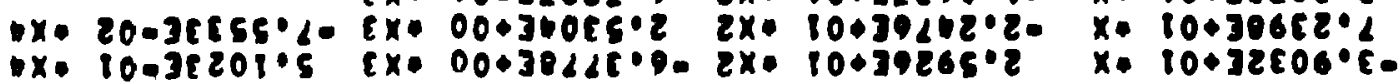

$2 x+10-310+8 \cdot 10 x+10+30262 \cdot 1$

$2 x+00+31022^{\circ} 8 \quad x+10+32608.5$

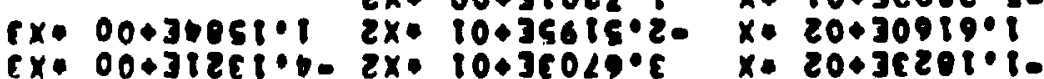

$\rightarrow x \cdot 10-35628 \cdot 1 \quad x+00+31261 \cdot 0.2 x \cdot 10+36020^{\circ}$

\section{$2 x \cdot 20-35166^{\circ} 2$}

$2 x+00+39600^{\circ} !$

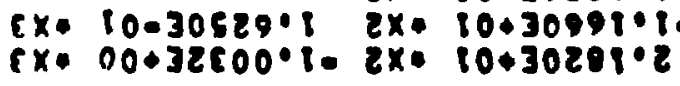

$x \cdot 00+3256 t^{\circ} 2$

$x=10+35005 \cdot 6$

$2 x \cdot 100311120^{\circ} 8$

$E \times 40.36202 \cdot 1 \cdot 2 x \cdot 00+36018 \cdot 5$

ex. $10-30920 \cdot$

$x=80+30055: t$
$x \cdot 20+35668 \cdot 1$

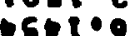
$x+10+30500$
$x=100$ $r x=10-36052 \cdot 1 \quad 2 x+c 0+351 E 0^{\circ} 6$

$x+10039906 \cdot 10$ $x=80+3 \angle 501.1$ $x \cdot 20+31<18 \cdot 1$

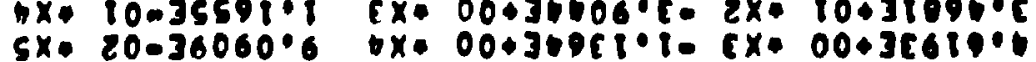

\section{$2 x \cdot 20-35960 \cdot 0$

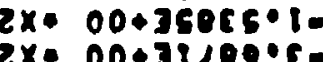

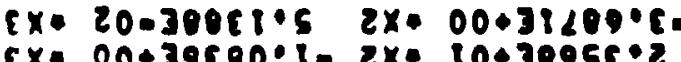

$c x+00+39600 \cdot 1-2 x \cdot 10+39956^{\circ}-2$

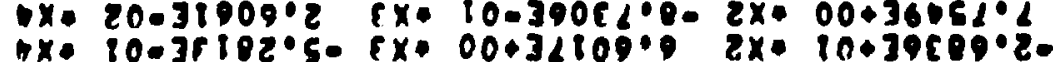

\section{$8 x+20-3017005-$}

$5 x \cdot 00+35065^{\circ}:-$

$1 \times+20030619.60-2 x+00+36260^{\circ} 9$

ix. $10-3951009-2 x+10+30000 \cdot 1$

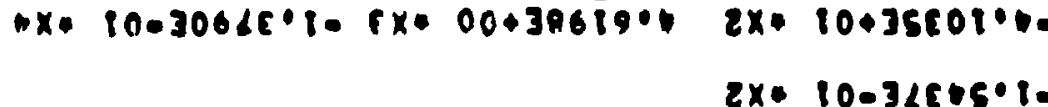

$2 x=10032060.6$

Ex: $10-31052 \cdot 1-8 x+00+35180.6$

$2 x \cdot 20.31095 \cdot 6 \cdot$

$2 x+00+32106 \cdot 2$

$x=00030500^{\circ}$

$x=00+30562^{\circ} \cdot 8$ $x=10+300 x^{\circ}$

$x=30+3201150$ $x=80+39115 \cdot 1$. $x+10+32060.0$

\section{$x \cdot 00+30952 \cdot 5$} $x=10+30082^{\circ}$ $x=10+32950.6$ $x * 10+356 E 0.60$

$x=20+30128 \cdot 1$

$6 x \cdot 00+34568$

$2 x+00+35160.60$

. 10+35609.1

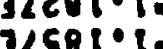
$x=20+31900^{\circ}$

$5 x \cdot 10-31052 \cdot t$

$2 x+10-3962<0,1$

$x \cdot 00+39216^{\circ} 6$

$2 x+10032190 \cdot 1$

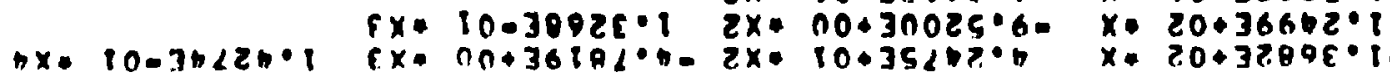

$20+36810010-(1-0,0) 4+$

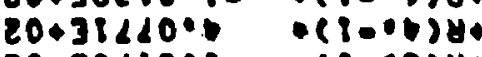

$20+30<10 \cdot 1-(10+8) y$.

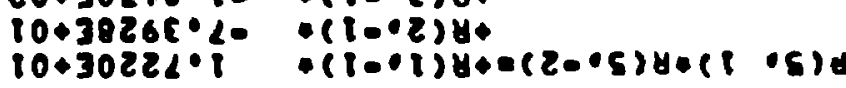

$20+30019 \cdot 10 \cdot(2-19) 4$

$20+30206 \cdot 6 \quad-(2-0.5) 40$

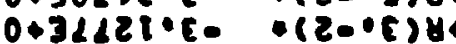

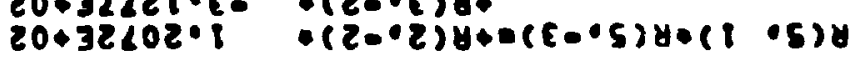

10+30101.6 $\quad(E-00) 4$.

$20+36516^{\circ} z \quad-(E-.5) 4$

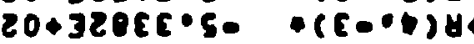

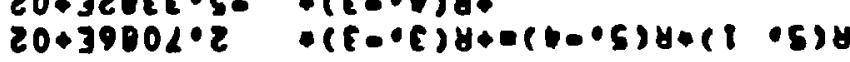

$20+39009 \cdot 2 \quad(1-0.0) 4$

$20+31216^{\circ} \mathrm{Co}+(\mathrm{coc}, \mathrm{siy}$

$50+36506 \cdot 5$ a

$20+36158 \cdot 1-(0 \cdot 9) y$

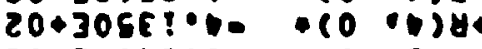

$20+3<041 \cdot 1 \quad(0.2) 4$

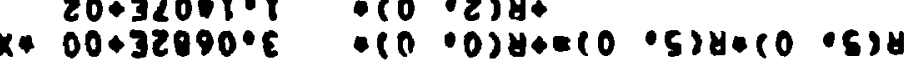

$\left.80+36560^{\circ}\right) \quad(1-19) 84$

$20+35110.60$ - (10.5)

$20+31009 \cdot 10$ - (100)

$20+32526^{\circ} z$ (I.-E)

$10+39055^{\circ}-(1-02) 4$

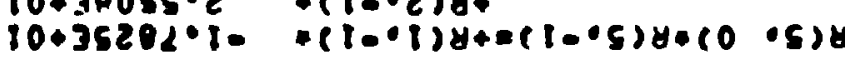

$10+35505.90 *(2009) 4$

$20+35110^{\circ} \mathrm{E} \cdot\left(20^{\circ} 5\right)^{\circ}$

$20+31051.6 \quad(20.0) 4$

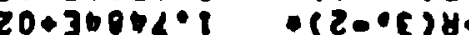

$20+32606 \cdot 1 \cdot+(2-02) \forall+=(2-\cdot 5) 4+(0 \cdot 5)$

$20+31600 \cdot 2-\quad-(80.09) 4+$

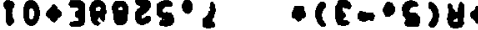

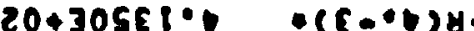

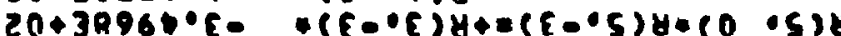

$20+36162 \cdot 10 \cdot(4-0) 4+40$

$20+361150.0(10-0) 4)^{2}$

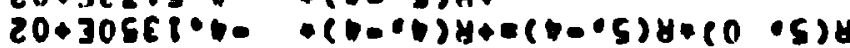

$20+39200^{\circ} 2 \quad \cdot(5-.9) H 4$

$20+31952 \cdot z-0 ! 5-\cdot 5) y+=(5-15) y+(0$ - 5$) 4$

$50+31<68 \cdot 1 \quad *(2-0.9) H+$

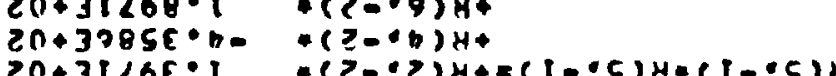


2x: 10-32<06. $10 x \cdot 10039950 \cdot 1$

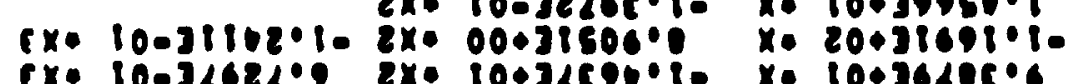

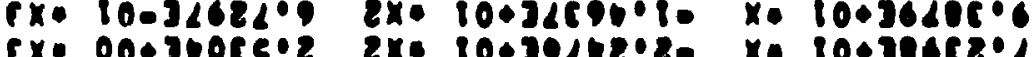

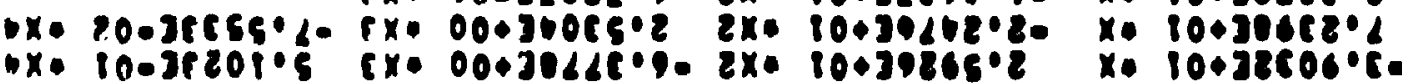

ax.

$x+20036 \angle 4002-2 x \cdot 00+32505$

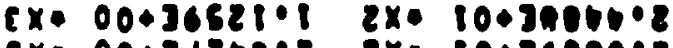

$6 x+20-\operatorname{anc} 60^{\circ} 0$ tx. $10-31<2 \alpha^{\circ}$

8x. $10+36000 \cdot 8$

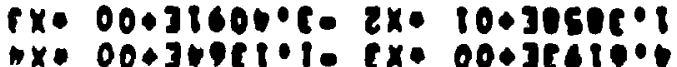

$2 x \cdot 80-35900^{1} \cdot-x \cdot 00+39996 \cdot 1$

8x. $00.3108201 \mathrm{x} \cdot 10+38602 \cdot 50$

$280.02 \times 0.00034$

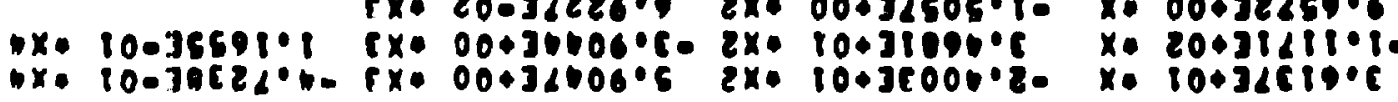

5x. $10035598 \cdot 1$

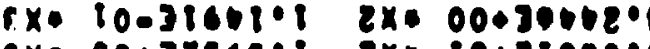

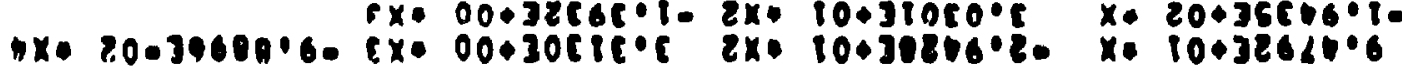

$2 x \cdot 10-36000 \cdot 2 \quad x+10+36590 \cdot 8$ $0+3<090.2$

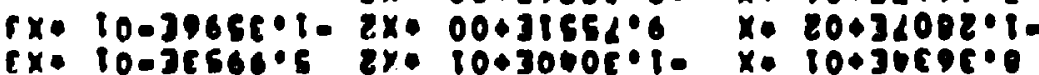

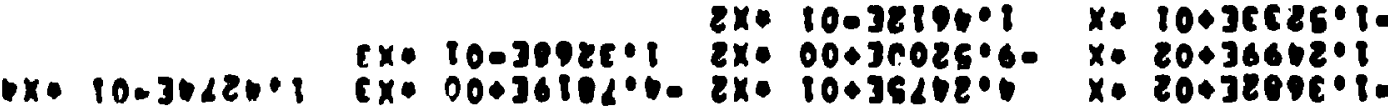

5x. 80035060.8 $x=00+30562.00$

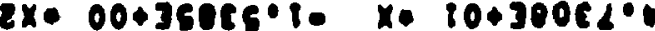

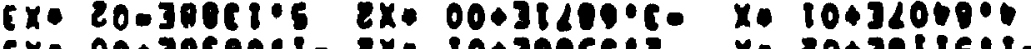

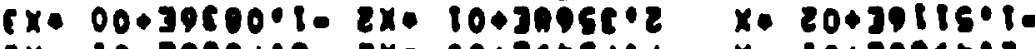

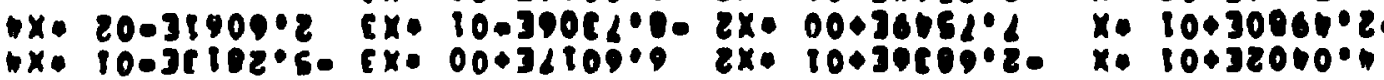

\section{$8 x+8003102800 \cdot x \cdot 0003605400$}

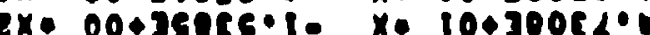

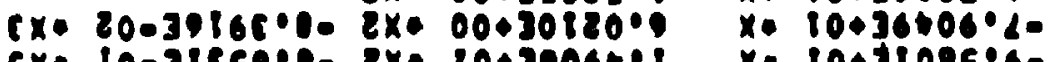
$6 x+10031650 \cdot 9-2 x \cdot 100390600$

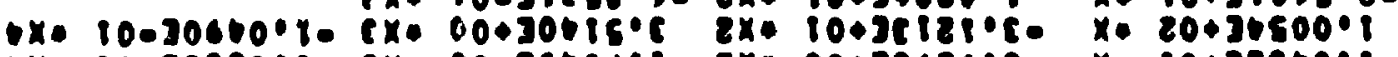

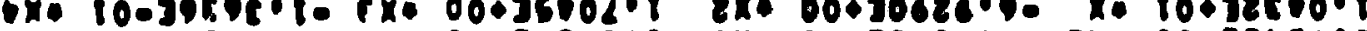

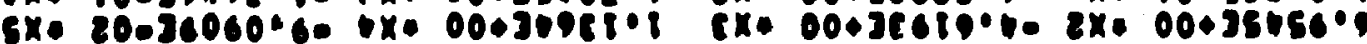

$80+30610 \cdot 10 \cdot(1 \cdot 0) 40$ $80+318100 \%$ of $18, y+$

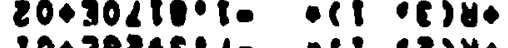

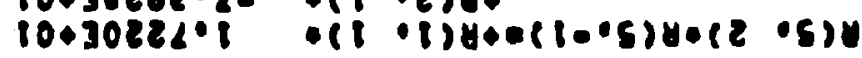

$20 \cdot 32099 \cdot 10 \cdot 10 \cdot 9140$

$20 \cdot 35110.6$ E 10.5$) 8$

$10+3916000 \%(0.6) 4$.

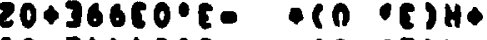

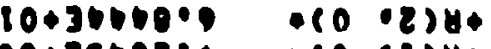

$00+35402^{\circ} \cdot 10 \cdot 1140$

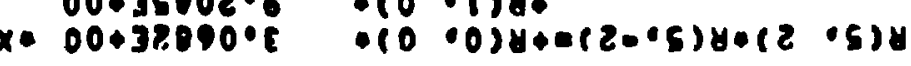

$10+31600 \cdot 20 \cdot(10 \cdot 9) 40$

$20+30<96 \cdot \%$ (1.05)4

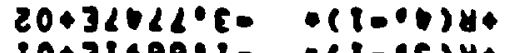

$10+31690 \cdot 10$ - (1-16)४

$20+3<041 \cdot 1 \quad(10-2) y+$

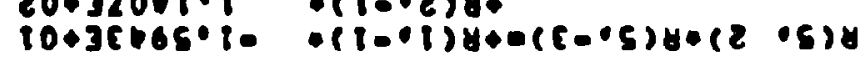

$20+36209 \cdot 1 \quad(20 \cdot 0) 40$

$20+31016^{\circ} 60 \quad(2000) 4$

$20+3919<\cdot 6 \quad(2005) 4$

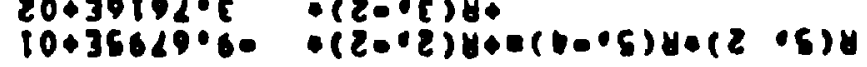

$20+31265 \cdot 2 \quad *(60.9) 4 *$

$20+32610 \cdot 6-(E-95) 4$

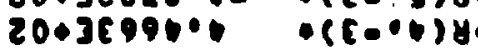

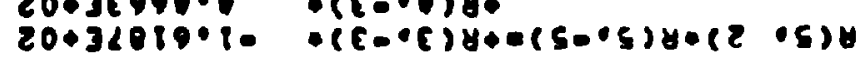

$20+31<60 \cdot 1 \quad(2 \cdot 9) 4$

$50+39056 \cdot 40-(8,0) 4$

$50+31<65 \cdot 1$ - 50 (5)

$20+35560 \cdot 1$ : $(1 \cdot 9) 40$

$20+35110^{\circ} 60$ - $(1.5) y$

$26+31009 \cdot 10$ a(1 - B ive

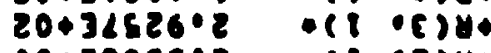

$10+30055^{\circ} z$ (1

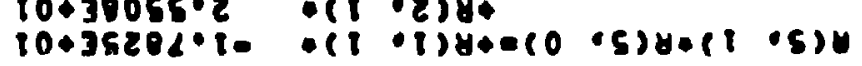

$10+30055 \cdot 50-(0 \cdot 9) 4$

20+3511008. - 10 .5)8

$20+39951^{\circ} 8$ - 10.6$) 40$

$\left.20+36050^{\circ}, 10 \cdot 18\right) 4$

$20+32920 \cdot 1$. $1(0.2) 4$.

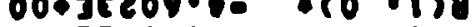

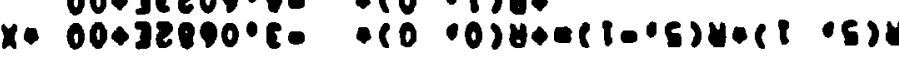




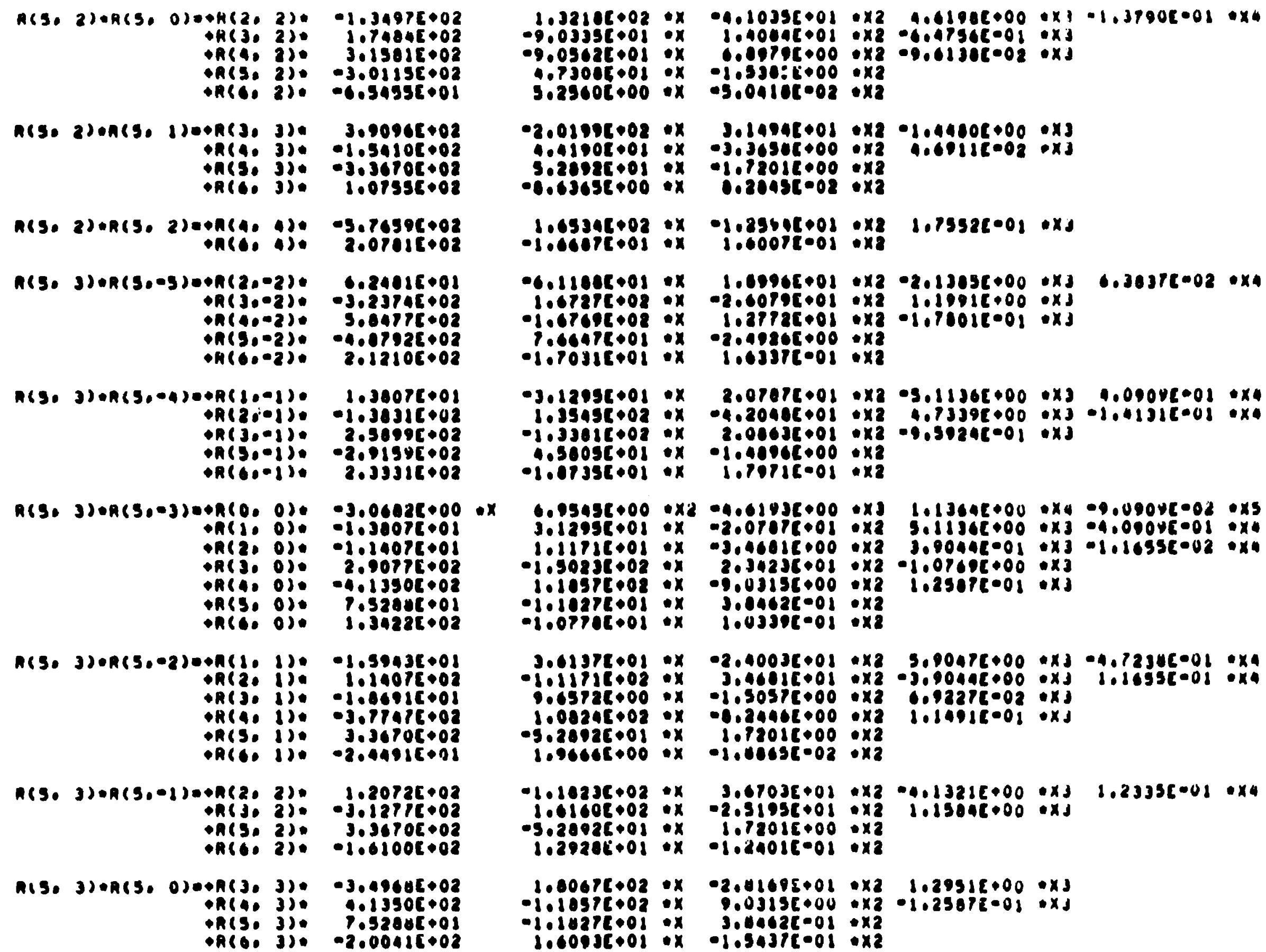

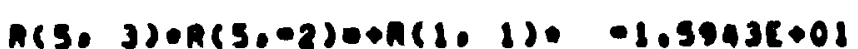
A(2. 1). 1.24078•02 (3)

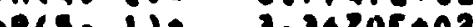
PR(s: 1): 3.3670ctoa

Q(5. 3$) \cdot A(3,01) 0+n(2,2)$, PA(S. 2): 3,36706002

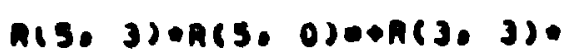
in(4. 3): R(s. 3). $1.13506+02$
$1.32045+01$ $-2.00416+02$

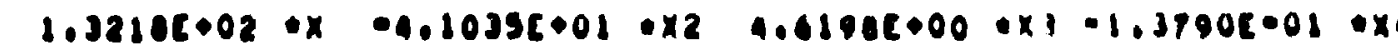

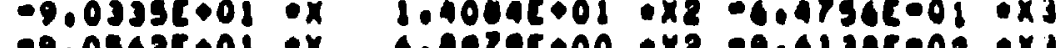

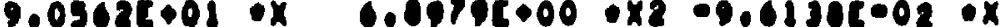

4.7305001 :x $-1,530: 1000$ ixz

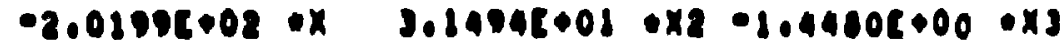

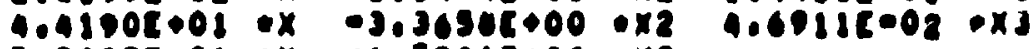

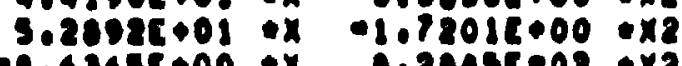

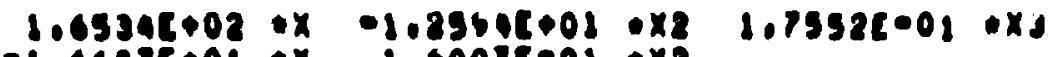

$01.007 c 001 \cdot x \quad 1.0001 \mathrm{c} \cdot 01 \cdot x^{2}$

$-0.11005+01 * x \quad 1.09965+01+x_{2} \cdot 2.1305\left[+00 * x_{3} \quad 0.30375002 \bullet x_{4}\right.$

$1.6727 c+02 * x-2.00796+01 \cdot x 21.10916+00 \times x J$

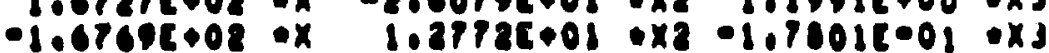

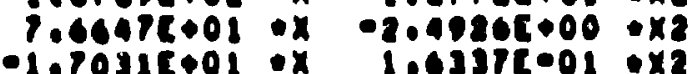

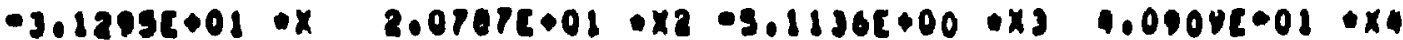

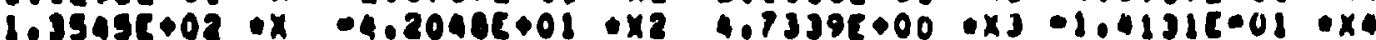

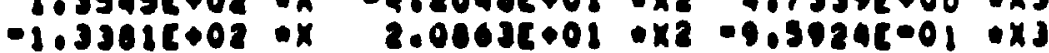

4.50056001 ox -104096000 ex

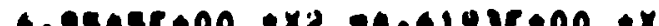

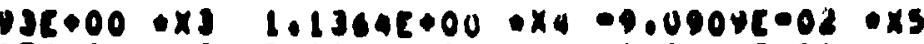

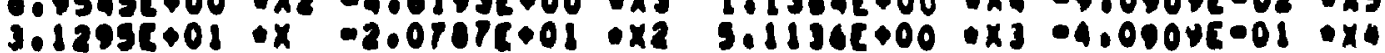

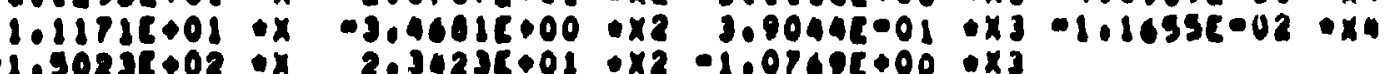
1. l.1.5. C.

1.04arcel $x$ $\begin{array}{ll}1.03396-01 & 0 \times 2\end{array}$

$3.01378001 \cdot x$

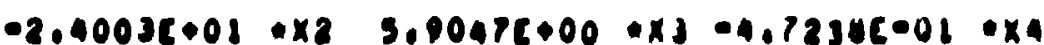
-

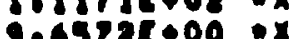
$1.08248+02$ : $x$ $-5.20026+01: x$ $1.9046000 \cdot x$

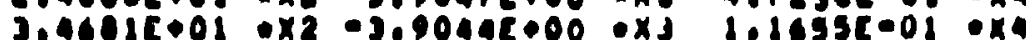

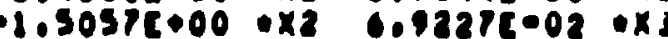
$0.2446 E+00$ ox 1.14016001 ox

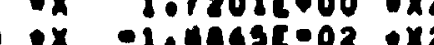

$-1 \cdot 10236+02+x$ $1.01606+02$ : $-502090601: x$

$3.0703[002 \times x 2 \cdot 0.13216+00 \cdot x 3 \quad 1.2335[001 \cdot x 4$

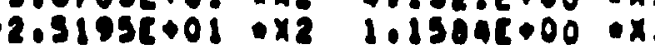

$1.12016+00 \times x$

$1.0047602 \times x-2.01095001 \times 2 \quad 1.29516000 \times x 3$

1.10576002 : 9.03156000 * $02-1.2587 E 001$. $x$

jocosiceos ax

$3.0462 c-01+x^{2}$ 


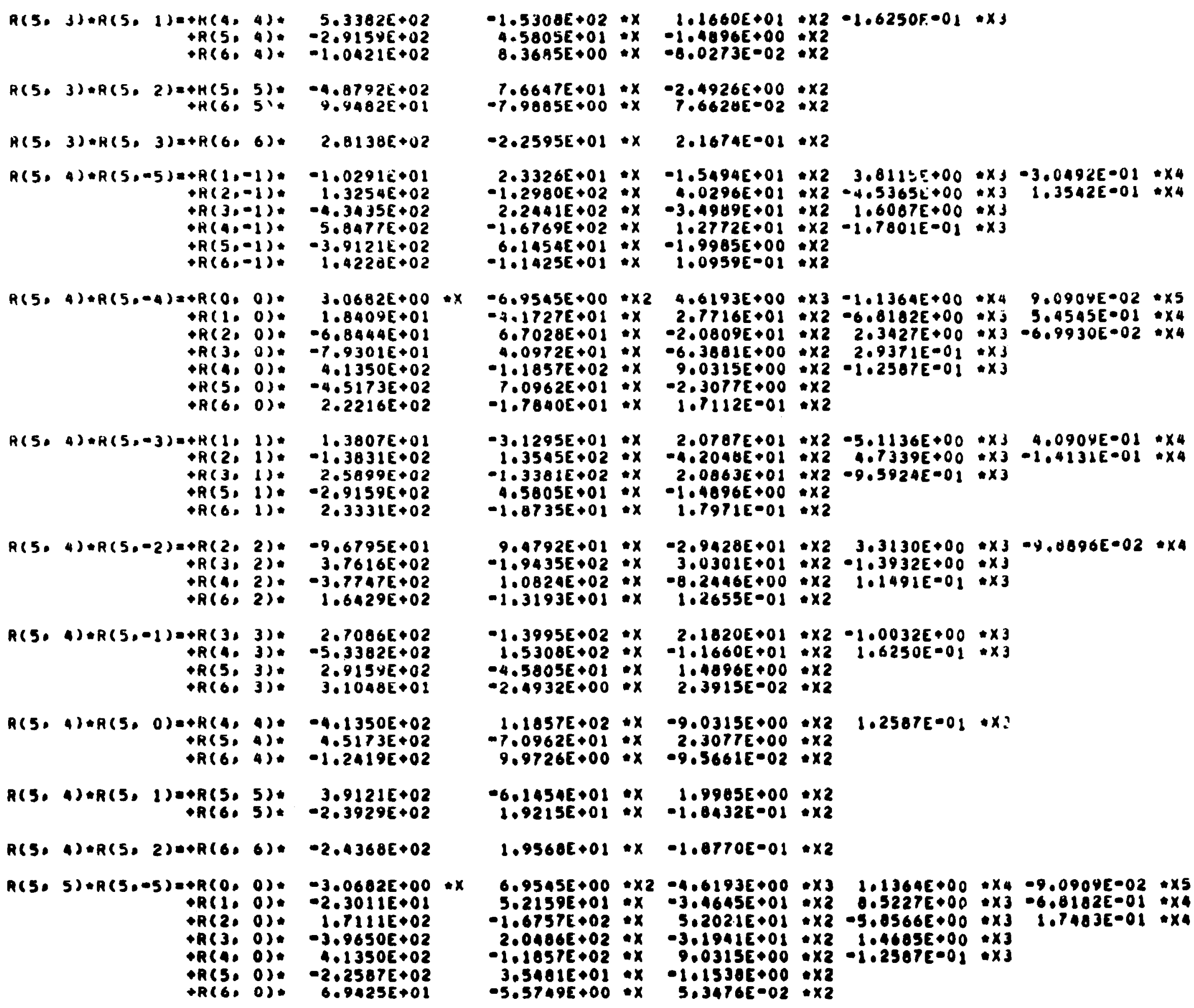

R(5. 3)*R(5. 1$)=+K(4,4) * 5.3362 E+02$ $R(5,4):=2$ $+R(6,4) *-1,0421 E+02$ $R(5,3)+R(5,2)=+H(5,5), \begin{array}{r}-4.8792 E+02 \\ +H(6,5), 9.9482 E+01\end{array}$

$A(5,3) * R(5,3)=+R(6,6) * 2.8138 E+02$

$R(5,4) \cdot R(5,-5)=+R(1,-1),-1.0291 c+01$ $\rightarrow R(2,-1)=1.3254 E+02$ $+R(3,-1) *-4.3 A 35 E+02$ $+R(5,-1) \quad-3.9121 E+02$ $+R(6,-1) * \quad 1.422 \theta E+02$

$R(5,4)+R(5,-4)=+R(0,0) * 3.0682 E+00 * X$ $\rightarrow R(1,0)=1.8409 E+0$ $+R(2,0):-6.8444 E+0$ $+R(3,0)=-7.9301 E+01$ $+R(4,0)=4.1350 E+02$ $\rightarrow R(5,0)-4.5173 E+02$

$R(5,4) \| R(5,-3)=+R(1,1): 1.3007 E+01$ $+R(2,1):-1,3831 E+02$ $\rightarrow R(3,1)=0.5899+02$ $+R(5,1):-2.9159 E+02$

$R(5,4) * R(5,-2)=4 R(2,2):-9.6795 E+01$ $A(3,2) .3 .7616 E+02$ $+R(4,2):-3.7747 E+02$

$R(5,4) \cdot R(5,-1)=0$ $+R(4,3) *-5.3382 E+02$ $+R(5,3):$ $\rightarrow R(6,3)$. 3.1048E+01

$R(5,4)+R(5,0)=+R(4,4)$ $R(5,4)=0.5173 E+02$ $\rightarrow R(6,4) \cdot-1.2419 E+02$

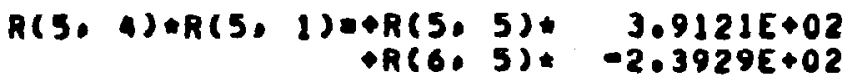

$R(5,4) * R(5,2) \oplus+R(6,6) *-2.4368 E+02$

$A(5,5) * R(5,-5)=+R(0,0) *-3.0602 E+00 * x$ $\rightarrow R(1,0) .-2.3011 E+01$ $\rightarrow R(2,0) * 1.7111 E+02$ $\rightarrow R(3,0)=-3.9650 E+02$ $\rightarrow R(A ., 0)$. A.1350E+02 $P R(5,0) .-2.2587 E+02$ $+R(6,0) .6 .9425 E+01$

$-1.5308 E+02 * x \quad 1.1660 E+01+x_{2}-1.6250 F-01 * x_{3}$

$4.5805 E+01: x \quad-1.9496 E+00 * \times 2$
$8.3695 E+00 * x \quad-6.0273 E=02: \times 2$

$7.6647 E+01 * x-2.4926 E+00 * \times 2$

$-2.25955+01 \cdot x \quad 2010745-010 x 2$

$2.3326 E+01 * x-1.5494 E+01 * x_{2} \quad 3.81125+00 * x_{3}-3.0492 E-01 \times x_{4}$ $-1.2980 E+02 \times x \quad 4.0296 E+01 \times \times 2-4.5365 i+00 * x 3 \quad 1.3542 E-01 \times x 4$ $2.2441 E+02 * x-3.4989 E+01 * \times 2 \quad 1.6007 E+00 * x J$

$-1.6769 E+02: x \quad 1.2772 E+01 * x 2-1.7801 E-01 * x 3$

$6.1454 E+01: x-1.9985 E+00 * x 2$
$-1.1425 E+01: x \quad 1.0959 E-01: \times 2$

$-6.9545 E+00 \times 2 \quad 4.6193 E+00+x 3-1.1364 E+00 * x 49.0904 E-02 * \times 5$ $-7.1727 E+01$ * $x \quad 2.7716 E+01 * x 2-6.8182 E+00 * x 3 \quad 5.4545 E-01 * x_{4}$ $6.7028 E+01 * x-2.0809 E+01 * x 2 \quad 2.3427 E+00 * x 3-6.9930 E-02 * x$ $4.0972 E+01 * x-6.3681 E+00 * x 2 \quad 2.9371 E-01 * x 3$

$-1.1857 E+02 * x \quad 9.0315 E+00 * \times 2-1.2507 E-01 * \times 3$

$7.0962 E+01$ :
$-1.7640 E+01$ : $-2.3077 E+00 * \times 2$

$-3.1295 E+01 * x \quad 2.0707 E+01 * \times 2-5.1136 E+00 * \times 3 \quad 4.0904 E-01 * \times 4$ $1.3545 E+02 * x-4.2046 E+01 * \times 2 \quad 4.7339 E+00 * \times 3-1.4131 E=01 * \times 4$ $-1.3381 E+02 * x \quad 2.0863 E+02 * \times 2-9.3924 E=01 * \times 3$

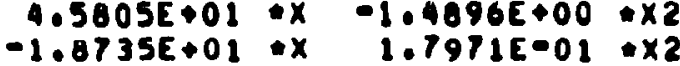

$9.9792 E+01$ ax $-2.9420 E+01 \times x 2-3.3130 E+00 \times x 3-4.0896 E=02=x 4$ $-1.9435 E+02 * x \quad 3.0301 E+01 \times x 2-1.3932 E+00 * x 3$

$1.0824 E+02 * x-8.2446 E+00 * x 2 \quad 1.1491 E-01 * x 3$

$-1.3193 E+01 \cdot x \quad 1.2655 E-01 \cdot \times 2$

$1.3995 E+02 \times x \quad 2.1820 E+01 * x 2-1.0032 E+00 * x 3$

$1.5300 E+02 * x-1.1660 E+01 * \times 2 \quad 1.6250 E=01 * x 3$

$\begin{array}{ll}-4.5805 E+01: x \quad 1.4896 E+00 & \times 2 \\ -2.4932 E+00 & \times \quad 2.3915 E-02 * x 2\end{array}$

$1.1837 E+02 * X \quad-9.0315 E+00 * \times 2 \quad 1.2587 E-01 * x ?$

$-7.0962 E+01$ * $x \quad 2.3077 E+00$ * $x 2$

$9.9726 E+00 * x \quad-9,5661 E-02 * x 2$

$-6.1454 E+01: x \quad 1.9905 E * 00 * \times 2$
$1.9215 E+01: x-1.8432 E-01 * x 2$

$1.9560 E+01 * x-1.0770 E-01 * \times 2$

$6.9545 E+00 * \times 2-4.6193 E+00 * x_{3} \quad 1.1364 E+00 * x_{4}-9.0900 E-02 * \times 5$ $5.2159 E+01 * x-3.4645 E+01 * x 20.5227 E+00+x 3-6.8182 E-01 \times x$ $-1.6757 E+02+x \quad 5.202 .1 E+01 * \times 2-5.0566 E+00 * x_{3} \quad 1.7483 E-01 * x 4$ $2.0406 E+02 * x-3.1941 E+01 * x 2 \quad 1.4685 E+00 * x 3$ $1.1857 E+02 * x \quad 9.0315 E+00 * \times 2-1.2587 E-01 * x 3$

$3.54016+01: x \quad-1.15305+00$ *X2
$-5.5749 E+00 * x \quad 5.3476 E-02 * \times 2$ 


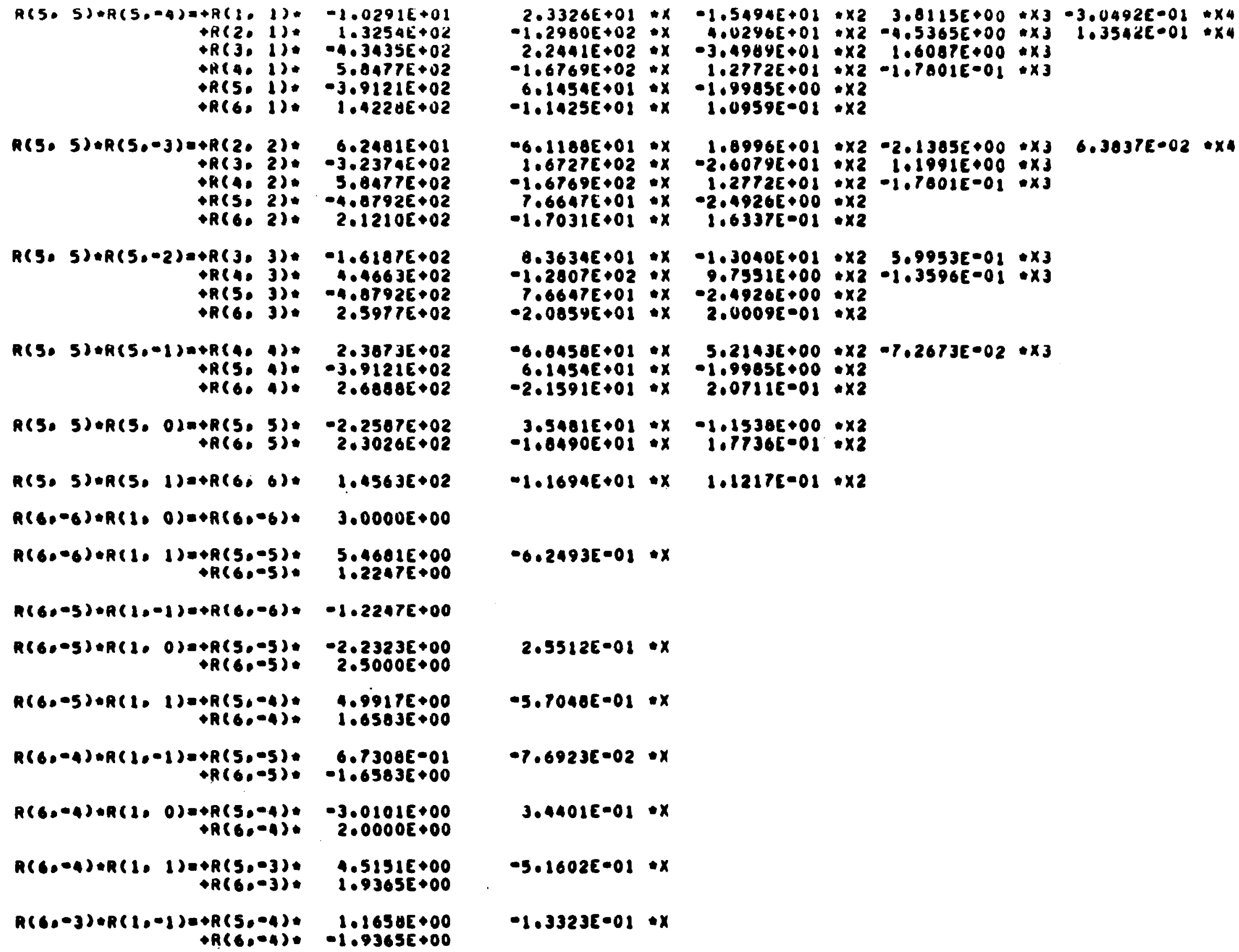

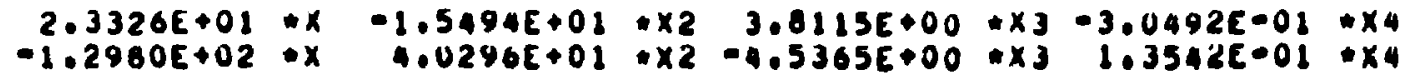
$2.2441 E+02: x-3.4949 E+01 * \times 2 \quad 1.6087 E+00 * x 3$

$-1.6769 E+02 * x \quad 1.2772 E+01 * x 2-1.7801 E-01 * x 3$

$6.1454 E+01$ \& $-1.9985 E+00+x 2$

$\begin{array}{rr}0.1454 E+01: x \quad-1.9985 E+00 & +\times 2 \\ -1.1425 E+01: x \quad 1.0959 E \cdot 01 * \times 2 & \end{array}$

$-6.1188 E+01: x$

$-1.6769 E+02 * x \quad 1.2772 E+01 * \times 2-1.7601 E-01 * x 3$

$-1.70315001 \cdot x-2.49265+00 * x 2$

$0.3634 E+01 * x-1.3040 E+01 * \times 2 \quad 5.9953 E-02 * x_{3}$

$-1.2807 E+02 * x \quad 9.7551 E+00 * x 2-1.3596 E-01 * x 3$

$7.6647 E+01: x \quad-2.4920 E+00$
$-2.0854 E+01: x \quad 2.0009 E-01: \times 2$

$-6.0458 E+01 * x \quad 5.2143 E+00+\times 2-7.2673 E-02 * \times 3$

$6.1454 E+01 * x-1.9965 E+00 * x 2$

$-2.15916+01 \times 2.0711 E-01 \times 2$

$3.5401 E+01 * x-1.1538 E+00 * \times 2$

$-1.8490 E+01 * x \quad 1.7736 E-01 * x 2$

$-1.1694 E+01 * x \quad 1.1217 E-01 * \times 2$

$-0.2493 E-01 \cdot x$

$2.5512 E-01 \cdot x$

$-5.7048 E-01 * x$

$-7.6923 E-02 \cdot x$

$3.4401 E-01 \cdot x$

$-5.1602 E-01 * x$

$-1.3323 E-01 \cdot x$ 


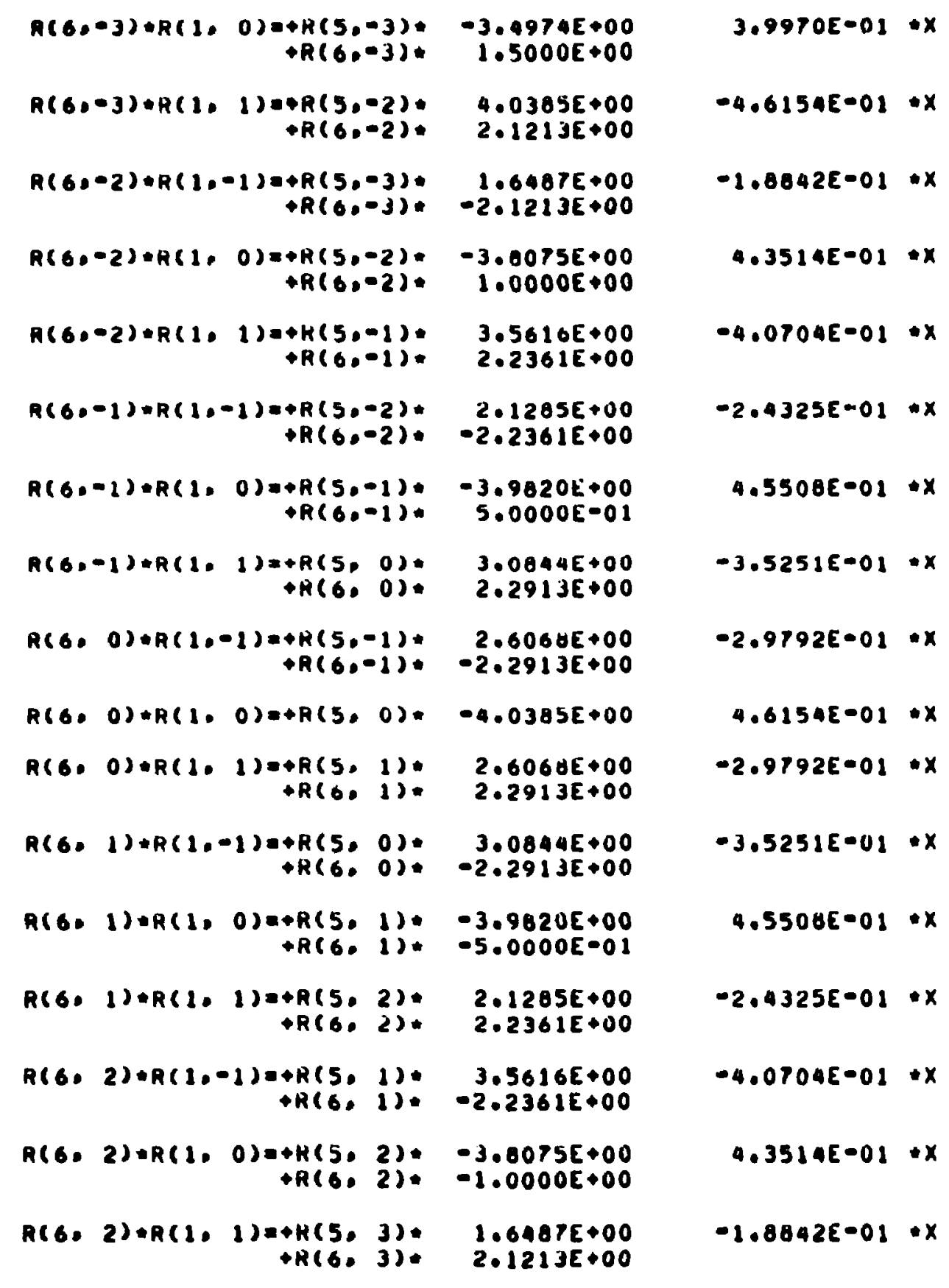




\begin{tabular}{|c|c|c|c|c|c|c|}
\hline$R(6)$ & $3) \cdot R(1$. & $\begin{aligned}-1)= & +R(5,2): \\
& +R(6,2)\end{aligned}$ & $\begin{array}{r}4.0365 E \bullet 00 \\
-2.1213 E \bullet 00\end{array}$ & $=0.6154 E=01$ & $\cdot x$ & \\
\hline A66. & $3) \cdot R(10$ & $\begin{array}{r}0)=+R(5,3) \\
+R(6,3)\end{array}$ & $\begin{array}{l}-3.4974 E+00 \\
-1.5000 E+00\end{array}$ & $3.9970 \mathrm{E} .01$ & $\bullet x$ & \\
\hline nces & $32=R(20$ & $\begin{array}{r}1)=+R(5,0) \\
+R(6,4)\end{array}$ & $\begin{array}{l}1.1658 E+00 \\
1.9365 E+00\end{array}$ & $-1,33235-01$ & $\cdot x$ & \\
\hline nese & 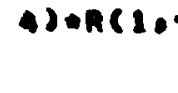 & $\begin{array}{r}-1)=+A(5,3) \\
+R(6.3)=\end{array}$ & $\begin{array}{r}+.5131 E+00 \\
-1.9365 E+00\end{array}$ & $-5.1602 E-01$ & $\bullet x$ & \\
\hline ne6. & A) ER(1. & $\begin{array}{r}0)=+R(5,1) \\
+R(6 .\end{array}$ & $\begin{array}{l}-3,0101 E+00 \\
-2,0000 E+00\end{array}$ & $3.4401 E-01$ & $\bullet x$ & \\
\hline n6es & $4) \cdot R(1)$ & $\begin{array}{r}1)=+R(5,5) * \\
+R(6,5) *\end{array}$ & $\begin{array}{l}6.7300 E-01 \\
1.6583 E+00\end{array}$ & $-7.6923 E=02$ & $\cdot x$ & \\
\hline R66: & $5) \cdot R(1.0$ & $\begin{aligned}-1) & +R(5,4)= \\
& +R(6,4)=\end{aligned}$ & $\begin{array}{r}1.9917 E+00 \\
-1.6583 E+00\end{array}$ & $-5.7040 E-01$ & $\bullet x$ & \\
\hline R660 & S) $: R(1$. & $\begin{aligned}0)= & +R(50: 5) \\
& +R(6.5)\end{aligned}$ & $\begin{array}{l}-2.2323 E+00 \\
-2.5000 E+00\end{array}$ & $2.55122 \cdot 01$ & $m x$ & \\
\hline nes. & $S) \cdot R(1$. & $1)=+R(6,6)=$ & $1.2247 E+00$ & & & \\
\hline Ac6. & $6) \cdot R(1$. & $\begin{aligned}-1) & =+R(5,5) \\
& +R(6,5)\end{aligned}$ & $\begin{array}{r}5 . \$ 681 E+00 \\
-1.2247 E+00\end{array}$ & $-6.24935-01$ & $\bullet x$ & \\
\hline Rese & $6) \cdot R(1$. & $020 \operatorname{Ro}(6,6)$ & $-3.0000 E+00$ & & & \\
\hline A660 & $-6) \cdot R(2$. & $03=+R(6 .-6)=$ & $1.2300 E+01$ & $-4.0000 E-01$ & $\bullet x$ & \\
\hline nc6.- & $-6)+R(2 \cdot$ & $\begin{aligned} 12= & +R(5,-5) \\
& +R(6,-5)\end{aligned}$ & $\begin{array}{l}2.3678 E+01 \\
0.6974 E+00\end{array}$ & $\begin{array}{l}-2.7060 E+00 \\
-2.0284 E-01\end{array}$ & $: x$ & \\
\hline RC6:- & $-6)+R(2$. & $\begin{aligned}2)= & +R(4,-4) \\
& +R(5,-4) \\
& +R(6,-4)\end{aligned}$ & $\begin{array}{l}2.4505 E+01 \\
1.4975 E+01 \\
3.7086 E+00\end{array}$ & $\begin{array}{l}-6.8046 E+00 \\
-1.7114 E+00 \\
-1.2060 E-01\end{array}$ & $\begin{array}{c}: x \\
: x \\
: x\end{array}$ & $\begin{array}{c}4.6675 E-01: \times 2 \\
.\end{array}$ \\
\hline Aco.e & $-5) * R(2$ & $-1)=+R(6,-6)$ & $-8.6974 E+00$ & $2.0204[-01$ & $\bullet x$ & \\
\hline R66.0 & $-5)+R(20$ & $\begin{aligned}0)= & +R(5,-5) \\
& \leftrightarrow R(6,-5)\end{aligned}$ & $\begin{array}{r}-1.6743 E+01 \\
6.1500 E+00\end{array}$ & $\begin{array}{r}1.91345+00 \\
-2.0000[-01\end{array}$ & $\ddot{x}$ & \\
\hline nis.o & $-5)=R(2$. & $\begin{aligned}1)= & +R(4,-4) \\
& +R(5,-4) \\
& +R(6,-4)\end{aligned}$ & $\begin{array}{r}-1.4140 E+01 \\
1.2969 E+01 \\
9.6352 E+00\end{array}$ & $\begin{array}{r}3.9748 E+00 \\
-1.4021 E+00 \\
-3.2334 E-01\end{array}$ & $: x$ & $-2.6940[-01 \cdot \times 2$ \\
\hline arese & $-5) \oplus R(2$. & $\begin{aligned}2) 0 & +R(1,-3) * \\
& +R(5,-3): \\
& +R(6,-3)\end{aligned}$ & $\begin{array}{l}2.0008 E \bullet 01 \\
1.0341 E \bullet 01 \\
5.86305 * 00\end{array}$ & $\begin{array}{l}-5.6213 E+00 \\
-2.0961 E+00 \\
-1.90695-01\end{array}$ & $\begin{array}{l}: x \\
: x \\
x \\
x\end{array}$ & $3.0110[-01 \times \times 2$ \\
\hline
\end{tabular}




\begin{tabular}{|c|c|c|c|c|c|}
\hline$A(6,--4)=H(2,-2)=+H(6,-6)=$ & $3.7080 E \bullet 00$ & $-1.2060 E-01$ & $\bullet x$ & & \\
\hline $\begin{aligned} R(6,-4) \bullet R(2,-1)= & +R(5,-5) \\
& \mapsto K(6,-5):\end{aligned}$ & $\begin{array}{r}0.7435 E \bullet 00 \\
-9.6352 E \bullet 00\end{array}$ & $\begin{array}{r}-9.9926 E=01 \\
3.1314 E=01\end{array}$ & $\bullet x$ & & \\
\hline $\begin{aligned} R(6,-4) \otimes R(2,0)= & +H(4,-4): \\
& +H(5,-4) \\
& +K(6,-4)\end{aligned}$ & $\begin{array}{r}1.3884 E \bullet 00 \\
-1.3061 E \bullet 01 \\
1.1182 E \bullet 00\end{array}$ & $\begin{array}{r}-2.0738 E+00 \\
2.0641 E+00 \\
-3.6364 E-02\end{array}$ & $\ddot{: x}$ & $1.4073 E-01$ & $\star \times 2$ \\
\hline $\begin{aligned} R(6,-4) \cdot R(2,1)= & +H(4,-3) \\
& +H(5,-3): \\
& +H(6,-3)=\end{aligned}$ & $\begin{array}{r}-1.706 J E \bullet 01 \\
3.9102 E+00 \\
8.7512 E+00\end{array}$ & $\begin{array}{r}4.7938 E+00 \\
-4.4688 E-01 \\
-2.8459 E-01\end{array}$ & $\begin{array}{l}: x \\
: x \\
: x\end{array}$ & $-3.2501 E-01$ & $\times 2$ \\
\hline $\begin{aligned} R(6,-4)+H(2,2)= & +R(4,-2): \\
& +H(5,-2) \\
& +H(6,-2)\end{aligned}$ & $\begin{array}{l}1.5961 E+01 \\
1.9156 E+01 \\
7.5010 E+00\end{array}$ & $\begin{array}{l}-4.4 B 42 E+00 \\
-2.1893 E+00 \\
-2.4393 E-01\end{array}$ & $: x$ & $3.0401 E-01$ & $\times 2$ \\
\hline $\begin{aligned} R(6,-3)+H(2,-2)= & +H(5,-5) \\
& +H(6,-5)\end{aligned}$ & $\begin{array}{r}-3.1927 E+00 \\
3.863 \forall E+00\end{array}$ & $\begin{array}{r}3.6480 E=01 \\
-1.9069 E=01\end{array}$ & $\ddot{* x}$ & & \\
\hline $\begin{aligned} R(6,-3)+R(2,-1)= & +H(4,-4) \\
& +H(5,-4) \\
& +R(6,-4)\end{aligned}$ & $\begin{array}{r}-3.3042 E+00 \\
1.3125 E+01 \\
-8.7512 E+00\end{array}$ & $\begin{array}{r}9.2832 E=01 \\
-1.5000 E+00 \\
2.8459 E=01\end{array}$ & $\begin{array}{l}: x \\
: x \\
: x\end{array}$ & $-6.2937 E-02$ & $\times 2$ \\
\hline $\begin{aligned} R(6,-3)+R(2,0) & +R(4,-3) \\
& +K(5,-3) \\
& +R(6,-3)\end{aligned}$ & $\begin{array}{r}1.1446 E+01 \\
-1.5730 E+01 \\
-2.7935 E+00\end{array}$ & $\begin{array}{r}-3.21506+00 \\
1.7987 E+00 \\
9.0909 E=02\end{array}$ & $\begin{array}{l}: x \\
: x \\
x\end{array}$ & $2.180<E-01$ & $0 \times 2$ \\
\hline $\begin{aligned} H(6,-3) \cdot R(2,1)= & +H(4,-2) \\
& +H(5,-2) \\
& +H(6,-2)\end{aligned}$ & $\begin{array}{r}-1.7484 E+01 \\
-3.4974 E+00 \\
6.0474 E+00\end{array}$ & $\begin{array}{r}4.9122 E+00 \\
3.9970 E-01 \\
-2.2260 E-01\end{array}$ & $: \begin{array}{l}x \\
: x \\
: x\end{array}$ & $-3.3303 E=01$ & $\times 2$ \\
\hline $\begin{aligned} A(6,-3)+A(2,2)= & +H(4,-1) \\
& +H(5,-1) \\
& +R(6,-1)\end{aligned}$ & $\begin{array}{l}1.2363 E+01 \\
1.0507 E+01 \\
0.6614 E+00\end{array}$ & $\begin{array}{l}-3.4735 E+00 \\
-2.1150 E+00 \\
-2.0167 E=01\end{array}$ & $: \begin{array}{l}x \\
: x \\
x\end{array}$ & $2.354 \times E-01$ & $\times 2$ \\
\hline $\begin{aligned} A(6,-2)+R(2,-2)= & +H(4,-4) \\
& +R(5,-4) \\
& +R(6,-4)\end{aligned}$ & $\begin{array}{r}1.1014 E+00 \\
-6.0571 E+00 \\
7.5010 E+00\end{array}$ & $\begin{array}{r}-3.0944 E-01 \\
6.9231 E-01 \\
-2.4393 E-01\end{array}$ & $\stackrel{x}{x}$ & $2.0974 E-02$ & $\cdot \times 2$ \\
\hline $\begin{aligned} R(6,-2) * R(2,-1)= & +H(4,-3): \\
& +R(5,-3) \\
& \mapsto R(0,-3)\end{aligned}$ & $\begin{array}{r}-6.23056+00 \\
1.5706 E+01 \\
-6.84746+00\end{array}$ & $\begin{array}{r}1.7505 E+00 \\
-1.79506+00 \\
2.2268 E-01\end{array}$ & $\begin{array}{ll}: x \\
: x \\
: x\end{array}$ & $-1.10606-01$ & $\star \times 2$ \\
\hline $\begin{aligned} A(6,-2) \cdot R(2,0)= & +H(4,-2) \\
& +R(5,-2) \\
& +R(6,-2)\end{aligned}$ & $\begin{array}{r}1.4270 E \bullet 01 \\
-1.1422 E+01 \\
-5.5909 E \bullet 00\end{array}$ & $\begin{array}{r}-4.0108 E+00 \\
1.3054 E+00 \\
1.0102 E-01\end{array}$ & $\begin{array}{l}: x \\
: x \\
x\end{array}$ & $2,71926 \cdot 01$ & $\cdot \times 2$ \\
\hline 1) $\begin{aligned} & +R(A,-1) \\
& +R(5,-1) \\
& +R(6,-1)\end{aligned}$ & $\begin{aligned}-1.6404 E+01 \\
-9.2533 E+00 \\
4.3307 E+00\end{aligned}$ & $\begin{array}{r}4.6313 E+00 \\
1.0575 E+00 \\
-1.4004 E-01\end{array}$ & $\begin{array}{ll}: x \\
: x \\
: x\end{array}$ & -3.13945001 & $-1 \times 2$ \\
\hline
\end{tabular}




\begin{tabular}{|c|c|c|c|c|c|c|}
\hline$H(6,-2), P(2$, & 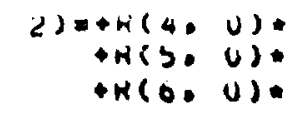 & $\begin{array}{l}9.215 U t+00 \\
1.6494 t+02 \\
9.3554 t+00\end{array}$ & $\begin{array}{l}-2.584 U E+U 0 \\
-1.4300 E+0 U \\
-3.0424 E=U 1\end{array}$ & $\begin{array}{l}: x \\
: x \\
* x\end{array}$ & $1 . / S S Z E-01$ &.$\times 2$ \\
\hline$R(6,-1) \oplus R(2$, & $\begin{aligned}-2)= & +K(4,-3) \\
& +K(5,-3) \\
& +K(6,-3)\end{aligned}$ & $\begin{array}{r}2.462 \forall E+00 \\
-4.030 \mathrm{JE}+00 \\
0.6614 E+00\end{array}$ & $\begin{array}{r}-6.9193 E-01 \\
1.0320 E+00 \\
-2.0167 E-01\end{array}$ & $\begin{array}{l}* x \\
: x \\
* x\end{array}$ & $0.0411 E-02$ &.$\times 2$ \\
\hline$R(6,-1)+A(20$ & $\begin{aligned}-1)= & +K(4,-2)= \\
& +R(5,-2)= \\
& +H(6,-2)=\end{aligned}$ & $\begin{array}{r}-9.2150 E+00 \\
1.6590 E+01 \\
-4.3307 E+00\end{array}$ & $\begin{array}{r}2.5890 E+00 \\
-1.8900 E+00 \\
1.40 H 4 E-01\end{array}$ & $\begin{array}{l}* x \\
: x \\
* x\end{array}$ & $-1.7552 E-01$ & $+\times 2$ \\
\hline$R(6,-1)=R(2$, & $\begin{aligned}(0)= & +H(4,-1) \\
& +R(5,-1) \\
& +H(0,-1)\end{aligned}$ & $\begin{array}{r}1.5961 E+01 \\
-5.9730 E+00 \\
-7.2692 E+00\end{array}$ & $\begin{array}{r}-4.4042 E+00 \\
6.4262 E-01 \\
2.3636 E-01\end{array}$ & $\begin{array}{l}: x \\
* x \\
* x\end{array}$ & $3.0401 E-01$ & $\cdot \times 2$ \\
\hline$R(6,-1)=R(2$, & (1)= $\begin{aligned}+R(4,0) \\
+R(5,0): \\
\\
+R(6,0)\end{aligned}$ & $\begin{array}{r}-1.4570 E+01 \\
-1.3356 E+01 \\
1.4792 E+00\end{array}$ & $\begin{array}{r}4.0935 E+00 \\
1.5264 E+00 \\
-4.0105 E-02\end{array}$ & $\begin{array}{l}: x \\
: x \\
: x\end{array}$ & $-2.175 J E-01$ & $\cdot \times 2$ \\
\hline$R(6,-1) \oplus R(2$, & $\begin{aligned}2)= & +R(4,1): \\
& +R(5,1): \\
& +R(6,1):\end{aligned}$ & $\begin{array}{l}6.5160 E+00 \\
1.4631 E+01 \\
9.5864 E+00\end{array}$ & $\begin{array}{l}-1.8307 E+00 \\
-1.6721 E+00 \\
-3.1173 E-01\end{array}$ & $\begin{array}{l}: x \\
: x \\
: x\end{array}$ & $1.2411 E-01$ & $\cdot \times 2$ \\
\hline$R(6,0) \oplus R(2$. & $\begin{aligned}-2)= & +K(4,-2) \\
& +R(3,-2) \\
& +H(6,-2)\end{aligned}$ & $\begin{array}{r}4.2657 E+00 \\
-1.1940 E+01 \\
9.3554 E+00\end{array}$ & $\begin{array}{r}-1.1945 E+00 \\
1.3652 E+00 \\
-3.0424 E=01\end{array}$ & $\begin{array}{l}: x \\
: x \\
x\end{array}$ & $0.1251 E-02$ & $+\times 2$ \\
\hline$R 66.0) \cdot R(2$. & $\begin{aligned}1)= & +R(4,-1): \\
& +R(5,-1): \\
& +R(6,-1)\end{aligned}$ & $\begin{array}{r}-1.2065 t+01 \\
1.5803 t+01 \\
-1.4792 E+00\end{array}$ & $\begin{array}{r}3.3098 E+00 \\
-1.8061 E+00 \\
4.8105 E-02\end{array}$ & $\begin{array}{l}x \\
-x \\
x\end{array}$ & $-2.2901 E-01$ & $+\times 2$ \\
\hline$R(6,0) * R(2)$ & $\begin{aligned}0)= & +R(a, 0) \\
& +K(6,0)\end{aligned}$ & $\begin{array}{r}1.6521 E+02 \\
-7.8273 E+00\end{array}$ & $\begin{array}{r}-4.6416 E+00 \\
2.5455 E-01\end{array}$ & $\cdot x$ & $3.1469 E-01$ & $-\times 2$ \\
\hline$R(6,0) \approx R(2$, & $\begin{aligned}1)= & +R(4,1): \\
& +R(5,1) \\
& +R(6,1)\end{aligned}$ & $\begin{array}{l}-1.2065 E+01 \\
-1.5803 E+01 \\
-1.9792 E+00\end{array}$ & $\begin{array}{l}3.3098 E+00 \\
1.8061 E+00 \\
4.8105 E-02\end{array}$ & $\begin{array}{l}: x \\
: x \\
: x\end{array}$ & $-2.2901 E-01$ & $\cdot \times 2$ \\
\hline$R(6,0) * R(2$. & $\begin{aligned}2)= & +R(4,2) \\
& +R(5 ; 2): \\
& +R(6,2)\end{aligned}$ & $\begin{array}{l}4.2657 E+00 \\
1.1946 E+01 \\
9.3554 E+00\end{array}$ & $\begin{array}{l}-1.1985 E+00 \\
-1.3652 E+00 \\
-3.0424 E=01\end{array}$ & $\begin{array}{l}x \\
x \\
x \\
x \\
x\end{array}$ & $0.1251 E-02$ & $1 \times 2$ \\
\hline$R(6,1) \star R C$ & $\begin{aligned} 2= & +R(0,0-1) \\
& +R(5,-1) \\
& +R(0,-1)\end{aligned}$ & $\begin{array}{r}6.5160 E+00 \\
-1.4631 E+01 \\
9.5864 E+00\end{array}$ & $\begin{array}{r}-1.8307 E+00 \\
1.6721 E+00 \\
-3.1175 E-01\end{array}$ & $\begin{array}{l}: x \\
: x \\
* x\end{array}$ & $1.2411 E-01$ & $1 \times 2$ \\
\hline$R(6,1) * R($ & $\begin{aligned} J= & +R(4,0) \\
& +R(5,0) \\
& +R(6,0)=\end{aligned}$ & $\begin{array}{r}-1.4570 E+01 \\
1.3356 E+01 \\
1.4792 E+00\end{array}$ & $\begin{array}{r}4.0935 E+00 \\
-1.5264 E+00 \\
-9.0105 E-02\end{array}$ & $\begin{array}{l}* x \\
x \\
x\end{array}$ & $-2.7753 E-01$ & $\times 2$ \\
\hline
\end{tabular}




\begin{tabular}{|c|c|c|c|c|c|c|c|c|}
\hline 260 & 2 , & $\begin{aligned}0)= & +R(400 \\
& +R(30 \\
& +R(60\end{aligned}$ & 1): & $\begin{array}{r}1.5961 E+01 \\
5.9730 E+00 \\
-7.2602 E+00\end{array}$ & $\begin{array}{r}-4.4842 E+00 \\
-6.8262 E-01 \\
2.3636 E=01\end{array}$ & $\begin{array}{l}: x \\
: x \\
x\end{array}$ & $3.0401 E$ & \\
\hline$R<60$ & 1) $) R(2$, & $\begin{aligned}1) & =+R(40 \\
& +H\left(55^{\circ}\right. \\
& +R(6 \circ\end{aligned}$ & $\begin{array}{l}2): \\
2):\end{array}$ & $\begin{array}{l}-9.2150 E+00 \\
-1.6590 E+01 \\
-1.3307 E+00\end{array}$ & $\begin{array}{l}2.5890 E+00 \\
1.8960 E+00 \\
1.4084 E-01\end{array}$ & $\begin{array}{l}x x \\
: x \\
x\end{array}$ & $-1.7552 E=01$ & $\cdot \times 2$ \\
\hline$R(6)$ & $1) \cdot R(2$ & $\begin{aligned}2)= & +R(4) . \\
& \mapsto R(5) \\
& +R(60\end{aligned}$ & $\begin{array}{l}3): \\
3):\end{array}$ & $\begin{array}{l}2.4620 E+00 \\
9.0303 E+00 \\
8.6614 E+00\end{array}$ & $\begin{array}{l}-6.9193 E-01 \\
-1.0320 E+00 \\
-2.8167 E-01\end{array}$ & $: \begin{array}{l}x \\
: x \\
x\end{array}$ & $4.6911 t-02$ & • \\
\hline A 60. & & $\begin{aligned}-2)= & +H(4) \\
& +R(5) \\
& +H(6)\end{aligned}$ & $\left.\begin{array}{l}0): \\
0 \\
0\end{array}\right)$ & $\begin{array}{r}Y .215 U E+00 \\
-1.6894 E+U 1 \\
\forall .3554 E+00\end{array}$ & $\begin{array}{r}-2.5890 E+00 \\
1.9308 E+00 \\
-3.0424 E=01\end{array}$ & $\begin{array}{l}: x \\
: x \\
: x\end{array}$ & $1.7552 E-01$ & $1 \times 2$ \\
\hline A 16. & $2) * R(2$ & $\begin{aligned}-1)= & +H(4) D \\
& +H(5) \\
& +H(6)\end{aligned}$ & $\begin{array}{l}\text { 1): } \\
\text { 1): } \\
1\end{array}$ & $\begin{array}{r}-1.64 \cap 4 E \bullet 01 \\
9.2533 E \gg 00 \\
4.3307 E \bullet 00\end{array}$ & $\begin{array}{r}4.6313 E+00 \\
-1.0575 E+00 \\
-1.4004 E-01\end{array}$ & $\begin{array}{l}: x \\
0 x \\
* x\end{array}$ & $-3.1394 E-01$ & \\
\hline$R 16$. & $2) \cdot R(2$ & $\begin{aligned}0)= & +H(4), \\
& +H(S) \\
& +H(6 .)\end{aligned}$ & $\begin{array}{l}2): \\
2):\end{array}$ & $\begin{array}{r}1.4270 E+01 \\
1.1422 E+01 \\
-5.5909 E * 00\end{array}$ & $\begin{array}{r}-4.0108 E+00 \\
-1.3054 E+00 \\
1.8102 E=01\end{array}$ & $\begin{array}{l}: x \\
: x \\
: x\end{array}$ & $2.714<E \cdot 01$ & 2 \\
\hline R 60. & $2) \bullet R(2$, & $\begin{aligned}1)= & +H(40, \\
& +K(550 \\
& +K(60\end{aligned}$ & $\begin{array}{l}3): \\
33:\end{array}$ & $\begin{array}{l}-6.2305 E+00 \\
-1.5700 E+01 \\
-6.8474 E+00\end{array}$ & $\begin{array}{l}1.7505 E+00 \\
1.7950 E+00 \\
2.2268 E-01\end{array}$ & $\begin{array}{l}: x \\
: x \\
: x\end{array}$ & $-1.1600 E-01$ & 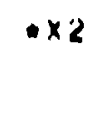 \\
\hline R 16. & $2) \cdot A(2$. & 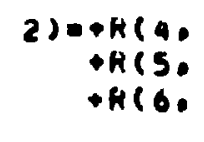 & $\begin{array}{l}4): \\
\text { 4): } \\
\text { 4): }\end{array}$ & $\begin{array}{l}1.1019 E+00 \\
6.0577 E+00 \\
7.5010 E+00\end{array}$ & $\begin{array}{l}-3.0944 E-01 \\
-6.92316-01 \\
-2.43936-01\end{array}$ & $\begin{array}{l}: x \\
: x \\
: x\end{array}$ & $2.047 \times E=02$ & 2 \\
\hline R66. & $3) \cdot R(2$. & $\begin{aligned}-2) & +R(4 a, \\
& \mapsto H(5) \\
& \bullet R(0 .\end{aligned}$ & $\begin{array}{l}\text { i): } \\
\text { i): }\end{array}$ & $\begin{array}{r}1.2363 E+01 \\
-1.89076+01 \\
0.6614 E+00\end{array}$ & $\begin{array}{r}-3.4735 E+00 \\
2.11506+00 \\
-2.8167 E=01\end{array}$ & $\begin{array}{l}: x \\
: x \\
: x\end{array}$ & $2 . J 54 Y E=01$ & \\
\hline 6. & & $\begin{aligned}-1)= & +K(410 \\
& +K(50 \\
& +11(60\end{aligned}$ & $\begin{array}{l}2): \\
2):\end{array}$ & $\begin{array}{r}-1.7484 E+01 \\
3.4974 E+00 \\
6.4474 E+00\end{array}$ & $\begin{array}{r}4.9122 E+00 \\
-3.9470 E=01 \\
-2.2268 C=01\end{array}$ & $\begin{array}{l}: x \\
: x \\
x\end{array}$ & -J.JJUSE-01 & $\times 2$ \\
\hline$R 16$. & $3)=4(2$. & $\begin{aligned}0)= & +K(4) \\
& +H(5 ; \\
& +4600\end{aligned}$ & $\begin{array}{l}3): \\
3):\end{array}$ & $\begin{array}{r}1.1496 E+01 \\
1.5730 E+01 \\
-2.7953 E+00\end{array}$ & $\begin{array}{r}-3.2150 E+00 \\
-1.7901 E+00 \\
9.09046-02\end{array}$ & $\begin{array}{l}: x \\
: x \\
x\end{array}$ & $2.180<t=01$ &.$\times 2$ \\
\hline 160 & $3) \bullet R(2$. & $\begin{aligned}1)= & +H(40, \\
& \bullet R(350 \\
& +H(60\end{aligned}$ & 1): & $\begin{array}{l}-3.3042 E+00 \\
-1.3125 E+01 \\
-H .7512 E+00\end{array}$ & $\begin{array}{l}9.20326=01 \\
1.30006+00 \\
2.80496-01\end{array}$ & $\begin{array}{l}: x \\
: x \\
: x\end{array}$ & $-0.2937 E=02$ & $x$ \\
\hline & . & $\begin{aligned}2) & =+K(5, \\
& +R(6)\end{aligned}$ & $\begin{array}{l}5): \\
55:\end{array}$ & $\begin{array}{l}3.1921 E+00 \\
5 . \forall 63 \forall E+00\end{array}$ & $\begin{array}{l}-3.6404 E=01 \\
-1.9004 E=01\end{array}$ & $: x$ & & \\
\hline
\end{tabular}




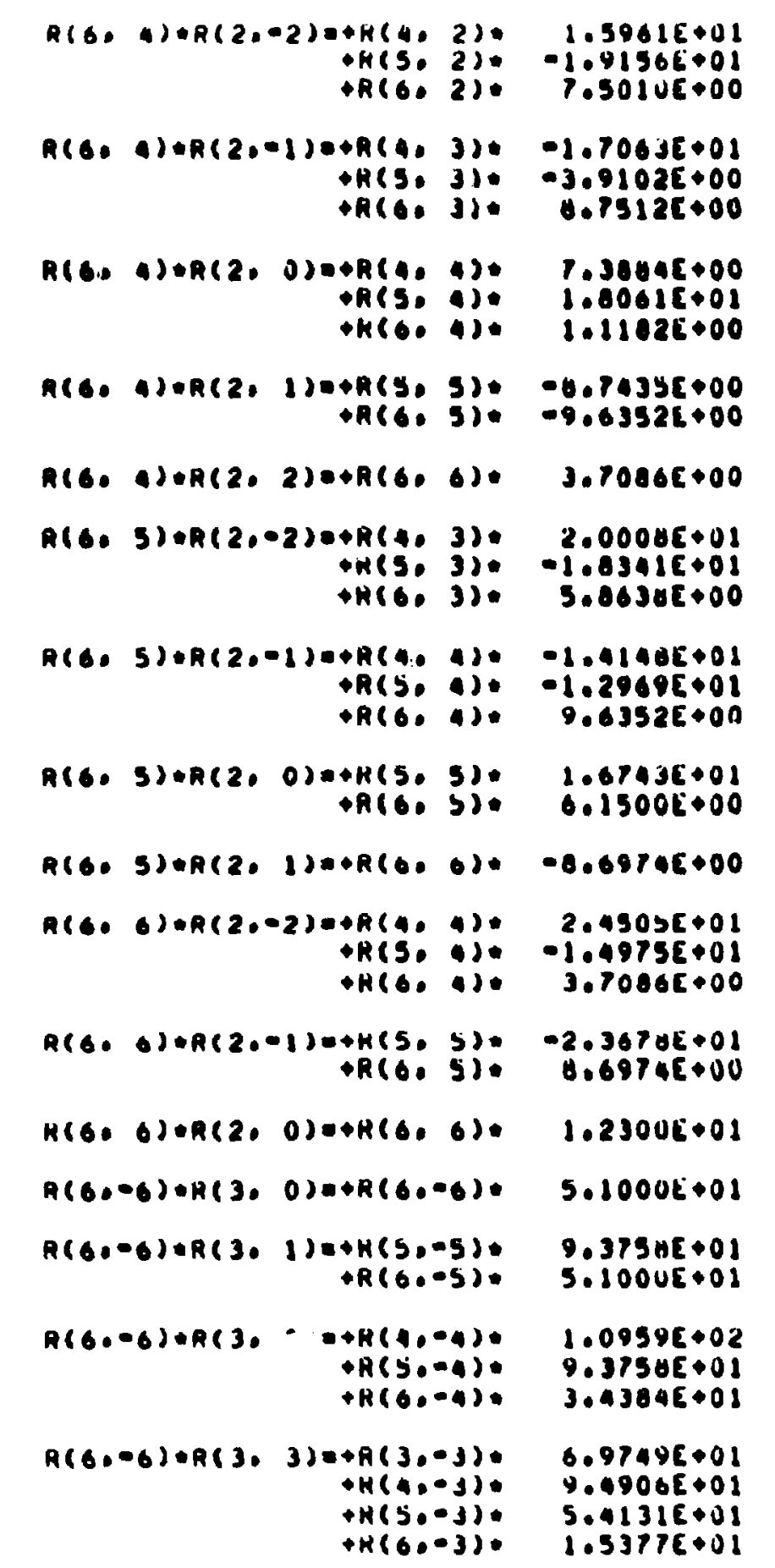

$-4.48428+00=x$ $\begin{aligned} 2.14936+00 & : x \\ -2.4303 E=01 & : x\end{aligned}$

$4.70386+00 \times x-3.25016001 \cdot x 2$ $4040006-01$ :x $-2.0439[-01 \cdot x$

$-2.07585+00 * x \quad 1.4073 E-01+x 2$ $-2.00016+00$ :x 0

$9.09205001 \quad x$ $3.1334 E=01$ Ox

$-1.2060601 \cdot x$

$-5.621] 5+00 * x$ $2.00616+00 \cdot x$ $-1.9064601: x$

$3.97400+00$ *x 1.40216000 :x 1.96216000
$-3.13346=01: x$
$* x$

$-1.913446+00 * x$ $-2.00006=01: x$ $2.0204[-0) \times x$

$-6.00405+00 \quad-x$ $1.71146+00$ ox $-1.2060 \varepsilon-01$ : $x$

$2.7060 \varepsilon+00 \div x$ $-4.00000-01$ *x $-3,0000 c+00$ :x $\begin{array}{lll}-1.29408+01 & : x \\ -3.0000 E+00 & : x\end{array}$ $-3.07096+01 * x$ $-1.29406+01: x$

$-3.0196 E+01$ :x $-2.6664 E+01$ :X $-7.4753 E+00$ *x $-4.0453 E=01$ :x

$3.04015001 \times \times 2$

$3.81106-01 \cdot \times 2$

$-2.0048[-01 \cdot \times 2$

$4.6675 E \cdot 01 * \times 2$ 


\begin{tabular}{|c|c|c|c|c|c|c|c|}
\hline$(6 .-5) * R(3 .-1)=+R(6 .-6)$. & $-5.100 u t+01$ & $3.0000 E+00$ & $+x$ & & & & \\
\hline$R(6,-5), R(3,0)=+H(5,-5) *$ & $-9.3758 E+01$ & $1.2948 E+01$ & $\cdot x$ & $-2.551<\varepsilon-01$ & $\times 2$ & & \\
\hline $\begin{aligned} R(6,-5) \times R(3,1)= & +R(4,-4) \\
& +K(6,-4)\end{aligned}$ & $\begin{array}{r}-1.0004 E+02 \\
3.7660 E+01\end{array}$ & $\begin{array}{r}2.0106 E+01 \\
-2.2156 E+00\end{array}$ & 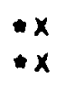 & $-1.9055 E+00$ & $\times 2$ & & \\
\hline $\begin{aligned} R(6,-5)+R(3,2)= & +K(3,-3) \\
& +R(4,-3) \\
& +R(5,-3) \\
& +K(6,-3)\end{aligned}$ & $\begin{array}{r}-4.932 U E+01 \\
2.2370 E+01 \\
7.655 J E+021 \\
4.3493 E+01\end{array}$ & $\begin{array}{r}2.7009 E+01 \\
-6.2040 E+00 \\
-1.0572 E+01 \\
-2.5584 E+00\end{array}$ & $\begin{array}{l}x \\
m x \\
: x \\
x\end{array}$ & $\begin{array}{r}-4.6345 E+00 \\
4.2609 t=01 \\
2.0831 E-01\end{array}$ & $\begin{array}{l}: \times 2 \\
: \times 2 \\
\times 2\end{array}$ & $2.5051 E=01$ & $+x_{3}$ \\
\hline $\begin{aligned} R(6,-5)=R(3,3)= & +R(3,-2) \\
& +R(4,-2) \\
& +R(5,-2) \\
& +K(6,-2)\end{aligned}$ & $\begin{array}{l}4.9320 E+01 \\
1.0251 E+02 \\
7.6553 E+U 1 \\
2.6634 E+01\end{array}$ & $\begin{array}{l}-2.7009 E+01 \\
-2.8800 E+01 \\
-1.0572 E+01 \\
-1.5667 E+00\end{array}$ & $\begin{array}{l}: x \\
: x \\
: x \\
x\end{array}$ & $\begin{array}{l}4.6345 E+0 U \\
1.4526 E+0 U \\
2.0831 E-01\end{array}$ & $\begin{array}{l}\times 2 \\
\times 2 \\
\times 2 \\
\times 2\end{array}$ & $-2.5051 E-01$ & $\times 3$ \\
\hline$R(6,-4)+R(3,-2)=+R(6,-6)$ * & $3.4384 E+01$ & $-2.0226 E+00$ & $\cdot x$ & & & & \\
\hline $\begin{aligned} R(6,-4) * R(3,-1)= & +H(5,-5) \\
& +H(6,-5)\end{aligned}$ & $\begin{array}{r}6.9245 E+U 1 \\
-3.7666 E+01\end{array}$ & $\begin{array}{r}-9.5624 E+00 \\
2.2156 E+00\end{array}$ & 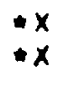 & $1.0842 L=01$ & $+\times 2$ & & \\
\hline $\begin{aligned} R(6,-4) \times R(3,0)= & +H(4,-4) \\
& +H(5,-4) \\
& +H(6,-4)\end{aligned}$ & $\begin{array}{r}7.3884 E+01 \\
-6.3212 E+01 \\
-2.7816 E+01\end{array}$ & $\begin{array}{r}-2.0758 E+01 \\
8.7293 E+00 \\
1.6364 E+00\end{array}$ & $\begin{array}{l}: x \\
: x \\
x\end{array}$ & $\begin{array}{r}1.4073 E+00 \\
-1.7201 E-01\end{array}$ & 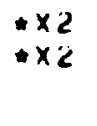 & & \\
\hline $\begin{aligned} R(6,-4)+R(3,1) & +H(3,-3) \\
& +H(4,-3) \\
& +R(5,-3) \\
& +H(6,-3)\end{aligned}$ & $\begin{array}{r}3.3252 E+01 \\
-7.5408 E+01 \\
-5.1612 E+01 \\
1.4661 E+01\end{array}$ & $\begin{array}{r}-1.8209 E+01 \\
2.1100 E+01 \\
7.1274 E+00 \\
-8.6244 E=01\end{array}$ & $\begin{array}{l}x \\
x \\
x \\
x \\
x\end{array}$ & $\begin{array}{r}3.1240 E+00 \\
-1.4363 E+00 \\
-1.4044 E-01\end{array}$ & $\begin{array}{l}* \times 2 \\
\times 2 \\
\times 2\end{array}$ & $-1.6890 E-01$ & $\times x$ \\
\hline $\begin{aligned} H(6,-4)+R(3,2) & +H(3,-2) \\
& +R(4,-2) \\
& +R(5,-2) \\
& +R(6,-2)\end{aligned}$ & $\begin{array}{r}-5.1513 E+01 \\
-3.5689 E+01 \\
3.9974 E+01 \\
4.1727 E+01\end{array}$ & $\begin{array}{r}2.8210 E+01 \\
1.0027 E+01 \\
-5.5209 E+00 \\
-2.4545 E+00\end{array}$ & $\begin{array}{l}: x \\
: x \\
x \\
x\end{array}$ & $\begin{array}{r}-4.8406 E+00 \\
-6.7980 E-01 \\
1.0874 E-01\end{array}$ & $\begin{array}{l}\times 2 \\
\times 2 \\
\times 2 \\
\times 2\end{array}$ & $2.6165 E-01$ & $\times 1$ \\
\hline $\begin{aligned} R(6,-4) * R(3,3)= & +R(3,-1): \\
& +R(4,-1) \\
& +K(5,-1) \\
& +K(6,-1)\end{aligned}$ & $\begin{array}{l}3.3252 E+01 \\
Y .2724 E+01 \\
0.6364 E+01 \\
3.5913 E+01\end{array}$ & $\begin{array}{l}-1.8209 E+01 \\
-2.6051 E+01 \\
-1.1926 E+01 \\
-2.1125 E+00\end{array}$ & $\begin{array}{l}x \\
: x \\
: x \\
x x\end{array}$ & $\begin{array}{l}3.1240 E+00 \\
1.7602 E+00 \\
2.3500 E-01\end{array}$ & $\begin{array}{l}\times 2 \\
\times 2 \\
\times 2\end{array}$ & $-1.6090 E-01$ & $\times s$ \\
\hline$R(6,-3) \notin R(3,-3)=+R(6,-6) \star$ & $-1.5377 E+01$ & $9.0453 E-01$ & $\cdot x$ & & & & \\
\hline $\begin{aligned} R(6,-3) * R(3,-2)= & +R(5,-5) \\
& +R(6,-5)\end{aligned}$ & $\begin{array}{r}-3.997 Y E+01 \\
4.3493 E+01\end{array}$ & $\begin{array}{r}5.5209 E+00 \\
-2.5584 E+00\end{array}$ & $x$ & -1.087 YE -01 &.$\times 2$ & & \\
\hline $\begin{aligned} R(6,-3) \oplus R(3,-1)= & +R(4,-4) \\
& +R(5,-4) \\
& +R(6,-4)\end{aligned}$ & $\begin{array}{r}-4.6726 E+01 \\
7.9957 E+01 \\
-1.4661 E+01\end{array}$ & $\begin{array}{r}1.3128 E+01 \\
-1.1042 E+01 \\
8.6244 E-01\end{array}$ & $\begin{array}{l}: x \\
: x \\
x\end{array}$ & $\begin{array}{r}-0.9006 E=01 \\
2.1757 E=01\end{array}$ & $\begin{array}{l}\times 2 \\
\times 2 \\
\times 2\end{array}$ & & \\
\hline
\end{tabular}




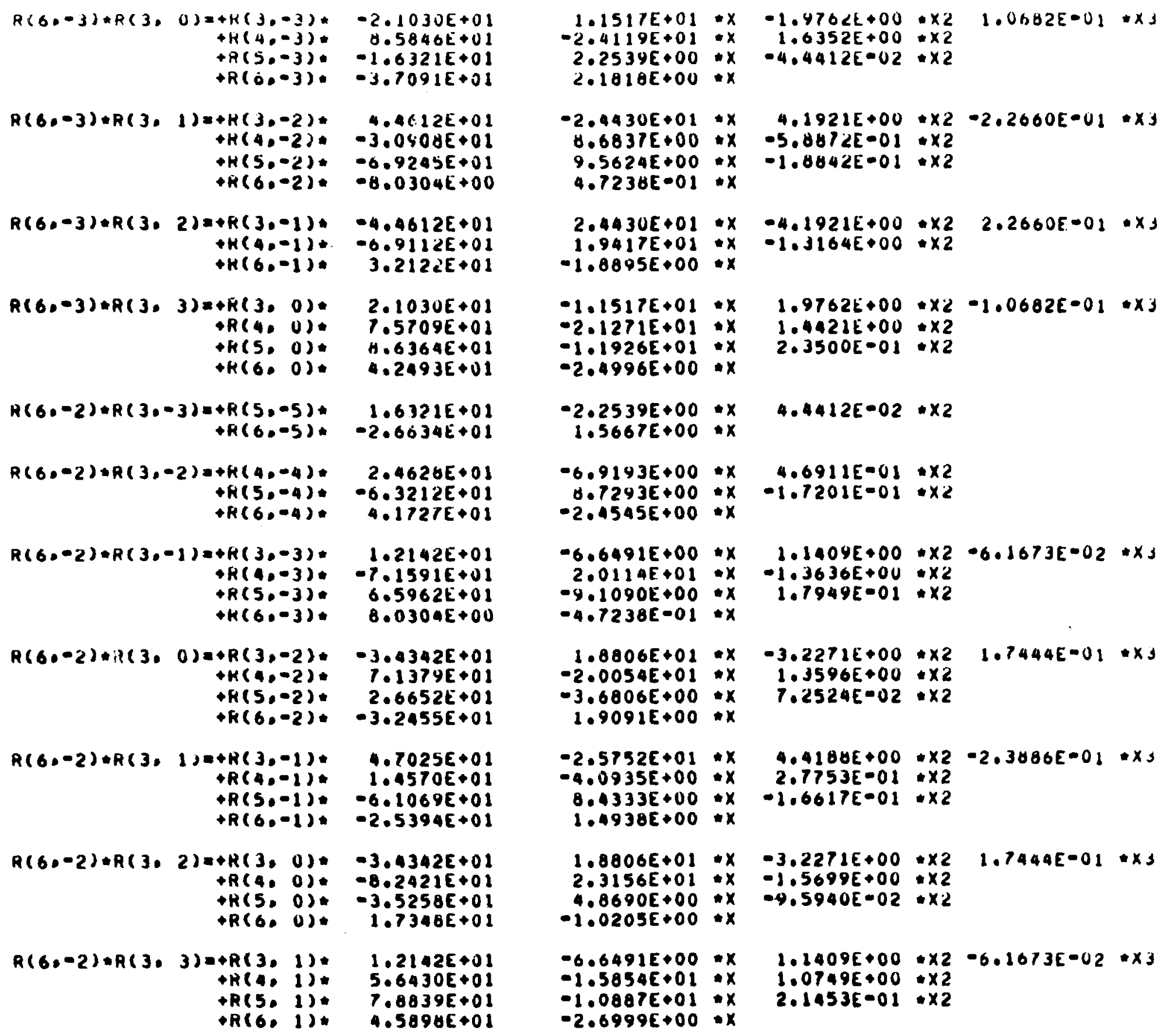
+ C $-6.1069 E+01$
$-2.5394 E+01$

$R(6,-2)+R(3,2)=+R(3,0) *$

$-3.4342 E+02$ $+R(4,0) *-8.2421 E+0)$ $+R(5,0):-3.5258 E+01$ $+R(6.0)$. 1.7346E+0)

$R(6,-2) * R(3,3)=+R(3,1) * 1.2142 E+01$ $+R(4,1)=5.6430 E+01$ $+R(5 ., 1): \quad 7.8839 E+01$ $\rightarrow R(6,1): \quad 9.5898 E+01$

$1.1517 E+01 * x$ $1.0302 t+00 * x 2$ $2.25345+00 * x-4.4412 E-02: x 2$ $2.101 \theta E+00 * x$

$-2.4430 E+01 * x$ $9.5624 E+00$ : $x$ - $1.0042 E=01$ : $x 2$ $9.5624 E+00$ ax $-1.0042 E=01$ ax

$2.4430 E+01 * x-4.1921 E+00 * \times 2 \quad 2.2660 E=01 \cdot x J$ $1.9417 E+01: x$ $-1.0895 E+00: x$

$-1,1517 E+01 * x$ $-2.1271 E+01 * x$ $-1.1926 E+01$ : $x$ $-2.4996 E+00 * x$

$-2.2539 E+00 * x$ $1.5667 E+00 * x$

$-6.9193 E+00 * x \quad 4.6911 E-01 \div \times 2$ $0.7293 E+00: x-1.7201 E=01 * x 2$
$-2.4545 E+00: x$

$-6.6491 E+00 * x$

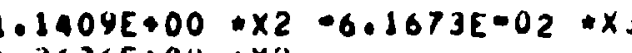

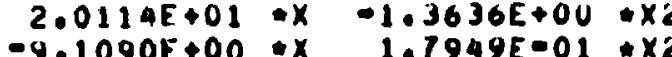
$-9.1090 E+00$ : $x$

$1.9762 E+00+\times 2-1.0682 E=01 * \times 3$ $1.4421 E+00 * \times 2$
$2.3500 E-01 * \times 2$

$4.4412 E-02 * \times 2$

$1000065+02=x$ $-2.0054 E+01$ : $x$ $3.6006 E+00 * x$ $1.9091 E+00 * x$

$-2.5752 E+01 * x$ $-4.0935 E+00=x$ $1.43305+00=x$

$1.8006 E+01 * X \quad-3.2271 E+00 * \times 2 \quad 1.7449 E-01 * x s$ $2.3156 E+01+x-1.5699 E+00 * x 2$ $4.0690 E+00 * x \quad-9.5940 E-02: x$.

$-6.6491 E+00 \cdots x$ -1.58545001 : $x$ $-1.0807 E+01 * x$
$-2.69996+00 * x$

$1.1409 E+00 \times 2-6.1673 E=02 \times \times 3$

$-3.2271 E+00 * \times 2 \quad 1.7444 E=01 * x 3$ $1.3596 E+00$ * $x 2$

$4.4180 E+00 \times 2-2.3886 E-01 \times x s$ $1.07496+00: \times 2$ 


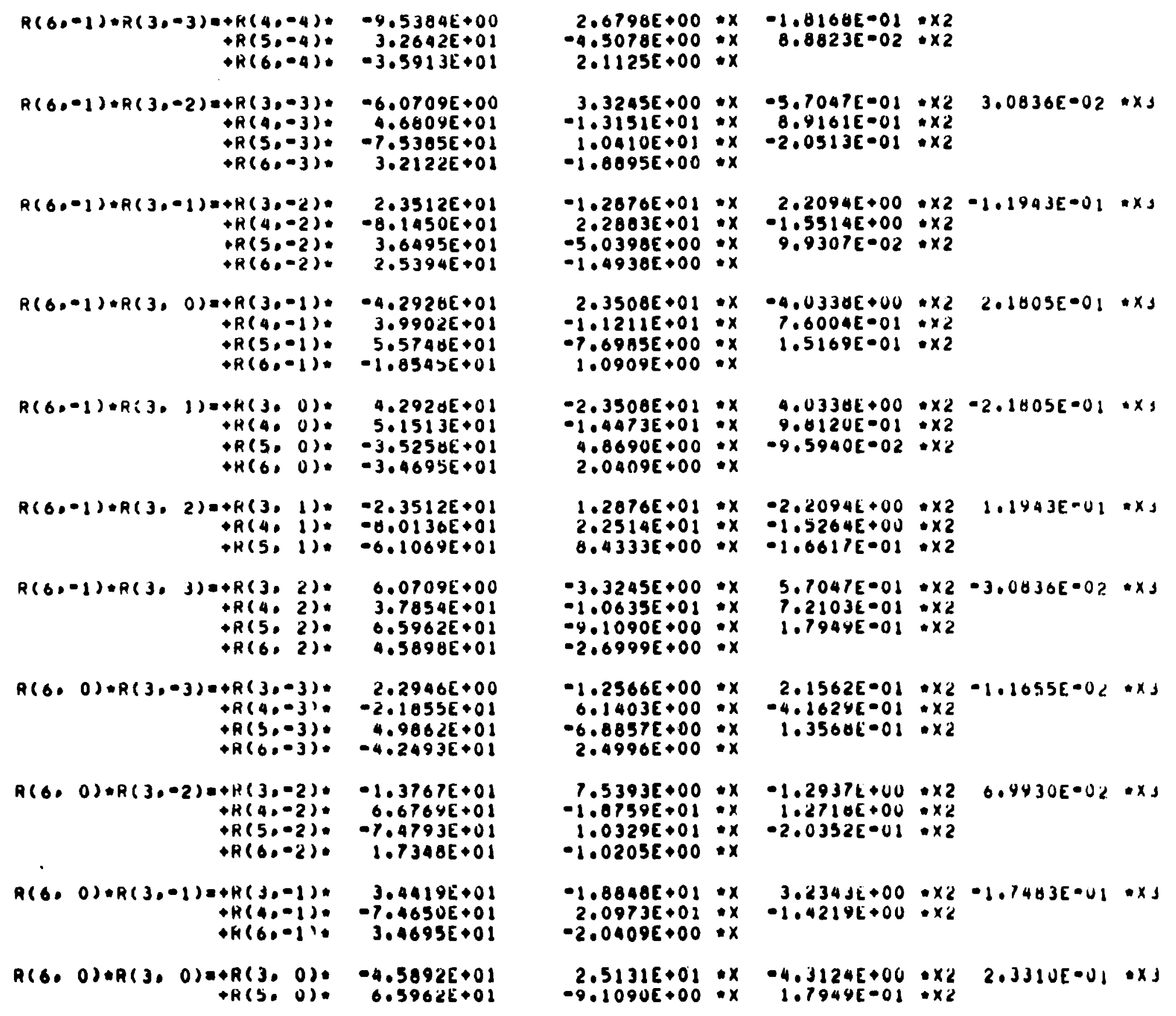




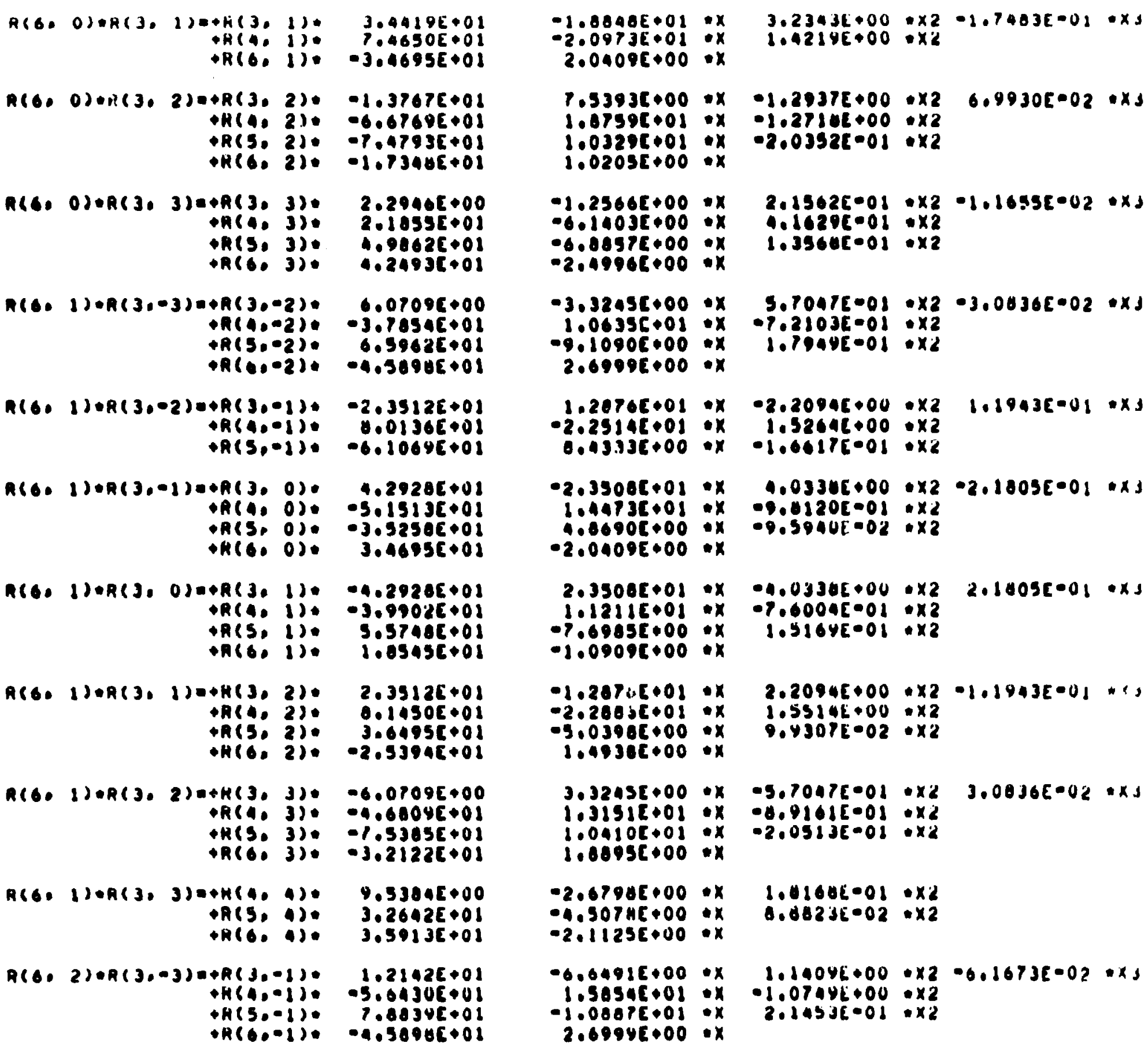
R(6. OSAR(3. 1)EPR(3. 1): 3.4419E O.01 $\rightarrow R(6$. 1). -3.4695E+01

$n(6,0)+R(3,2)=+R(3,2),-1.3767 E+0)$ H(A, 2). $-6.6760 E+01$ $+1 \mathrm{C6}, 250-1,73406+01$

R(6, O)+R(3, 3)=+A(3, 3), $2.2900 E+00$ AC(A. 3) $2.1055 E+01$

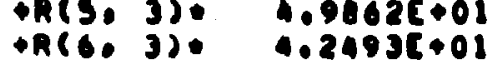

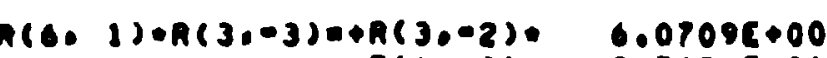
PA( (n) $4 A(0,-2) \cdot-4.38945+0)$

$R(6,1) \cdot R(3,-2) 0+A(3,-1),-2,3512 E+0)$ $\begin{array}{ll}+R(A,-1) & 0.0136 E+01 \\ * R(5,-1) & -6.1069 E+01\end{array}$

R(O. 1$)+R(3,-1)=+A(3,0), \quad 4.2928 C+0)$

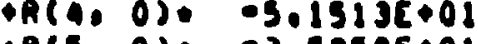

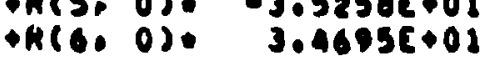

R(6, 1)०R(3, O)D०R(3. 1), -4.20285+01 R(A. 1). -3.90026+0)

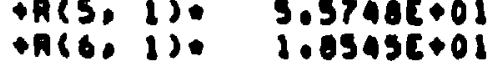

A(6. 1)०A(3, 1)=+H(3, 2), 2.3512C+0) $+R(A, 2) \circ 0.1450 E+01$ $+H(6,2)=-2.3394 E+02$

$R(6,1) \cdot R(3,2)=+H(3,3),-6.0 P 095+00$ R(4. 3): $-4.00045+01$ $+K(S, 3)=-1.5305 E+0)$ $+R(6,3):-3,21226+01$

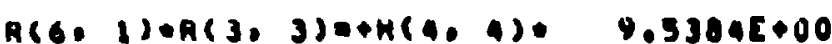
$+A(5$, A $) \quad 3.2642 E+01$
$+R(6, A): 3.5013 E+01$

$R(6,2) \cdot R(3,-3)++R(3,-1)=1.21426+01$ $+H(4,-1):-5.04306001$

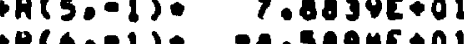

$-1.8048 E+01$ :x $2.0973 E+01$ :x $2,04096+00 \cdot x$

$r .53936 .00 * x$ $1.67595001: x$ $\begin{array}{ll}1.03296+01 & * x \\ 1.0205 E+00 & : x\end{array}$

$-1.25605+00 * x$ $0.14035+00$ :x $-6.08576+00$ ix $02.49966+00$ * $K$

$0.32455+00 \cdot x$ $1.06356+02 \div x$ $9.10906+00$ :x $2.69996+00 * x$

$1.20705+01$ :x $-2.25146+01$ : $x$ $0.03 .336+00$ : $x$

$-2.3500[+01 * x$ $1 \cdot 9473 c+01$ ix $0.06906+00$
$-2.0409 E 000: x$

$2.35008+01: x$ $2.35006+01$
$1.12116+01$ $.7 .6985 E 000$ ix $1,09096+00 \cdot x$

$-1.287,[001 \cdot x$ - 2.26036008 ox $-5.03986 .00: x$

$3.32455+00 \cdot x$

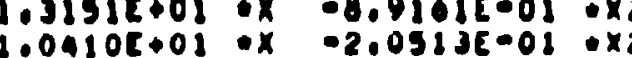
$1.00056 .00 \cdot x$

-2.67905000 ax $-4.507 H E+00$ :x 0.50746000 :x

$0.64915000 \cdot x$

l. -1.008 repol : $x$ $2.69946 .00 \cdot x$

$3.23436+00 \div \times 2-1.7403 E-01-x 3$

$1.2937 E+00 \times 2 \quad 6.9930[002 \times x J$

$1.2710 E+00$ : $\times 2$
$-2.03525-01: \times 2$

$2.15625 \cdot 01 \cdot \times 2 \cdot 1.16555 \cdot 02 \cdot x d$ $1.16206=01$ : $x 2$

$5.70470001 \times 2 \cdot 3.0430[002 \times x J$ 1.70005 001 - $x 2$

$0.20946 .00 \cdot \times 2 \quad 1.19436001 \times 1$ $1.52646+00 \cdot x 2$

$4.03305 .00 \div \times 2-2.1805 E-01 \times x 3$ $9.0120<-01$ ix $x$

$-4.03305000+\times 2 \quad 2.1405 E-01$ *xs 07.60092001 ix

$2.20846+00+\times 2-1.1943 E=01$ क $1.5314 E+00$ : $x_{2}$

$5.7047 E-01+\times 2 \quad 3.0036 E-02 * x J$

\section{$1.01008-01 \cdot x$} $0.08235=02 . \times 2$

$1.14046000 \cdot \times 2-0.16736=02 \times 1$ 1.07496000 - $x 2$ 


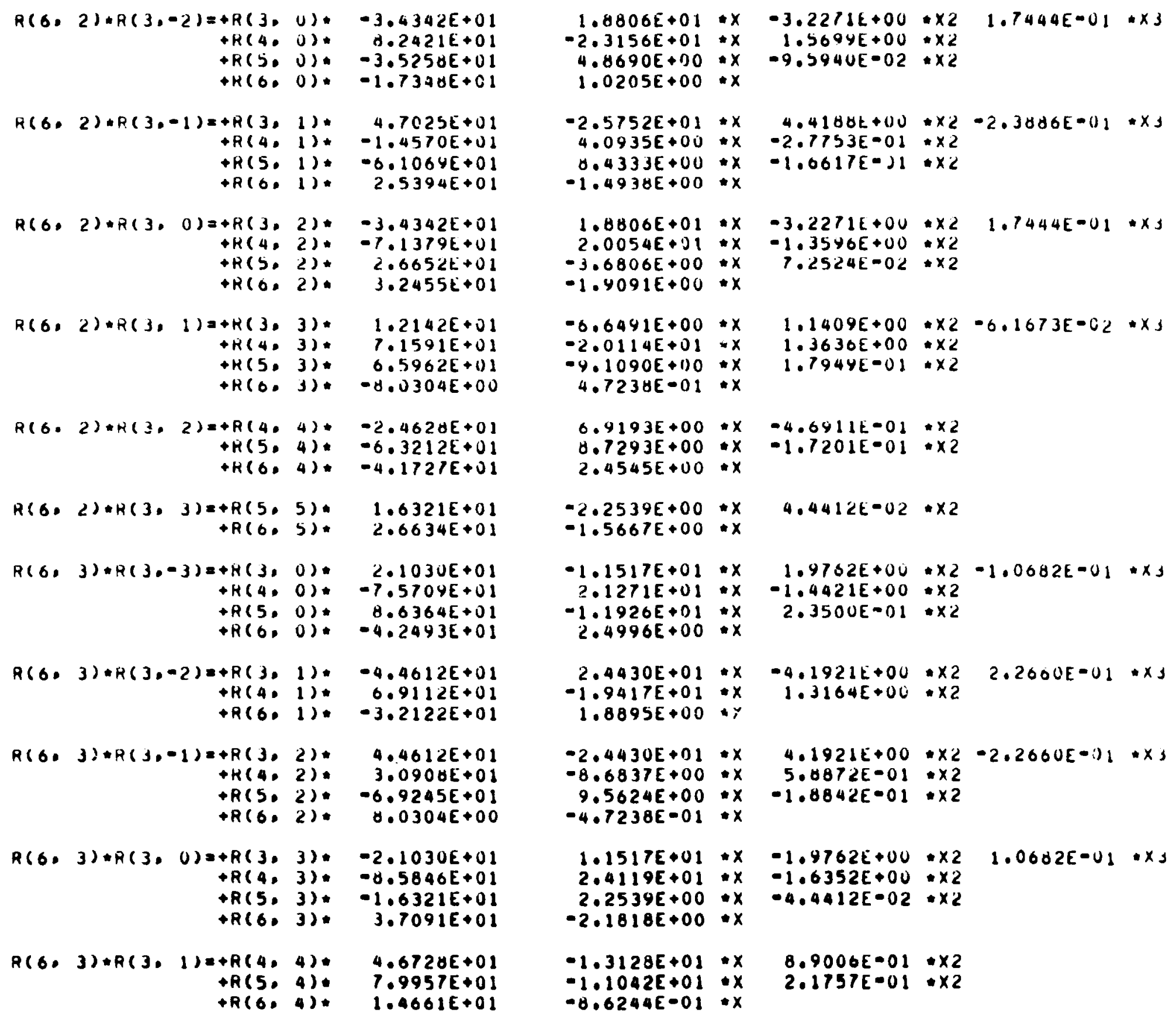

$R(6,3) \sqcap R(3,0)=+R(3,3):-2.1030 E+01$ $+R(4,3)=-0.5846 E+01$ $+R(5,3)=-1.6321 E+01$ $+R(6,3)$. 3.7091E+01

$R(6,3) * R(3,1)=+R(4,4), 4.6720 E+0)$ $+R(5,4) \cdot \quad 7.9957 E+0)$ $+R(6,4): 1.4661 E+0)$

$1.8806 E+01 * x-3.2271 E+00 * \times 2 \quad 1.7444 E-01 * \times 3$ $-2.3156 E+01 * x \quad 1.569 y E+00 * x$ $4.8690 E+90 * x-9.5940 E-02 * x$ $1.0205 E+00 * x$

$-2.5752 E+01 * x \quad 4.4188 t+00 * x 2-2.38 d 6 E-01 * x$ $4.0935 E+00 \times x \quad-2.7 r 53 E-01 \times x$ $0.4333 E+00: x-1.0617 E-J 1$ i $x$

$1.0800 E+01 * x \quad-3.2271 E+00 * x 2 \quad 1.7444 E-01 * x s$ $2.0054 E+11=x-1.35 y 6 E+00: x$

$-1.6806 E+00=x$

$-6.6491 E+00 * x \quad 1.1409 E+00 * \times 2-6.1673 E-C 2 * x$ $-4.10905+110$ : $x$ $4.723 \forall E-01 * x$

$0.9193 E+00 * x \quad-4.6911 E-01 * \times 2$ $0.7293 E+00: x-1.7201 E-01: x 2$ $2.4545 E+00: x$

$-2.2539 E+00 * x \quad 4.4412 E-02 * \times 2$

$-1.5667 E+00 \cdot x$

$-1.1517 E+01 * x \quad 1.9762 E+00 * x^{2}-1.0682 E-01 * x_{3}$ $2.1271 E+01: x$ $2.1271 E+01: x$
$-1.1920 E+01: x$ $-1.1926 E+01: x$

$-1.4421 E+00: \times 2$

$2.3500 E=01 * 2$

$2.4430 E+01 * x-4.1921 E+00 \times 2 \quad 2.2600 E=01 * x J$ $-1.9417 E+01 * x \quad 1.3164 E+00 * \times 2$

$1.8895 E+00$. $Y$

$-2.4430 E+01 * x \quad 4.1921 E+00 * 22-2.2600 E-01 * x 3$ $-8.6837 E+00 * x \quad 5.8872 E-01 * \times 2$

$-4.7238 E-01: x$

$1.1517 E+01 * x-1.9762 E+00 * \times 2 \quad 1.0602 E-U 1 * x J$ $2.4119 E+01 * x-1.6352 E+00 * \times 2$ $2.2539 E+00 * x-4.4412 E-02 * x 2$

\section{$-1.3128 E+01 * x \quad 8.9006 E=01 * x 2$}

$1.1042 E+01+x \quad 2.1757 E=01 * x 2$ 


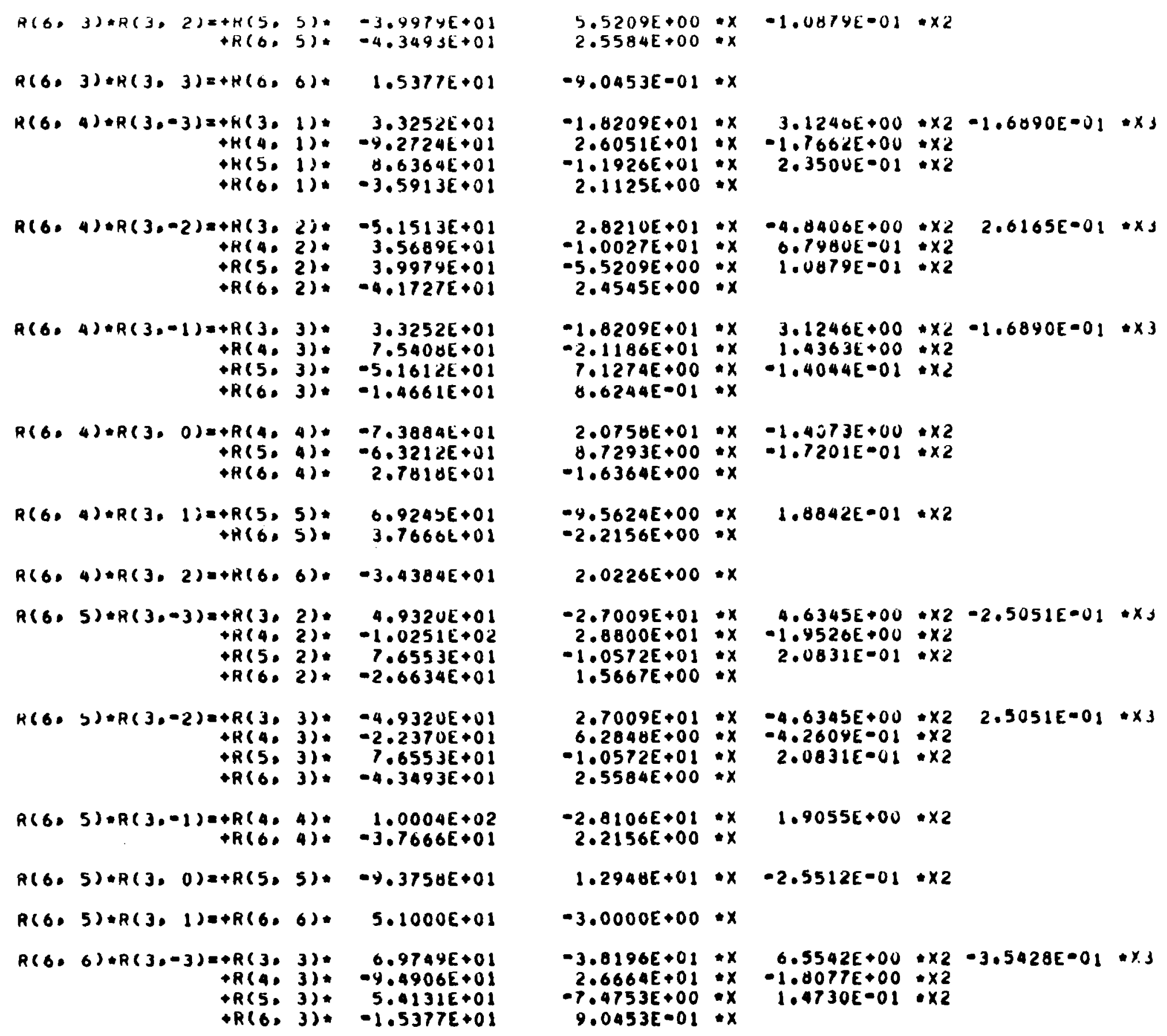

$5.5209 E+00: x$
$2.5584 E+00: x$

$-9.0453 E-01+x$

$-1.6209 E+01 \cdot x$

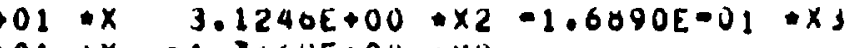
$2.6051 E+01 * x-1.7662 E+00 \div x 2$ $-1.1926 E+01 * x \quad 2.350 U E-01 * x 2$

$2.8210 E+01 * x$ $-1.0027 E+01 * x$ $-5.5209 E+00 * x$
$2.4545 E+00 * x$

$0.02095+01 \cdot x$

$-1.8209 E+01: x$
$-2.1186 E+01: x$

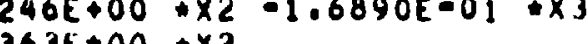
$7.1274 E+00: x-1.4044 E=02: x 2$ $0.6244 E-01 * x$

$2.075 \theta E+01 * x-1.4573 E+00 \div 2$ $0.7293 E+00 \div x-1.7201 E-01: x 2$ $-1.6364 E+00 * x$

$-9.5624 E+00: x$ $2.0226 E+00 * x$

$1.8842 E-01 * \times 2$

$-2.7009 E+01 * x$ $2.8800 E+01: x$ $-1.0572 E+01: x$
$1.5667 E+00: x$

\section{$2.7009 E+01+x$ $6.2848 E+00: x$} $-1.0572 E+01: x$
$2.5584 E+00: x$

$-2.8106 E+01: x$ $2.2156 E+00 * x$ $1.2948 E+01 \cdot x$ $-3.0000 E+00 * x$

$-3.8196 E+01 * x$ $2.6664 E+01 \div x$ $-7.4753 E+00: x$ $9.0453 E-01 * x$

$4.6345 E+00 \times 2=2.5051 E=01 * x 3$ $-1.9526 E+00: \times 2$
$2.0831 E-01: \times 2$

$-4.6345 E+00: \times 2 \quad 2.5051 E-01 * x 3$ $-4.2609 E-01: \times 2$ $2.0831 E-01 \cdot \times 2$

$1.9055 E+00 * \times 2$

$-2.5512 E-01 * \times 2$

$6.5542 E+00+x 2-3.5428 E-01 * x .3$ $-1.0077 E+00 \times \times 2$ $1.4730 E=01: \times 2$ 


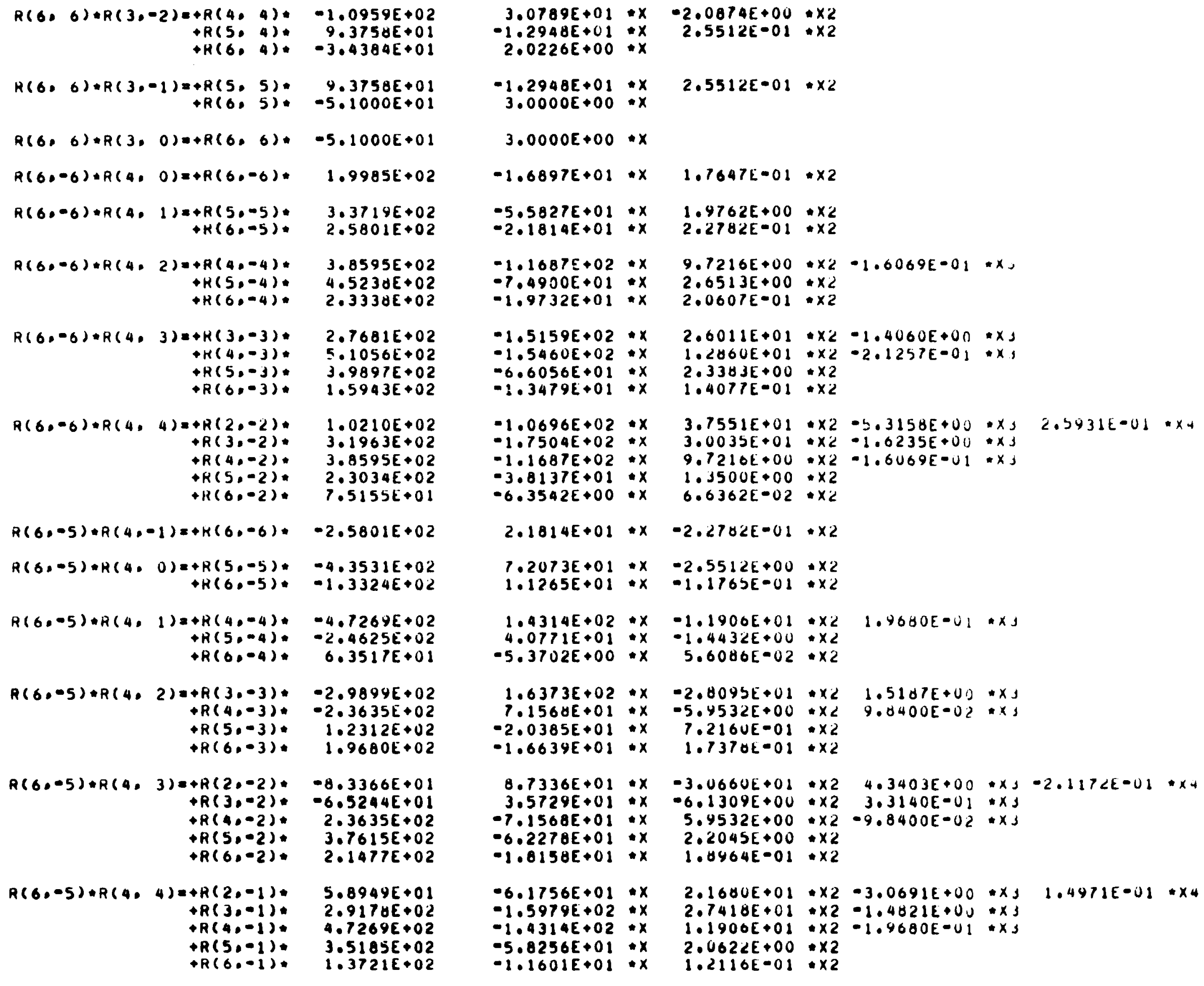




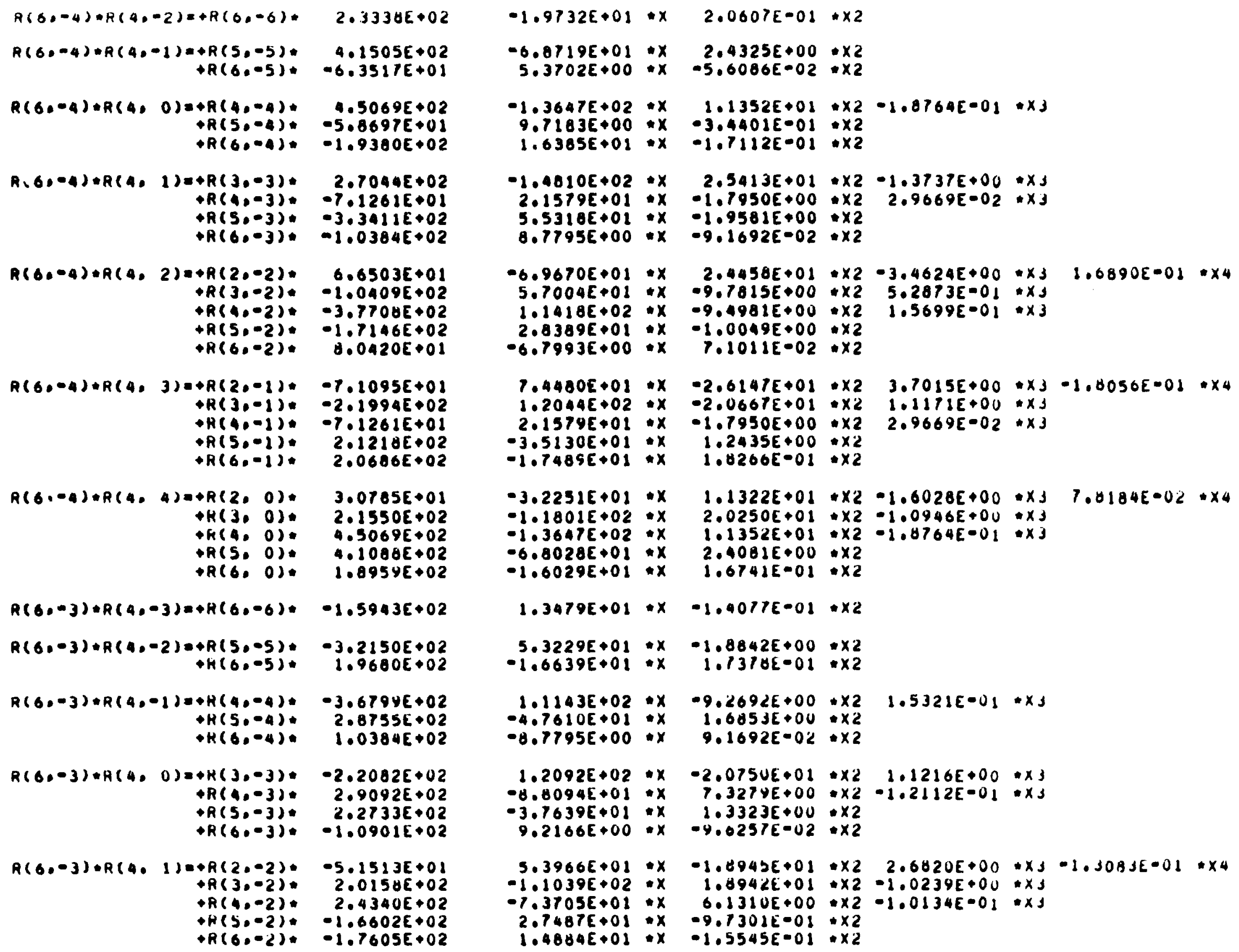




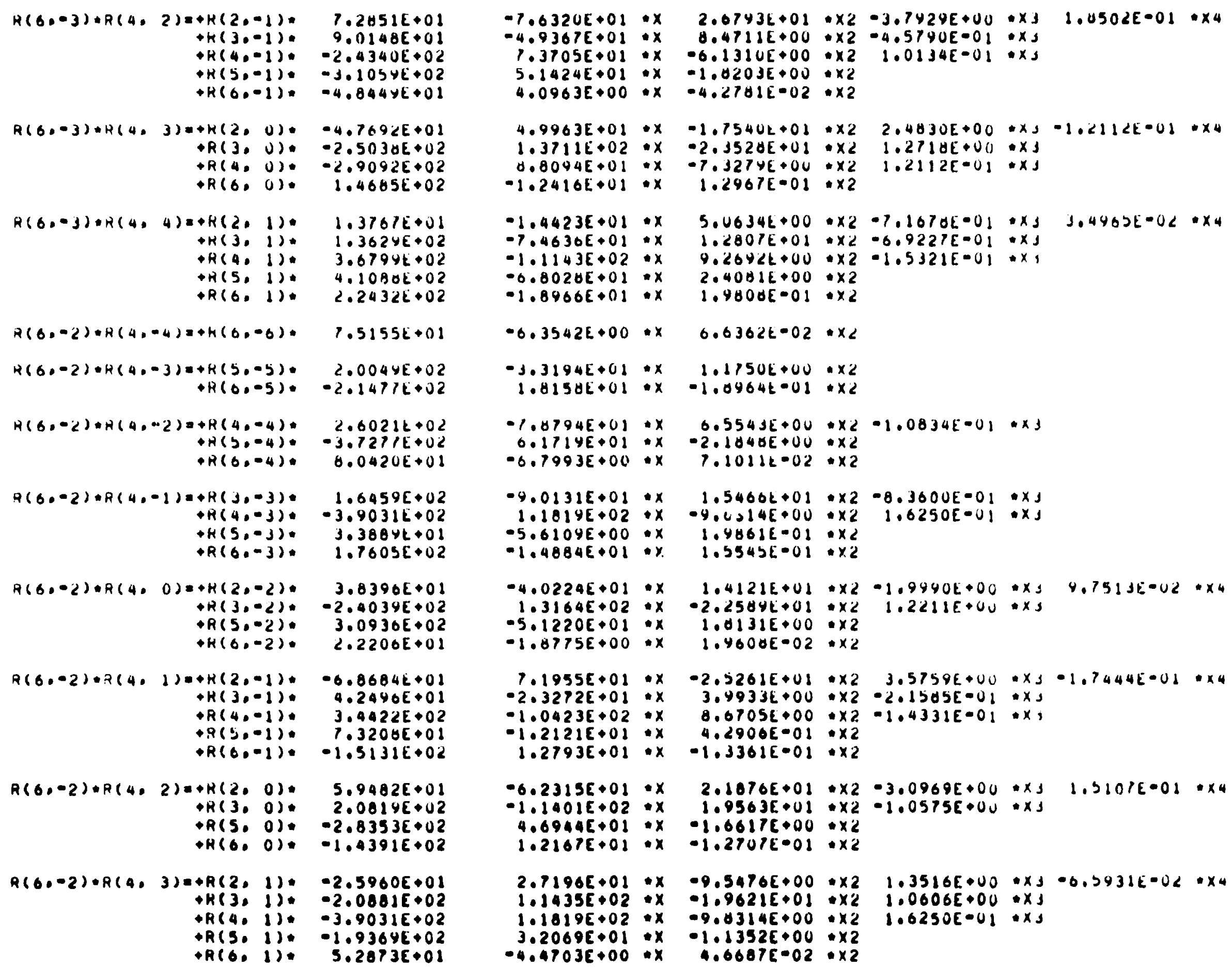




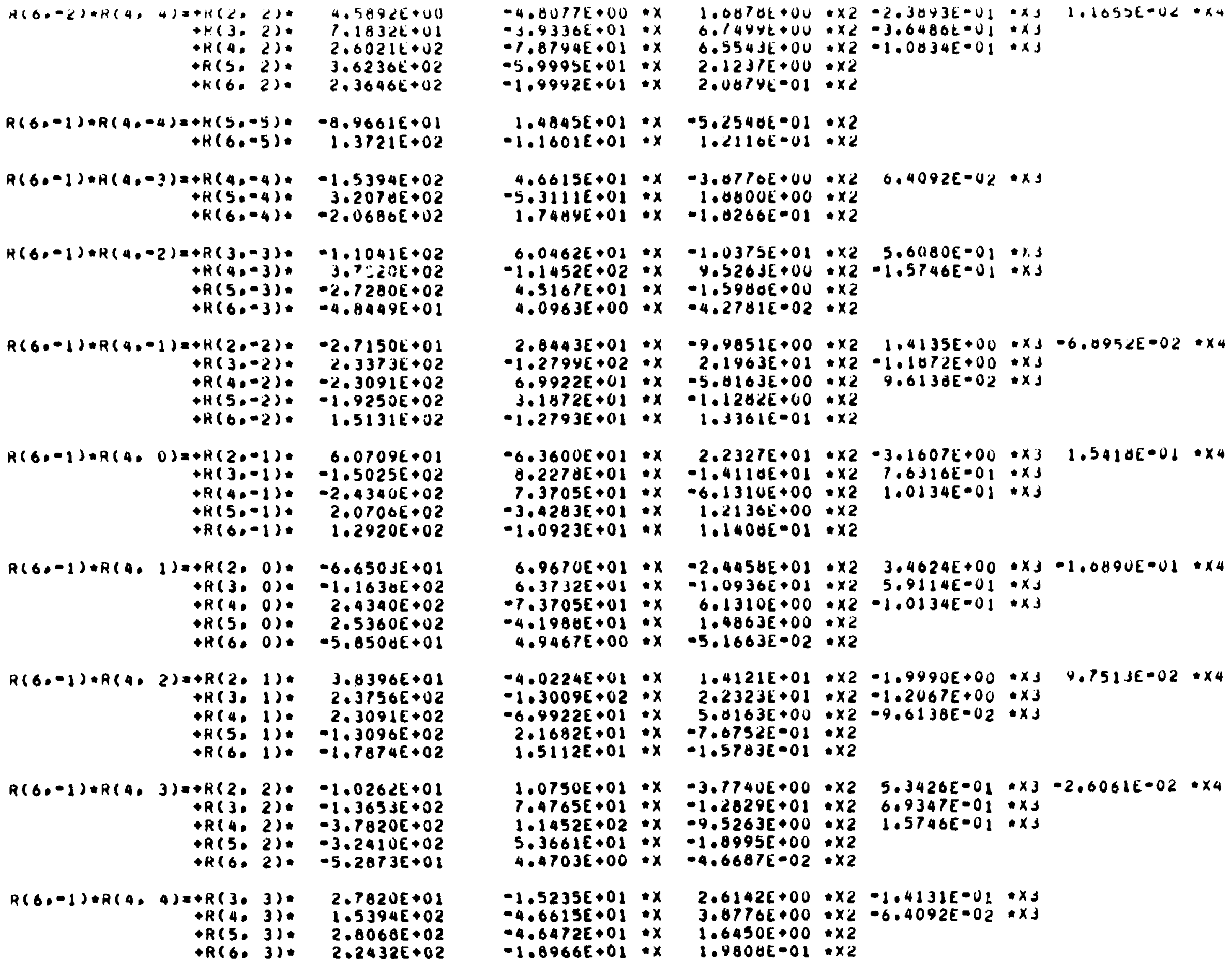

$A(6,-2)+R(4,4)=+K(2, \angle) * \quad 4.5 \theta 92 E+U 0$ $+H(3,2) * 7.1832 k+U)$ - $R(4,2) * 2.6021 k+02$ R $(5,2)=3.6230 E+0$ $+k(6,2) .2 .3646 E+02$ $-4.9661 E+0)$ $+k(0.05)=1.3721 E+02$

$R(6,-1) * A(4,-?)=+R(4,-4):$ $+R(5,-4)$ $+K(6,-4)$.

$R(6,-1) * R(4,-2)=+R(3,-3) \cdot$ $+R(4,-3)$ * $+R(5,-3)$. $\rightarrow R(6,-3)$.

$R(6,-1) * R(4,-1)=+H(2,-2) *$ $+R(3,-2)$. $\rightarrow R(0,-2)$. $+H(5,-2)$. $+R(6,-2)$.

$1.5394 E+02$ $3.2070 E+02$ $-2.0680 E+02$

$-1 \cdot 1041 E+02$ $3.7,-6,6 c+05$ $-2.7280 E+02$ $-4.0499 E+01$

$-2.7150 E+01$ $2.33736+02$
$-2.30916+02$ $-1.9250 E+02$ $1.9250 E+02$
$1.5131 E+02$

$R(6,-1) \cap R(4,0)=+R(2,-1)$. $+R(3,-1)$ P $R(40-1)-2.4340 \varepsilon+02$ $+R(0,-1), \quad 1.2920 E+02$

$R(6,-1) * R(4,1)=+R(2,0)$. $-6.650 J E+01$ $+A(3,0) *-1.1630 E+02$ - $R(5.0)$ 2.4340E+02 A

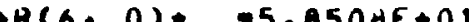

$R(6,-1) * R(4,2)=+R(2,1) * 3.8396 E+01$ $+R(3,1)=2.3756 E+02$ R(4. 1): 2.3091E+02 $+R(5,1) *-1.3096 E+02$

$R(6,-1)+R(4,3)=+R(2,2) *$ (45. 2). $\rightarrow R(6,2)-5.2073 E+01$

$R(6,-1) \oplus R(4,4)=+R(3,3)=$ (4. 3): $1.53945+02$ 1.5Jg4E०02 $\rightarrow R(6,3): 2.2432 E+02$

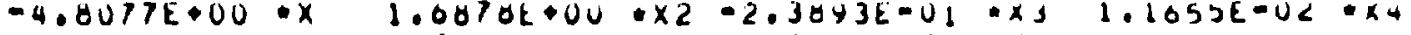
$-1.9336 \varepsilon+01 * x \quad 6.144 y k+0 u * x<-3.6480 k=01 * x J$

$-5.9995 E+01 * x \quad 2.12 J 1 E+00 * x^{2}$
$-1.9992 E+01 * x \quad 2.0474 t-01 * x^{2}$

$1.4845 E+01 * x-5.2540 E-01 * x 2$

$4.6615 E+01 * x-3.0770 E+00+\times 2 \quad 6.4092 E-02 * x 1$

$-5.3111 E+01 * x \quad 1.0800 E+00 * x^{2}$

$6.0462 E+01 * x-1.0375 E+01 * \times 2 \quad 5.6080 E-01 * 1.3$

$-1.1452 E+02 \times x \quad y .5263 E 000 \times 2-1.57465-01 . x 3$

$4.5167 E+01 * x-1.5980 E+00=x 2$

$4.0963 E+00 * x-4.2781 E=02 \times x 2$

$2.0443 E+01 * x-9.9851 E+00 \times \times 2 \quad 1.4135 E+00 \times x J-6.0952 E-02 * \times 4$ $-1.57905002 \cdot x$ 2.1963E+01*x2-1.1872E+00*xJ

(1) $-5.8163 E+00 * x 2 \quad 9.6133 E=02 * x J$

$1.1 .1242 E+00 * x 2$

$-6.3600 E+01 * x$ $1,13616=01 * \times 2$ $0.2278 E+01 * x-1.4110 E+01 * x 2 \quad 1.6316 E-01 * x J$

$7.3705 E+01 * x-6.1310 E+00 * \times 2 \quad 1.0234 E+01 * x 3$

$-3.4283 E+01 * x \quad 1.2136 E+00 * \times 2$ $-1.0923 E+01 * x \quad 1.1400 E=01 * \times 2$

$6.9670 E+01 * x \quad-2.4456 E+01 * \times 2 \quad 3.4624 E+00 * x_{3} \cdot 1.0890 E \cdot 01 * \times 4$ $6.3732 E+01 * x-1.0936 E+01 * x 2 \quad 5.9114 E-01 * x J$ - $.3705 E+01: x \quad 6.1310 E+00 \times 2=1.0134 E=01 * x 3$

$-4.1988 E+01: x \quad 1.4863 E+00 * x z$

$4.9467 E+00 * x-5.1663 E-02 * x 2$

$1.4121 E+01 * \times 2-1.9990 E+00 * x_{3} 9.751 \mathrm{JE}-02 * \times 4$

$-6.9922 E+01: x$

$5.0163 E+00: \times 2-9.6138 E-02 \times x 3$

$2.1602 E+01 * x-7.0752 E=01 * x 2$

$1.0750 E+02 * x-3.7740 E+00 \times 2 \quad 5.3426 E-01 * x 3-2.6061 E-02 * \times 4$

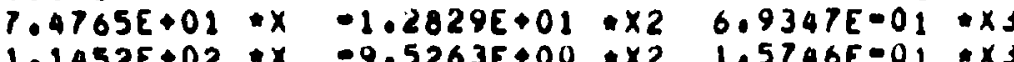

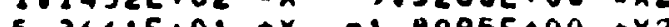
$4.4703 E+00 \cdot x-4.6687 E-02: \times 2$

$-1.5235 E+01 \cdot x$ $-4.6472 E+01: x$

$2.6142 E+00+x 2-1.4131 E=01 * x 3$

$3.8776 E+00 \times x 2-6.4092 E=02 \times x 3$

$1.6450 E+00: \times 2$

$1.98005-01: x 2$ 


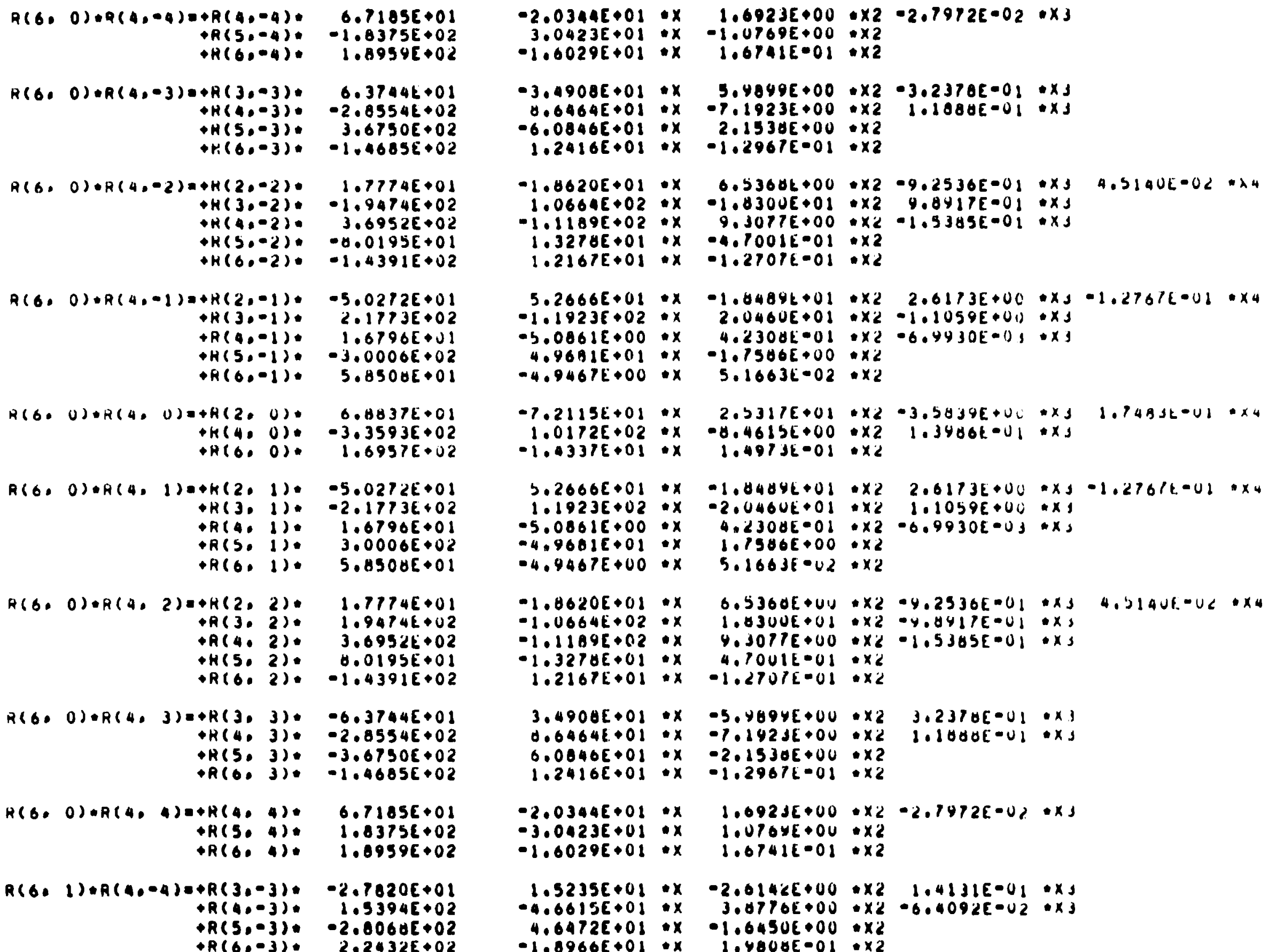

$R(6,0)+Q(4,-0)=+R(4,-4) * 6.7105 E+0)$ $+R(5,-4):-1.8375 E+02$
$+H(6,-4) * 1.8959 E+02$

$R(6,0)+R(4,-3)=+R(3,-3) *$ H(5.-3) - $2.8554 E+02$ $+H(6,-3)=-1.4685 E+02$

$A(6,0) \bullet A(4,-2)=+H(2,-2) \bullet$ $\rightarrow H(3,-2)$.

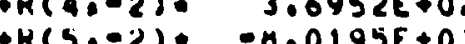
$+H(6,0-2) .-1,43918+02$

$R(6,0)+R(4,-1) a+R(2,-1)$. $\rightarrow R(3,-1)$. $+R(4.0-1)$. $2.1773 E+02$ H $(5,-1) .-3.0000 E+02$ R $(6,-1)$. $5.8500 E+01$

$R(6,0)+R(4,0)=+A(2,0)=$ $+K(4,0)$

$0.8837 E \cdot 01$ $-3.35936 .02$ $1.6957 E \cdot 02$

$R(6,0) \bullet R(4,1)++K(2,1)$. $+R(3,1):$ $\rightarrow R(4,1):$ $+R(6,1)$.

$-5.0272 E+01$ $-2.17736402$ $1.67905 \div 01$ $3.0006 E+02$ $5.85085+01$

$A(6,0) \cup R(4,2)=+R(2,2)$. $+R(3,2)$ $\rightarrow A(4,2)$ $+H(5,2)$

$1.7774 E+01$ $1.9474 E+02$ $3.6952 E .02$ 0.01952001

$R(6,0)+R(4,3)=+R(3,3)$. $+H(4,3)$. $-3.07505+02$ $\rightarrow R(0,3)=-1.4685 E+02$

$R(6,0)=R(4,0)=+R(40,4)=$ $\rightarrow R(5,4): 1.83756+02$

$R(6,1) \cup R(4,-4)=+R(3,-3)$ $R(40-3), \quad 1.53945+02$ $R(50-3)=2000605002$ $-R(5,03) \cdot-2.0060 E+02$

$-2.0344 E+01 * x \quad 1.6923 E+00 \cdot \times 2-2.7912 E-02 * \times 3$

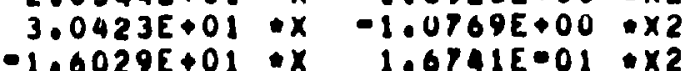

$-3.4908 E+01 * x \quad 5.4899 E+00 \div \times 2-3.2370 E-01 * \times J$ $8.6464 E+01 * x-7.1923 E+00 \times x 21.1080 E=01 \times x J$ $-6.0846 E+01: x \quad 2.153 \forall E+00 \times x 2$

$06.00465+01$ ox 2015305000 : $x 2$

$-1.8620 E+01 * x \quad 0.5360 t+00 * \times 2-9.2536 E=01 * x J \quad 4.5140 E=02 * A_{4}$ $1.0664 E+02 * x-1.8300 E+01 \times x 2 \quad 9.8417 E=01 \times x J$

$-1.1189 E+02 * x \quad 9.3077 E+00 \times 2 \times 2-1.5385 E=01 \times x$

$1.3278 E+01$ : $-4.7001 E-01: x 2$

$5.2666 E+01 \cdot x-1.0489 k+01 * \times 2 \quad 2.6173 E+00 \times x=1.27676 \cdot 01 * \times 4$ $\left.-1.1923 E+02 * x \quad 2.0400 E+01 * x_{2}-1.1059 E+01\right) \times x^{2}$

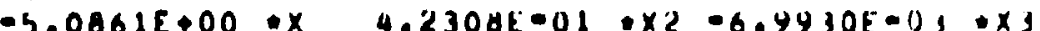

4.96915001 : $x-1,15065000$ : $x x^{2}$

$-4.0467 E+00 * x \quad 5.16636-02 * x 2$

$-7.2115 E+01 * x \quad 2.3317 E+01 * x 2-3.5839 E+U 0 * x J 1.743 J E-U 1 \cdot \times 4$ $1.0172 E+02 * x-4.46156+00 * x 2 \quad 1.34806-01 * x s$

$-1.433\} E+01$ : $x$ 1.497JE-01:x2

$3.2666 E+01 * x-1.8444 E+01 * x_{2} \quad 2.6173 E+0 U=X_{1}-1.276 / t-01 * x_{4}$ $1.1923 E+02$ * $x-2.0460 E+01 * x 2 \quad 1.1059 E+00$ * $x 1$

-5.08015000 : $x$

$-4.9601 E+01: x \quad 1.1986 E+00: x 2$

$-4.9467 E+00 \cdot x \quad 5.16635002+x 2$

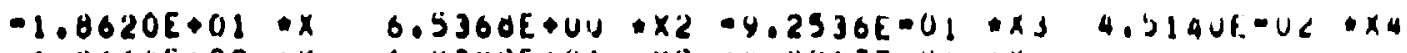
$-1.0664 E+02 * x \quad 1.8300 E+01 * x 2-4.4417 E-01 * x s$

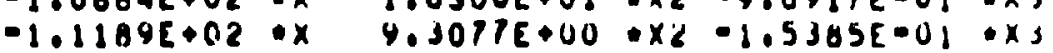

$-1.3278 E+01$ : $x$, POU1E-01 $x$.

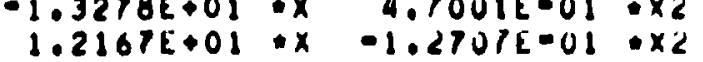

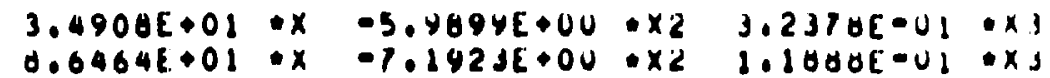

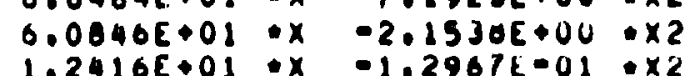

$-2.0344 E+01 \times x \quad 1.092 J E+00+x 2-2.7972 E-0 ?+x 3$

$-3.0023 E+01$ : $x \quad 1.076 y E+00$ : $x 2$

$\begin{array}{ll}-3.0023 E+01: x & 1.076 y E+00 \\ -1.6029 E+01: x \quad 1.0741 E-01: x 2 & \end{array}$

$1.5235 E+01 * x-2.0142 E+00 * x 2 \quad 1.4131 E-01 * x\}$

$4.6615 E+01: x \quad 3.67765+00 \cdot x 2.0$

$4.6472 E+01: x \quad-1.64506+00$ * $\times 2$ 


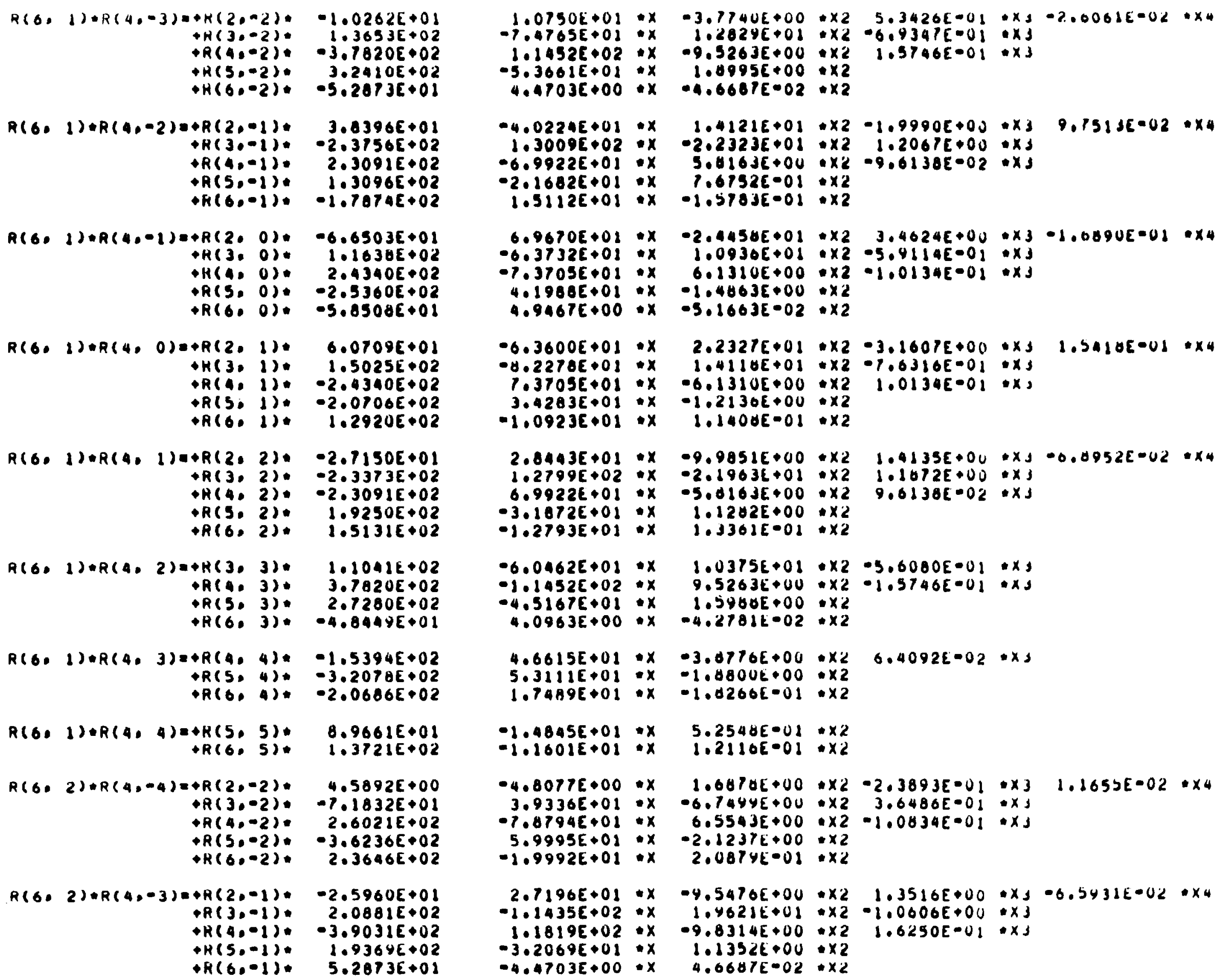




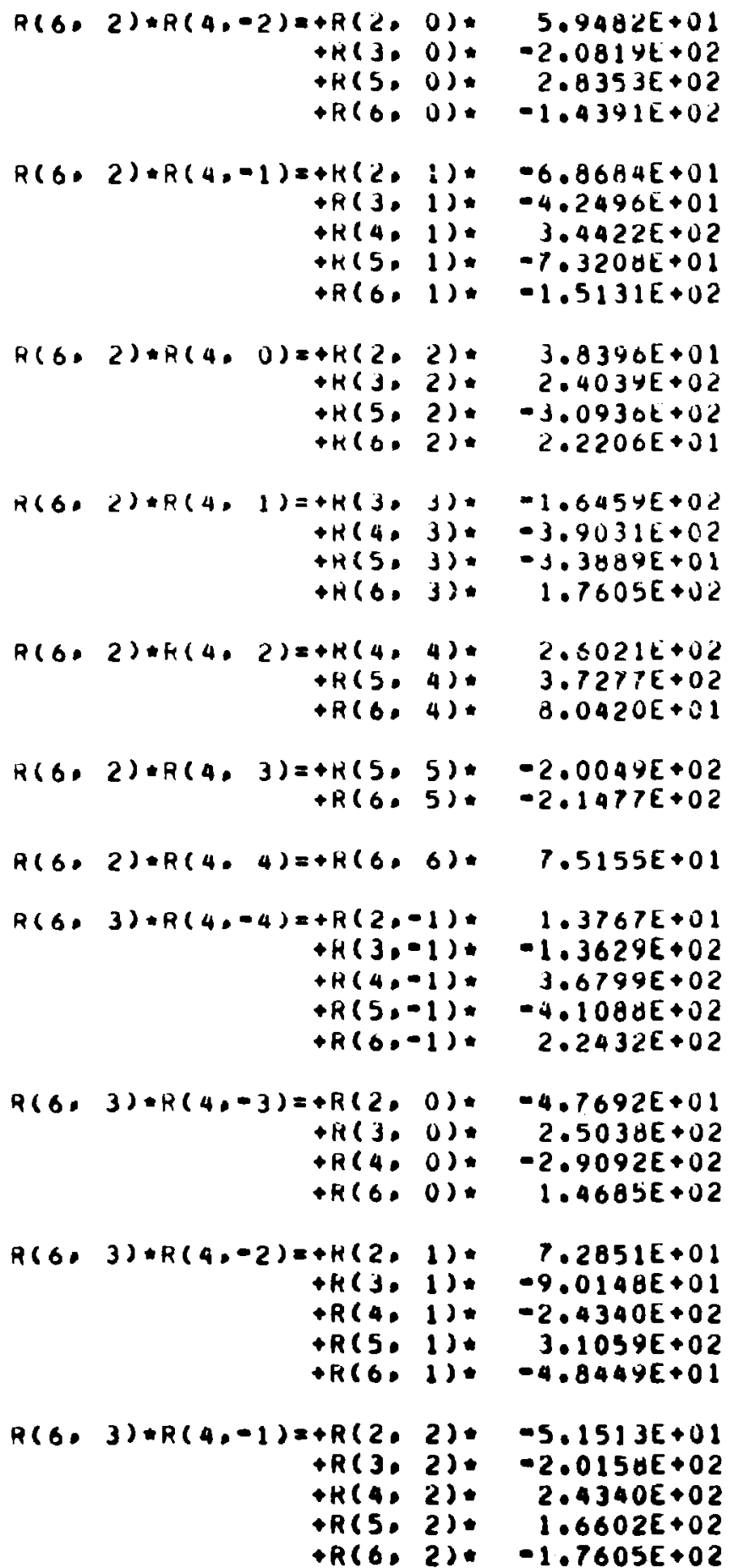
$+R(5,1) * 3.1039 E+02$

$R(6,3)=R(4,-1)=+R(2,2) *$ $+R(3,2)=-2.0158 E+02$ AH(4) 2): 2.4340E+02 $+R(6,2) . \quad-1.7605 E+02$ 


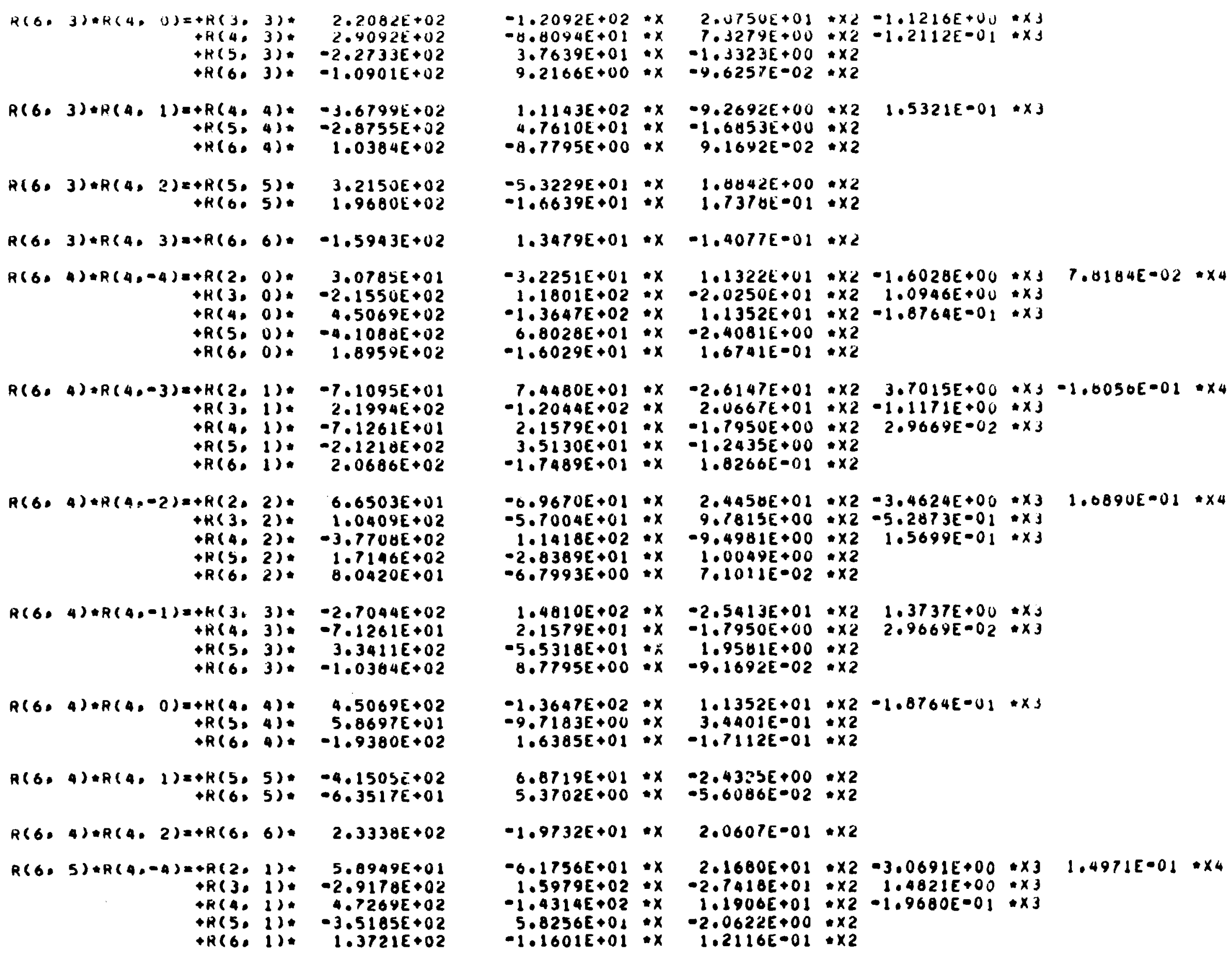

$R(6,3) \cdot R(4,0)=+R(3,3) * 2.2082 E+02$ पA(4. 3): $209092 E+02$ $+R(603)=0.0100901 E+02$

$R(6,3)+R(4,1)=+R(4,4) *-3.6799 E+02$ $+R(5,4)$ $+M(604)=1.0384 E+02$

$R(6,3) * R(4,2)=+R(5,5) *$ $+K(6,5)$.

$3.2150 E+02$ $1.9680 \mathrm{E}+02$

$R(6,3)=R(4,3)=+R(6,6) \cdot$

$R(6,4)+R(4,-4)=+R(2,0)$. $\rightarrow K(3,0)$. $\rightarrow R(4,0)$. $\rightarrow R(5,0)$.

$-1.5943 E+02$

$3.0785 E+01$ $-2.1550 E+02$ $4.5069 E+02$ $-4.100 \partial E+02$ $1.8959 E+02$

$R(6,4)=R(4,-3)=+H(2,1)=-7.1095 E+01$ $+R(3,1) * 2.1994 E+02$ $\rightarrow R(4,1) .-7.1261 E+01$ $+R(5,1) *-2.1210 E+02$ $+R(6,1)$. $2.0686 E+02$

$R(6,4)+R(4,-2)=+R(2,2), 6.6503 E+01$ $+K(3,2) .1 .0409 E+02$ $+R(4,2):-3.7708 E+02$ $+R(S, 2): \quad 1.7146 E+02$

$R(6,4)+R(4,-1)=+K(3,3)=-2,7044 E+02$ $+R(4,3)=-7.1261 E+0$ $+R(5,3): 3.3411 E+02$ $+R(6,3)=-1.0364 E+02$

$R(6,4) \| R(4,0)=+K(4,4): 4.5069 E+02$ $+R(5.4): \quad 5.8697 E+01$
$+R(6.9):-1.9380 E+02$

$\begin{array}{rlr}R(6,4)+R(4,1)= & +R(5,5), & -4,1505 \bar{c}+02 \\ & +R(6,5):-6,3517 E+01\end{array}$ $R(6,4) \div R(4,2)=+R(6,6)$. $2.3338 E+02$ $R(6,5) * R(9,-4)=+R(2,1) * 5.0949 E+01$ $+R(3,1):-2.917 \theta E+02$ $+R(4,1)$ 1. 4.7269E+02 $\rightarrow R(5,1) .-3.5185 E+02$ $+R(6,1)$. 1.3721E+02

$-1.2092 E+02: x$
$-8.8094 E+01: x$ $-0.8094 E+01: x$ $0.2166 E+00 . x$ $.2366 E+00 * x$

$1.1143 E+02: x$ $4.7610 E+01 * x$ $-8.7795 E+00 * x$

$-5.3229 E+01: x$ $-1.6639 E+01 \cdot x$

$1.3479 E+01 \cdot x$

$-3.2251 E+01: x$ $1.1801 E+02 * x$ $-1.3647 E+02 * x$ $6.8028 E+01: x$
$-1.6029 E+01: x$

$7.4480 E+01: x-2.6147 E+01 * x 2 \quad 3.7015 E+00 * x_{3}=1.6050 E=01 * x_{4}$ $-1.2044 E+02: x$ $2,1579 E+01: x$ $3.5130 E+01: x$
$-1.7489 E+01: x$

$-0.9670 E+01 * x$ $-5.7004 E+01: x$ $1.1418 E+02 * x$ $-2.8369 E+01: x$
$-6.7993 E+00: x$

$1.4010 E+02 * x$ $2.1579 E+01 \div x$ $-5.5318 E+01 . \hat{x}$ B.7795E+00:x

$-1.3647 E+02 * x$ $-9.7183 E+00=x$ $0.07196+01+x$ $5.3702 E+00: x$

$-1.9732 E+01 * x$

$-0.1756 E+01+x$ $1.5979 E+02 \div x$ $-1.4314 E+02+x$ $5.8256 E+01: x$
$-1.1601 E+01: x$

$2.0750 E+01 * x 2-1.1216 E+0 u * x J$ $-1.3323 E+00 \times 2$ $-1.3323 E+00: \times 2$

$-9.2692 E+00 * \times 2 \quad 1.5321 E-01 * \times 3$ $0.6053 \mathrm{E}+00 \div \times 2$

$1.0842 E+00 \times 2$ $1.7370 E-01 \div 2$

$-1.4077 E-01 * \times 2$

$1.1322 E+01 \times \times 2-1.6028 E+00 \times X_{1} \quad 7.0184 E-02 \times x_{4}$ $-2.0250 E+02 \times \times 2 \quad 1.0946 E+0 u * x 3$

$1.1352 E+01: \times 2-1.8764 E=01 * \times 3$

$-2.4081 E+00+x 2$

$2.0667 E+01 * \times 2-1.1171 E+00 \times x$

$-1.7950 E+00: \times 2 \quad 2.9669 E-02: \times 3$

$-1.2435 E+00 * \times 2$

$1.8266 \mathrm{E}-01: \times 2$

$2.4458 E+01 \div \times 2-3.4624 E+00 * \times 3 \quad 1.0890 E=01 * \times 4$ $9.7815 E+00 \div \times 2-5.2873 E=01: x 3$

$-9.4981 E+00 * \times 2 \quad 1.5699 E-01 * \times 3$

$1.0049 E+00: \times 2$
$7.1011 E-02: \times 2$

$-2.5413 E+01=x 2 \quad 1.3737 E+00=x 3$ $-1.7950 E+00 * 2 \quad 2.9669 E-02 * x 3$ $1.9581 E+00 \times 2$

$-9.1692 E-02: \times 2$

$1.1352 E+01 * \times 2-1.8764 E-01 * x 3$ $3.4401 E-01: \times 2$
$-1.7112 E-01: \times 2$

$-2.4323 E+00 * \times 2$
$-5.6006 E=02 * \times 2$

$2.0607 E-01 * \times 2$

$2.1680 E+01 * \times 2-3.0691 E+00 * \times 3$

$1.4971 E=01 * x_{4}$

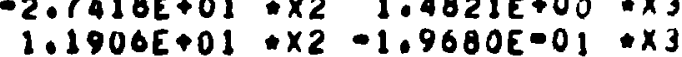

$-2.0622 E+00 * \times 2$

$1.2116 E-01 \div \times 2$ 


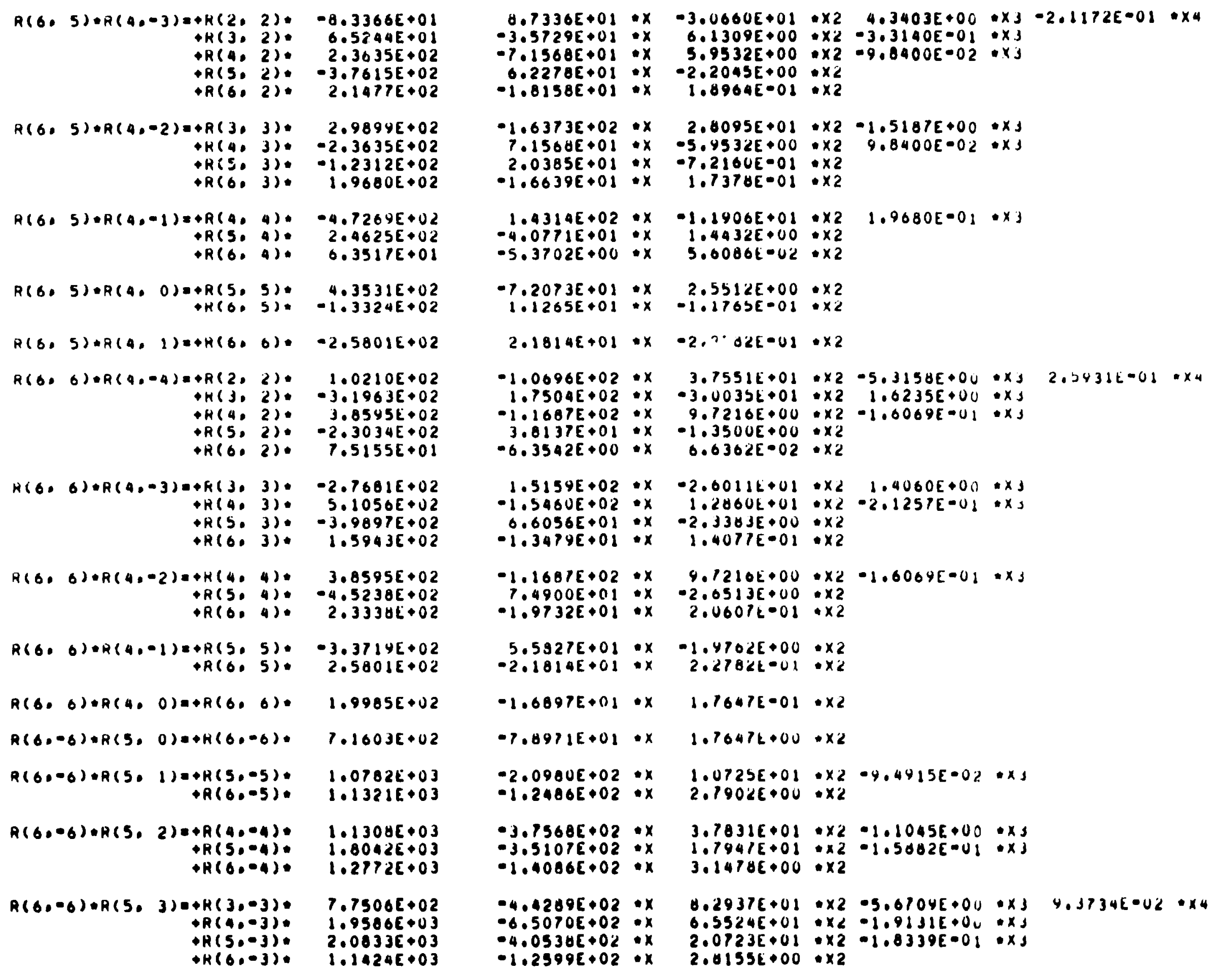




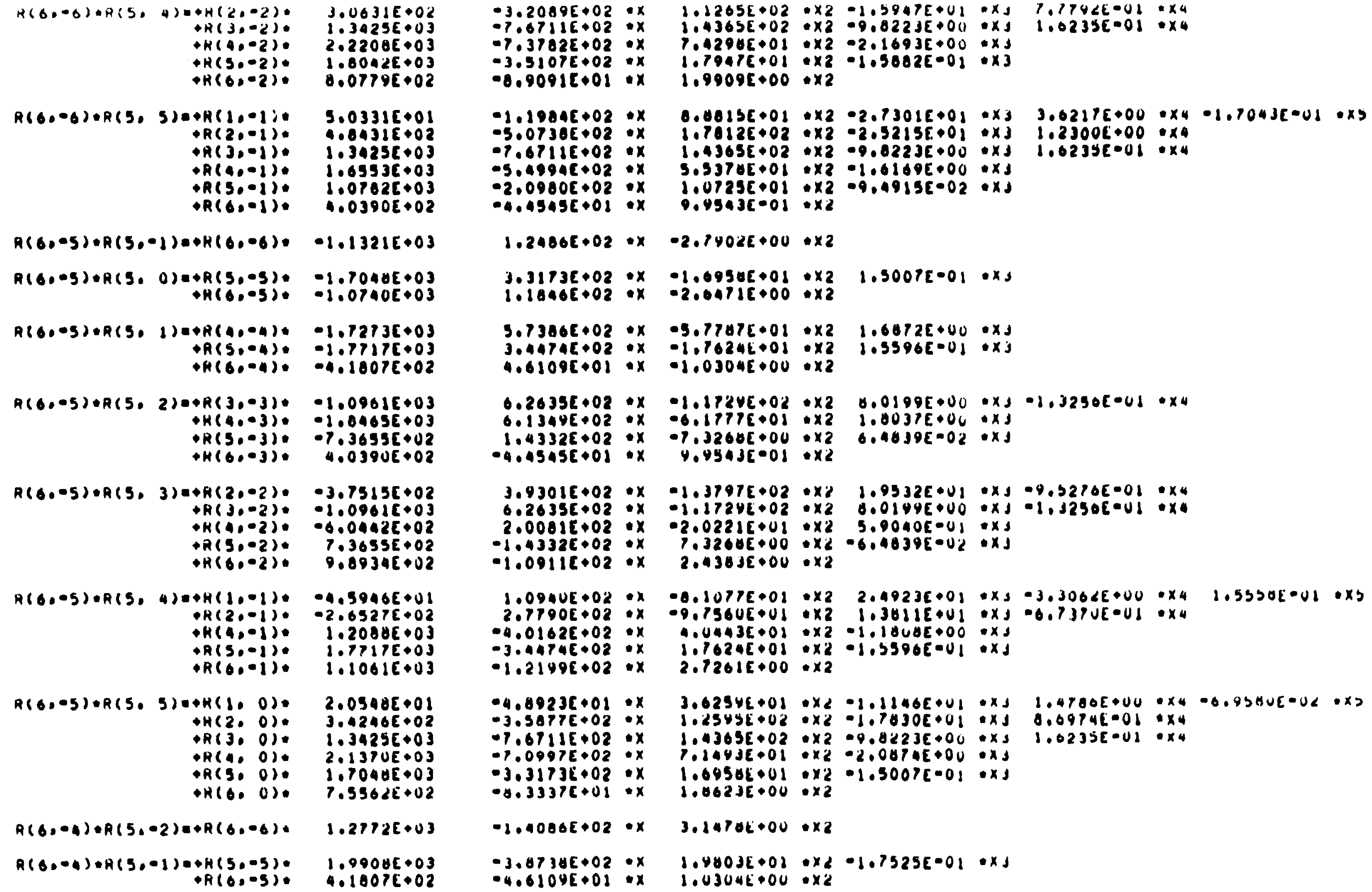

$H(6,-0) \cdot R(5,4)=H(2,-2)$. TR(3.-2) $\rightarrow R(4,-2)$ $+H(5,-2):$

$3.0631 E+02$ 1.3455603 2.22086003 $1.80425+03$ $8.0779 E \bullet 02$

$R(0,06) \cdot R(5,5)=+R(1,-1)$ $\rightarrow(2,-1)$ - $R(3,-1)$ $\rightarrow(4,-1)$ $\rightarrow R(5,-1)$ $\rightarrow R(6,-1)$

$R(6,-5) \cdot R(5,-1) \cdot+H(0,-6)$.

$\begin{aligned} R(6,-5) \cdot R(5,0)= & +R(5,-5) \\ & +H(6,-5)\end{aligned}$ $-1.0 .05) \cdot-1.0740 E+03$

$R(6005) \cdot R(5,1)=+R(40-4) \cdot$ $\leftrightarrow R(5,04) \cdot$ $4.04316+02$ $1.34256+03$ 1.65536003 $1.0762[\bullet 03$ $1.0390 E+02$

$-1 \cdot 1321[\cdot 03$

$R(6,05) \cdot R(5,2) \cdot P R(3,-3) \cdot-1.096) E+03$ CH $(4,-3)$. $+H(6,03)$ :

$-1.7273[003$ $-1.77176003$ $-7.36535+02$ $4.0390 E+02$

$A(6,-5) \cdot A(5,3)=+R(2,-2)$ $+R(3,-2):$ a $(5,02)$ $\rightarrow H(B,-2)$.

$A(6,-5) \cdot A(5,4)=+4(1,-1)=$ - $R(2 \cdot-1)$. $\rightarrow H(4,-j)$ $+R(5,-1)$ $\rightarrow R(0,-1)$.

$R(6,05) \cdot R(5,5) 0+H(1,0)=$ $+H(2,0)$. + A(3. O). $\rightarrow R(4.0)$. - R(S. O) R $(0,0)$

$R(6,-14) * R(5,-2)=+R(6,-6)$.

$A(0,-4)+A(5,-1) \cdot+H(5,-5)$. - $A(0,-5)$.

-3.20896002 : $x$ $-7.67112+02: x$ $-7.37028+02$ ax $0.5107 e+02$ ax $-8.90916+01 \cdot x$

$-1.1984 E+02$ *x $-5.0730 E+02$ *x $-7.67116+02$ : $x$ $-5.48946+02 \div x$ $-2.0980 E+02 \div x$ - $4.05456+01$ *x

$1.24060+02 * x$

$3.31736+02 * x-1.09502+01 \cdot \times 2 \quad 1.3007 E-01 \cdot x \mathrm{~s}$

$5.7306 E+02 * x-5.7787 E+01 * x 21.6072 E+00 * x J$ $3.4474 E+02 * x-1.76246+01 \cdot x 2 \quad 1.5396 E-01 \cdot x j$

$4.61095+01 \cdot x-1.03045+00 * \times 2$

$6.26356+02 * x-1.17246+02 * x 2 \quad 0.0199 E+00 * x]=1.3250[001 \cdot x 4$ $0.1344 E+02 * x \quad-6.1777 E+01 * x 2 \quad 1.0037 E+00: x J$

$-4.0545 E+01 * x \quad y, 9543 E-01+x^{2}$

$3.9301 E+02 * x-1.3797 E+02 * x z \quad 1.9332 E+01 \cdot x]=9.3270 E=01 \cdot x_{4}$

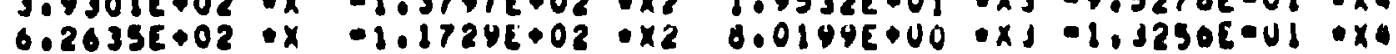
$2.00815+02 * x$ $-1.43325+02 * x$

$3.7315[0.02$ $-1.09616+03$ $7.04025+02$
$7.36535+02$ 0.09345002

$-1.0911 E+02 \cdot x$ $0 \times 2 \cdot 6.48395002+x 1$

$2.438 J E+00 \cdot \times 2$

$1.0900 E+02 \cdot x$ $2.7790 E+02$ : $x$ $-4.0162 E+02 * x$ $-3.4474 E+02$ :x $-1.21996+02$ : $x$ 1.7?1780 $1.10612+03$

$2.0398 E \bullet 01$ $3.4246 E \bullet 02$
$1.3425 E \bullet 03$ $2.13700 \bullet 03$ $1.7040 E \bullet 03$ $7.55625+02$ $1.27725 \cdot 03$ $1.99006+03$ $4.10076+02$

$-4.89236 .01 \cdot x$ $-3.5 a 77 E+02 \cdot x$ $-7.6711 E+02$ ix $-7.09976002 \cdot x$ $-3.3173602 \cdot x$ $0.3337 c+01 \cdot x$

$-1.00065+02 \cdot x$ $-3.87346+02 \cdot x$ $.4 .61095+01 . x$

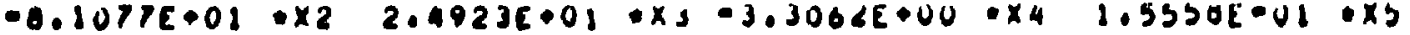

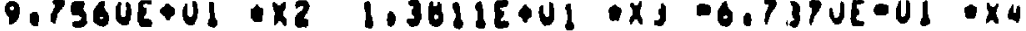

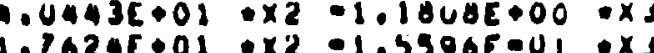

$2.72615+00 \times 2$

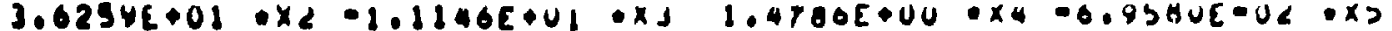
$1.2345 E+02+x 2-1.7830 E+01$ : $x J \quad 0.6974 E=01: x 4$

$1.4365 E+02 \cdot \times 2-9.8223 E+00 * x J 1.0235 E-01 \cdot x$

$1.1443 E+01 \cdot \times 2-2.0870 E+00$ - $x J$

$1.6956 \mathrm{E} \cdot 01 \cdot \times 2 \cdot 1.5007[-01 \cdot x \mathrm{~J}$

$1.0623 E+00 \cdot \times 2$

$3.1470 k \cdot 00 \cdot \times 2$

$1.80036002+x 2 \cdot 1.7525 E-01 \cdot x$

$1.0304 E 000$. $\times 2$ 


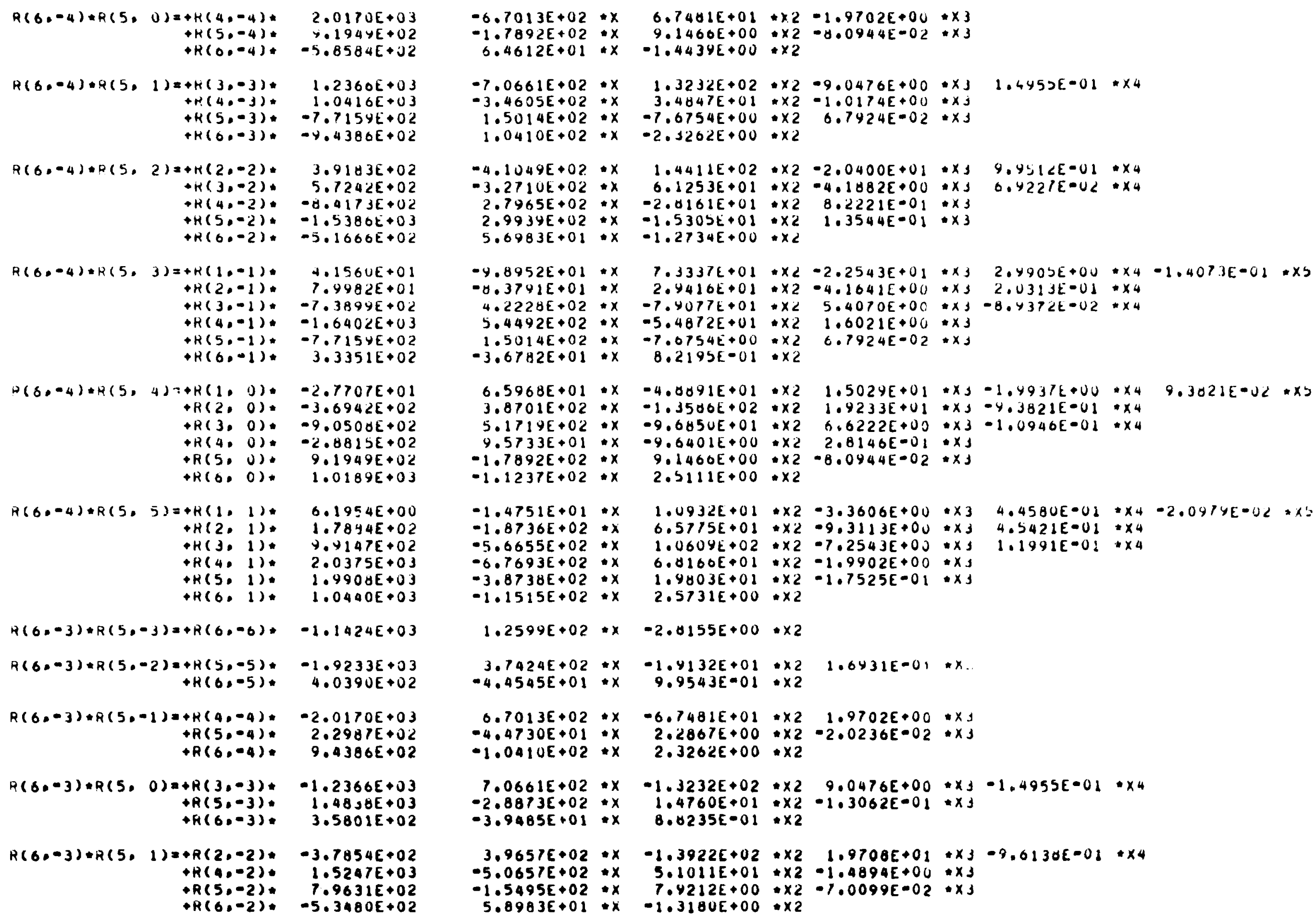

$R(6,-4) * R(5,0)=+H(4,-4): \quad 2.0170 E+03$ $+K(5,-4) * \quad-1.1944 E+02$
$+R(0,-4) *-5.8584 E+J 2$

$R(6,-4) \cdot R(5,1)=+R(3,-3) *$ $+R(40-3) *-1.0416 E+03$ $-7.715 Y E+02$

$R(6,-4) \cdot P(5,2)=+K(2,-2) * 3.9183 E+02$ $\rightarrow H(3,-2), \quad 5.7242 E+0$ - $R(4,-2)$ $-5.1600 \mathrm{E}+0$ 2

$R(6,-4) * R(5,3)=+R(1,-1) * \quad+156 \cup E+01$ $+R(2,-1) * \quad 7.9982 E+0$ $+K(30-1)=-7.3899 E+0$ $+R(4,-1)=-1.6402 E+U$ $+R(5,-1) *-7.715 Y E+02$ $\rightarrow R(6,-1) * 3.3351 E+02$

$D(8,-4)+R(5,4)=+R(1,0),-2.7707 E+01$ $+R(2,0)=-3.6942 E+02$ $+R(3,0) .9 .0500 E+02$ $+R(4,0)=-2.8825 E+02$ $+R(5,0)=9.1949 E+02$ $+R(0,0) * \quad 1.0189 E+03$

$R 66,-4) * R(5,5)=+R(1,1) *$ $+H(2,1):$ $+R(3,1):$ $+R\left(40^{\circ} 1\right):$ $+H(5 \circ)$

$6.1954 E+00$ $1.7894 E+02$
$9.4147 E+02$ $9.9147 E+02$
$2.0375 E+03$ $0.0375 E+03$
$1.9908 E+03$ $1.0440 E+03$

$R(6,-3) * R(5,-3)=+R(6,-6) *$ $-1.1424 E+03$

$R(6,-3) * R(5,-2)=+R(5,-5) *-1.9233 E+03$ $+H(6,-5) .4 .0390 E+O$

$R(6,-3) * R(5,-1)=+R(4,-4)$. $+R(5,-4)$. $+R(6,-4)$.

$-2.0170 E+03$ $2.2987 E+U 2$ $9.4386 E+02$

$R(6,-3) * R(5,0)=+R(3,-3)$ $+R(5,-3)$.
$+F(6,-3)$

$-1.2366 E+03$ $1.48,8 E+03$ $3.5801 E+02$

$R(6,-3)+R(5,1)=+R(2,-2)$ *

$-3.7854 E+02$ $+H(4,-2): \quad 1.5247 E+03$ $+R(5,-2) * 7.9631 E+02$ $\rightarrow R(6,-2):-5,3480 E+02$

$-6.7013 E+02 * x \quad 6.7481 E+01 * \times 2-1.9702 E+00 * x 3$ $-1.7892 E+02: x \quad 9.1460 E+00 \times x 2-8.0944 E-02 * x 3$ $0.4612 E+01: x$

$-7.0661 E+02 \cdot x$ $1.5014 E+02 \% x$ $1.5014 E+02: x$
$1.0410 E+02: x$

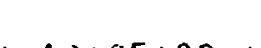
$-4.1049 E+0 ?: x$ $-3.2710 k+02: x$ $2.9939 E+02: x$ $5.6983 E+01: x$ $1.4439 E+00 \div \times 2$

1.32J2E+02 $\times 2=9.0476 E+00 * x J \quad 1.445 \supset E=01 * \times 4$ $3.4847 E+01 * x 2-1.0174 E+00 \div x$

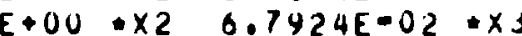
$-2.3262 E+00 * \times 2$

$1.4411 E+02 \times 2-2.0400 E+01 \times x J \quad 9.951 \angle E=01 * \times 4$ $6.1253 E+01 * \times 2=4.1882 E+00 * x J \quad 0.4227 E-U 2 * \times 4$ $2221 E=01: x 3$ $1.3544 E=01 \cdot \times 3$

$-9.8952 E+01: x$ $1.2734 E+00 \times 2$ $4.2228 E+02: x$ $5.4492 E+02 \cdot x$ $1.5014 E+02: x$ $1.5014 E+02: x$
$-3.67 \mathrm{BLE}+01: x$

$7.3337 E+01 * x 2-2.2543 E+01 * \times 3 \quad 2.4905 E+00 * \times 4-1.4073 E-02 * \times 3$ $2.9410 E+01 \times 22-4.2641 E+00 * x 3 \quad 2.0313 E=01 \times \times 4$

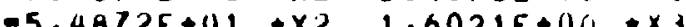
$-7.0754 E+00 \times x=0.7924 E-002 \times x$ $0.0754001 \times 2$

$6.5968 E+01 * x$

$-4.0091 E+01 * \times 2 \quad 1.5029 E+01 * x 3-1.4937 E+00 * \times 4 \quad 9.3021 E-02 \times X 3$ $3.8701 E+02 \cdot x$ $5.1719 E+02: x$ $1.7892 E+02: x$ $-1.78926+02$ ax

$-1.3506 E+02 * x 2 \quad 1.9233 E+U 1: x 3-4.3821 E-U 1: x 4$

$-9.685 U E+01 * \times 2 \quad 6.6222 E+00 * \times 3-1.0946 E-01 * \times 4$

$-9.6401 E+00 \div 2 \quad 2.8146 E-01: \times 1$

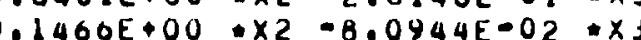

$2.5111 E+00 \times 2$

$-1.4751 \varepsilon+01 * x$ $-1.8736 E+02=x$ $-5.6655 E+02: x$

$1.0932 E+01 * \times 2-3.3606 E+00 * x 3 \quad 4.4580 E-01 * x 4-2.0974 E-02 \times x^{\prime}=$ $6.57 / 5 E+01 * \times 2-9.3113 E+00 * x 3 \quad 4.5421 E-01=x$ $1.0609 E+02 * x 2-7.2543 E+00 * x 3$

$6.8160 E+01$ : $x 2-1.9902 E+00$ ax

$-3.8738 E+02: x$

$-1.1515 E+02 * x \quad 2.5731 E+00 * \times 2$

$1.2599 E+02 * x-2.0155 E+00 * 2$

$3.7424 E+02 * x-1.4132 E+01 * x 2 \quad 1.6431 E-01 * x$ $-4.4545 E+01 * x \quad 9.9543 E=01 * \times 2$

$0.7013 E+02 * x-6.7481 E+01 * \times 2 \quad 1.9702 E+00 * X$

$-4.4730 E+01 * x \quad 2.2867 E+00 * \times 2-2.0236 E-02 * x$

$-1.0410 E+02 \div X \quad 2.3262 E+00 * x 2$

$7.0661 E+02 * x-1.3232 E+02 * x_{2} \quad 9.0476 E+00 * x_{3}-1.4955 E-01 * x_{4}$

$-2.8873 E+02: x \quad 1.4760 E+01: \times 2-1.3062 E-01 * x J$
$-3.9485 E+01: x \quad 8.0235 E-01: \times 2$

$3.9657 E+02 * x-1.3922 E+02 * \times 2 \quad 1.9700 E+01+x_{3}=9.0130 E-01 * x$

$-5.0657 E+02 * x \quad 5.1011 E+01 * \times 2-1.4894 E+00 * x$

$-1.5495 E+02 * x \quad 7.4212 E+00 * x 2-1.0099 E-02 * x J$

$5.8983 E+01 \cdot x-1.3180 E+00 \times 2$ 


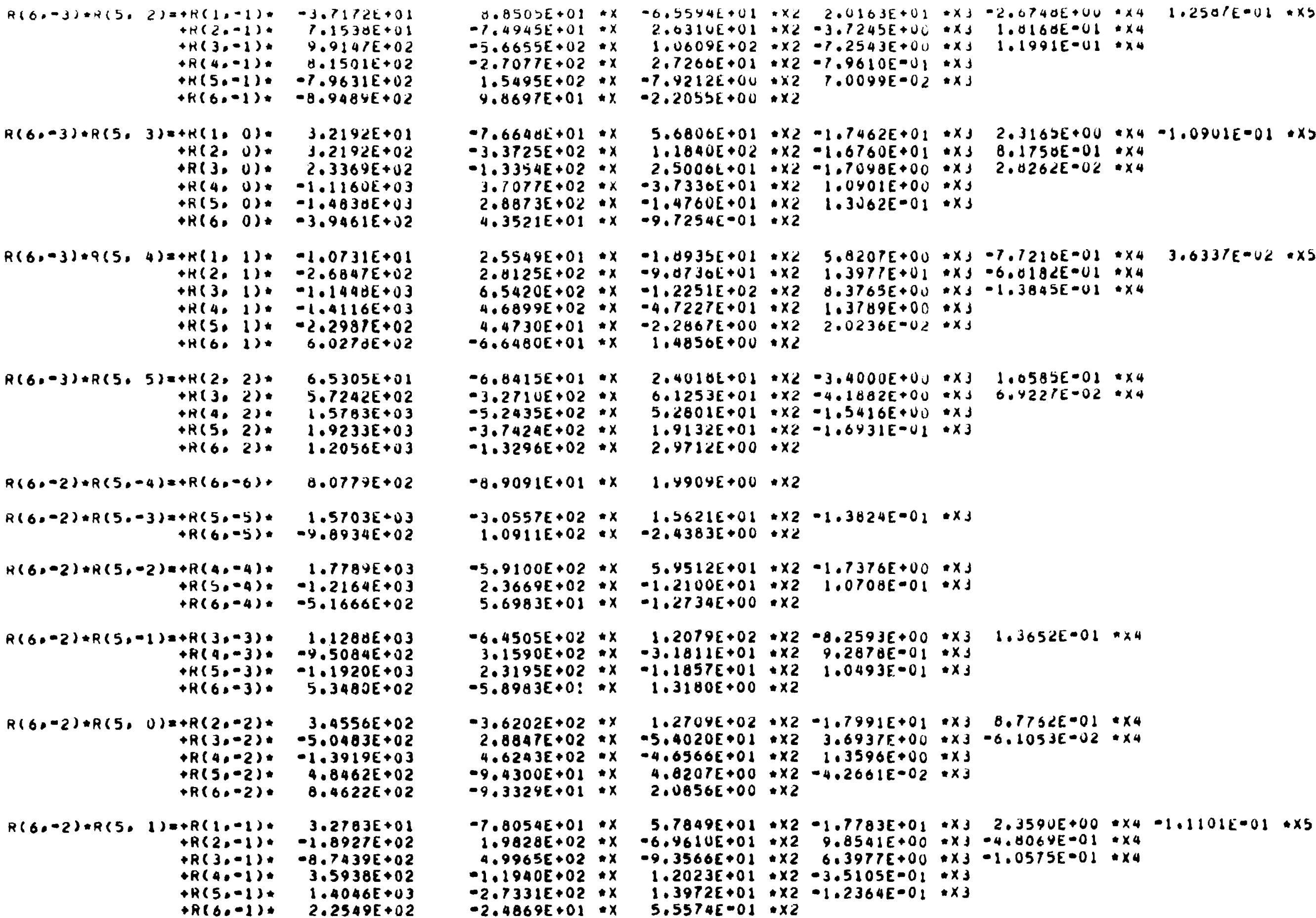

$0.850 \supset E+01 * x$ $-1.4945 E+01: x$ $-5.6655 E+02: x$ $-2.7077 E+02: x$ $1.5495 E+02: x$ $9.8697 E+01 * x$

$x \quad 5.6800 E+01 * x 2-1.7462 E+01 * x 1 \quad 2.3162 E+00 * x 4-1.0902 E=01 * x 3$ $-3.3725 E+02 * x \quad 1.1840 E+02 * x 2-1.6760 E+01 * x J \quad 8.1750 E-01: x^{2}$

$3.7077 E+02: x-3.7330 E+01: \times 2 \quad 1.0901 E+00: x j$

$2.8873 E+02 * x-1.4760 E+01 * \times 2 \quad 1.3 J 62 E-01 * x 3$

$4.3521 E+01 \times x-9.7254 E-01 \times 2$

$2.5549 E+01 * x-1.0935 E+01 * x 2 \quad 5.8207 E+00 * x 1-7.7210 E=01 * \times 4$ $2.8125 E+02 * x-9.0730 E+01 * x 2 \quad 1.3977 E+01 \times x J-6.0182 E=01 \times x_{4}$ $6.5420 E+02 \times x-1.2251 E+02 \times 2 \quad 0.3765 E+00 \times x_{3}=1.3845 E-01 * x_{4}$ $4.6899 E+02 * x-4.7227 E+01 * x 2 \quad 1.3789 E+00 * x J$ $4.4730 E+01 * x-2.2867 E+00 * x 2 \quad 2.0236 E-02 * x 3$

$-6.6480 E+01 * X \quad 1.4850 E+00 * X 2$

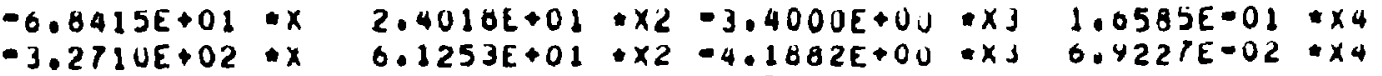
$-5.2435 E+02 * x$ $-3.7424 E+02: x$

$-1.3296 E+02 * x$

$-0.9091 E+01 * x$ $5.2801 E+01 \div \times 2-1.5416 E+U 0 \div \times J$ $1.9132 E+01 \div \times 2-1.6931 E=01 * x 3$ $2.9712 E+00 \times 2$

$x \quad 1.4904 E+00 \times 2$

$3.0557 E+02: x \quad 1.5621 E+01 * x 2-1.3624 E-01 * x J$
$1.0911 E+02: x-2.4383 E+00 * x 2$

$5.9512 E+01+\times 2-1.7376 E+00: \times J$ $2.3669 E+02 * x-1.2100 E+01 * x 2 \quad 1.0708 E-01 * x 3$ $5.6983 E+01 \div x-1.2734 E+00 \div 2$

$-6.4505 E+02 * x \quad 1.2079 E+02+x 2-8.2593 E+00 * x 3 \quad 1.3652 E=01 * x 4$ $3.1590 E+02: x-3.1811 E+01 * x 2 \quad 9.287 \theta E=01 * x J$ $2.3195 E+02 * x-1.1857 E+01 * x 2 \quad 1.0493 E-01 * x 3$ $-5.8983 E+0: \times x \quad 1.3180 E+00 \times \times 2$

$-3.6202 E+02 * x \quad 1.2709 E+02 * \times 2-1.7991 E+01 * \times 3 \quad 0.7752 E-01 * \times 4$ $2.8847 E+02 * x \quad-5.4020 E+01 * x 2 \quad 3.6937 E+00 * x 3-6.1053 E=02 * x$ $4.6243 E+02 * x-4.6566 E+01 * x 2 \quad 1.3596 E+00 * x 3$

$-9.4300 E+01: x \quad 4.0207 E+00 * x 2-4.2661 E-02 * x$

$9.3324 E+01$

$-7.8054 E+01 * x \quad 5.7849 E+01 * \times 2-1.7783 E+01 * x 3 \quad 2.3590 E+00 * x 4-1.1101 E-01 * \times 5$ $9.8541 E+00=x 3-4.0064 E-01 * x 4$ $6.3977 E+00 * 3=1.0575 E=01 * x$

105E-01: $x 3$

- $x \quad 1.3972 E+01=x 2-1.2364 E-01=x 3$

$5,5574 E-01: \times 2$

$3.6337 E-42 \times 5$

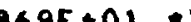




\begin{tabular}{|c|c|c|c|c|c|c|c|c|c|c|c|c|c|}
\hline$F, 6,-2) \backsim R(5$, & $\begin{aligned}2)= & +R(1) \\
& +R(2) \\
& +R(3) \\
& +R(4) \\
& +R(5) \\
& +R(6)\end{aligned}$ & $\begin{array}{l}0) \text { : } \\
\text { 0): } \\
\text { J): } \\
0 \text { ): } \\
0 \text { ): } \\
0 \text { ): }\end{array}$ & $\begin{array}{r}-3.5046 E+01 \\
-2.3364 E+02 \\
3.8162 E+02 \\
1.5187 E+03 \\
4.8462 E+02 \\
-6.4439 E+02\end{array}$ & $\begin{array}{r}8.3444 E+01 \\
2.4477 E+02 \\
-2.1807 E+02 \\
-5.0456 E+02 \\
-9.4300 E+01 \\
7.1070 E+01\end{array}$ & $\begin{array}{l}: x \\
: x \\
: x \\
: x \\
x \\
x \\
x\end{array}$ & $\begin{array}{r}-6.1843 E+01 \\
-8.5928 E+01 \\
4.0835 E+01 \\
5.0808 E+01 \\
4.8207 E+00 \\
-1.5882 E+00\end{array}$ & $\begin{array}{l}+\times 2 \\
\times 22 \\
x \times 2 \\
\times 22 \\
\times 22 \\
x 2 \\
\times 22\end{array}$ & $\begin{array}{r}1.9010 E+01 \\
1.2164 E+01 \\
-2.7922 E+00 \\
-1.4834 E+00 \\
-4.2661 E=02\end{array}$ & 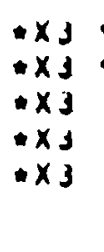 & $\begin{array}{r}-2.521 \forall E+0 U \\
-5.433 \Delta E=01 \\
4.6151 E=02\end{array}$ & $\begin{array}{l}: \times 4 \\
: \times 4 \\
x \times 4\end{array}$ & $1.1860 E=01$ & מ \\
\hline$R(6,-2): R(5)$ & $\begin{aligned}3)= & +R(1) \\
& +H(2) \\
& +R(3) \\
& +R(4) \\
& +R(5) \\
& +R(6 .\end{aligned}$ & $\begin{array}{l}\text { 1): } \\
\text { 1): } \\
\text { 1): } \\
\text { 1): } \\
\text { 1): } \\
\text { 1): }\end{array}$ & $\begin{array}{r}1.5175 E+01 \\
3.2126 E+02 \\
9.445 E+02 \\
1.6636 E+02 \\
-1.1920 E+03 \\
-8.5245 E+02\end{array}$ & $\begin{array}{r}-3.6132 E+01 \\
-3.3656 E+02 \\
-5.3969 E+02 \\
-5.5271 E+01 \\
2.3195 E+02 \\
9.4017 E+01\end{array}$ & $\begin{array}{l}x \\
: x \\
x \\
x \\
x \\
x \\
x\end{array}$ & $\begin{array}{r}2.6779 E+01 \\
1.1815 E+02 \\
1.0106 E+02 \\
5.5657 E+00 \\
-1.1857 E+01 \\
-2.1009 E+00\end{array}$ & $\begin{array}{l}\times 22 \\
\times 2 \\
\times 22 \\
\times 22 \\
\times 22 \\
\times 2 \\
\times 22\end{array}$ & $\begin{array}{l}-8.2317 E+00 \\
-1.6726 E+01 \\
-6.91103 E+00 \\
-1.6250 E-01 \\
1.0493 E=01\end{array}$ & 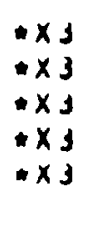 & $\begin{array}{l}1.0520 E+00 \\
8.158 Y E=U 1 \\
1.1422 E=01\end{array}$ & $\begin{array}{l}x \times 4 \\
=x 4 \\
x \times 4\end{array}$ & $-5.1300 E=02$ & S5: \\
\hline$R(6,-2) \otimes R(5)$ & $\begin{aligned}4)= & +R(2) \\
& +R(3) \\
& +R(4) \\
& +R(5)\end{aligned}$ & $\begin{array}{l}2): \\
2): \\
2): \\
2)=\end{array}$ & $\begin{array}{l}-1.2391 E+02 \\
-9.0508 E+02 \\
-1.8300 E+03 \\
-1.2164 E+03\end{array}$ & $\begin{array}{l}1.2981 E+02 \\
5.1719 E+02 \\
6.079 \forall E+02 \\
2.3669 E+02\end{array}$ & $\begin{array}{l}x \\
: x \\
: x \\
x\end{array}$ & $\begin{array}{l}-4.5570 E+01 \\
-9.6850 E+01 \\
-6.1223 E+01 \\
-1.2100 E+01\end{array}$ & $\begin{array}{l}: \times 2 \\
: \times 2 \\
x 2 \\
\times 2\end{array}$ & $\begin{array}{l}6.4510 E+00 \\
6.6222 E+00 \\
1.7875 E+00 \\
1.0708 E-01\end{array}$ & 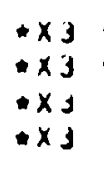 & $\begin{array}{l}-3.1469 E-01 \\
-1.0940 E-01\end{array}$ & $\begin{array}{l}\times 4 \\
\times 4 \\
\times 4\end{array}$ & & \\
\hline$R(s+2) \cdot R(5)$ & $\begin{aligned}5)= & +R(3) \\
& +R(4) \\
& +R(5) \\
& +R(6 .\end{aligned}$ & $\begin{array}{l}3): \\
3): \\
32: \\
3):\end{array}$ & $\begin{array}{l}2.3369 E \bullet 02 \\
9.8421 E \diamond 02 \\
1.5703 E \bullet 03 \\
1.2056 E \bullet 03\end{array}$ & $\begin{array}{l}-1.3354 E+02 \\
-1.2699 E+02 \\
-3.0557 E+02 \\
-1.3296 E+02\end{array}$ & $\begin{array}{l}: x \\
: x \\
: x \\
x\end{array}$ & $\begin{array}{l}2.5006 E+01 \\
3.2927 E+01 \\
1.5621 E+01 \\
2.9712 E+00\end{array}$ & $\begin{array}{l}\times 2 \\
\times 2 \\
\times 2 \\
\times 2 \\
\times 2\end{array}$ & $\begin{array}{l}-1.709 \forall E+00 \\
-9.6138 E=01 \\
-1.3824 E=01\end{array}$ & 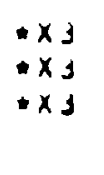 & $2.0262 E=02$ & $\cdot x_{4}$ & & \\
\hline$R(6,-1) \cdot R(5$. & $-5)=+R(6,-$ & -6). & $-4.0390 E+02$ & $4.4545 E+01$ & $x$ & $-9.9543 E-01$ & $\times 2$ & & & & & & \\
\hline$P(6,-1) \notin R(5$, & $\begin{aligned}-3)= & +R(4,-= \\
& +R(50= \\
& +R(60=\end{aligned}$ & $\begin{array}{l}-4): \\
\text { (4): } \\
\text {-4): }\end{array}$ & $\begin{array}{r}-1.3779 E+03 \\
1.7274 E+03 \\
-3.3351 E+02\end{array}$ & $\begin{array}{r}4.5778 E+02 \\
-3.3612 E+02 \\
3.6782 E+01\end{array}$ & $\begin{array}{l}x \\
: x \\
: x\end{array}$ & $\begin{array}{r}-4.6098 E+01 \\
1.7183 E+01 \\
-8.2195 E-01\end{array}$ & $\begin{array}{l}: \times 2 \\
: \times 2 \\
: \times 2\end{array}$ & $\begin{array}{r}1.345 y E+00 \\
-1.5206 E=01\end{array}$ & 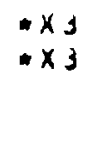 & & & & \\
\hline$R(6,-1)=R(5$, & $\begin{aligned}-2)= & +R(3,0- \\
& +R(4,0 \\
& +R(5,0 \\
& +R(6,0-\end{aligned}$ & $\begin{array}{l}\text {-3): } \\
\text {-3): } \\
\text {-3): }\end{array}$ & $\begin{array}{r}-9.4445 E+02 \\
1.59111 E+03 \\
1.0133 E+02 \\
-8.9489 E+02\end{array}$ & $\begin{array}{r}5.3964 E+02 \\
-5.2860 E+02 \\
-3.5284 E+01 \\
9.8697 E+01\end{array}$ & $\begin{array}{l}: x \\
: x \\
: x \\
x\end{array}$ & $\begin{array}{r}-1.0106 E+02 \\
5.3230 E+01 \\
1.0037 E+00 \\
-2.2053 E+00\end{array}$ & $\begin{array}{l}* \times 2 \\
\times 2 \\
\times 2 \\
\times 2 \\
\times 2\end{array}$ & $\begin{array}{r}6.9103 E+00 \\
-1.5541 E+00 \\
-1.5962 E-02\end{array}$ & $\begin{array}{l}\times 3 \\
\times \times 3 \\
1 \times 3 \\
1 \times 3\end{array}$ & $-1+1422 E=01$ & $n \times 16$ & & \\
\hline$R(6,-1) \otimes R(5$ & $\begin{aligned}-1)= & +R(2,0 \\
& +R(3,-= \\
& +R(4,0 \\
& +R(5,-0 \\
& +R(6.0\end{aligned}$ & $\begin{array}{l}-2): \\
-2): \\
-2) \\
2) \\
2)\end{array}$ & $\begin{array}{r}-2.9926 E+02 \\
0.7439 E+02 \\
6.4288 E+02 \\
-1.3430 E+03 \\
-2.2549 E+02\end{array}$ & $\begin{array}{r}3.1352 E+02 \\
-4.9965 E+02 \\
-2.1359 E+02 \\
2.6133 E+02 \\
2.4869 E+01\end{array}$ & $\begin{array}{l}: x \\
: x \\
: x \\
: x \\
: x\end{array}$ & $\begin{array}{r}-1.1000 E+02 \\
9.3566 E+01 \\
2.1500 E+01 \\
-1.336 U E+01 \\
-5.5574 E-01\end{array}$ & $\begin{array}{l}* \times 2 \\
\times \times 2 \\
=\times 2 \\
=\times 2 \\
\times 2\end{array}$ & $\begin{array}{r}1.5581 E+01 \\
-6.3977 E+0 U \\
-6.2797 E=01 \\
1.1023 E=01\end{array}$ & $\begin{array}{l}\times 3 \\
03 \\
\times 1 \\
\times 3 \\
\times 3\end{array}$ & $\begin{array}{r}-7.0004 E-01 \\
1.057 \mathrm{JE}-01\end{array}$ & $: x_{4}$ & & \\
\hline$R(6,-1) * R(5$. & $\begin{aligned}0)= & +R(1,0 \\
& +R(2,0 \\
& +R(3,0 \\
& +R(4,0 \\
& +R(5,0 \\
& +R(6,0\end{aligned}$ & $\begin{array}{l}1 \\
1 \\
1 \\
1): \\
1): \\
1) \\
1): \\
1):\end{array}$ & $\begin{array}{r}-2.0391 E+01 \\
2.7319 E+02 \\
5.0463 E+02 \\
-1.2449 E+03 \\
-0.1092 E+02 \\
6.5094 E+02\end{array}$ & $\begin{array}{r}6.7597 E+01 \\
-2.8620 E+02 \\
-2.0847 E+02 \\
4.1361 E+02 \\
1.5779 E+02 \\
-7.1791 E+01\end{array}$ & $\begin{array}{l}: x \\
: x \\
: x \\
: x \\
: x \\
: x\end{array}$ & $\begin{array}{r}-5.009 y E+01 \\
1.0047 E+02 \\
5.4020 E+01 \\
-4.1650 E+01 \\
-0.0666 E+00 \\
1.6043 E+00\end{array}$ & $\begin{array}{l}+\times 2 \\
\times 2 \\
\times 2 \\
\times 2 \\
\times 2 \\
+\times 2 \\
\times 2\end{array}$ & $\begin{array}{r}1.5400 E+01 \\
-1.4223 E+01 \\
-3.6937 E+00 \\
1.2161 E+00 \\
7.1386 E=02\end{array}$ & 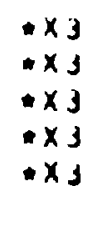 & $\begin{array}{r}-2.042 Y E+O U \\
6.9382 E=01 \\
6.105 \mathrm{JE}=02\end{array}$ & $: x 4$ & $9.6130 E=02$ &.$\times 5$ \\
\hline
\end{tabular}




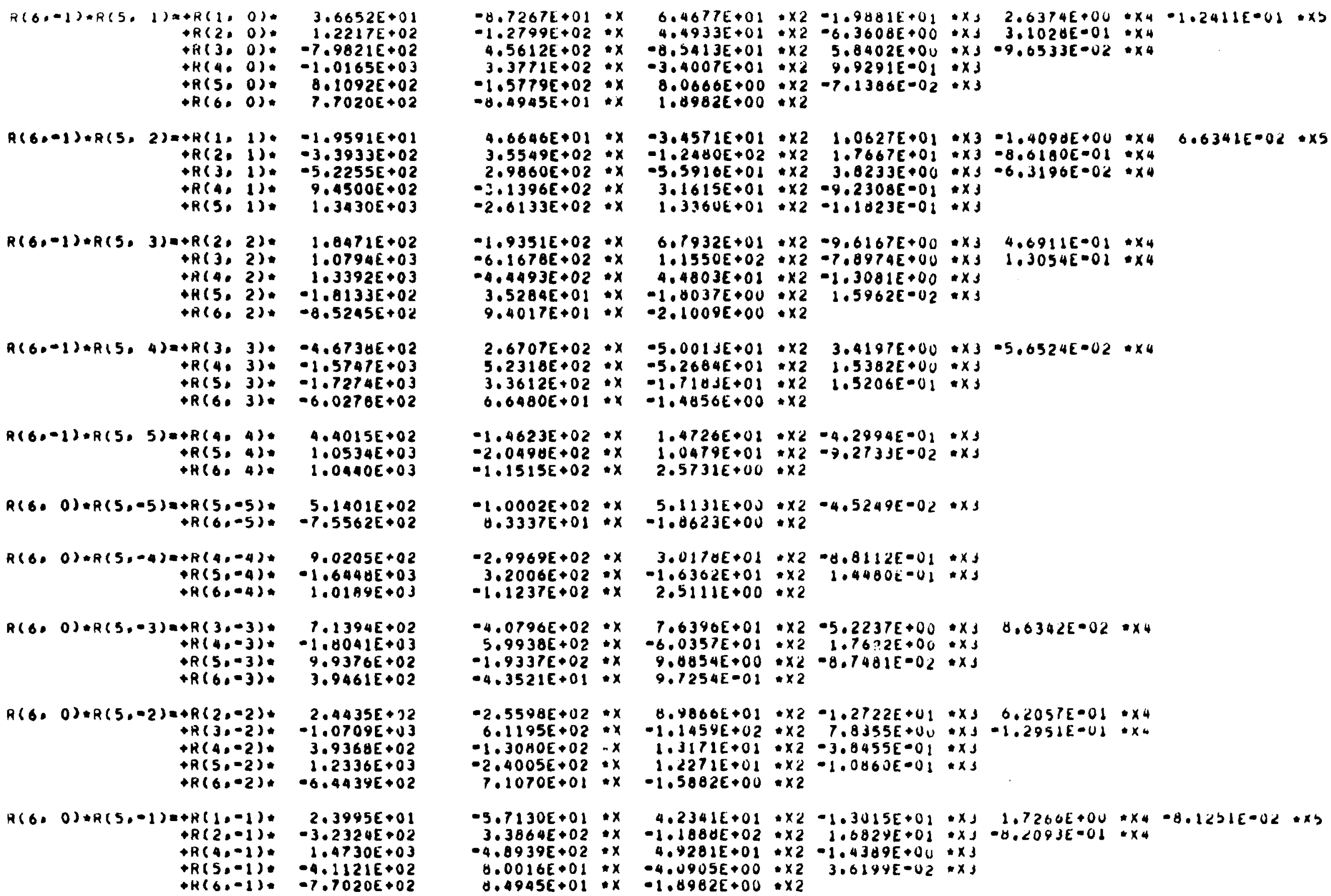

$-8.7267 E+01 * x \quad 6.4677 E+01 \times x^{2}-1.9881 E+01 * x 1 \quad 2.6374 E+00+x_{4}-1.2411 E+01 \times x^{2}$ $x$

$3.37715+02, x$

$-1.57795002, x$

$-1.57+98+02: x$

$4.6646 E+01 * x-3.4571 E+01 \times 2 \quad 1.0627 E+01 \times 3-1.4090 E+00=\times 4 \quad 6.6342 E-02 \times 5$ $3.5549 E+02 \times x-1.2440 E+02 \times x 2 \quad 1.7667 E+01 \times x 3=8.0100 E-01=x 4$

$2.9860 E+02 * x-5.5916 E+01: \times 2 \quad 3.0233 E+00 \times x=-6.3196 E=02 \times x 4$

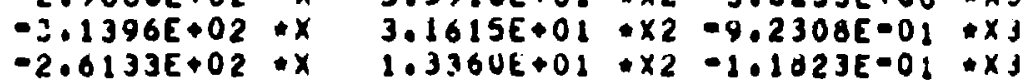

$-1.9351 E+02 * x \quad 6.7932 E+01 * \times 2-9.6167 E+00 * x_{3} \quad 4.6911 E-01 * x_{4}$

$-4.4493 E+02 * x \quad 4.1550 \varepsilon+02 * x 2=7.0274 \varepsilon+x=x 3$

$3.5284 E+01 * x-1,0037 E+00 * x 21.5962 E-02 * x 3$

$9.4017 E+01 * x-2.1009 E+00 * x 2$

$2.6707 E+02 * x \quad-5.001 J E+01 * \times 2 \quad 3.4197 E+00 * x 3-5.6524 E-02 * \times 4$ $5.2318 E+02 * x \quad-5.2684 E+01 * x 2 \quad 1.5382 E+00 * x J$ $3.3612 E+02 * x-1.710 J E+01 * x^{2} \quad 1.5206 E=01 * x 3$

$6.6480 E+01 * x-1.4856 E+00 * \times 2$

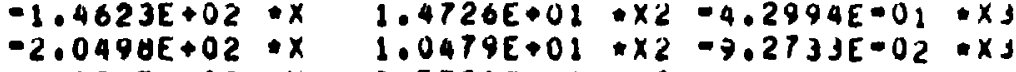
$-1.1515 E+02 * x \quad 2.5731 E+00 * x 2$

$-1.0002 E+02 * x \quad 5.3131 E+00+\times 2-4.5249 E=02 * x 3$ $0.3337 E+01 \times x-1.0623 E+00 \div \times 2$

$-2.9969 E+02 * x \quad 3.0170 E+01 \times 2-8.8112 E-01 * x J$

$\begin{array}{rr}3.2006 E+02: x & -1.6362 E+01: \times 2 \\ -1.1237 E+02 * x \quad 2.5111 E+00: \times 2 & \end{array}$

$-4.0796 E+02 * x \quad 7.6396 E+01 * \times 2-5.2237 E+00 * x_{J} \quad 8.0342 E-02=x$

$5.9938 E+02 * x-6.0357 E+01 * x 2-1.76 ? 2 E+00 * x J$

$-1.9337 E+02 * x \quad 9.0854 E+00 * x 2-8.7481 E-02 * x J$

$4.3521 E+01$ : $x$

$-2.5598 E+02 \times x \quad 0.9860 E+01 * x 2-1.2722 E+U 1 * x J \quad 6.2057 E-01 * x 4$

$6.1195 E+02 * x-1.1459 E+02 * x 27.8355 E+00 * x J-1.2951 E-01 * x$

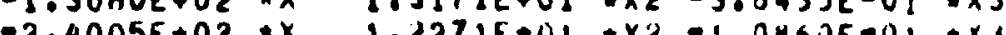

$7.1070 E+01: x-1.50025+00$ a $x 2=10$

$-5.7130 E+01 * x \quad 4.2341 E+01 * x 2-1.3015 E+01 * x 1 \quad 1.7260 E+00 * x 4-8.12 b 1 E-02 \times x b$

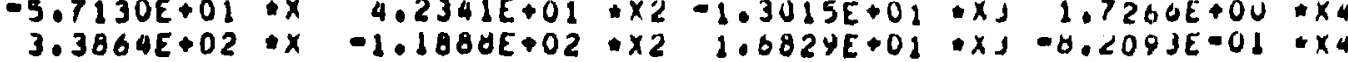

$-4.8939 E+02 * x \quad 4.9281 E+01 * x 2-1.43 \theta 9 E+00 * x 3$

$0.0016 E+01 * x-4.0905 E+00 \div 2 \quad 3.6199 E=02 \times x J$

$0.4945 E+01: x-1.0982 E+00: x 2$ 
$R(6,0)+R(5,0)=+K(1,0)=-3.7172 E+01$ $+R(3,0): \quad 9.4445 E+02$
$+R(5,0)=-1.3701 E+03$

$R(6,0) \because R(5,1)=+R(1,1)=2.3995 E+01$ $K(2.1)=1.2324 E+02$ $R(4.1)=-1.4730 E+03$ $+R(3,1):-4.1121 E+02$
$+R(6,1): 1.7020 E+02$

$R(6,0) \cdot R(5,2)=+H(2,2),-2.4433 E+02$ $+H(3,2)=-1.070 Y E+03$ $\rightarrow H(4.2)=3.9368 E+02$ $+K(5,2): \quad 1.2330 E+03$
$+K(6,2): \quad 6.4439 E+02$

$R(6,0) * R(5,3)=+R(3,3)=$ $\rightarrow R(4,3)$ - $R(5,3):$

$7.1394 E+02$ $1.8041 E+03$

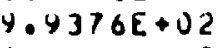
- $K(6,3)=-3.9461 E+U 2$

$R(6,0)+R(5,4)=+K(4,4)=-4.0205 E+02$ - $K(5,4)=-1.6448 E+U 3$ H(6.4). -1.0189E+03

$R(6,0) * R(5,5)=R(5,5) * 5.1401 E+02$ - $R(6,5) . \quad 7.5562 E+02$

$R(6,1), 4(5,-5)=+K(4,-4),-4.4015 E+02$ $+K(5,-4): \quad 1.0534 E+03$
$+K(6,-4):-1.0440 E+03$

$F(6,1)+K(5,-4)=+K(3,-3),-4.673 d E+02$ K(a,-3) $\quad 1.5747 E+03$ $+K(5,-3):-1.7274 E+03$
$+R(6,-3)=6.0270 E+02$

$1.8471 E+02$ $-1.8471 E+02$
$1.0794 E+03$
$-1.0392 E+03$ $-1.3392 E+03$ $\begin{array}{rr}+R(4,-2):-1.3392 E+03 \\ +R(5,-2):-1.8133 E+02 \\ +R(6,-2) & \quad 0.5245 E+02\end{array}$ $+R(6,-2)$

$-1.9591 E+01$

$R(6,1) * R(5,-2)=+R(1,-1) *$ $+R(2,-1)$. $-5.3255 E+02$ $R(4,-1) \quad-9.4500 E+02$ $\rightarrow R(5,-1)$. $1.343 \cup E+03$

$R(6,1) * R(5,-1)=+R(1,0)$ $+R(2,0) *-1.2211 E+02$ R(3.0) -7.9821E+02 PR(4. 0) 1.0165E+03 $+R(5,0), 8.1092 E+02$
$0.0505 E+02: x$ $-5.3969 E+02 * X$ $2.6672 E+02 * x$

$-5.7130 E+01 \cdot x$ $3.3864 E+02 * x$ $4.0939 E+02$ ix $8.0016 E+01: x$
$-8.4945 E+01 * x$ .8 .49458 $2.5598 E+02 * x$ $0.1195 E+02 * x$ $1.30 R U E+02: x$
$-2.4005 E+02: x$ $-2,4005 E+02: x$
$-7.1010 E+01: x$

$-7.1070 E+01: x$

$-4.0796 E+02 \cdot x$ $-3.9938 E+02: x$ $1.9337 E+02 * x$ $4.3521 E+01 \cdot x$

$2.9969 E+02 \cdot x$ $2.99695+02 * x$
$3.2006 E+02 * x$
$1.1237 E+02 * x$ $1.1237 E+02 * x$

$-1.0002 E+02 * x$ $-8.3337 E+01 * x$ $1.4623 \varepsilon+02 * x$ $-2.0498 E+02 \div x$ $1,1515 E+02 \cdot x$

$2.6707 E+02 * x$ $5.2318 E+02$ : $x$

$-3.3612 E+02: x$

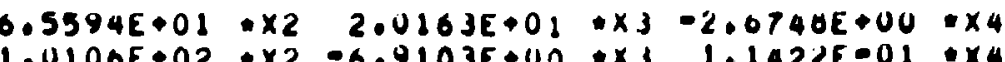

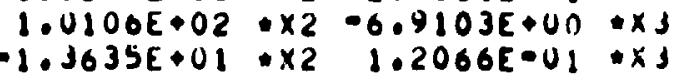

$4.2341 E+01 * x 2-1.3015 E+01 * x J \quad 1.7260 E+00+x 4-0.1251 E-02 * x b$ $1.1880 E+02 \times \times 2-1.6829 E+01 \times x y \quad 0.2093 E-01 \cdot \times 4$ $4.9281 E+01 \times 2 \quad 1.4389 E+00 \div x J$

$-4.0905 E+00: \times 2$
$1.8982 E+00: \times 2$

$-8.9860 E+01 * x<\quad 1.2722 E+01 * x 3-0.2057 E=01 \cdot \times 4$ $1.1454 E+02 * x_{2} \quad 7.8355 E+00 * x_{3}-1.2951 E-01 * x_{4}$ $1.3172 E+01 * x 2 \quad 3.0455 E-01 * x s$

$1.2271 E+01 * x 2-1.0060 E-01 * x 3$

$1,5802 E+00 \times x$

$7.63906+01 * \times 2-5.2237 E+00 * x 3 \quad 0.03426=02 * x^{4}$ $6.0357 E+01 * x 2-1.7622 E+00 * x 1$ $9.0854 E+00 \times 2=8.7481 E=02 \times x y$ $-9.7254 E-01 \times 2$

$3.0178 E+01 \times 2 \quad 0.0112 E=01 * \times 1$ $1.0362 E+01: \times 2 \quad 1.4480 E=01 \times 3$

$5.1131 E+00 * \times 2-4.5249 E-02 * \times J$ $1.8623 E+00: \times 2$

$-1.4726 E+01 \times 2 \quad 4.2944 E=01 * \times 3$

1.047 YE+0: $x<=9.2733 E=02: x J$

$1.0479 E+01: \times 2$
$-2.5731 E+00: \times 2$

$-5.0013 E+01 * x 2 \quad 3.4197 E+00 \times x,-5.0524 E-02 \times x 4$

$5.2684 E+01 \times 2-1.5302 E+00: x 3$

$1.7183 E+01 \times 2 \quad 1.5200 E=01 \times x 3$

$1.9351 E+02 * x \quad-6.7932 E+01 * x 2 \quad 9.6167 E+00 * x_{1}-4.0911 E=01 * x_{4}$ 4.94935002 a

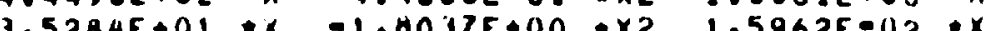

$0.401750010 x \quad 2.100950000 \times 2$

$4.6646 E+01 * x-3.4571 E+01 * \times 2 \quad 1.0627 E+01 * x 3-1.4090 E+00 \cdot \times 4 \quad 0.6341 E \cdot 02 . x$ $2.1009 E+00 \times 2$ $-3.5549 E+02 * x \quad 1.248 U E+02 * x_{2}-1.7667 E+01 * x_{3} 0.0100 E=01 * x_{4}$ $2.9860 E+02+x-5.59165+01 \div \times 2 \quad 3.8233 E+00 \div x 3-6.3196 E-0: 2 \times 4$

$3.1396 E+02 * x-3.1615 E+01 * x 29.2308 E-01 * x$

$-2.6133 E+02: x \quad 1.3360 E+01: x 2-1.1023 E-01: x J$

$0.7267 E+01 \cdot x$ $1.2799 E+02: x$ $-3.3771 E+02 * x$ $-1.5779 E+02$ : $x$ $0.4945 E+01: x$

$6.4677 E+01+x_{2}-1.9881 E+01 * x_{3} \quad 2.03741 .00+x_{4}-1.2411 E+01 * x_{3}$

$0.4933 E+01: x 2 \quad 6.3600 E+00: x 3-3.1020 E=01: x_{4}$

$-8.5413 E+01: \times 2 \quad 3.8402 E+00: x J=9.653 J E=02 * x^{4}$

$3.4007 E+01 \times 2-9.9291 E=01 * x 3$

$0.06005+00 \times 2=7,13965=02 \times x J$

$1.89825+00: \times 2$ 


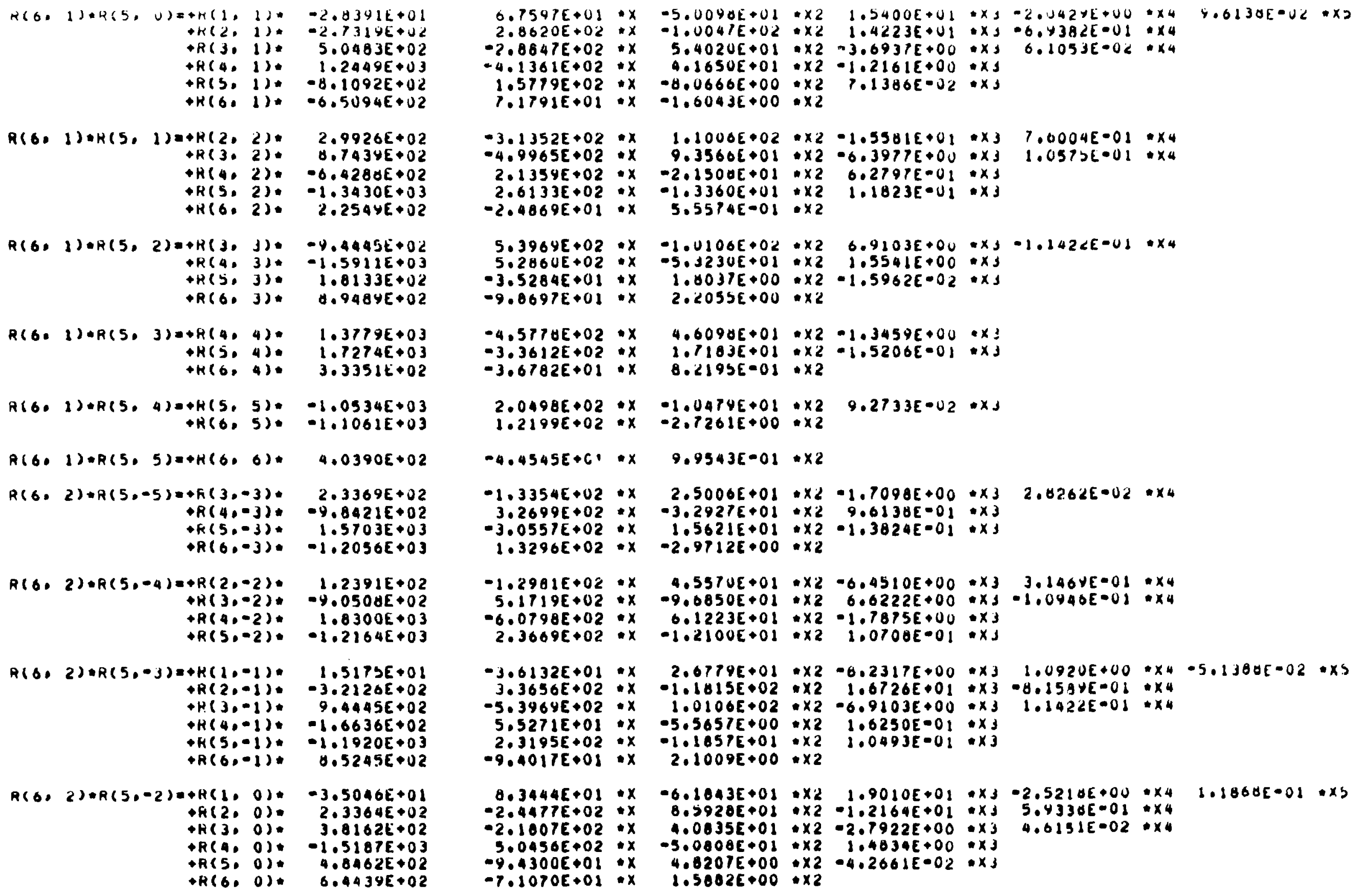

$-4.5770 E+02 * x \quad 4.6090 E+01 \times 2-1.3459 E+00 \times x$ $-3.3612 E+02 * x \quad 1.7183 E+01 \times \times 2=1.5200 E=01 * x$ $-3.6782 E+01: x \quad 8.2195 E=01: \times 2$

$2.0498 E+02 * x-1.0474 E+01 * \times 2 \quad 9.2733 E-02 * x J$ $1.2199 E+02: x-2.7261 E+00: \times 2$

$-4.4545 E+C, \times 9.9543 E-01 * \times 2$

$-1.3354 E+02 \times x \quad 2.5006 E+01 \times 22-1.7098 E+00 * x 3 \quad 2.0262 E=02 \times \times 4$

$0.0138 \varepsilon=01 \cdot x 3$

$3.2699 E+02 * x-3.2927 E+01 * x 2-9.6138 E-01 * x$

$1.052965+02 \times x-2.97125000 \times x=$

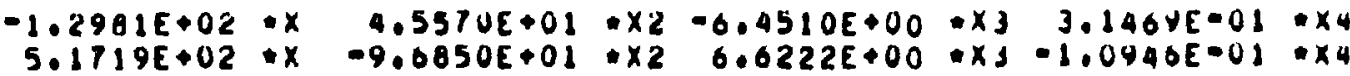
$-6.0798 E+02 * x \quad 6.1223 E+01 * x 2-1.7875 E+00 * x^{3}$

$-3.6132 E+01 * x \quad 2.0779 E+01 * x 2-0.2317 E+00 * x 3 \quad 1.0920 E+00 * x 4-5.1380 E-02 * x 5$ $3.3656 E+02 * x-1.1815 E+02 * x 2 \quad 1.6726 E+01 * x 3-8.859+E=01 * x 4$ $-5.3969 E+02 * x \quad 1.0106 E+02 * \times 2-6.9103 E+00 * x 31.1422 E-01 * x_{4}$ $5.5271 E+01 * x-5.5657 E+00 * x 2 \quad 1.6250 E=01 * x$ $2.3195 E+02 * x-1.1857 E+01 * x 2 \quad 1.0493 E=01 * x 3$ $-9.4017 E+01 \times 2.1009 E+00 \times 2$

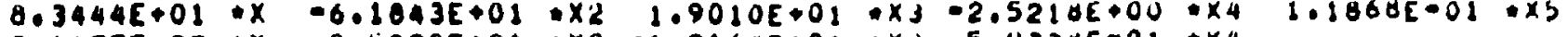
$-2.4477 E+02$ * $x \quad 8.5928 E+01 \times x 2-1.2164 E+01 * x 3 \quad 5.9330 E-01 \times x$ $-2.1007 E+02$ * $x \quad 1.0835 E+01 * x 2-2.7922 E+00$ * $\times 3 \quad 4.0151 E=02+x$ $0456 E+02: x$ $9.4300 E+01 * x \quad 4.8207 E+00 \div x=-4.2661 E-02: x$ $-7.1070 E+01 \times x \quad 1.5002 E+00: x 2$ 
$R(6,2)+R(5,-1)=+R(1,1)+3.2703 E+01$ $+R(2,1)=1.8927 E+02$ $+R(3 \circ) 1)-8.7439 E+02$ R(A. 1)a - J.5930E402 $+R(5,1): \quad-1.4046 E+03$
$+R(6,1):-2.2549 E+02$

$R(6,2)+R(5,0)=+R(2,2)=-3.4550 E+02$ $4 R(3,2)=-5.0483 E+02$ A $+R(5,2): \quad 4.8462 E+02$
$+R(6,2), \quad-8.4622 E+02$

$R(6,2)+R(5,1)=0 R(3,3)$ $\rightarrow R(403)=$ $1.120 A E+03$ $R(6,3)=-5.3480 E+02$

$R(6,2) * R(5,2)=+R(4,4)$. $R(5,4),-1.2164 E+03$

$5.1666 E+02$

$R(6,2)+R(5,3)=+R(5,5)=$ $+R(6,5)$

$R(6,2) \cdot R(5,4)=+R(6,6)$.

$R(6,3) \oplus R(5,-3)=+R(2,-2)$ $R(3,-2)$ P( $50-2)$ $R(0,-2)$

$1.5703 E+03$ $9.8934 E+02$

$-8.0779 E+02$

$-6.5305 E+01$ $5.7242 E+02$ $-1.573 \mathrm{E}+03$ $-1.9233 E+03$

$R(6,3) \oplus R(5,-4)=+R(1,-1)$. $+R(2,-1)$ ( $40-1)$ $4(50-1)$ $H(5,-1)$

$-1.0731 E+01$ $2.6847 E+02$ $-1.1448 E+03$ 1.4 $-2.2987 E+02$

$+R(60,1)$

$R(6,3) \bullet R(5,-3)=+R(1,0)$ $+R(2,0),-3.2192 E+0$ $\rightarrow R(3,0):$ $\rightarrow R(4.0)$ $\rightarrow R(6,0)$.

$2.3369 E+02$ $1.1160 E+03$ $3.9461 E+02$

$R(6,3) \cup R(5,-2)=+R(1,1)$. $+R(2,1)=-7.1538 E+0$ $+R(3.1) \quad 9.9147 E+02$ R $+R(6,1)=8.9409 E+02$
$-7.0054 E+01: x$ $-1.9828 E+02 * x$ $4.9965 E+02: x$
$1.1940 E+02: x$ $-2.7331 E+02: x$ $2.4069 E+01: x$

$3.6202 E+02 * x$ $3.6202 E+02: x$
$2.8847 E+02 * x$ $-4.6243 E+02: x$ $-9.9300 E+01: x$ $9.3329 E+01: x$

$-6.4505 E+02 \cdots x$ $-3.1590 E+02 \cdot x$ $2.3195 f+02: x$ $5.0903 E+01 \cdot x$

$5.9100 E+02 * x$ $5.9100 E+02: x$
$2.3669 E+02: X$ $2.3669 E+02: x$
$-5.6903 E+01: x$ $-3.0557 E+02: x$
$-1.0911 E+02: x$ $0.9091 E+01 \cdot x$ $6.0415 E+01: x$ $5.2435 E+02: x$ $-3.7424 E+02: x$ $1.3296 \varepsilon+02: x$

$2.5549 E+01 \cdot x$ $-2.0125 E+02: x$ 0.020 $4.47305+01 . x$ $4.973050010 x$

$1.06485+01+x$

$7.6648 E+01 * x$ $3.3725 E+02$ : $x$ $-1.33546+02: x$ $-3.7077 c+05 * x$ $-4.3521 E+01=x$

$0.0505 E+01 * x$ $7.4945 E+01: x$ $3.6655 E+02: x$ $1.5495 E+02: x$ $-9.8607 E+01: x$
$5.7849 E+01 * \times 2-1.7703 E+01 * \times 3 \quad 2,35906+00 \times x 4 \cdot 101101 E \cdot 01 \times \times 5$ $6.9610 E+01: \times 2-9.8541 E+00 * x 3 \quad 4.8069 E-01: x_{4}$ $-9.3566 E+01 \times 2 \quad 6.3977 E+00 \times x=1.0575 E=01 \times x$

$1.2023 E+01 \times 2 \quad 3.5105 E-01+x 3$

$1.39725 \times 01$ : $x$. $-1.2364 E-01 * x 3$

$-1.2709 E+02 * \times 2 \quad 1.7991 E+01 * x_{3}-0.7762 E-01 \times 44$ $5.4020 E+01: x^{2} \quad 3.6937 E+00$ : $x y-6.1053 E-02 * x_{4}$ $4.6566 E+01: \times 2-1.3596 E+00 \quad x j$

$4.0207 E+00 * x 2-4.2662 E=02 * x^{3}$

$-2.0856 E+00: \times 2$

$1.2079 E+02 * \times 2-0.2593 E+00 * \times 3 \quad 1.3652 E=01 * \times 4$ $3.1811 E+01 * \times 2-9.2878 E=01 * x 3$

$1.1857 E+01: \times 2 \quad 1.0493 E \cdot 01 \times x$

$-5.9512 E+01 * \times 2 \quad 1.7376 E+00 * \times 3$

$1.2100 E+01 \div \times 2 \quad 1.07006-01: \times 3$ $1.2734 E+00: \times 2$

$1.5621 E+01 \cdot \times 2-1.3824 E-01 * x 3$

$2.43836 \cdot 00 \cdot \times 2$

$-1.990 Y E+00 * 2$

$-2.4016 E+01 * 2 \quad 3.4000 E+0 u \cdot x 3-1.0585 E-01 \cdot \times 4$ $6.1253 E+01 * x^{2}-4.1082 E+04 * x 3 \quad 0.4227 E=02 * x_{4}$ 1.41325001 : $x^{2}-1.09315=01$ a

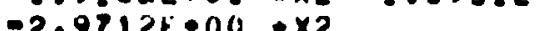

$-1.0935 E+01 * \times 2 \quad 5.8207 E+00 * x_{3}-7.7210 E-01 * \times 4 \quad 3.6317 E-02 * \times S$ $9.8736 E+01: x 2-1.3977 E+01 * x y \quad 6.0182 E-01 \cdot x 4$

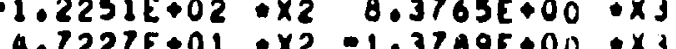

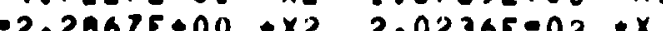

$1.0856 \mathrm{E}+00 \times 1 \times 2$

$5.0006 E+01 \times 22-1.7462 E+01 \times x_{1} \quad 2.1165 E+00 \times x 4-1.0901 E-01 \times x 3$ $1.1040 E+02: x 2 \quad 1.6760 E+01: x 3-8.1750 E-01: x_{4}$

$2.5006 E+01 \div \times 2-1.7090 E+00 \div x 32.0262 E-02 * x$

$3.7330 E+01 \times 2-1.0901 E+00 * x$

$1.4760 E+01: \times 2 \quad 1.3062 E-01: x$

$9.1254 E-01: \times 2$

$6.5594 E+01 * \times 2 \quad 2.0163 E+01 \cdot \times 3-2.0740 E+00 * \times 4 \quad 1.2501 E-01 \times 3$ $2.6310 E+01 * x 2 \quad 3.7245 E+00 * x 3-1.0160 E-01 * x 4$

$1.0609 E+02 \times x 2=7.2543 E+00$ xJ $1.1991 E=01$ * $x 4$

$-2.7260 E+01: \times 2 \quad 7.9610 E-01 * x J$

$7.009 y E=02$ * $x$ 


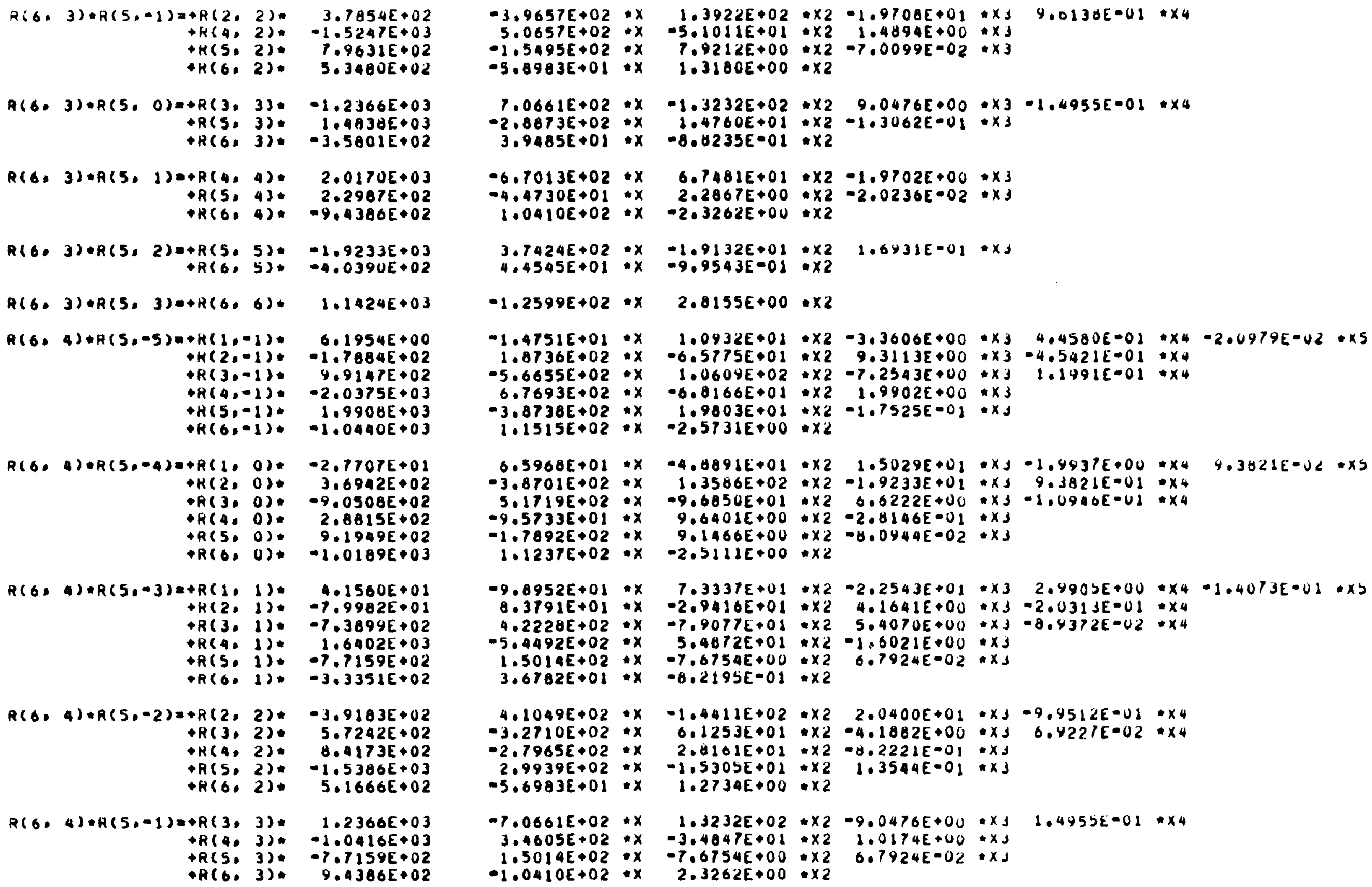

$-3.9657 E+02 * x \quad 1.3922 E+02 * \times 2-1.9700 E+01 * x 3 \quad 9.0138 E-01 * \times 4$ $5.0657 E+02 * x \quad-5.1011 E+01 \times x 2 \quad 1.4494 E+00 \times x 3$

$-1.5495 E+02 \cdot x \quad 7.9212 E+00 \times x 2-7.0099 E-02 \times x 3$

$-5.8983 E+01+x \quad 1.3180 E+00 \times 2$

$7.0661 E+02 * x-1.3232 E+02 * \times 2 \quad 9.0476 E+00 * \times 3-1.4955 E-01 * \times 4$ $-2.8873 E+02 * x \quad 1.4760 E+01: \times 2-1.3062 E-01 \times x 3$

$3.9485 E+01 * x-8.8235 E \cdot 01 * x 2$

$-6.7013 E+02+x \quad 6.7481 E+01 * \times 2-1.9702 E+00 * \times 3$

$-4.4730 E+01 * x \quad 2.2867 E+00 * x 2-2.0236 E-02 * x$

$1.0410 E+02 * x-2.3262 E \cdot 00 * \times 2$

$3.7424 E+02 * x \quad-1.9132 E+01: \times 2 \quad 1.6931 E-01 * x 1$
$4.4545 E+01 * x \quad-9.9543 E-01: \times 2 \quad$

$-1.2599 E+02 * x \quad 2.0155 E+00 \times 2$

$-1.4751 E+01 * x \quad 1.0932 E+01 \times 2=3.3606 E+00 * x 3 \quad 4.4580 E-01 \times x 4-2.0979 E-02 \times \times 5$ $1.8736 E+02 * x \quad-6.5775 E+01 * \times 2 \quad 9.3113 E+00 * x 3-4.5421 E-01 * x 4$ $-5.6655 E+02 * x \quad 1.0609 E+02 * \times 2-7.2343 E+00 * x 3 \quad 1.1991 E-01 * x 4$

$6.7693 E+02 * x-6.0166 E+01: \times 2 \quad 1.9902 E+00 * x 3$

$1.9003 E 02: \times 2-1.7525 E=01 * x$

$1.1515 E+02 \cdot x \quad-2.5731 E+00 \times x 2$

$6.5968 E+01 * x$ $-3.8701 E+02 \cdot x$ $5.1719 E+02: x$
$-9.57335+01: x$ $-9.5733 E+01: x$
$-1.7892 E+02: x$ $-1.7892 E+02: x$
$1.1237 E+02: x$

$-4.0091 E+01 * \times 2 \quad 1.5029 E+01 * x J-1.9937 E+00 * x_{4} \quad 9.3821 E=0<* \times S$ $1.3586 E+02 * x 2-1.9233 E+01 * x 39.3821 E=01 * x$ $9.6050 E+01 * \times 2 \quad 0.6222 E+00 * x 3-1.0940 E-U 1 * x$

$9.6401 E+00 \times 2-2.8146 E-01 * x$

$9.1466 E+00+x 2-8.0944 E-02+x 3$

$1.1237 E+02 * x-2.5111 E+00 \times 2$

$-9.0952 E+01 * x \quad 7.3337 E+01 * x 2-2.2543 E+01 * x 3 \quad 2.9903 E+00 \times x 4-1.4073 E-01 * x b$ $8.3791 E+01 * x \quad-2.9416 E+01 * x 2 \quad 4.1641 E+00 * x 3=2.031 \mathrm{JE}=01 * x_{4}$ $4.2228 E+02: x-7.9077 E+01: \times 2 \quad 5.4070 E+00 * x J=8$

$-5.4492 E+02: x \quad 5.4672 E+01$ x $x 2-1.6021 E+00 \times x J$

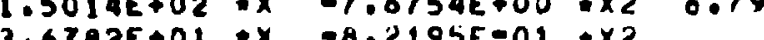

$4.1049 E+02 \times x-1.4411 E+02 \times 22 \quad 2.0400 E+01 * x 3-9.9512 E-01 \times \times 4$ $-3.2710 E+02 * x \quad 6.1253 E+01 * x 2-4.1882 E+00 * x J \quad 0.4227 E-02 * x 4$

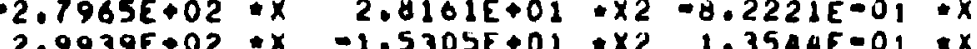
$-5.6983 E+01 * x-1.2734 E+00, x 2$

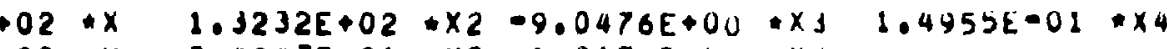
$3.4605 E+02 * x-3.4847 E+01 * \times 2 \quad 1.0174 E+00 * x J$ $1.5014 E+02 * x-7.6754 E+00 * x 2 \quad 6.7924 E-02 * x J$ 


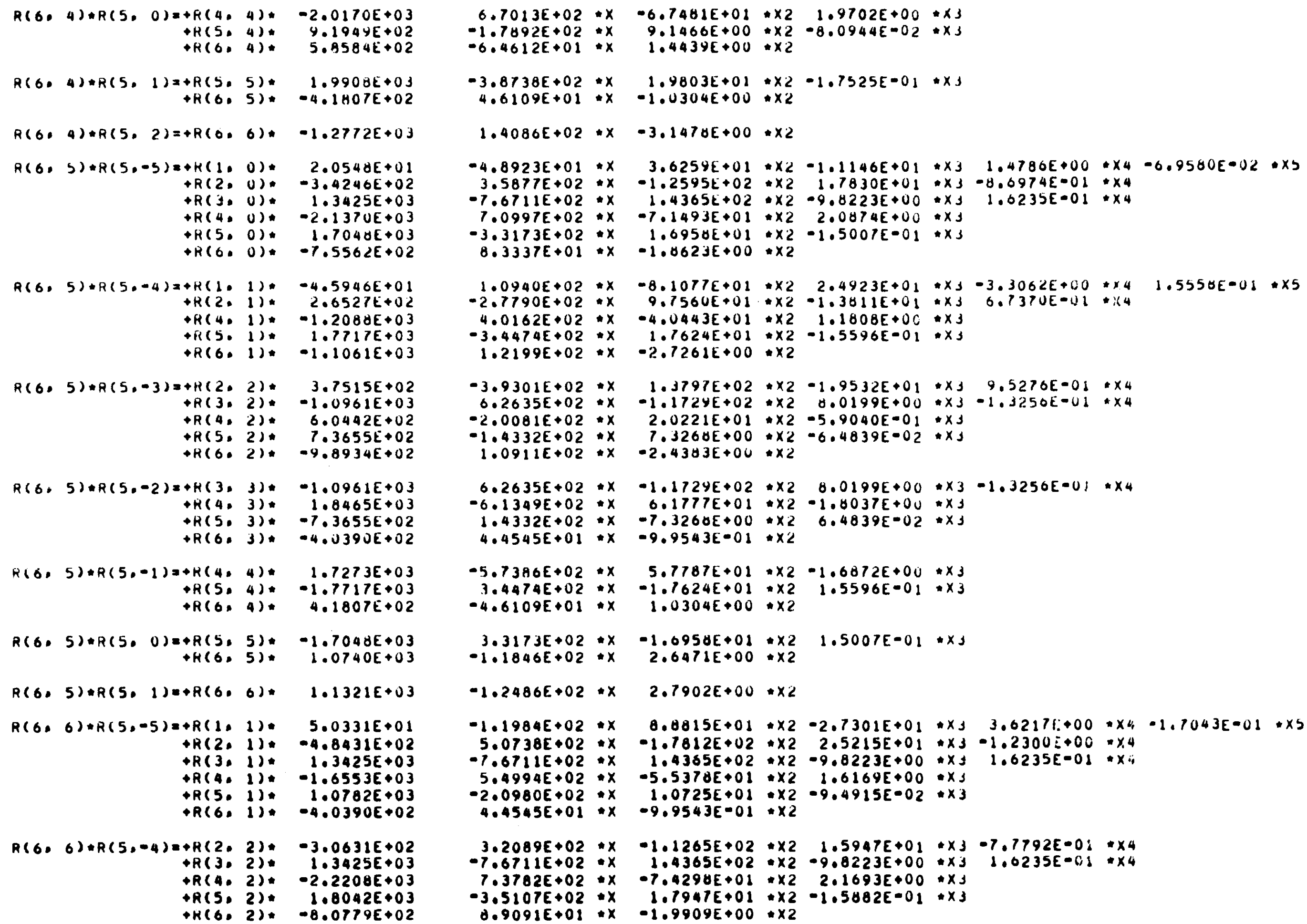

$1.0961 E+03$

$R(6,5) * R(5,-2)=+H(3,3)$ $R(4,3)$. $R(5,3)=-7.3655 E+02$ $+R(6,3):-4.0390 E+02$

$R(6,5) * R(5,-1)=+R(4,4) * 1.7273 E+03$

$+R(5,4):-1.7717 E+03$
$+R(6,4) .4 .1807 E+02$ $R(6,4)$.

$R(6,5): R(5,0)=+R(5,5),-1.7048 E+03$ $H(6,5) * 1.0740 E+03$

$R(6,5) \# R(5,1)=+R(6,6) * 1.1321 E+03$

$R(6,6) * R(5,-5)=+R(1,1) * 5.0331 E+01$ $+R(2,1):-4.8431 E+02$ $+R(3,1)$ 1.3425E+03 $+R(4.1)=-1.6553 E+03$ $\rightarrow R(5$. 1): $1.0782 E+03$ $\rightarrow R(6,1):-4.0390 E+02$

$R(6,6)+R(5,-4)=+R(2,2):-3.0631 E+02$ $+R(3,2): \quad 1.3425 E+03$ $+R(4,2):-2.2208 E+03$ $+R(5,2) .1 .0042 E+03$ $+R(5,2): \quad 1,8042 E+03$
$+K(6,2):-8.0779 E+02$

$6.7013 E+02 * x \quad-6.7481 E+01 * \times 2 \quad 1.9702 E+00 * x 3$ $-1.7492 E+02 * x \quad 9.1466 E+00 * x 2-0.0944 E-02 * x$

$-6.4612 E+01 * X \quad 1.4439 E+00 * x 2$

$-3.8738 E+02 * x \quad 1.9803 E+01 * x 2-1.7525 E-01 * x$ $4.6109 E+01 * x-1.0304 E+00 * \times 2$

$1.4086 E+02 * x-3.1478 E+00 * \times 2$

$-4.8923 E+01 * x \quad 3.6259 E+01 * x 2-1.1146 E+01 * x 3 \quad 1.4786 E+00 * x 4-6.9580 E-02 * X_{3}$ $3.5877 E+02 * x-1.2595 E+02 * x 2 \quad 1.7830 E+01 * x 3=8.6974 E=01 * x 4$ $-7.6711 E+02 * x \quad 1.4365 E+02 * x 2-9.8223 E+00 * x J 1.6235 E=01 * x 4$

$7.0997 E+02 * x-7.1493 E+01 * x 2 \quad 2.0874 E+00 * x J$

$-3.3173 E+02 * x \quad 1.6950 E+01 * x 2-1.5007 E-01 * x$

$0.3337 E+01 * x-1.0623 E+00 * x 2$

$1.0940 E+02 * x-8.1077 E+01 * \times 2 \quad 2.4923 E+01 * x 3-3.3062 E+00 * \times 4 \quad 1.5558 E=01 * \times 5$ $-2.7790 E+02: x \quad 9.7560 E+01 \cdot \times 2-1.3611 E+01 * x 3 \quad 6.7370 E-41 *: 14$

$4.0162 E+02 * x-4.0443 E+01 * x 2 \quad 1.1808 E+0 C * x 3$

$-3.4474 E+02 * x \quad 1.7624 E+01 * x 2-1.5596 E=01 * x$

$1.2199 E+02 * x-2.7261 E+00 * \times 2$

$-3.9301 E+02 * x \quad 1.3797 E+02 * \times 2-1.9532 E+01 * x 39.5276 E-01 * x 4$ $6.2635 E+02 * x-1.172 y E+02 * x 2 \quad 0.0199 E+00 * x 3-1.3250 E=01 * x$ $-2.0001 E+02 * x \quad 2.0221 E+01 * \times 2-5.9040 E=01 * x J$

$-1.4332 E+02 * x \quad 7.3268 E+00 * x 2-6.4839 E-02 * x$

$1.0911 E+02 * x-2.4343 E+00 * x 2$

$6.2635 E+02 * x-1.1729 E+02 * x 2 \quad 0.0199 E+00 * x 3-1.3256 E=01 * x 4$ $-6.1349 E+02 * x \quad 0.1777 E+01 * x 2-1.8037 E+00 * x s$

$1.4332 E+02 * x-7.3260 E+00 * x 26.4839 \varepsilon=02 * x$

$4.4545 E+01 * x-9.9543 E-01 * x^{2}$

$-5.7386 E+02 * x \quad 5.7787 E+01 * \times 2-1.6872 E+00 * x 3$

$3.4474 E+02 * x-1.1624 E+01 * \times 2 \quad 1.5596 E-01 * \times 3$

$-4.6109 E+01 * x \quad 1.0304 E+00 * x 2$

$3.3173 E+02 * x-1.0958 E+01 * \times 2 \quad 1.5007 E-01 * x$

$-1.1846 E+02 * x \quad 2.6471 E+00 * \times 2$

$-1.2486 E+02 * 2.7902 E+00 * 2$

$-1.1984 E+02 * x \quad 0.0815 E+01 * x 2-2.7301 E+01 * x J \quad 3.62171:+00 * x 4-1.7043 E=01 * x b$ $5.0738 E+02 * x-1.7812 E+02 * \times 2 \quad 2.5215 E+01 * x 3-1.23005+00 * \times 4$

$-7.6711 E+02: x \quad 1.4365 E+02: x 2-9.8223 E+00 * x\} 1.6235 E-01 * x$

$5.4994 E+02: x-5.5370 E+01 * x 2 \quad 1.6169 E+00 * x 1$

$-2.0980 E+02 * x \quad 1.0725 E+01 * x 2-9.4915 E-02 * x 3$

$4.4545 E+01: x-9.9543 E-01$ : $x$

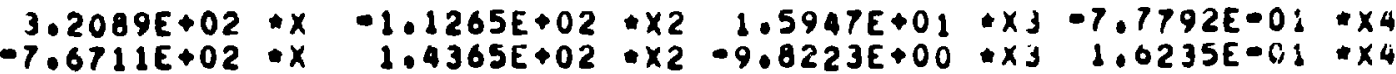

$7.3782 E+02 * x-7.4298 E+01 \times x 2 \quad 2.1693 E+00 * x$

$-3.5107 E+02 * x \quad 1.7947 E+01: \times 2-1.5882 E-01: x 3$

$0.9091 E+01: x-1.9909 E+00: x 2$ 


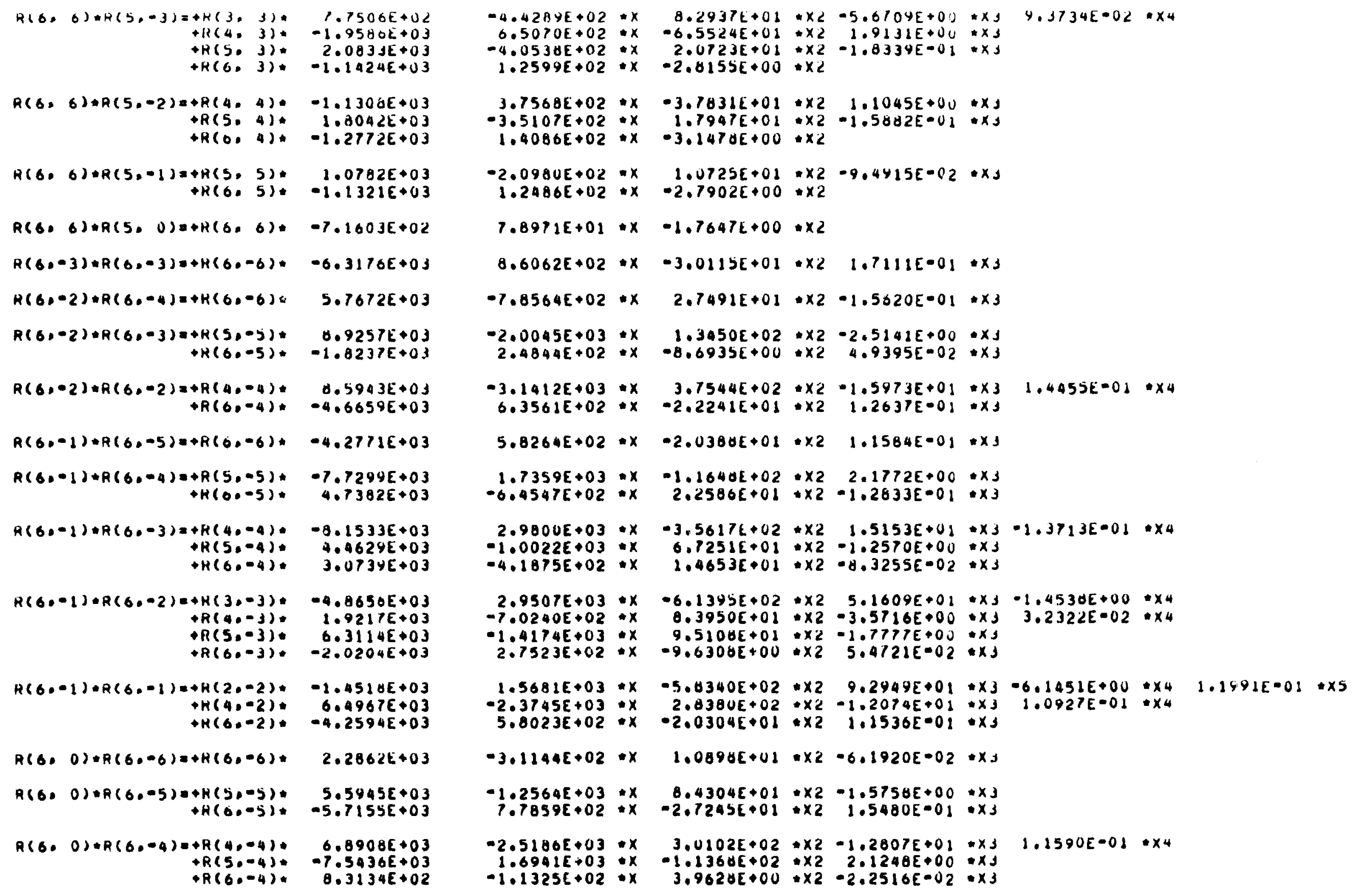

$-4.42 B Y E+02 * x \quad 8.2937 t+01 * x 2-5.6109 E+00 * x y \quad 9.1734 E-02 * \times 4$ $0.5070 E+02 * x-6.5524 E+01 * x 2 \quad 1.9131 E+00 * x$ $-4.0536 E+02 * x \quad 2.0723 E+01 * x 2-1.8339 E-01 * x J$

$1.2599 E+02 * x-2.8155 E+00 * 2$

$3.7568 E+02 * x-3.7831 E+01 * x 21.1045 E+00 * x J$ $-3.5107 E+02 * x \quad 1.7947 E+01 * x 2-1.5802 E-01 * x s$ $1.4086 E+02 \cdot x-3.2470 E+00 \cdot \times 2$

$-2.0980 E+02 * x \quad 1.0725 E+01 * \times 2-9.4415 E-02 * x J$ $1.2486 E+02 \times x-2.7902 E+00 \times 2$

$7.8911 E+01 * x-1.7647 L+00 * 2$

$8.6062 E+02 * x-3.0115 E+01 * \times 2 \quad 1.7111 E-01 * x 3$

$-7.8564 E+02 * x \quad 2.7491 E+01 * \times 2-1.5620 E-01 * \times 3$

$-2.0045 E+03 * x \quad 1.3450 E+02 * \times 2-2.5141 E+00 * x J$

$2.4844 E+02 * x-8.6935 E+00 * x 2 \quad 4.9395 E-02 * x 3$

$-3.1412 E+03 * x \quad 3.7544 E+02 * x 2=1.5973 E+01 * x 3 \quad 1.4455 E-01 \times x 4$ $6.3561 E+02 * x-2.2241 E+01 * x 2 \quad 1.2637 E-01 * x 3$

$5.8264 E+02 * x-2.0380 E+01+x 2 \quad 1.1584 E-01 * x 3$

$1.7359 E+03 * x-1.1640 E+02 * \times 2 \quad 2.1772 E+00 * x 3$

$2.9000 E+03 * x-3.5617 t+02 * x_{2} \quad 1.5153 E+01 * x 3-1.3713 E-01 * \times 4$

$-1.0022 E+03: x \quad 6.1251 E+01 * \times 2-1.2570 E+00 * x 3$

$2.9507 E+03 * x-6.1395 E+02 * x_{2} \quad 5.1609 E+02 * x_{3}-1.4530 E+00 * x_{4}$ $-7.0240 E+02 * x \quad 0.3950 E+01 * \times 2-3.5716 E+00 * x J \quad 3.2322 E=02 * \times 4$

$-1.4174 E+03: x \quad 9.5100 E+01 * x 2-1.7777 E+00 * x 3$

$1.5681 E+03 * x \quad-5.0340 E+02 * \times 2 \quad 9.2949 E+01 * \times 3-6.1451 E+00 * \times 4 \quad 1.1591 E=01 * \times 5$ $-2.3745 E+03 * x \quad 2.0380 E+02 * x 2-1.2074 E+01 * x 3 \quad 1.0927 E=01 * x_{4}$ $5.8023 E+02 \cdot x \cdot 2.0304 E+01 * \times 2 \quad 1.2536 E-01 * x$

$-3.1144 E+02 * x \quad 1.0898 E+01 \times 2-6.1920 E-02 * x$

$\begin{array}{rlrr}-1.2564 E+03 & * x & 8.4304 E+01 * x 2-1.5758 E+00 * x 3 \\ 7.7859 E+02 * x & -2.7245 E+01 * \times 2 & 1.5400 E-01 * x 3\end{array}$

$-2.5106 E+03 * x \quad 3.0102 E+02 * x 2-1.2807 E+01 * x 3 \quad 1.1590 E-01 * x 4$

$-1.6941 E+03 * x$
$-1.1325 E+02 * x \quad 3,9620 E+00 * x 2-2.2516 E-02 * x 3$ 


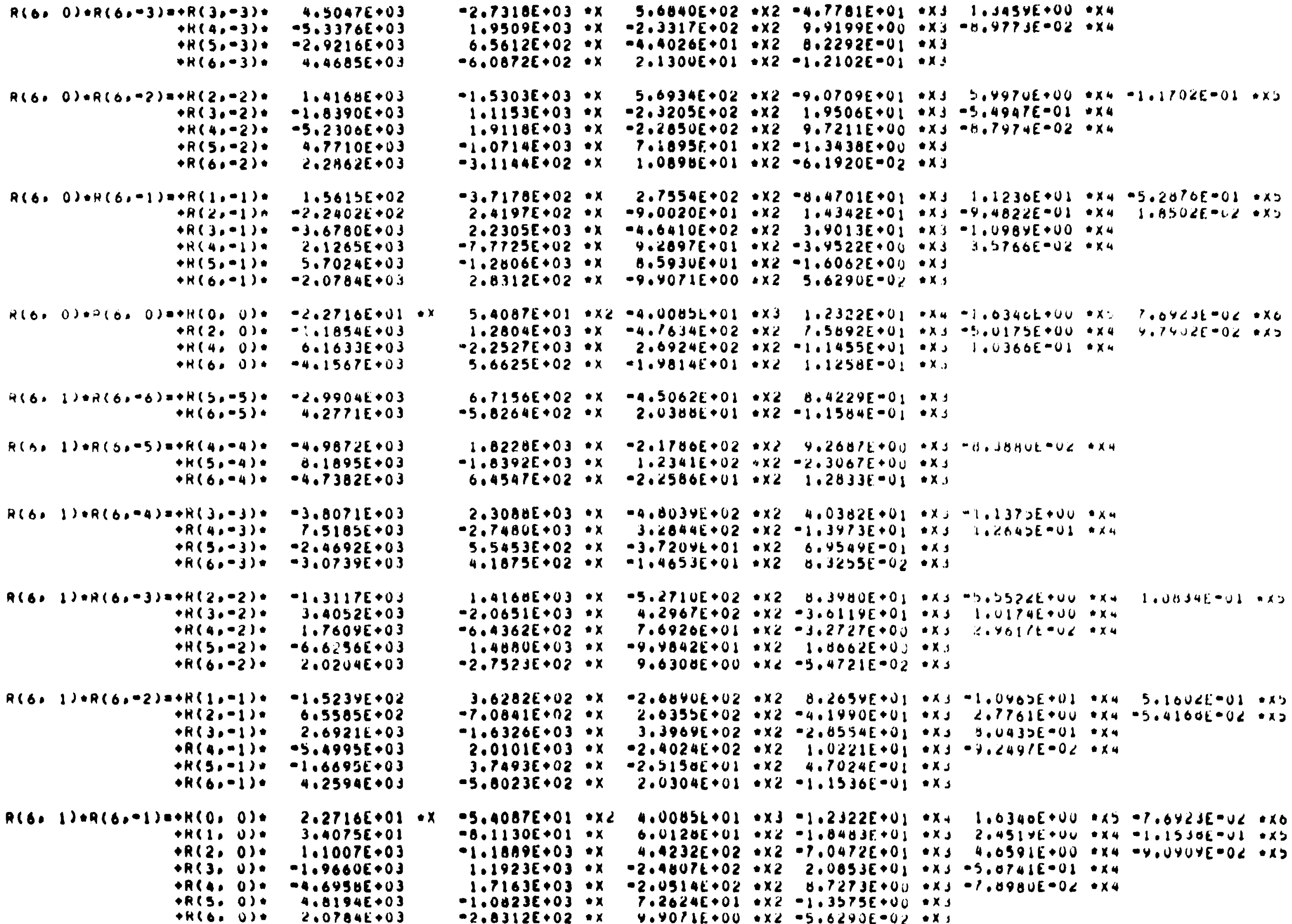

$R(6,0)+R(6,-3)=+R(3,-3) * \quad 405047 E+03$ H $(4,-3)$. $-5,3376 E+03$ $\rightarrow R(5,-3),-2.9216 E+03$ - $R(6,-3) . \quad 4.46855+05$

$R(6,0) \cdot R(0,-2)=+R(2,-2)$. $\rightarrow R(3,-2)$. $\rightarrow R(4,-2)$. $\rightarrow R(5,-2)$. $\rightarrow R(6,-2)$.

$1.91686+03$ $-1.8390 E+03$ $-5.23005+03$ $4.7710 E+03$ $2.2462 E+03$

$R(6,0) \bullet R(6,-1)=0 R(1,-1)$ $\rightarrow R(2,-1) \theta^{2}$ $\rightarrow R(3,-1)$. $+R(4,-j)$. $+K(5,-1)$. $+K(60 \cdot 1)$.

$1.5613 E+02$ $-2.2402 E+02$ $-3.6780 E+03$ $2.1265 E+03$ $5.7024 E+03$ $-2.0784 E+0.3$

$R(0,0) .2(0,0)=0 R(0,0)$. $+R(2,0)$ $\rightarrow H(0.0)$. $-4.1567 E+03$

$-2.2716 E+01 \cdot x$ $\because 1854 E+03$ $0.1633 E+03$

$A(6,1)+A(6,-6)=+R(5,-5)$.

$R(3,1) \bullet A(5,-5)=+R(4,-4)$. R $(6,-4)$.

$R(6,1)+R(6,-4)=+K(3,-3)$. $\rightarrow R(4,-3)$ $+R(5.03)$. A $(60-3)$.

$A(6,1) \cdot A(6,-3)=+H(2,-2)$. $R(3,-2):$ H $(5,-2)$. $\rightarrow R(6,-2)$.

$4.98725+03$ $0.1095 E+03$ $.4 .7382 E+03$

$-3.8071 E+03$ $7.3185 E+03$ $-2.46925+03$
$-3.07396+03$

$-1.31176+05$ $3.40525 \bullet 03$ $1.7604 E+03$ $-6.6236[+03$ $2.0204 E+03$

$R(6,1)+R(6,-2)=4 R(1,-1)$. $H(2,-1)$. $\rightarrow R(3,-1):$ $+H(5,-1)$. - $R(6,-1)$.

$R(6,1) \cdot R(6,01)=+K(0,0)$. H $(1,0)=$ $\rightarrow R(2,0)$. (R i - H(O. O).

$-1.523 y[+02$ $6.5585 E+02$ $2.00216+03$ 05.09950 .03 $-1.6095 E+03$ $4.25946+03$

$2.27165+01 \cdot x$ $3.260752+01$ $1.10075+03$ $-1.96005+03$ $-1.96606+03$ $0.69506+03$ $2.8948 \div 03$

$-2.7318 E+03 * x \quad 5.6040 E+02 * x 2-4.7781 E+01 * x J \quad 1.545 y E+00 * x_{4}$ $1.9509 E+03 * x-2.3317 E+02 * \times 2 \quad 9.9199 E+00 * x-8.977 J E=02 * x_{4}$ $6.5612 E+02 \div x-4.4026 E+01 \times x 2 \quad 0.2292 E-01 * x 3$

$-6.0872 E+02 * x \quad 2.1300 E+01 * \times 2-1.2102 E-01 * x$

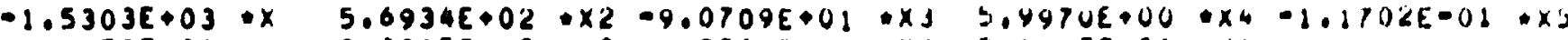
$1.11536+03: x$ $1.9118 E+03 * x$

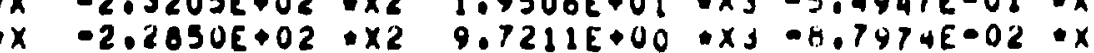

$-3.1144 E+02: x \quad 1.80956+01: \times 2-1.3438 E+00: x 3$

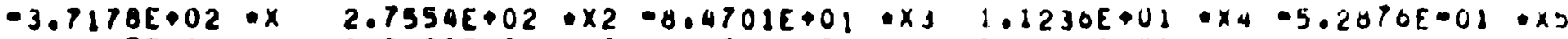
$2.4197 E+02 \cdot x-9.0020 E+01 \cdot \times 2 \quad 1.4342 E+0,: x j-9.4822 E-01 \cdot x 4 \quad 1.0502 E-12 \cdot x$ $2.2305 E+03 * x \quad-4.6410 E+02 \times x 2 \quad 3.9013 E+01 * x 3-1.096 \times E+00 * x_{4}$

$-7.7725 E+02$ * $x \quad 9.2097 E+01 * x 2-3.4522 E+00 * x 3 \quad 3.5766 E=02 * x_{4}$

$.2806 E+03 * x \quad 1.5930 E 001+x 2-1.6062 E+01 \cdot x$

$2.8312 E+02 * x-9.4071 E+00 \times 25.6290 E-02 * x$

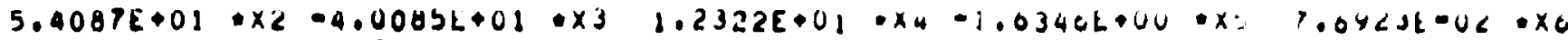

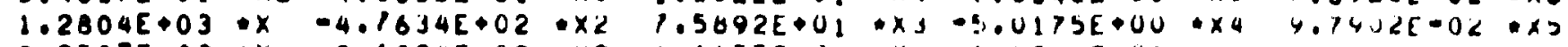
$-2.2527 E+03 * x \quad 2.0924 E+02 * x 2-1.1455 E+U 1 * x J 1.0360 E=01 . x 4$

$5.6625 E+02 \cdot x-1.9814 E+01 \cdot \times 21.1258 E-01 \cdot x$.

$6.7156 E+02 * x \quad-4.5062 E+01+x 2 \quad 8.4229 E-01 * x y$

$\left.1.0228 E+03 * x-2.1780 E+02 * x^{2} \quad 9.2607 k+01\right) \times 3-1.5840 t=02 \times x_{4}$

$-1.0392 E+03 * x \quad 1.2341 E+02 \times x 2-2.3067 E+0 u * x$

$6.4547 E+02 * x-2.2586[+01 * x 2 \quad 1.2833 E=01 \cdot x$

$2.3080 E+03 * x-4.003 y E+02 * x_{2} \quad 4.0302 E+01 * x j-1.23 Y 2 E+00 \cdot x_{4}$

$-2.7480 E+03 * x \quad 3.2044 E+02 * x 2-1.3473 E+01 * x J \quad 1.6442 E-U 1 * x 4$

$5.5453 E+02: x \quad-3.720 y E+01: x 2 \quad 6.4549 E-01: x 1$

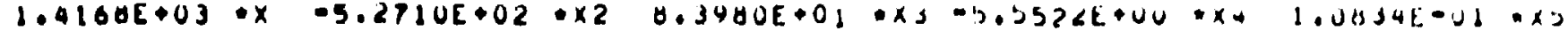
$-2.0651 E+03 * x \quad 4.2967 E+02 * x 2-3.0119 E+0 j * x 3 \quad 1.0174 E+U 0 * x 4$

$-6.4362 E+02 * x \quad 7.6920 E+01 * x 2-3.2727 E 00 j: x s$

$-2.7523 E+02 * x \quad 9.6304 E+00+x<-5.4721 E-02: x 3$

$\left.\left.3.6282 E+02 * x-2.0840 E+02 \times x^{2} \quad 0.2654 E+01 * x\right]-1.0405 E+01 * x_{4} \quad 5.160<E-01 * x^{\prime}\right)$

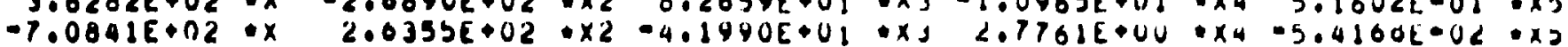
$-1.63265+03: x$ $2.01016003 \cdot x$ $3.74935002 \cdot x$ $3.7493 E+02: x$
$-5.8023 E+02: x$

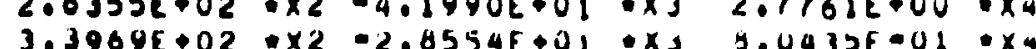
$-2.4024 E+02: x^{2} \quad 1.0221 E+01: x J-7.2441 E=02: x_{4}$ $-2.5150 E+01: x 2 \quad 4.7024 E-01: x 3$

$-5.4007 E+01 \div \times 2$ $2.0304 E+01 \cdot x^{2} \cdot 1.1536 E-01 \cdot x_{3}$

$-8.11302+01: x$

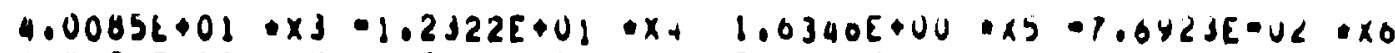

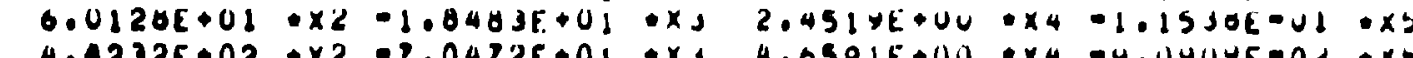
$1.1923 E+03: x-2.94075+020 x 2$ - $x$.

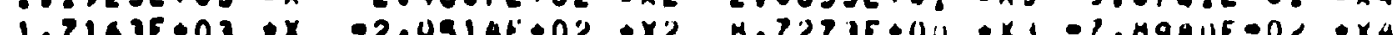

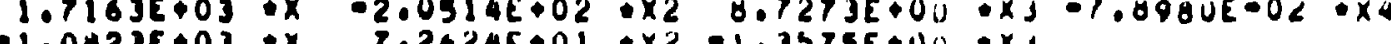

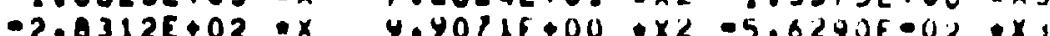




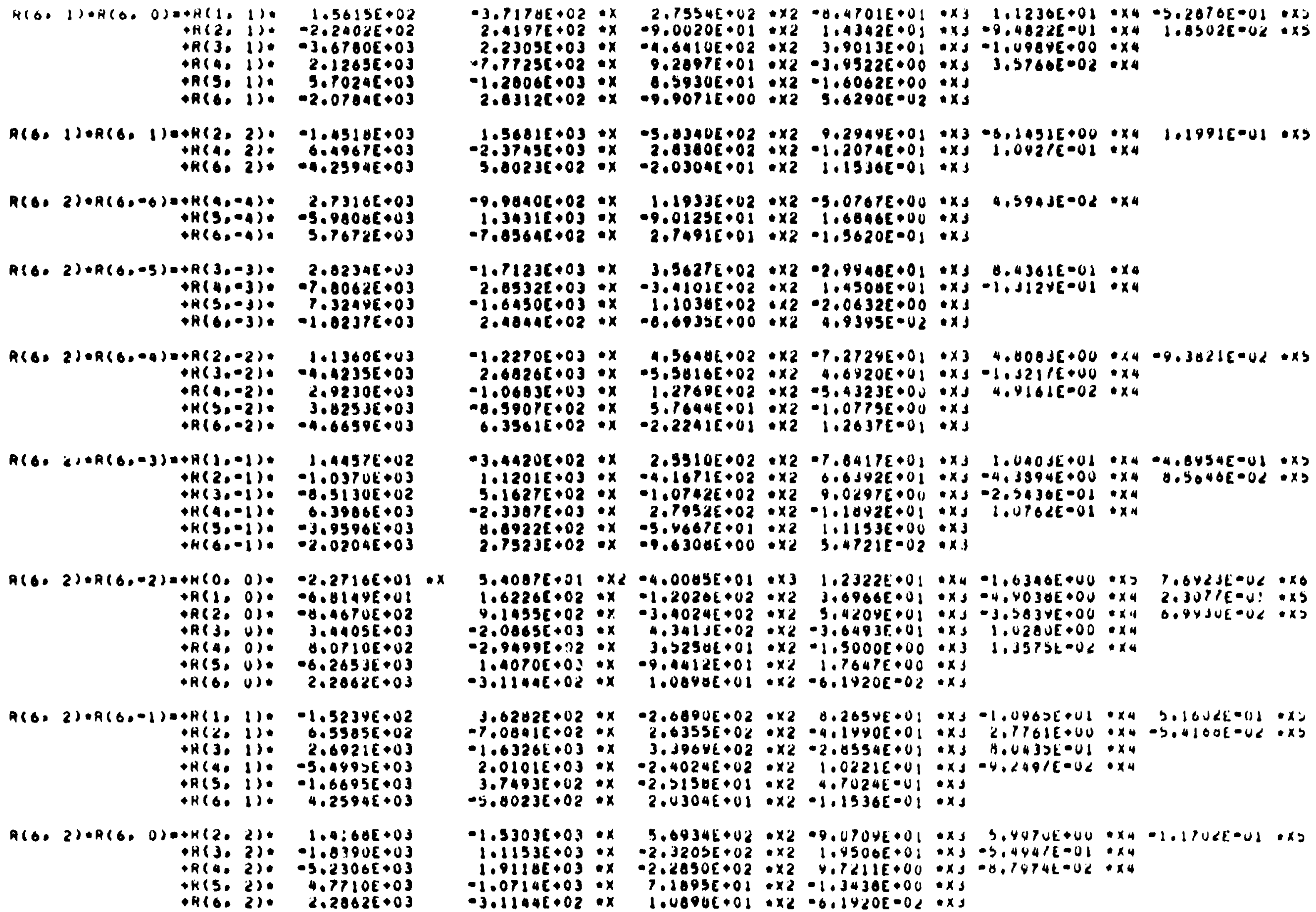

$A(6,2) \cdot A(6,-1)=0+H(1,1)$ $\rightarrow H(2,1)$

$-1.5234 E+02$ $+R(3) 1) \quad$. - H(4. 1) - $-5.449>E+03$ $+H(6$.$) i) 4.2594 E+03$

A(6, 2) $R(6,0)++H(2,2), 1.4: 60[+03$ H(3, 2$):$ $+R(4,2) \cdot$ $+k(5,2):$ $1.83908+03$ $4.77106+03$ $2.28626 * 03$

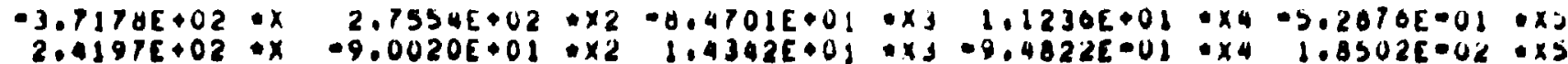

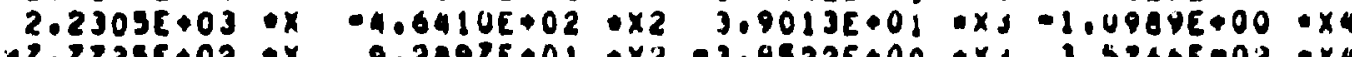

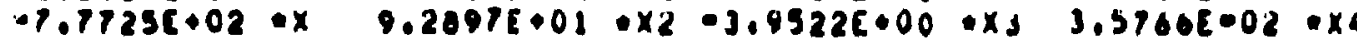

$1.2006[003 * x \quad 0.5930 E+01 * x 2+1.60025+00 * x J$

$\bullet x-9.9071 E+00 * x 2$

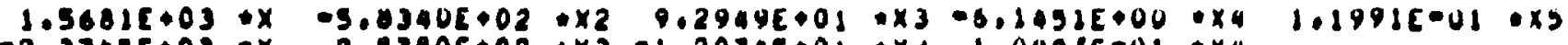

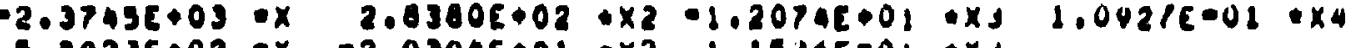

$5.0023 E+02 \cdot x-2.0304 E+02 \times 2 \quad 1.1530 E-01 \cdot x$

$-9.9040 E+02 \times x \quad 1.1933 E+02 \times \times 2-5.0707 E+00 * x 3 \quad 4.594 J E \cdot 02 \times \times 4$

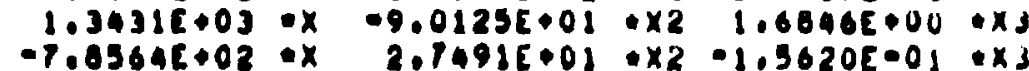

$-1.71236+03 \times x \quad 3.5627 E+02 \times 2-2.9940 E+01 \times x J 0.4361 E=01 \times x$

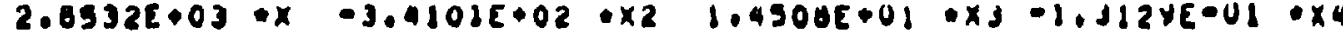

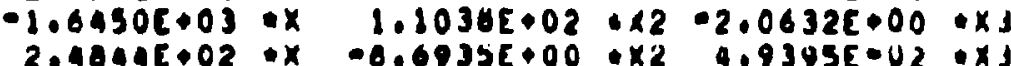

$-1.2270 E+03 * x \quad 4.5640 E+02 \cdot \times 2-7.2729 E+01 * \times 3 \quad 4.000 J E+00 \cdot \times 4-9.3821 E-02 \times x b$ $2.6826 E+03 * x-5.5816 E+02 * \times 2 \quad 4.6420 E+01 \cdot x]-1.321 / E+00 \cdot x 4$

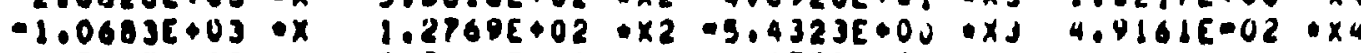

$0.5907 E+02 \times x \quad 5.7644 E+01 \times x 2-1.0773 E+00 \cdot x$

$6.3561 E+02 \times x-2.2241 E+01 \times 2 \quad 1.2637 E=01: x J$

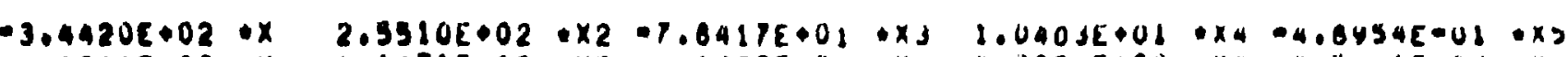

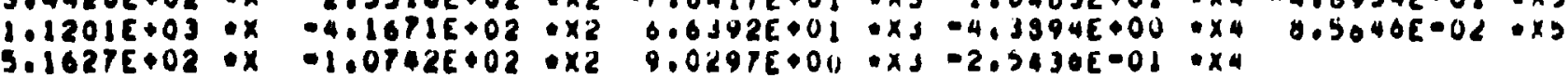
$-5.33076+03: x$

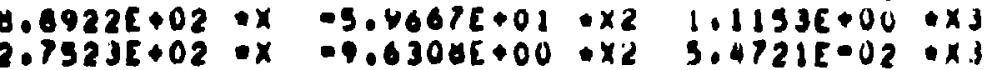
$1.0742 E+02 \times x 2 \quad 0.0297 E+00, x J-2.3430 E-01 * x$

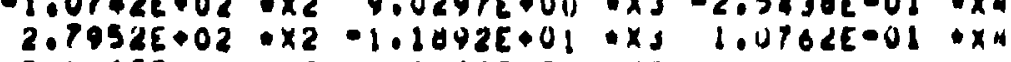

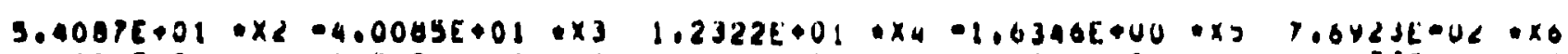

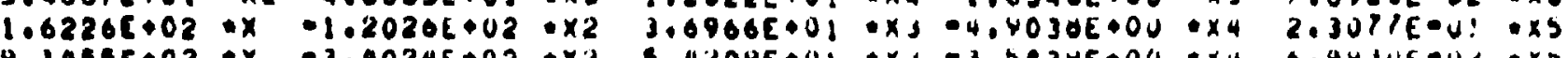

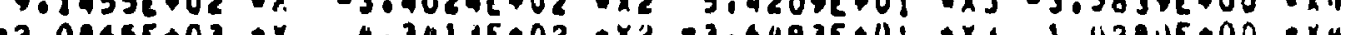

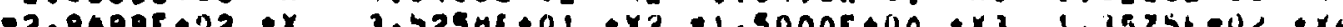

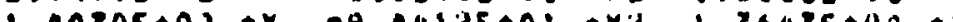

$-3.11446+02+x \quad 1.00406+01: \times 2-6.1920 E-02: x$

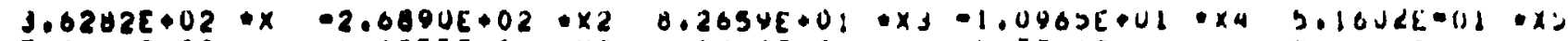

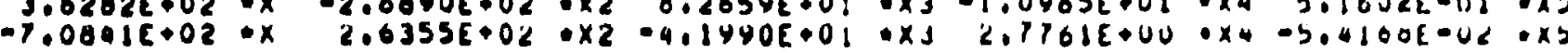
$-1.63268 .03 \cdot x$ $2.01016+03: x$ $3.7493 E+02: x$
$-5.00236+02: x$

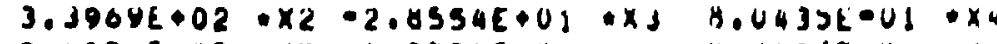

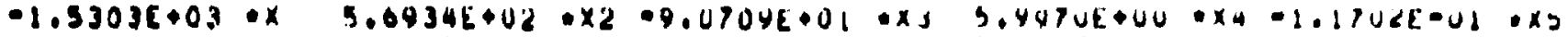
$1.1153 E+03 * x \quad-2.3205 E+02 * x 2 \quad 1.4500 E+01 * x 3=5,444 / E-01: x_{4}$

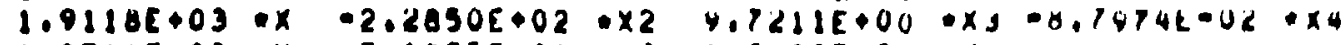

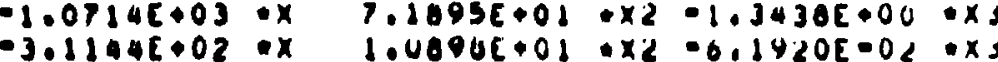




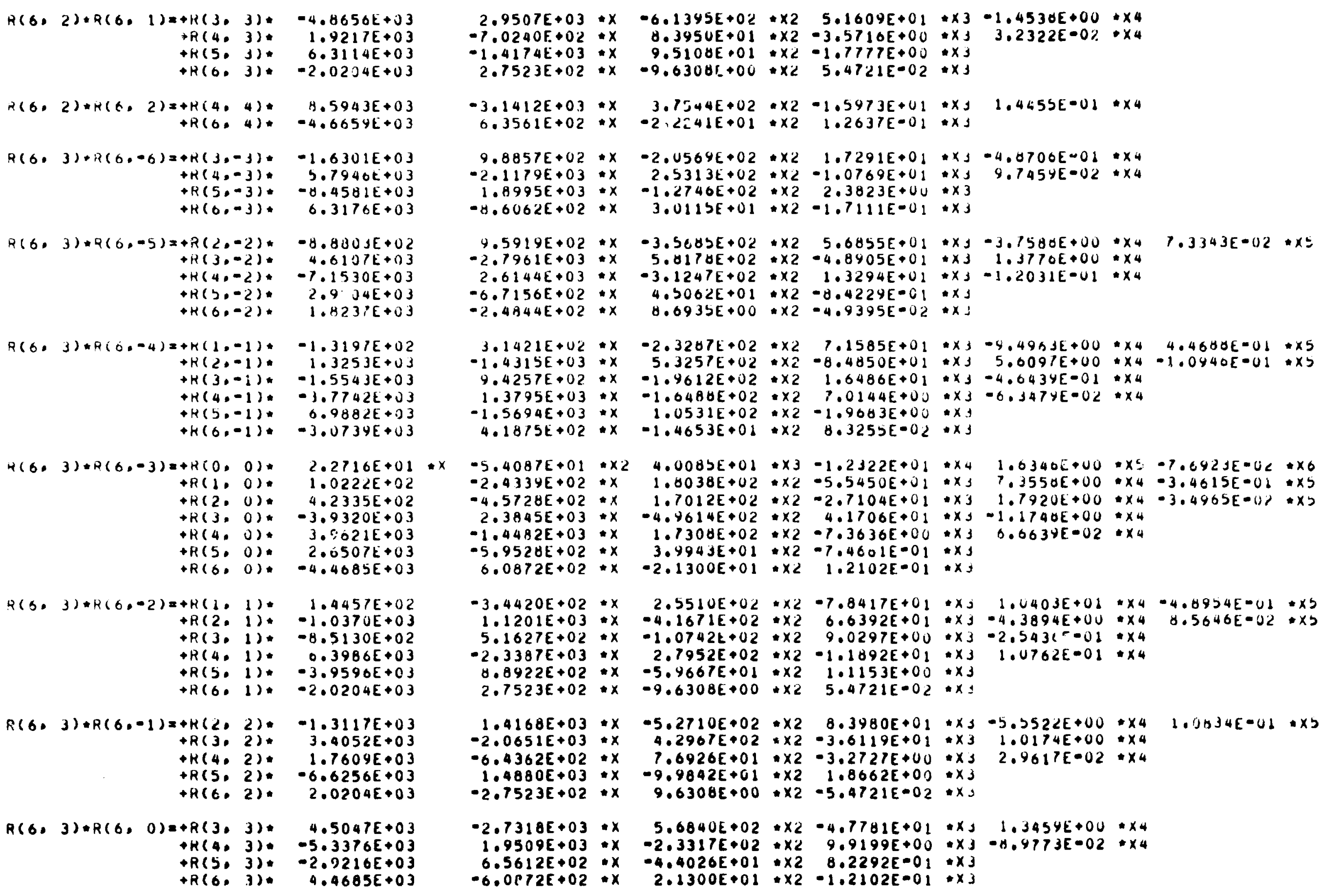

$R(6,2) * R(6,1)=+R(3,3) *=4.8650 E+J 3$ $\rightarrow R(4,3)=1.9217 E+03$ $+R(5,3):-6.3114 E+03$

$\begin{aligned} R(6,2)+R(6,2)= & +R(4,4), \quad H .5943 E+03 \\ & +R(6,4) \quad-4.6654 E+03\end{aligned}$ $+R(6,4) *=4.6654 E+03$

$R(6,3)+R(6,-6)=+R(3,-3) *$ $\rightarrow R(4,-3):$ $3.7940 t+03$ $-8.4581 E+03$ $+K(6,-3)=6.3176 E+03$

$R(6,3) \cdot R(6,-5)=+R(2,-2)=$ $+R(3,-2)$ $-7.1530 E+03$ $+R(5,-5)$. $+H(6,-2) * 1.823 ; E+03$

$R(6,3) * R(0,-4)=+K(1,-1) *-1.3197 E+02$ $\rightarrow K(2,-1):-1.3253 E+U 3$ $+K(3,-i) *-1.5243 E+03$ $+K(4,-1) *-1.7742 E+U 3$ $+h(5 .-1) \quad 0.9882 E+03$ $+H(6,-1) .-3.0739 E+U 3$

$4(6,3)+R(6,-3)=+R(0,0)=$ $+R(1,0)$ $+R(2,0)$ $+R(3,0)$ $+R(4,0)$ $+R(5,0): \quad 2.06507 E+03$
$+R(6,0):-4.4085 E+03$ $2.2716 E+01$ $1.0222 E+02$ $4.2335 E+02$ $-3.9320 \mathrm{E}+03$ $3.5021 E+03$ $2.0507 E+03$

$R(5,3)+R(6,-2)=+R(1,1)+1.4457 E+02$ $+R(2,1) *-1.0370 E+03$ $+R(3.1)=-8.5130 E+02$ $+R(4.11)=0.3986 E+03$ $+R(5 ., 1)=-3.9590 E+03$ $+R(6,1) \cdot-2.0204 E+03$

$R(6,3) * R(6,-1) x+R(2,2) *-1.3117 E+03$ $+R(3,2) * 3.4052 E+03$ $+K(4,2): 1.7609 E+03$ $+R(5,2):-6.6256 E+03$
$+R(6,2): \quad 2.0204 E+03$

$R(6,3)=R(6,0)=+R(3,3) * 4.5047 E+03$ $\rightarrow R(4,3)=-5.3376 E+03$ $+R(5,3)=-2.9216 E+03$ $+R(6,3)=4.4605 E+03$

$2.9507 E+03 * x-6.1345 E+02 * x 2 \quad 5.1609 E+01 * x 3-1.4536 E+00 * x 4$

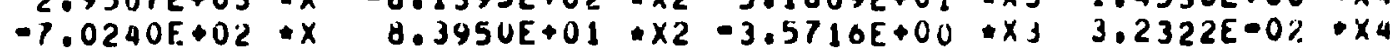

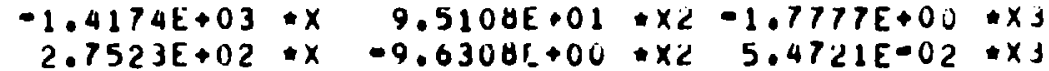

$-3.1412 E+0.3 * x \quad 3.1544 E+02 * x 2-1.5973 E+U 1 * x 31.4455 E-01 * \times 4$ $6.3561 E+02 * x-2,2241 E+01 * x 2 \quad 1.2637 E=01 * x 3$

$9.8857 E+02 * x-2.0569 E+02 * x 2 \quad 1.7291 E+01 * x 3-4.0700 E-01 * x_{4}$ $-2.1179 E+03 * x \quad 2.5313 E+02 * x 2-1.0769 E+01 * X_{3} \quad 9.7454 E=02 * \times 4$ $1.8995 E+03 * x \quad-1.2740 E+02 * \times 2 \quad 2.3823 E+U U: \times 3$
$-8.6062 E+02 * x \quad 3.0115 E+01 * \times 2-1.7111 E=01 * x 3$

$9.5919 E+02 \times x-3.5605 E+02 * \times 2 \quad 5.6855 E+01 * x y-3.7580 E+00 \times \times 4 \quad 7.3343 E=02 * x=$ $-2.7961 E+03 * x \quad 5.0178 E+02 * x 2-4.8905 E+01 * x 3 \quad 1.3770 E+00 * x 4$ $2.6144 E+03: x-3.1247 E+02 * x 2 \quad 1.3294 E+U 1 * x 3-1.2031 E=U 1 * x_{4}$ $-6.7156 E+02 * x \quad 4.3062 E+01 * x 2-0.4229 E=01 * x J$
$-2.4444 E+02 * x \quad 0.6935 E+00 * x 2-4.9395 E-02 * x j$

$3.1421 E+02 * x-2.3287 E+02 * x 2 \quad 7.1585 E+01 * x 3-9.495 J E+00 * \times 4 \quad 4.4680 E=01 * \times 5$ $-1.4315 E+03 * x \quad 5.3257 E+02 * x 2-8.4850 E+01 * x J \quad 5.0097 E+00 * x 4-1.0440 E=01 \times x b$ $9.4257 E+02 * x-1.9612 E+02 * x 2 \quad 1.6486 E+01 * x J-4.0434 E-01 * x 4$

$1.3795 E+03: x-1.0466 \varepsilon+02: \times 2 \quad 7.0144 E+00 \times 3=6.347 \times E=02 * x 4$

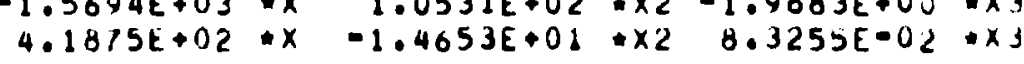

$-5.4087 E+01 * \times 2 \quad 4.0085 E+01 * x 3-1.2 J 22 E+01 * x 4 \quad 1.6340 E+00=x 5=7.692 J E=0<* x 0$ $-2.4339 E+02 \times x \quad 1.8038 E+02 * x 2-5.5450 E+01 * x_{3} \quad 7.3550 E+00 * x 4-3.4615 E-01 * x 5$ $-4.5728 E+02 * x \quad 1.7012 E+02 * x 2-2.7104 E+01 * x 3 \quad 1.7920 E+00 * x 4-3.4965 E-(1) * x b$

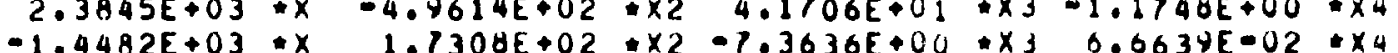
$-1.4482 E+03 \times x \quad 1.7300 E+02 * x 2=7.3636 E+00 * x 30.0637 E=02 * x$

$5.9528 E+02 * x \quad 3.9943 E+01 * x 2-7.4601 E-01 * x$

$6.0872 E+02 * x-2.1300 E+01 * x 21.2102 E=01 * x$

$-3.4420 E+02 * x \quad 2.5510 E+02 * \times 2-7.8417 E+01 * x 3 \quad 1.0403 E+01 * \times 4-4.8954 E-U 1 * x 5$ $1.1201 E+03 * x-4.1671 E+02 * x 2 \quad 0.6392 E+01 * x 3-4.3894 E+00 * x 4 \quad 8.5646 E-02 * x J$ $5.1627 E+02: x-1.0742 E+02 * x 2 \quad 9.0297 E+00 * x 3-2.5432=01: x 4$

$2.3387 E+03=x \quad 2.7952 E+02 * x 2-1.1892 E+01 * x J-1.0762 E-01 * x 4$ $0.8922 E+02: x \quad-5.9667 E+01 * x 2 \quad 1.1153 E+00 * x J$ $2.7523 E+02 * x-9.6308 E+00 * x 2 \quad 5.4721 E-02 * x=$

$1.4168 E+03 * x-5.2710 E+02 * \times 2 \quad 8.3980 E+01 \times x 3-5.3522 E+00 \times \times 4 \quad 1.0034 E-01 \times x b$ $-2.0651 E+03 * x \quad 4.2967 E+02 \times \times 2-3.6119 E+01 * x 3-1.0174 E+00 * x$ $0.4362 E+02 * x \quad 7.6926 E+01 * x 2-3.2727 E+00 * x s \quad 2.9617 E=02 * x 4$

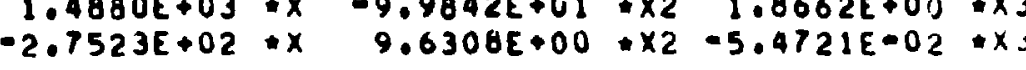

$-2.7318 E+03 * x \quad 5.6840 E+02 * x 2-4.7781 E+01 * x J 1.3454 E+00 \times x 4$ $1.9509 E+03 * x \quad-2.3317 E+02 * x_{2} \quad 9.9199 E+00 * x_{3}-8.9773 E-02 * x_{4}$

$\begin{array}{rrrr}6.5612 E+02 * x & -4.4026 E+01 * x 2 & 8.2292 E-01 * x 3 \\ -6.0172 E+02 * x & 2.1300 E+01 * x 2-1.2102 E-01 * x\end{array}$ 


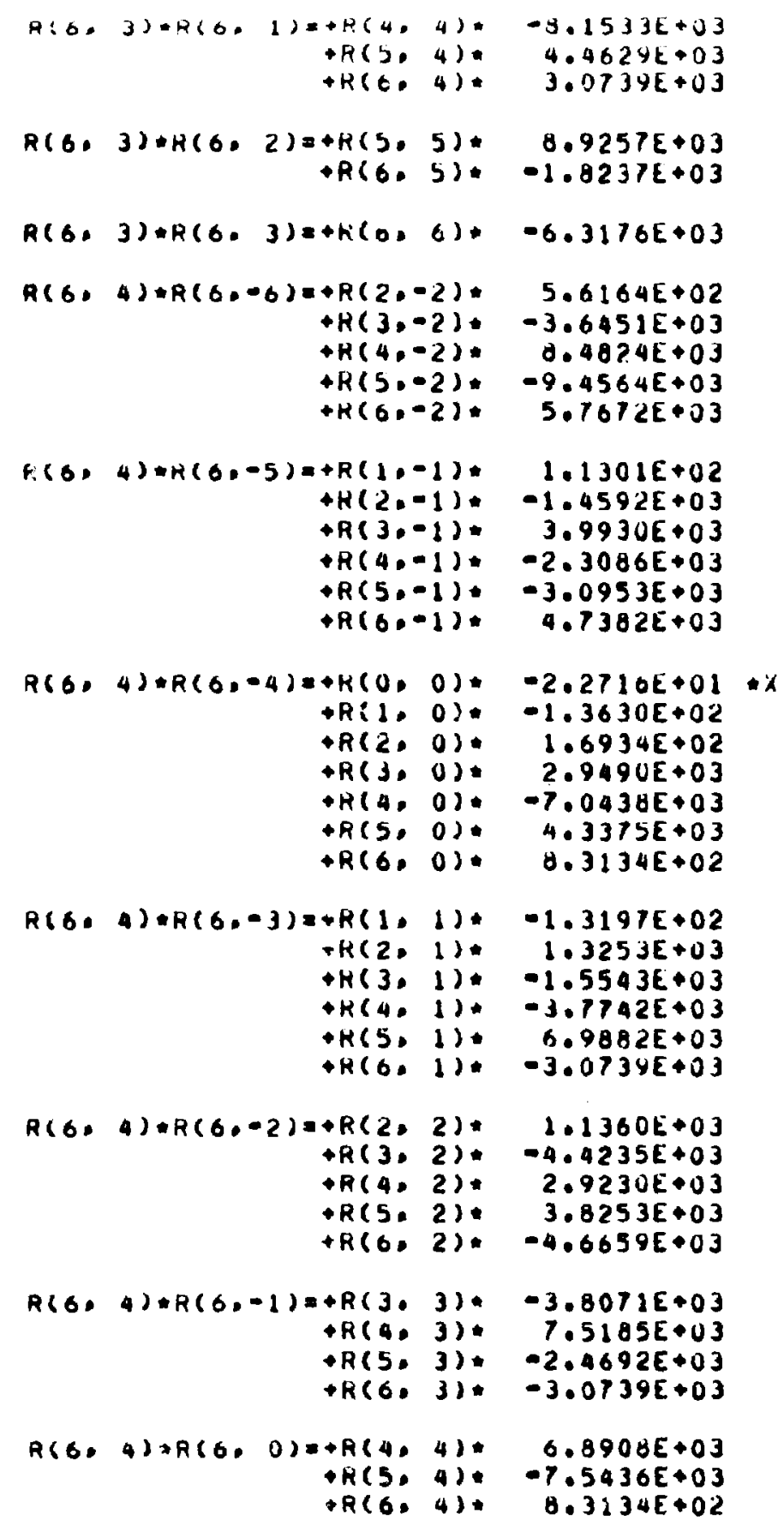




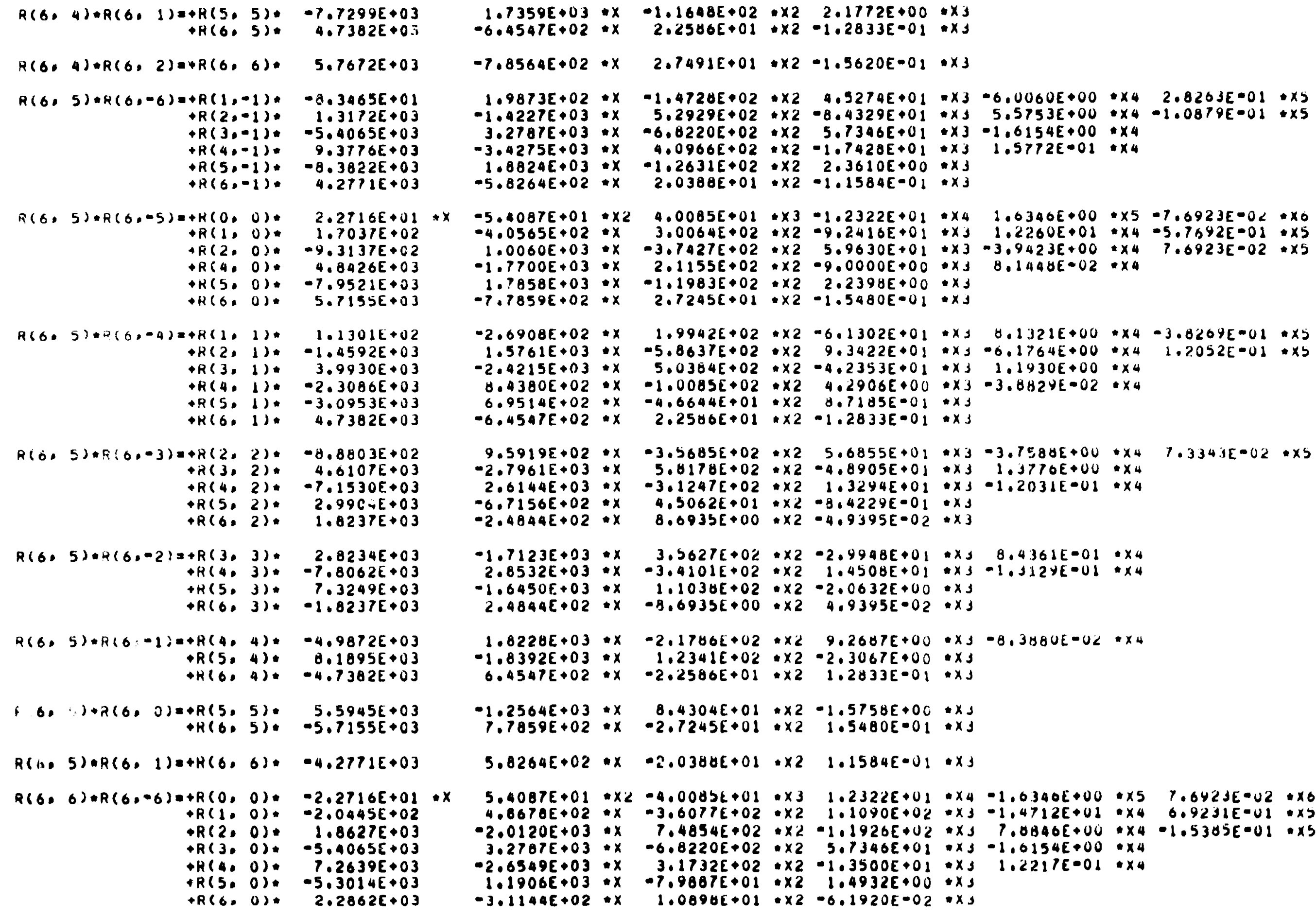
$\begin{aligned} R(6,4) * R(6,1)= & +R(5,5): \begin{array}{r}-7.7299 E * 03 \\ +R(6,5):\end{array} \quad 4.7382 E+0.7\end{aligned}$

$R(6,4) * R(6,2)=4 R(6,6)$.

$R(6,5) * R(6,-6)=+R(1,-1)$ $R(2,-1)$ $+R(3,-1)$ $+R(5,-1)$. $+R(6,-1)$

$R(6,5) * R(6,-5)=+H(0,0)$ $+R(1,0)$ $A R(2,0)$ $+R(4,0)$. $+R(5,0)$

$5.7672 E+03$

$-2.3465 E+01$ $1.3172 E+03$ $1.3172 E+03$
$-5.4065 E+03$ $9.3776 E+03$ $-8.3822 E+03$ $4.2771 E+03$

$2.2716 E+01$ $1.7037 E+02$ $-9.3137 E+C 2$ $4.8426 E+03$ $-7.9521 E+03$
$5.7155 E+03$

$R(6,5), P(6,-4)=+R(1,1)=$ $\rightarrow R(2,1)$. $\rightarrow R(3,1)$. R $R(4,1)$, 1 $\rightarrow K(6,1)$

$1.1301 \mathrm{E}+02$ $1.4592 \mathrm{E}+03$ $3.9930 E+03$ $-2.3086 E+03$ $-3.0953 E+03$ $4.7382 E \bullet 03$

$\begin{aligned} R(0,5)+R(0,-3)= & +R(2,2): \\ & +R(3,2) \\ & +R(4,2)\end{aligned}$ $+R(3,2)$. $+R(4,2):$ $+R(5,2):$
$+R(6,2):$

$-8.8803 E+02$ $4.6107 E+03$ $-7.1530 E+03$ $2.99 C-E+03$ $1.8237 E+03$

$R(6,5)+R(6,-2)=+R(3,3) *$ $+R(4,3):$
$+R(5,3)$ $+R(5,3):$
$+R(6,3):$ $2.8234 E+03$
$-7.8062 E+03$
$-7.3249 E+03$ $7.3249 E+03$
$-1.8237 E+03$

$R(6,5) * R(6:-1)=+R(4,4) *-4.9872 E+03$ $+R(5.4)=0.1895 E+03$ $\rightarrow H(6,4)=-4.7382 E+03$

$\begin{aligned}(6, \cdot)+R(6,0)=+R(5,5), & 5.5945 E+03 \\ & +R(6,5),-5.7255 E+03\end{aligned}$ $R(1,5) \cdot R(6,1)=+R(6,6),-4.2771 E+03$ $\begin{aligned} R(6,6) * R(6,-6)+ & +R(0,0):-2.2716 E+01 * x \\ & +R(1,0) *-2.0445 E+02\end{aligned}$ $\rightarrow R(2,0): 1.8627 E+03$ $+R(3.0)=-5.4065 E+03$ $+R(4.0)=7.2639 E+03$ $+R(5.0)=-5.3014 E+03$ $+R(6,0) .2 .28626+03$

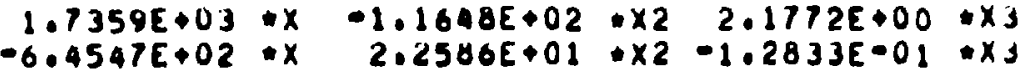

$-7.8564 E+02 * x \quad 2.7491 E+01 * \times 2-1.5620 E-01 * 3$

$1.9873 E+02 * x-1.4724 E+02 * \times 2 \quad 4.3274 E+01=x 3-6.0060 E+00 * x 4 \quad 2.0263 E+01 * x 5$ $-1.4227 E+03: x \quad 5.2929 E+02 * x 2-8.4329 E+01 * x J 5.5753 E+00 * x 4-1.0879 E-01 * \times 5$ $3.2787 E+03 * x-6.0220 E+02 * x 2 \quad 5.7346 E+01+x 3-1.6154 E+00 * x^{2}$

$100060+02 * x 2-1.7428 E+01 * x_{3}-1.5772 E=01 * x_{4}$

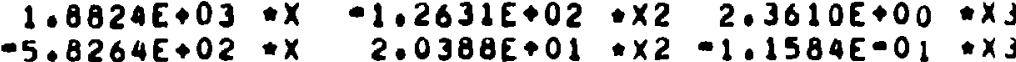

-5.4087E+01*X2 4.0085E+01*X3-1.2322E+01*x4 $1.0340 E+00 * \times 5-7.6923 E-02 * \times 0$ $-4.0565 E+02 * x \quad 3.0064 E+02 * \times 2-9.2416 E+01 * x 3 \quad 1.2260 E+01 * \times 4-5.7642 E-01 * \times 5$ $1.0060 E+03 * x-3.7427 E+02 * \times 2 \quad 5.9630 E+01 * x 3-3.9423 E+00 * \times 4 \quad 7.6923 E-02 * \times 5$ $-1.7700 E+03 * x \quad 2.1155 E+02 * \times 2-9.0000 E+00 * x 3 \quad 8.1448 E=02: \times 4$

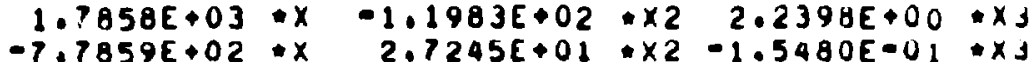

$-2.6908 E+02 * x \quad 1.9942 E+02 * \times 2-6.1302 E+01 * x 3 \quad 0.1321 E+00 * \times 4-3.8209 E-01 * \times 5$ $1.5761 E+03 * x-5.8637 E+02 * x 29.3422 E+01 * x 3-0.1764 E+00 * x 4 \quad 1.2052 E-01 * x b$ $-2.4215 E+03 * x \quad 5.0304 E+02 * x 2-4.2353 E+01 * x 3=1.1930 E+00 * x 4$ $0.4380 E+02 * x-1.0085 E+02 * x 24.2906 E+00 * x 3=3.8 B 2 y E=02 * x$

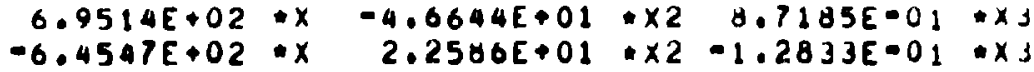

$9.5919 E+02 * x-3.5685 E+02 * \times 2 \quad 5.6855 E+01 * x 3-3.7588 E+00 * \times 4 \quad 7.3343 E-02 * \times 5$ $-2.7961 E+03 * x \quad 5.8178 E+02 * x 2-4.8905 E+01 * x 3 \quad 1.3770 E+00 * x$

$2.6144 E+03 * x-3.1247 E+02 * x 2 \quad 1.3294 E+01$ *XJ $-1.2031 E=01 * x$

$\begin{array}{lll}-6.7156 E+02 * x & 4.5062 E+01 * x 2-8.4229 E-01 * x \\ -2.4 B 44 E+02 * x & 8.6935 E+00 * x 2-4.9395 E-02 * x 3\end{array}$

$-1.7123 E+03 * x \quad 3.5627 E+02 * x 2-2.9998 E+01 * x y-0.4361 E=01 * x$

$2.8532 E+03 * x-3.4101 E+02 * x 2 \quad 1.4508 E+01 * x 3-1.312 Y E=01 * x$

$-1.6450 E+03: x \quad 1.1036 E+02 * x 2-2.0632 E+00 * x J$

$2.4844 E+02 * x-8.6935 E+00 * x 2 \quad 4.9395 E-02 * x$

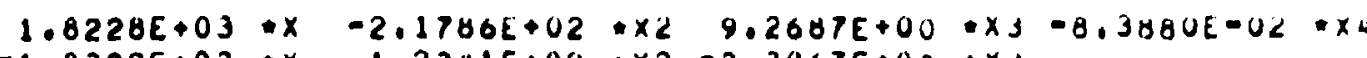

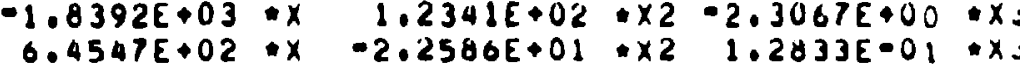

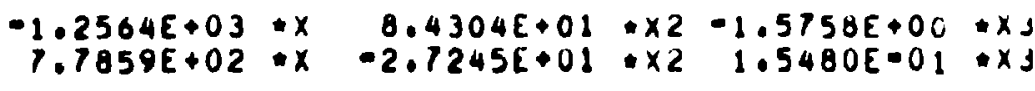

$5.0264 E+02 * x \quad-2.03846+01 \cdot \times 2 \quad 1.1584 E-01 \cdot x J$

$5.4087 E+01: \times 2-4.00056+01 * x 3 \quad 1.2322 E+01 * x 4-1.0340 E+00 * \times 5 \quad 7.642 J E-02 * \times 0$ $4.8670 E+02 \times x-3.6077 E+02 \times x 2 \quad 1.1090 E+02 \times x 3-1.4712 E+01 * x 40.9231 E-01 * x$ $-2.0120 E+03: x \quad 7.4854 E+02 \times x 2-1.1926 E+02 \times x J 7.0840 E+00 \times x 4-1.5305 E=01 \times x 5$ $3.2787 E+03 \times x \quad-6.8220 E+02 \times x 2 \quad 5.7346 E+01 * x 3-1.0154 E+00 * x 4$ $-2.6549 E+03 * x \quad 3.1732 E+02 * x 2-1.3500 E+01 * x 3 \quad 1.2217 E=01 * x$

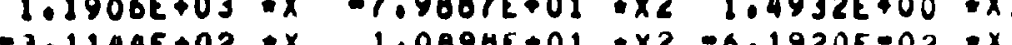
$-3.1144 E+02 * x \quad 1.0896 E+01 * x 2-0.1920 E-02 * x$ 


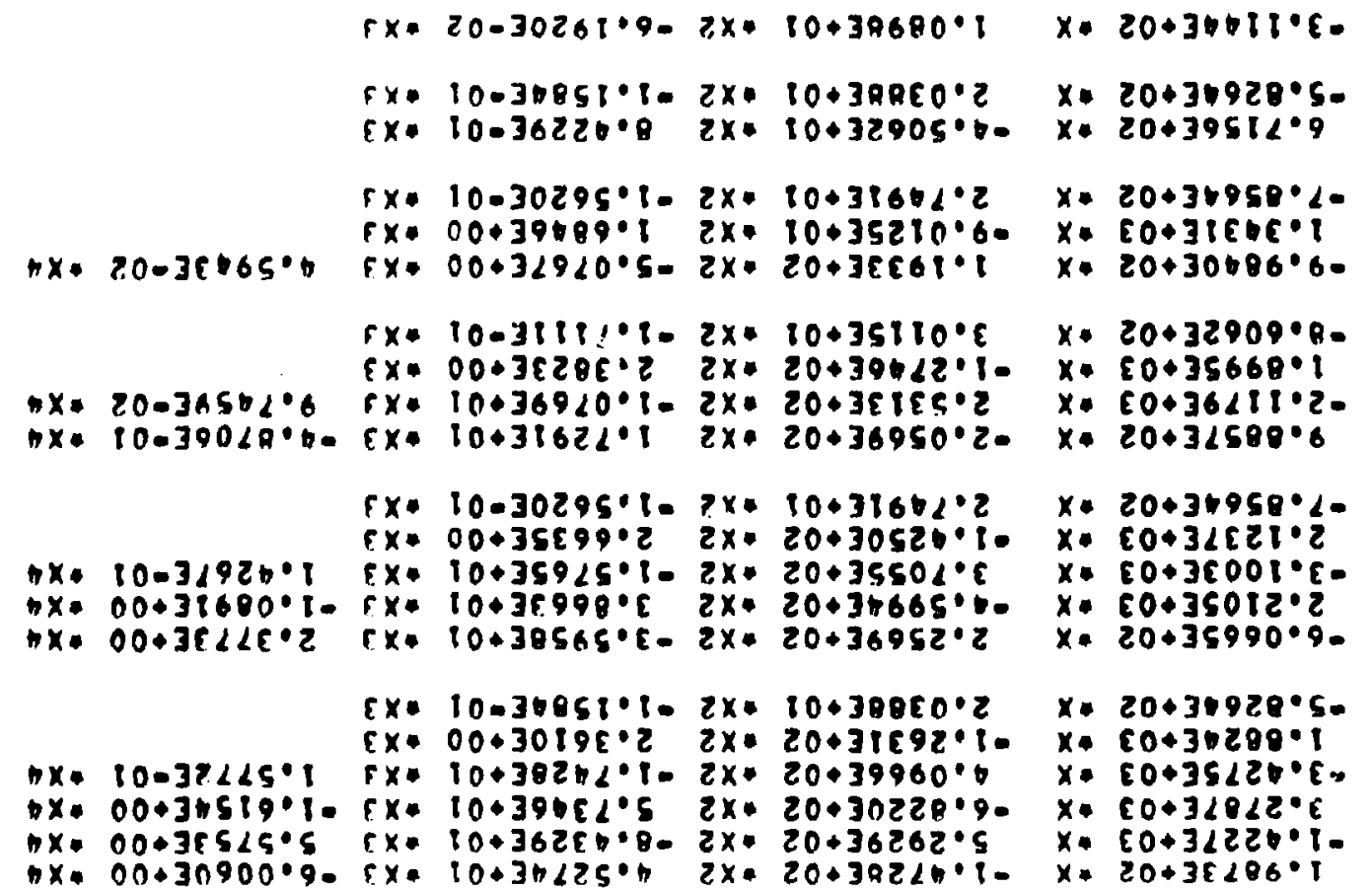

$6 x+20-30261 \cdot 9-z x+10+39680 \cdot 1 \quad x+20+36611 \cdot 6=$

$x+20+32909 \cdot 8=$

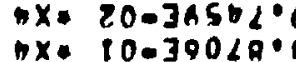

$x \cdot x \cdot 60+3565105$

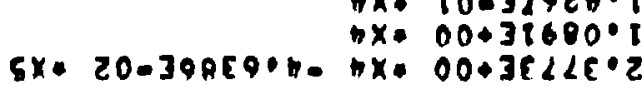

SX. 20.39AC

tx. $00+3+519^{\circ}$

$8 x=3003008002$

$5 x \cdot 10.36280 \cdot 1$

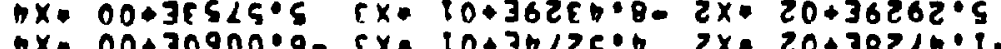

\author{
$(0+32902 \cdot 2 \quad *(9 \cdot 9) 4+010 \cdot 9) 8 \cdot(9 \cdot 9) 4$

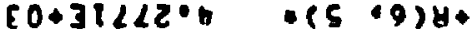 \\ $\left(0+34066^{\circ} z=0(5 \cdot 5) y+=(1-09) 4 *(9 \cdot 0) 8\right.$

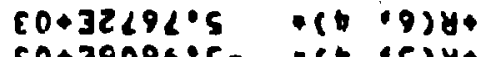

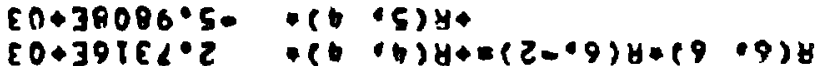 \\ $60+39218.0 \quad(6.9) 40$

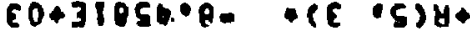

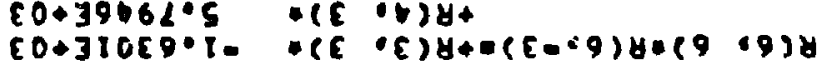 \\ $60+32<92 \cdot 5 \quad(2 \cdot 9) 4 \cdot$ \\ $60+3626.60=(5-2) 4$.

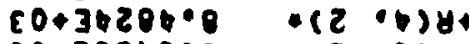

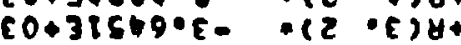

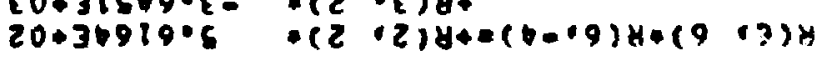 \\ $\cos 31<2 z \cdot 0$ - (1 99$) \mathrm{y}$

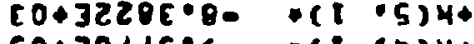 \\ CO+3922द. 6 (1) 0$)$ H

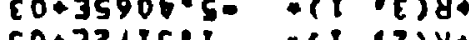

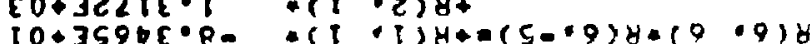




\begin{tabular}{|c|c|c|c|c|c|}
\hline$O(10 C) \cdot 0(10 C)=$ & $\begin{array}{l}-0<00 \mathrm{C}: \\
+0120 \mathrm{C}:\end{array}$ & $\begin{array}{l}3.3333 E=01 \\
3.5333 E=01\end{array}$ & $\cdot x$ & & \\
\hline $0(110) * 0(10 C)=$ & $\begin{array}{l}+\log (115) \\
+0(216)\end{array}$ & $\begin{array}{r}-5.0000 E-01 \\
.0000 E+00\end{array}$ & & & \\
\hline $0(11 C) \cdot 0(11 C)=$ & $\begin{array}{l}+0(00 C) \\
+\quad 0(20 C) \\
+\quad 0(22 C)\end{array}$ & $\begin{array}{r}3.3333 E=01 \\
-1.0691 E=01 \\
5.000 U E-01\end{array}$ & $\cdot x$ & & \\
\hline $0(20 C) \cdot 0(10 C)=$ & $\begin{array}{l}O(10 C) \\
+O(306)\end{array}$ & $\begin{array}{r}-6.0000 E=01 \\
6.0000 E=01\end{array}$ & & $0.0000 E-01$ & $\cdot x$ \\
\hline $0(206) \cdot 0(11 C)=$ & $\begin{array}{l}+0(116) \\
+10(215) \\
+0(316)\end{array}$ & $\begin{array}{l}3.0000 E-01 \\
3.6000 E+00 \\
6.0000 E-01\end{array}$ & & $-4.0000 E-01$ & $\cdot x$ \\
\hline $0(21 C) \cdot 0(10 C)=$ & $\begin{array}{l}+0(11 C): \\
+10(215) \\
+0(31 C)\end{array}$ & $\begin{array}{r}-1.5000 E-01 \\
-5.0000 E-01 \\
2.0000 E=01\end{array}$ & & $2.0000 E-01$ & $\cdot x$ \\
\hline $0(216) \cdot 0(116)=$ & $\begin{array}{l}+0(106): \\
+10(225) \\
+0(30 C) \\
+0(32 C)\end{array}$ & $\begin{array}{r}-1.5000 E-01 \\
2.5000 E=01 \\
-1.0000 E=01 \\
5.0000 E=01\end{array}$ & & $2.0000 E=01$ & $\cdot x$ \\
\hline $0(226) \cdot 0(10 C)=$ & $\begin{array}{l}+10(225) \\
+0(326)\end{array}$ & $\begin{array}{r}-1.0000 E+00 \\
1.000 D E+00\end{array}$ & & & \\
\hline $0(22 c) 00(110)=$ & $\begin{array}{l}+0(116): \\
+0(215) \\
+0(31 C) \\
+0(33 C)\end{array}$ & $\begin{array}{r}-3.0000 E-01 \\
1.0000 E+00 \\
-1.0000 E-01 \\
5.0000 E-01\end{array}$ & & $4.0000 E-01$ & $\cdot x$ \\
\hline $0(20 C)+0(20 C)=$ & $\begin{array}{l}+O(0 O C): \\
+\quad U(20 C) \\
+\quad O(40 C)\end{array}$ & $\begin{array}{r}-6.0000 E=01 \\
-2.1429 E+00 \\
2.5714 E=01\end{array}$ & $\cdot x$ & $\begin{array}{l}8.0000 E-01 \\
5.7143 E-01\end{array}$ & $: x^{x}$ \\
\hline $0(21 C) * 0(20 C)=$ & $\begin{array}{l}+10(115)= \\
+0(216) \\
+10(315) \\
+0(116) .\end{array}$ & $\begin{array}{r}1.5000 E-01 \\
-1.0714 E+00 \\
-6.0000 E-01 \\
1.2037 E-01\end{array}$ & & $\begin{array}{r}-6.00006-01 \\
2.8571 E-01\end{array}$ & $\begin{array}{l}x \\
: x\end{array}$ \\
\hline $0(210) * 0(216)=$ & $\begin{array}{l}+O(00 C) \\
+O(20 C) \\
+O(22 C) \\
+O(A 0 C) \\
+O(A 2 C)\end{array}$ & $\begin{array}{l}-5.0000 E=02 \\
-8.9286 E=02 \\
-2.6786 E=01 \\
-1.4286 E=02 \\
7.1429 E-02\end{array}$ & $\cdot x$ & $\begin{array}{l}6.6667 E-02 \\
2.36106-02 \\
7.1429 E-02\end{array}$ & $\begin{array}{l}x z \\
: x \\
: x\end{array}$ \\
\hline
\end{tabular}




\begin{tabular}{|c|c|c|c|c|}
\hline$a(220)+0(20 c)=$ & $\begin{array}{l}+0(220) \\
+10(325): \\
+0(426)\end{array}$ & $\begin{array}{r}2.1429 E+U 0 \\
-6.0000 E+00 \\
4.2957 E=U 1\end{array}$ & $-5.7143 E-01$ & $* x$ \\
\hline $0(22 C) \cdot 0(216)=$ & 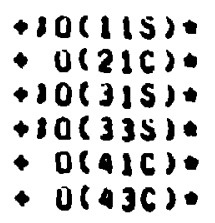 & $\begin{array}{r}1.5000 E-01 \\
-1.0714 E+00 \\
3.0000 E-01 \\
-5.0000 E-01 \\
-7.1429 E-02 \\
5.0000 E-01\end{array}$ & $\begin{array}{r}-2.0000 E=01 \\
2.8571 E=01\end{array}$ & $: x$ \\
\hline $0(22 c) \cdot u(22 c)=$ & $\begin{array}{l}+0(00 C): \\
+\quad 0(20 C): \\
+0(40 C): \\
+0(44 C)\end{array}$ & $\begin{array}{l}-2.0000 E-01 \cdot x \\
7.1429 E-01 \\
1.0206 E-02 \\
5.0000 E-01\end{array}$ & $\begin{array}{r}2.6667 E=01 \\
-1.9068 E=01\end{array}$ & $: x 2$ \\
\hline $0(300)=0(100)=$ & $\begin{array}{l}O(20 C) \\
-O(40 C)\end{array}$ & $\begin{array}{r}-8.5714 E=01 \\
1.4266 E=01\end{array}$ & $4.2057 E-01$ & $* x$ \\
\hline$J(30 C)=0(11 C)=$ & $\begin{array}{l}0(216): \\
+\log (315): \\
+\quad 0(416):\end{array}$ & $\begin{array}{l}1.71 \backsim 3 E+00 \\
1.5000 E+00 \\
7.1429 E-01\end{array}$ & $-n .5714 E-01$ & $\bullet x$ \\
\hline $0(310)=0(100)=$ & $\begin{array}{l}+0(216): \\
+\operatorname{O}(315): \\
+0(416) .\end{array}$ & $\begin{array}{r}-2.2057 E+00 \\
-5.0000 E=01 \\
7.1429 E=01\end{array}$ & $1.1429 E+00$ & $\cdot x$ \\
\hline $0(316) * 0(110)=$ & $\begin{array}{l}+0(206): \\
0(226) \\
+0(325) \\
+0(406) \\
+0(42 C)\end{array}$ & $\begin{array}{r}-5.7143 E-01 \\
2.8571 E-01 \\
2.5000 E+00 \\
-7.1129 E-02 \\
3.5714 E-01\end{array}$ & $\begin{array}{r}2.8571 E-01 \\
-1.4280 E=01\end{array}$ & $\begin{array}{l}x \\
x \\
x\end{array}$ \\
\hline $0(32() \cdot 0(10 C)=$ & $\begin{array}{l}+O(226): \\
+\quad 0(325): \\
+\quad 0(426):\end{array}$ & $\begin{array}{r}-2.0571 E-01 \\
-1.0000 E+00 \\
1.4286 E-01\end{array}$ & $1.4286 E-01$ & $\| x$ \\
\hline $0(32()+0(116)=$ & $\begin{array}{l}\quad 0(21 C): \\
+10(315): \\
+\quad 0(33 S): \\
+0(416): \\
+0(43 C):\end{array}$ & $\begin{array}{r}-5.7143 E-01 \\
2.5000 E-01 \\
2.5000 E-01 \\
-7.1429 E-02 \\
3.0000 E-01\end{array}$ & $2.8571 E-01$ & $\cdot x$ \\
\hline$O(33 C)+0(10 C)=$ & $\begin{array}{l}+10(335): \\
+0(436):\end{array}$ & $\begin{array}{r}-1,3000 E+00 \\
1.0000 E+00\end{array}$ & & \\
\hline $0(33 C)+0(11 C)=$ & $\begin{array}{l}+0(22 C): \\
+10(325): \\
+0(42 C): \\
+0(446):\end{array}$ & $\begin{array}{r}-8.5714 E-01 \\
1.5000 E+00 \\
-7.1429 E-02 \\
5.0000 E-01\end{array}$ & $4.2057 E-01$ & $* x$ \\
\hline
\end{tabular}




\begin{tabular}{|c|c|c|c|c|c|c|}
\hline o(30C)=0r $\quad: j$, & $\begin{array}{l}+0(10 C): \\
+0(30 C) \\
+0(50 C)\end{array}$ & $\begin{array}{r}1.5429 E+00 \\
-4.4000 E+00 \\
2.3810 E-01\end{array}$ & $\begin{array}{r}-2.8286 E+00 \\
5.3333 E=01\end{array}$ & $: x$ & $1.0266 E+00$ & $+\times 2$ \\
\hline $0(300)=0(210$ & $\begin{array}{r}0(11 C) \\
+20(215) \\
0(316) \\
+0(15) \\
\partial(51 C)\end{array}$ & $\begin{array}{r}-2.5714 E-01 \\
-3.4286 E+00 \\
-5.5000 E-01 \\
1.0714 E+00 \\
2.3810 E-01\end{array}$ & $\begin{array}{l}4.7143 E=01 \\
1.7143 E+00 \\
6.6667 E=02\end{array}$ & $\begin{array}{l}: x \\
: x \\
: x\end{array}$ & $-1.7143 E-01$ & $\times 2$ \\
\hline $0(300) \cdot 0(226)=$ & 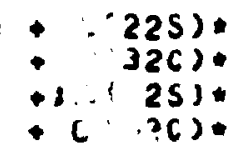 & $\begin{array}{l}1.7143 E+00 \\
1.1000 E+01 \\
2.1429 E+00 \\
1.6667 E+00\end{array}$ & $\begin{array}{l}-8.5714 E=01 \\
-1.3333 E+00\end{array}$ & $\cdot x$ & & \\
\hline $0(3 \& C) * 0(20 C)=$ & $\begin{array}{l}+0(11) \\
+10(25) \\
+0(3) \\
+10(415) \\
+0(516)\end{array}$ & $\begin{array}{r}1.0286 E+30 \\
6.8571 E+00 \\
-3.3000 E+00 \\
-2.1429 E+00 \\
7.1429 E-01\end{array}$ & $\begin{array}{r}-1.8857 E+00 \\
-3.4206 E+00 \\
4.0000 E=01\end{array}$ & $\begin{array}{l}=x \\
x x \\
=x\end{array}$ & $6.8571 E-01$ & $\times 2$ \\
\hline $0(32() \cdot 0(2)=)=$ & $\begin{array}{l}+0(100) \\
+10(225) \\
+0(306) \\
+0(326) \\
+10(425) \\
+0(506) \\
+0(526)\end{array}$ & $\begin{array}{r}3.4286 E=01 \\
-8.5714 E=02 \\
-3.6667 E=01 \\
.2 .7500 E+00 \\
1.7657 E=01 \\
.9683 E=02 \\
.3333 E=01\end{array}$ & $\begin{array}{r}-6.285 T E=01 \\
4.2857 E=01 \\
4.4444 E=02 \\
3.3333 E=01\end{array}$ & $\begin{array}{l}: x \\
4 x \\
: x \\
x\end{array}$ & $2.2857 E \cdot 01$ & $-4 \times 2$ \\
\hline $0(310)=0(220)=$ & $\begin{array}{l}+O(116) \\
+10(215) \\
+O(31 C) \\
+0(336) \\
+10(915) \\
+10(435) \\
+0(516) \\
+0(53 C)\end{array}$ & 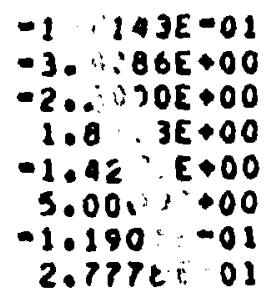 & $\begin{array}{r}3.1429 E-01 \\
1.7143 E+00 \\
2.6667 E=01 \\
-2.2222 E-01\end{array}$ & $\begin{array}{l}* x \\
: x \\
: x \\
* x\end{array}$ & $-1.2420 E-01$ &.$\times 2$ \\
\hline $0(32 C) \oplus 0(20 C)=$ & $\begin{array}{l}+10(225): \\
+10(425) \\
+0(526)\end{array}$ & $\begin{array}{l}1.7143 E \\
-8.5714 E- \\
1.0000 E+1\end{array}$ & $-8.5714 E=02$ & $-x$ & & \\
\hline $0(3 c()+0(21 C)=$ & 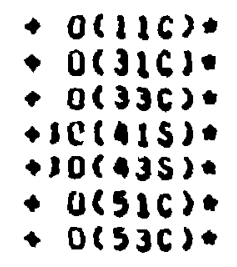 & $\begin{array}{r}0.5714 E=0 E \\
-2.7500 E=01 \\
-1.5833 E=01 \\
2.3000 E=01 \\
-2.5000 E=01 \\
-2.3810 E-02 \\
5.5556 E-02\end{array}$ & $\begin{array}{r}-1.5714 E-01 \\
3.3333 E-02 \\
5.5556 E-02\end{array}$ & $\begin{array}{l}-x \\
: x \\
: x\end{array}$ & $5.714 \mathrm{JE}-02$ & $\times 2$ \\
\hline
\end{tabular}




\begin{tabular}{|c|c|c|c|c|c|c|c|}
\hline 0.32()$=0(22())=$ & $\begin{array}{l}+0(100) \\
+0(306) \\
+10(445): \\
+0(50 C): \\
+0(546)\end{array}$ & $\begin{array}{l}1.7143 E=01 \\
7.3333 E-01 \\
5.0000 E=01 \\
7.9365 E=03 \\
5.0000 E-01\end{array}$ & & $\begin{array}{l}-3.14<E-01 \\
-8.88 t+t^{-0}-02\end{array}$ & $x_{0 x}^{* x}$ & $1.1424 E-01$ & $1 \times 2$ \\
\hline $0(336) \div 0(20 C)=$ & $\begin{array}{l}+O(336) \\
+10(435) \\
+O(536)\end{array}$ & $\begin{array}{r}5.5000 E+00 \\
-9.0000 E+00 \\
3.3333 E-01\end{array}$ & & $-0.6667 E-01$ & $\bullet x$ & & \\
\hline $0(336)=0(216)=$ & $\begin{array}{l}+80(225): \\
+0(32 C): \\
+0(425): \\
+10(445) . \\
+0(526) \\
+0(54 C) .\end{array}$ & $\begin{array}{r}0.3714 E-01 \\
-2.7500 E+00 \\
3.2143 E-01 \\
-7.5000 E-01 \\
-1.6667 E-01 \\
5.0000 E-01\end{array}$ & & $\begin{array}{r}-4.2857 E=01 \\
3.3333 E=01\end{array}$ & $: x$ & & \\
\hline $0(33 C)=0(22 C)=$ & $\begin{array}{l}+0(11 C) . \\
+\quad 0(215) \\
+0(31 C) \\
+0(115) \\
+0(51 C) . \\
+0(55 C) .\end{array}$ & $\begin{array}{r}3.1429 E-01 \\
-3.4206 E+00 \\
1.1000 E+00 \\
-4.2057 E-01 \\
2.3010 E=02 \\
5.0000 E-01\end{array}$ & & $\begin{array}{r}-9.4286 E-01 \\
1.7143 E+00 \\
-1.3333 E-01\end{array}$ & $\begin{array}{l}x \\
: x \\
x\end{array}$ & $\therefore .4286 E=01$ & 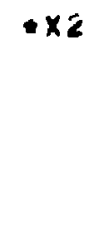 \\
\hline $0(300)=0(30 C)=$ & $\begin{array}{l}+0(00 C) \\
+0(20 C) \\
+\quad 0(40 C) \\
+\quad 0(60 C)\end{array}$ & $\begin{array}{r}0.5114 E-01 \\
6.20576+00 \\
-1.9870 E+00 \\
1.0023 E-01\end{array}$ & $\cdot x$ & $\begin{array}{r}-1.571 A E+00 \\
-3,9040 E+00 \\
1.1688 E-01\end{array}$ & $\begin{array}{l}: x 2 \\
: x \\
: x\end{array}$ & $\begin{array}{l}5.7143 E=01 \\
3.0095 E-01\end{array}$ & $\begin{array}{l}: \times 3 \\
: \times 2\end{array}$ \\
\hline $0(31 C)+0(30 C)=$ & $\begin{array}{l}+80(115) . \\
+0(216) \\
+8(315) \\
+0(416) . \\
+80(515) \\
+0(616) .\end{array}$ & $\begin{array}{r}-2.5714 E+00 \\
6.2857 E+00 \\
9.0000 E+00 \\
-6.6234 E+00 \\
-1.7857 E+00 \\
7.5758 E-01\end{array}$ & & $\begin{array}{r}1.7143 E+00 \\
-3.9048 E+00 \\
-2,00005+100 \\
3.0961 E-01\end{array}$ & $\begin{array}{l}* x \\
: x \\
* x \\
* x\end{array}$ & $\begin{array}{r}-1.71436+00 \\
3.8095 E-01\end{array}$ & $\begin{array}{l}: \times 2 \\
02\end{array}$ \\
\hline $0(316) \cdot 0(31 C)=$ & 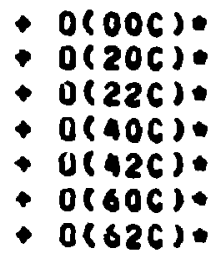 & $\begin{array}{r}5.71436-01 \\
3.14296+00 \\
6.2857 E+00 \\
-2.2078 E-01 \\
-4.4156 E+00 \\
-5.4113 E-02 \\
3.7879 E-01\end{array}$ & $\cdot x$ & $\begin{array}{r}-1.0476 E+C O \\
-1.952 A E+C O \\
-3.9040 E+C O \\
1.2967 E-02 \\
2.3974 E-O 1\end{array}$ & $\begin{array}{l}: x 2 \\
: x \\
: x \\
: x \\
: x\end{array}$ & $\begin{array}{l}3.8095 E-01 \\
1.9040 E=01 \\
3.0095 E-01\end{array}$ & $\begin{array}{l}\times 3 \\
\times 1 \times 2 \\
: \times 2\end{array}$ \\
\hline $0(32 C) * 0(30 C)=$ & $\begin{array}{l}+U(22 C): \\
+10(325) \\
+0(126) \\
+10(525) \\
+0(826)\end{array}$ & $\begin{array}{r}-3.142 Y E+00 \\
9.0000 E+00 \\
6.6234 E-01 \\
-5.0000 E+00 \\
1.5152 E-01\end{array}$ & & $\begin{array}{r}1.9524 E+00 \\
-2.0000 E+00 \\
-3.0961 E-02\end{array}$ & $\begin{array}{l}: x \\
: x \\
: x\end{array}$ & $-1.9048 E-01$ &.$\times 2$ \\
\hline
\end{tabular}




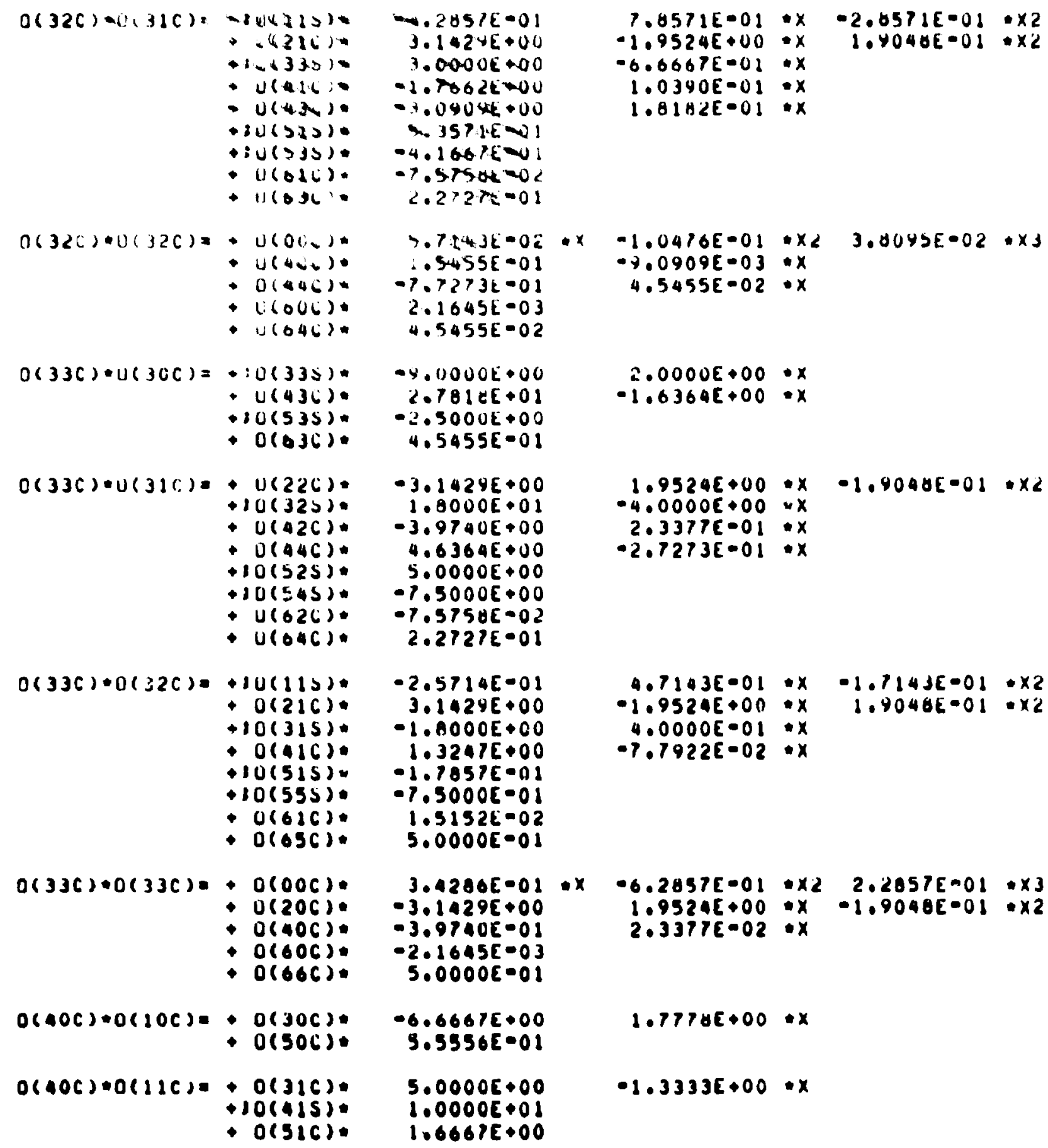

\section{$-6.2857 E-01 * x^{2} \quad 2.2057 E-01 * x 3$ $1.9524 E+00: x-1.9040 E-01: x 2$} $2.3377 E-02$ : $x$

$1.77786+00 \cdot x$

$\bullet 1.3333 E \bullet 00 \cdot x$ 


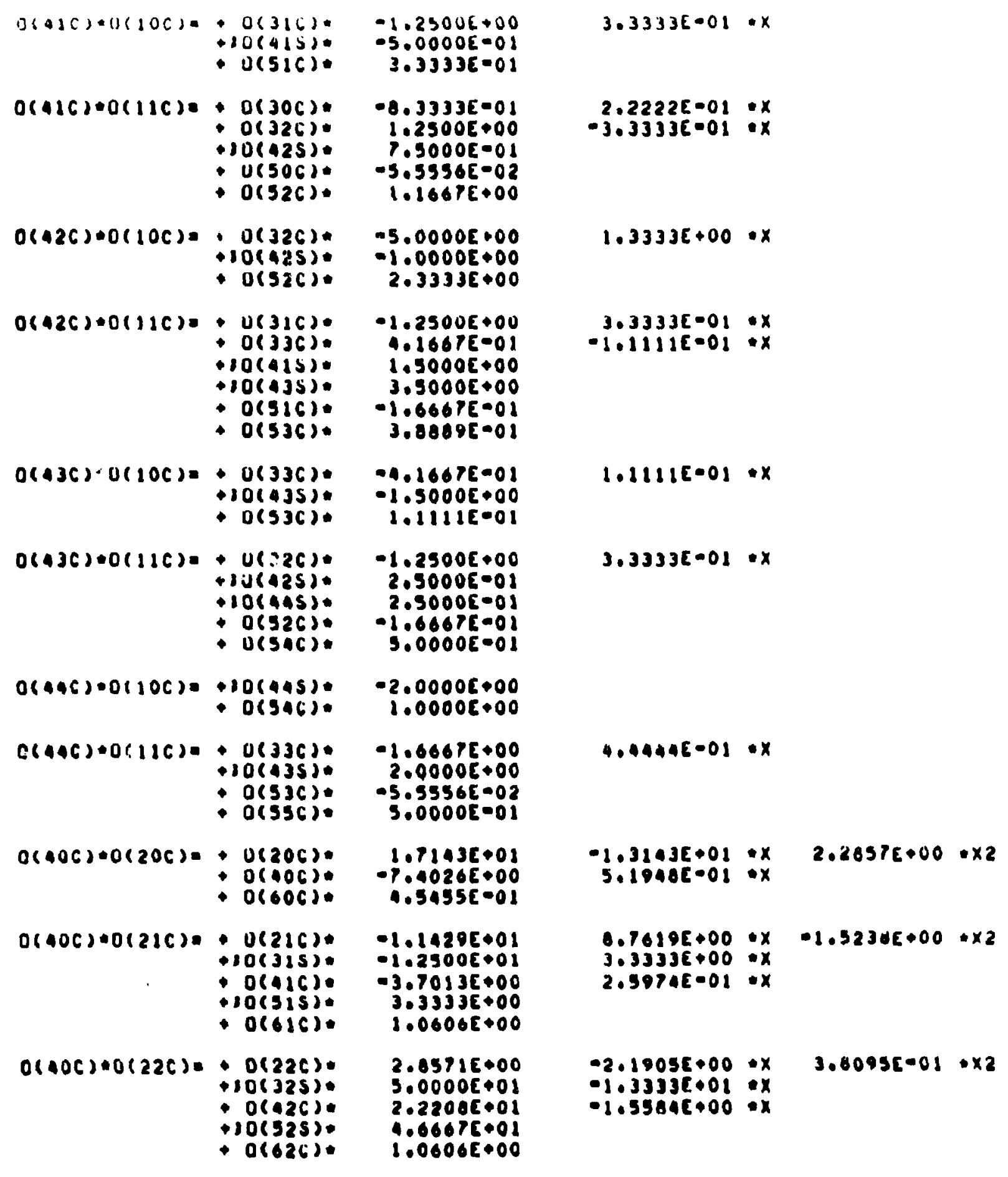




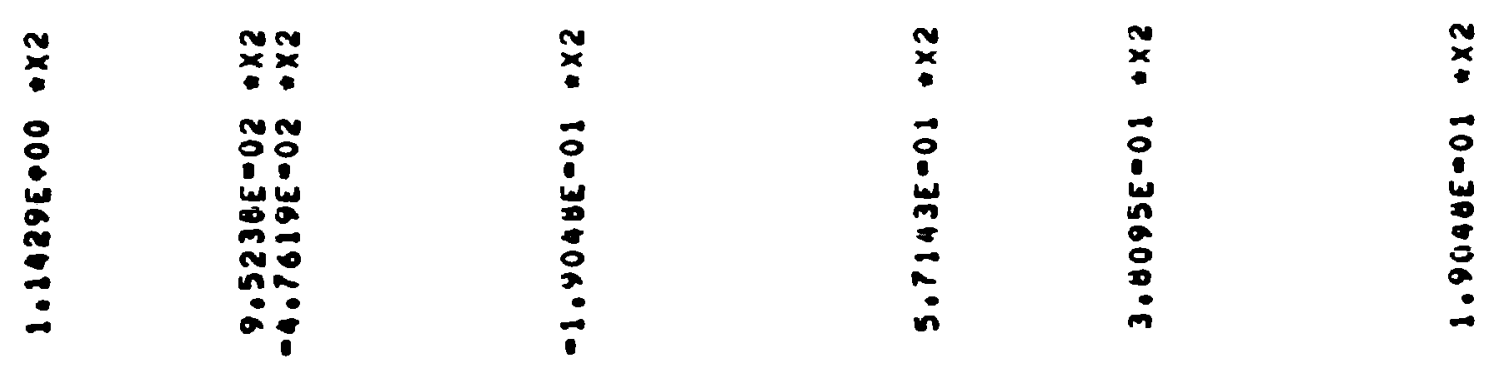

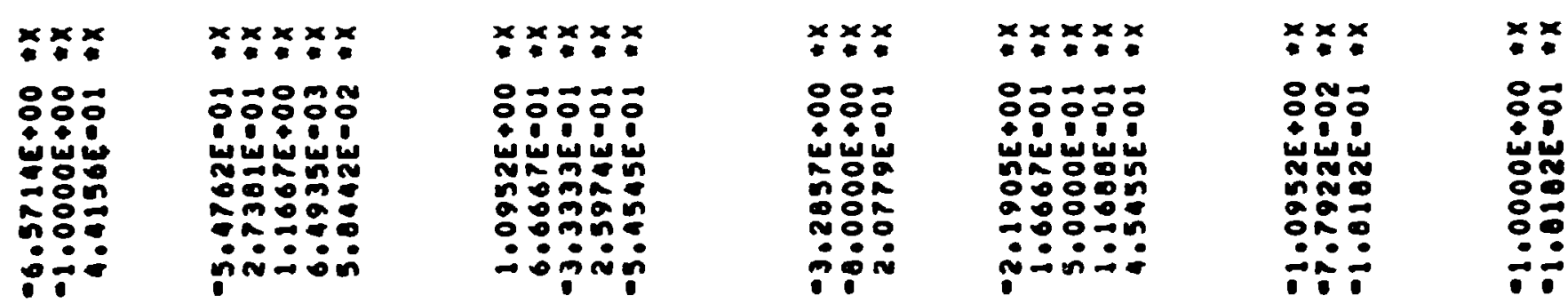
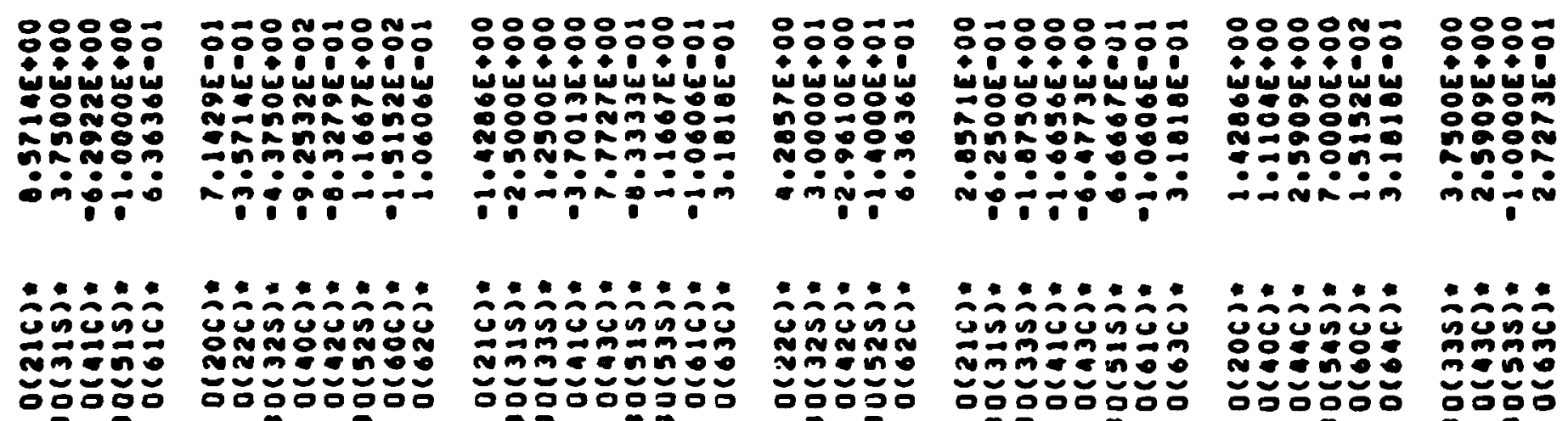

추눈 응으. ธоำㅇํำ

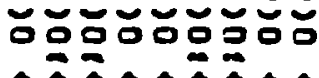

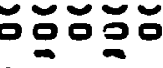

:

$\frac{a}{0}$

¿ัญ

ญ̊

ปั)

: 


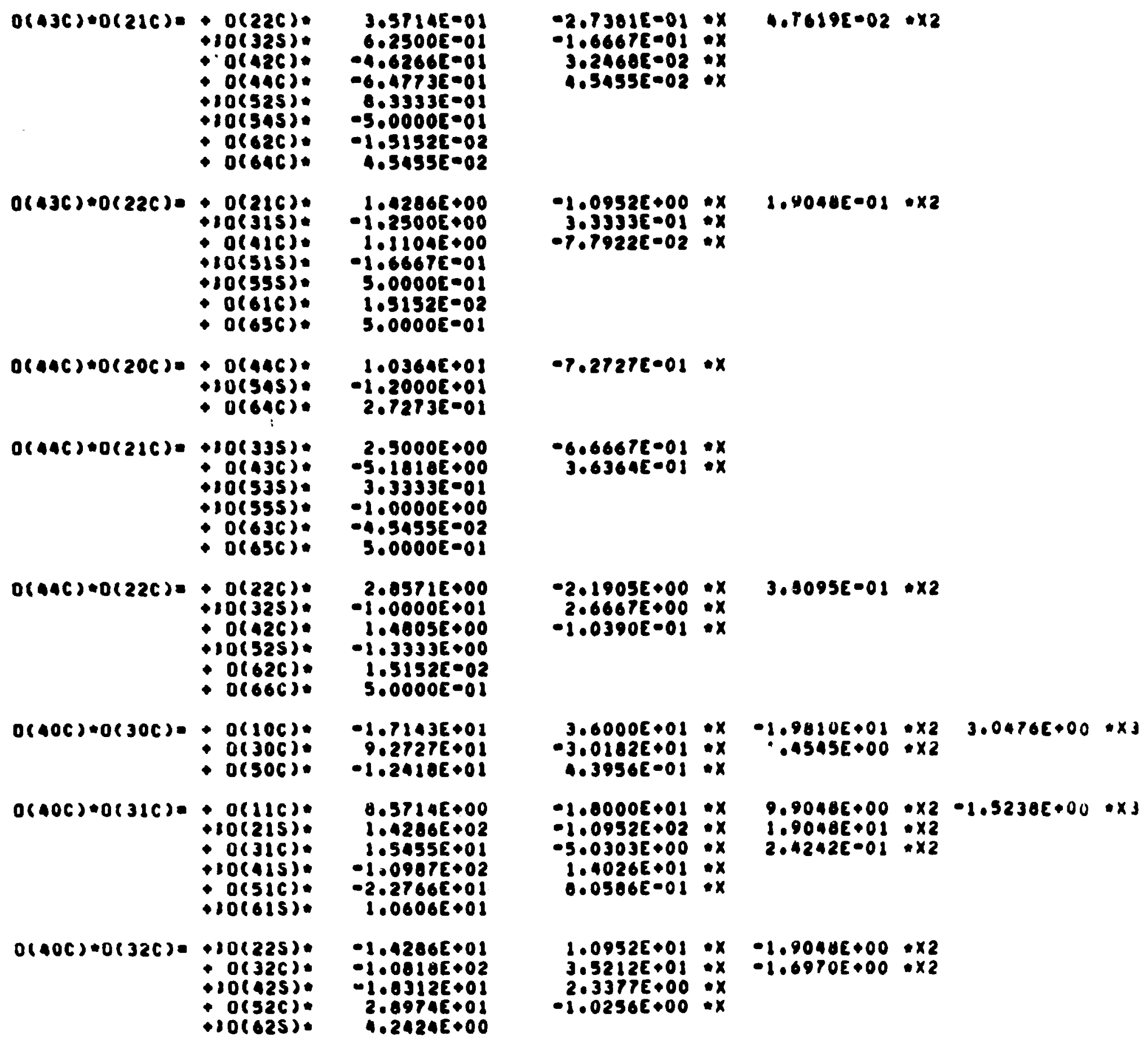

$-2.73018-01$-x

$1.06676-01$ :x

$\begin{array}{ll}3.24685-02 & : x \\ 4.54555-02 & +x\end{array}$

4.54558002

$1.09526+000 x$ $3.3333 E 001: x$ $-7.7922 E-02$ ix

$-7.2727 E-01 \cdot x$

0.66676001 : $x$ $3.6364 \varepsilon-01: x$

$-2.1905 E+00 * x \quad 3.5095 E-01 * \times 2$ $2.6667 E+00$ *x $-1.0390 E-01 \cdot x$

$1090406-01 * 2$ $. .76195 \cdot 02 \cdot \times 2$

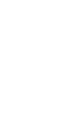




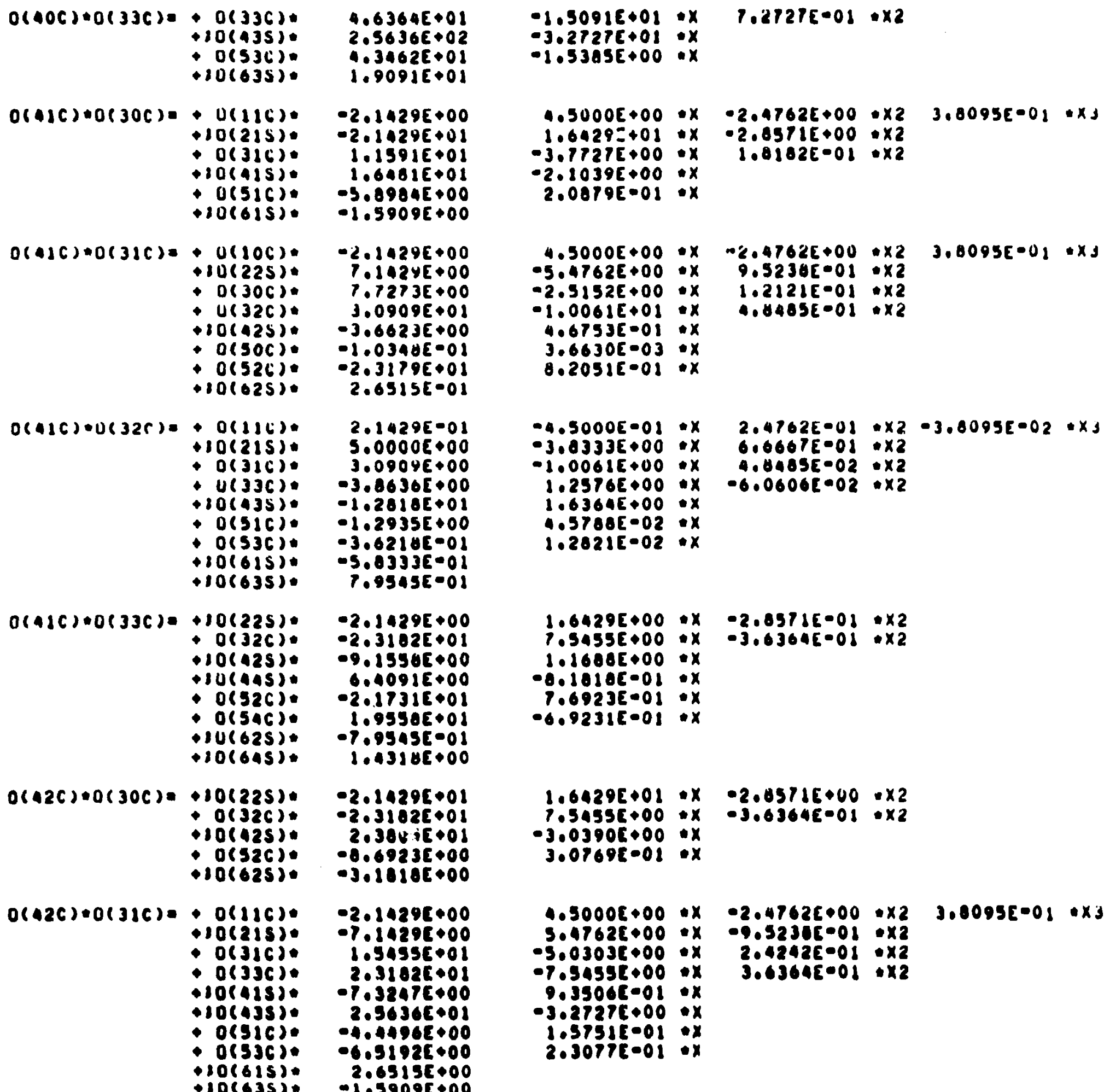

$-2.1429 E+00$ $7.142 \times E+00$ $7.7273 E+00$ $3.0909 E+01$ $-3.66235+00$ $-1.03906-01$ $-2.31796+01$ .

$0(416) \cdot 0(32 r)=0(116)=$ $10(215)$.

$0(316)$

$+20(435)$

- o(sic).

$+0(536)$

$10(6) 5)$

$+10(635)$.

$2.1429 E-01$

$5.0000 E+00$

$3.0904 E+00$

$-3.0636[+00$

$-1.28185+01$

$-1.2935 E+00$

$-3.62186-01$

$-5.03336-01$

$0(18 C) 00(33 C)=+10(225)=$

$-2.14295+00$

$-2.310$

io(azs).

$-2.17315+01$

$1.9550 E+01$

- o(5ac).

$+10(645)$.

$-7.9545 E-01$

.

$0(12 C)=0(30 C)=+10(225)=$

$10(425)$.

$-2.14295+0$

$2.384 i 5+01$

$-8.6923 E \bullet 00$

$0(526): \quad-0.6923 E+00$
$+10(625), \quad-3.18185+00$

$O(42 C) \cdot 0(316)=+0(116)=$

$-2.14298+00$

$+10(215)$

$-7.14208+00$

O(3)

$1.54556+01$

0(33c).

Ho(nis).

2.31026001

1.386reson

- iocasss. 2.5636600

- Orsics.

- Orsscj. 0.5192500

$10(615)$ 2.6515E.00

$-1.50916001 * x \quad 1.2727 E-01 \cdot \times 2$

$-3.2727 E+01: x$

$1.5000 E+00 * x \quad-2.4762 E+00 * x 2 \quad 3,0095 E-01 * x 3$

$1.6429=+01 * x-2.8571 E+00 * x 2$

$-3.7727 E+00 \cdot x \quad 1.8182 E-01 \cdot \times 2$

$-2.1039 E+00: x$

$2.0879 E=01 \cdot x$

$4.5000 E+00 * x \times \% .4762 E+00 \times 2 \quad 3.8095 E-02 \times x J$

$-5.47625+00: x \quad 9.52345-01: \times 2$

$-2.5152 E+00 * x \quad 1.2121 E-01 \times x 2$

$-1.0061 E+01: x \quad \square .0405 E-01: x 2$

$4.6753 E-01: x$

$3.66305-03: x$

$0.20515001: x$

$-4.5000 E-01 * x \quad 2.4762 E-01 * x_{2}-3.8095 E-02 * x_{3}$

$-3.0333 E+00$ \& $6.6667 E-01$ x2

$-1.00615+00$ \& $4.8485 E-02 \times x 2$

$1.23765+00$ : $x \quad-6.0606[-02: \times 2$

$1.63645+00 * x$

$0.57805-02 \cdot x$

$1,28215002 \cdot x$

$1.6429 E+00: x-2.0571 E-01 * x 2$

$1.5455 E+00 * x-3.6364 E-01 * \times 2$

$1,1608 E+00 \cdot x$

$-0.10106-01 \cdot x$

$7.6023 E \cdot 01 \cdot x$

$-6.92316-01$ : $x$

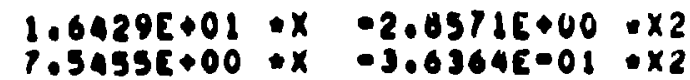
$-3.03906+00: x$ $3.07608-01 \cdot x$

4.50008000 .

$-2 \cdot 47626 \cdot 00 \cdot \times 2$

5.4762E +00 :x $-9.52306-01 \quad \times 2$

$-5.03035+00$ : $x \quad 2.42426-01$ *x

-

- . 3506e-01 ux

- 3.272ripoo $x$

2.30775001 : $x$ 


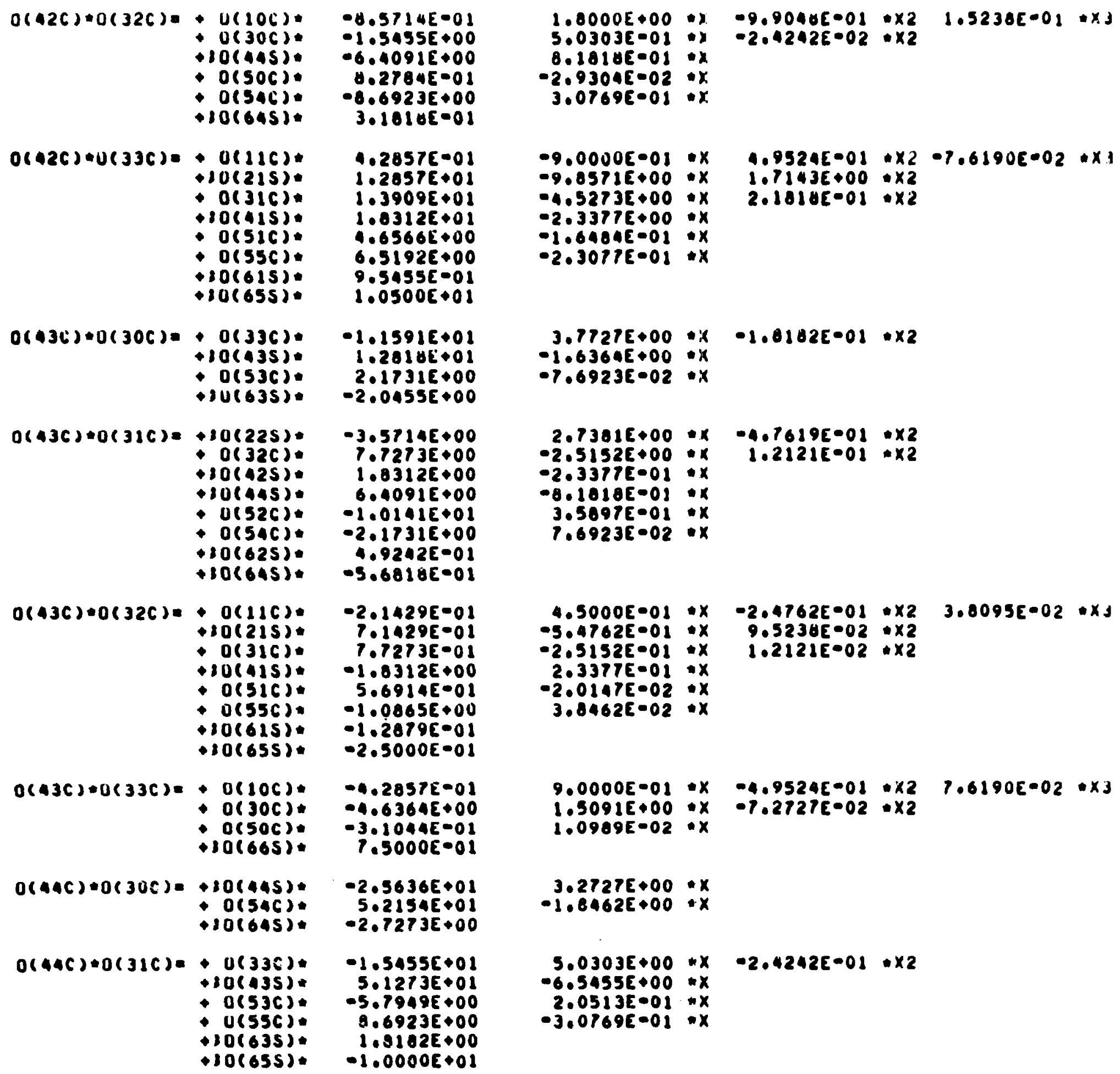

$-2.1429 E-01$

$7.1429 E-01$

7.72736001

$-1.8312 E+00$

$5.6914 E-01$

- 10065 .

$-1.28796-0$

$-2.5000 E-01$

$O(43 C)=0(33 C)=\quad O(10 C)$

$+10(665)$ P.5000E-01

$0(A 4 C)+0(300)=+10(445)$

o(c5s) -2.56joerol $+0(54 C): \quad-5.2154 E+01$
$+10(645):-2.7273 E+00$

$0(44 C)+0(31 C)=+0(335)=$ $+10(435)=$ $+0(53 C)$.

U (5sc).

$\rightarrow 10(655)$.

$1.5455 E+0$ $5.1273 E \bullet 01$ $-5.7949 E+00$

$1.0000 E+00$ $5.03036-01$ ix 8.181 -0.01 *x $-2.9304 E-02$ ix $-2.93046-02$ :x
$3.07696-01: x$

$9.9040[0.01 \div \times 2$

$1.52385=02+\times 3$

$-9.0000 E-01 * x$ $9.0571 E+00$ ix - $052735+00$ : $x$ $-2.337 r e+00$ ax

-1.6400601 ax

$3.7727 E+00 * x-1.0102 E-01 * \times 2$

$-1.6360+00$ ix

$-7.6923 E-02 * x$

$2.73816+00 * x-4.76195-01 * \times 2$ $-2.5152 E+00 * x \quad 1.2121 E-01 * \times 2$ $-2.3377 E-01$ : $x$

$0.1018 E-01$ : $x$

$7.6923 E-02 * x$

$\left.4.5000 E-01 * x \quad-2.4762 E-01 * x_{2} \quad 3.0095 E-02 * x\right]$ $-5.4762 E-01$ : $x$

$2.5152 E-01 * x \quad 1.2121 E=02 * x 2$

$2.337 T E-01$ : $x$

$-2.0147 E-02$ : $x$

$3.0462 E-02 * x$

$9.0000 E-01 * x \quad-4.9524 E-01 \times \times 2 \quad 7.6190 E-02 * \times 3$ $1.5091 E+00 * x \quad-7.2727 E-02 * x 2$

$.0909 E-02 \cdot x$

$3.2727 E+00 * x$

$-1.6462 E+00+x$

$5.0303 E+00 * x \quad-2.4242 E-01 * \times 2$

$-6.5455 E+00 * x$

$2.0513 E=01 * x$
$-3.0769 E=01 * x$

$09545=01+x 2-7.61905=02 \times x 1$ $1.71436+00 \div \times 2$
$2.18145001: \times 2$ 


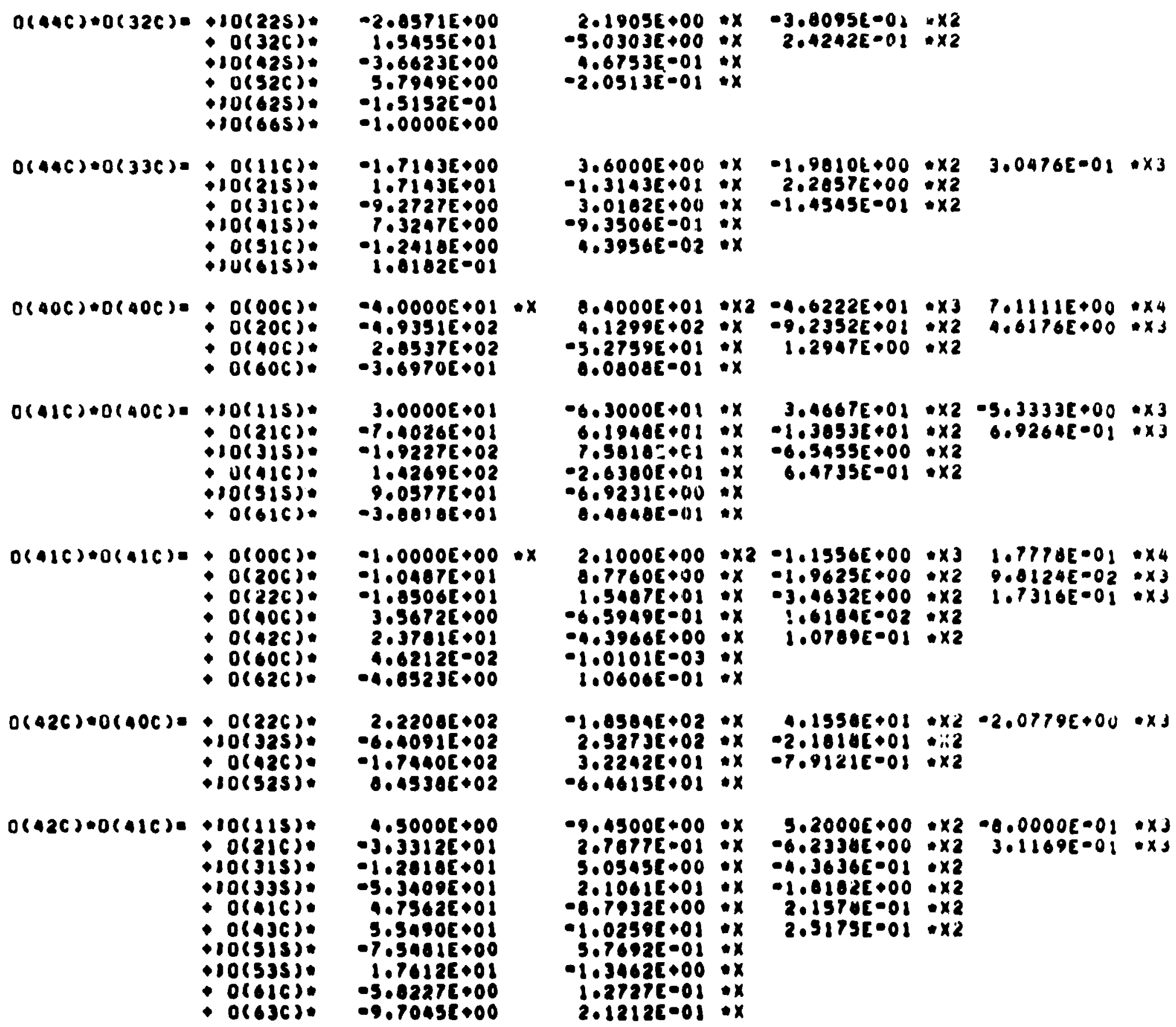

$2.10056+00 * x-3.00956-02 \times x 2$ $-5.0303 E+00 \cdot x \quad 2.4242 E-01: \times 2$ $4.6753 E=01$ : $x$ $3=01 \cdot x$

$3.6000 E+00 * x-1.9810 E+00 * \times 2 \quad 3.0476 E-01 * \times 3$ $1.31436+02$ : $x \quad 2.20575+00: x$ $3.0102 E+00 \div x$ $0.30565=02$ ax

$0.4000 E+01 * \times 2-4.6222 E+01 * \times 3 \quad 7.1111 E+00 * \times 4$ $4.12995+02 * x-9.2352 E+01$ * $\times 2 \quad 4.61765+00 \times x$ $-5.2759 E+01 * x \quad 1.2947 E+00: \times 2$ $0.08085001 \cdot x$

$-6.3000 E+01 * x \quad 3.4667 E+01 \times 2-5.3333 E+00 \times x 3$ $6.1940 E+01 * x-1.3053 E+01 * x 2 \quad 6.9264 E-01 * x 3$ $7.5010=+C 1$ in $x$ $-2.63805+01$ : $x$

$6.92315+00 * x$

$2.1000 E+00 * 2-1.1556 E+00 * \times 3 \quad 1.7778 E-01 * \times 4$ $0.7760 E+00 * x-1.9625 E+00 * x 2 \quad 9.8124 E-02 * x 3$ $1.5467 E+01 \times x-3.4632 E+00 \times 2 \quad 1.7316 E-01 \times x 3$ $-6.5949 E-01$ *X $\quad 1.6104 E-02$ * X2 $-4.39665+00$ *X $1.0709 E-01 \times x 2$

1.01015003 : $x$ $1.0600 E-01 \cdot x$

$1.0584 E+02 * x \quad 4.1558 E+01 * 22-2.0779 E+00 * x J$ $2.5273 E+02 \div x \quad-2.1014[+01 * i \pi 2$ $3.22426+01 * x-7.9121 E-01 * x 2$ -6.4615501 *x $2.7877 E-01$ : $x \quad-6.2330 E+00 \times x 2$ 3.1169E-01 *x $5.05455 .00 * x-4.3636[-01 \cdot \times 2$ $-8.7932 E 000$ * 2.157 UE-01 $-\times 2$ $1.0259 E+01 * x \quad 2.3175[-01 * x 2$ $5.76825001: x$ $1,34625+00$ :x l.2727e-01 : $x$ $2.12120001: x$ 


\begin{tabular}{|c|c|c|c|c|c|c|c|c|c|}
\hline $0(+2 C) \cdot 0(42 C)=$ & 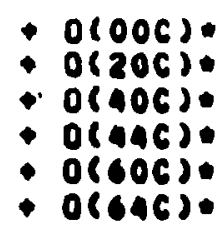 & $\begin{array}{r}-2.00005+00 \\
-9.0701 E+00 \\
-0.7190 E+00 \\
0.3234 E+01 \\
2.0333 E+00 \\
-1.16455+01\end{array}$ & $\bullet x$ & $\begin{array}{r}1.20005+00 \\
1.25975+00 \\
1.61216+00 \\
-1.53805+01 \\
-4.44446002 \\
2.54556-01\end{array}$ & $\begin{array}{l}: x 2 \\
: x \\
: x \\
: x \\
: x \\
: x\end{array}$ & $\begin{array}{r}-2.31116000 \\
-1.84706000 \\
-3.95608=02 \\
3.7862 E-01\end{array}$ & $\begin{array}{l}-\times 3 \\
\times \times 2 \\
\times 2 \\
\times 2\end{array}$ & $\begin{array}{l}3.5536 E \bullet 01 \\
0.2352 E \bullet 02\end{array}$ & $\begin{array}{l}0 \times 4 \\
0 \times 3\end{array}$ \\
\hline $0(436) \div 0(406) 0$ & 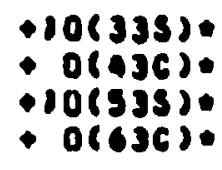 & $\begin{array}{r}1,0602 E+02 \\
-3.3294 E+02 \\
1.0064 E+01 \\
2.7727 E+01\end{array}$ & & $\begin{array}{r}-4.2121 E+01 \\
6.15525 \circ 01 \\
-7.6923 E-01 \\
-6.06060-01\end{array}$ & $\begin{array}{l}: x \\
: x \\
: x \\
\cdot x\end{array}$ & $\begin{array}{r}3.63645 \$ 00 \\
-1.3105 E+00\end{array}$ & $\begin{array}{l}\times 2 \\
\times 2\end{array}$ & & \\
\hline $0(436) \cdot 0(1) 6)=$ & 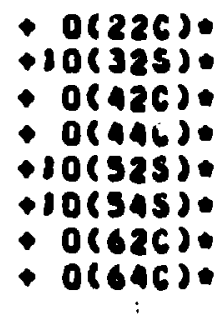 & 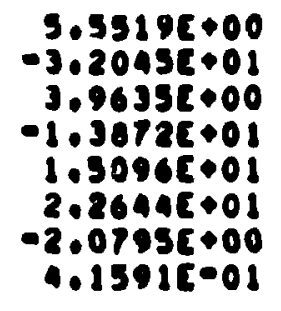 & & 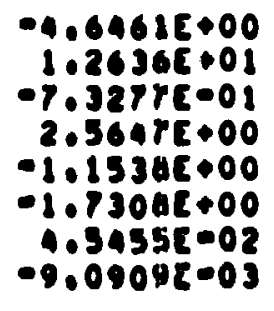 & $\begin{array}{l}: x \\
: x \\
: x \\
: x \\
: x \\
: x \\
: x \\
: x\end{array}$ & $\begin{array}{r}1.03905+00 \\
-1.09098+00 \\
1.79025-02 \\
-6.29375-02\end{array}$ & $\begin{array}{l}: \times 2 \\
: \times 2 \\
: \times 2 \\
\times 2\end{array}$ & $-5.1048 c-02$ & . $x J$ \\
\hline $0(436) \cdot 0(426) 0$ & $\begin{array}{l}+10(115): \\
+0(216) \\
+10(315) \\
+0(116) \\
+10(515) \\
+10(555) \\
+0(616) \\
+0(656)\end{array}$ & $\begin{array}{r}1.5000 E+00 \\
-1.85065+01 \\
6.4091 E+00 \\
7.9271 E+00 \\
-7.5481 E+00 \\
1.3587 E+01 \\
3.6045 E+00 \\
-9.1500 E+00\end{array}$ & & 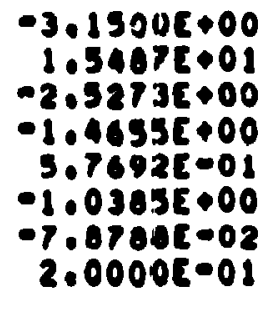 & $\begin{array}{l}: x \\
: x \\
: x \\
: x \\
: x \\
: x \\
: x \\
: x\end{array}$ & $\begin{array}{r}1.73335+00 \\
-3.46325+00 \\
2.10185-01 \\
3.59645-02\end{array}$ & $\begin{array}{l}: \times 2 \\
: \times 2 \\
: \times 2 \\
\times 2\end{array}$ & $\begin{array}{r}-2.6667 E-01 \\
1.7316 E-01\end{array}$ & $\begin{array}{l}0 \times 3 \\
0 \times 3\end{array}$ \\
\hline $0(436)=0(43 C)=$ & 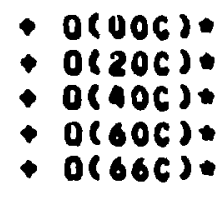 & $\begin{array}{r}-1.4206 E-01 \\
6.1600 E-01 \\
-1.18911+00 \\
-1.1223 E-01 \\
-1.3250 E+00\end{array}$ & $\cdot x$ & $\begin{array}{r}3.0000 E-01 \\
-5.1623 E-01 \\
2.1983 E-01 \\
2.4531 E-03 \\
3.3333 E=02\end{array}$ & $\begin{array}{l}: x 2 \\
: x \\
: x \\
: x \\
: x\end{array}$ & $\begin{array}{r}-1.6508 E-01 \\
1.1544 E-01 \\
-5.3946 E-03\end{array}$ & $\begin{array}{l}: \times 3 \\
: \times 2 \\
\times \times 2\end{array}$ & $\begin{array}{r}2.5397 E-0.2 \\
-5.7720 E=03\end{array}$ & $\begin{array}{l}-\times 4 \\
\times \times 3\end{array}$ \\
\hline $0(4 A C)+0(40 C) 0$ & $\begin{array}{l}\forall U(44 C): \\
+10(545): \\
+U(646)\end{array}$ & $\begin{array}{r}2.21965+02 \\
-1.24625+02 \\
6.6545 E+01\end{array}$ & & $\begin{array}{r}-4.1035 E+01 \\
5.5385 E \bullet 01 \\
-1.4545 E+00\end{array}$ & $\begin{array}{l}: x \\
: x \\
: x\end{array}$ & $1.0070 E \bullet 00$ & $\times 2$ & & \\
\hline$O(A+C) \oplus O(A 1 C)=$ & $\begin{array}{l}+10(335) \\
\bullet \quad 0(436) \\
+10(535) \\
10(555) \\
+0(636) \\
+0(65 C)\end{array}$ & $\begin{array}{r}2.1364 E+01 \\
-1.1098 E+02 \\
2.0120 E+01 \\
-1.0115 E+01 \\
-1.1091 E+01 \\
3.6600 E+01\end{array}$ & & $\begin{array}{r}-0.4242 E+00 \\
2.0517 E+01 \\
-1.5385 E+00 \\
1.3846 E+00 \\
2.4242 E-01 \\
-0.0000 E-01\end{array}$ & $\begin{array}{l}: x \\
: x \\
: x \\
: x \\
: x \\
: x\end{array}$ & $\begin{array}{r}7.2727 E=01 \\
-5.0350 E-01\end{array}$ & $\begin{array}{r}\times 2 \\
\times \times 2 \\
\times 2\end{array}$ & & \\
\hline $0(A 4 C) \oplus 0(42 C)=$ & $\begin{array}{l}\quad 0(22 C) \\
+10(325) \\
+0(42 C) \\
+\quad 0(525) \\
+0(62 C) \\
+0(66 C)\end{array}$ & $\begin{array}{r}1.1805 E+01 \\
-1.2818 E+02 \\
1.7562 E+01 \\
-1.207 T E+02 \\
5.5455 E+00 \\
1.2200 E \diamond 01\end{array}$ & & $\begin{array}{r}-1.2390 E+01 \\
5.0545 E \bullet 01 \\
-8.7932 E+00 \\
9.2308 E+00 \\
-1.2121 E-01 \\
-2.6667 E-01\end{array}$ & $\begin{array}{l}: x \\
: x \\
: x \\
: x \\
: x \\
: x\end{array}$ & $\begin{array}{r}2.7700 E+00 \\
-4.3636 E+00 \\
2.1570 E-01\end{array}$ & $\begin{array}{l}: \times 2 \\
: \times 2 \\
: \times 2\end{array}$ & $-1.38535-01$ & $\cdot \times 3$ \\
\hline
\end{tabular}




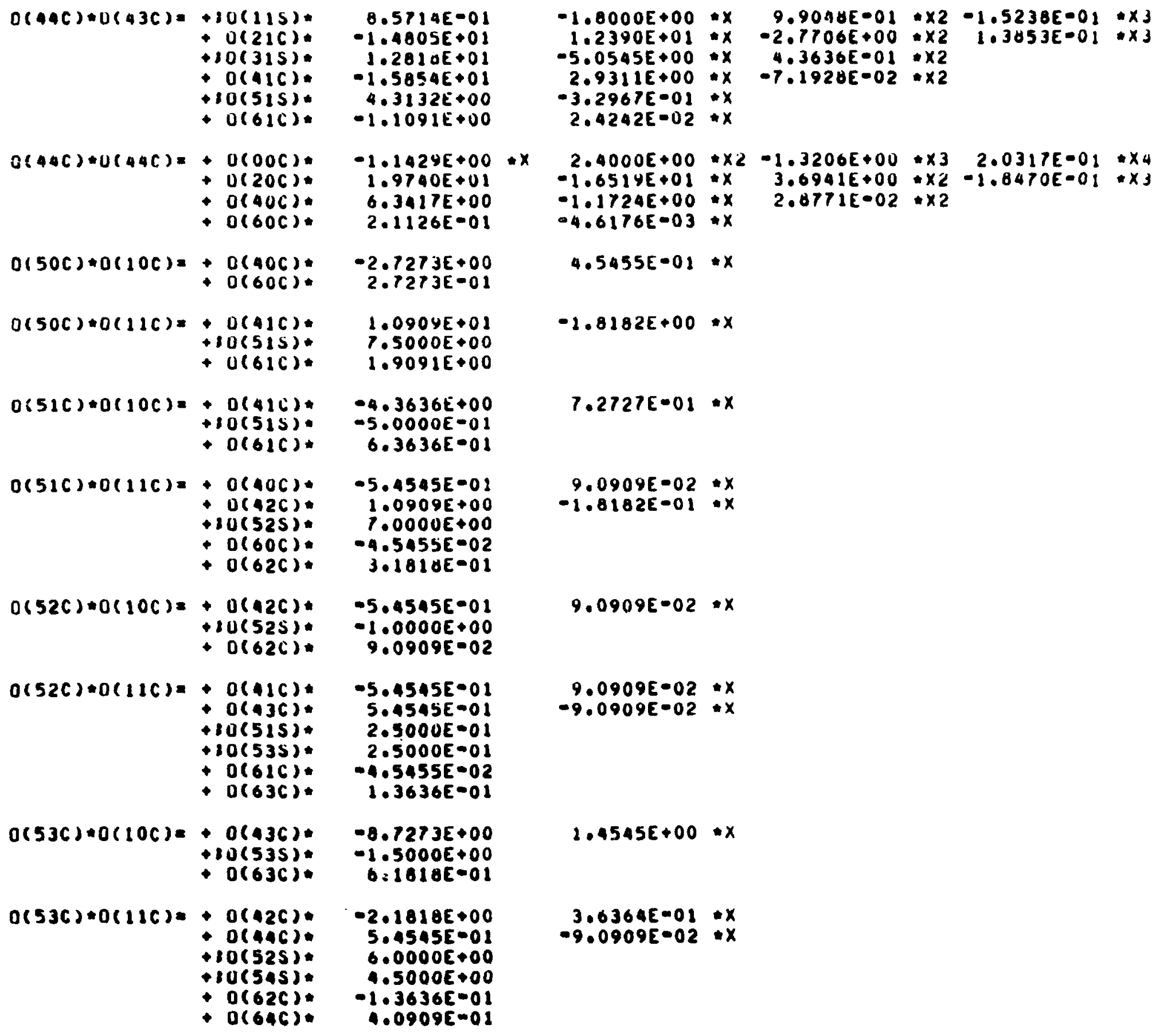
$+10(535)=-1.5000 E+00$ $+0(63 C)$ b:

$0(53 C) * 0(116)=+0(42 C)--2.1818 E+00$ D(446). 5.45A5E-01
$+10(525)$ 6.0000E+00 + $10(525)$ (54) $4.0000 E+00$ - O(62C). -1.3636E-01 $3.6364 E-01 * x$
$-9.09095002 * x$ 


\begin{tabular}{|c|c|c|c|c|c|c|}
\hline $0(54 C) \cdot 0(10 C)=$ & $\begin{array}{l}+0(44 C) " \\
+30(545) " \\
+0(64 C) .\end{array}$ & $\begin{array}{r}-5.4545 E-01 \\
-2.0000 E+00 \\
Y .0909 E-02\end{array}$ & Y.0909E-02 & $\cdot x$ & & \\
\hline$O(5+C) \cdot 0(11 C)=$ & $\begin{aligned} & 0(436) \\
+ & 10(535): \\
+ & 0(555) \\
+ & 0(636) \\
+ & 0(656)\end{aligned}$ & $\begin{array}{r}-2.1810 E+00 \\
2.5000 E-01 \\
2.5000 E-01 \\
-4.5455 E=02 \\
5.0000 E-01\end{array}$ & $3.6364 E-01$ & $a x$ & & \\
\hline $0(55 C)+0(10 C)=$ & $\begin{array}{l}+10(555) * \\
+0(656) *\end{array}$ & $\begin{array}{r}-2.5000 E \\
1.0000 E\end{array}$ & & & & \\
\hline $0(55 C) \times 0(11 C)=$ & $\begin{array}{l}+0(44 C): \\
+8(545): \\
+U(64 C): \\
+0(66 C) .\end{array}$ & $\begin{array}{r}-2.7273 E+00 \\
2.5000 E+00 \\
-4.5455 E=02 \\
5.0000 E-01\end{array}$ & $4.5455 E-01$ & $+x$ & & \\
\hline $0(50 C) \bullet 0(20 C)=$ & $\begin{array}{l}+O(30()) \\
+O(50 C)\end{array}$ & $\begin{array}{r}5.4545 E+01 \\
-1.1154 E+01\end{array}$ & $\begin{array}{r}-2.3636 E+01 \\
5.1282 E=01\end{array}$ & $\begin{array}{l}x \\
x\end{array}$ & $2.4242 E+00$ & $\times 2$ \\
\hline $0(50 C)=0(21 C)=$ & $\begin{array}{l}+0(316) * \\
+0(415) * \\
+0(516) * \\
+80(615) *\end{array}$ & $\begin{array}{r}-1.3636 E+01 \\
-3.2727 E+01 \\
-2.7885 E+00 \\
4.7727 E+00\end{array}$ & $\begin{array}{l}5.9091 E+00 \\
5.4545 E+00 \\
1.2021 E-01\end{array}$ & $\begin{array}{l}: x \\
: x \\
: x\end{array}$ & $-6.0600 E-01$ & $\cdot \times 2$ \\
\hline $0(50 C)=0(22 C)=$ & $\begin{array}{l}+U(32 C): \\
+10(425) \\
+0(526) \\
+10(625) .\end{array}$ & $\begin{array}{l}2.7273 E+01 \\
3.2727 E+01 \\
1.5615 E+02 \\
9.5455 E+00\end{array}$ & $\begin{array}{l}-1.1818 E+01 \\
-5.4545 E+00 \\
-7.1795 E+00\end{array}$ & $\begin{array}{l}: x \\
: x \\
-x\end{array}$ & $1.2121 E+00$ &.$\times 2$ \\
\hline$O(51 C)+0(20 C)=$ & $\begin{array}{l}+0(316) * \\
+80(115) \\
+0(516) \\
+80(615) .\end{array}$ & $\begin{array}{r}1.6364 E+01 \\
1.3091 E+01 \\
-1.0038 E+01 \\
-1.9091 E+00\end{array}$ & $\begin{array}{r}-7.0909 E+00 \\
-2.1810 E+00 \\
4.6154 E-01\end{array}$ & $\begin{array}{l}: x \\
: x \\
: x\end{array}$ & $7.2727 E-01$ & $+\times 2$ \\
\hline $0(510) * 0(210)=$ & $\begin{array}{l}+0(306) * \\
+0(326) \\
+10(425) \\
+0(506) \\
+0(526) \\
+10(625)\end{array}$ & $\begin{array}{r}3.6364 E+00 \\
-5.4545 E+00 \\
-4.3636 E+00 \\
-1.8590 E=01 \\
-7.8077 E+00 \\
-.7727 E-01\end{array}$ & $\begin{array}{r}-1.5758 E+00 \\
2.3636 E+00 \\
7.2727 E=01 \\
0.5470 E-03 \\
3.5997 E-01\end{array}$ & $\begin{array}{l}: x \\
: x \\
: x \\
: x \\
: x\end{array}$ & $\begin{array}{r}1.6162 E=01 \\
-2.4242 E=01\end{array}$ & $\begin{array}{l}\times 2 \\
\times 2 \\
\times 2\end{array}$ \\
\hline $0(516) * 0(22 C)=$ & $\begin{array}{l}* 0(316) \\
+0(336) \\
+10(415) \\
+10(435) \\
+0(516) \\
+0(536) \\
+10(615) \\
+10(635)\end{array}$ & $\begin{array}{r}-2.7273 E+00 \\
9.0909 E+01 \\
-1.0909 E+01 \\
1.5273 E+01 \\
-5.57696+00 \\
5.2051 E+00 \\
-1.9091 E+00 \\
3.6102 E+00\end{array}$ & $\begin{array}{r}1.1818 E+00 \\
-3.9394001 \\
1.8182 E+00 \\
-2.545 E+00 \\
2.5611 E-01 \\
-2.3932 E-01\end{array}$ & $\begin{array}{l}: x \\
: x \\
: x \\
: x \\
: x \\
: x\end{array}$ & $\begin{array}{r}-1.2121 E=01 \\
4.0404 E-02\end{array}$ & $\begin{array}{l}\times 2 \\
\times 2 \\
\times 2\end{array}$ \\
\hline
\end{tabular}




\begin{tabular}{|c|c|c|c|c|c|c|}
\hline $0(526)=0(200)=$ & $\begin{array}{l}+0(326) * \\
+10(425) * \\
+0(526) * \\
+10(625)\end{array}$ & $\begin{array}{r}8.1818 E+00 \\
3.2727 E+00 \\
-6.0923 E+00 \\
-5.4545 E-01\end{array}$ & $\begin{array}{r}-3.5455 E+00 \\
-3.4545 E-01 \\
3.07695=01\end{array}$ & $\begin{array}{l}\cdot x \\
: x \\
: x\end{array}$ & $3.63646=01$ & $-\times 2$ \\
\hline $0(52 C)=0(21 C)=$ & $\begin{array}{l}+0(316): \\
+0(33 C): \\
+10(415) \\
+10(435) \\
+0(516) \\
+0(53 C) \\
+10(615) \\
+10(635)\end{array}$ & $\begin{array}{r}6.8102 E-01 \\
-2.2727 E-01 \\
-5.4545 E-01 \\
-2.1273 E+00 \\
-2.7005 E=01 \\
-4.6474 E-01 \\
2.0455 E-01 \\
6.0102 E-02\end{array}$ & $\begin{array}{r}-2.9545 E=01 \\
9.8485 E=02 \\
9.0909 E-02 \\
1.5455 E=01 \\
1.2821 E=02 \\
2.1360 E=02\end{array}$ & $\begin{array}{l}: x \\
: x \\
: x \\
: x \\
: x \\
: x\end{array}$ & $\begin{array}{r}3.0303 E-02 \\
-1.0101 E=02\end{array}$ & $\begin{array}{l}\times 2 \\
\times 2 \\
\times 2\end{array}$ \\
\hline $0(52 C) \cdot 0(22 C)=$ & $\begin{array}{l}+0(306): \\
+10(445) \\
+0(50 C) \\
0(546) \\
+10(645)\end{array}$ & $\begin{array}{l}9.0909 E=01 \\
5.4545 E=01 \\
3.7179 E=01 \\
3.3462 E+00 \\
4.0909 E=01\end{array}$ & $\begin{array}{l}-3.9394 E=01 \\
-9.0909 E=02 \\
-1.7094 E=02 \\
-1.5385 E=01\end{array}$ & $\begin{array}{l}\cdot x \\
: x \\
: x \\
: x\end{array}$ & $4.0404 E 0.02$ &.$\times 2$ \\
\hline $0(536)=0(206)=$ & $\begin{array}{l}-0(336) \\
+10(435) \\
+0(536) \\
+10(635)\end{array}$ & $\begin{array}{r}1.0909 E+01 \\
7.0545 E+01 \\
-1.1154 E+00 \\
-7.3636 E+00\end{array}$ & $\begin{array}{r}-4.7273 E+00 \\
-1.3091 E+01 \\
3.1282 E=02\end{array}$ & $\begin{array}{l}\cdot x \\
: x \\
: x\end{array}$ & $4.0485 E-01$ & $-x^{2}$ \\
\hline $0(53 c)=0(216)=$ & $\begin{array}{l}+0(32 C): \\
+10(445): \\
+0(52 C): \\
+0(54 C) . \\
+0(625) . \\
+0(645) .\end{array}$ & $\begin{array}{r}1.0909 E+01 \\
-3.2727 E+00 \\
-1.1154 E+01 \\
-1.1712 E+01 \\
1.5000 E-01 \\
-2.0435 E-01\end{array}$ & $\begin{array}{r}-4.7273 E+00 \\
5.4505 E=01 \\
5.1202 E-01 \\
5.3046 E-01\end{array}$ & $\begin{array}{l}: x \\
: x \\
: x \\
: x\end{array}$ & $4.0485 E .01$ & $-\times 2$ \\
\hline $0(53()=0(226)=$ & 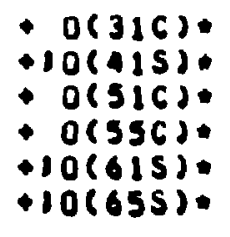 & $\begin{array}{r}5.4545 E+00 \\
-1.3091 E+01 \\
1.4615 E+00 \\
3.3462 E+00 \\
-1.0909 E+00 \\
9.0000 E+00\end{array}$ & $\begin{array}{r}-2.3636 E+00 \\
2.1010 E+00 \\
-2.0513 E=01 \\
-1.5385 E=01\end{array}$ & $\begin{array}{l}: x \\
: x \\
: x \\
: x\end{array}$ & $2.4242 E \cdot 01$ & $1 \times 2$ \\
\hline $0(54 C) \cdot 0(20 C)=$ & $\begin{array}{l}+10(445): \\
+0(346): \\
+10(645)=\end{array}$ & $\begin{array}{r}6.5455 E+00 \\
6.6923 E+00 \\
-1.0909 E+00\end{array}$ & $\begin{array}{l}-1.0909 E+00 \\
-3.0769 E=01\end{array}$ & $\begin{array}{l}-x \\
-x\end{array}$ & & \\
\hline $0(\operatorname{sac})=0(21 C)=$ & $\begin{array}{l}+0(336) \\
+10(435) \\
+0(336) \\
+0(556) \\
+10(635) \\
+10(655) .\end{array}$ & $\begin{array}{r}9.0904 E-02 \\
2.1810 E+00 \\
-6.3064 E-01 \\
-8.3654 E-01 \\
2.9545 E=01 \\
-7.5000 E-01\end{array}$ & $\begin{array}{r}-3.9394 E-01 \\
-3.6364 E-01 \\
2.9915 E=02 \\
3.0462 E=02\end{array}$ & $\begin{array}{l}\cdot x \\
: x \\
: x \\
x \\
x\end{array}$ & $4.0404 E=02$ &.$\times 2$ \\
\hline
\end{tabular}




\begin{tabular}{|c|c|c|c|c|c|c|c|c|}
\hline $0(5+C)=0(22 c)=$ & 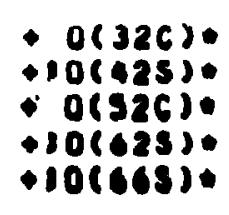 & $\begin{array}{r}5.4545 E+00 \\
-2.10105+00 \\
1.4615 E+00 \\
-1.3630 E-01 \\
5.0000 E-01\end{array}$ & $\begin{array}{r}-2.3636[000 \\
3.63645-01 \\
-2.0513[-01\end{array}$ & $: x$ & $2.4242 C-01$ & $-\times 2$ & & \\
\hline$\dot{o}(556) \bullet 0(206) 0$ & $\begin{array}{l}\text { Oo(ssc). } \\
\text { - io(sess). }\end{array}$ & $\begin{array}{r}1.57312+01 \\
-1.50006+01\end{array}$ & $-7.0923[-01$ & $\cdot x$ & & & & \\
\hline $0(556) \cdot 0(216) 0$ & $\begin{array}{l}+10(445)= \\
+0(546)= \\
+10(645)= \\
+10(605) .\end{array}$ & $\begin{array}{r}\text { S. AsAsE }+00 \\
-8.36546+00 \\
3.40916-02 \\
-1.25006+00\end{array}$ & $\begin{array}{r}-9.0009 c-01 \\
3.0462[-01\end{array}$ & $\stackrel{x}{* x}$ & & & & \\
\hline $0(556) \backsim 0(226)=$ & $\begin{array}{l}+0(336): \\
+10(435): \\
+0(536) \\
+10(635)\end{array}$ & $\begin{array}{r}2.09096+00 \\
-2.10140001 \\
1.05905000 \\
-4.34550-01\end{array}$ & $\begin{array}{r}-3.93946+00 \\
3.83646000 \\
-8.54706-02\end{array}$ & $\begin{array}{l}0 x \\
0 x \\
0 x\end{array}$ & 0.04040001 & $1 \times 2$ & & \\
\hline $0(306) 00(306) 0$ & 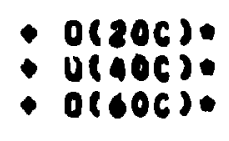 & $\begin{array}{r}-7.79226+01 \\
6.09596+01 \\
-6.00915+00\end{array}$ & $\begin{array}{r}r .2727 c \circ 01 \\
-1.23106+01 \\
2.12125-01\end{array}$ & $\begin{array}{l}: x \\
: x \\
0 x\end{array}$ & $\begin{array}{r}-2.03406+01 \\
3.5964001\end{array}$ & $\begin{array}{l}\times 2 \\
1 \times 2\end{array}$ & $1,73160+00$ & $\cdot \times 3$ \\
\hline $0(300) 00(316) 0$ & 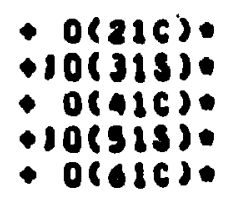 & $\begin{array}{r}1.5504 c+02 \\
2.04556+02 \\
2.03206+01 \\
-1.2923 c+02 \\
-3.5030 c+01\end{array}$ & 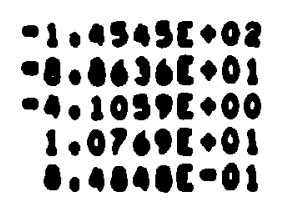 & $\begin{array}{l}: x \\
: x \\
: x \\
0 x \\
0 x\end{array}$ & $\begin{array}{l}1.06935 \cdot 01 \\
0.09005 .00 \\
1.19005-01\end{array}$ & $\begin{array}{l}\times 1 \times 2 \\
1 \times 2 \\
0 \times 2\end{array}$ & $-3.16326 \cdot 00$ & $\cdot \times 3$ \\
\hline $0(300) \cdot 0(326) 0$ & $\begin{array}{l}+0(226) \\
+10(325): \\
+\quad 0(426) \\
+10(525) \\
+\quad 0(526)\end{array}$ & $\begin{array}{r}-7.7922 c+00 \\
-1.6364+02 \\
-1.12796+01 \\
-1.2923 c+02 \\
0.00011+00\end{array}$ & $\begin{array}{r}1.2727 c+00 \\
1.09006+01 \\
1.64246+01 \\
1.07096+01 \\
-2.1212 c-01\end{array}$ & $\begin{array}{l}0 x \\
0 x \\
0 x \\
0 x \\
0 x \\
0 x\end{array}$ & $\begin{array}{l}-2.0346 c+00 \\
-7.2727 E+00 \\
-4.10526-01\end{array}$ & $\begin{array}{l}1 \times 2 \\
1 \times 2 \\
1 \times 2\end{array}$ & 1.73160 .01 &.$\times 3$ \\
\hline $0(506) \cdot 0(3)())=$ & $\begin{array}{l}+10(335) \\
+0(436) \\
+10(435) \\
+0(636)\end{array}$ & 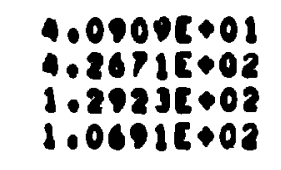 & $\begin{array}{l}-1.7127 c+01 \\
-0.62240+01 \\
-1.07690001 \\
-2.54550+00\end{array}$ & $\begin{array}{l}0 x \\
0 x \\
0 x \\
0 x\end{array}$ & $\begin{array}{l}1.01425+00 \\
2.5175 E+00\end{array}$ & $\begin{array}{l}\times 2 \\
-\times 2\end{array}$ & & \\
\hline $0(516) 00(306) 0$ & 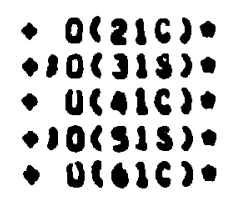 & $\begin{array}{r}-6.2330 c+01 \\
-4.09096+01 \\
1.72155+01 \\
2.5046001 \\
-1.7010 t+01\end{array}$ & $\begin{array}{r}5.9102 c+01 \\
1.7727+01 \\
-1.56025+01 \\
-2.1530 c+00 \\
4.24246-01\end{array}$ & $\begin{array}{l}: x \\
: x \\
0 x \\
0 x \\
0 x\end{array}$ & $\begin{array}{r}-1.02716+01 \\
-1.01026+00 \\
\text { A.sgsue-01 }\end{array}$ & $\begin{array}{l}0 \times 2 \\
0 \times 2 \\
0 \times 2\end{array}$ & $1.3453 E \cdot 00$ & $\cdot x$ \\
\hline$n(516) \cdot 0(316) 0$ & 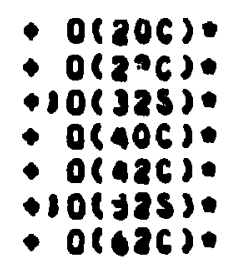 & 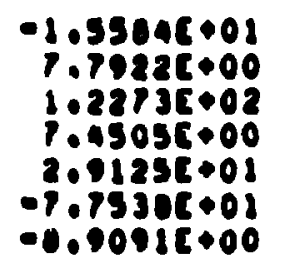 & 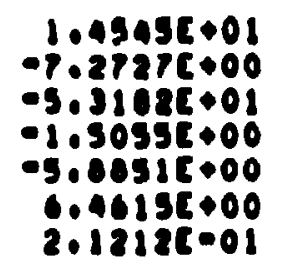 & $\begin{array}{c}: x \\
: x \\
0 x \\
: x \\
: x \\
0 x \\
0 x\end{array}$ & 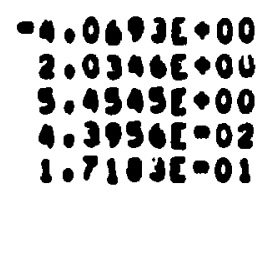 & $\begin{array}{r}-\times 2 \\
0 \times 2 \\
0 \times 2 \\
0 \times 2 \\
0 \times 2 \\
02\end{array}$ & $\begin{array}{r}3.46326=01 \\
-1.73168-01\end{array}$ & os \\
\hline
\end{tabular}




\begin{tabular}{|c|c|c|c|c|c|c|c|c|}
\hline $0(51 C)+0(326)=$ & $\begin{array}{l}+0(216): \\
+10(315) \\
+10(335) \\
+0(416) \\
+0(436) \\
+30(535) \\
+0(61 C)\end{array}$ & $\begin{array}{r}0.2338 E+00 \\
1.2273 E+01 \\
-6.8182 E+00 \\
1.6933 E+01 \\
-5.2154 E+01 \\
-8.6154 E+00 \\
-3.5636 E+00\end{array}$ & $\begin{array}{r}5.8182 E+00 \\
-5.3182 E+00 \\
2.9545 E+00 \\
-3.4216 E+00 \\
1.0538 E+01 \\
7.1795 E=01 \\
8.4848 E=02\end{array}$ & $\begin{array}{l}: x \\
: x \\
: x \\
: x \\
: x \\
: x \\
x\end{array}$ & $\begin{array}{r}1.6277 E+00 \\
5.4545 E=01 \\
-3.0303 E=01 \\
9.9900 E=02 \\
-3.0764 E=01\end{array}$ & $\begin{array}{l}\times 2 \\
\times 2 \\
: \times 2 \\
\times 2 \\
\times 2 \\
\times 2 \\
\times 2\end{array}$ & $-1.3653 E=01$ & 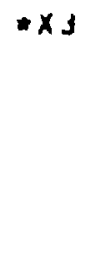 \\
\hline $0(510) 0(33 c)=$ & $\begin{array}{l}+0(22 C): \\
+10(325) \\
+0(42 C) \\
+0(44 C) \\
+10(525) \\
+10(545) \\
+0(62 C) \\
+0(64) .\end{array}$ & $\begin{array}{r}-1.5584 E+00 \\
-4.0907 E+01 \\
-3.0480 E+01 \\
1.4224 E+01 \\
-1.2923 E+02 \\
7.7530 E+01 \\
-8.9091 E+00 \\
1.0691 E+01\end{array}$ & $\begin{array}{r}1.4545 E+00 \\
1.7727 E+01 \\
6.15 B 8 E+00 \\
-2.8741 E+00 \\
1.0769 E+01 \\
-6.4615 E+00 \\
2.1212 E-01 \\
-2.5455 E-01\end{array}$ & $\begin{array}{l}: x \\
: x \\
: x \\
: x \\
x \\
: x \\
: x \\
x \\
x\end{array}$ & $\begin{array}{r}-4.0693 E=01 \\
-1.0182 E+00 \\
-1.7982 E=01 \\
0.3916 E=02\end{array}$ & $\begin{array}{l}: \times 2 \\
\times 2 \\
\times 2 \\
\times 2 \\
x 2\end{array}$ & $3.463 ! E=02$ & $x_{3}$ \\
\hline $0(52 C) \cdot 0(30 C)=$ & $\begin{array}{l}+0(22 C): \\
+10(32 S): \\
+0(42 C): \\
+10(525): \\
+0(62 C) .\end{array}$ & $\begin{array}{r}-3.8961 E+00 \\
-4.0909 E+01 \\
2.0320 E+00 \\
4.2462 E+01 \\
-1.2727 E+00\end{array}$ & $\begin{array}{r}3.6364 E+00 \\
1.7727 E+01 \\
-4.1059 E-01 \\
-3.5385 E+00 \\
3.0303 E-02\end{array}$ & $\begin{array}{l}x \\
: x \\
: x \\
: x \\
: x\end{array}$ & $\begin{array}{r}-1.0173 E+00 \\
-1.0182 E+00 \\
1.1980 E-02\end{array}$ & $\begin{array}{l}x 2 \\
x 2 \\
x 2 \\
\times 2\end{array}$ & $8.6580 E-02$ & $\cdot \times 1$ \\
\hline $0(52 C) \cdot 0(310)=$ & 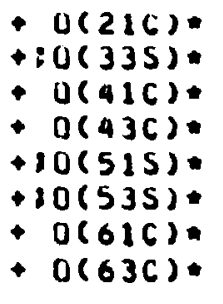 & $\begin{array}{r}-7.7922 E+00 \\
6.8162 E+00 \\
1.0837 E+01 \\
3.3189 E+01 \\
-2.7692 E+00 \\
3.0769 E-01 \\
-1.2727 E+00 \\
-3.8182 E+00\end{array}$ & $\begin{array}{r}7.2727 E+00 \\
-2.9545 E+00 \\
-2.189 \forall E+00 \\
-6.7063 E+00 \\
2.3077 E-01 \\
-2.5641 E-02 \\
3.0303 E-02 \\
9.0909 E-02\end{array}$ & $\begin{array}{l}: x \\
: x \\
: x \\
: x \\
: x \\
: x \\
: x \\
: x\end{array}$ & $\begin{array}{r}-2.0346 E+00 \\
3.0303 E=01 \\
6.3936 E-02 \\
1.4500 E-01\end{array}$ & $\begin{array}{l}: \times 2 \\
: \times 2 \\
1 \times 2 \\
x 2\end{array}$ & $1.7316 E-01$ & $* x_{3}$ \\
\hline $0(52 C) \cdot 0(32 C)=$ & $\begin{array}{l}+0(20 C) \\
+\quad 0(40 C) \\
+0(44 C) \\
+\quad 0(545) \\
+0(60 C) \\
+0(64 C) .\end{array}$ & $\begin{array}{l}-7.7922 E-01 \\
-3.3866 E-01 \\
-2.3706 E+00 \\
-8.3077 E+00 \\
1.2727 E=01 \\
-3.8182 E=01\end{array}$ & $\begin{array}{r}7.2727 E=01 \\
6.8432 E=02 \\
4.7902 E=01 \\
6.9231 E=01 \\
-3.0303 E=03 \\
9.0909 E=03\end{array}$ & $\begin{array}{l}* x \\
: x \\
: x \\
: x \\
: x \\
* x\end{array}$ & $\begin{array}{l}=2.0346 E=01 \\
-1.9980 E=03 \\
-1.3986 E=02\end{array}$ & $\begin{array}{l}: \times 2 \\
: \times 2 \\
\times \times 2\end{array}$ & $1.7316 E-02$ & $=3$ \\
\hline $0(52 C) \cdot 0(33 C)=$ & 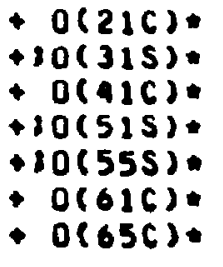 & $\begin{array}{l}1.5504 E+00 \\
4.0909 E+00 \\
1.0160 E+01 \\
4.6154 E+00 \\
2.7692 E+00 \\
1.7818 E+00 \\
6.4000 E+00\end{array}$ & $\begin{array}{l}-1.4545 E+00 \\
-1.7727 E+00 \\
-2.0529 E+00 \\
-3.0462 E-01 \\
-2.3077 E=01 \\
-4.2424 E-02 \\
-2.0000 E-01\end{array}$ & $\begin{array}{l}: x \\
: x \\
: x \\
: x \\
: x \\
: x \\
: x \\
x\end{array}$ & $\begin{array}{l}4.0693 E=01 \\
1.8182 E=01 \\
5.9940 E=02\end{array}$ & $\begin{array}{l}\times 2 \\
\times 2 \\
\times 2 \\
\times 2\end{array}$ & $-3.4632 E-02$ & $* x_{3}$ \\
\hline $0(53 C) \cdot 0(30 C)=$ & $\begin{array}{l}+10(335) . \\
+0(436) . \\
+0(535) . \\
+0(636) .\end{array}$ & $\begin{array}{r}-8.1618 E+01 \\
-1.7069 E+02 \\
\$ .0615 E+01 \\
7.6364 E+00\end{array}$ & $\begin{array}{r}3.5455 E+01 \\
3.4490 E+01 \\
-3.3846 E+00 \\
-1.0182 E=01\end{array}$ & $\begin{array}{l}x \\
0 x \\
0 x \\
x\end{array}$ & $\begin{array}{l}-3.6364 E \odot 00 \\
-1.0070 E \bullet 00\end{array}$ & $\begin{array}{l}\times 2 \\
\times 2 \\
\times 2\end{array}$ & & \\
\hline
\end{tabular}




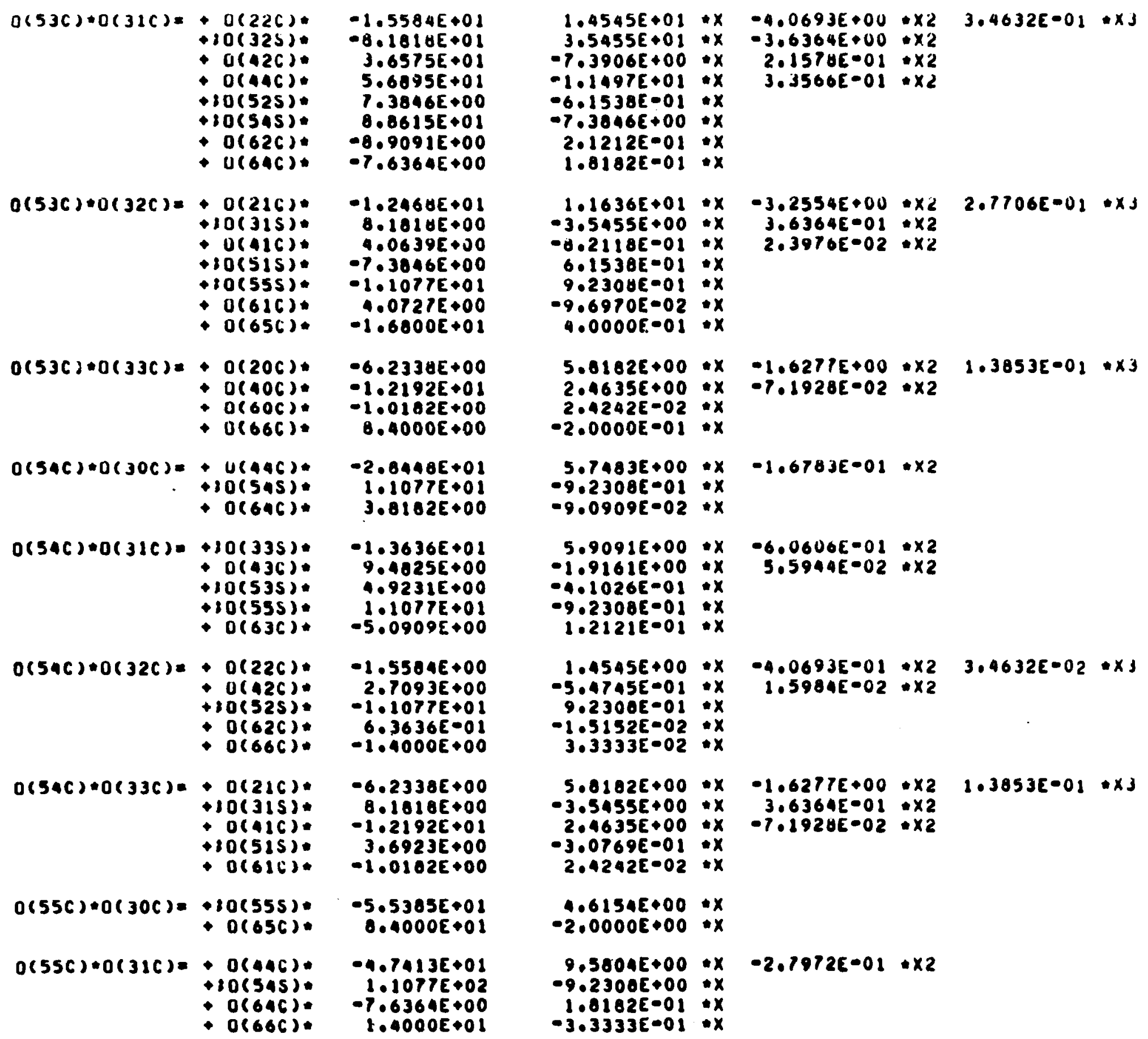

$-1 \cdot 3636 E+01$

$4.9231 E+00$

$1.1077 E+01$
$-5.09095+00$

$O(5 A C) \times O(32 C)=+O(22 C)$ $+10(525)$ $+0(62 C)$.

$-1.5504 E+00$ $2.7093 E+00$ $-1.1077 E+01$ $6.3636 E-01$
$-1.4000 E+00$

$0(54 C) \cdot 0(336)=+0(21 C)$ o(aic) $+10(515)$.

$-6.2338 E+00$ $8.1010 E+00$ $-1.2192 E+01$ $3.6923 E+00$ $-1.0102 E+00$

$0(55 C)+0(30 C)=+10(555)$

$-5.5305 E+01$
$0.4000 E+01$

$0(55 C) * 0(31 C)=+0(44 C)$

$-4.74135+01$ $1.10775+02$ o(66C). $\begin{array}{lll}1.4545 E+01 * X & -4.0693 E+00 & \times 2 \\ 3.5455 E+01 & -3.6364 E+00 & \times 2\end{array}$ $x-3.6364 E+00-x 2$

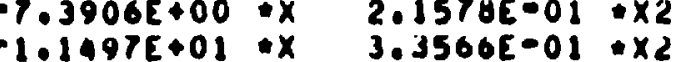

$-6.1538 E-0$ $-7.3846 E+00$. $2.1212 E-01 * x$ $1.8182 E-01$ *x

$1.1636 E+01 * x$ $-3.5455 E+00: x$ $-0.2118 E-01: x$ $6.1538 E-01: x$ $9.23086-01$ : $x$ $-9.6970 E-02 * x$

$5.0182 E+00 * x-1.627 P E+00 * \times 2 \quad 1.3853 E-01 * \times 3$ $2.4635 E+00 * x-7.1928 E-02 * \times 2$

$2.4242 E-02 * x$ $-2.0000 E-01$ : $x$

$5.7483 E+00 * x-1.6783 E-01 * \times 2$ $-9.23086-01 \div x$

$-9.0909 E-02 * x$

$5.9091 E+00 * x-6.0606 E-01 * \times 2$ $-1.9161 E+00 * x \quad 5.5944 E-02 \times 2$ $-4.10265-01$ : $x$ $-9.2308 E-01$ : $x$ $1.2121 E-01: x$

$1.4545 E+00 * x \quad-4.0693 E-01 * \times 2 \quad 3.4632 E-02 * x 1$ $-5.4745 E-01 * x \quad 1.5984 E-02 * x 2$

$9.2300 E-01: x$ $-1.5152 E-02: x$ $5.0182 E+00+x \quad-1.6277 E+00 * x 2 \quad 1.3853 E-01 * x J$ $-3.5455 E+00: x \quad 3.6364 E-01: x 2$ $2.4635 E+00 * x-7.1928 E-02 * x 2$ $-3.0769 E-01+x$ $2.4242 E-02+x$

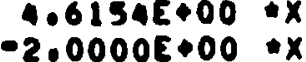

$9,5804 E+00 * x-2.7972 E-01 * \times 2$

$0.23005+00$ : $x$ $1.0182 E-01$ : $x$ $-3.33335001: x$ 


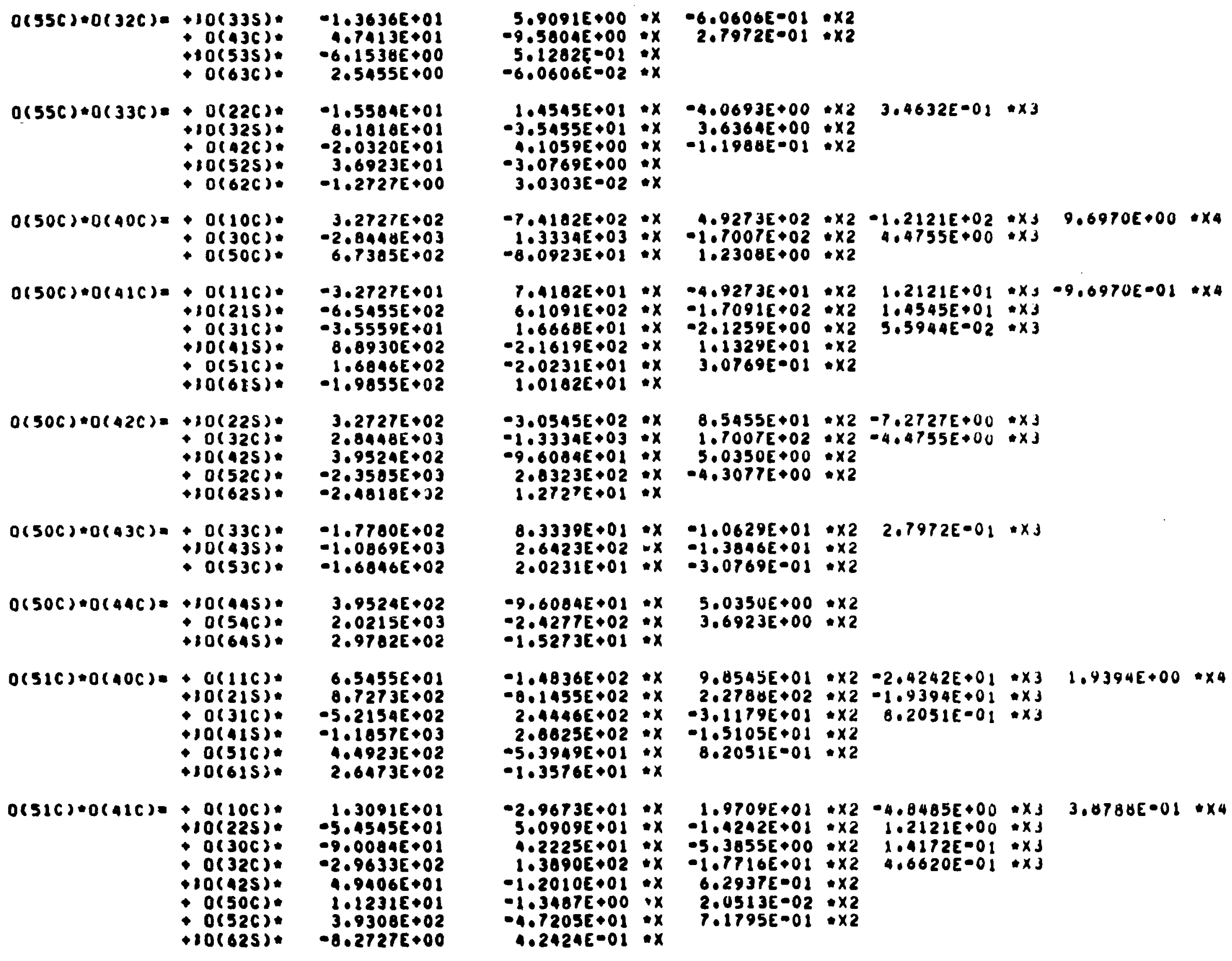


?า?

유웅

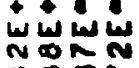

>0

눙요

vi⿺辶⿻丷木

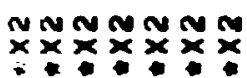

ํํํ:००:

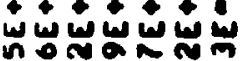

ind

ที่ํํㅇํํำ

i: $: \div \div 0 \therefore$

$x \times x \times x \times x$

뭉뭉ㅁㅇㅇㅇㅇㅇㅇㅇ

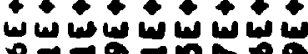

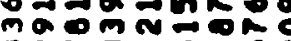

oxim

$\because \therefore \div: \because \div \div \div$ :

\section{x.}

施

然

जि:

$\therefore:$

iก

XXXXXX

유뭉웅

山必岕岁

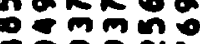

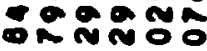

บํ:: : :

xxxxxx

후뭉훙ํㅇ뭉ㅇㅇ

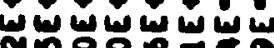

=0응

ㅍำลำลำ

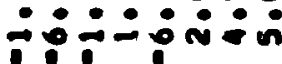

$x$
$\vdots$
0
0
$\vdots$
$\vdots$
$\vdots$
$\vdots$
$\vdots$

บบำ

:유:

내에용

iñ

$\because: 0$ :

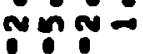

$\times \times \times \times x \times$

뭉ㅎㅇㅇㅇㅇㅇㅜ

ww山w山

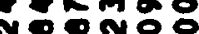

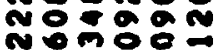

i०: :

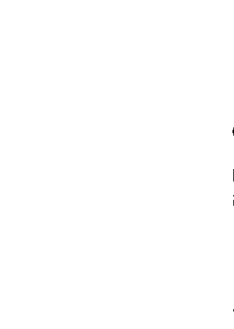

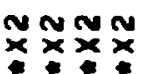

$x \times x \times$

둥ㅎㅇㅇㅇㅇㅇ

岕岕岕岕

$\rightarrow$ 웅웅

$0 \div 0 \mathrm{~m}$

$\div \div \div \div \dot{0}$

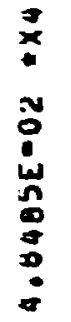

르

ㅁ 뮤유

: ::요

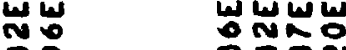

초

:ं।

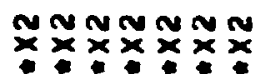

무유 융ㅇㅇㅇ

w

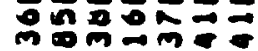

m:mą:

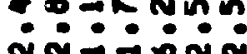

$\because \div$

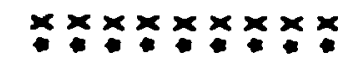

유유:유유

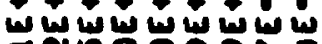

o으의.

웅용ㅇㅇ:

$\because \div \dot{0} \div \div \div \therefore:$

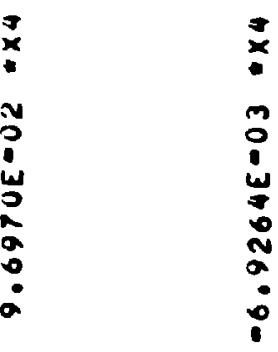

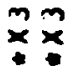

xख⿱

$\therefore \%$

$\underset{\sim}{\omega}$

in

$\because i$

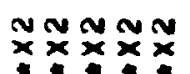

무유:

w出山心

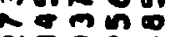

ก⿻ำ

:

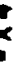

:

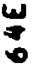

$\stackrel{2}{0}$

$\times \times \times \times x$

융ㅇㅇ웅

w山w w

주음ำ

- Nㅜ승ㅇㅇㅇ

ำ뭉

뭉

U⿺乚一匕

in $\overrightarrow{0}$ 范

$\because:=$

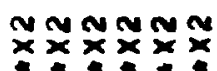

웅우융우

w㟧

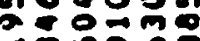

กีว:ㅇํำ

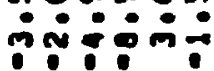

$x \times x \times x \times x$

웅융ㅇㅇㅇㅇㅇㅇㅜ

ww山w山w

○ N.

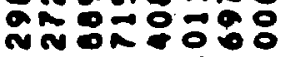

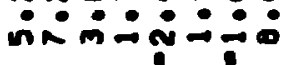

ํํㅇํㅇㅇํㅇํ유유

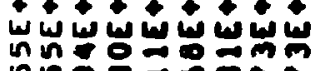
nñ.0.0

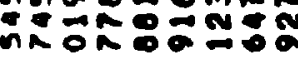

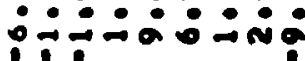
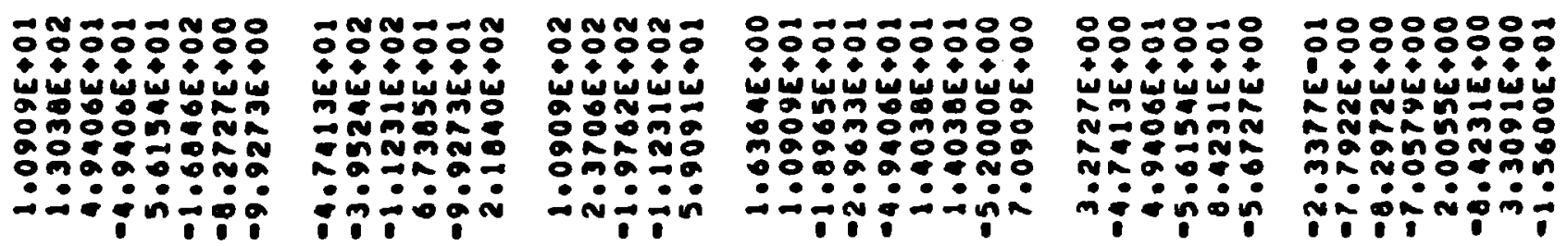

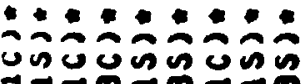

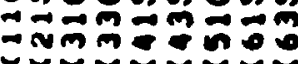

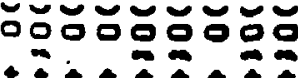
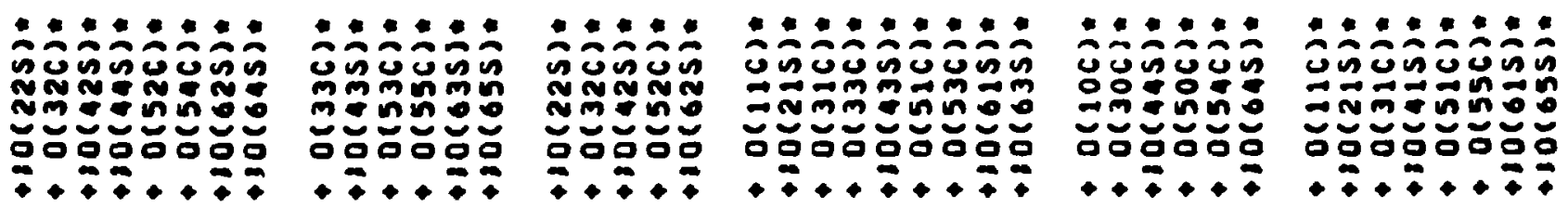

ปั

5.

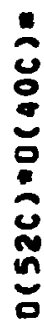

0
$\vdots$
$\vdots$
$\vdots$
0
0
0

ڤ̇

彳ิ 


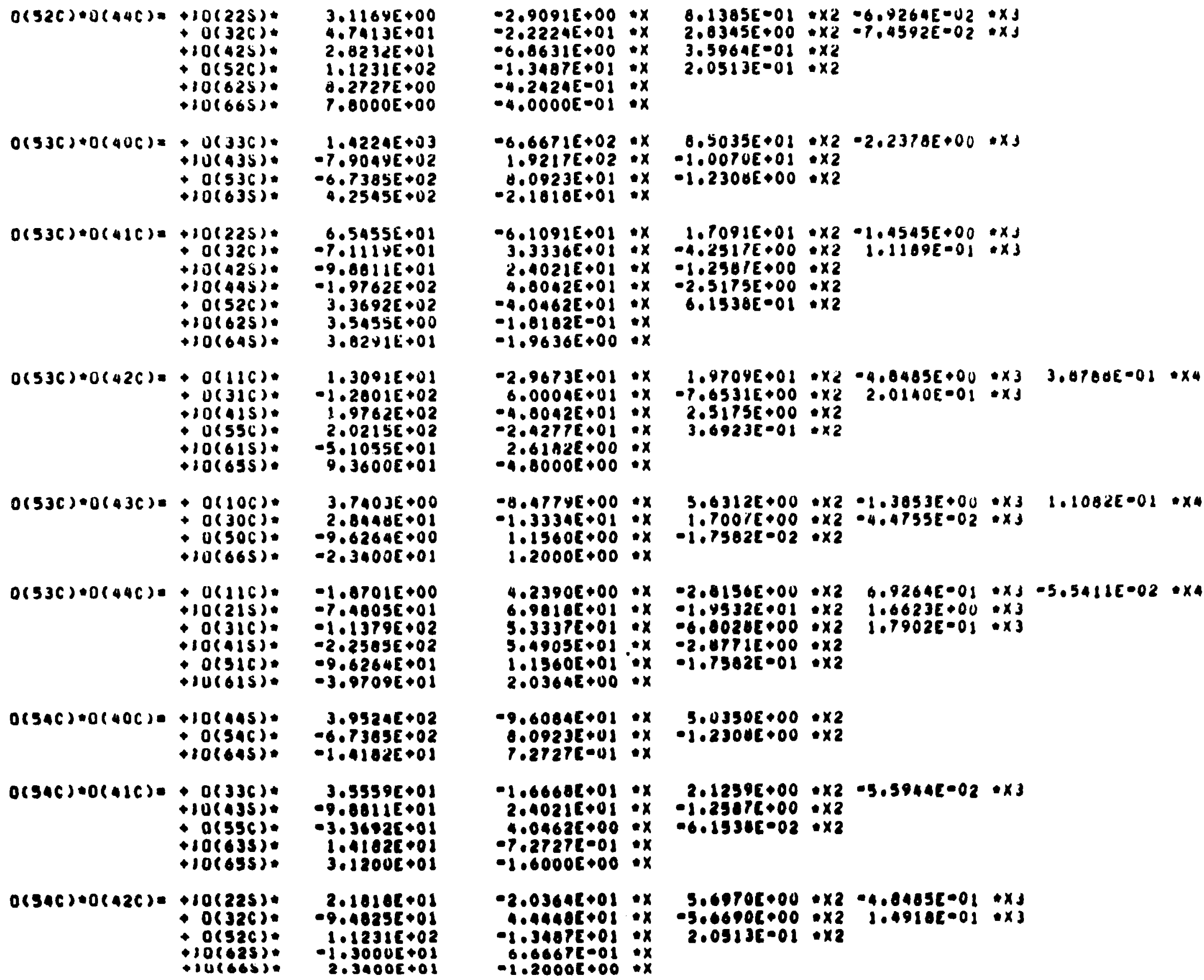

$3.116 y E+00$ $4.7113 E+01$ $2.823<E+01$ $0.2727 E+00$ a.2727E+00

$0(53 C)+0(40 C)=\quad O(33 C)$. $10(435)$ $+0(536)$.

$1.4224 E+03$ $-7.4224 E+03$ $-6.7385 E+02$
$4.2545 E+02$

$0(53()+0(41 C)=+10(225)=$ o( - O(52C). - 10(025). $10(045)$

$6.5455 E+01$ -7.111YE 01 $-9.80116+01$ $-1.97625+02$ $-1.97622+02$ $3.36928+02$ 3.

1.30015001

$0(53 C) \cdot 0(42 C)=0(116)$ o(31C). - O(55). 10 (615). $10(655)$. $-1.2001 E+02$ $3.9762 E+02$ $-5.10555001$ $9.1055 E+01$
$9.3600 E+01$

$3.7403 E+00$

$0(53 C) \cdot 0(43 C)=+0(10 C)$ O(30C). 10(065) $2.84486+01$
$-9.62646+00$ $-9.6264 E+00$
$-2.34005+01$

$-1.07015+00$

$0(53 C) \cdot 0(44 C)=+0(116)=$ $10(215)$. - iocils 0(5ic). $\rightarrow 10(615)$. $-7.4005 E+01$ $-1.13796+02$ $-2.2505 E+02$ $-9.62645+02$

$3.9524 \mathrm{C}+02$ $3.9529 c+02$
$-6.7365 E+02$ $-1.41026 \circ 02$ o(sac)

$3.55598 \bullet 01$

$O(s a c) \cdot 0(416)=0(336)$ $+10(435)$ a(s5c) -3.36.2E+0 1.4182(+02

$0(340) \cdot 0(426)=+10(225)$ O(326). $0(326)$. ?10(6)s):

$-2.9091 E+00 * x$ $-2.2224 E+01 \cdot x$ $0.8631 E+00 * x$ $1.3407 E+01$ I $X$ $-4.2424 E-01: x$ $.0000 E-01 \cdot x$

$-6.66716+02 \cdot x$ $1.9217 E+02 * x-1.0070 E+01 * x 2$

$-2.1010 E+01: x$

$-6.10912+01: x$ $3.3336 E+01: x$ $-4.2517 E+00: \times 2 \quad 1.4545 E+00 * x J$ $0.4021 E+01 * x-1.2581 E+00$ *x2

- 15385001 . $x$

$-4.04625+01$ ix $x$

-1.90365000 : $x$

$-2.96735+01 * x$ $6.00035001 * x$ $-4.0042 E+01 * x$ $-2.4277 E+01$ : $x$ $2.61 A 2 E+00$ ix $-4.8000 E+00: x$

$-8.47748+00$ : $x$ $-1.33346+01 * x$ $1.15606+00$ : $x$ $.20002+00 \cdot x$

$4.2390 E+00 * x-2.0156 E+00 \div \times 2 \quad 0.9264 E-01 * x J-5.5411 E-02 \cdot x 4$

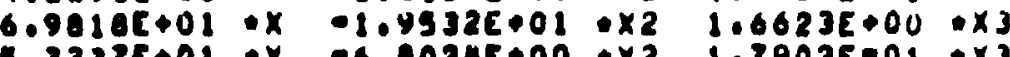

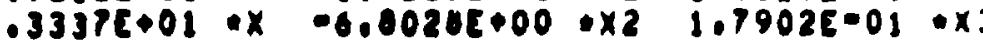

$3.4905 E+01 * x-2.47715+00 \div \times 2$

$2.03640+00 * x$

$-9.6084 E+01 \cdot x \quad 5.0350 E+00 \bullet \times 2$

$0.0923 E+01$ * $-1.2300 E+00 \div \times 2$

i.2727E-01 :x

$-1.06605+01 \cdot x$ $2.40216+01: x$ $4.04625000: x$ -7.27270001 :x

$3.12006+01$

$2.10106+01$ $9.48255+01$ $1.12316+02$ $1.3000 E+01$
$2.3400 E+01$

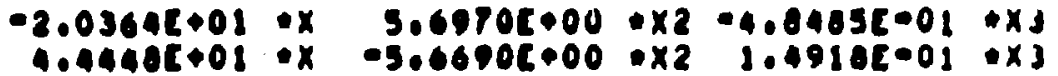

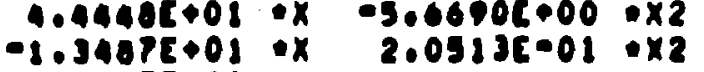

$2.12596+00 \times 2-5.59446-02 \times \times 3$

$1.25076+00 \div \times 2$

$6.66675-01$
-1.2000000

$02+x 2=6.9264 E-02 \cdot x J$

$1.97045+02 \times \times 2-4.04855+00 \div \times 3$

$3.0700 E=01 \cdot \times 4$ 


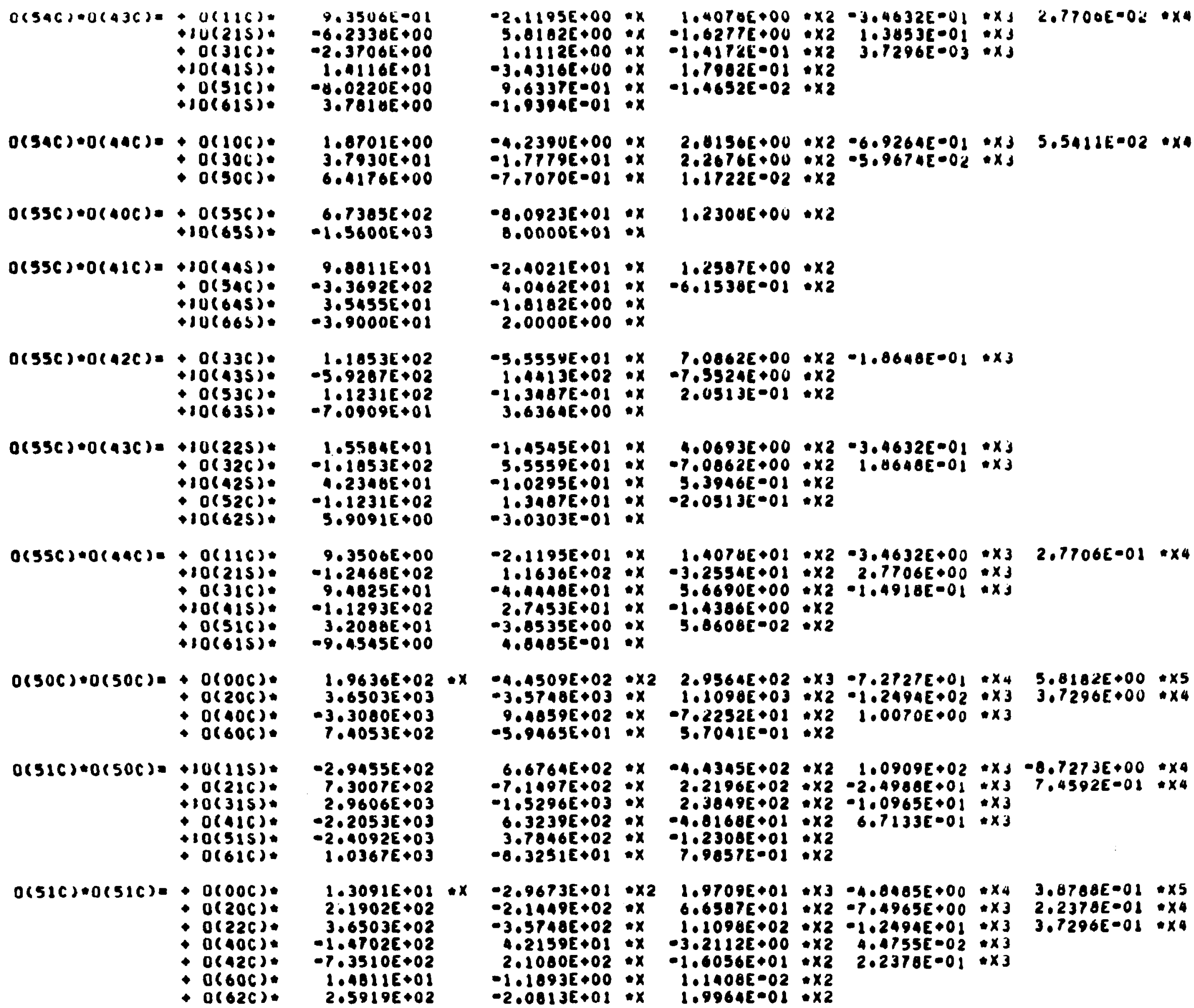

$O(\operatorname{sac} c) 0(43 c)=\quad u(126)$. - lu(2) 15$).$ $+10(4 i s)$. - O(Sic). $\rightarrow 0(516)$.

$O(s 4 C) \cdot 0(A 4 C)=+O(10 C)$ $+0(300)$ - o(soc).

$0(556) \times 0(100)=+0(556)$ $+10(655)$

$0(556) \cdot 0(416)=+10(445)=$ o(5ac). ilvess.

$0(55 C) \cdot 0(12 C)=+0(33 C)$ $+0(536)$ $+10(635)$.

Q. $35006-01$ $-6.23386+00$ $1.0116 E+01$ $-8.02205+00$ $0.0220 E+00$

$1.87016+00$ $3.79306+01$ $6.41765+00$

$6.7305 E+02$ $-1.5600 E+03$

$9.00115+01$ $-3.36926+02$ $3.5455 E+01$

$1.1853 E+02$ $-5.9267 E+02$ $1.1231 E+02$ $1.5504 E+01$
$-1.1053 E+02$ $-1.1053 E+02$ A.23 $368+01$

$-1.1231 E+02$
$5.9091 E+00$ ios

$0(55 C)+0(44 c)=+0(116)$ $+10(215)$ iociss. ( $+0(516)$.
$+10(615)$.

$9.3506 E+00$ $-1.2468 E+02$ $9.4025 E+01$ $1.12936+02$ $3.2088 E+01$

$1.96365+02 . x$

$O(50 C) \times O(50 C)=+O(00 C)$ $0(206)$ $0(40 C)$.
$0(60 C)$. $3.6503 E+03$
$-3.3000 E+03$ $-3.3000 E+03$
$7.4053 E+02$

$O(51 C) \div O(50 C)=+10(115)=$ $0(216)$. - o(4ic). $+10(515)$.

$-2.9455 E+02$ $7.3007 E+02$ $-2.20535+03$ $-2.00925+03$

$1.03675+03$

- 0(616).

$0(51 C) 00(51 C)=+0(00 C)$

- $O(20 C) *$

$0(226)$

- $0(425)$.

- $0(600)$

$+0(62 C)$. $1.3091 E+01$
$2.1902 E+02$ . $19025+02$ $3.6503 E+02$
$-1.0702 E+02$ $-7.35105+02$ $1.0811 E+01$ $2.5919 \varepsilon+02$

$-2.11956+00 * x$ 1.8102E+00 :x $-3.4316 E+00$ : $x$ $9.6337 E-01 * x$ $9.63375=01$
$-1.03946=01: x$

$-4.23906+00 \cdot x$ $-1.7779 E+01$ : $x$ $-1.77796+01$
$-7.7070 E-01$

$-0.0923[+01 * x$ $8.0000 E+01 \cdot x$

$-2.4021 E+01 \cdot x$ $0.0462 E+01 * x$ $1.8182 E+00$ ix 1.441380 1.44136002 ox

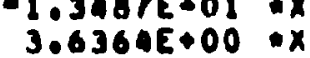

$-1.4545 E+01 * x$ $5.3539 E+01$ : $x$ $103075+01$ ox $1.3407 E+01: x$
$-3.0303 E-01: x$ $-2.1195 E+01$ *x $1.1636 E+02$ : $x$ $2.74536+02: x$ $4.8405 E-01: x$

$1.40705+00 \times \times 2-3.4632 E=01 \times x J$ $2.77006-00 . \times 4$ $10021.3853 E=01$ : $x J$

$1.79025001 \times x^{2}$

$1.4652 E-02 \cdot \times 2$

$2.0150 E+00 * \times 2 \cdot 609264 E-01 \times \times 3 \quad 5.5411 E=02 \cdot \times 0$ $2.26765+00 \cdot \times 2 \cdot 0.96745002 \cdot x^{2}$ $2.26765+00: \times 2$
$1.17225-02: \times 2$

$1.23005+00 \times 2$

$1.2507 E+00 \cdot \times 2$ $-6.1538 E \cdot 01 \div \times 2$

$7.0862 E+00 * \times 2-1.0640 E-01 * \times 3$ $1.02505+00 \times x$

$4.0693 E+00 \cdot \times 2-3.4632 E-01 * x 3$ $-7.0062 E+00: \times 2 \quad 1.4640 E=01: \times 3$ $5.3946 E-01 \times x 2$ $-2.0513 E=01: x^{2}$

$1.40785+01 * \times 2-3.4632 E+00 * \times 3 \quad 2.7706 E-01 * \times 4$ $3.6690 E+00 \cdot x 2 \cdot 1.4918 E-01 * x$ $1.43865+00: \times 2$

$-4.4509 E+02 * \times 2 \quad 2.9564 E+02 * \times 3 \cdot 7.2727 E+01 * x_{4}$ $9.4859 E+02$ ax -7.22525001 ax 1.0070 $5.7041 E-01 \cdot \times 2$

$6.6764 E+02 * x$

$-4.4345 E+02 * \times 2 \quad 1.0909 E+02 * x J-0.7273 E+00 * \times 4$ .1497E+02 *X $2.2196 E+02 * \times 2-2.4980 E+01 * x 3 \quad 7.4592 E-01 * \times 4$

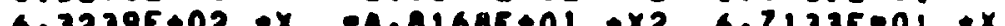
3.7000802 ax 01.023005001 $-8.3251 E+01: x \quad 7.9857 E-01: x 2$

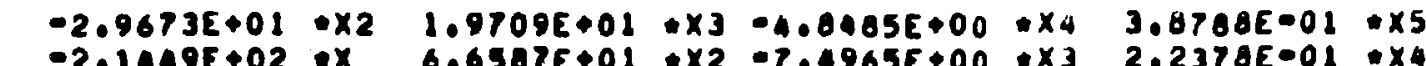

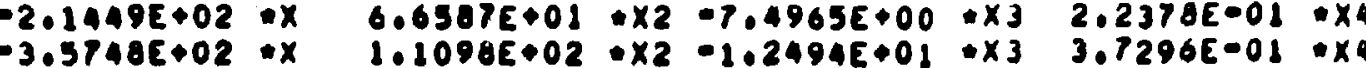
$2.1000 E+02: x-1.6056 E+01: x 2 \quad 2.2370 E-01: x 3$

$-1.1893 E+00 * x \quad 1.1408 E-02 * x$

$-2.0013 E+01$ * $x \quad 1.9964 E-01: \times 2$ 


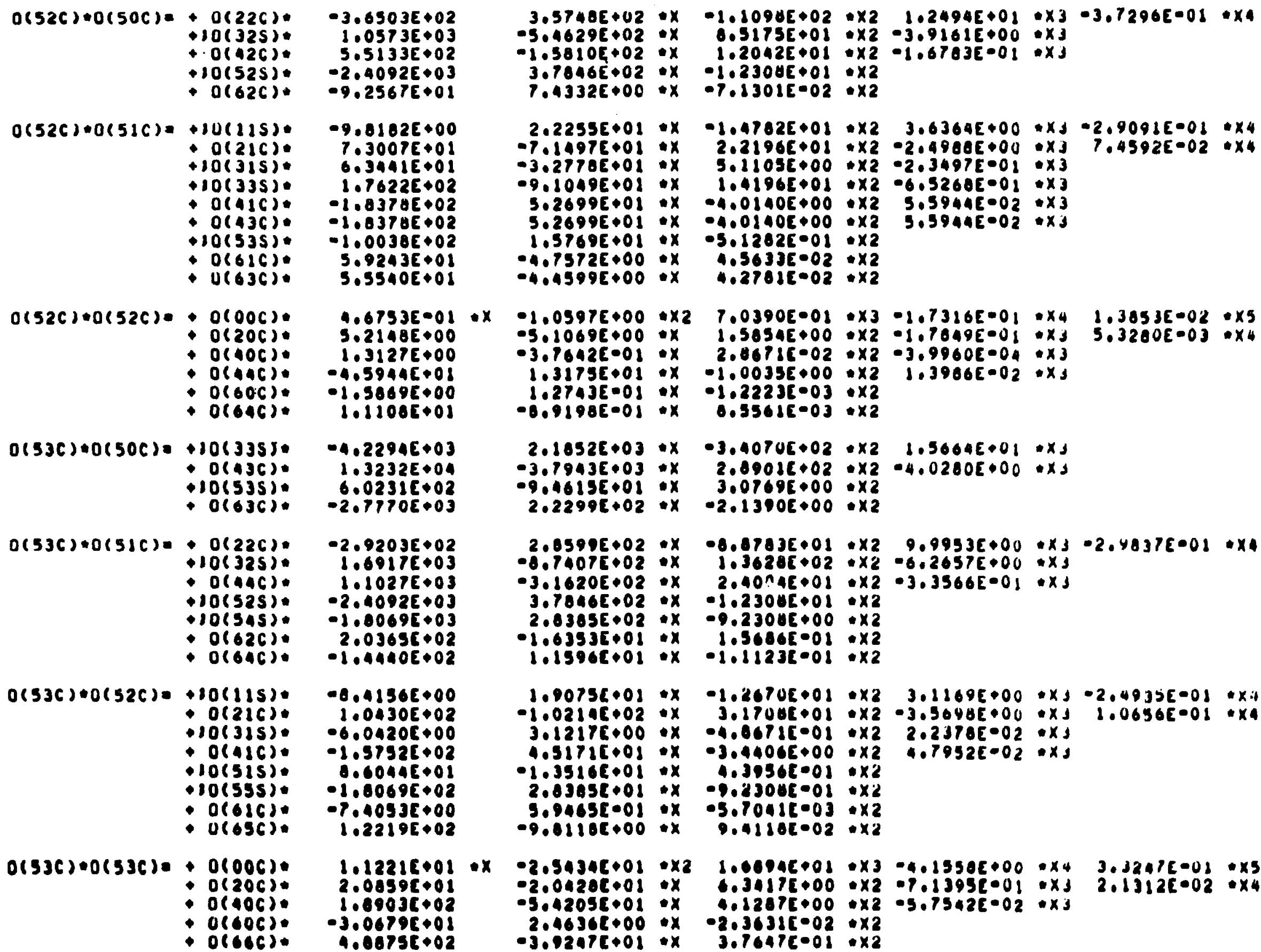

$0(52 C) \cdot 0(50 C)=+0(22 C)$ $10(325)$. $+10(525)$.

$+0(62 C)$.

$0(52 C) \cdot 0(51 C)=+10(115) \cdot$ $+0(216)$. $+10(315)$ $+10(335)$

- O(436).

$10(535)$.

: $0(6) 36)$ (6)

$0(52 C) \cdot 0(52 C)=0(00 C) \circ$ - $0(20 C)$

$+O(A O C)$

- O(Aac).

$+0(60 \mathrm{C})$.

$0(336) \div 0(50 C)=+10(335)=$

$0(436):$
$+10(535):$

- $0(636)$.

$O(53 C) \times 0(5) C)=O(22 C)$ $+10(325)$. $+10(525)$ - 10(sas). - O(62C). - O(64C).

$0(53 C)+0(52 C)=+10(115)$. $0(216)$.
$-10(3 i s)$. - o(aic). $10(515)$. - i0(sssi. - $0(616)$. - o(osci.

$0(536) \cdot 0(536)=0(00 C)$ - $O(20 C)$. O $(606)$.

$-3.6503 E \bullet 02$ $1.0573 E \bullet 03$ $-2.4092 E \bullet 03$ $-9.2567 E \bullet 01$

$-9.01825+00$ $1.3007 E+01$ . 34 Ale 01 $1.0370 E \bullet 02$
0.03760002 $1.8370 E+02$ $1.0038 E+02$ $5.92435 \bullet 01$ $3.5540 E+01$

$4.6753 E=01$ * $5.2148 E+00$ $1.3127 E+00$ $-4.59445+01$ $-1.58695+00$ $1.11085+01$

$-4.22946+03$ $1.3232 E+04$ $6.02316+02$

$-2.92035 \bullet 02$ $1.6917 E+03$ 1.10275003 $-2.4092 E+03$ $-1.00695003$ 2.03655002 $-1.4410 E+02$

$-0.4156 E+00$ $1.04305+02$ - $1.5752 E+02$

- 004 E०01 $-1.0069 E+02$ $-7.00535+00$ $7.4053 E+00$
$1.2219 E+02$

$1.12216+01$ - $x$ $2.00596+01$ $1.00036+02$ $-3.06796+01$ $4.0675 E+02$

$3.57405+02 * x-1.10985+02 \times x 2 \quad 1.2494 E+01 * x 3-3.72965=01 * \times 4$ $-5.4629 E+02 \cdot x \quad 0.5175 E+01 \cdot x 2-3.9161 E+00 * x 3$

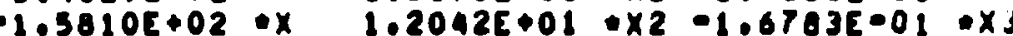

$3.7046 E+02 * x \quad-1.2304 E+01 * x 2$

$2.2255 E+01 * x-1.4702 E+01 * \times 2 \quad 3.6364 E+00 * x_{1}-2.9091 E-01 * x_{4}$ $-7.1497 E+01 * x \quad 2.2196 E+01 \times 2-2.4900 E+00 \cdot x 37.4592 E=02 \times x$ $-3.2770 E+01: x \quad 5.1103 E+00+\times 2-2.3497 E \bullet 01 * x^{3}$

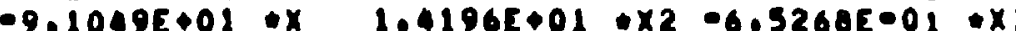
$5.2690 E+01 * x-4.0140 E+00 \div \times 2 \quad 3.5944 E-02 \times x 3$ $5.2699 E+01 * x-4.0140 E+00 * x 25.5944 E 002 * x j$ $1.3769 E+01: x-5.1202 E-01 * x 2$ $-4.7572 E+00 * x \quad 4.36335 \cdot 02 * x 2$

$-4.4999 E+00 \cdot x \quad 4.2701 E-02 \times x 2$

$-1.05975+00 \times 2$ $-5.10696+00 * x$ $-3.76425001: x$ 1.31735001 : $x$ $1.27435001: x$ $1.2743 E-01: x \quad-1.2223[-03: \times 2$
$-8.9198 E-01: x \quad 0.3561 E-03: \times 2$

$7.0390 E-01 \cdot x_{3}-1.7316 E=01 \times x_{4} \quad 1.3853 E-02 \times x^{2}$ $1.5854 E+00 \times x 2-1.7049 E=0, \times 3 \quad 5,3280 E-03: x 4$

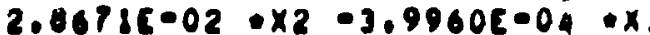

$2.1052 E+03 * x-3.40706+02 \times x^{2} 1.5604 E+01 * x_{3}$ $-3.7943[003 * x \quad 2.09016+02 * x 2 \cdot 4.0280[+00 * x \mathrm{~J}$

$0.46155+01$ * $3.0769 E+00 \times x^{2}$ $2.22995+02 * x-2.1300 E+00 * x 2$

$2.0599 E+02 * x-8.0783 E .01+x 2 \quad 9.9953 E+00 \times x_{3}-2.4837 E-01 \times x_{4}$ $-8.7407 E+02 * x \quad 1.3628 E+02 * x 2-6.2657 E+00 * x j$ $-3.1620 E+02 * x \quad 2.40 n 4 E+01 * x 2-3.3566 E-01 * x J$

$3.7046 E+02 * x-1.2300 E * 01 * x 2$

$2.0305 E+02 * x-9.2300 E \bullet 00 * x 2$

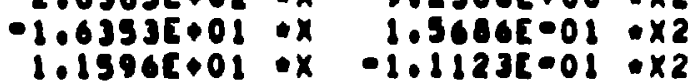

$1.90756+01 \times x-1.26705001 \times x 2 \quad 3.11695000 \times x 1-2.4935 E-01 * x: 1$ $1.02146+02 \cdot x$ $3.12175+00 \times x$ $0.5171[+02 \cdot x$ $-1.3516 c 001$ ix $2.03058001: x$ $3.94655001 \cdot x$ $3.17005001 \cdot \times 2-3.50485000$ * $x 3$ $0.06715-01 \times 2 \quad 2.2378 E-02$ * $x$

$0.44065+00 \cdot x 2 \quad 1.70525=02 \times x$

$9.41102 .02: \times 2$

$-2.5434 c+01 \cdot \times 2$

$1.00946001+x_{3}=4.15506+00 \times x_{4}$ $-2.04206+01 \times x \quad 6.34175+00 * x 2-7.13956001 \times x d$ $-5.4205 E+01 * x \quad 0.12875000 * x 2-5.75425 \cdot 02 * x$

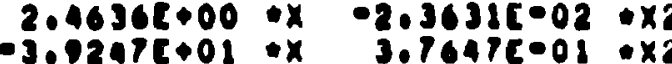

$3.32476003 \cdot x 5$ 


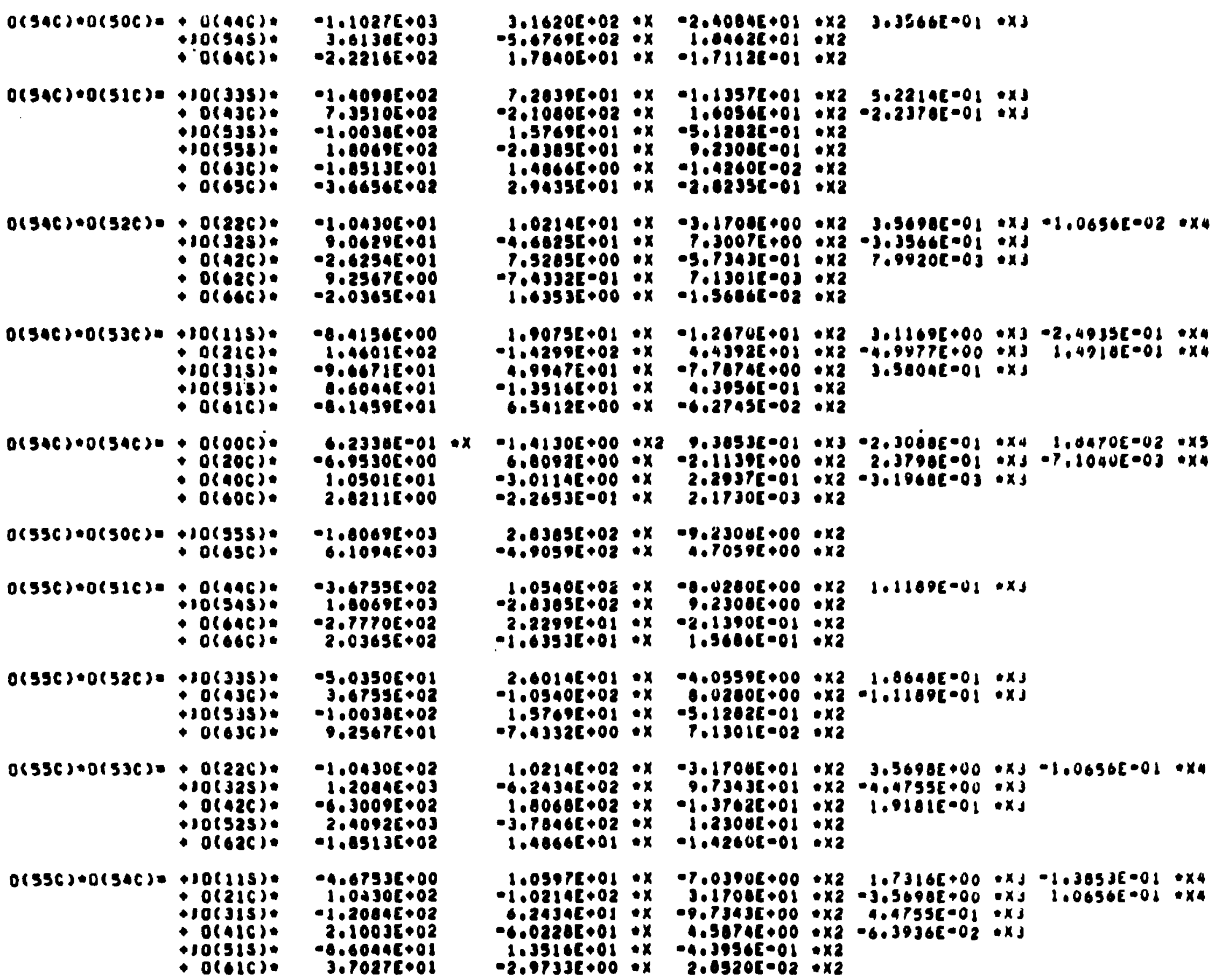

$0(540) \cdot 0(500)=0(446)$. $10(545)$.
$0(646)$.

$0(5+6) \cdot 0(516)=+10(335)=$ $\rightarrow 0(436)$ $10(535)$

iorsss

O $0(636)$.

$0(346)=0(526) 0+0(226) 0$ $10(325)$ - Orazs

orces.

$0(346) \cdot 0(336)+.0(115)$ - O(2ic). io(sis).

- o(bic).

$0(5) C) 0(54 C) 0+0(006)$ O(20c). O O 906$)$ $7.35105 \cdot 02$ $-1.00306 .02$ 100068002 $-1.85135+01$
-3.66506 .02

10090508 9.06296002 - 06254001 $-2.03650 .08$

$-8.41506+00$ $2 \cdot 46016+02$ 0.06715001 $-0.14396008$

$0.23305-01 \cdot x$ $-6.9530[+00$ $1.0501 E \bullet 01$

$-1.0069[0.03$ + $0(056)$. $6.10946 \bullet 03$

$-3 \cdot 6755 c+02$ 1.60695003 $-2.77706+02$
$2.03636+02$ o(sec)

-

$0(536) \cdot 0(526)=+10(335)=$ O(436).

io(ejc)

3.675

$-1.0030 \mathrm{c}+02$

$-1.0038 c+02$
$9.25676+01$

$0(55 C) \times 0(53 C)=0(226)=$

o(4ac).

$\rightarrow 10(323)$

$-1.04305+02$ $1.20046+03$ $-63009[\bullet 02$ $-1.05135002$

$0(356)+0(3 \circ C)=P(0(12 s)=$ $\rightarrow 0(216)$ - o(4if). $\because 0(5 i s)$. 1.04306 .02 $-1.20845+02$ $2.10035 \cdot 02$ $-0.6004 c+01$ $3.70276 \cdot 01$

$3.1620[002 \times x-2.4004[001+x 23.3566[-01 * x]$

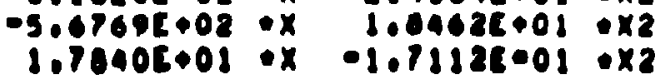

$1.3030[001 \cdot x-1.13572001 \cdot \times 2 \quad 5.22108001$

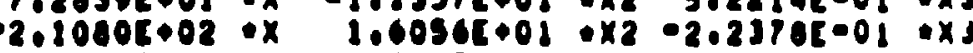

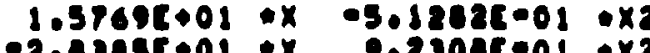

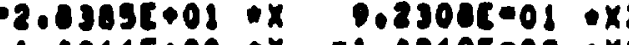

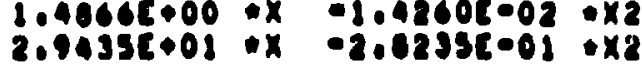

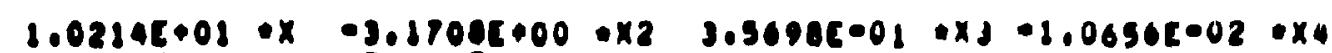

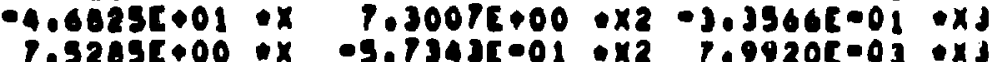

$-7.4335[0.01$ : $x$ P.130ic-0j : $x 2$

$1.63535+00$ ix -1.36060 .02 : $x 2$

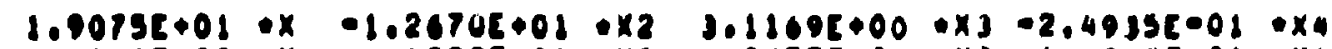

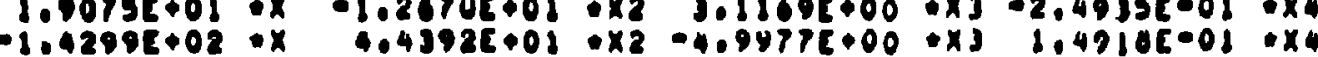

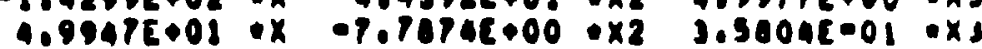

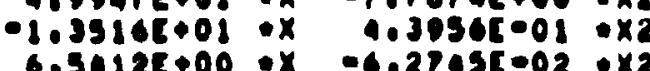

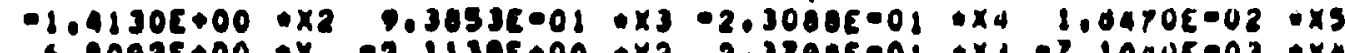

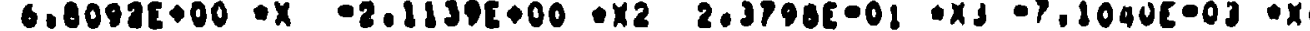

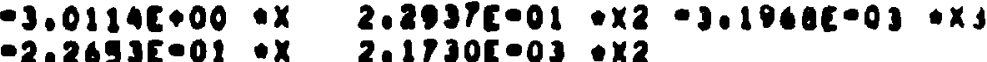

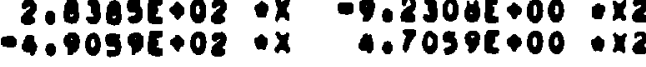

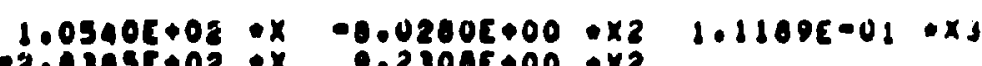

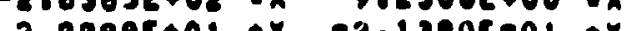

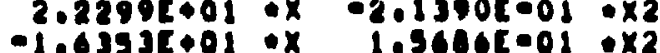

$2.60146+01 * x-4.05596000 * \times 2 \quad 1.86408-01 * x 3$

$-1.0540[+02$ \& $\quad .0280 E+00 * x 2-1.1180[-01 * x J$

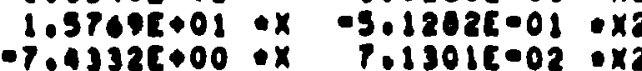

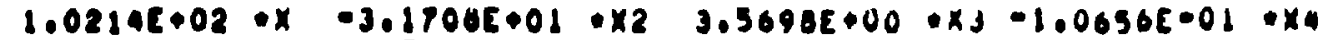
$-6.2434[+02$ \& $9.7343[0.01: \times 2-4.4755 E+00: x 3$

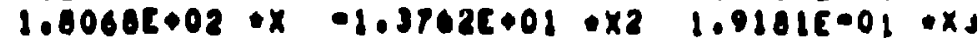

$-3.7846 E+02$ \& $1.2300 E+01$ *xa

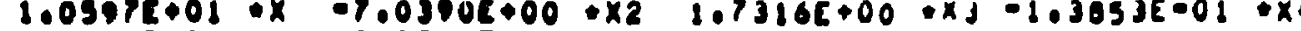

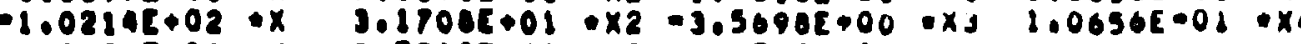

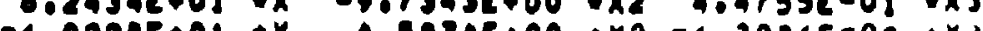
$6.02205001 \times x \quad 0.59746+00 * \times 2-6.39366-02 * x J$

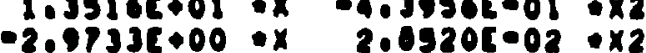




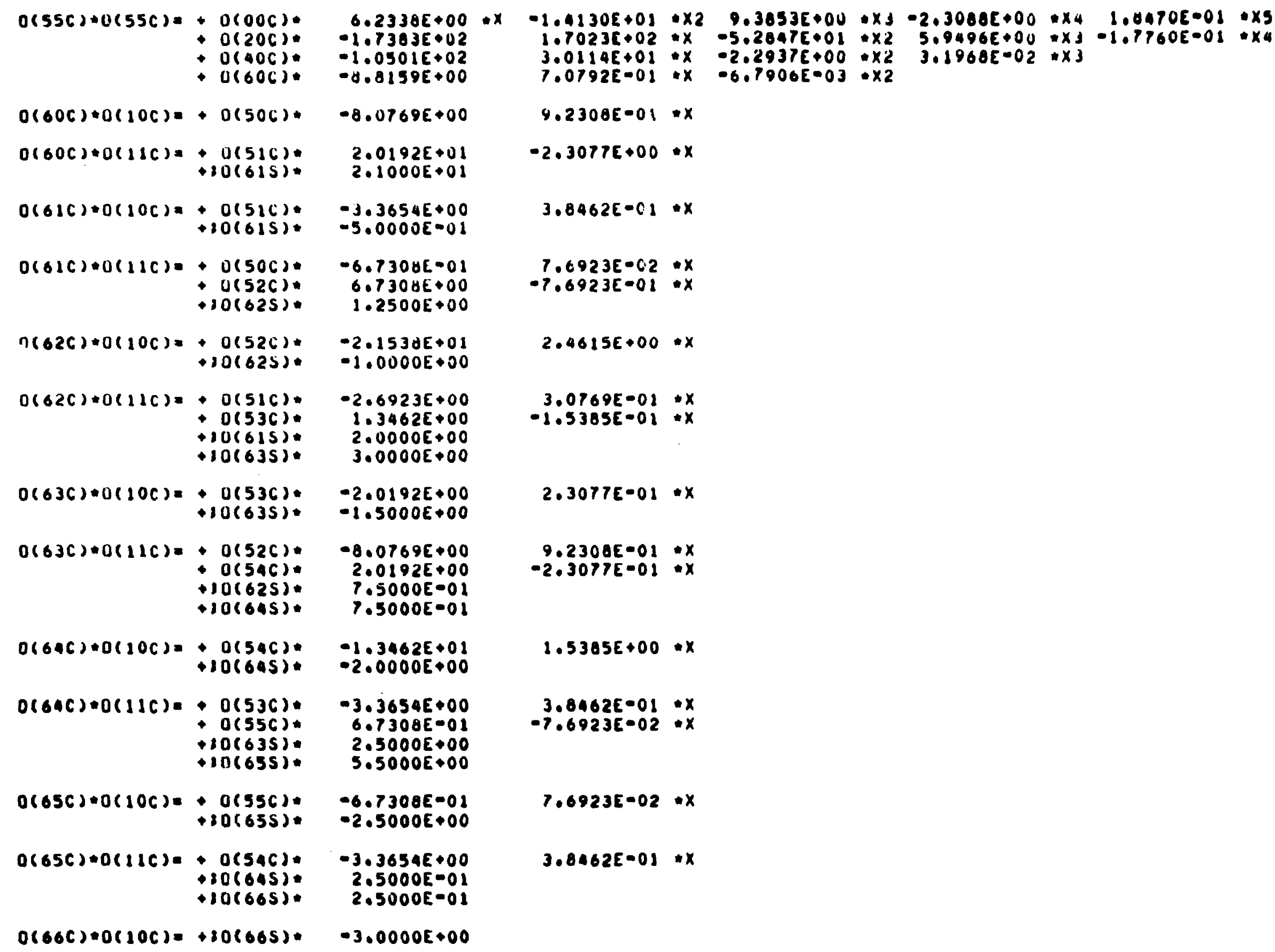

$-2.6923 E+00$

$1.3462 E+00$ $2.0000 E+00$ 3.0000E +00

$-2.0192 E+00$ $-1.5000 E+00$

$-1.4130 E+01 \times 2 \quad 9.3853 E+00 * x_{3}-2.3008 E+00 \times x_{4} \quad 1.0470 E-01 * \times 5$ $1.7023[+02 * x \quad-5.2847 E+01 * x 25.9496 E+00 \times x 3-1.7 r 60 E=01 * x$

$\begin{array}{llll}3.0114 E+01: x & -2.2937 E+00 & \times 2 & 3.1960 E-02 \\ 7.0792 E-01: x & -6.7900 E-03: \times 2 & \end{array}$

$9.2308 E-01 \cdot x$

$-2.30776+00 \cdot x$

$3.8462[-01 \cdot x$

$7.6923 E-02+x$

$-7.6923 E-01 * x$

$2.4615 E+00 * x$

$3.0769 E-02 \div x$

$-1.5385 E-01 * x$

$2.3077 E \cdot 01 \cdot x$

$-8.0769 E+00$

$2.0192 E+00$

$7.5000 E-01$

$7.5000 E 001$

$\begin{aligned} 0(64 C) \cdot O(10 C)= & +0(54 C): \\ & +10(645)\end{aligned}$

$-2.0000 E \bullet 00$

$O(64 C) * 0(11 C)=+0(53 C)$

$-0(556)$.

. $3654 E+00$

$6.7308 E-01$

$2.5000 E+00$ $+10(655)$ 5.5000E +00

$9.2300 E-01: x$

$-2.3077 E-01: x$

$1.5385 E+00: x$

$3.8462 E-01 \cdot x$

$-7.6923 E-02: x$

$0.73085-08$

$-2.5000 E+00$

$7.6923 E-02 \cdot x$

$+30(655)$.

$-3.3654 E+00$

$2,5000 E-01$

$3.8462 E-01: x$

$0(66 C)+0(10 C)=+80(665) \cdot-3.0000 E+00$ 


\begin{tabular}{|c|c|c|c|c|c|c|}
\hline $0(66 C)=0(11 C)=$ & $\begin{array}{l}+0(55()= \\
+10(655)=\end{array}$ & $\begin{array}{r}-4.0385 E+00 \\
3.0000 E+00\end{array}$ & $4.6154 E-01$ & $x$ & & \\
\hline $0(60 C) \cdot 0(20 C)=$ & $\begin{array}{l}+0(40 C) \\
+0(006)\end{array}$ & $\begin{array}{r}6.6004 E+01 \\
-1.5655 E+01\end{array}$ & $\begin{array}{r}-1.8566 E+01 \\
5.0909 E-01\end{array}$ & $\begin{array}{l}x \\
: x\end{array}$ & $1.2587 E \diamond 00$ &.$\times 2$ \\
\hline $0(60 C)+0(216)=$ & $\begin{array}{l}+O(416)= \\
+10(515) \\
+0(61 C) .\end{array}$ & $\begin{array}{l}-8.8112 E+01 \\
-7.0673 E+01 \\
-7.8273 E+00\end{array}$ & $\begin{array}{l}2.4755 E+01 \\
0.0769 E \gg 00 \\
2.5455 E-01\end{array}$ & $\begin{array}{l}x \\
: x \\
x\end{array}$ & $-1.6783 E+00$ & $\times 2$ \\
\hline $0(60 C)=0(226)=$ & $\begin{array}{l}+O(426) * \\
+0(525) * \\
+O(626) *\end{array}$ & $\begin{array}{l}1.4056 E+01 \\
5.6530 E+02 \\
7.8273 E+01\end{array}$ & $\begin{array}{l}-1.2378 E+01 \\
-6.4615 E+01 \\
-2.5455 E+00\end{array}$ & $\begin{array}{l}x \\
: x \\
x\end{array}$ & $8.3916 E-01$ & $\times 2$ \\
\hline $0(616)+0(206)=$ & $\begin{array}{l}+O(416) \\
+10(515) \\
+0(616)\end{array}$ & $\begin{array}{r}1.4056 E+01 \\
1.0096 E+01 \\
-1.4536 E+01\end{array}$ & $\begin{array}{r}-1.2378 E+01 \\
-1.1536 E+00 \\
4.7273 E-01\end{array}$ & $: x$ & $0.3916 E-01$ & $\pm \times 2$ \\
\hline $0(616) * 0(21())=$ & $\begin{array}{l}+O(40 C) \\
+0(42 C) \\
+\quad 0(525) \\
+0(60 C) \\
+0(62 C)\end{array}$ & $\begin{array}{r}1.0357 E+00 \\
-3.6713 E+00 \\
-3.0288 E+01 \\
-9.3182 E-02 \\
-1.3977 E+00\end{array}$ & $\begin{array}{r}-5.1573 E-01 \\
1.0315 E+00 \\
3.4615 E+00 \\
3.0303 E-03 \\
4.5455 E-02\end{array}$ & $\begin{array}{l}: x \\
: x \\
x \\
x \\
x \\
x\end{array}$ & $\begin{array}{r}3.4965 E=02 \\
-6.9930 E=02\end{array}$ & $\begin{array}{l}\times 2 \\
\times 2 \\
\times 2\end{array}$ \\
\hline $0(6)()=0(22 C)=$ & $\begin{array}{l}+0(416) . \\
+0(436) \\
+\quad 0(515) . \\
+10(535) . \\
+0(616) . \\
+0(636) .\end{array}$ & $\begin{array}{r}-7.3427 E+00 \\
7.34276+00 \\
-1.0096 E+01 \\
6.7308 E+00 \\
-7.8273 E+00 \\
1.1182 E+01\end{array}$ & $\begin{array}{r}2.0629 E+00 \\
-2.0629 E+00 \\
1.1538 E+00 \\
-7.6923 E-01 \\
2.5455 E-01 \\
-3.6364 E-01\end{array}$ & $\begin{array}{l}x \\
: x \\
: x \\
: x \\
: x \\
x\end{array}$ & $\begin{array}{r}-1.3986 E-01 \\
1.3986 E-01\end{array}$ & $\begin{array}{l}* \times 2 \\
\times 2\end{array}$ \\
\hline $0(62 C)=0(20 C)=$ & $\begin{array}{l}+0(426) . \\
+10(525) . \\
+0(626)\end{array}$ & $\begin{array}{r}3.5245 E+01 \\
1.2923 E+02 \\
-1.1182 E+01\end{array}$ & $\begin{array}{r}-9.9021 E+00 \\
-1.4769 E+01 \\
3.6364 E-01\end{array}$ & $\begin{array}{l}: x \\
: x \\
x\end{array}$ & $6.71335-01$ & $\times 2$ \\
\hline $0(62 c)=0(21 C)=$ & 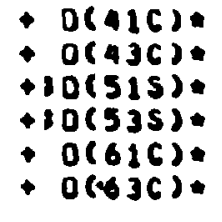 & $\begin{array}{r}1.1748 E+01 \\
-1.1748 E+01 \\
-1.0385 E+00 \\
-7.0038 E+00 \\
-2.23640+00 \\
-5.5909 E+00\end{array}$ & $\begin{array}{r}-3.3007 E+00 \\
3.3007 E+00 \\
1.6154 E-01 \\
8.0615 E-01 \\
7.2727 E-02 \\
1.0182 E-01\end{array}$ & $\begin{array}{l}: x \\
: x \\
: x \\
* x \\
* x \\
* x\end{array}$ & $\begin{array}{r}2.2378 E-01 \\
-2.2378 E-01\end{array}$ & $\begin{array}{l}: \times 2 \\
\times 22\end{array}$ \\
\hline $0(62 C) * 0(22 C)=$ & $\begin{array}{l}P(406): \\
+O(44 C): \\
+O D(545): \\
+O(80 C): \\
+O(64 C):\end{array}$ & $\begin{array}{l}2.9371 E+00 \\
1.9685 E+00 \\
2.4231 E+U 1 \\
1.4909 E+00 \\
6.7091 E+00\end{array}$ & $\begin{array}{l}-8.2517 E-01 \\
-4.1259 E-01 \\
-2.7692 E+00 \\
-4.0405 E-02 \\
-2.10108-01\end{array}$ & $\begin{array}{l}: x \\
: x \\
: x \\
: x \\
x\end{array}$ & $\begin{array}{l}5.5944 E-02 \\
2.7972 E-02\end{array}$ & $\begin{array}{l}\times 2 \\
\times 2\end{array}$ \\
\hline$O()=$ & $\begin{aligned} & O(436) \\
+ & 0(535): \\
+ & 0(636)\end{aligned}$ & $\begin{array}{r}5.2067 E+01 \\
1.0173 E+01 \\
-5.5909 E+00\end{array}$ & $\begin{array}{r}-1.0053 E+01 \\
-2.0769 E+00 \\
1.0102 E-01\end{array}$ & $\begin{array}{l}x \\
x \\
x \\
x\end{array}$ & $1.0070 E * 00$ & $\times 2$ \\
\hline
\end{tabular}




\begin{tabular}{|c|c|c|c|c|c|c|c|}
\hline $0(63 C) * 0(21 C)=$ & 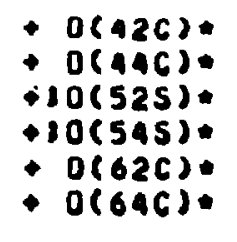 & $\begin{array}{r}1.056 E+00 \\
-1.1014 E+00 \\
-1.0385 E+00 \\
-1.3125 E+01 \\
-1.3977 E+00 \\
-1.9568 E+00\end{array}$ & $\begin{array}{r}-1.2378 E+00 \\
3.0944 E=01 \\
4.6154 E=01 \\
1.5000 E+00 \\
1.5455 E=02 \\
6.3636 E=02\end{array}$ & $\begin{array}{l}: x \\
: x \\
x \\
x \\
x \\
x \\
x \\
x\end{array}$ & $\begin{array}{r}0.3916 E-02 \\
-2.0979 E-02\end{array}$ & $\begin{array}{l}: \times 2 \\
: \times 2\end{array}$ & \\
\hline $0(63 c)=0(22 C)=$ & $\begin{array}{l}+0(416): \\
+D(515): \\
+10(555): \\
+0(616): \\
+0(656) .\end{array}$ & $\begin{array}{r}8.8112 E+00 \\
-8.0769 E+00 \\
2.0192 E+00 \\
1.4727 E+00 \\
1.2300 E+01\end{array}$ & $\begin{array}{r}-2.4755 E \bullet 00 \\
9.2300 E-01 \\
-2.3077 E-01 \\
-1.4545 E-01 \\
-4.0000 E-01\end{array}$ & $\begin{array}{l}: x \\
: x \\
: x \\
x \\
x \\
x\end{array}$ & $1.6703 E=01$ & $\times 2$ & \\
\hline $0(64 C) \cdot 0(20 C)=$ & $\begin{array}{l}0(446) \\
+10(545) \\
+0(64 C)\end{array}$ & $\begin{array}{l}2.2028 E \bullet 01 \\
1.6154 E+02 \\
2.236 \oplus E \bullet 00\end{array}$ & $\begin{array}{l}-6.1808 E+00 \\
-1.8462 E+01 \\
-7.2727 E-02\end{array}$ & $\begin{array}{l}: x \\
: x \\
: x\end{array}$ & $4.8958 E-01$ & $\times 2$ & \\
\hline $0(64 C) \cdot 0(21 C)=$ & $\begin{aligned} & O(436): \\
&+ 0(535): \\
&+O(555): \\
&+O(636): \\
&+O(656)\end{aligned}$ & $\begin{array}{r}2.9371 E \bullet 01 \\
1.6827 E \bullet 00 \\
-5.0481 E+00 \\
-6.5227 E+00 \\
-1.8450 E \bullet 01\end{array}$ & $\begin{array}{r}-0.2517 E+00 \\
-1.9231 E-01 \\
5.7692 E-01 \\
2.2212 E-01 \\
6.0000 E-01\end{array}$ & $\begin{array}{l}: x \\
: x \\
* x \\
* x \\
x \\
x\end{array}$ & $5.5944 E-01$ & $\cdot \times 2$ & \\
\hline $0(64 C)-0(22 C)=$ & $\begin{array}{l}+O(42 C) * \\
+D(525) \\
+0(62 C) \\
+0(666)\end{array}$ & $\begin{array}{r}1.4685 E \bullet 01 \\
-8.0769 E \mapsto 01 \\
5.5909 E \mapsto 00 \\
1.2000 E+00\end{array}$ & $\begin{array}{r}-4 \cdot 1259 E+00 \\
9.2300 E+00 \\
-1.0102 E-01 \\
-1.3333 E-01\end{array}$ & $\begin{array}{l}* x \\
* x \\
* x \\
* x\end{array}$ & $2.7972 E-01$ & $\times 2$ & \\
\hline $0(656) * 0(20 C)=$ & $\begin{array}{l}+10(555) . \\
+0(656) .\end{array}$ & $\begin{array}{l}1.0096 E \bullet 01 \\
1.2300 E \bullet 01\end{array}$ & $\begin{array}{l}-1.1538 E+00 \\
-4.0000 E-01\end{array}$ & $\frac{x}{x}$ & & & \\
\hline$D(65 C) \cdot 0(21 C)=$ & $\begin{array}{l}+0(446) \\
+\quad 0(545) \\
+0(64 C) \\
+0(666)\end{array}$ & $\begin{array}{r}1.8357 E+00 \\
5.0401 E+00 \\
-8.3064 E-01 \\
-1.0250 E+00\end{array}$ & $\begin{array}{r}-5.1573 E-01 \\
-5.7692 E-01 \\
2.7273 E-02 \\
3.3333 E-02\end{array}$ & $\begin{array}{l}\cdot x \\
: x \\
: x \\
: x\end{array}$ & $3.4965 E-02$ & $\times 2$ & \\
\hline $0(65 C)+0(22 C)=$ & $\begin{aligned} & O(436): \\
+ & 0(535) \\
+ & 0(636):\end{aligned}$ & $\begin{array}{r}1.4685 E+01 \\
-3.3654 E+00 \\
1.8636 E+00\end{array}$ & $\begin{array}{r}-4.1259 E+00 \\
3.8462 E-01 \\
-6.0606 E-02\end{array}$ & $\begin{array}{l}: x \\
: x \\
: x\end{array}$ & $2.7972 E-01$ & $\times 2$ & \\
\hline $0(66 C)+0(20 C)=$ & $+0(66 C)$ & $2.4600 E+01$ & $-8.0000 E-01$ & $\bullet x$ & & & \\
\hline $0(66 C)=0(21 C)=$ & $\begin{array}{l}+10(555): \\
+0(656)\end{array}$ & $\begin{array}{r}1.0096 E+01 \\
-1.2300 E+01\end{array}$ & $\begin{array}{r}-1.1530 E+00 \\
1.0000 E-01\end{array}$ & $\begin{array}{l}* x \\
x\end{array}$ & & & \\
\hline $0(660) * 0(22 C)=$ & $\begin{array}{l}+O(446): \\
+10(545): \\
+O(846) .\end{array}$ & $\begin{array}{r}2.2028 E+01 \\
-4.0305 E+01 \\
2.2364 E+00\end{array}$ & $\begin{array}{r}-6.1088 E+00 \\
1.6154 E+00 \\
-7.2727 E=02\end{array}$ & $\begin{array}{l}x x \\
x \\
x \\
x \\
x\end{array}$ & $4.89585=01$ & $\times 2$ & \\
\hline $0(606) \cdot 0(300)=$ & $\begin{array}{l}-O(306)= \\
+0(506)=\end{array}$ & $\begin{array}{r}-7.3427 E+02 \\
2.6385 E+02\end{array}$ & $\begin{array}{r}4,0210 E+02 \\
-3.6436 E+01\end{array}$ & $\stackrel{* x}{x}$ & $\begin{array}{r}-6.0998 E+01 \\
7.8795 E-01\end{array}$ & $\begin{array}{l}: \times 2 \\
\times 2 \\
\times 2\end{array}$ & $3.72965 \$ 00 \times 3$ \\
\hline
\end{tabular}




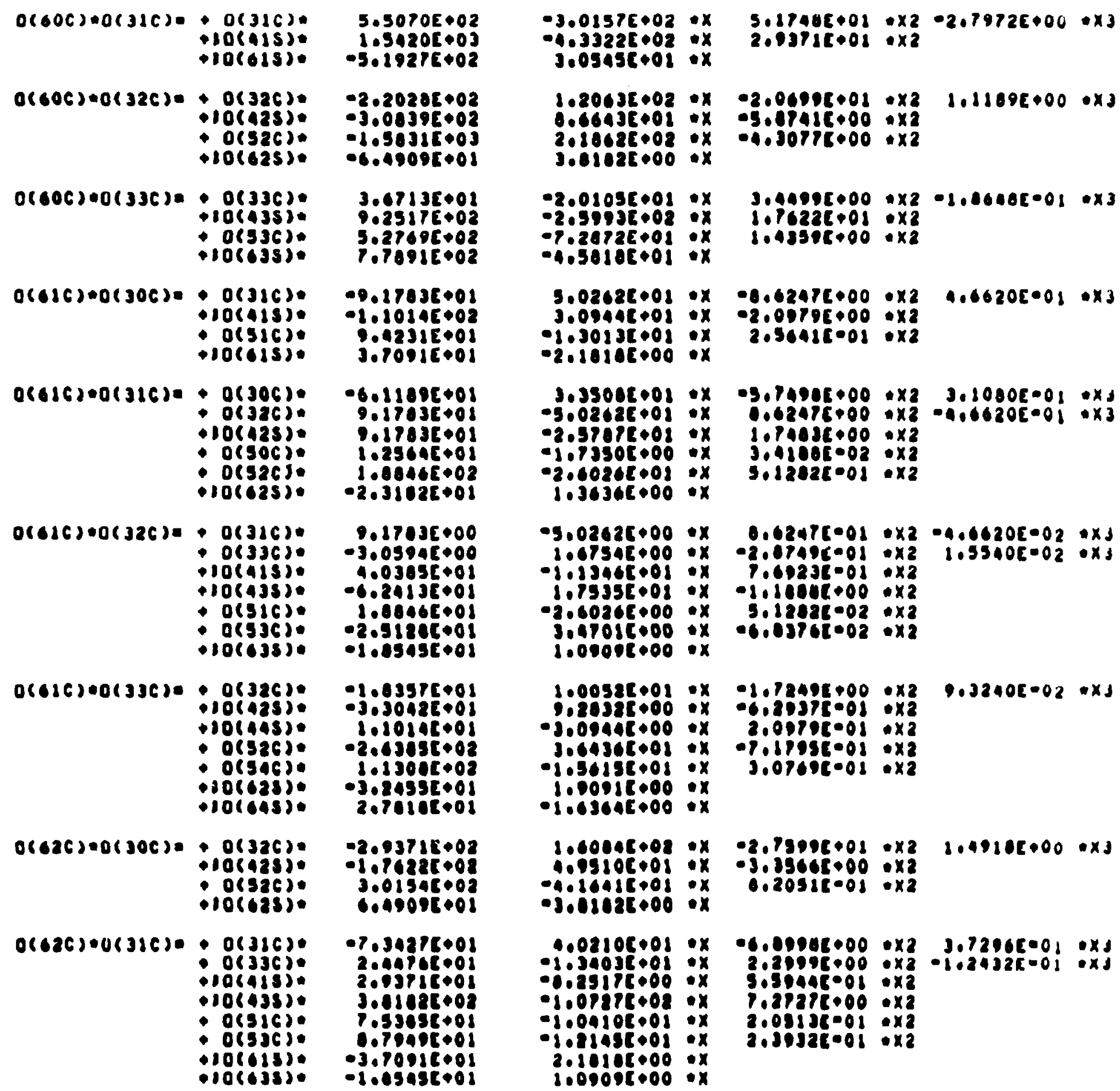

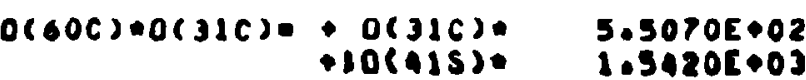
10(615). -5.192rE+02

$0(606) \cdot 0(326)=+0(326)=$ io( $+10(625)$.

$0(600) 00(3) 6)=0(336)$ - $10(435)$ O(536).

$-2.2028[\bullet 02$ $-3.08396002$ -1.56315003
$-6.4900[001$

3.07135001 g.5siretos $5.27696+02$
P.7091E॰02

$0(016) \times 0(306)=0(316)$ - $10(415)$. $\rightarrow$ o(sic).

$0.0 .17635+01$ $-1.1014 E \cdot 02$ $0.931 E \bullet 01$
0.70015001 j.rovicod

$0(616)+0(316)=0(306) 0$ io(s) - oesocj. o(s26).

$-6011895+01$ $0.17835 \circ 01$ $1.2564 E+01$ $1.00465+02$

$0(016) \cdot 0(326)=0(316)=$ O(336): $10(415)$. - lo(ajs). - o(sic): - iocojss.

$9.17035 \bullet 00$ $-3.05945+00$ $4.0305 E+01$ 1.0846 col $-2.51806008$ $-1.05456+01$

$0(436) \cdot 0(336)=0(0(326)=$ $10(425) .0$
$+10(445) .0$

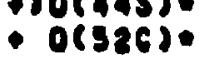
io(sugs: i. io(6s)

- 10 .6357e+01 $1.10 i+6+01$ $-2.0345[+02$ $1.13005+02$ $-3.84535 \cdot 01$
2.70105001

$0(62 c)=0(305)=0(3)=0(0)$ $10(425)=-1.76225+02$ 10(326): 3.0154c.02

$0(626) 00(326) \circ 0(316)$ - $0(336)$ - $10(413)$. $10(435)$. - o(sic)

$-7.34375+02$ 2.90705002 3.01025002 P.s3ose+01 - $7040 \mathrm{cos}$ $\rightarrow$ iocessio.

$-3.01575002 * x$ $\begin{array}{rl}-0.3322 E+02 & * x \\ 3.05456+02 & -x\end{array}$

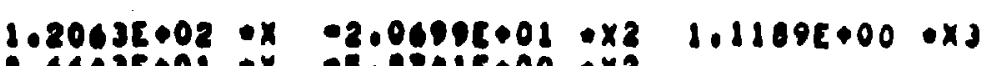
0.66435002 ox -5.97416000 ix 2.16625002 ox $-4,307 r 600 * x^{2}$ $3.81025 \cdot 00 \cdot x$

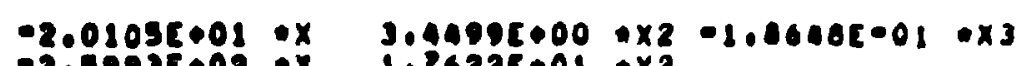

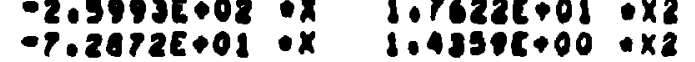

$-4.5010[+01$ ix

$3.02682 \cdot 02 \cdot x$

$00.62475+00 \cdot \times 2 \quad 0.0620[\bullet 01 \times x 3$ - $02.0970 E+00 \times x 2$

-1.30135001 :x $2.564 i c 02: x 2$

-2.18105000 : $x$

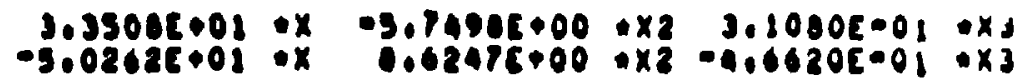
$-2.5707<+02 \cdot x$ $-1.7350[000 \cdot x$

$-5,02625+00$ *x $0.62476-01 \cdot x 2-4,06205-02$ ax

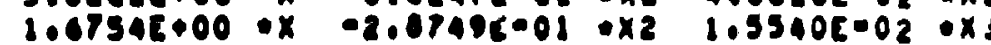

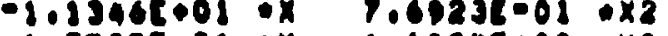
1.75355008 : $x$

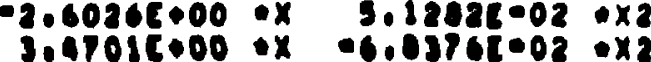

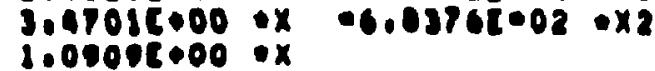

$1.00525+01$ : $x \quad-1.72495+00$ - $\times 2$

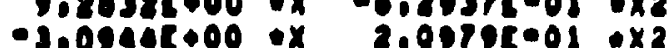

- Mogatcoo ex 2.0979600 ex

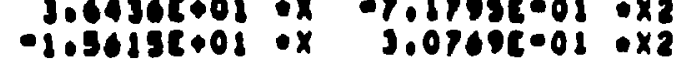
$1.009 i c+00 \cdot x$ 01.0304 E.00 : $x$

$1.00045 \cdot 02$ :x

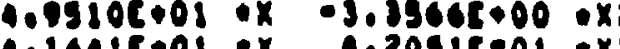

-30103000 : $x$

$0.02105001 \cdot x$ -1.31038001 ox

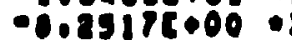
- 1 oparteos of $-1.04102+01$ : $-1.8105 t+01 * x$ $2.10106000 * x$
$100005800 * x$

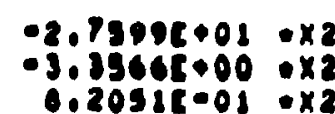

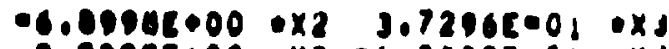
$3.20996000 \times x^{2}-1,24325=01: x d$

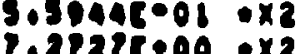

2.09136001 : $x$

$2.30326008: x 2$ 


\begin{tabular}{|c|c|c|c|c|c|c|c|c|}
\hline $0(62 C)+0(32 C)=$ & $\begin{array}{l}+0(30 C): \\
+\quad 0(44 S) \\
+0(50 C) \\
+0(54 C) \\
+10(645)\end{array}$ & $\begin{array}{l}-1.9560 E+01 \\
-1.4685 E+01 \\
-5.0256 E+00 \\
-1.1308 E+02 \\
-1.6691 E+01\end{array}$ & $\begin{array}{l}1.0723 E+01 \\
4.1259 E+00 \\
6.9402 E=01 \\
1.5615 E+01 \\
9.8182 E-01\end{array}$ & $\begin{array}{l}: x \\
x \\
x \\
x \\
x \\
x \\
x\end{array}$ & $\begin{array}{l}-1.8399 E-00 \\
-2.7972 E-01 \\
-1.3675 E-02 \\
-3.0769 E-01\end{array}$ & $\begin{array}{l}\times 2 \\
\times 22 \\
\times 22 \\
\times 2 \\
\times 2\end{array}$ & $9.9456 E \cdot 02$ & $\cdot \times 3$ \\
\hline $0(62 C) \cdot 0(33 C)=$ & $\begin{array}{l}+0(316): \\
+10(415): \\
+0(516): \\
+0(556) \\
+10(815) \\
+10(655)\end{array}$ & $\begin{array}{l}1.4685 E+01 \\
8.8112 E+01 \\
1.5385 E+01 \\
2.2615 E+01 \\
5.1927 E+01 \\
1.2240 E+02\end{array}$ & $\begin{array}{l}-8.0420 E+00 \\
-2.4755 E+01 \\
-1.0410 E+01 \\
-3.1231 E+00 \\
-3.0545 E+00 \\
-7.2000 E+00\end{array}$ & $\begin{array}{l}: x \\
: x \\
x \\
x \\
x \\
x \\
x \\
x\end{array}$ & $\begin{array}{l}1.3800 E+00 \\
1.6783 E+00 \\
2.0513 E \circ 01 \\
6.1538 E=02\end{array}$ & $\begin{array}{l}\times 12 \\
\times \times 2 \\
\times 22 \\
\times 22\end{array}$ & $-7.4592 E-02$ & $+\times 3$ \\
\hline $0(63 C)+0(30 C)=$ & $\begin{array}{l}+0(336): \\
+10(435) \\
+0(53 C) \\
+10(635)\end{array}$ & $\begin{array}{r}-3.6713 E+01 \\
-3.9650 E+02 \\
-1.8846 E+01 \\
7.4162 E+01\end{array}$ & $\begin{array}{r}2.0105 E+01 \\
1.1140 E+02 \\
2.6026 E+00 \\
-4.3636 E+00\end{array}$ & $\begin{array}{l}: x \\
: x \\
: x \\
x\end{array}$ & $\begin{array}{l}-3.4499 E+00 \\
-7.5524 E+00 \\
-5.1282 E-02\end{array}$ & $\begin{array}{l}* \times 2 \\
x \times 2 \\
x \times 2\end{array}$ & $1.06485=01$ & $\cdot \times 3$ \\
\hline $0(63 C)+0(3)()=$ & $\begin{array}{l}+0(32 C): \\
+10(425): \\
+10(445): \\
+0(52 C): \\
0(54 C): \\
+10(625): \\
+10(645) .\end{array}$ & $\begin{array}{r}-1.1014 E+02 \\
-2.2028 E+01 \\
4.4056 E+01 \\
2.2615 E+02 \\
2.2615 E+02 \\
-4.6364 E+00 \\
9.2727 E+00\end{array}$ & $\begin{array}{r}6.0315 E+01 \\
6.1808 E+00 \\
-1.2378 E+01 \\
-3.1231 E+01 \\
-3.1231 E+01 \\
2.7273 E-01 \\
-5.4545 E-01\end{array}$ & $\begin{array}{l}: x \\
: x \\
: x \\
x \\
x \\
x \\
x \\
x \\
x\end{array}$ & $\begin{array}{r}-1.0350 E+01 \\
-4.1958 E-01 \\
8.3916 E-01 \\
6.1538 E-01 \\
6.1538 E-01\end{array}$ & 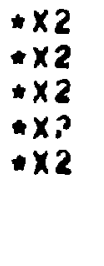 & 5.59445 .01 & $-\times 3$ \\
\hline$O(63 C)=0(32 C)=$ & $\begin{array}{l}+0(316): \\
+10(415): \\
+0(556): \\
+10(615)= \\
+10(655)=\end{array}$ & $\begin{array}{r}-1.1014 E+01 \\
2.2028 E+01 \\
-1.1300 E+01 \\
-7.4182 E+00 \\
-4.0000 E+01\end{array}$ & $\begin{array}{r}6.0315 E+00 \\
-6.1880 E+00 \\
1.5615 E+00 \\
4.3636 E-01 \\
2.4000 E+00\end{array}$ & $\begin{array}{l}x \\
x \\
x \\
x \\
x \\
x \\
x\end{array}$ & $\begin{array}{r}-1.0350 E+00 \\
4.1958 E-01 \\
-3.0769 E-02\end{array}$ & $\begin{array}{l}\times 2 \\
\times \times 2 \\
\times 2\end{array}$ & $5.5 Y 44 E-02$ & $\pm \times 3$ \\
\hline $0(63 C)=0(33 C)=$ & $\begin{array}{l}+0(30 C)= \\
+0(50 C)= \\
+10(66 S)=\end{array}$ & $\begin{array}{r}-1.4685 E+01 \\
-1.5077 E+01 \\
1.0200 E+01\end{array}$ & $\begin{array}{r}8.0420 E+00 \\
2.0821 E+00 \\
-6.0000 E-01\end{array}$ & $\begin{array}{l}* x \\
: x \\
* x\end{array}$ & $\begin{array}{l}-1.3800 E+00 \\
-4.1026 E-02\end{array}$ & $\begin{array}{l}: \times 2 \\
: \times 2\end{array}$ & $1.4592 E-02$ & $\cdot \times 3$ \\
\hline $0(64 C)=0(30 C)=$ & $\begin{array}{l}+10(445): \\
+0(54 C): \\
+10(645):\end{array}$ & $\begin{array}{r}-2.2028 E+02 \\
-5.6538 E+02 \\
5.5636 E+01\end{array}$ & $\begin{array}{r}6.1808 E+01 \\
7.8077 E+01 \\
-3.2727 E+00\end{array}$ & $\begin{array}{l}: x \\
: x \\
x\end{array}$ & $\begin{array}{l}-4.1958 E+00 \\
-1.5365 E+00\end{array}$ & $\begin{array}{l}* \times 2 \\
\times 2\end{array}$ & & \\
\hline $0(64 C)=0(316)=$ & $\begin{array}{l}+0(33 C) \\
+10(435): \\
+0(53 C) \\
+0(556) \\
+10(635) \\
+10(655)\end{array}$ & $\begin{array}{r}-6.1189 E \bullet 01 \\
-3.6713 E \bullet 02 \\
6.2021 E \bullet 01 \\
1.1308 E \bullet 02 \\
3.0909 E \bullet 01 \\
2.0400 E \bullet 02\end{array}$ & $\begin{array}{r}3.3508 E+01 \\
1.0315 E+02 \\
-8.6752 E+00 \\
-1.5615 E+01 \\
-1.6162 E+00 \\
-1.2000 E+01\end{array}$ & $\begin{array}{l}: x \\
: x \\
: x \\
: x \\
: x \\
x \\
x\end{array}$ & $\begin{array}{r}-5.7498 E+00 \\
-6.9930 E+00 \\
1.7094 E=01 \\
3.0769 E=01\end{array}$ & $\begin{array}{l}+\times 2 \\
\times 2 \\
\times 2 \\
\times 2 \\
\times 2\end{array}$ & $3.1080 E-01$ & $\cdot \times 3$ \\
\hline$O(64 C)=O(32 C)=$ & $\begin{array}{l}P(326): \\
+10(425): \\
+0(526): \\
+10(625): \\
+10(665) *\end{array}$ & $\begin{array}{r}-7.3427 E+01 \\
1.4605 E+01 \\
7.5305 E+01 \\
-1.3909 E+01 \\
-1.7000 E+01\end{array}$ & $\begin{array}{r}1.0210 E+01 \\
-1.1259 E+00 \\
-1.010 E+01 \\
8.1018 E-01 \\
1.0000 E+00\end{array}$ & $\begin{array}{l}: x \\
: x \\
: x \\
x \\
x \\
x\end{array}$ & $\begin{array}{r}-6.89905-00 \\
2.79725=01 \\
2.0513 E-01\end{array}$ & $\begin{array}{l}: \times 2 \\
: \times 2 \\
: \times 2\end{array}$ & $3.7296 E=01$ & $\cdot \times 3$ \\
\hline
\end{tabular}




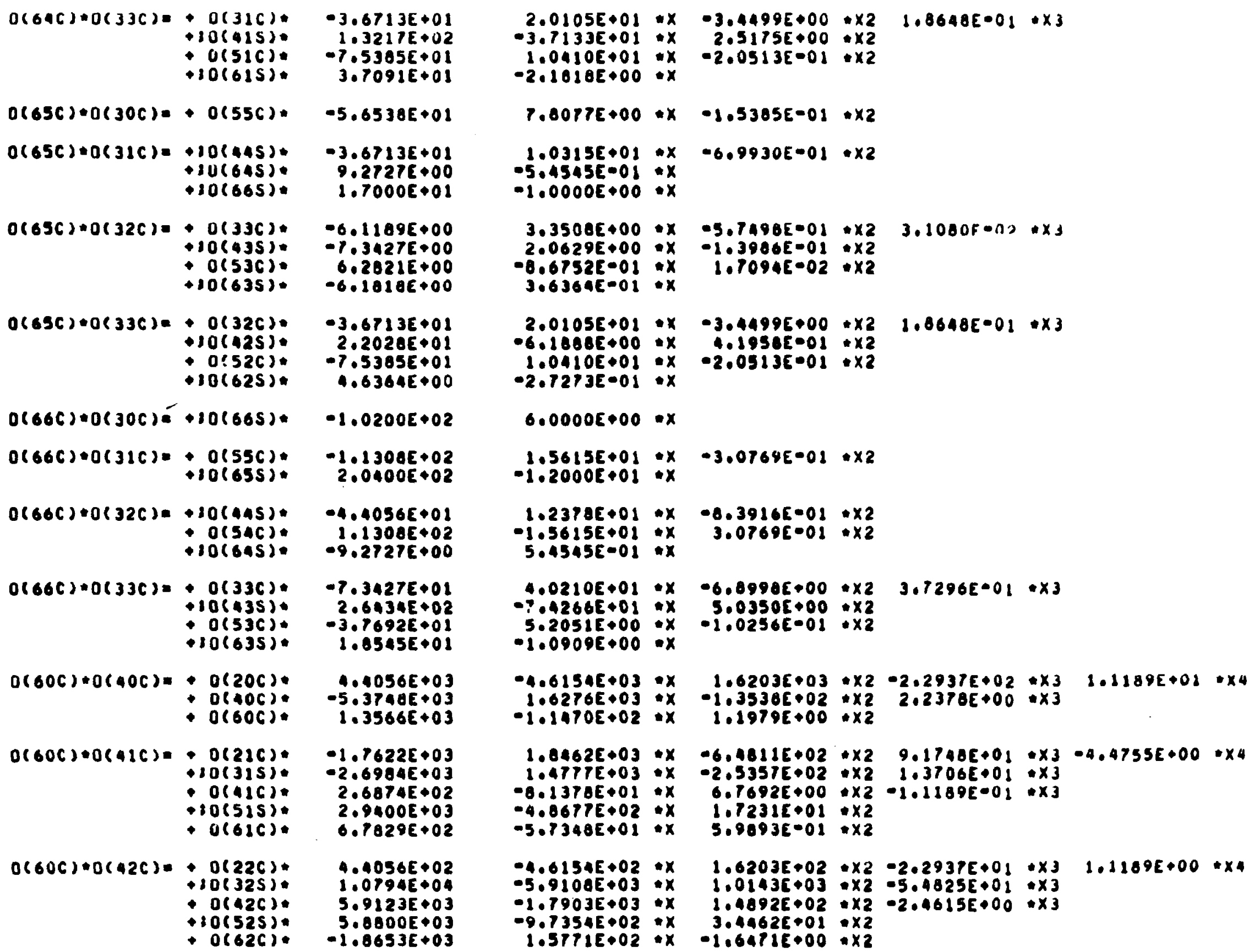

$2.0105 E+01 * x-3.4499 E+00 * x 2$

$1.8648 E \cdot 01 * \times 3$ $1.0410 E+01 * x-2.0513 E-01 \div x 2$

$-2.1010 E+00 \cdot x$

$7.00776+00 * x-1.5305 E-01 * \times 2$

$1.0315 E+01 * x \quad-6.9930 E-01 * x 2$

$-5.45456001 \cdot x$

$1.00002+00$ : $x$

$3.35005400 * x-5.74985-01 * \times 2 \quad 3.10000 \times 02 * x]$ $2.06292+00$ : $x$ - $1.39062=01$ : $x 2$

$3.6364-01$ : $x$

$2.0105 E+01 * x-3.4499 E+00+\times 2 \quad 1.8648 E-01+\times 3$ $-6.1808 E+00 * x \quad 4.1950 E-01+x 2$

$1.0410 E+01: x \quad-2.0513 E-01+x 2$
$-2.7273 E-01: x$

$6.0000 E+00 \cdot x$

$1.5615 E+01 * x-3.0764 E-01 * 2$

$-1.2000 E+01 \cdot x$

$1.2370 E+01 * x-0.3916 E-01 * x 2$

$-1.5615 E+01$ : $x \quad 3.0769 E-01 \times \times 2$

$5.45455-01 \cdot x$

\section{$4.0210 E+01 * x-6.0990 E+00 * \times 2 \quad 3.7296 E * 01 * x 3$} $4.4266 E+01 * x \quad 5.0350 E+00 * x 2$
$5.2051 E+00 * x-1.0256 E-01 * x 2$

$5.2051 E+00: x$
$-1.0009 E+00: x$

$-4.61546+03=x$

$1.6276 E 003$ a $\quad 1.6203 E+03 * \times 2-2.2937 E+02 * x 3$

$-1.1470 E+02 * x \quad-1.3538 E+02 * x 2 \quad 2.2378 E+00 * x$

$1.1189 E+01 \cdot \times 4$

$1.0462 E+03 * x-6.4811 E+02+\times 2 \quad 9.1740 E+01 * \times 3-4.4755 E+00 * x 4$ 1.477fE+03 *x $-2.5357 E+02 * x 2 \quad 1.3706 E+01 * x$

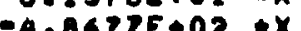

$-5.734 \theta 5+01 \times x \quad 1.72312+01 \times x 2$

$-4.6154 E+02 * X \quad 1.6203 E+02 \times \times 2-2.2937 E+01 * \times 3 \quad 1.1189 E+00+x 4$

$-5.9100 E+03: x \quad 1.0143 E+03: \times 2-5.4825 E+01: x 3$

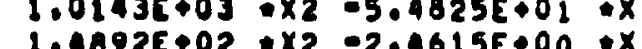

$-9.7354 E+02: x \quad 304462 E+01: \times 2$ 


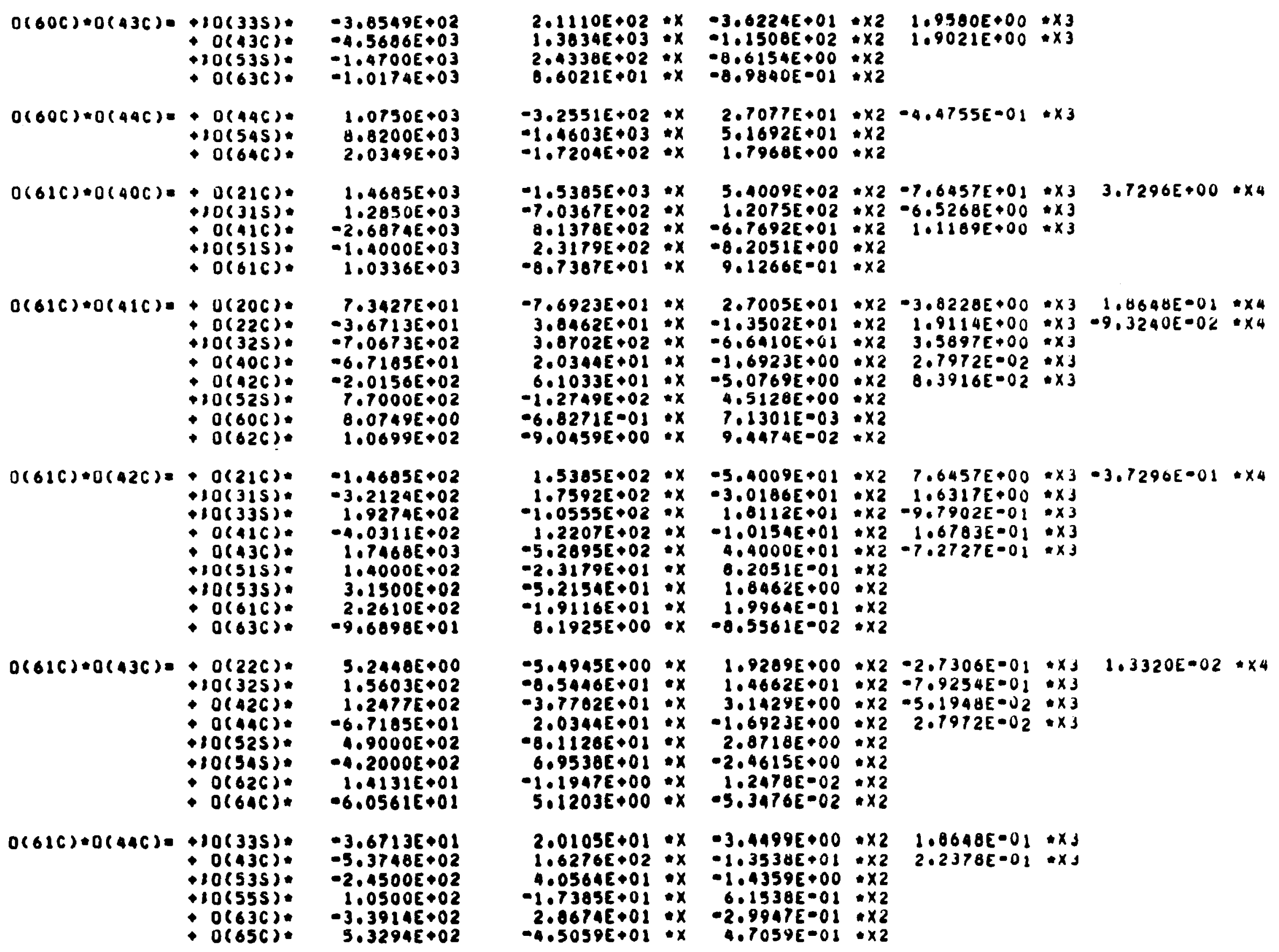

$0(600)+0(436) 0+10(335)$ $+0(436)$.

$+0(535)$.
$+0(63 C)$.

$O(60 C) * 0(44 C)=+O(44 C)=$ $+8(545):$
$+0(64 C)$.

$0(61 C)=0(40 C)=+0(216)=$ $+10(315)$. $0(416):$
$+10(515)$. $+10(515)$.
$+0(616)$.

$O(61 C) \cdot 0(41 C)=+O(20 C)$ $+D(22 C)$. $+10(325)$ $+0(126)$ $10(525)$ $+0(60 C)$.

$O(61 C)+0(42 C)=+0(216)=$ $10(315)$ $10(335)$. $0(416)=$
$-0(436)$ $0(436)$.
$+10(515)$ $10(535)$. $+0(61 C)$

$0(61 C)+0(43 C)=+0(22 C)$ $10(325)$. - $0(426)$. - O(4ac). 10(5as). ro(sas) - $0(626)$ O(64C).

$0(61 C) \cdot 0(44 C)=+10(335)=$ $0(43 C)$. $10(535)$. $-10(555)$. O(63C).

$-3.0549 E+02$ $-4.5606 E+03$ $-1.4700 E+03$ $-1.0174 E+03$

$1.0750 E+03$ . $8200 E+03$ $2.0349 E+03$

$1.4685 E+03$ $1.2850 E+03$ $-2.6874 E+03$ $-1.4000 E+03$ $1.0336 E+03$

$7.3427 E+01$ $-3.6713 E+01$ - $7.0673 E+02$ $-6.7165 E+01$ $-2.0156 E+02$ r.rovoc?02 $0.0749 E+00$ $1.0699 E+02$

$-1.4605 E+02$ $-3.2124 E+02$ $1.9274 E+02$ - $4.0311 E+02$ 1.74686003 $1.4000 E+02$ $3.1500 E+02$ $2.2610 E+02$
$-9.6898 E+01$

$3.2448 E+00$ $1.5603 E+02$ $1.24775+02$ $-6.7185 E+01$ $4.9000 E+02$

$-4.2000 E+02$

$1.4131 E+01$
$-6.0561 E+01$

$-3.67136+01$ $-5.3748 E+02$

$-2.4500 E+02$

$1.0500 E+02$

$5.32945+02$

$2.1110 E+02 * x-3.6224 E+01 \times \times 2 \quad 1.9580 E+00 * x 3$ $1.3834 E+03 * x-1.1508 E+02 * \times 2 \quad 1.9021 E+00 * x 3$ $2.4338 E+02 * x \quad-8.6154 E+00 * \times 2$
$8.6021 E+01 * x \quad-8.9840 E-01 * \times 2$

$-3.2551 E+02 * x \quad 2.7077 E+01 * \times 2-4.4755 E-01 * x 3$ $\begin{array}{lll}-1.4603 E+03 * x & 5.1692 E+01 * \times 2 \\ -1.7204 E+02 * x \quad 1.7968 E+00 * \times 2 & \end{array}$

$-1.5385 E+03 * x \quad 5.4009 E+02 * \times 2-7.6457 E+01 * x \quad 3.7296 E+00+x 4$ $-7.0367 E+02 \times x \quad 1.2075 E 002 \times x 2-6.5268 E+00 * x$ $0.1378 E+02 * x-6.7692 E+01 * x 21.1189 E+00 * x$ $\begin{array}{rrr}2.3179 E+02 * x & -8.2051 E+00 & * x 2 \\ -8.7387 E+01 * x \quad 9.1266 E-01 * \times 2\end{array}$

$-7.6923 E+01 * x \quad 2.7005 E+01 \times \times 2-3.8228 E+00 \times 3 \quad 1.8648 E=02 \times x 4$ $3.0462 E+01 * x \quad-1.3502 E+01 * \times 2 \quad 1.9114 E+00 * x 3-9,3240 E-02 * x$ $30.0702 E+02 * x \quad-6.6410 E+01$ : $x 2 \quad 3.5897 E+00 * x 3$

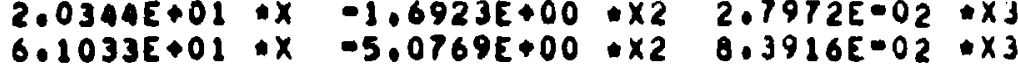

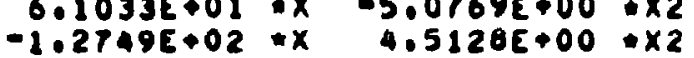
$-6.8271 E-01$ : $\mathrm{T}, 1301 E=03: \times 2$ $\begin{array}{rl}-6.8271 E-01 * x & 7.13015-03 \\ -9.0459 E+00 * x \quad 9.4474 E-02 * x 2 & \end{array}$

$1.5385 E+02 * x-5.4009 E+01 * \times 2 \quad 7.6457 E+00 * 3-3.7290 E-01 * \times 4$ $1.7592 E+02 * x-3.0186 E+01 * \times 2 \quad 1.6317 E+00 * x$

1.05sse02 ax 1.01125001 axa $-9.7902 E 01$ ax

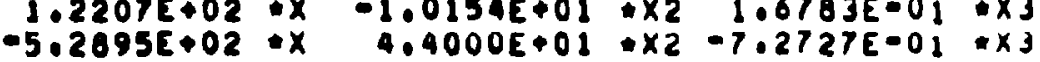

$-5.2895 E+02$ : $x$ Q.4000

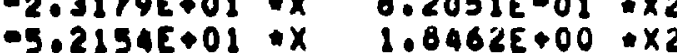
$-1.9116 E+01: x \quad 1.9964 E=01: x 2$

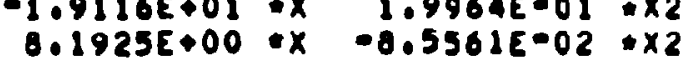

$-3.4945 E+00 * x \quad 1.9209 E+00 \times \times 2-2.7306 E-01 * \times 3 \quad 1.3320 E-02 * \times 4$ $-8.5446 E+01 \times x \quad 1.4662 E+01 \times \times 2-7.9254 E-01 \times x 3$

$3.7702 E+01$ : $x$

$2.0344 E+01 * x-1.6923 E+00 * x 2 \quad 2.7972 E-02 * x J$

$0.1126 E+08 \times x \quad 2.0710 E+00 \times x$

$0.9530 E+01 \times x-2.4615 E+00 \times x 2$

$-1.1947 E+00 * x \quad 1.2478 E-02 * \times 2$
$5.1203 E+00 * x \quad-5,3476 E-02 * \times 2$

$2.0105 E+01 \div x-3.4499 E+00 \div \times 2$

$1.0048 E-01 * x J$

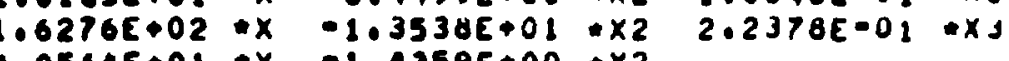

$4.05646+01$ : $x \quad 1.4359 E+00$ : $x 2$

$1.7385 E+01 * x \quad 6.1538 E-01 * \times 2$

$\begin{array}{rr}2.0674 E+01 * x & -2.9947 E=01 * \times 2 \\ -4.5059 E+01 * x \quad 4.7059 E-01 * x 2 & \end{array}$ 


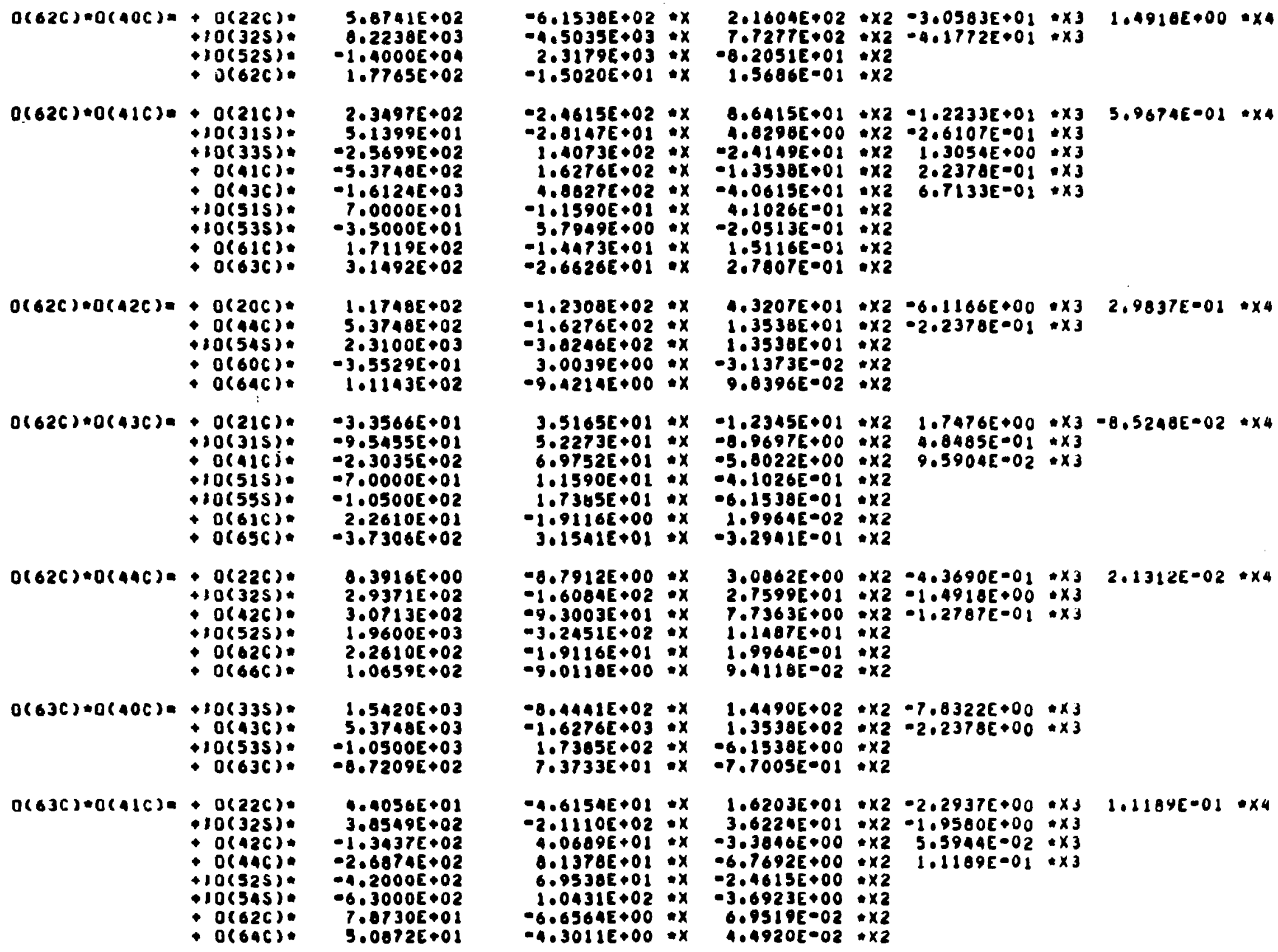

$-8.7912 E+00 \times x \quad 3.0062 E+00 \times 2-4.3690 E-01 * \times 3 \quad 2.1312 E-02 \times \times 4$ $-1.6084 E+02 * x \quad 2.7599 E+01 * \times 2-1.4918 E+00 * x 3$ $0.03003 E+01: x \quad 7.7363 E+00: \times 2-1.2787 E-01: \times 3$

$-3.2451 E+02: x \quad 1.1407 E+01: \times 2$

$-1.9116 E+01: x \quad 1.9964 E-01: \times 2$

$-8.4441 E+02 * x \quad 1.4490 E+02 * \times 2-7.8322 E+00 * x 3$

$-1.6276 E+03 * x \quad 1.3538 E+02 * x 2-2.2378 E+00 * x 3$

$1.7305 E+02 * x \quad-6.1538 E+00 * \times 2$

$-4.6154 E+01 * x \quad 1.6203 E+01 * x 2-2.2937 E+00 * x J \quad 1.2189 E-01 * x 4$ $-2.11105+02 \cdot x$ $4.0609 E+01 * x-3.3846 E+00 * x 25.5944 E-02 * x 3$ $0.1378 E+01 * x-6.7692 E+00 * \times 2 \quad 1.1189 E-01 * \times 3$

$6.9530 E+01$ * $-2.9615 E+00 * x 2$

$1.0431 E+02 * x \quad-3.6923 E+00 * \times 2$

$-6.6564 E+00 * X \quad 6.9519 E-02 * \times 2$
$-4.3011 E+00 * x \quad 4.4920 E-02 \times \times 2$ 


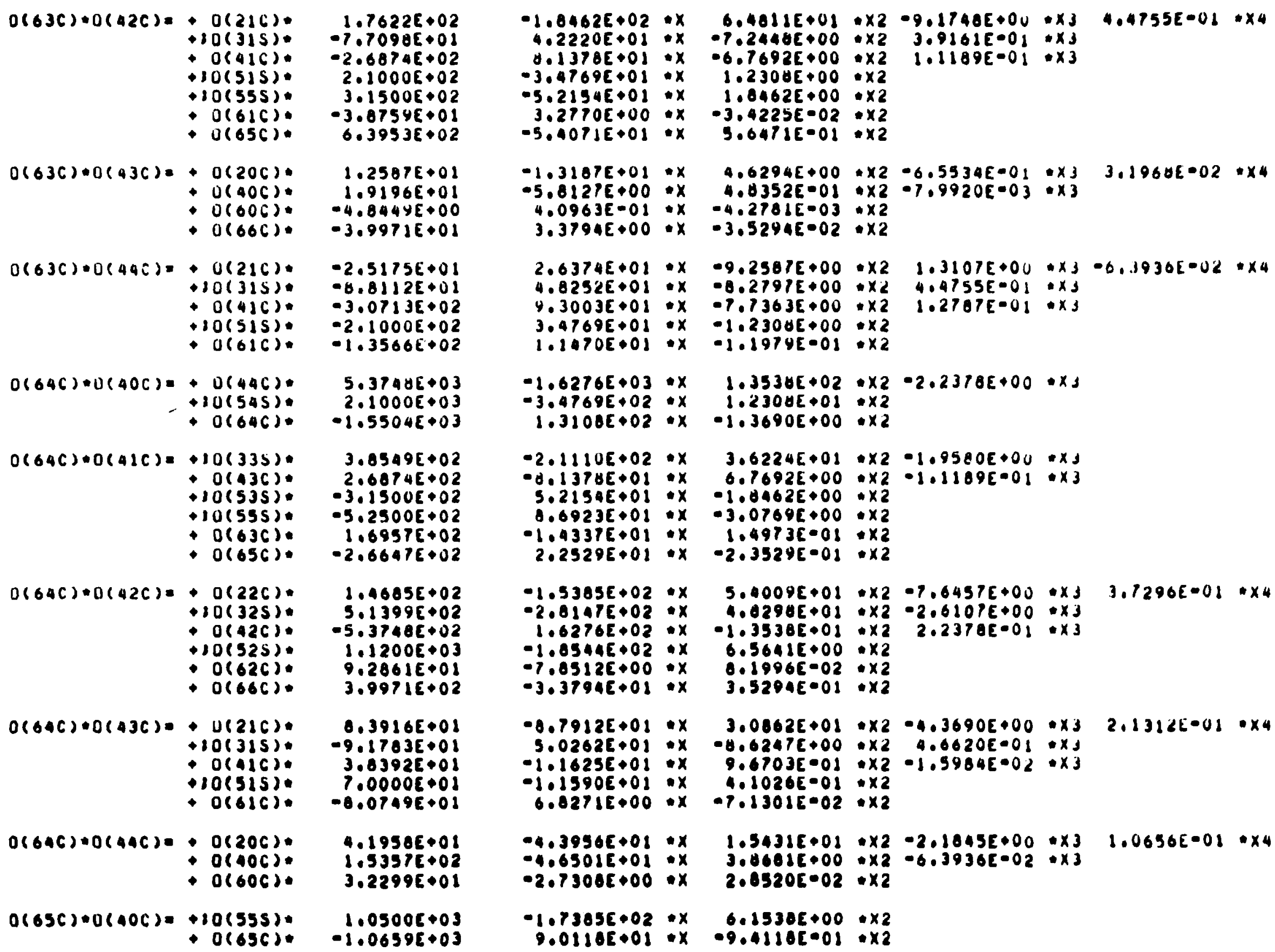

2.09055०02

$1.4605 E+02$ $5,1399 E+02$ $+10(325)$ $-0(426)$. $+10(525)$ - $0(62 C)$ $-5.37406+02$ $1.1200 E+03$ $9.2861 E+01$ $3.9971 E+02$

$O(64 C) \cdot 0(43 C)=+U(21 C)$ $+10(315)$. - $0(416)$. $+0(616) .0 .07496+01$

$O(64 C) \times O(44 C)=0(20 C): 0(40 C)$ $0(600)$

$4.19586+01$ $1.53576+02$ $3.2299 E \bullet 01$

$0(65 C)+0(40 C)=+10(555):$

$1.05006+03$ $0(656) \cdot$ 


\begin{tabular}{|c|c|c|}
\hline $0(65 C)+0(416)=$ & $\begin{array}{l}+0(44 C): \\
+10(545): \\
+0(64 C): \\
+0(66 C) .\end{array}$ & $\begin{array}{r}1.3437 E+02 \\
-2.1000 E+02 \\
-1.2112 E+01 \\
-6.6610 E+01\end{array}$ \\
\hline $0(65 C)=0(426)=$ & $\begin{array}{l}+10(335): \\
+0(436) \\
+10(535) \\
+0(63 C)\end{array}$ & $\begin{array}{r}1.2850 E+02 \\
-2.6874 E \bullet 02 \\
-3.5000 E+01 \\
9.6898 E+01\end{array}$ \\
\hline $0(65 C) \times 0(43 C)=$ & $\begin{array}{l}+0(22 C) \\
+\quad 0(325) \\
+0(426): \\
+0(525) \\
+0(62 C)\end{array}$ & $\begin{array}{r}1.0490 E+01 \\
-1.8357 E+01 \\
-1.9196 E+01 \\
1.4000 E+02 \\
-1.0131 E+01\end{array}$ \\
\hline $0(65 C)+0(44 C)=$ & $\begin{array}{l}+0(21 C): \\
+80(315): \\
+0(416) \\
+10(515): \\
+0(61 C) .\end{array}$ & $\begin{array}{r}4.1950 E+01 \\
-7.3427 E+01 \\
1.5357 E+02 \\
-7.0000 E+01 \\
3.2299 E+01\end{array}$ \\
\hline$D(60 C)+0(40 C)=$ & $+0(66 C)$ & $1.5988 E+03$ \\
\hline $0(66 C)+0(41 C)=$ & $\begin{array}{l}+10(55 S): \\
+0(65 C) .\end{array}$ & $\begin{array}{r}3.1500 E+02 \\
-7.9941 E+02\end{array}$ \\
\hline $0(66 C) \neq 0(42 C)=$ & $\begin{array}{l}+U(44 C): \\
+10(545): \\
+\quad 0(646) .\end{array}$ & $\begin{array}{r}5.3748 E \bullet 02 \\
-1.8900 E+03 \\
2.1802 E+02\end{array}$ \\
\hline $0(66 C)=0(43 C)=$ & $\begin{array}{l}+80(335) \\
+0(436) \\
+10(535) \\
+0(636)\end{array}$ & $\begin{array}{r}1.1014 E+02 \\
-5.3746 E+02 \\
1.0500 E+02 \\
-7.2674 E+01\end{array}$ \\
\hline$O(66 C)=0(A 4 C)=$ & $\begin{array}{l}+O(226) \\
+10(32 S) \\
+0(42 C) \\
+10(52 S) \\
+\quad O(626)\end{array}$ & $\begin{array}{r}1.2507 E+02 \\
-8.8112 E+02 \\
3.0713 E+02 \\
-8.4000 E+02 \\
4.8449 E+01\end{array}$ \\
\hline $0(60 C)=0(50 C)=$ & $\begin{array}{l}+O(106) \\
+0(306) \\
+0(506)\end{array}$ & $\begin{array}{r}-4.7500 E+03 \\
6.0445 E+04 \\
-2.1931 E+04\end{array}$ \\
\hline $0(60 C) \oplus 0(51 C)=$ & $\begin{array}{l}+0(116) \\
+0(215) \\
+10(415) \\
+0(516) \\
+10(615)\end{array}$ & $\begin{array}{r}7.9301 E+02 \\
1.8503 E+04 \\
-3.8487 E+04 \\
-6.5794 E+03 \\
1.4581 E+04\end{array}$ \\
\hline
\end{tabular}

$-4.0609 E+01 \times x \quad 3.3046 E+00 \times 2-5.59445-02 * x 3$ $3.47696+01: x \quad-1.2306 E+00$ * $x 2$

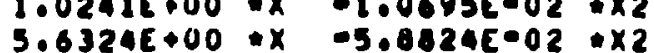

$-7.03676+01 \cdot x$

$-7.0367 E+01 \cdot x$ $-8.1925 E+00 \div x \quad-2.0513 E-01: x 2$

$-1.0989 E+01 * x \quad 3.8378 E+00 * \times 2-5.4612 E-01+x 3 \quad 2.0640 E=02 * \times 4$ $1.0052 E+01: x-1.7249 E+00: x 2 \quad 0.3240 E-02: x J$ $5.8127 E+00: x \quad-9.0352 E-01 * x 2 \quad, .9920 E-03 * x\}$

$-2.31796+01 \cdot x \quad 0.2051 E-01$ : $x 2$

$1.1947 E+00 * x-1.2470 E-02$ *x

$-4.3956 E+01 * x \quad 1.5431 E+01 * \times 2-2.1845 E+00 * x 3 \quad 1.0650 E-01 * x 4$

$4.0210 E+01 * x-6.8998 E+00 * x 2 \quad 3.7296 E=01 * x 3$

$-4.6501 E+01 * x \quad 3.0681 E+00 \times x 2-6.3936 E-02 * x 3$

$1.15905+01 * x-4.20265-01 * x 2$

$-2.7308 E+00 * x \quad 2.8520 E-02 * x 2$

$-1.3518 E+02 * x \quad 1.4118 E+00 \times 2$

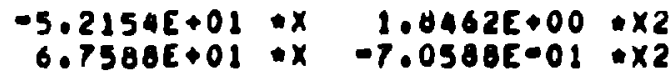

$-1.6276 E+02 * x \quad 1.3538 E+01 * \times 2-2.2378 E-01 * x 3$

$3.1292 E+02: x \quad-1.1077 E+01+x 2$
$-1.8433 E+01: x \quad 1.9251 E-01 * \times 2$

$-6.0315 E+01 * x \quad 1.0350 E+01 * \times 2-5.5944 E+01 * x 3$

$1.6276 E+02 * x-1.3538[+01 * x 2 \quad 2.2370 E-01 * x 3$

$-1.7385 E+01: x \quad 6.1538 E-01 * x 2$

$-1.3187 E+02 * x \quad 4.6294 E+01 * \times 2-6.5534 E+00 * \times 3 \quad 3.1960 E-01 \times \times 4$

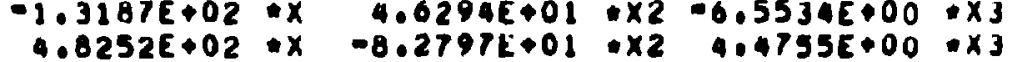

$-9.3003 E+01: x \quad 7.7363 E+00: \times 2-1.2787 E-01: x 3$

$1.3900 E+02: x-4.9231 E+00: x 2$

$-4.0963 E+00: x \quad 4.2781 E-02: \times 2$

$1.1329 E+04 * x-8.3961 E+03 * \times 2 \quad 2.5809 E+03 * \times 3-3.4238 E+02 * \times 4 \quad 1.6112 E+01 * \times 5$ $-3.4540 E+04 * x \quad 6.4680 E+03 * x 2-4.4220 E+02 * x 37.3100 E+00 * x$

$4.2675 E+03 * x-2.1816 E+02 * \times 2 \quad 1.9306 E+00 * x 3$

$-1.8 B 81 E+03 * x \quad 1.3993 E+03 * \times 2-4.3015 E+02 * x 3 \quad 5.7063 E+01 * x 4-2.6853 E+00 * \times 5$ $-1.9385 E+0 * \times \quad 6.8052 E+03 * \times 2-9.6336 E+02 * x 3 \quad 4.6993 E+01 * \times$

$1.2787 E+04=x-1.28762+03 * \times 2 \quad 3.7594 E+01 * x 3$

$1.2803 E+03 * x-6.5448 E+01 * \times 2 \quad 5.7919 E=01 * \times 3$

$-1.6001 E+03 * x \quad 3.5936 E+01 * \times 2$ 


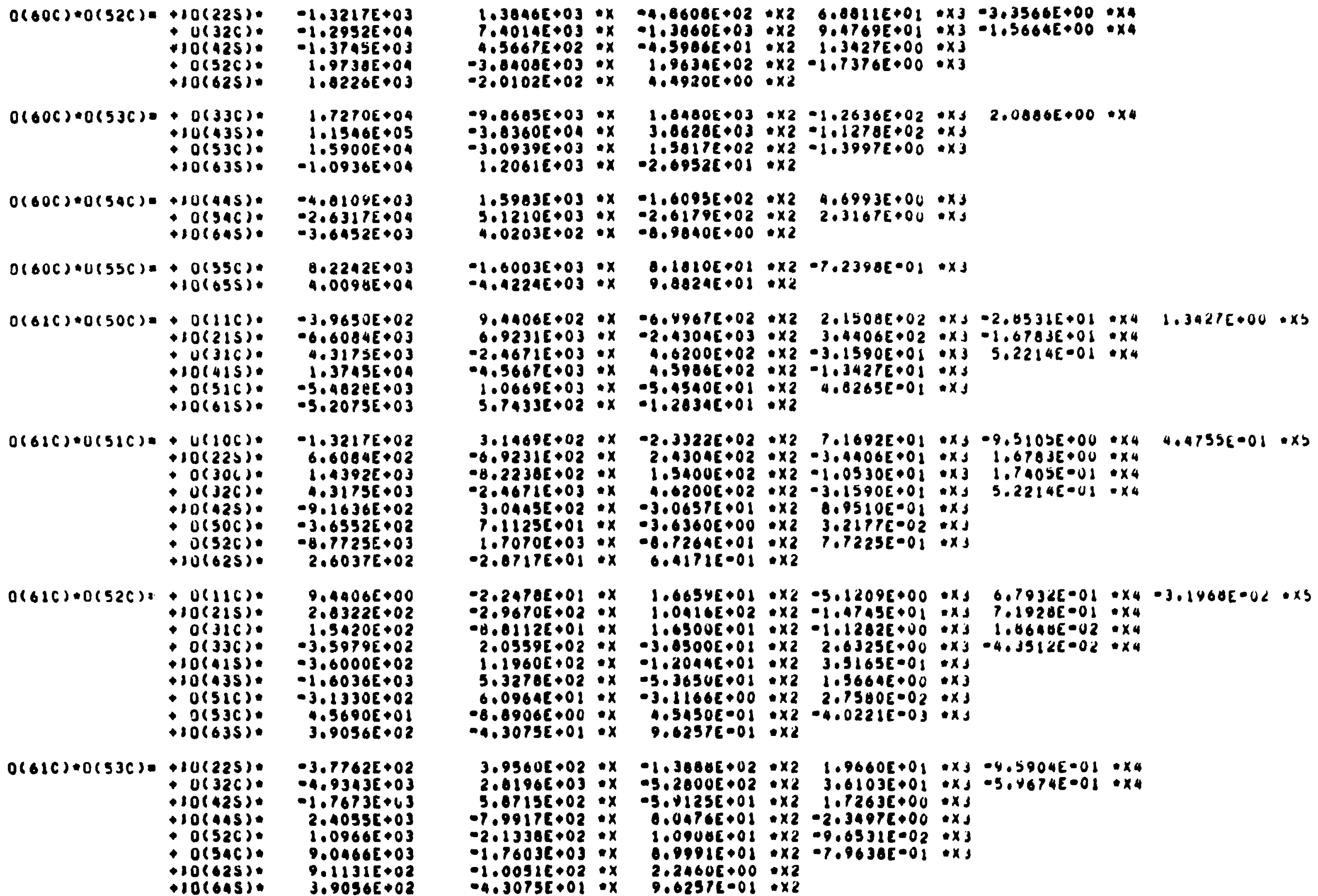

$0(80 C)=0(32 C)=+10(225)$ $+U(326)$ o(s2c) $+10(625)$

$-1 \cdot 3217 E+03$ $-1.29525004$ $1.37 A 5 E+03$ $1.8226[\bullet 03$

$0(60 C) \cdot 0(53 C)=+0(33 C)$ $+10(435):$ $+10(635)$.

$1.7270 E \bullet 04$ $1.15460+05$ $1.5900 E+04$ $-1.09365+04$

$0(600) 00(54 C)=+10(445)$. $+10(605)$.

4.01095003 $-3.6317 E+0$

$0(60 C) \cdot 0(55 C)=+0(55 C):$

$4.00985+0$

$0(61 C)+0(50 C)=0(11 C)$ (0) $10(415)$. - o(sic).

$-3.9630 E+02$ $-6.6084 E+03$ $4.3175 E+03$ $1.37455+04$ $-5.482 \mathrm{eE}+03$ $-5.2075 E+03$

$0(626) 00(51 C) \cdot U(106)$ $10(225)$ o(jac) $-10(425)$. - o(soc). - o(52C).

$-1.3217 E+02$ $6.6084 E+02$ $1.4392 E \bullet 03$ $4.31756+03$ $-9.16365+02$ $-3.65325+02$ $-8.77255003$ $2.6037 E+02$

$0(616) 00(52 C), 0(116)$ $-10(215)$. - o(3ic). - o(336). $+10(415)$. $+10(435)$. - $0(516)$. $0(536)$ $9.44066+00$
$2.03226+02$ $1.3420 E+02$ 3.5979E $\$ 02$ $-3.6000 E+02$

$-1.6036 E+03$

$-3.1330 E+02$ $4.56005+0$ $3.9056[+02$

$0(6)(C)=0(53 C)=-10(225)$ $0(326)$ $10(425)$ $10(445)$ o(5ac) $10(625)$ $\rightarrow 10(645)$

$1.3646 E+03 * x \quad-4,8608 E+02 * x 2 \quad 600111 E+01 * x^{3}-3,3566\left[+00 * x_{4}\right.$ . $4.5667 E+02 * x-4.5906 E+01 * x 2 \quad 1.3427 E \cdot 00 * x 3$

$-2.01025+02 * x \quad 1.9634 E+02 * x 2-1.7376[+00 * x 3$

$-9.0605 E+03 * x \quad 1.8480 E+03 * x 2=1.2636 E+02 * x d \quad 2.0806 E+00 * x 4$ $-3.8360[+04 * x \quad 3.0620 E+03 * x 2 \cdot 1.1278 E+02 * x 3$

$-3.0939 E+03 * x \quad 8.5817 E+02 * x 2 \cdot 1.3997 E+00 * x 3$

$1.20616+03 * x-2.09526+01 * \times 2$

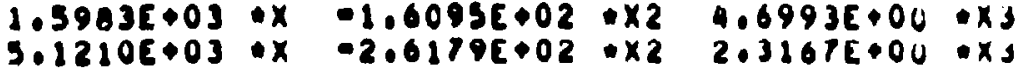

$4.0203 E+02 \cdot x-8.90405+00 * \times 2$

$-1.6003 E+03: x \quad 0.1010 E \div 02 \div \times 2-7.2390 E-01 * \times 3$

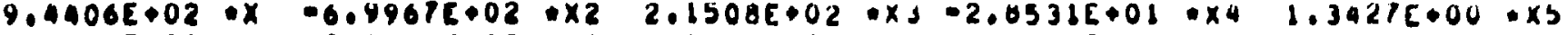
$6.9231 E+03 * x-204304 E+03: \times 2 \quad 3.4406 E+02 * x]-1.6783 E+01 \cdot \times 4$ $-2.4671 E+03 \cdot x \quad 4.6200 E+02 \times x 2-3.1500 E+01 * x 3 \quad 5.2214 E=01 \times x^{4}$ $-4.5667 c+03 * x \quad 9.5986 E+02 * x 2-1,3427 E+01 * x_{3}$ $1.0669 E+03 * x-5.45406+01 * x 2-4.0263 E 001 * x_{3}$ $3.74335+02 \cdot x-1.2834 c+01 \cdot \times 2$

$3.14698+02 \cdot x$ $-6,92316+02: x$ $-8.22385+02 * x$ $-2.46716+03 * x$ $3.04456+02 * x$ $7.1125 C+01 * x$ $1.70705 \cdot 03$ :x $-2.0717 E+01 \cdot x$

$-2.24706+02: x$ $-2.96705+02 \cdot x$ $\bullet .01126+01: x$ $2.05595+02 \div x$ $1.19605+02: x$ $6.0964 E+01: x$ $-8.8906 E+00 \cdot x$ $-4.3075 E+01 \cdot x$

$-2.3322 E+02 * x 2 \quad 7.1692\left[+01 \quad * x_{3}-9.5105 E+00 * x_{4}\right.$ $4 \cdot 475560.01 \cdot \times 5$

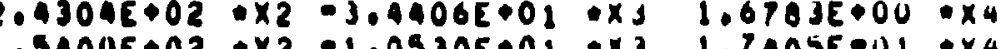

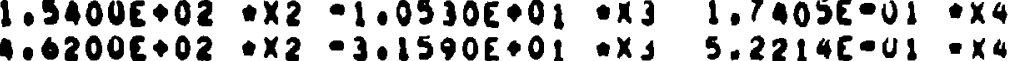
$-3.0657 E+01: x 2$ : $0.05105001: x$ $-3.6360 E+00: \times 2 \quad 3.21776002 \times x j$ $-0.7264 E+01 \times x^{2} \quad 7.7225 E-01: x 1$ $0.41715-01 * 2$

$1.66545+01 \cdot x_{2}-5.1209 E+00 * x_{3} \quad 6.7932 E-01 * x_{4}-3.1460 E-02 \cdot \times 5$ $1.0416 E+02 \times x^{2}-1.4745 E+01 * x J 7.1928 E-01 * x_{4}$ $1.6500 E+01 \cdot \times 2 \cdot 1.1282 E+00 * x 3 \quad 1.6640 E-02 * \times 4$

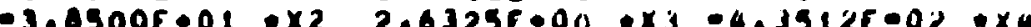

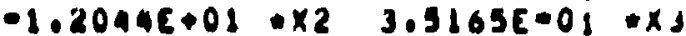

$-5.3650 E+01 * x 2 \quad 1.5664 E+00 * x 3$

$0.11605000=x 2 \quad 2.75005002 \times x j$

$0.54505001 \cdot x^{2}=4.02215=03 \cdot x J$

$9.6257 E-01 \cdot \times 2$

$3.9960 E+02 * x$

$-3.77625+02$

$04.93435+03$

$0.1 .76735+43$

$2.4055[003$

$2.40555+03$

$9.04665+03$

$0.11315+02$

$9.11316+02$
$3.9056 E+02$

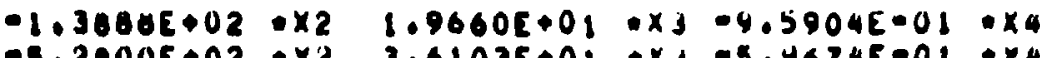

$5.07158 .02: x$

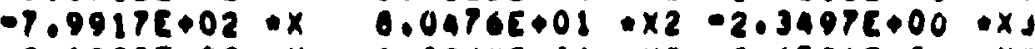

$-2.1338 E+02 \times x \quad 1.0900[001 \times \times 2-0.0331 E-02 * x 3$

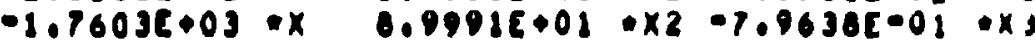

$-1.00516+02 * x \quad 2.20606+00 * x 2$ 


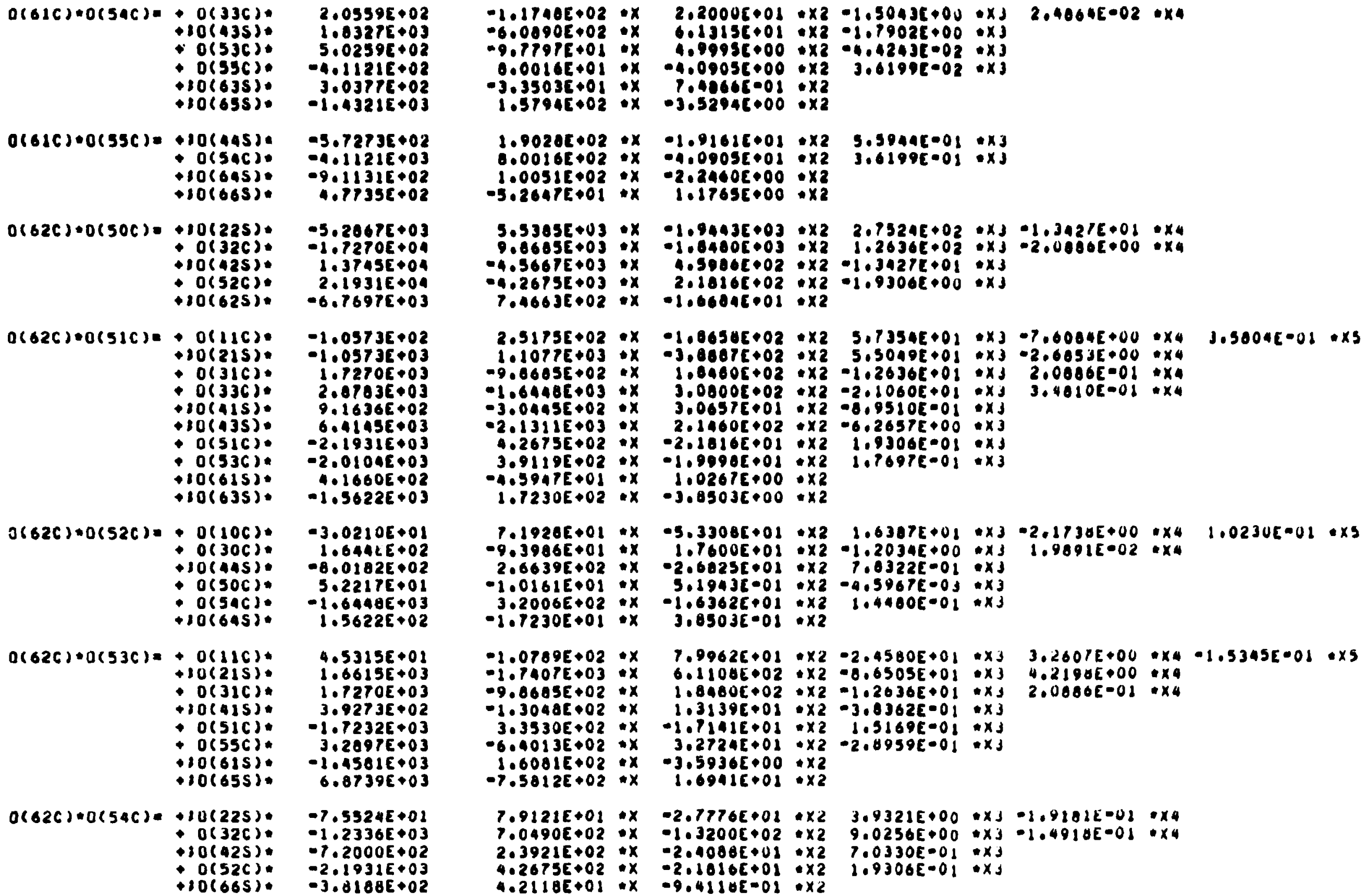

$0(61 C)+0(5 A C)=0(33 C) \quad 2.05595+02$ $+10(435)$ - o(536). - D(S5C) - $-4.11218+02$ $+10(635)$ 3.0377E+02

$0(616) \cdot 0(55 C)=+10(445)$ O(SAC). $+10(645)$.

$-5.72735+02$ $-4.11215+03$ $-9.1131$ 1.7P35E+02

$0(82 C)+0(30 C)=+10(225)$ $+0(326)$. $+10(425):$ $+0(526):$
$+10(625)$ $-3.20672+03$ - $1.7270 \mathrm{cos}$ 1.3745 $31 E+0$ $-6.76975+03$

$0(62 C)=0(51 C)=+0(11 C)$ $+10(215)$ $+0(316)$. + o(336). $+10(415)$. O(51C). - $0(536)$ (0) $+10(635)$.

$3(62 C) * 0(52 C)=0(10 C)$ O $\rightarrow 0(50 \mathrm{c})$. - o(sac).

$-1.0573 E+02$ $-1.05736+03$ $1.72706+03$ $2.0783 E+03$ $9.16365+02$ 6 .4lasetos $-2.01005003$ 4.16005002 $4.16605+02$

$-3.02105+0$ $-1.64 A L E+02$ $5.2217 E+01$ $-1.6440 E+03$ $1.56225+02$

$0(626) \cdot 0(53 C)=0(1 / C)$ $+10(215)$.

$4.5315 E+01$ $1.6615 E+03$ $1.7270 E+03$ $3.9273 E \div 02$ $-1.72325+03$

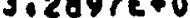
$-1.45016 \div 03$ $6.8739 \varepsilon+03$ $10(655)$.

$0(62 C) \cdot 0(54 C)+10(225)$ $0(326)$ $+30(425)$. $+\quad 0(526):$

$-1.17405+02 * x \quad 2.2000[+01 * x 2-1.5043[+00 * x J$ $-6.0090 E+02 * x \quad 6.13156+01 * x 2-1.7902 E+00 * x 3$

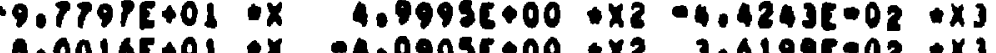

$-3.3503 E+01$ : $x$

$1.57945+02$ :x

$1.00200 .02: x \quad-1.91615001: \times 2 \quad 3,5944 c-01: x 3$

$1.0051 E+02$ *x $-2.29002+00 \times x$

$-5.264 P E+01+x \quad 1.1765 E+00 \times x$

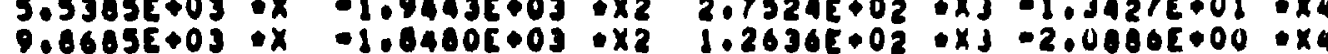

$0.0605 E+03 * x$

$-4.5667 E+03$ ix

$7.4663 E+02: x$

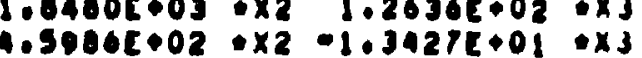

$2.80166+02 \div \times 2-1.93006+00: x j$

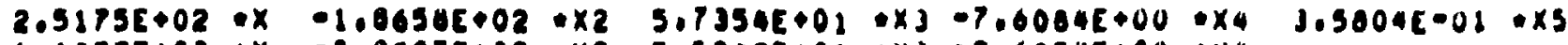

$1.107 r e+03$ : $x$ a

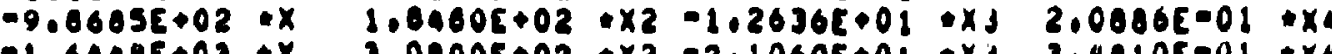

$-1.6448 E+03 \times x \quad 3.0000 E-02+x 2-2.1060 E+01$ *x $3.4010 E=01 \times x$

$-3.04456+02$ : $x$

0.0

$3.91105002 \times x$

$-4.5947 E+01 \cdot x$

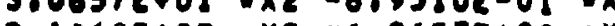

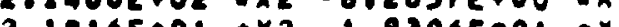

$\$ .0267 E+00 \times \times 2$

$-3.85036000 \cdot \times 2$

$1.19205+01 \cdot x$

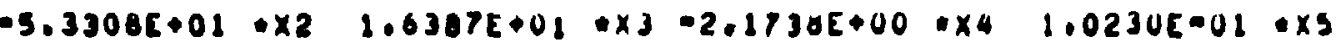

$-0.1920 E+01: x$

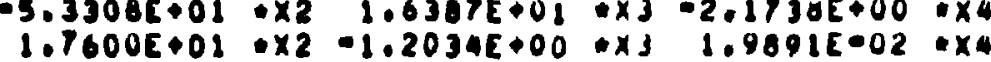

$2.66395+02: x-2.6023[01 \times x 27.03225-01: x J$

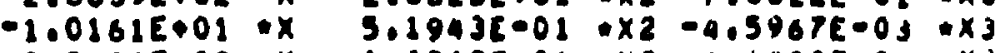

$3.2006 E+02: x-1.6362 E+01 * x 2 \quad 1.4400 E-01 * x 3$

$-1.0769 E+02 * x \quad 7.9962 E+01 * x 2-2.4580 E+01 * x 3 \quad 3.2607 E+00 * x 4 \cdot 1.5345 E-01 \cdot \times 5$

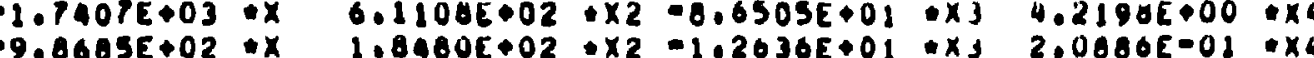

$1.3048 \varepsilon+02: x$

$3.3530 E+02 \times x-1.1141 E+01 \times x 2+1.5169 E-01 \times x J$

$-6.0013 E+02 \times x \quad 3.2724 E+01 \times 2-2.8959 E-01 \times x J$

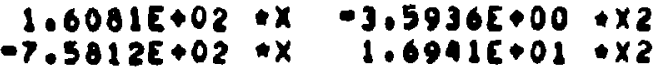

$7.9121 E+01 * x-2.7776 E+01+x 2 \quad 3.9321 E+00 * x 3-1.9181 E-02 \times x+4$

$7.0490 E+02 * x-1.3200 E+02 * \times 2 \quad 9.0256 E+00 * x 3-1.4918 E-01 * x$

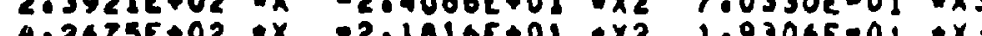

$4.2675 E+02$ : $x \quad-2.10162+01$ : $x 2 \quad 1.9306 E=01$ * $x$ 


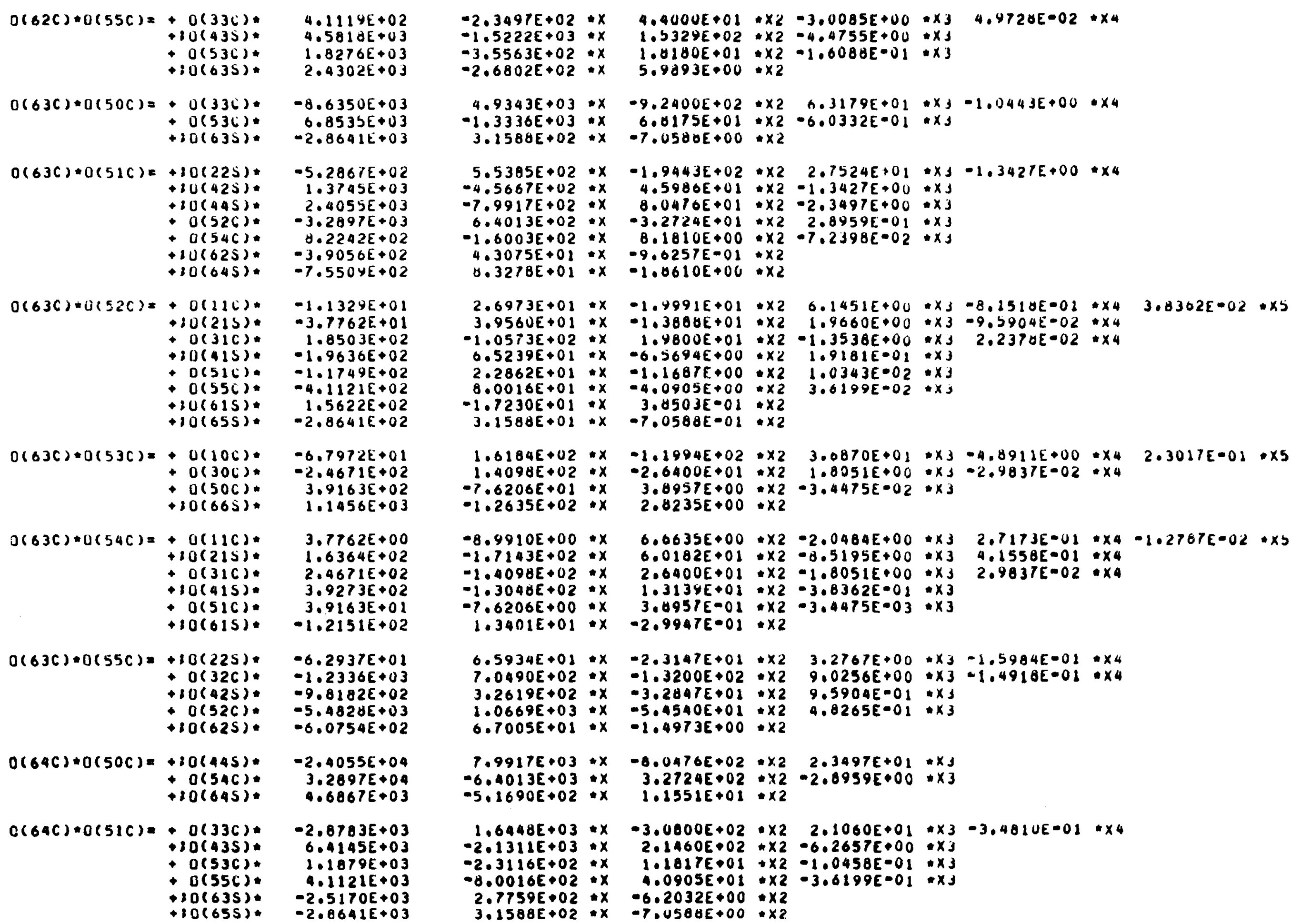
$\begin{aligned} 0(82 C) * 0(55 C)= & +0(33 C) \\ & +10(435)\end{aligned}$ $+0(53 C)$.
$+i 0(635)$.

4. $1119 E+02$ $4.5818 E+U 3$ $1.8276 E+03$ $2.4302 E+03$

$O(63 C) * 0(50 C)=\quad 0(33 C)$ $+: 0(635)$

$-8.6350 E+03$ $6.8535 E+U 3$
$-2.8641 E+03$

$0(63 C) \cdot 0(51 C)=+10(22 S)=$ $+10(425)$. $+20(445)$. + $0(526)$. $+0(54 C)$. $+10(625)=$

$-5.2867 E+02$ $1.3745 E+03$ $2.4055 E+03$ $-3.2897 E+0$ $0.2242 \varepsilon+02$ $-3.9056 E+02$ $-7.55 O$ Y +02

$O(63 C)+0(52 C)=+O(11 c)$ $+i 0(215)$ io(4ls) $+0(516)$ - $0(556)$ $+10(615)$ $+10(655)$.

$0(63 C) * 0(53 C)=+O(10 C)$ $+0(300)$. $0(50 C)$ $30(665)$

$O(63 C)+0(54 C)=+O(11 C)$ $+10(215)$ $+0(316)$. Ho(als). $0(51 C)$ $10(615)$

$-1.1329 E+01$ $-3.7762 E+01$ $1.8503 E+02$ $-1.9636 E+02$ $-1.1749 E+02$ $1.5622 E+02$ $-2.8641 E+02$

$-6.7972 E+01$ $-2.4671 E+02$ $3.9163 E+02$
$1.1456 E+03$

$3.7762 E+00$ $1.6364 E+02$ $2.4671 E+02$ $3.9273 E+02$ $3.9163 E+01$

$-1.2151 E+02$

$0(63 C)+0(55 C)=+10(22 S)=$ $+0(32 C)$ $10(425)$. - $0(52 C)$. $+10(625)$. $-1.2336 E+03$ $-9.8182 E+02$ $-5.4828 E+03$ $-6.0754 E+02$

$O(64 C) * 0(50 C)=+10(145)$. $+0(546):$
$+10(645)$.

$-2.4055 E+04$ $3.2897 E+04$
$. .6867 E+03$

$0(64 C)+0(51 C)=+0(33 C)$ $+10(435)$ $+0(53 C)$. $+0(55 C)$ $+10(635)$.
$+10(655)$

$-2.3497 E+02 * x$ $-1.5222 E+03: x$ $-3.5563 E+02: x$
$-2.6802 E+02: x$

$4.9343 E+03 * x-9.2400 E+02 * x 2 \quad 0.3179 E+01 * x+-1.13443 E+00 * x_{4}$ $-1.3336 E+03 * x \quad 6.8175 E+01 * x 2-6.0332 E=01 * x$

$3.1588 E+02 * x-7.0580 E+00 * \times 2$

$5.5385 E+02 * x$ $-4.5667 E+02 * x$ $-7.9917 E+02: x$ $6.4013 E+02 * x$ $-1.6003 E+02 \div x$ $4.3075 E+01=x$
$\forall .3278 E+01=x$

$2.6973 E+01 * x$ $3.9560 E+01 * x$ $-1.0573 E+02 * x$ $0.5239 E+01: x$ $2.2862 E+01$ : $x$ $\begin{array}{ll}2.0016 E+01 & * x \\ -1 & x\end{array}$ $-1.7230 E+01: x$
$3.1588 E+01 * x$

$1.6184 E+02 * x$ $1.4098 E+02 * x$ $-7.6206 E+01$
$-1.2635 E+02 * x$

$-8.9910 E+00 * x$ $-1.7143 E+02 \div x$ $-1.4098 E+02$.

$-1.3040 E+02 * x$

$-7.6206 E+00 * x$ 1.3401E+01 * $X$

$6.5934 E+01=x$ $7.0490 E+02$ : $x$ $3.2619 E+02: x$ $1.0669 E+03: x$
$6.7005 E+01: x$

$7.9917 E+03 * x$ $-6.4013 E+03: x$

$1.6448 E+03 \cdot x$ $-2.1311 E+03: x$ $-2.3116 E+02 * x$ $-8.0016 E+02: x$ $2.7759 E+02: x$
$3.1588+02: x$

$4.4000 E+01 * \times 2-3.0085 E+00 * \times 3 \quad 4.9720 E=02 * \times 4$ $1.5329 E+02$ \& $x 2-4.4755 E+00$ X $x$ $1.0200 E+01 * x 2-1.0088 E=01 * x$

$5.9293 E+00 * \times 2$

$1.9443 E+02 * \times 2 \quad 2.7524 E+01 * \times 1$

$4.5980 E+01 * \times 2-1.3427 E+00$ : $x J$

O.0APOE+01*X2-2.3497E\$0O $\times 3$

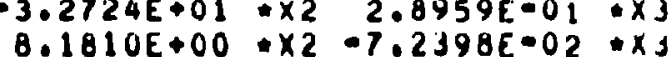

$-9.6257 E-01$ * $x$

$-1.9991 E+01 * \times 2 \quad 6.1451 E+00 * x 3=8.2518 E-01 * x 4$ $-1.3808 E+01 \times x 2 \quad 1.9660 E+00 * x 3-9,5904 E-02 * x 4$ $1.9800 E+01 * x 2-1.3538 E+00 * x 3 \quad 2.2370 E-02 * x 4$

$-6.5694 E+00 * x 2 \quad 1.9181 E=01 * x J$

$\begin{array}{lll}-1.1687 E+00 * \times 2 & 1.0343 E-02 * \times 3 \\ -4.0905 E+00 * \times 2 & 3.6299 E-02 * x 3\end{array}$

$3.0503 E=01: x$

$-7.0588 E-02: \times 2$

$-1.1994 E+02 * \times 2 \quad 3.0870 E+01 * x 3-4.8911 E+00 * \times 4 \quad 2.3017 E-01 \times \times 5$ $-2.6400 E+01 \times 2 \quad 1.8051 E+00 \times x 3-2.9837 E-02 \times x$ $3.8957 E+00: \times 2-3.4475 E-02 * x 3$
$2.8235 E+00: \times 2$

$6.6635 E+00 * x 2-2.0484 E+00 * x 3 \quad 2.7173 E-01 * x 4-1.2787 E=02 \times x 5$ $6.0182 E+01 * x 2-8.5195 E+00 * x 3 \quad 4.1558 E-01 * x$ $2.6400 E+01 * x_{2}-1.8051 E+00 * x_{3} \quad 2.9637 E-02 * x 4$

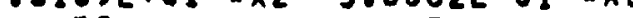
$3.6957 E-01 \times 2=3.4475 E-03 \times x$

$-2.9947 E-01 \times 2$ $6.41458+10$ 1.1879840 4.112 $-2.5170 E+03$
$-2.8641 E+03$

$\begin{array}{lll}-2.3147 E+01 & \times 2 & 3.2767 E+00 * x_{3}-1.5984 E-01: \times 4 \\ -1.3200 E+02 * x 2 & 9.0256 E+00 * x 3-1.4918 E-01: \times 4\end{array}$

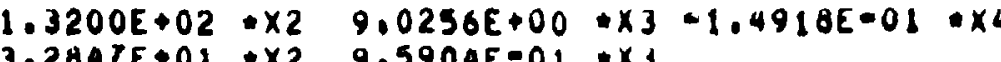

$\begin{array}{lll}-5.4540 E+01: \times 2 & 9.5904 E-01: \times 1 \\ -5.0265 E-01: x^{2} & \end{array}$

$-1.4973 E+00: \times 2$

$-8.0476 E+02 \times 2 \quad 2.3497 E+01 * \times 1$ $3.2724 E+02 * x 2-2.8959 E+00 * x$

$1.1551 E+01 * \times 2$

$-3.0000 E+02 * \times 2 \quad 2.1060 E+01 * \times 3-3.4010 E=01 * \times 4$

$2.1460 E+02 * \times 2-6.2657 E+00 * x 3$

$1.1817 E+01+x 2-1.0458 E=01 * x 3$

$1.0905 E+01 \times 2-3.6199 E=01 * x 3$

$-6.2032 E+00$
$-7.05 \theta 8+00 * \times 2$ 


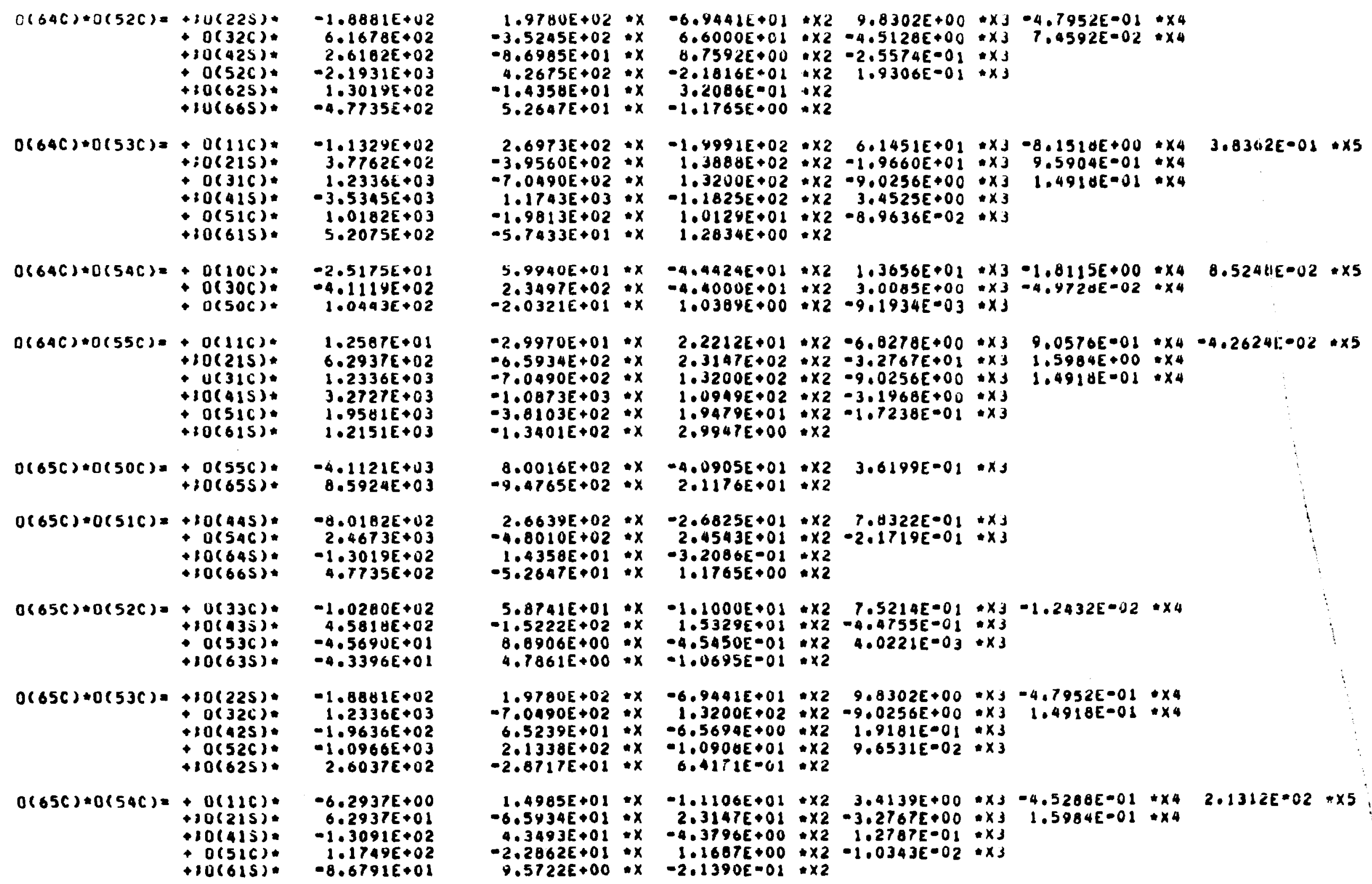

$O(64 C)+0(52 C)=+3 U(22 S)+\quad-1.8881 E+U 2$ $+0(326) * \quad 6.1678 E+02$ $+10(425)=2.6102 E+02$ $+0(52 C)-2.1931 E+03$ $+10(625): \quad 1.3019 E+02$

$O(64 C)+0(53 C)=+O(11 C) *$ + io(215): - $0(415)$. - $0(516)$.

$-1.1329 E+02$ $3.7762 E+0$ $1.2336 \varepsilon+03$
$-3.5345 E+03$ $1.0182 E+03$ $5.2075 E+02$

$+10(615)$.

$O(64 C) * D(54 C)=+D(10 C) *$ $+0(50 C)$.

$0(64 C)+0(55 C)=+0(116) *$ $+10(215)$ $+0(31 \mathrm{c})$. $+10(415)$. $+0(516)$.
$+10(615)$.

$-2.5175 E \cdot 01$ $-4.1119 E+02$

$1.2587 E+01$ $6.2937 E+02$ $1.2336 E+03$ $3.2727 E+03$ $1.9581 E+03$ $1.2151 E+03$

$0(65 C)+0(50 C)=+0(55 C)$. $+\% 0(655)$

$\begin{aligned} 0(65 C)=0(51 C)= & +10(445) . \\ & +0(54 C)\end{aligned}$ $+10(645)$.

$-4.1121 E+U 3$ $8.5924 E+03$ $130(665):$

$0(65 C) \cdot 0(52 C)=+0(33 C)$ $+0(536)$. $\rightarrow 10(635)$.

$0(65 C) \div 0(53 C)=+10(225)=$ $+0(326)$. $+10(425):$
$+0(526)$. $+10(526)$.
$+10(625)$. $-8.0182 E+02$ $0.1 .30105+02$ $-1.3019 E+02$
$4 . T 735 E+02$

$-1.0280 E+02$ $4.581 \forall E+02$ $-4.5690 E+0$ $-4.3396 E+01$

$-1.8881 \varepsilon+02$ $1.2336 \varepsilon+03$ $-1.9636 E+02$ $1.0966 E+03$
$2.0037 E+02$

$0(65 C) \div 0(54 C)=$ $0(126):$ $+10(415)=$ $+0(510)$. $+10(615)$.

$1.9780 E+02 * x \quad-6.9441 E+01 * x 2 \quad 9.8302 E+00 * x J-4.7952 E=01 * \times 4$ $-3.5245 E+02 * x \quad 6.6000 E+c 1 * x 2=4.5128 E+00 * x 3 \quad 7.4592 E=02 * x$

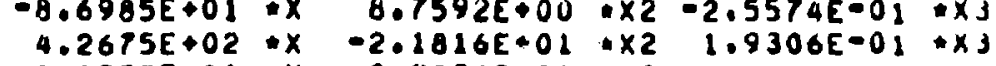

$-1.4358 E+01 \times x \quad 3.2086 E=01+\times 2$

$2.6973 E+02 * x-1.4991 E+02 * \times 2 \quad 6.1451 E+01 * x 3-8.1520 E+00 * \times 4 \quad 3.8362 E-01 * \times 5$ $-3.9560 E+02 * x \quad 1.3888 E+02 \div \times 2-1.9660 E+01 * x 3 \quad 9.5904 E=01 * x 4$

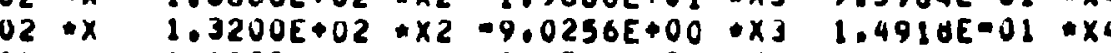

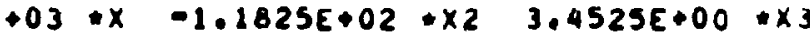

$-1.9813 E+02 * x \quad 1.0129 E+01 * \times 2-8.9636 E-02 * \times 3$

$-5.7433 E+01 * x \quad 1.2834 E+00: x 2$

$5.9940 E+01 * x-4.4424 E+01 * \times 2 \quad 1.3656 E+01 * \times 3-1.0115 E+00 * \times 4 \quad 8.52441 E-02 * \times 5$ $2.3497 E+02 * x-4.4000 E+01 * \times 2 \quad 3.0085 E+00 * x 3-4.4720 E=02 * x 4$

$-2.0321 E+01 * x \quad 1.0369 E+00 * x 2-9.1934 E-03 * x 3$

$-2.9970 E+01 * x \quad 2.2212 E+01 * \times 2-6.0278 E+00 * x 3 \quad 9.0570 E=01 * x 4=4.26241:-02 * \times 5$ $-0.5934 E+02 * x \quad 2.3147 E+02 * x 2-3.2767 E+01 * x 3 \quad 1.5984 E+00 * x 4$

$-7.0490 E+02 * x \quad 1.3200 E+02 * x 2-9.0256 E+00 * x J 1.4918 E=01 * x$

$-1.0873 E+03 * x \quad 1.0949 E+02 \times x 2-3.1960 E+00 \times x J$

$-3.8103 E+02 * x \quad 1.9479 E+01 * \times 2-1.7238 E-01 * x 3$

$-1.3401 E+02 * X \quad 2,9947 E+00 * x 2$

$0.0016 E+02 * x-4.0905 E+01 * \times 2 \quad 3.6199 E-01 * \times 3$ $-9.4765 E+02 * x \quad 2.1176 E+01 * x 2$

$2.6639 E+02 * x-2.6825 E+01 * x 2 \quad 7.4322 E-01 * x 3$

$0.0010 E+02 \times x \quad 2.4543 E+01 \times x 2-2.1719 E=01 * x 3$

$\begin{array}{rl}1.4358 E+01 * x & -3.2086 E-01 * x \\ -5.2647 E+01 * x & 1.1765 E+00+x 2\end{array}$

$5.8741 E+01 * x-1.1000 E+01+x 2 \quad 7.5214 E=01 * x 3-1.2432 E-02+\times 4$

$-1.5222 E+02: x \quad 1.5329 E+01 \times 2-4.4755 E-01 \times x$

$0.6906 E+00 * x-4.5450 E=09 \times 2 \quad 4.0221 E=03 * x 3$

$4.7861 E+00 * x-1.0695 E=01 * \times 2$

$1.9780 E+02 \times x-6.9442 E+01 \times \times 2 \quad 9.8302 E+00 \times \times 3-4.7952 E-01 \times x 4$ $-7.0490 E+02 * x \quad 1.3200 E+02 * \times 2=9.0256 E+00 * x 3 \quad 1.4918 E=01 * x$ $6.5239 E+01 * x-6.5694 E+00 * x 2 \quad 1.9181 E=01 * x 3$

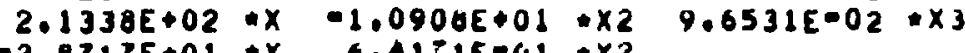

$1.4985 E+01 * x-1.1106 E+01+x 2 \quad 3.4139 E+00 * x 3-4.5280 E-01 * \times 4 \quad 2.1312 E=02 * \times 5$

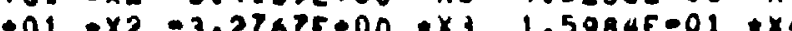

$0.3403 E+01, x$

$-2.2862 E+01 * x \quad 1.1687 E+00 * x 2-1.0343 E-02 * x_{3}$

$9.5722 E+00: x-2.1390 E-01: \times 2$

$1.1749 E+02$
$-8.6791 E+01$ 


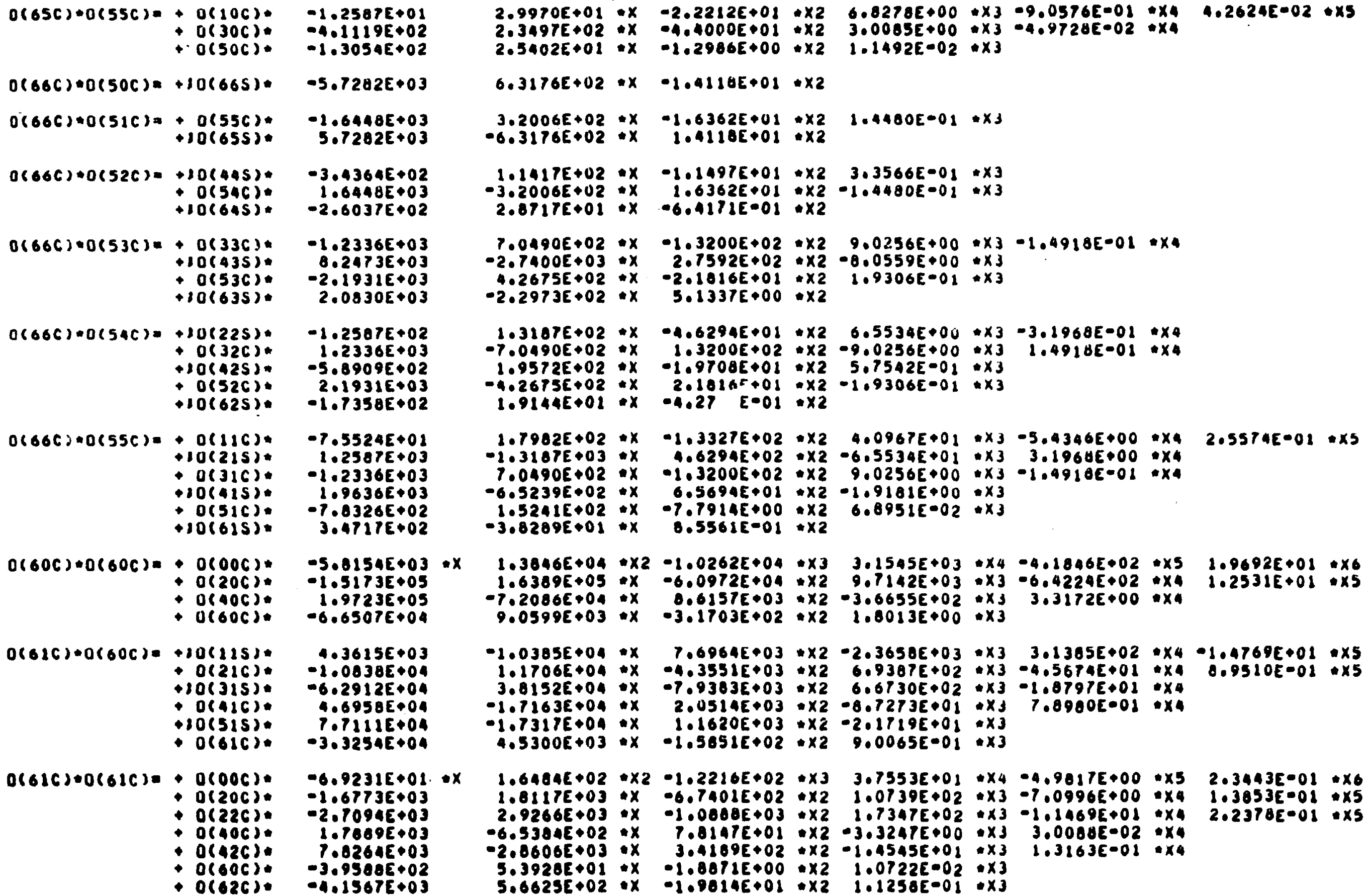

$0(65 C) * 0(55 C)=+0(10 C)$. $+0(30 C)$. $+0(506)$.

$O(66 C) \cdot 0(50 C)=+10(665)=$

$0(66 C) \times 0(516)=+0(55 C) *$ $+10(655)$

$0(66 C)+0(52 C)=+10(445)=$ $+0(546):$
$+10(645)$.

$0(66 C)=0(53 C)+0(33 C) *$ $+10(435)$ $+0(536)$. $+10(635)$.

$0(666)=0(540)=+10(225)=$ $+0(32 C)$ $+0(326)$. $+10(625)$.

$0(66 C) \times 0(55 C)=0(11 C)$. $+10(215)$. $\rightarrow 0(316)$. + o(sic). $+10(6) 5)$.

$O(60 C)+0(60 C)=+O(O 0 C)$ $+0(200)$. $+0(40 C)$

$0(616)+0(60 C)=+80(125)=$ $+0(216)$. $+10(315)$. $\rightarrow 0(515)$.

- O(6ic).

$0(616)=0(616)=0(006)$ - $0(206)$. - $0(226)$. - O(40C). - $0(426)$. - O(60C).

$-1.2587 E+0$ $0.1119 E \bullet 02$

$-5.7282 E \bullet 03$

$-1.6448 E+03$

$-3.43645+02$ $1.6448 E+03$ $-2.6037 E+02$

$-1.2336 E+03$ $6.2473 E+03$ $-2.1931 E+03$ $2.0830 E+03$

$-1.2507 E+02$ $1.2336 E+03$ $-5.8909 E+02$ $2.19316+03$ $-1.7338 E+02$

$-7.5524 E+01$ $1.2587 E+03$ $-1.2336 E+03$ $-7.0326 E+02$ $3.4717 E+02$

$-5.0154 E+03 * x$ $-1.5173 E+05$ $1.9723 E+05$ $-6.6507 E+04$

$1.3615 E+03$ $1.0038 E+04$

$4.6958 E+04$

$7.71115+04$

$-3.3254 E+04$

$-6.9231 E+01 \cdot x$ $-1.67736+03$ $-2.70945+03$

1.70095003

$1.0268+03$
$0.02640+03$

$7.0264 E+03$
$-3.95005+02$

$-3.95806+02$
$-4.1567 E+03$

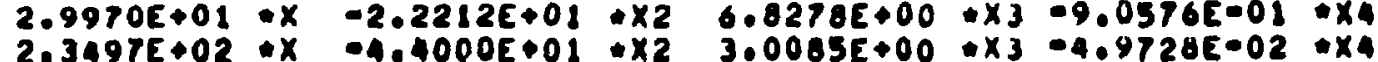
$2.5402 E+01 * x-1.2986 E+00 * \times 2 \quad 1.1492 E-02 * x 3$

$0.3176 E+02 * x-1.4110 E+01 * \times 2$

$3.2006 E+02 * x-1.6362 E+01 * \times 2 \quad 1.4400 E-01 * x 3$

$6.3176 E+02 * x \quad 1.4118 E+01 * \times 2$

$1.1417 E+02 * x-1.1497 E+01 * \times 2 \quad 3.3566 E-01 * \times 3$

$-3.2006 E+02 \cdot x \quad 1.6362 E+01 \cdot \times 2-1.4480 E-02 \times x 3$

$2.8717 E+01 * x-6.1171 E=01 * \times 2$

$7.0490 E+02 * x-1.3200 E+02 * x 29.0256 E+00 \times x+1.4918 E-01 * x 4$

$-2.7400 E+03 * x \quad 2.7592 E+02 * x 2-0.0559 E+00 * x 3$

$4.2675 E+02 * x-2.1016 E+01 * \times 2 \quad 1.9306 E-01 * x$

$-2.2973 E+02 * x \quad 5.1337 E+00 \times x$

$1.3107 E+02 * x-4.6294 E+01 * x 2 \quad 6.5534 E+00 * x 3-3.1960 E-01 * x Q$ $-7.0490 E+02 * x \quad 1.3200 E+02 * x 2-9.0256 E+00 * x 3 \quad 1.4918 E-01 * x_{4}$ $1.9572 E+02 * x-1.9708 E+01 * \times 2 \quad 5.7542 E-01 * x 3$

$-1.2675 E+02 * x \quad 2.10165+01 * x 2-1.9306 E-01 * x_{3}$

$1.9144 E+01 * X-4.27 \quad E=01 * \times 2$

$1.7902 E+02 * x-1.3327 E+02 * x 2 \quad 400967 E+01 * x 3-5.4346 E+00 \times x 4 \quad 2.5574 E-01 * \times 5$ $-1.3187 E+03 * X \quad 4.6294 E+02 * \times 2-6.5534 E+01 * \times 3 \quad 3.1964 E+00 \times \times 4$

$7.0490 E+02 * x-1.3200 E+02 * x 29.0256 E+00 * x 3-1.4910 E=01 \times x 4$

$-6.5239 E+02 * x \quad 6.5694 E+01 * \times 2-1.9161 E+00 * x 3$

$1.5241 E+02 * x-7.7914 E+00 * \times 2 \quad 6.8951 E 002 * x 3$

$-3.8289 E+01 * x \quad 0.5561 E=01 * \times 2$

$1.3846 E+04 * x 2-1.0262 E+04 * x 3 \quad 3.1545 E+03 * \times 4-4.1846 E+02 * \times 5 \quad 1.9692 E+01 * \times 6$

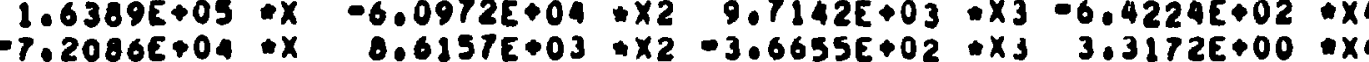

$9.05995+03 * x-3.1703 E+02: \times 2 \quad 1.8013 E+00 * x 3$

$-1.0385 E+04 \times x \quad 7.6964 E+03 * x 2-2.3650 E+03 * x 3 \quad 3.1365 E+02 * x 4-1.4769 E+01 * x 5$ $1.1706 E+04 \times x-4.3531 E+03 * x 2609387 E+02 * x 3-4.5674 E+01 \times x 40.9510 E-01+x 5$ $3.8152 E+04 \times x-7.9303 E+03 \times \times 2 \quad 6.6730 E+02+x 3-1.0797 E+01 * x$

$-1.7163 E+04 \times x \quad 2.0514 E+03 * \times 2-8.7273 E+01 * x J \quad 7.8900 E-01 * x 4$

$1.7317 E+04 \times x \quad 1.1620 E+03 * x 2-2.1719 E+01 * x 3$

$4.53005+03 * x-1.5851 E+02 * \times 29.0065 E-01 * x 3$

$1.6404 E+02 \times \times 2-1.2216 E+02 * \times 3 \quad 3.7553 E+01 * x 4-4.9017 E+00 * \times 5 \quad 203443 E-01 * \times 6$ $1.8117 E+03 \times x-6.7401 E+02 * \times 2 \quad 1.0739 E+02 * \times 3-7.0996 E+00 \times \times 41.3053 E-01 * \times 5$ $2.9266 E+03 \times x-1.0008 E+03 * \times 2 \quad 1.7347 E+02 * \times 3-1.1469 E+01 * \times 42.2370 E-01 * \times 5$ $-6.5304 E+02: X \quad 7.8147 E+01 \times 2-3.3247 E+00 \times 3 \quad 3.00885-02 \times x$

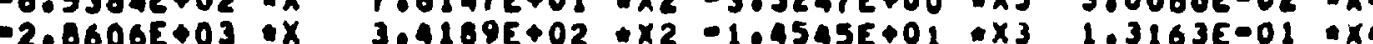

$5.3928 E+01 * x \quad-1.8071 E+00 * x 2 \quad 1.0722 E 002 \times x$

$5.6825 E+02: x-1.0014 E+01: \times 2 \quad 1.1258 E-01: \times 3$ 


\begin{tabular}{|c|c|c|}
\hline $0(62 C) \cdot 0(60 C) 0$ & $\begin{aligned} & 0(226) \\
+ & 0(325) \\
+ & 0(426) \\
+ & 0(525) \\
+ & 0(626)\end{aligned}$ & $\begin{array}{r}4.33516+04 \\
-1.2502 E+05 \\
-1.0331 E+05 \\
4.31826+05 \\
3.65796 \bullet 04\end{array}$ \\
\hline $0(62 C)+0(6) C)=$ & $\begin{array}{l}+30(115): \\
+0(216) \\
+10(315) \\
+10(335) \\
+0(416) \\
+0(436) \\
+10(515) \\
+10(535) \\
+0(616) \\
+0(636)\end{array}$ & $\begin{array}{r}4.1530 E+02 \\
-3.0965 E+03 \\
-4.4937 E+03 \\
-1.0405 E+04 \\
1.1051 E+04 \\
1.0057 E+04 \\
2.20325+03 \\
9.9963 E+03 \\
-6.6507 E+03 \\
-4.90001003\end{array}$ \\
\hline $0(62 C) \cdot 0(62 C)=$ & 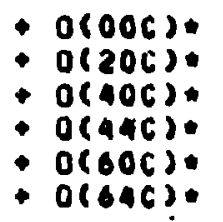 & $\begin{array}{r}-1.1077 E+02 \\
-2.0643 E+03 \\
4.9195 E+02 \\
2.1914 E+04 \\
6.9674 E+02 \\
-7.9809 E+03\end{array}$ \\
\hline $0(63 C) * 0(60 C))$ & $\begin{array}{l}+10(335) . \\
+0(436) \\
+10(535) \\
+0(636)\end{array}$ & $\begin{array}{r}6.2912 E \bullet 04 \\
-1.9723 E+05 \\
-2.6989 E+04 \\
7.1495 E+04\end{array}$ \\
\hline $0(63 C) * 0(61 C)=$ & 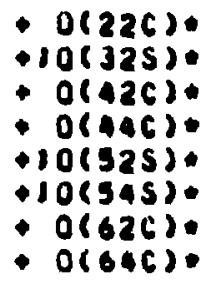 & $\begin{array}{r}1.5403 E+03 \\
-8.9874 E+03 \\
-1.3417 E+03 \\
-8.21771+03 \\
2.3133 E+04 \\
1.3494 E+04 \\
-1.2470 E+03 \\
2.0704 E+03\end{array}$ \\
\hline $0(03()+0(62 C)=$ & 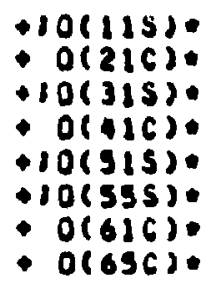 & 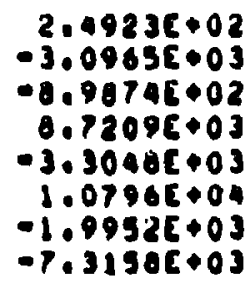 \\
\hline $0(630) 0(6) 30)$ & 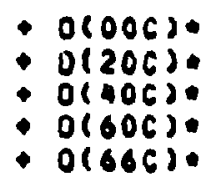 & $\begin{array}{r}-2.7692 E+01 \\
-2.9806002 \\
-6.03756+02 \\
3.10456+02 \\
-3.65795+03\end{array}$ \\
\hline
\end{tabular}

$-9.6825 E+04 * x \quad 1.7420 E+04 \times x 2-2.7755 E+03 * x 3 \quad 1.4350 E+02 * x 4-3.5004 E+00 \cdot \times 5$

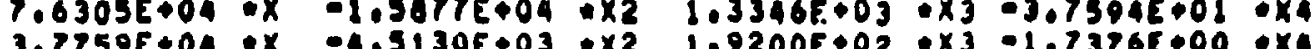

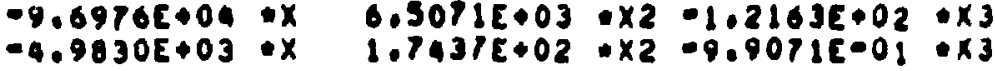

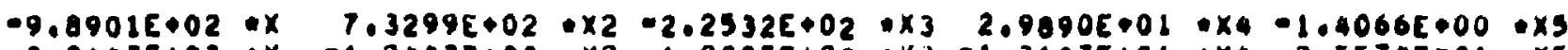
$3.3447 E+03 \times x-1.2443 E+03 \div \times 2 \quad 1.9825 E+02 \times K 3-1.3107 E+01 * x 4205574 E-01: x 5$ $2.72525+03 * x$ $6.3587 E+03 * x$ $-4.3317 E+03+x$ $-4.0048 E+03 * x$ $-4.9478 E+02: x$ $-2.0203 E+03 * x$ $9.0599 E+02: x$
$6.7950 E+02: x$ $-5.6702 E+02 * x 2 \quad 4.7664 E+01 * x 3-1.3427 E+00 * x_{4}$ $1.3230 E+03 * x_{2} \quad 1,1122 E+02 * x_{3}-1.1329 E+00 * x_{4}$

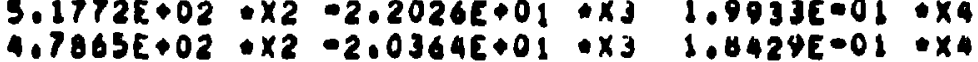
$3.3200 E+01 \times \times 2-6.2050 E-01 * x 3$ $1.35578+02 \times x 2=2.5339 E+00 \div \times 3$

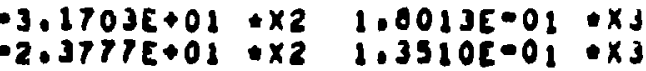

$2.6374 E+02 \div \times 2-8$
$2.2290 E+03: x$ $-1.45065+02 * \times 3 \quad 6.0085 E+01 * \times 4-7.9707 E+00+\times 5$ $2.22960+03: x$
$-1.20015 \div 02: x$ $-8.0095+03: x$
$-1.7901 E+02$ $0.00956+03$ : $x$ $0.4914 E+01: x$ $-8.2955 E+02: \times 2 \quad 1.3217 E+02: x_{3}-0.1379 E+00 * x 4$

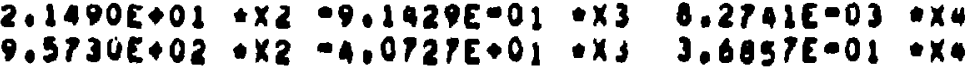

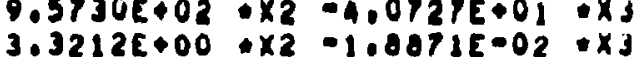

$-3.8192 E+04 * x \quad 7.9383 E+03 * \times 2-6.6730 E+02 * x 3 \quad 1.4707 E+01 * x_{4}$ $7.2006[004 \times x-0.61575+03 * x 2 \quad 3.6655 E+02 * x]-3031726+00 * x_{4}$ $6.06106+03 \times x-4.06906+02 \times x 27,6010 E+00 * x]$

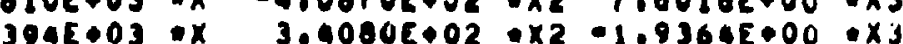

$3.7509 E-01: \times 6$
$1.7050 E-01: \times 5$

$0.22165+02+\times 2-9.9125 E+01 * \times 3 \quad 6.5534 E+00 \cdot \times 4-1.2707 E-01 * \times 5$ (.) $3.0036 E+03: x$ $-5,19525003$ in $-3.03058+03: x$ $1.69076+02: x$
$-2.03126+02: x$ $-1.1340 E+03 * \times 2 \quad 9.5329 E+01 \times 3-2.6053 E+00 * x$ $9.0610 E+01 * x 2 \quad 2.4935 E+00: x 3-2.2560 E-02: x 4$

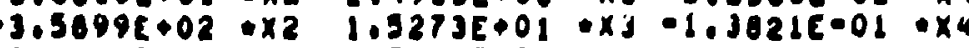

$3.9060 E+02 \times 2 \times 2-6.5150 E+00 \times x$

$3.03358+02+x 2-3,0009 E+00 * x$

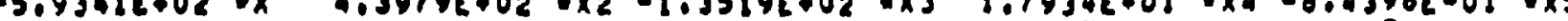
$3.34476+03 * x-1.2443 E+03 * \times 2 \quad 1.9425 E+02 * x 3-1.3107 E+01 * x 4 \quad 2.5574 E-01 \cdot x 5$

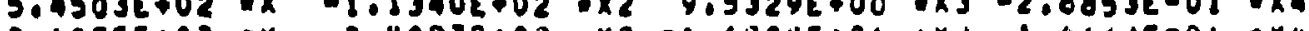

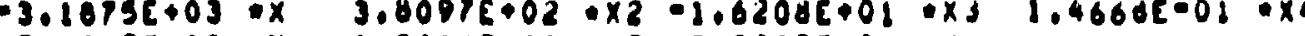

$1.4217 \mathrm{C}+02: x$

$-2.42446+03: x$

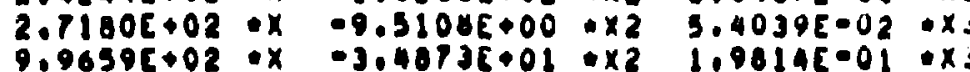

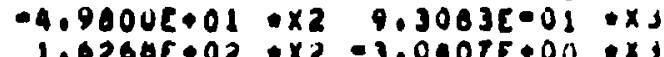

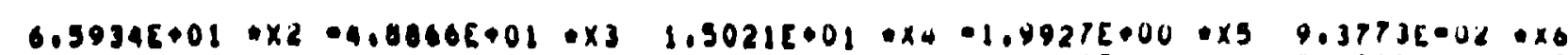

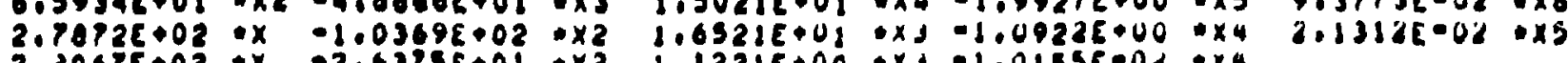

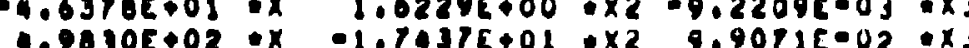




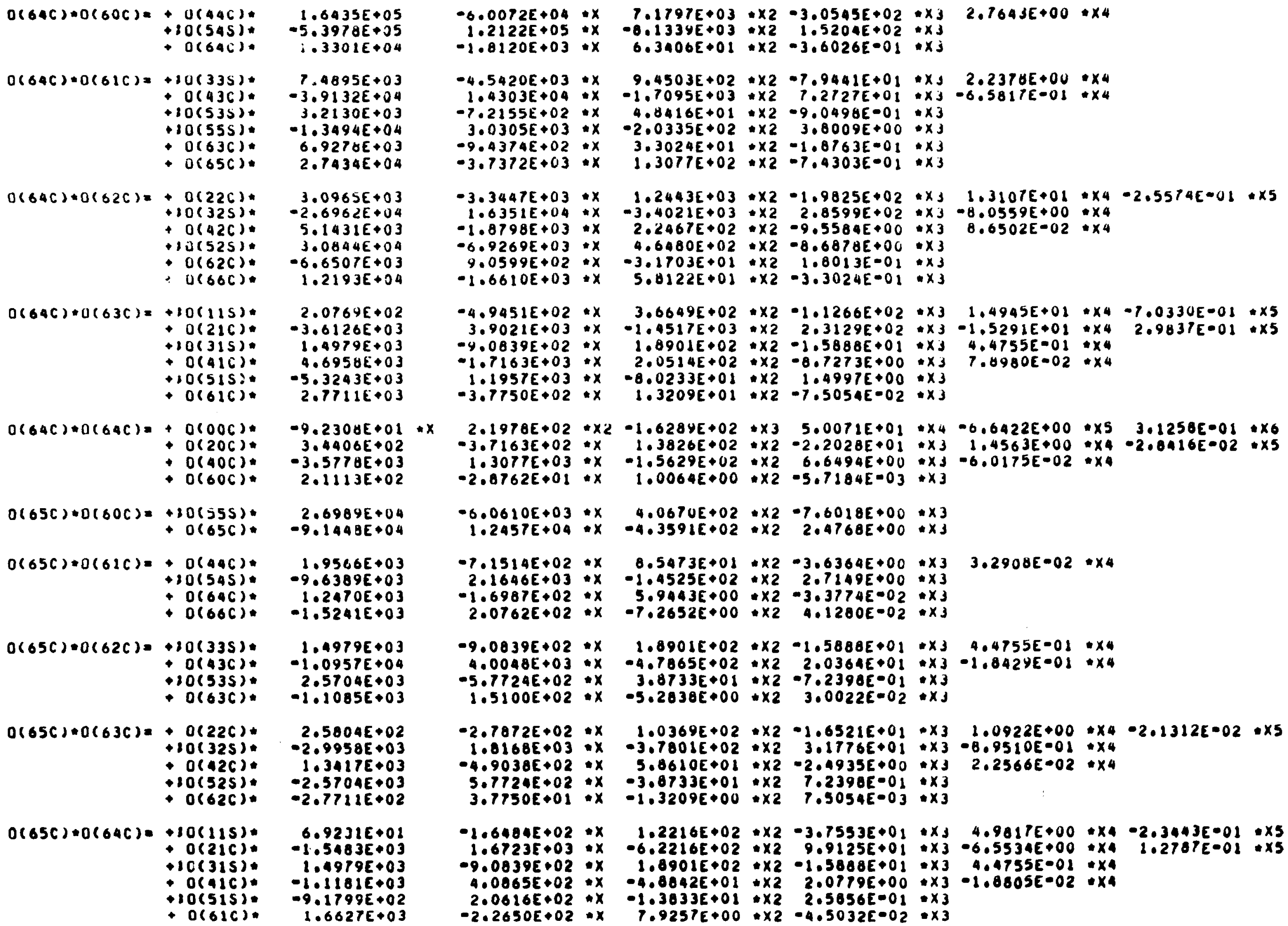

$0(64 C)+0(60 C)=+0(44 C)$ $+30(545)$ $+0(64 c)$.

$1.6435 E+05$ $-5.3978 E+05$

$7.4095 E+03$

$0(64 C)+0(61 C)=+10(335)$ $+30(535)$. $+10(555)$. $+0(636)$.

- $0(65 C)$. $3.2130 E+03$ $-1.3494 E+04$ $6.927 \forall E+03$ $2.7434 E+04$

$0(64 C)+0(62 C)=+0(22 C)$. $+10(325)$. $+0(42 C)$. - $0(626)$. $0(660)$.

$3.0965 E+03$ $-2.6962 E+04$ $5.1431 E+03$ $3.0844 E+04$ $-6.6507 E+03$ $1.2193 E+O 4$

$0(64 C)+0(63 C)=+10(115)$ $+0(216)$. $+10(315)$. $+0(416)$. $+10(515):$

$2.0769 E+02$ $-3.6126 E+03$ $1.4979 E+03$ $4.6958 E+03$ $-5.3243 E+03$ $2.7711 E+03$

$O(64 C)+0(64 C)=+O(00 C)$ $+0(206)$. $+0(40 C)$.

$-9.2308 E+01$. $3.4406 E+02$ $-3.5778 E+03$
$2.1113 E+02$

$2.69095+04$

$0(65 C)+0(60 C)=+30(555)$

65( )

$0(65 C)+0(61 C)=+0(44 C)$ $+0(64 C)$.

$+0(66 C)$.

$0(65 C)=0(62 C)=+10(335)$ $\rightarrow 0(436)$. $\rightarrow 0(63 C)$.

$0(65 C) * 0(63 C)=+0(22 C)$ $+10(425)$ $+10(525)$. $+0(62 C)$.

$0(65 C)+0(64 C)=+10(115) *$ $+0(216)$. $+10(315)$. $+0(416)$. $+10(515)$. $9.1448 E+04$ $1.9566 E+03$
$0.953895+03$ $-9.6389 E+03$ $1.2470 E+03$
$-1.5241 E+03$

$1.4979 E+03$ $-1.0957 E+04$ $2.5704 E+03$ $-1.1085 E+03$

$2.5004 E+02$ $-2.9950 E+03$ $1.34176+03$ $-2.5704 E+03$ $-2.7711 E+02$

$6.92 J 1 E+01$ $-1.5483 E+03$

$1.49795+03$

$-1.11816+03$ $-9.1799 E+02$

$1.6027 E+03$

$-6.0072 E+04 * x \quad 7.1797 E+03 * \times 2-3.0545 E+02 * x 3$

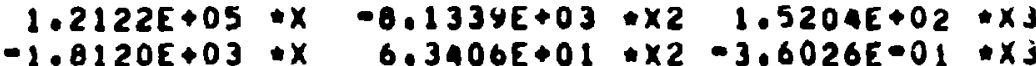
$6.3406 E+01 * \times 2-3.6026 E-01 * x J$

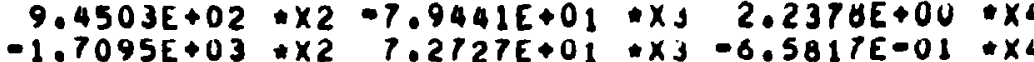
$1.4303 E+04: x$ $-7.2155 E+02: x$ $3.0305 E+03: x$ $-9.4374 E+02: x$ $-3.7372 E+03: x$ $4.0416 E+01 \div \times 2-9.0498 E-01 \div \times 3$ $-2.0335 E+02 * \times 2 \quad 3.0009 E+00 * x 3$ $3.3024 E+01+\times 2-1.0763 E=01: \times 3$
$1.3077 E+02 \div \times 2-7.4303 E=01: \times 3$

$-3.3447 E+03 * x \quad 1.2443 E+03 * \times 2-1.9825 E+02 * x 3 \quad 1.3107 E+01 * \times 4-2.5574 E=01 * \times 5$ $1.0351 E+04 * x-3.4021 E+03 * x 2 \quad 2.8599 E+02 * x 3-8.0559 E+00 \times x 4$ $-1.8798 E+03 \times x \quad 2.2467 E+02 * \times 2-9.5584 E+00 * x 3 \quad 8.6502 E-02 * \times 4$

$-6.9269 E+03 * X \quad 4.6480 E+02 * \times 2-8.6878 E+00 * x 3$

$9.0599 E+02 * x-3.1703 E+01 * \times 2 \quad 1.8013 E-01 * x 3$

$-1.6610 E+03+x \quad 5.0122 E+01 * \times 2-3.3024 E-01 * x$

$-4.9451 E+02 * x \quad 3.6649 E+02 * x 2-1.1266 E+02 * x 3 \quad 1.4945 E+01 * x 4-7.0330 E-01 * x 5$ $3.9021 E+03 * x-1.4517 E+03 * x 2 \quad 2.3129 E+02 * x 3-1.5291 E+02 * x 4 \quad 2.9837 E-01 * x 5$ $-4.0839 E+02 * x \quad 1.8901 E+02 * x 2-1.5888 E+01 * x 3 \quad 4.4755 E-01 * x 4$

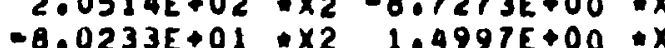

$-3.7750 E+02 * x \quad 1.3209 E+01: \times 2-7.5054 E-02 * x$

$2.1978 E+02 * \times 2-1.6284 E+02 * \times 3 \quad 5.0071 E+01 * \times 4-6.6422 E+00 * \times 5 \quad 3.1258 E-01 * \times 6$ $-3.7163 E+02 * x \quad 1.3826 E+02 * x 2-2.2028 E+01 * x 3 \quad 1.4563 E+00 * x+-2.0416 E-02 * x 5$ $1.3077 E+03 * x-1.5629 E+02 * x 2 \quad 6.6494 E+00 * x J-6.0175 E-02 * x 4$

$-2.8762 E+01 * x \quad 1.0064 E+00 * \times 2-5.7184 E-03 * \times 3$

$\begin{array}{rrrr}-6.0610 E+03 * x & 4.0670 E+02 * \times 2 & -7.6018 E+00 & * \times 3 \\ 1.2457 E+04 * x \quad-4.3591 E+02 * \times 2 & 2.4768 E+00 & \times 3\end{array}$

$-7.1514 E+02 * x \quad 8.5473 E+01 * 2-3.6364 E+00 * \times 3 \quad 3.2908 E-02 * \times 4$

$2.1646 E+03 * x \quad-1.4525 E+02 * x 2 \quad 2.7149 E+00 * x 3$

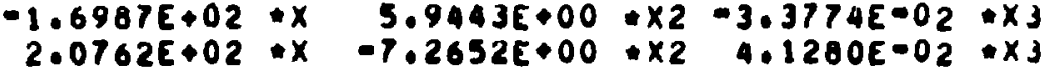

$-9.0039 E+02 * x \quad 1.8901 E+02 * x 2-1.5880 E+01 * x 34.4755 E-01 * x 4$

$4.0048 E+03 * x \quad-4.7865 E+02 * \times 2 \quad 2.0364 E+01 * x 3-1.842 y E-01 * \times 4$

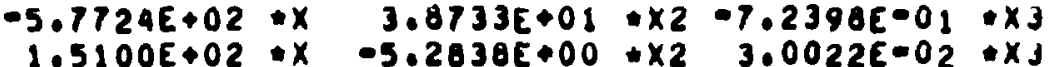

$-2.7872 E+02 * x \quad 1.0369 E+02 * x 2-1.6521 E+01 * x 3 \quad 1.0922 E+00 * x 4-2.1312 E=02 * \times 5$ $1.8168 E+03 \times x-3.7801 E+02 \times x_{2} 3.1776 E+01 \times x_{3}-6.9510 E-01 * x_{4}$

$-4.9038 E+02 * x \quad 5.8610 E+01 * \times 2-2.4935 E+00 * x 3 \quad 2.2566 E-02 * \times 4$

$5.7724 E+02 * x-3.8733 E+01 * \times 2 \quad 7.2398 E-01 * x 3$

$-1.6484 E+02 * x \quad 1.2216 E+02 * \times 2-3.7553 E+01 * x J \quad 4.9017 E+00 * \times 4-2.3443 E-01 * \times 5$ $1.6723 E+03 * x-6.2216 E+02 * \times 2 \quad 9.9125 E+01 * x 3-6.5534 E+00 * x 4 \quad 1.2787 E-01 * \times 5$

$-9.0839 E+02 * x \quad 1.8901 E+02 * \times 2-1.5808 E+01 * \times 3 \quad 4.4755 E-01 * \times 4$

$4.0065 E+02 * x-4.8042 E+01 * \times 2 \quad 2.0779 E+00 * x 3-1.8005 E-02 * x 4$

$2.0616 E+02 * x-1.3633 E+01 * \times 2 \quad 2.5656 E-01 * x 3$

$-2.2650 E+02 * x \quad 7.9257 E+00 * x 2-4.5032 E-02=x 3$ 


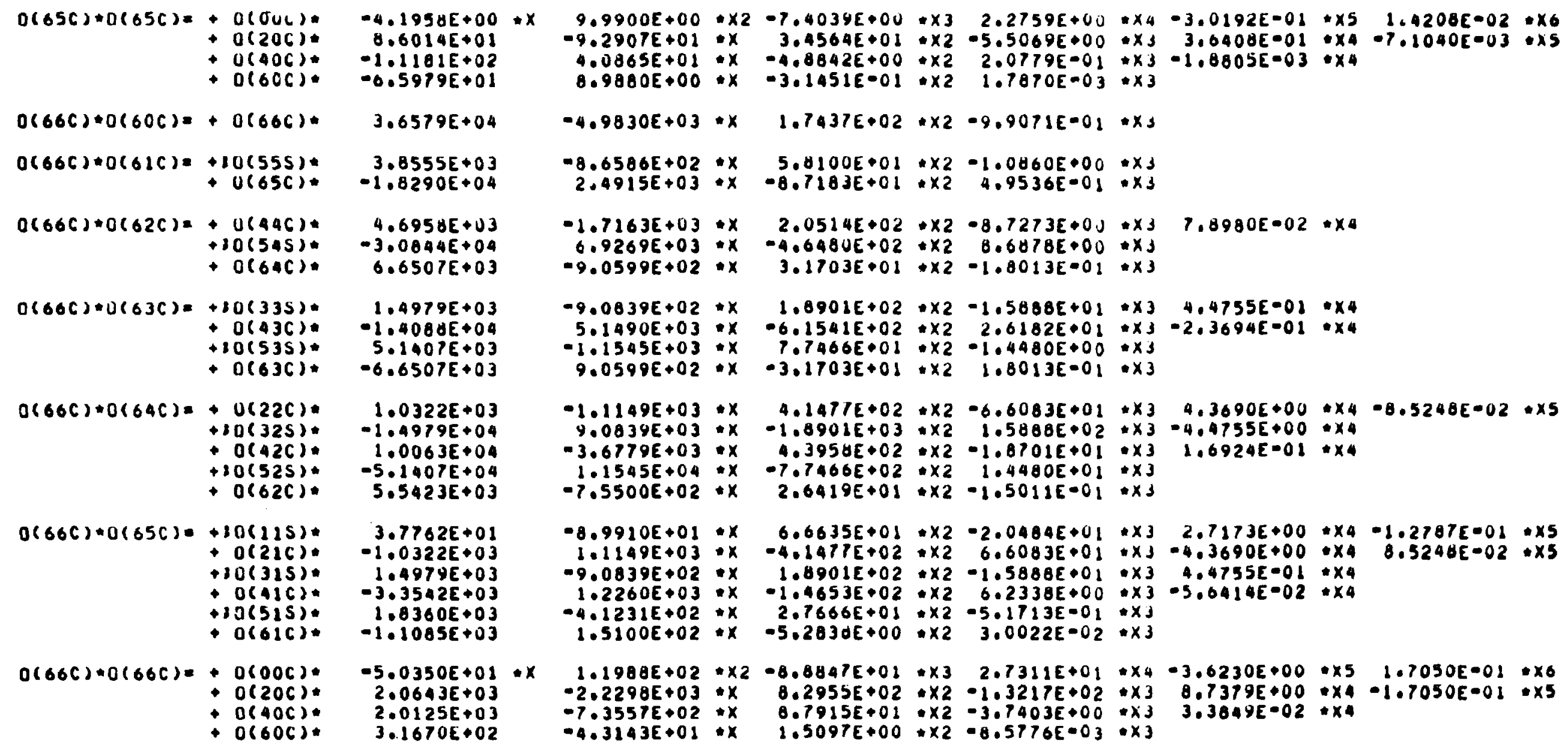

$0(65 C) * 0(65 C)=+0(\sigma u c)$ $+0(206)$. $+0(40 C)$.

$+0(60 C)$.

$0(66 C)+0(60 C)=+0(66 C)=$ $\begin{aligned} 0(66 C)+0(61 C)= & +10(555) * \\ & +0(65 C)\end{aligned}$

$Q(66 C) * 0(62 C)=+U(94 C)$ $+30(545)$.

$0(606) * 0(63 C)=+30(335)=$ $+0(43 C)$ $+30(535)$.

$O(66 C) * O(64 C)=+O(22 C)=$ $+10(325)$ $+0(42 C)$. $+80(525):$
$+0(62 C)$.

$0(66 C) * 0(65 C)=+30(125) *$ $0(216)$ o(aic).

$+10(515)$.

- 0(6)

$O(66 C) * 0(66 C)=+O(00 C)$ $0(400)$ $+0(60 C)$.

$-4.1958 E+00$ $0.6014 E+0$ $-0.5979 E+01$

$3.6579 E+04$

$3.8555 E+03$
$-1.8290 E+04$

$4.6958 E+U 3$ $-3.0044 E+04$ $6.6507 E+03$

$1.4979 E+03$ $-1.40806+0$ $5.1407 E+03$ $-6.6507 E+03$

$1.0322 E+03$ $-1.4979 E+04$ $-50063 E+04$ $-5.1407 E+04$

$3.7762 E+01$ $-1.03222+03$ $1.4979 E+03$

$1.83605+03$

$-1.1005 E+03$

$-5.0350 E+01$ $2.0643 E+03$ $2.0125 E+03$
$3.1670 E \bullet 02$

$9.9900 E+00 \times 2-7.4039 E+00 * \times 3 \quad 2.2759 E+00=\times 4-3.0192 E-01 * \times 5 \quad 1.4208 E-02 * \times 6$

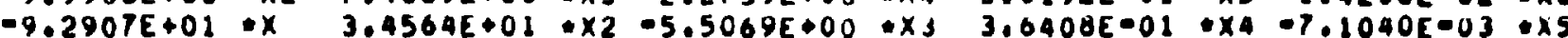

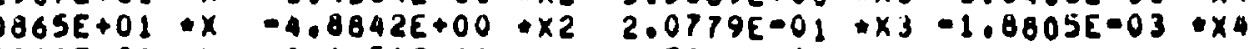

$8.9800 E+00 * x-3.1451 E-01 * \times 2 \quad 1.7870 E-03 * x 3$

$-4.9830 E+03 * x \quad 1.7437 E+02 * \times 2-9.9071 E=01 * \times s$

$-0.6506 E+02 * x \quad 5.8100 E+01 * \times 2-1.0060 E+00 * x_{3}$

$-1.7163 E+03 * x \quad 2.0514 E+02 * \times 2-8.7273 E+0 J * \times 3 \quad 7.8980 E-02 * x 4$ $6.9269 E+03: x-4.6480 E+02+x 2 \quad 8.6878 E+00 * x 3$

$-9.0839 E+02 * x \quad 1.0901 E+02 * \times 2-1.5888 E+01 * x 3 \quad 4.4755 E=01 * x 4$ $5.1490 E+03: x-6.1541 E+02: \times 2 \quad 2.6182 E+01 * x 3-2.3694 E-01 * x 4$

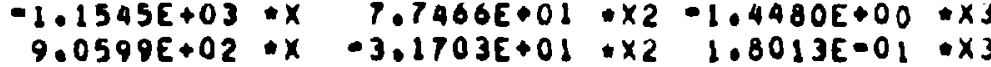

$-1.1149 E+03 * x \quad 4.1471 E+02 * \times 2-6.6083 E+01 * x 3 \quad 4.3690 E+00 * \times 4-0.5248 E-02 * \times 5$ $9.0039 E+03 * x-1.8901 E+03 * x 2 \quad 1.588 B E+02 * x 3-4.4755 E+00 \times x_{0}$

$-3.6779 E+03 * x \quad 4.3958 E+02 * x 2-1.8701 E+01 * x 3 \quad 1.6924 E=01 * x 4$

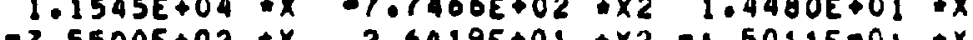

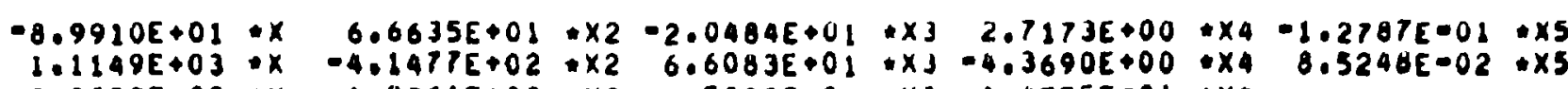
$-9.0839 E+02: x \quad 1.8901 E+02+x 2-1.588 B E+01 * x 3-4.4755 E-01 * \times 4$

$1.5100 E+02: x-5.283 d E+00 \times x 23.0022 E=02 \times x 3$

$1.1900 E+02 * \times 2-8.8447 E+01 \times \times 3 \quad 2.7311 E+01 * \times 4-3.6230 E+00 * \times 5 \quad 1.7050 E-01 * \times 6$ $-2.2298 E+03 * x \quad 8.2955 E+02 * x 2-1.3217 E+02 * x 3 \quad 0.7379 E+00 * x 4-1.7050 E-01 * \times 5$ $-7.3557 E+02 * x \quad 0.7915 E+01 * x 2-3.7403 E+00 * x 3 \quad 3.3049 E-02 * x 4$

$-4.3143 E+01 * x \quad 1.5097 E+00 * x 2-8.5776 E=03: x 3$ 

$0(115) \cdot 0(115)=+0(006) \quad 3.3333 E-0) * x$
$0(206) \quad-1.6667 E_{-01}$
$+0(226) \quad-5.0000 E-02$

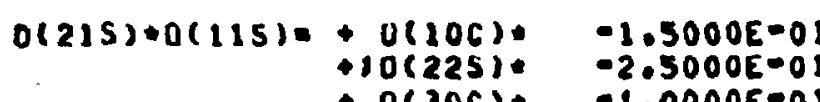
- $0(306)-1.0000 E=0$
$2.0000 E-01 * x$ 


\begin{tabular}{|c|c|c|c|c|c|}
\hline $0(225)=0(115)=$ & $\begin{array}{l}+0(116) . \\
+0(215) . \\
+0(31 C) . \\
+0(336) .\end{array}$ & $\begin{array}{r}-3.0000 E-01 \\
1.0000 E+00 \\
-1.0000 E-01 \\
-5.0000 E-01\end{array}$ & & $4.0000 E-01$ & $x$ \\
\hline$D(215) \backsim 0(215)=$ & $\begin{array}{l}+O(O O C) \\
+O(20 C) \\
+O(226) \\
+\quad O(40 C) \\
+O(42 C)\end{array}$ & $\begin{array}{r}-5.0000 E-02 \\
-8.9286 E-02 \\
2.6706 E-01 \\
-1.4286 E-02 \\
-7.1429 E-02\end{array}$ & $-x$ & $\begin{array}{r}6.6667 E-02 \\
2.3810 E=02 \\
-7.1429 E=02\end{array}$ & $\begin{array}{l}x_{2} 2 \\
: x \\
x\end{array}$ \\
\hline $0(225) \cdot 0(215) \theta$ & $\begin{array}{l}+10(115): \\
+0(216): \\
+10(315): \\
+0(335) \\
+0(416) \\
+0(436):\end{array}$ & $\begin{array}{r}1.5000 E-01 \\
-1.0: 14 E+100 \\
3.0000 E-01 \\
5.0000 E-01 \\
-7.11429 E-02 \\
-5.0000 E-01\end{array}$ & & $\begin{array}{r}-2.0000 E-01 \\
2.0571 E-01\end{array}$ & $: x$ \\
\hline $0(225)+0(225)=$ & $\begin{array}{l}+O(O O C): \\
+0(20 C) . \\
+O(40 C) \\
+O(44 C)\end{array}$ & $\begin{array}{r}-2.0000 E-01 \\
7.1429 E-01 \\
1.142068-02 \\
-5.0000 E-01\end{array}$ & $x$ & $\begin{array}{r}2.6667 E=01 \\
-1.90404=01\end{array}$ & $: x 2$ \\
\hline $0(315)=0(125)=$ & $\begin{array}{l}O(206): \\
+O(226): \\
+10(325): \\
+O(406) \\
+O(426) .\end{array}$ & $\begin{array}{l}-5.7143 E-01 \\
-2.8571 E-01 \\
-2.5000 E+00 \\
-7.1429 E-02 \\
-3.5714 E-01\end{array}$ & & $\begin{array}{l}2.8571 E-01 \\
1.4266 E-01\end{array}$ & $\begin{array}{l}x \\
x \\
x\end{array}$ \\
\hline $0(325)=0(215)=$ & $\begin{array}{l}+0(216) \\
+10(315) \\
+\quad 0(335) \\
+O(416) \\
+O(436)\end{array}$ & $\begin{array}{r}-5,7143 E-01 \\
2.5000 E-01 \\
-2.5000 E-01 \\
-7.1429 E-02 \\
-3.0000 E-01\end{array}$ & & 2.85716001 & $* x$ \\
\hline $0(335)=0(125)=$ & $\begin{array}{l}+0(226) \\
+10(325) \\
+0(426) \\
+0(94 C)\end{array}$ & $\begin{array}{r}-6.5714 E-01 \\
1.5000 E+00 \\
-7.14296-02 \\
-5.0000 E=01\end{array}$ & & 4.285 re $=01$ & $x$ \\
\hline
\end{tabular}




\begin{tabular}{|c|c|c|c|c|c|c|}
\hline $0(315)+0(215)=$ & $\begin{aligned} & 0(10 C) \\
+ & 0(225) \\
+ & 0(30 C) \\
+ & 0(32 C) \\
+ & 0(425) \\
+ & 0(50 C) \\
+ & 0(526)\end{aligned}$ & $\begin{array}{r}3.9280 E-01 \\
0.5714 E-01 \\
-3.6667 E-01 \\
2.7500 E+00 \\
-1.7857 E=01 \\
-3.9603 E-02 \\
-8.3333 E-01\end{array}$ & $\begin{array}{r}-6.2857 E-01 \\
-4.2857 E-01 \\
4.4444 E-02 \\
-3.3333 E-01\end{array}$ & $\begin{array}{l}: x \\
: x \\
: x \\
x\end{array}$ & $2.2857 E-01$ & $\times 2$ \\
\hline $0(315) \cdot 0(225)=$ & 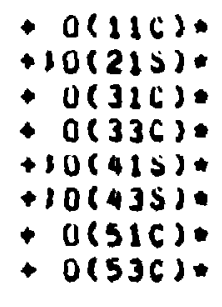 & $\begin{array}{l}-1.7143 E=01 \\
-3.4286 E+00 \\
-2.2000 E+00 \\
-1.8333 E+00 \\
-1.4286 E+00 \\
-5.0000 E+00 \\
-1.1905 E-01 \\
-2.7778 E=01\end{array}$ & $\begin{array}{l}3.1429 E-01 \\
1.7143 E+00 \\
2.6667 E \bullet 01 \\
2.2222 E-01\end{array}$ & $\begin{array}{l}: x \\
: x \\
: x \\
x\end{array}$ & $-1.14296-01$ & $\times 2$ \\
\hline $0(325)=0(215)=$ & $\begin{array}{l}+0(116) \\
+0(316) \\
+0(336) \\
+10(415) \\
+0(435) \\
+0(516) \\
+0(536)\end{array}$ & $\begin{array}{r}8.5114 E-02 \\
-2.7500 E=01 \\
4.5833 E=01 \\
2.5000 E=01 \\
2.5000 E=01 \\
-2.3610 E-02 \\
-5.5556 E-02\end{array}$ & $\begin{array}{r}-1.5714 E=01 \\
3.3333 E=02 \\
-5.3556 \varepsilon=02\end{array}$ & $\begin{array}{l}x \\
: x \\
x\end{array}$ & $5.7143 E-02$ & $\cdot \times 2$ \\
\hline $0(325) * 0(225)=$ & $\begin{array}{l}+0(10 C) \\
+0(306) \\
+0(445) \\
+0(50 C) \\
+0(54 C)\end{array}$ & $\begin{array}{r}1.7143 E-01 \\
7.3333 E-01 \\
-5.0000 E-01 \\
7.9365 E-03 \\
-5.0000 E-01\end{array}$ & $\begin{array}{l}-3.1429 E-01 \\
-0.0009 E-02\end{array}$ & $: x$ & $1.2429 E-01$ & $\times 2$ \\
\hline $0(335)+0(215) 0$ & $\begin{array}{l}+10(225) \\
+0(326) \\
+10(425) \\
+0(445) \\
+0(52 C) \\
+0(546)\end{array}$ & $\begin{array}{r}0.5714 E-01 \\
-2.7500 E+00 \\
3.2143 E=01 \\
7.5000 E-01 \\
-1.6667 E-01 \\
-5.0000 E-01\end{array}$ & $\begin{array}{r}-4.2857 E-01 \\
3.3333 E-01\end{array}$ & $: x$ & & \\
\hline $0(335)+0(225)=$ & $\begin{array}{l}+0(116) \\
+10(215) \\
+0(316) \\
+10(185) \\
+0(516) \\
+0(556)\end{array}$ & $\begin{array}{r}3.1429 E-01 \\
-3.1286 E+00 \\
1.1000 E+00 \\
-4.2057 E-01 \\
2.3810 E-02 \\
-5.0000 E-01\end{array}$ & $\begin{array}{r}-9.12065-01 \\
1.7143 E+00 \\
-1.3333 E-01\end{array}$ & $\begin{array}{l}x \\
x \\
x\end{array}$ & $3.42460-01$ &.$\times 2$ \\
\hline
\end{tabular}




\begin{tabular}{|c|c|c|c|c|c|c|c|}
\hline$O(315) \cdot 0(315)=$ & $\begin{array}{l}+O(00 C) \\
+0(206) \\
+0(22 C) \\
+0(006) \\
+0(42 C) \\
+0(60 C) \\
+0(62 C)\end{array}$ & $\begin{array}{r}5.7143 E-01 \\
3.1429 E+00 \\
-6.2357 E+00 \\
-2.2076 E=01 \\
4.4156 E+00 \\
-5.4113 E=02 \\
-3.7879 E-01\end{array}$ & $\bullet x$ & $\begin{array}{r}-1.0476 E+00 \\
-1.9524 E+00 \\
3.9048 E+00 \\
1.2987 E=02 \\
-2.3974 E-01\end{array}$ & $\begin{array}{l}: x 2 \\
: x \\
: x \\
: x \\
x\end{array}$ & $\begin{array}{r}3.0095 E-01 \\
1.4048 E-01 \\
-3.0095 E-01\end{array}$ & $\begin{array}{r}\times 3 \\
\times 2 \\
\times 2 \\
\times 2\end{array}$ \\
\hline $0(325)+0(315)=$ & $\begin{array}{l}+10(115): \\
+0(216): \\
+0(335): \\
+0(416): \\
00(436): \\
+80(515): \\
+0(535): \\
+0(616): \\
+0(636)\end{array}$ & $\begin{array}{r}-4.2857 E-01 \\
3.1629 E+00 \\
-3.0000 E+00 \\
-1.7662 E+00 \\
3.0900 E+00 \\
5.3571 E-01 \\
-1.1667 E-01 \\
-7.5758 E-02 \\
-2.2727 E-01\end{array}$ & & $\begin{array}{r}7.8571 E-01 \\
-1.9524 E+00 \\
6.6667 E-01 \\
1.0390 E-01 \\
-1.8182 E-01\end{array}$ & $\begin{array}{l}: x \\
: x \\
: x \\
: x \\
: x\end{array}$ & $\begin{array}{r}-2.0571 E-01 \\
1.9048 E-01\end{array}$ & $: \times 2$ \\
\hline $0(325) * 0(325)=$ & 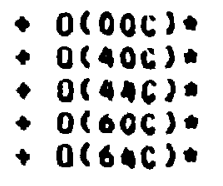 & $\begin{array}{r}5.7143 E-02 \\
1.545 E=01 \\
7.7273 E-01 \\
2.1645 E-03 \\
-4.5455 E-02\end{array}$ & $\cdot x$ & $\begin{array}{l}-1.0476 E=01 \\
-9.0909 E=03 \\
-4.5455 E-02\end{array}$ & $\begin{array}{l}x 2 \\
: x \\
x\end{array}$ & $3.0045 E-02$ & $* \times 3$ \\
\hline $0(335)+0(315)=$ & $\begin{array}{l}+0(226): \\
+10(325) \\
+0(426) \\
+0(446) \\
+10(525) \\
+10(545) \\
+0(626) \\
+0(646)\end{array}$ & $\begin{array}{r}-3.1429 E+00 \\
1.8000 E+01 \\
-3.9740 E+00 \\
-4.6364 E+00 \\
5.0000 E+00 \\
7.5000 E+00 \\
-7.9750 E-02 \\
-2.2727 E-01\end{array}$ & & $\begin{array}{r}1.9524 E+00 \\
-4.0000 E+00 \\
2.3377 E-01 \\
2.7273 E-01\end{array}$ & $\begin{array}{l}: x \\
: x \\
: x \\
x \\
x\end{array}$ & $-1.4048 E-01$ &.$\times 2$ \\
\hline $0(335)=0(325)=$ & $\begin{array}{l}+10(115): \\
+0(216) \\
+10(315) \\
+0(416) \\
+10(515) \\
+10(355) \\
+0(616) \\
+0(656)\end{array}$ & $\begin{array}{r}-2.5714 E-01 \\
3.1 .29 E+00 \\
-1.4000 E+00 \\
1.3247 E+00 \\
-1.7057 E-01 \\
7.3000 E-01 \\
1.5152 E-02 \\
-5.0000 E-01\end{array}$ & & $\begin{array}{r}4.7143 E-01 \\
-1.9324 E+00 \\
4.0000 E-01 \\
-7.7922 E-02\end{array}$ & $\begin{array}{l}: x \\
: x \\
: x \\
* x\end{array}$ & $\begin{array}{r}-1.7143 E=01 \\
1.9040 E-01\end{array}$ & $\begin{array}{l}: \times 2 \\
\times 2\end{array}$ \\
\hline $0(335) * 0(335)=$ & $\begin{array}{l}+0(00 C) \\
+0(20 C) \\
+0(40 C) \\
+0(60 C) \\
+0(66 C)\end{array}$ & $\begin{array}{r}3.4206 E=01 \\
-3.1429 E+00 \\
-3.9740 E-01 \\
-2.1643=-03 \\
-5.0000 E-01\end{array}$ & $+x$ & $\begin{array}{r}-6.2857 C-01 \\
1.9524 E+D 0 \\
2.337 T C-02\end{array}$ & $\begin{array}{l}: x^{2} \\
: x \\
: x\end{array}$ & $\begin{array}{r}2.2857 E-01 \\
-1.9048 E-01\end{array}$ & $\begin{array}{l}\times \times 3 \\
\times \times 2\end{array}$ \\
\hline
\end{tabular}




\begin{tabular}{|c|c|c|c|c|c|c|}
\hline $0(415) \cdot 0(115)=$ & $\begin{array}{l}+0(30 C): \\
+ \text { o }(32 C): \\
+10(425): \\
+0(30 C): \\
+0(52 C):\end{array}$ & $\begin{array}{l}-1.3333 E-01 \\
-1.2500 E+00 \\
-7.5000 E-01 \\
-5.5536 E-02 \\
-1.1667 E+00\end{array}$ & $\begin{array}{c}2.22225=01 \\
3.33335001 \\
.\end{array}$ & $: x$ & & \\
\hline $0(425) \div 0(215)=$ & $\begin{array}{l}+0(316): \\
+0(336) \\
+10(415) \\
+10(435) \\
+0(516) \\
+0(53 C) .\end{array}$ & $\begin{array}{r}-1.2500 E+00 \\
-4.1867 E-01 \\
1.5000 E+00 \\
-3.5000 E+00 \\
-1.6067 E-01 \\
-3.8089 E-01\end{array}$ & $\begin{array}{l}3.33336-01 \\
1.11118-01\end{array}$ & $: x$ & & \\
\hline $0(135)+0(115)=$ & $\begin{array}{l}\text { D } 0(32 C): \\
+10(425) \\
+10(145): \\
+0(52 C): \\
+0(54 C) .\end{array}$ & $\begin{array}{r}-1.2500 E+00 \\
2.5000 E-01 \\
-2.50008-01 \\
-1.6667 E-01 \\
-3.0000 E-01\end{array}$ & $3.33335 \cdot 01$ & $\cdot x$ & & \\
\hline $0(445)=0(115)=$ & $\begin{array}{l}+0(336) * \\
+10(435): \\
+0(536): \\
+0(556) *\end{array}$ & $\begin{array}{r}-1.6667 E+00 \\
2.0000 E+00 \\
-5.5556 E-02 \\
-5.0000 E-01\end{array}$ & $1.04445-01$ & $\cdot x$ & & \\
\hline $0(115) \cdot 0(215) 0$ & $\begin{array}{l}0(20 C): \\
+0(22 C): \\
+10(325): \\
+0(40 C): \\
0(426): \\
+10(525): \\
+0(60 C): \\
+0(62 C):\end{array}$ & 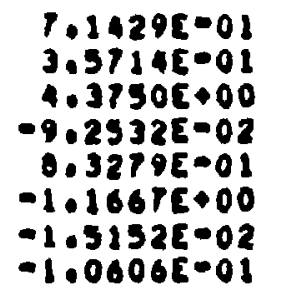 & $\begin{array}{r}-5.17625=01 \\
-2.73815=01 \\
-1.16675+00 \\
6.4935 E=03 \\
-5.04425=02\end{array}$ & $\begin{array}{l}: x \\
: x \\
: x \\
: x \\
x \\
x\end{array}$ & $\begin{array}{l}9.52302=02 \\
1.7619 E-02\end{array}$ & $\begin{array}{l}: \times 2 \\
1 \times 2\end{array}$ \\
\hline $0(415)+0(225)=$ & 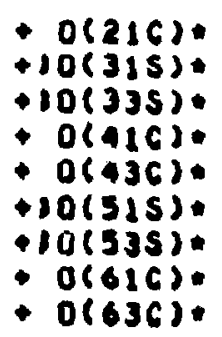 & $\begin{array}{l}-1.1206 E+00 \\
-2.3000 E+00 \\
-1.2500 E+00 \\
-3.7013 E+00 \\
-7.1727 E+00 \\
-8.3333 E-01 \\
-1.1867 E+00 \\
-1.06068=01 \\
-3.1810 E=01\end{array}$ & $\begin{array}{l}1.09525+00 \\
6.6667 E-01 \\
3.33335=01 \\
2.59745=01 \\
5.4545 E-01\end{array}$ & $\begin{array}{l}: x \\
: x \\
: x \\
: x \\
: x\end{array}$ & $-1.9048 E=01$ & $* \times 2$ \\
\hline
\end{tabular}




\begin{tabular}{|c|c|c|c|c|c|c|}
\hline $0(425) \cdot 0(215)=$ & $\begin{array}{l}+0(21 C): \\
+10(315): \\
+10(335): \\
+0(416): \\
+0(436): \\
+10(515): \\
+0(616): \\
+0(63 C):\end{array}$ & $\begin{array}{r}2.8571 E+00 \\
-6.2500 E-01 \\
1.0750 E+00 \\
-1.6656 E+00 \\
6.4773 E+00 \\
6.6667 E-01 \\
-1.0606 E-01 \\
-3.1018 E-01\end{array}$ & $\begin{array}{r}-2.1905 E+00 \\
1.6667 E=01 \\
-5.0000 E=01 \\
1.1688 E-01 \\
-4.5455 E-01\end{array}$ & $\begin{array}{l}: x \\
: x \\
0 x \\
x \\
x \\
x\end{array}$ & $3.0095 E=01$ & $-\times 2$ \\
\hline $0(425)=0(225)=$ & $\begin{array}{l}+O(20 C): \\
+O(40 C): \\
+\quad 0(44 C) \\
+0(545) \\
+\quad 0(60 C) \\
+O(64 C)\end{array}$ & $\begin{array}{r}1.4206 E \bullet 00 \\
1.1104 E+00 \\
-2.5909 E \times 00 \\
-7.0000 E+00 \\
1.5152 E-02 \\
-3.1818 E-01\end{array}$ & $\begin{array}{r}-1.0952 E+00 \\
-7.7922 E-02 \\
1.0182 E-01\end{array}$ & $\begin{array}{c}x \\
x \\
x \\
x\end{array}$ & $1.9048 E .01$ & $\times 2$ \\
\hline$O(435)=0(215)=$ & $\begin{array}{l}+0(22 C): \\
+10(325): \\
+0(42 C) \\
+0(94 C) \\
+10(525) \\
+10(545): \\
+0(62 C) \\
+0(64 C)\end{array}$ & $\begin{array}{r}3.5714 E=01 \\
6.2500 E-01 \\
-4.6266 E=01 \\
6.9773 E=01 \\
0.3333 E-01 \\
5.0000 E-01 \\
-1.5152 E-02 \\
-4.5455 E-02\end{array}$ & $\begin{array}{r}-2.7381 E-01 \\
-1.6667 E-01 \\
3.2468 E-02 \\
-4.5455 E-02\end{array}$ & $\begin{array}{l}* x \\
* x \\
: x \\
* x\end{array}$ & $4.7619 \varepsilon \cdot 02$ & $\times 2$ \\
\hline $0(435)=0(225)=$ & $\begin{array}{l}+0(216): \\
+10(315): \\
+0(416): \\
+10(515): \\
+0(555) \\
+0(616): \\
+0(656):\end{array}$ & $\begin{array}{r}1.4286 E+00 \\
-1.2500 E+00 \\
1.1104 E+00 \\
-1.6667 E=01 \\
-5.0000 E=01 \\
1.5152 E-02 \\
-5.0000 E-01\end{array}$ & $\begin{array}{r}-1.0952 E+00 \\
3.3333 E-01 \\
-7.7922 E=02\end{array}$ & $\begin{array}{l}: x \\
: x \\
x\end{array}$ & $1.9048 E-01$ & $\cdot \times 2$ \\
\hline $0(445) \cdot 0(215)=$ & $\begin{array}{l}+10(335): \\
+0(436): \\
+10(535): \\
+10(555) \\
+0(636) \\
+0(65 C):\end{array}$ & $\begin{array}{r}2.5000 E+00 \\
-5.1010 E+00 \\
3.3333 E-01 \\
1.0000 E+00 \\
-4.5455 E=02 \\
-5.0000 E-01\end{array}$ & $\begin{array}{r}-6.6667 E=01 \\
3.6364 E-01\end{array}$ & $\ddot{x}$ & & \\
\hline $0(445)=0(225)=$ & $\begin{array}{l}+0(22 C): \\
+10(325): \\
+0(42 C) \\
+0(525) \\
+0(62 C) \\
+0(66 C) .\end{array}$ & $\begin{array}{r}2.8571 E+00 \\
-1.000 E+01 \\
1.4805 E+00 \\
-1.3333 E+00 \\
1.5152 E-02 \\
-5.0000 E-01\end{array}$ & $\begin{array}{r}-2.1905 E+00 \\
2.6667 E+00 \\
-1.0390 E-01\end{array}$ & $\begin{array}{l}: x \\
: x \\
: x\end{array}$ & $3.0095 E-01$ & $+\times 2$ \\
\hline
\end{tabular}




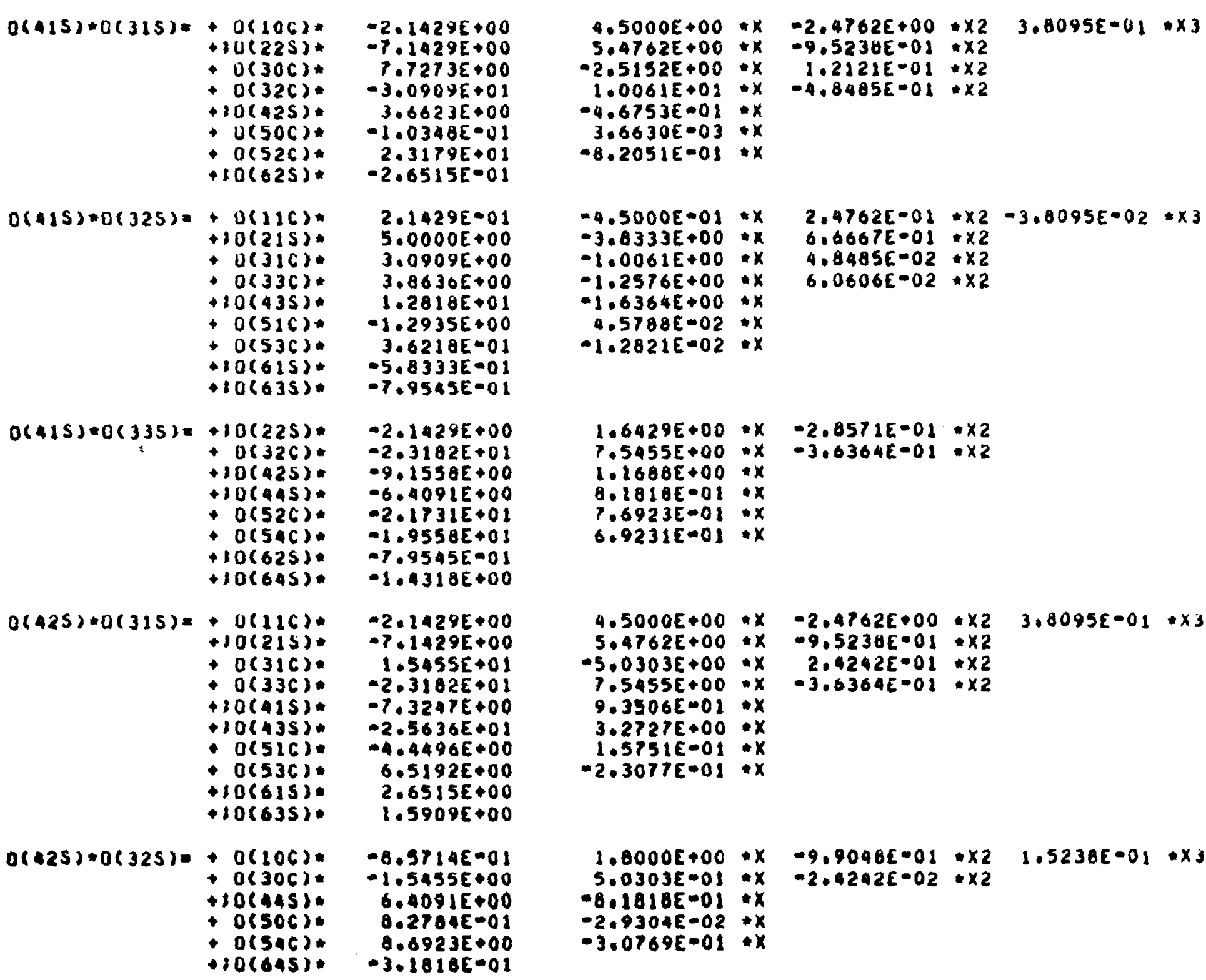

$0(425) * 0(325)=+0(106) *$

$+10(a 45)$

$6.5714 E-01$
$1.5455 E+00$

t o(soc).

$6.4091 E+00$

- o(s+c).

$0.6923 E+00$

$+10(645)$.

$4.5000 E+00 * x \quad-2.4762 E+00 * \times 2 \quad 3.0095 E=01 \times 3$ $5.4762 E+00$ * $x$ - $9.5236 E=0 J$ : $x 2$

$-2.5152 E+00 \times x \quad 1.2 j 21 E-01: x 2$

$1.00612+02 x$

$4.6753 E-01$ : $x$

$-8.2051 E-01$ : $x$

$-4.5000 E=01 * x \quad 2.4762 E-01 * \times 2-3.0095 E-02 * \times 3$

$-3.6333 E+00$ : $x \quad 6.6667 E-01: x 2$

$-1.0061 E+00 \div x \quad 4.8485 E-02 \div \times 2$

$-1.2576 E+00 \times x \quad 6.0606 E-02+\times 2$

$-1.6364 E+00$ : $x$

$4.5788 E-02$ : $x$

$-1.2821 E-02: x$

$1.6429 E+00 * x=2.8571 E-01 * \times 2$

$7.5455 E+00 * x-3.6364 E-01 * x 2$

$1.2608 E+00 \neq x$

6.1818E-01 $x$

$7.6923 E=01: x$
$6.9231 E=01: x$

$4.5000 E+00 * x \quad-2.4762 E+00 * x 2 \quad 3.8095 E-01 * x 3$

$5.4762 E+00+x-9.5230 E-01 * x 2$

$-5.0303 E+00 * x \quad 2.4242 E=01 * x 2$

$3.5455 E+00 * x-3.6364 E=02 \div x^{2}$

$9.3506 E=01 * x$

$1.57515=01: x$

$-2.3077 E-01 \cdot x$

$1.0000 E+00 * x=9.9048 E-01 * \times 2 \quad 1.523 B E-01 * x$

$5.0303 E-01: x-2.4242 E-02: x 2$

$-6.1818 E-01: x$

$-2,9304 E-02 * x$ 


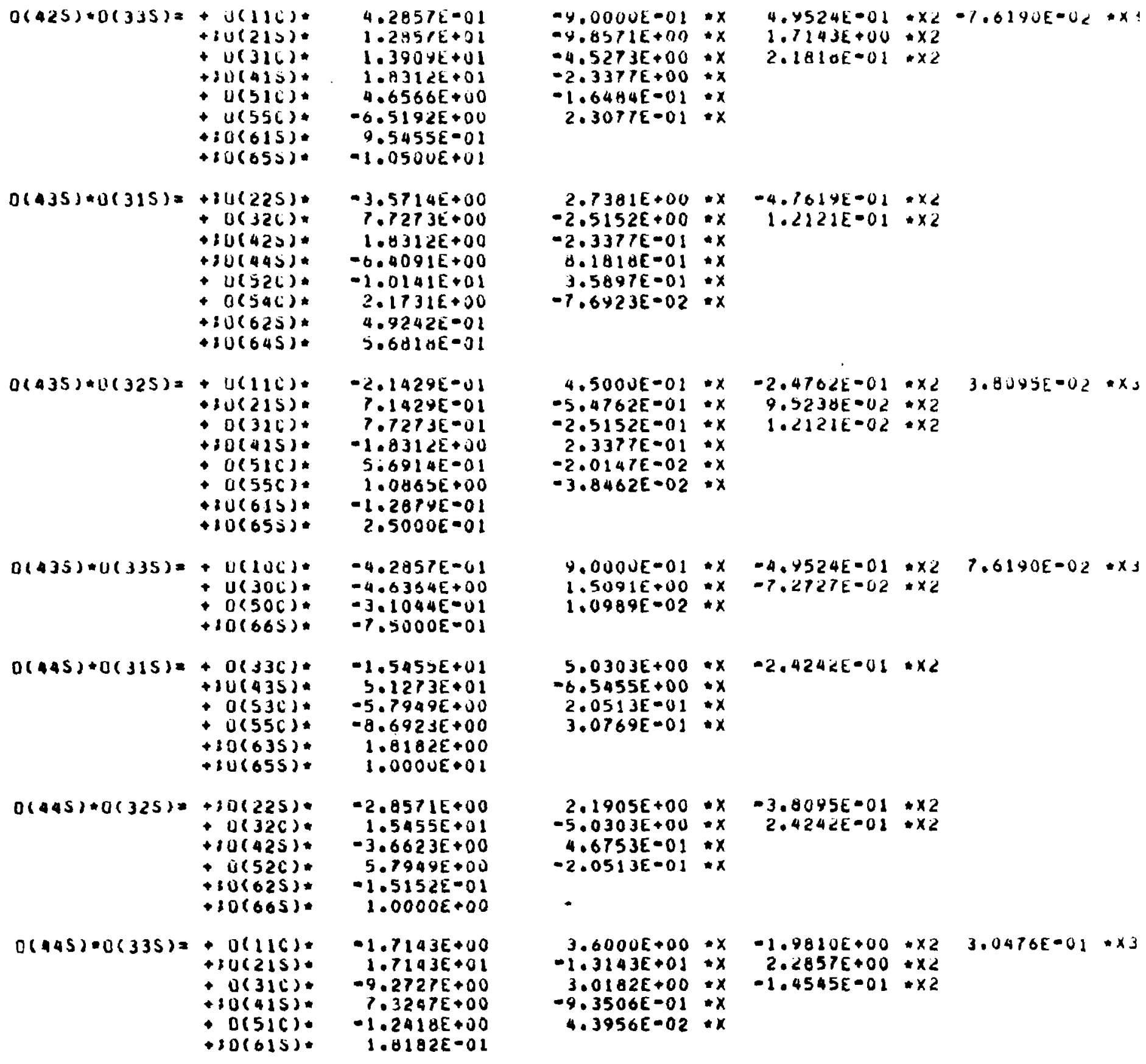

$1.7143 E+00$ $1.7193 E+01$ $-9.2727 E+00$ $7.3247 E+00$ $-1.2418 E+00$ $1.0182 E-01$

$-y .0000 E-01 * x \quad 4.4524 E=01 * x 2=7.6190 E=02 * x$ $-9.8571 E+00 * x \quad 1.71435+00 * x$ $-4.5273 E+00 * x \quad 2.1810 E=01 * x^{2}$

$-2.33 P T E+00 * x$ $-1.64 \mathrm{HAE}-01: x$ 2.30 TrE-O1 $+X$

$2.7381 E+00 * x-4.1619 E-01 * x 2$ $-2.5152 E+00 * x \quad 1.2121 E=01 * x 2$ $3.58978-01: x$ $\begin{array}{rl}-7.6923 \varepsilon-02: x & x\end{array}$

$4.5000 E-01 * x-2.4702 E-01 \times x^{2} 3.8095 E-02 * x_{3}$ $-5.4762 E-01 * x \quad 9.523 y E=02 * x 2$ $-2.5152 E-01 * x \quad 1.2121 E=02 * x 2$

$2.3377 E-01 * x$

$-3.8462 E-02=x$

$9.0000 E-01 * x \quad-4.4524 E-01 * \times 2 \quad 7.6190 E-02 * x_{3}$ $-7.2727 E-02 \times 2$

$1.0989 E-02 * x$

$5.0303 E+00 * x \quad-2.424 K E=01 * \times 2$

$-6.5455 E+00 * x$

$2.0513 E=01 * x$
$3.0769 E-01 * x$

$2.1905 E+00 * x \quad-3.8095 E=01 * \times 2$ $-5.0303 E+00=x$ $-2.0513 E-01 \times x$

$3.6000 E+00 * x-1.9880 E+00 * \times 2 \quad 3.0476 E-01 * \times 3$ $-1.3143 E+01 * x \quad 2.2857 E+00 \div \times 2$ $3.0182 E+00 * x-1.4545 E-01 * x$

$-9.3506 E-01: x$ $4.3956 E-02 * x$ 


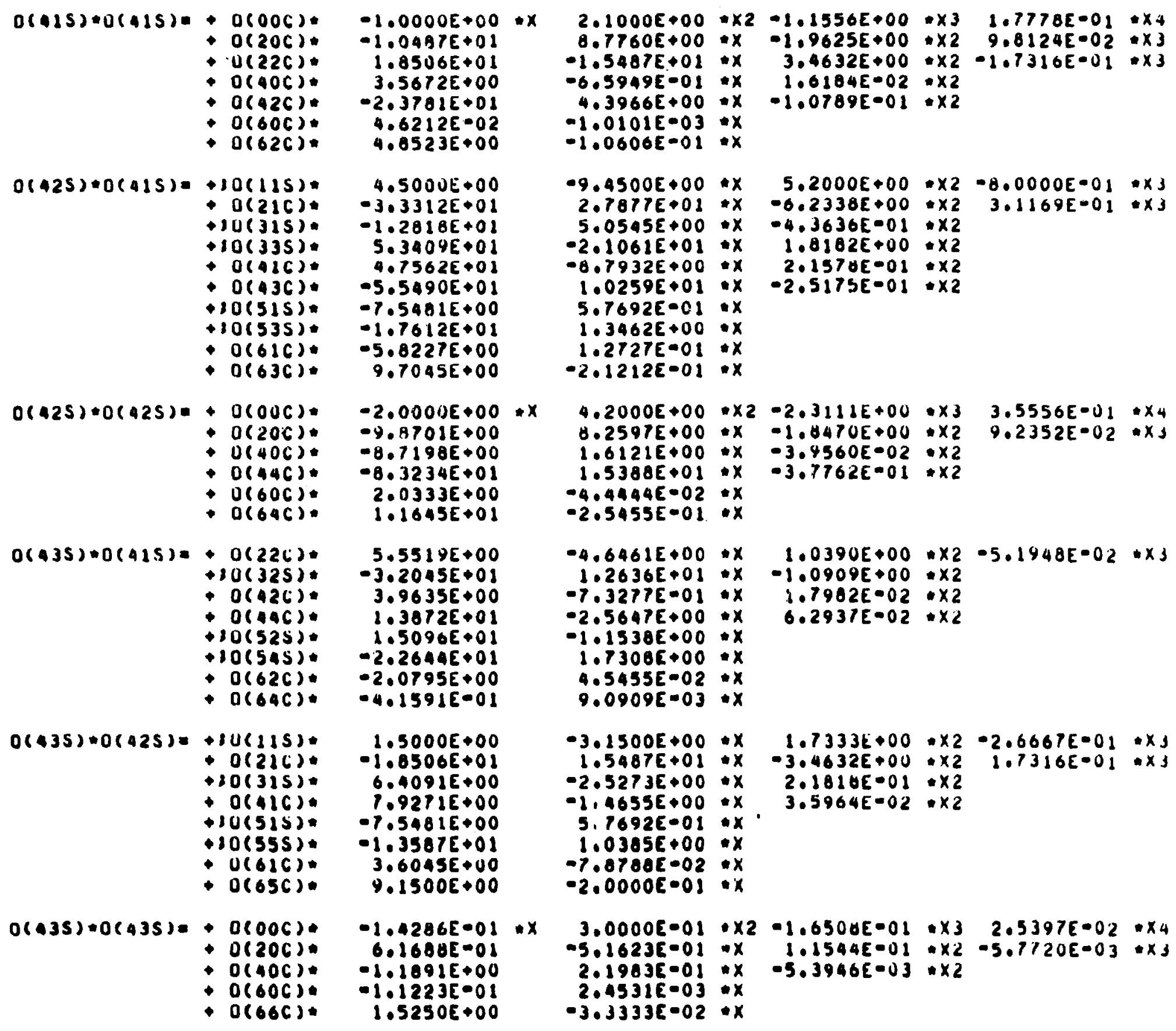

$4.5000 E+00$ $-3.3312 E+0$ $-1.2818 E+01$ $5.3409 E+01$ 4.7562501 $-7.54906+01$ - r.5anie+00 $-1.7612 E+01$ $-5.8227 E+00$

$0(425)=0(425)++0(00 C)=$ - $0(200)$ $-2.000016+00$. $-9.8701 E+00$ $-8.71986+00$ $-0.32345+01$

$O(60 C)$

$2.0333 E+00$

$O(435) \neq D(415)=-O(226)=$ $10(325)$ - $0(42())$ 0(4ac). 10(5as). 0(5as). $0(62 C)$

$5.5519 E+00$ $3.9635 E+00$ $1.96356+00$ $1.3072 E+01$ 1. $-2.2644 E+01$ $-2.0795 E+00$

$O(435) * O(425)=+1 U(115)=$ $0(216)$ $+0(416):$ 10(5) (u(s) $+0(616)$.
$+0(65 C)$.

$1.5000 E+00$ $1.5000 E+00$
$-1.8506 E+01$ $6.4091 E+00$ $\because .9271 E+00$ $-7.5481 E+00$ $-7.51812+00$ $-1.65075+01$ $3.6045 E+00$

$O(435)=0(435)=+0(006)+$ $O(20 C)$ - $O(\triangle O C)$.

$+0(60 C)$

$2.1000 E+00 * x 2-1.1556 E+00 * x 3 \quad 1.7778 E-01 * x 4$ $0.7760 E+00 * x-1.9625 E 000 \times x 2$ $9.8124 E-02 \times x 3$

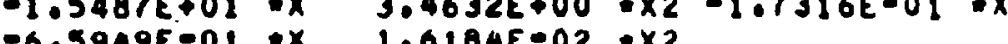
$-6.5949 E-01: x \quad 1.6184 E-02 * x 2$ $-1.01015-03 \cdot x$ $-1.01012003: x$ $0.9500 E+00: x$ $2.7877 E+01 * x$
$5.0545 E+00 * x$ $-2.1001 E 001+x$ $-8.7932 E+00 * x$ $1.0259 E+01: x$ $5.7692 E-01: x$ $5.76925-01: x$ $1.2727 E-01$ : $x$ $-2,1212 \varepsilon-01: x$

$5.2000 E+00 * \times 2-8.0000 E-01 \cdot \times 3$ $-6.2338 E+00 \times \times 2 \quad 3.1169 E-01 * \times 3$ $1.36365-01 * x 2$

$1.01028+00 \times 2$ $-2.15706-01: \times 2$

$4.2000 E+00 * x_{2}-2.3111 E+00 * x_{3} \quad 3.5556 E-01 * x_{4}$ $8.2597 E+00 * x \quad=1.8470 E+00 * x_{2} \quad 9.2352 E-02 * x s$ $1.6121 E+00 * x-3.4560 E-02 * x 2$

1.53802001 : $x$

$\begin{aligned} & -4.4444 E=02 \\ & -2.5455 E-01\end{aligned}-x$

$-4.6461 E+00 * x \quad 1.0390 E+00 * \times 2-5.1948 E-02 * \times 3$ $1.2636 E+01 * x-1.0909 E+00 \times \times 2$ $1.7902 E-02 \div \times 2$ $-2.56472+00 \cdot x$

$-1.1538 E+00$ $1.73006+00: x$ 4.5455E-02 *x $x$

$-3.1500 E+00 * x \quad 1.7333 E+00 * \times 2-2.6667 E-01 * x J$ $-1.5487 E+01 * x-3.4632 E+00 * x 2 \quad 1.7316 E=01 * x J$ $-1.5275 E+00 \times x \quad 2.1816 E-01$ : $x 2$ $-1.4655 E+00 \cdot x, 3.5964$ S. 16922001 a $x$ $-7.03056000: x$ $-2.0000 E-01: x$

$-1 \cdot 4286 E-01 \cap x$ $6.16805-01$ $-1.1091 E+00$ $-1.1223 E-0$ $1.5250 E+00$

$3.0000 E-01 \times 2-1.6500 E-01 \times 3 \quad 2.5397 E-02 \times \times 4$ $-5.1623 E-01$ : $x \quad 1.1544 E-01: x 2-5.7720 E-03 \times x 3$ $2.1903 E-01 * x \quad-5.3946[-03 * \times 2$

$2.45316-03 \cdot x$

$-3.3333 E-02 \cdot x$ 


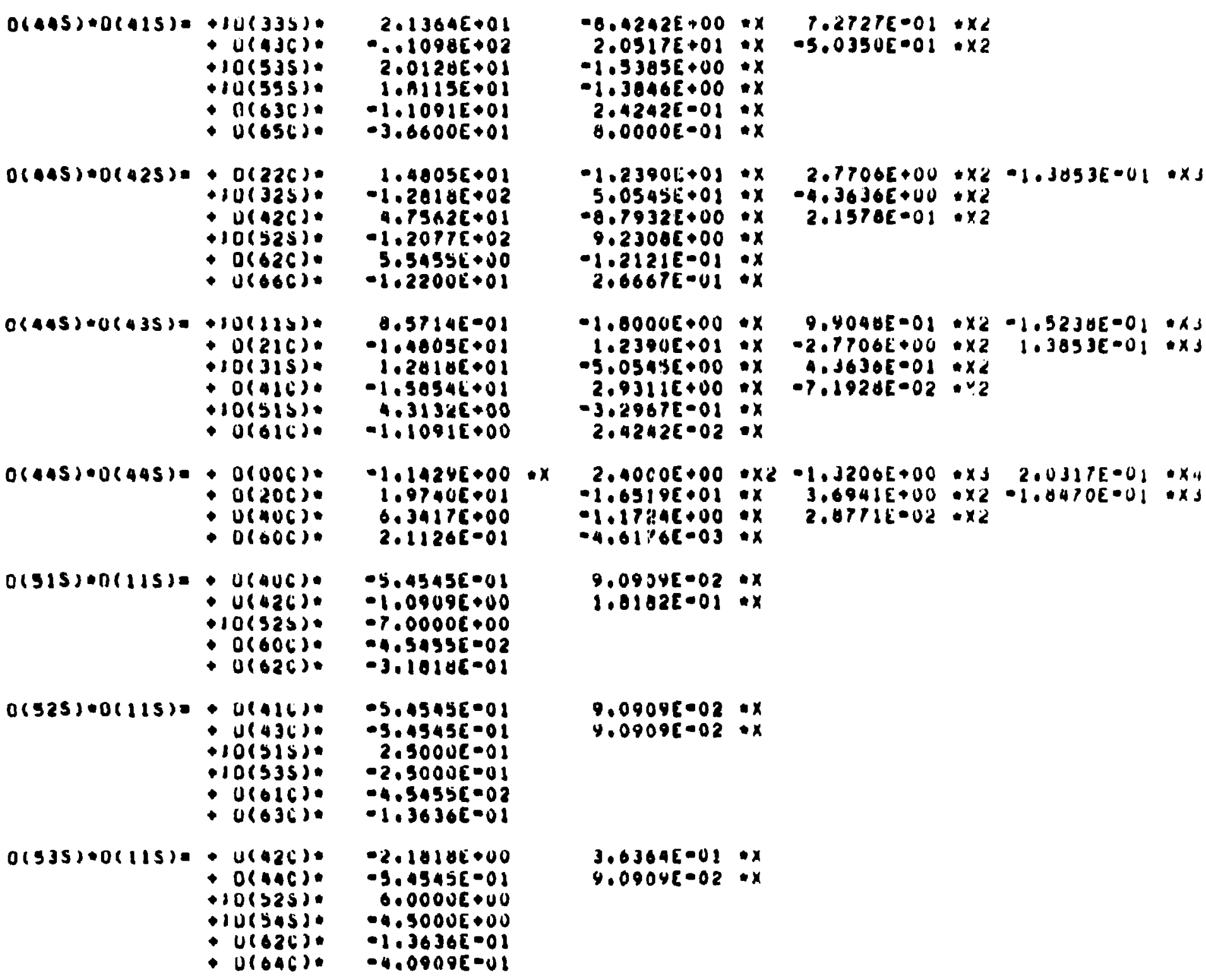

$0(405)+0(415)=+10(335)=$

- U(A3c).

$10(535)$.

- O(63c).

- vesco.

$0(045) \cdot 0(425) \cdot 0(226) \cdot$

- ivrass.

U

D(62C).

- vercio

$0(445) \cdot 0(435)=+10(213) *$

$+0(210)$.

- ido(3is).

- O(4)

orgicio

$0(445) 00(445) \cdot 0(006)$.

- $0(200)$

- U(nOC):

$0(515)+0(115)=-$ o(AUC).

- U(4ac).

$10(525)$.

- $0(606)$

$0(525) 00(115)=0(416) 0$

$-10(436):$
$-10(5) s 50$.

- $10(33 s)$.

- $0(016)=$

- $0(036)$.

$0(535) \cdot 0(115)=0(42 c)$

io(32s):

ivesasja

- v(6z)

- $0(046)$.

$2.13645+0$

$.01098 E+02$

$2.0120 E+01$

loniseror

$-3.6600 E+01$

$1.4805 E+01$

$1.2018 E+02$

$4.75625+01$

(1.20

5.

0.57145001

1.4805

$1.28106+01$

1.30546008

$4.31326+00$

$-1.10915+00$

$1.1429 E+00$

$1.97406 \times 01$

c.jaliceos

$2.11266-01$

$-5.4545 E \cdot 0$ I

$-1.09096+00$

$-7.0000 C+00$

$-3.10106-01$

$-5.0545 E-01$

$-5.45456-01$

0.50001

$-4.54556=02$
$-1.36365-01$

$-2.18186+00$

$-9.4545[-01$

$0.00006+00$

-9.5000 C 00

$-1.3636 E-01$

$-4.0000 E-01$

$-6.4242 E+00: x \quad 7.2727 E-01 * x 2$
$2.0517 E+01: x \quad-5.0350 E-01 * \times 2$

$-1.3385 E+00$

$1.3046 E+00$ :x

$2.42426-01: x$
$0.0000 E-01: x$

$-1.2380[+01 \times x \quad 2.7700 E+00+x 2-1.3053 E=01$ * $x J$

$5.0545 E+01 x \quad x \quad x \quad x$

.

90

$2.2121 E=01: x$

$-1.0000 E+00 * x \quad 9.4048 E=01 \times 2-1.5230 E=01 * x J$

$1.2390 E+01 * x-2.7706 E+00 \times 2 \quad 1.3853 E=01 * x J$

$-5.0545 E+00 * x \quad 4.36305001 \times x 2$

$2.93116+00 * x-7.1920 E-02 \div 2$

$3.2967 E-01: x$
$2.42426-02: x$

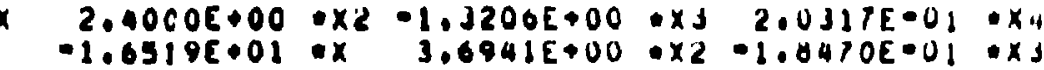

$-1.17946+00$ : $\quad 2.07116=02 \times x^{2}$

$9.09345002 \cdot x$

$1.01025001 \cdot x$

$9.09095-02 \cdot x$

$4.09095-02 \cdot x$

$3.63645-01: x$
$9.09045002: x$ 


\begin{tabular}{|c|c|c|c|c|c|c|}
\hline$O(545) \cdot 0(115)=$ & $\begin{array}{l}+u(43 C) \\
+10(535) \\
+\ln (555) \\
+U(636) \\
+u(65 C)\end{array}$ & $\begin{array}{r}-2.1810 E+00 \\
2.5000 E=01 \\
-2.5000 E=01 \\
-4.5455 E-02 \\
-5.0000 E-01\end{array}$ & $3.6364 E-01$ & *x $x$ & & \\
\hline $0(555)+0(115)=$ & $\begin{array}{l}+U(440) \\
+\quad U(545) \\
+\quad 0(64 C) \\
+\quad U(66 C)\end{array}$ & $\begin{array}{r}-2.7273 E+00 \\
2.5000 E+00 \\
-4.5455 E=02 \\
-5.0000 E-01\end{array}$ & $4.5455 E-01$ & 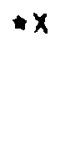 & & \\
\hline $0(515) * 0(215)=$ & $\begin{array}{l}+0(30 c) \\
+0(32 C) \\
+10(425) \\
+0(50 C) \\
+0(52 C) \\
+80(625)\end{array}$ & $\begin{array}{r}3.6364 E+00 \\
5.4545 E+00 \\
4.3636 E+00 \\
-1.85908-01 \\
7.8077 E+00 \\
-4.7727 E=01\end{array}$ & $\begin{array}{r}-1.5758 E+00 \\
-2.3636 E+00 \\
-7.2727 E=01 \\
8.5470 E=03 \\
-3.5897 E-01\end{array}$ & $\begin{array}{l}: x \\
: x \\
: x \\
: x \\
x\end{array}$ & $\begin{array}{l}1.0162 E-0 \mathrm{i} \\
2.4242 E-01\end{array}$ & $\begin{array}{l}\times 2 \\
\times 2 \\
\times 2\end{array}$ \\
\hline$: 515)+0(225)=$ & $\begin{array}{l}+0(316): \\
+0(336)= \\
+0(415): \\
+0(435) \\
+0(516) \\
+0(536) \\
+0(615) \\
+80(635)\end{array}$ & $\begin{array}{l}-2.72: 3 E+00 \\
-9.0904 E-01 \\
-1.0909 E+01 \\
-1.5273 E+01 \\
-5.5769 E+00 \\
-5.2051 E+00 \\
-1.9091 E+00 \\
-3.8182 E+00\end{array}$ & $\begin{array}{l}1.1818 E+00 \\
3.9394 E-01 \\
1.8182 E+00 \\
2.5455 E+00 \\
2.5641 E-01 \\
2.3932 E-01\end{array}$ & $\begin{array}{l}: x \\
: x \\
: x \\
: x \\
: x \\
: x\end{array}$ & $\begin{array}{l}-1.2121 E=01 \\
-4.0404 E=02\end{array}$ & $\begin{array}{l}: \times 2 \\
\times 2\end{array}$ \\
\hline $0(52 S) \bullet 0(22 S)=$ & $\begin{array}{l}+0(316) \\
+0(336) \\
+0(415) \\
+10(435) \\
+0(516) \\
+0(536) \\
+0(615) \\
+10(635 .\end{array}$ & $\begin{array}{r}6.8182 E-01 \\
2.2727 E-01 \\
-5.4545 E-01 \\
2.7273 E-00 \\
-2.7895 E-01 \\
4.6474 E-01 \\
2.0455 E-01 \\
-6.8182 E-02\end{array}$ & $\begin{array}{r}-2.9545 E-01 \\
-9.8485 E-02 \\
9.0909 E-02 \\
-4.5455 E-01 \\
1.2821 E-02 \\
-2.1368 E-02\end{array}$ & $\begin{array}{l}: x \\
: x \\
: x \\
: x \\
: x \\
: x\end{array}$ & $\begin{array}{l}3.0303 E-02 \\
1.0101 E-02\end{array}$ & $\begin{array}{l}\times 2 \\
\times 2\end{array}$ \\
\hline $0(525) \cdot 0(225)=$ & $\begin{array}{l}+0(30 C) \\
+10(445) \\
+0(500) \\
+0(54 C) \\
+10(645)\end{array}$ & $\begin{array}{r}9.0909 E-01 \\
-5.4545 E-01 \\
3.7179 E-01 \\
-3.3462 E+00 \\
-4.0909 E-01\end{array}$ & $\begin{array}{r}-3.9394 E=01 \\
9.0909 E-02 \\
-1.7094 E-02 \\
1.5385 E-01\end{array}$ & $\begin{array}{l}x \\
: x \\
: x \\
: x\end{array}$ & $4.0404 E=02$ & $+\times 2$ \\
\hline
\end{tabular}




\begin{tabular}{|c|c|c|c|c|c|c|c|}
\hline $0(535)+0(215)=$ & $\begin{array}{l}+0(32 C) * \\
+\quad 0(445): \\
+0(526) \\
+0(54 C) \\
+30(625): \\
+30(645) *\end{array}$ & $\begin{array}{r}1.0909 E+01 \\
3.2727 E+00 \\
-1.1154 E+01 \\
1.1712 E+01 \\
7.5000 E-61 \\
2.0455 E-01\end{array}$ & $\begin{array}{r}-4.7273 E+00 \\
-5.4545 E=01 \\
5.1282 E=01 \\
-5.3846 E=01\end{array}$ & $\begin{array}{l}: x \\
: x \\
: x \\
: x\end{array}$ & $4.8485 E-01$ & $1 \times 2$ & \\
\hline $0(535)+0(225)=$ & $\begin{array}{l}+0(316) \\
+10(415) \\
+0(526) \\
+0(556) \\
+10(615) \\
+10(655)\end{array}$ & $\begin{array}{r}5.4545 E+00 \\
-1.3091 E+01 \\
4.4615 E+00 \\
-3.3462 E+00 \\
-1.0909 E+00 \\
-9.0000 E+00\end{array}$ & $\begin{array}{r}-2.3636 E+00 \\
2.1818 E+00 \\
-2.0513 E-01 \\
1.5385 E-01\end{array}$ & $\begin{array}{l}: x \\
: x \\
: x \\
x\end{array}$ & $2.4242 E-01$ & $\times 2$ & \\
\hline $0(545)=0(215)=$ & $\begin{array}{l}+0(33 C): \\
+10(435): \\
+0(536): \\
+0(55 C) \\
+10(635): \\
+10(655) *\end{array}$ & $\begin{array}{r}9.0909 E-01 \\
2.1816 E+00 \\
-6.5064 E-01 \\
9.3654 E-01 \\
2.9545 E-01 \\
7.5000 E-01\end{array}$ & $\begin{array}{r}-3.9394 E-01 \\
-3.6364 E=01 \\
2.9915 E-02 \\
-3.8462 E-02\end{array}$ & $\begin{array}{l}x \\
i x \\
x \\
x \\
x\end{array}$ & $4.0404 E-02$ & $\times 2$ & \\
\hline $0(545)+0(225)=$ & $\begin{array}{l}+0(32 C): \\
+10(425): \\
+0(526): \\
+80(625) \\
+10(665)\end{array}$ & $\begin{array}{r}5.4545 E+00 \\
-2.1818 E+00 \\
4.4615 E+00 \\
-1.3636 E-01 \\
-5.0000 E-01\end{array}$ & $\begin{array}{r}-2.3636 E+00 \\
3.6364 E=01 \\
-2.0513 E=01\end{array}$ & $\begin{array}{l}x \\
x \\
x\end{array}$ & $2.4242 E-01$ & $1 \times 2$ & \\
\hline $0(555)+0(215)=$ & $\begin{array}{l}+3 U(44 S): \\
+\quad 0(54 C): \\
+30(645): \\
+10(665):\end{array}$ & $\begin{array}{r}5.4545 E+00 \\
-8.3654 E+00 \\
3.4091 E-01 \\
1.2500 E+00\end{array}$ & $\begin{array}{r}-9.0909 E=01 \\
3.8462 E=01\end{array}$ & $x$ & & & \\
\hline $0(55 S)+0(225)=$ & $\begin{array}{l}+0(33 c): \\
+20(435): \\
+\quad 0(536) \\
+10(635)\end{array}$ & $\begin{array}{r}9.0909 E+00 \\
-2.1816 E+01 \\
1.8590 E+00 \\
-4.5455 E-01\end{array}$ & $\begin{array}{r}-3.9394 E+00 \\
3.6364 E+00 \\
-8.5470 E-02\end{array}$ & $\begin{array}{c}x \\
x \\
x \\
x\end{array}$ & $4.0404 E-01$ & $+\times 2$ & \\
\hline $0(515)=0(315)=$ & $\begin{aligned} & 0(20 C): \\
+ & 0(22 C): \\
+ & 0(325) \\
+ & 0(40 C) \\
+ & 0(42 C) \\
+ & 0(525) \\
+ & 0(62 C)\end{aligned}$ & $\begin{array}{r}-1.5584 E+01 \\
-7.7922 E+00 \\
-1.2273 E+02 \\
7.4505 E+00 \\
-2.9125 E+01 \\
7.7538 E+01 \\
8.9091 E+00\end{array}$ & $\begin{array}{r}1.4545 E+01 \\
7.2727 E+00 \\
5.3182 E+01 \\
-1.5055 E+00 \\
5.8051 E+00 \\
-6.4615 E+00 \\
-2.1212 E-01\end{array}$ & $\begin{array}{l}: x \\
: x \\
: x \\
: x \\
: x \\
: x \\
-x\end{array}$ & $\begin{array}{r}-4.0693 E+00 \\
-2.0346 E+00 \\
-5.4545 E \bullet 00 \\
4.3956 E=02 \\
-1.7183 E-01\end{array}$ & $\begin{array}{l}\times 2 \\
\times 2 \\
\times 2 \\
\times 2 \\
\times 2 \\
\times 2\end{array}$ & $\begin{array}{l}3.4632 E=01: \times 3 \\
1.7316 E=01: \times 3\end{array}$ \\
\hline
\end{tabular}




\begin{tabular}{|c|c|c|c|c|c|c|c|c|}
\hline $0(515) * 0(325)=$ & $\begin{aligned} & O(216) \\
+ & 10(315) \\
+ & 10(335) \\
+ & 0(416) \\
+ & 0(43 C) \\
+ & 10(535) \\
+ & 0(61 C)\end{aligned}$ & $\begin{array}{r}6.2338 E+00 \\
1.2273 E+01 \\
6.8182 E+00 \\
1.6933 E+01 \\
5.2154 E+01 \\
9.6154 E+00 \\
-3.5636 E+00\end{array}$ & $\begin{array}{r}-5.8182 E+00 \\
-5.3182 E+00 \\
-2.9545 E+00 \\
-3.4210 E+00 \\
-1.0538 E+01 \\
-7.1795 E-01 \\
0.4848 E-02\end{array}$ & $\begin{array}{l}: x \\
: x \\
: x \\
: x \\
: x \\
: x \\
: x\end{array}$ & $\begin{array}{l}1.6277 E+00 \\
5.4545 E-01 \\
3.0303 E-01 \\
9.9900 E-02 \\
3.0769 E-01\end{array}$ & $\begin{array}{l}\times 2 \\
\times 2 \\
\times 22 \\
\times 2 \\
\times 22 \\
\times 2\end{array}$ & $-1.3853 E-01$ & $\times 3$ \\
\hline $0(515) * 0(335)=$ & $\begin{array}{l}+0(22 C) \\
+10(32 S) \\
+0(42 C) \\
+0(44 C) \\
+10(52 S) \\
+10(54 S) \\
+0(62 C) \\
+0(64 C)\end{array}$ & $\begin{array}{l}-1.5584 E+00 \\
-4.0909 E+01 \\
-3.0440 E+01 \\
-1.4224 E+01 \\
-1.2923 E+02 \\
-7.7538 E+01 \\
-8.9091 E+00 \\
-1.0641 E+01\end{array}$ & $\begin{array}{l}1.4545 E+00 \\
1.7727 E+01 \\
6.1588 E+00 \\
2.8741 E+00 \\
1.0769 E+01 \\
6.4615 E+00 \\
2.1212 E-01 \\
2.5455 E-01\end{array}$ & $\begin{array}{l}: x \\
: x \\
: x \\
: x \\
: x \\
: x \\
: x \\
: x\end{array}$ & $\begin{array}{l}-4.0693 E=01 \\
-1.8182 E+00 \\
-1.7982 E=01 \\
-6.3916 E-02\end{array}$ & $\begin{array}{l}* \times 2 \\
\times 2 \\
\times \times 2 \\
\times 2\end{array}$ & $3.4632 E-02$ & $\bullet \times 3$ \\
\hline $0(525)+0(315)=$ & $\begin{array}{l}+0(21 C) \\
+10(335) \\
+O(41 C) \\
+0(43 C) \\
+10(515) \\
+10(535) \\
+0(61 C) \\
+0(63 C)\end{array}$ & $\begin{array}{r}-7.7922 E+00 \\
-6.8182 E+00 \\
1.0837 E+01 \\
-3.3189 E+01 \\
-2.7692 E+00 \\
-3.0769 E-01 \\
-1.2727 E+00 \\
3.8102 E+00\end{array}$ & $\begin{array}{r}7.2727 E+00 \\
2.9545 E+00 \\
-2.1898 E+00 \\
6.7063 E+00 \\
2.3077 E-01 \\
2.5641 E-02 \\
3.0303 E-02 \\
-9.0909 E=02\end{array}$ & $\begin{array}{l}: x \\
: x \\
: x \\
: x \\
: x \\
: x \\
: x \\
: x\end{array}$ & $\begin{array}{r}-2.0346 E+00 \\
-3.0303 E=01 \\
6.3936 E=02 \\
-1.9580 E-01\end{array}$ & $\begin{array}{l}\times 2 \\
\times 2 \\
\times 2 \\
\times 2 \\
\times 2\end{array}$ & $1.7316 E=01$ & $\times 3$ \\
\hline $0(525) \cdot 0(325)=$ & $\begin{array}{l}+C(20 C) \\
+\quad O(40 C) \\
+O(44 C) \\
+10(545) \\
+O(60 C) \\
+0(64 C)\end{array}$ & $\begin{array}{r}-7.7922 E-01 \\
-3.3866 E-01 \\
2.3706 E+00 \\
8.3077 E+00 \\
1.2727 E-01 \\
3.8182 E-01\end{array}$ & $\begin{array}{r}7.2727 E-01 \\
6.8432 E=02 \\
-4.7902 E-01 \\
-6.9231 E-01 \\
-3.0303 E=03 \\
-9.0909 E-03\end{array}$ & $\begin{array}{l}: x \\
: x \\
: x \\
: x \\
: x \\
: x\end{array}$ & $\begin{array}{r}-2.0346 E=01 \\
-1.9980 E=03 \\
1.3986 E=02\end{array}$ & $\begin{array}{l}\times 2 \\
\times 2 \\
\times 2 \\
\times 2\end{array}$ & $1.7316 E=02$ & $\times 3$ \\
\hline $0(525)=0(335)=$ & $\begin{array}{l}+O(216) \\
+10(315) \\
+0(41 C) \\
+30(515) \\
+0(555) \\
+0(616) \\
+O(656)\end{array}$ & $\begin{array}{r}1.5584 E+00 \\
4.0909 E+00 \\
1.0160 E+01 \\
4.6154 E+00 \\
-2.7692 E+00 \\
1.7810 E+00 \\
-8.4000 E+00\end{array}$ & $\begin{array}{r}-1.4545 E+00 \\
-1.7727 E+00 \\
-2.0529 E+00 \\
-3.0462 E-01 \\
2.3077 E=01 \\
-4.2424 E=02 \\
2.0000 E-01\end{array}$ & $\begin{array}{l}: x \\
: x \\
: x \\
: x \\
: x \\
: x \\
: x \\
x\end{array}$ & $\begin{array}{l}4.0693 E-01 \\
1.8182 E=01 \\
5.9940 E=02\end{array}$ & $\begin{array}{l}\times 2 \\
\times 2 \\
\times 2 \\
\times 2\end{array}$ & $-3.4632 E-02$ & $+\times s$ \\
\hline $0(535: 0(315)=$ & $\begin{array}{l}+O(22 C) \\
+O(325) \\
+O(42 C) \\
+O(44 C) \\
+10(525) \\
+10(545) \\
+0(62 C) \\
+0(64 C)\end{array}$ & $\begin{array}{r}-1.5584 E+01 \\
-8.1818 E+01 \\
3.6575 E+01 \\
-5.6895 E+01 \\
7.3846 E+00 \\
-8.6615 E+01 \\
-8.9091 E+00 \\
7.6364 E+00\end{array}$ & $\begin{array}{r}1.4545 E+01 \\
3.5455 E+01 \\
-7.3906 E+00 \\
1.1497 E+01 \\
-6.1530 E-01 \\
1.3846 E+00 \\
2.1212 E-01 \\
-1.8102 E-01\end{array}$ & $\begin{array}{l}: x \\
: x \\
: x \\
: x \\
: x \\
: x \\
: x \\
: x\end{array}$ & $\begin{array}{r}-4.0693 E+00 \\
-3.6364 E+00 \\
2.1570 E=01 \\
-3.3560 E=01\end{array}$ & $\begin{array}{l}\times 1 \times 2 \\
\times 2 \\
\times 2 \\
\times 2 \\
\times 2\end{array}$ & $3.4632 E=01$ & $\times 1$ \\
\hline
\end{tabular}




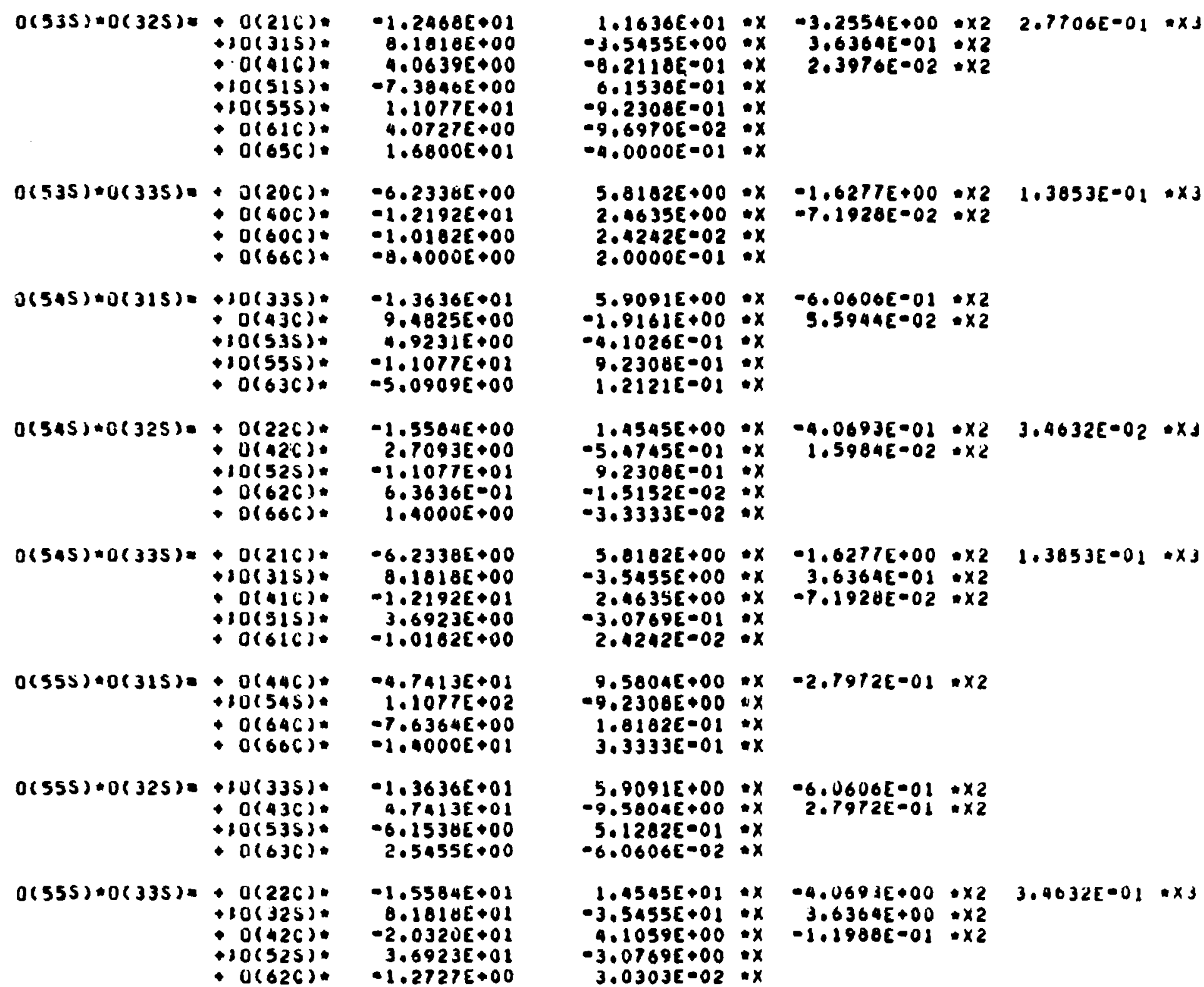

$-1.36365+01$ $.6 .1538 E+00$ $2.54556+00$

$-1.5584 E+0$ $0.1818 E+0$ $3.0923 E+01$ $-1.2727 E+00$ 


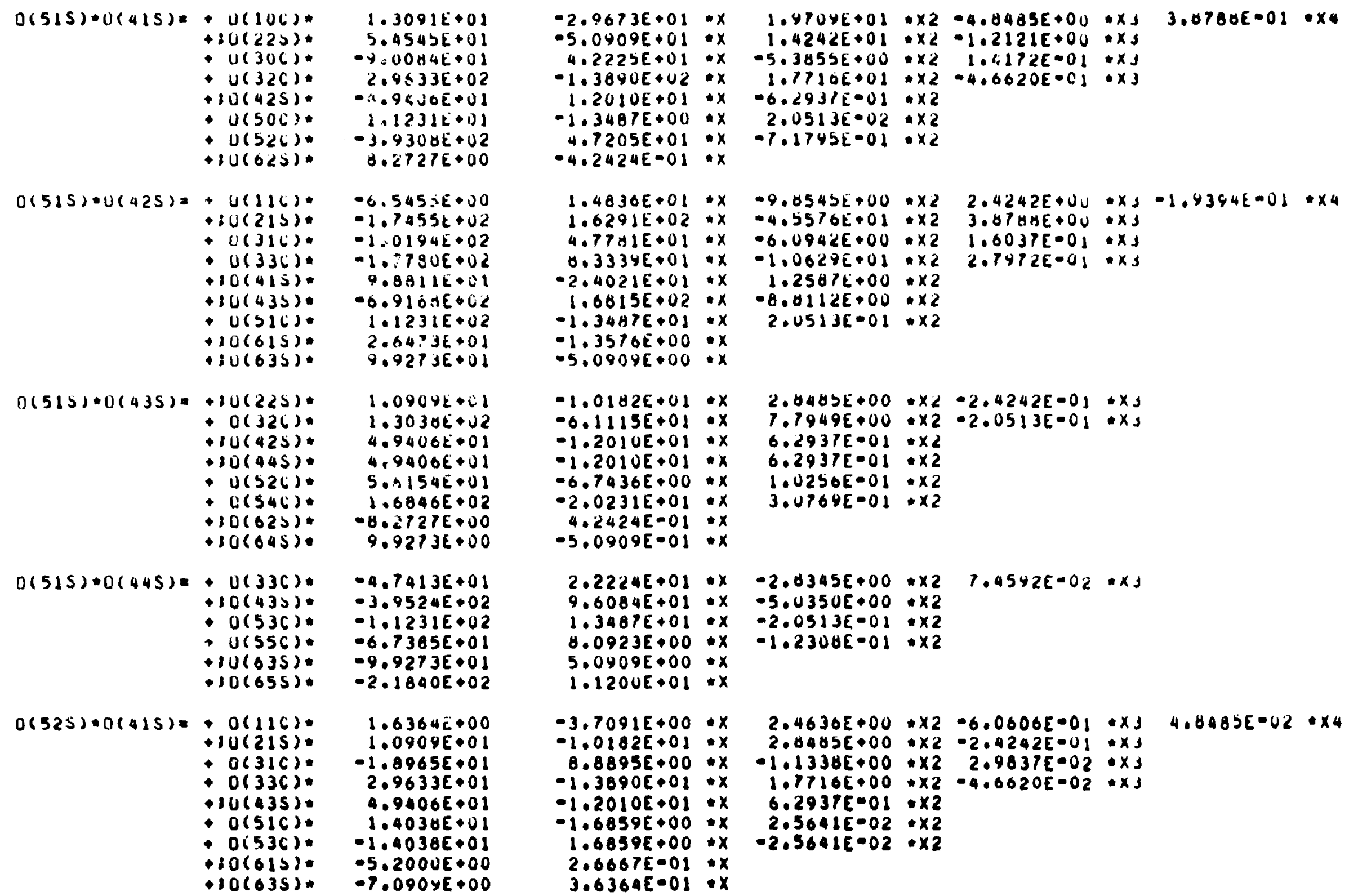

$1.0904 k+i 1$

$1.3030 t+12$

$4.94065+01$

$4,94006+01$

5.1545 .01

$1.6846 E+02$

$-8.2727 E+00$

$9.92736+00$

$O(515)+0(445)=+O(33 C)$ $+30(435)$ $+0(536)$ $+10(635)$.

$-4.7413 E+01$ $-3.9524 E+02$ $-1.1231 E+02$ $-6.7365 E+01$

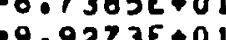
$-9.9273 E+01$ $+10(655)$.

$O(325) * 0(415)=+O(11()+$ $+10(215)$ O $0(316)$ : $+10(435)$

$1.6364 \vec{c}+00$ $1.0909 E+01$ $1.8965 E+01$ $.9633 E+01$ $9.9406 E+01$ $1.4038 E+01$ $+0(536):$

$\begin{array}{ll}+10(615) . & -5.2000 E+00 \\ +10(635) . & -7.090 Y E+00\end{array}$

$-2.9673 E+01 * x \quad 1.470 y E+01 * \times 2-4.0405 E+00 * x 3 \quad 3.0780 E-01 * x$ $1.4242 E+01: x 2-1.2121 E+00: x 3$

$4.2225 E+01 * x \quad-5.3855 E+00 * x 2 \quad 1.4172 E=01 * x J$

$-1.3890 E+42 * x \quad 1.7710 E+01 * \times 2-4.6620 E-01 * \times 3$

$1.2010 E+01 * x-6.293 / E=01 * x 2$

$-1.3487 E+00: x \quad 2.0513 E=02: \times 2$

$4.7205 E+01: x-7.1745 E=01 * x$

$-4.2424 E-01: x$

$1.4836 E+01 * x \quad-9.0543 E+00 * \times 2 \quad 2.4242 E+0 U * x J-1.4344 E-01 * \times 4$ $1.6291 E+02 * x \quad-4.5576 E+01 * \times 2 \quad 3.8745 E+0 u * x J$

$4.7741 E+01 * x \quad-6.0942 E+00 \times 2 \quad 1.6037 E=01 * x J$

$0.3334 E+01 * x-1.0629 E+01 * \times 2 \quad 2.7972 E=01 * x 3$

$-2.4021 E+01 * x \quad 1.2587 \varepsilon+00 \times 2$

$1.6815 E+02 \times x-8.0112 E+00 \times 2$

$-1.34 A 7 E+01 * x \quad 2.0513 E-01 \times x 2$

$-1.3576 E+00$ : $x$

$-5.0909 E+00: x$

$-1.0182 E+01 * x \quad 2.0485 E+00 * x_{2}-2.4242 E-01 * x J$

$-0.1115 E+01 * x \quad 7.7949 E+00 * x 2-2.0513 E=01 * x s$

$-1.20105+01 \cdot x$

$1.20105+01$. $x$

$-6.7436 E+00 \cdot x$

$-2.0231 E+01: x$

$4.2424 E-01: x$

$6.2937 E-01: x 2$

$6.2937 E-01: x$

$1.0250 E-01: x$

$3.07695-01 \div \times 2$

$-5.0909 E-01 * x$

$2.2224 E+01 * x-2.0345 E+00 * x 27.4542 E-02 * x y$

$9.6084 E+01: x-5.03505000+x 2$

$1.3487 E+01 * x-2.0513 E-01 * x 2$

$8.0923 E+00 * x \quad-1.2308 E-01 * x 2$

$5.0909 E+00 * x$

$1.1200 E+01 * x$

$-3.7091 E+00 * x$

$1.0282 E+01 * x$

$0.8895 E+00$

$-1.3890 E+01$ * $x$

$-1.2010 E+01 . x$

$-1.6859 E+00=x$

$1.6859 E+00 * x$

$2.6667 E-01$.

$3.6364 E-01: x$

$2.46305+00 * \times 2-6.0006 E-01 * x J 4.8485 E-02 * \times 4$

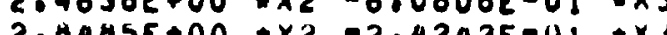

$-1.1338 E+00 * \times 2 \quad 2.9837 E=02 \times x 3$

$1.7716 E+00 * \times 2-4.6620 E-02 * x_{3}$

$6.2937 E-01$ : $x 2$

$2.5641 E=02 \times \times 2$ 


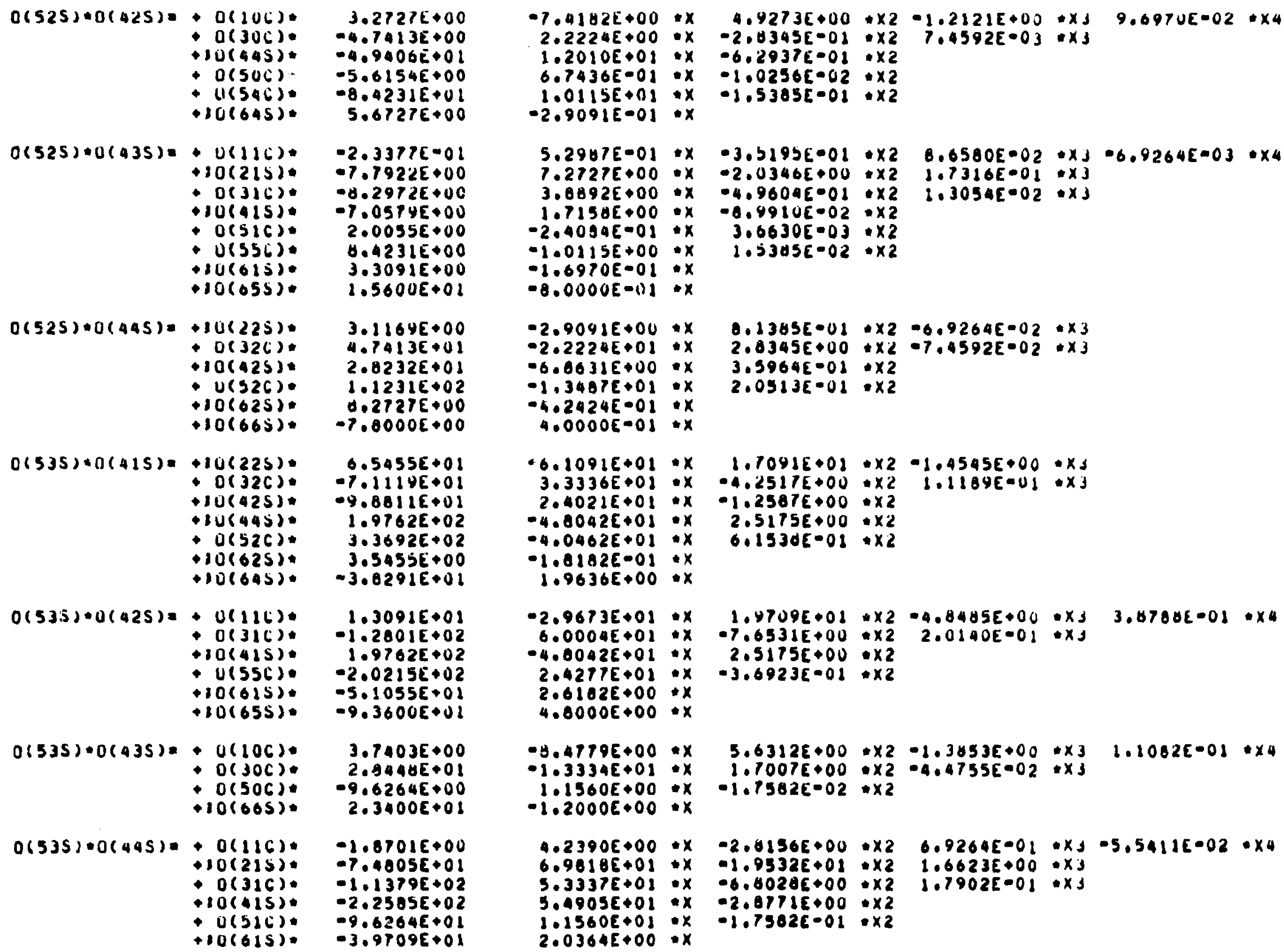

$5.2907 E-01+x-3.5195 E-01+x 2$

$6.6580 E=02+x_{3}=6.9264\left[=03 \cdot x_{4}\right.$ $7.2727 E+00 * x \quad-2.0346 E 000$ *x $1.7316 E=01 * x]$

$1.7158[+00 * x-0.90106-02 \times x 2$

$-1.02155+00: x \quad 3.6630 E=03: x 2$

$-1.6970 E-01: x$

$-8.00005-111+x$ $-2.9041 E+00: x \quad 0.1385 E=01: \times 2=6.9264 E=02: \times 3$

$-6.0631 E+00$ : $x$

$-1,3407 E+01 \cdot x$

$-4.2424 E-01: x$
$-0000 E-01: x$

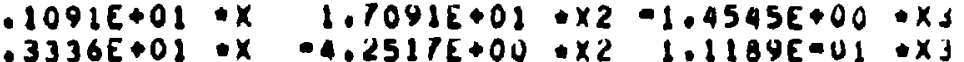
作

$2.40212+01$ : $x$ - $1.2567 E+00$ : $x 2$

$-4.0042 E+01 * x \quad 2.5175 E+00$ * $x 2$

$-4.0402 E+01 * x \quad 6.1530 E-01 * x 2$

$-1.01826001 \div x$

$1.9636 E+00: x$

$-2.9673 E+01 * x \quad 1.4709 E+01 * \times 2-4.8485 E+00 * x 3 \quad 3.8700 E-01 * \times 4$ $6.0004 E+01: x-7.6531 E+00 * x 2 \quad 2.0140 E=01 * x$

$-4.0042 E+01: x \quad 2.5175 E+00 \cdot x 2$

2.427TE+01: $x$

2.6

$-5.4779 E+00 \times x \quad 5.6312 E+00 * \times 2-1.3853 E+00 \times \times 3 \quad 1.1082 E-01 \times \times 4$

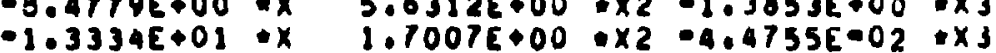

$1.1560 E+00 * x-1.7582 E-02 \times 2$

$-1.2000 E+00 \cdot x$

$0.2390 E+00 * x-2.8156 E+00 * x 2 \quad 0.9264 E-01 * x 3-5.5411[-02 * x 4$ $6.9818 E+01 * x-1.9532 E+01 * x 21.6623 E+00 * x 3$ $5.3337 E+01 * x \quad-6.80206+00 \div \times 2 \quad 1.7902 E-01 * x 3$

$3.4905 E+01: x \quad-2.0771 E+00 * \times 2$

$1.1560 \varepsilon+01: x-1.7582 E-01: \times 2$ 


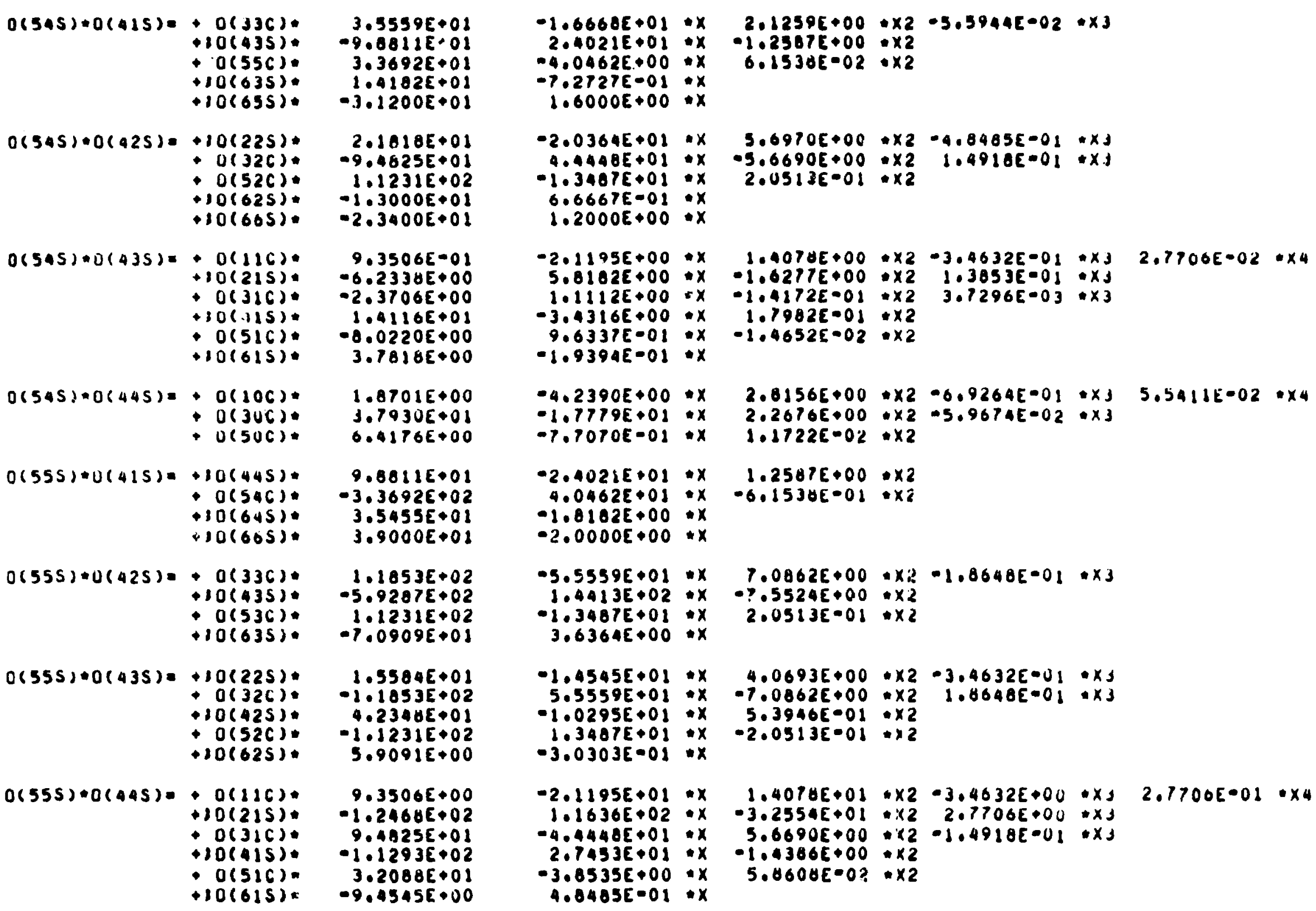

$O(545)+0(415)=+0(336) \cdot \quad 3.5559 E+02$ $+10(435) .9 .8811 E+01$ $+0(55 C) .3 .3692 E+01$ $+10(635): \quad 1.4182 E+01$
$+10(655) * \quad-3.1200 E+01$

$0(545) \cdot 0(925)=+10(225) * 2.1018 E+01$ $-0(326)=9.4625 E+01$ - $0(52 \mathrm{C}) .1231 \mathrm{1}+02$ $+10(625)=-1.3000 E+01$

$0(545) \cdot 0(435)=+0(126) \quad 9.3506 E-0)$ $\rightarrow 0(215) \quad-6.2334 E+00$ - O(jicjo - 30(nis). 1.4116e+01 $+10(615)$ 3.78)8E+00

$0(54 S)=0(445)=0(106) * 1.8701 E+00$ O $0(306)+3.7930 E+01$ - 0.41500$)=06+00$

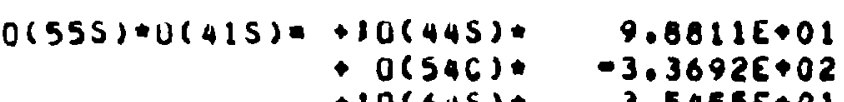
$+10(645): \quad 3.5455 E+01$
$+10(605) * \quad 3.9000 E+01$

$0(55 S)=0(42 S)=+0(336) * \quad 1.1853 E+02$ $+10(435)-5.9287 \varepsilon+02$ $\begin{array}{rr}0(536): \quad 1.1231 E+02 \\ +10(635) & -7.0909 E+01\end{array}$

$0(55 S)+0(435)=+10(225)+1.5584 E+01$ - $0(326) .-1.1853 E+02$ $+10(425) .4 .2348 \varepsilon+01$ $+0(52 C):-1.1231 E+02$
$+10(625): \quad 5.9091 E+00$

$0(555) \cdot 0(445)=+0(126)$. $10(215)$. - D(jic). $+10(4) 5)$. - $0(516)=$ $+10(615)$.

$-1.0608 \varepsilon+01 \cdot x$ $2.4021 E+02 * x$ $-4.04626+00: x$ $-7.2727 E-01$
$1.6000 E+00: x$

$-2.0364 E+01 * x \quad 5.6970 E+00 \times 2-4.8485 E-01 * x 3$ $4.4448 E+01 * x \quad-5.6690 E+00 \div \times 2 \quad 1.4910 E-01+x$. $1.3407 E+01$ ox $2.0513 E=01$ x

$6.6667 E-01$ :x

$-2.1195 E+00 * x$ $5.8102 E+00=x-1.6277 E+00 \times x 2 \quad 1.3053 E-01: x J$ $1.1112 E+00$ ox $1.4172 \varepsilon=01$ ax $3.1296 \varepsilon=03 x$ $0.43162+00 \times x-1.0952 E-03 \times x 2$ $9.03350010 x$

$-4.2390 E+00 * x$ $1.7779 E+02: x$ -7. rOTOE-01 *

$2.8156 E+00 \times 2-0.9264 E-01 \times \times 3 \quad 5.5411 E=02 \times \times 4$ $2.26765+00 \times 2-5.96745002 \times x 3$ $1.17226-02 \times 2$

$0.4021 E+01 * x \quad 1.2587 E+00 * x 2$

$4.0462 E+01 \cdot x-6.1530 E-01 * x a$

$-1.8102 E+00 \cdot x$

$2.0000 E+00 * x$

$-5.5559 E+01 * x \quad 7.0062 E+00 * x_{2}-1.8648 E-01 * x_{3}$ $\begin{array}{rr}1.4413 E+02: x & -? .5524 E+00 \\ -1.3487 E+01 * x & 2.0513 E-01: \times 2\end{array}$

$1.3487 E+01$ : $x$

$-1.4545 E+01 * x \quad 4.0693 E+00 * \times 2-3.4632 E-01 * x s$ $5.5559 E+01 * x-7.0862 E+00 * x 2 \quad 1.8648 E-01 * x J$ $-1.0295 E+01: x \quad 5.3946 E-01 * x 2$

$1.3407 E+01: x$
$-3.0303 E-01: x$

$-2.1195 E+01 * x$

$1.4078 E+01 * \times 2-3.4632 E+00 * x 3$ $1.1636 E+02 \cdot x-3.2554 E+01 * x 2 \quad 2.7706 E+00 * x$ $-4.4448 E+01: x \quad 5.6690 E+00 * x 2-1.4918 E-01 * x$

$2.7433 E+01: x-1.43365+00 * x 2$

$-3.6535 E+00 \times x \quad 5.0600 E-0 ? \times 2$

$1.24605+02$

$1.1293 E+02$

$3.2088 E+01$

$4.0405 E-01$ * $x$

$2.7700 E-02 \cdot \times 4$ 


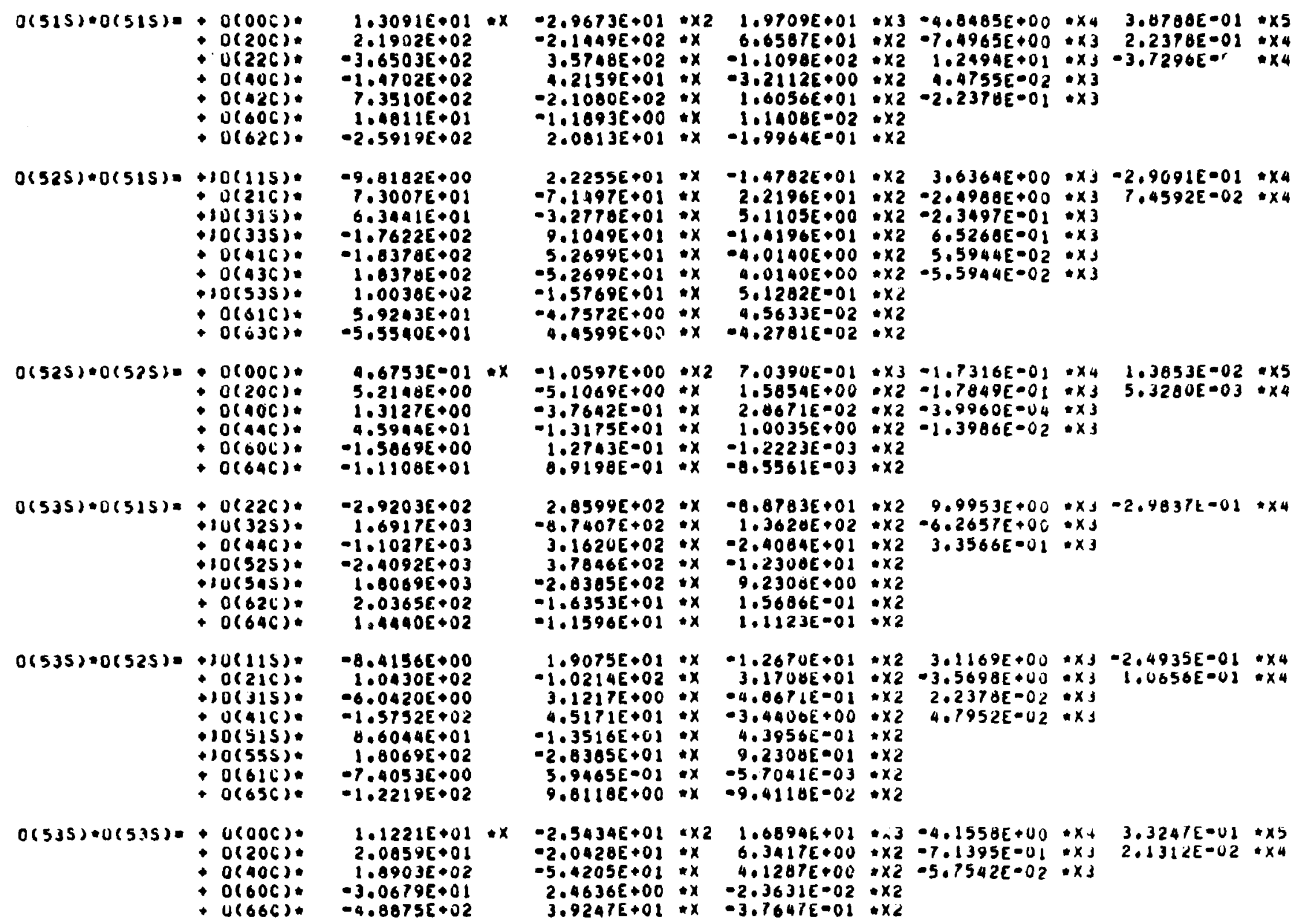

$-2.9203 E \bullet 02$ $1.69176+03$ $-1.1027 E+03$ $-2.40926+03$ $1.00696+03$ $2.03656+02$

$8.4156 \varepsilon+00$ $1.0430 E+02$
$-6.04206+00$ $-1.5752 E+02$ $6.60446+01$ $1.8069 E+02$ $-7.4053 E+00$
$-1.2219 E \bullet 02$

$\times \quad-2.9673 E+01 * \times 2 \quad 1.9709 E+01 \times x 3-4.0485 E+00 \times x_{4} \quad 3.4700 E-01 \times \times 5$ $-2.1449 E+02+x \quad 6.6587 E+01 * x 2-7.4965 E+00 \div x_{3} \quad 2.2378 E=01 * x_{4}$ $3.5748 E+02 * x-1.1098 E+02 * x 2 \quad 1.2494 E+01 * x 3-3.7296 E=0 \quad * x$ $4.2159 E+01: x-3.2112 E+00 * x 2 \quad 0.47555002 * x 3$

$-2.1000 E+02 * x \quad 1.6056 E+01 * x 2-2.237 \forall E-01 * x 3$

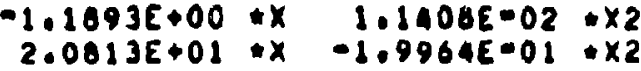

$2.2255 E+01 \times x-1.4782 E+01 \times 2 \quad 3.6364 E+00 \times x-2.9091 E-01 * x 4$

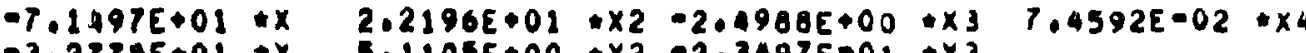
-

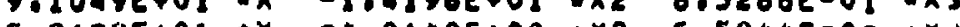

5.

$-1.57698+01$ : $x$

$0.75725+00+x$

$4.4599 E+00 * x \quad-4.2781 E=02 * x 2$

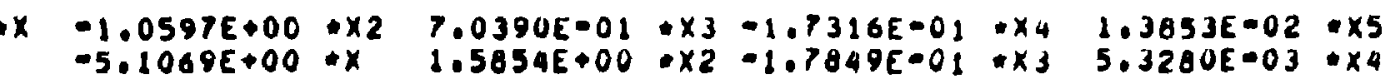
$-3.7642 E=01 * x \quad 2.0671 E=02 * x 2-3.9960 E=04: x^{3}$ $-1.3175 E+01 * x \quad 1.0035 E+00 * 2-1.3986 E-02 \times \times 3$

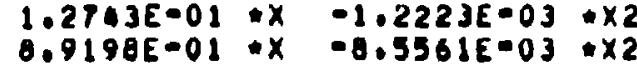

$2.8599 E+02 * x-8.0783 E+01 * x 2 \quad 9.9953 E+00 * x \leq-2.9837 k-01 * x$ $-8.7407 \varepsilon+02$ *x $1.3020 E+02$ *x $-6.2651 E+00$ *xJ

$3.1620 E+02 \times x \quad-2.4004 E+01 \div \times 2 \quad 3.3566 E-01 * x 3$

$3.7846 E+02 * x-1.2300 E+01 * x 2$

$-2.0365 E+02$ \& $\quad 9.2300 E+00$ ix

$-1.6353 E+01: x \quad 1.5606 E-01: \times 2$

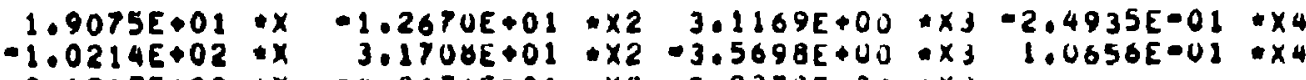
$3.1217 E+00 * x-4.0671 E-01 * x 2 \quad 2.2378 E-02 \times x j$ $4.5171 E+01 * x-3.4400 E+00 * x_{2} 4.7952 E-02 * x_{3}$ $-1.3516 E+01 * x \quad 4.3956 E-01 * x$ $-2.8305 E+01 * x \quad 9.2308 E \cdot 01 * x$ $3.9465 E-01: x \quad-5.7041 E-03 * \times 2$
$9.8118 E+00 * x \quad-9.4110 E=02 * \times 2$

$1.1221 E+01 * x$ $2.00596+01$

$1.0903 E+02$

$-3.0679 E+01$
$-4.8675 E+02$

$-2.5434 E+01 \times x 2 \quad 1.0894 E+01 * 33-4.1558 E+00 * x_{4} \quad 3.3247 E=01 * \times 5$ $2.0428 E+01 * x \quad 6.3417 E+00 * x 2-7.1395 E=01 * x J 2.1312 E=02$ * $x$ $-5.4205 E+01 * x \quad 4.1287 E+00 * x 2-5.7542 E-02 * x 3$

$2.4636 E+00$ : $x \quad-2.3631 E=02$ * $x 2$ 


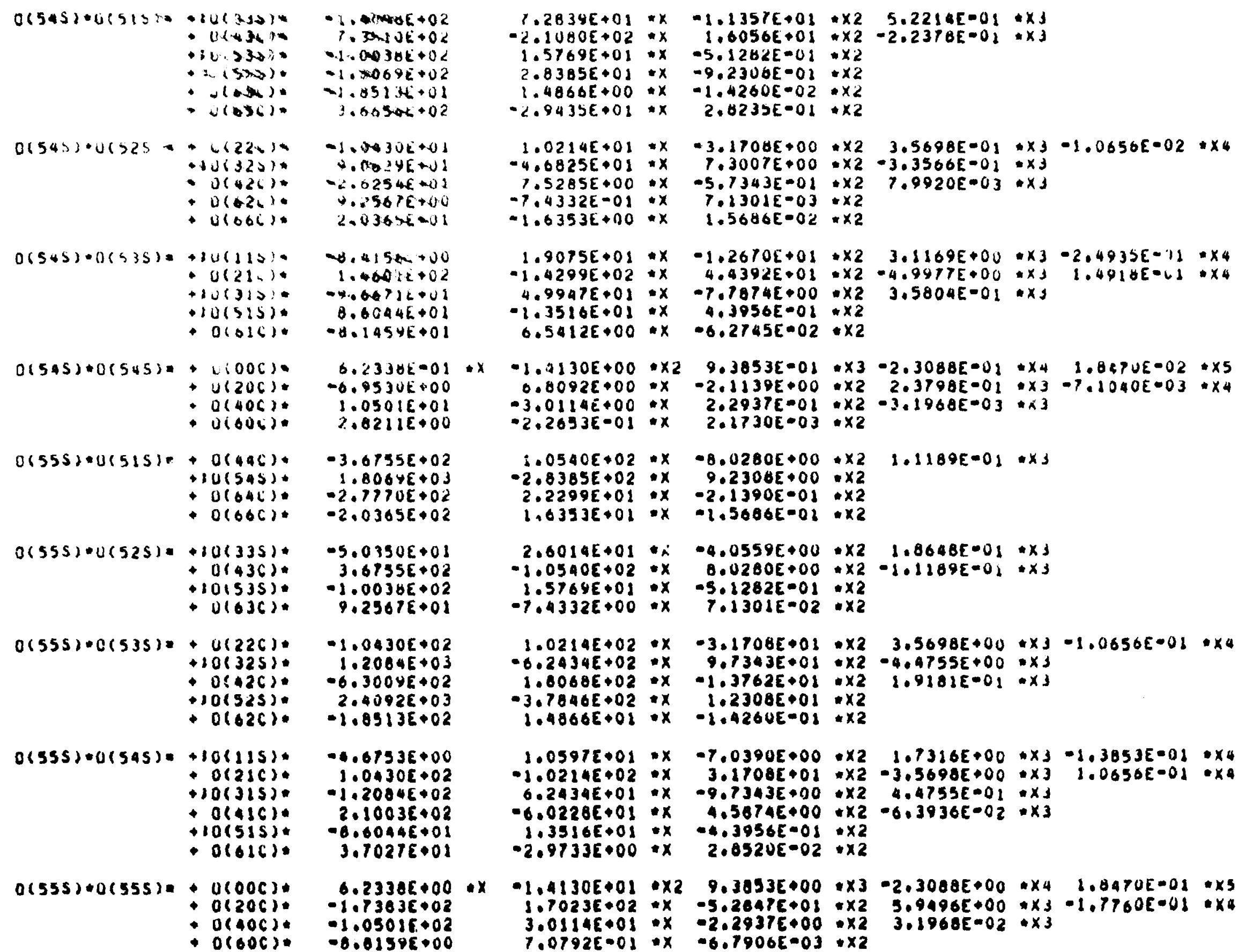

$-3.6755 E+02$

$1.806+E+03$ $-2,77702+02$
$-2,0365 E+02$

$0(555) \cdot 0(525)+10(335)$ $+0(436)$.
$+10(535)$

$-5.0350 E+01$ $3.6755 E+02$ $-1.0038 E+02$
$9.2567 E+01$ $+0(636)$

$.2567 E+01$

$0(555)+0(535)=+u(22 C)$ $+10(325)$. - 10 (525)

$-1.0430 E+02$ $1.2004 E+03$ 0.0005 $2.4092 E+03$
$-1.0513 E+02$ $+0(62 C)$.

$1.05136+02$

$0(355) \cdot 0(545)=+10(115)$ - o(a)c + io(jisjo.

0(4ici.

$+10(515)$.

$1.0430 E+02$

$1,2084 E+02$

2.1003E402

- o(61C).

$6.60446+01$
$3.7027 E \bullet 01$

$0(555) \times 0(555)=0(00 C)$ $-0(406)$ $+0(600)$

6.23306000 .x $-1.73838 .02$ $-1.05016+02$
$-6.01508+00$

$1.2839 E+01 * x-1.1357 E+01 * x 2 \quad 5.2214 E-01 * x 1$ $-2.1000 E+02 \times x \quad 1.6056 E+01 \times \times 2-2.2376 E=01 * x J$ $1.5769 E+01 * x-5.1262 E=01 * x \cdot 2$

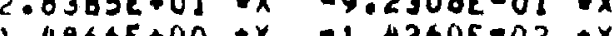
$-2.9435 E+01 * x \quad 2.0235 E=01 * x 2$

$1.0214 E+01 * x-3.1708 E+00 * 2 \quad 3.5698 E-01 * \times 3-1.0656 E-02 * \times 4$ $-4.0825 E+01: x$ $7.3007 E+00 \times 2=3.3566 E-01 \div x 3$

$-7.4332 E-01: x \quad-5.7343 E=01 * x 2 \quad 7.9920 E-03 * x J$

$-1.6353 E+100 \times \quad 1.1301 E-03 \div \times 2$

$1.9075 E+01 * x-1.2670 E+01 * \times 2 \quad 3.8169 E+00 * x 3-2.4935 E-11 * x^{4}$ $-1.4299 E+02 \times x \quad 4.4392 E+01=\times 2=4.9917 E+00 \times x+1.4916 E=61 * x_{4}$ $4.9947 E+01=x-7.7874 E+00 * \times 2 \quad 3.5804 E-01 * x 3$

$-1.3516 E+01 * x \quad 4.3056 E=01 * \times 2$

$-1.9130 E+00 \times 2 \quad 9.3853 E=01 \times 3=2.3088 E=01 \times x 4 \quad 1.847 U E=02 * \times 5$ $0.0092 \varepsilon+00 * x \quad-2.1130 E+00 * x 2 \quad 2.3798 E-01 * x 3=7.1040 E=03 * x 4$

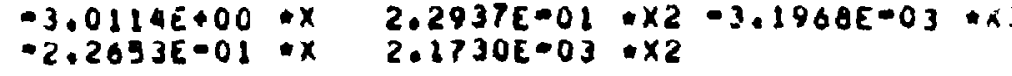

$\therefore .0540 E+02 \times x \quad-8.0280 E+00 * 2 \quad 1.1189 E-01 * \times 3$ $-2.8385 E+02: x \quad 9.2308 E+00: \times 2$ $2.2299 E+01 \times x-2.1390 E-01 \times 2$ $1.6353 E+01 * x-1.5606 E-01: \times 2$

$2.6014 E+01 * A-4.0559 E+00+x 2 \quad 1.864 B E=01 * X S$ $-1.0540 E+02 * x \quad 0.0280 E+00 * x 2-1.1289 E=01 * x$ $-1.5764 E+01: x-5.1282 E-01 * x 2$

$1.0214 E+02 \times x-3.1708 E+01 \times 2 \quad 3.5698 E+00 \times 3-1.0656 E-01 \times 4$ $-8.02434 E+02: x \quad 9.7343 E \div 01: \times 2-4.4755 E+00 \times x 3$ $1.8068 E+02 * x-1.3762 E+01 * x 2 \quad 1.9181 E-01 * x 3$

$-3.7846 E+02$ * $x \quad 1.2300 E+01 * x 2$ $1.4866 E+01 \cdot x-1.4260 E-01 \cdot \times 2$

$1.0597 E+01 \times x-7.0390 E+00 \times \times 2 \quad 1.7316 E+00 \times \times 3-1.3853 E=01 \times \times 4$ $-1.0214 E+02 \times x \quad 3.1708 E+01 \times \times 2-3.5690 E+00 \times x \quad 1.0650 E-01 \times x 4$

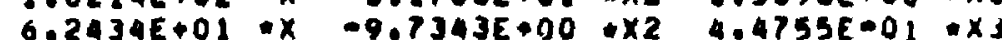
$-6.0228 E+01 \div x \quad 4.56746+00 * x 2-6.3936 E-02 * x 3$

$1,3536 E+01$ : $\quad-1.3956 E-01: \times 2$

$0.97335000 * x \quad 2.0520 \varepsilon=02 * \times 2$

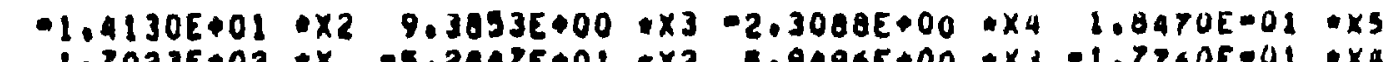
$1.7023 E=02 \times x \quad-5.2647 E+01 * \times 2 \quad 5.9496 E+00 * x 3-1.7760 E-01 \times x$ $3.01146+01: x-2.2937 E+00 * x 2 \quad 3.1968 E-02 * x 3$ 


\begin{tabular}{|c|c|c|c|c|c|c|}
\hline $0(615)+0(125)=$ & $\begin{array}{l}+0(502): \\
+0(526) \\
+10(625)\end{array}$ & $\begin{array}{l}-0.730 \text { - }=01 \\
-6.730 \Delta E+00 \\
-1.2500 E+00\end{array}$ & $\begin{array}{l}7.6923 E=02 \\
7.6923 E=01\end{array}$ & $: x$ & & \\
\hline $0(625)=0(115)=$ & $\begin{array}{l}+0(51 C): \\
+0(536): \\
+10(615) \\
+10(635)\end{array}$ & $\begin{array}{r}-2.092 J E \bullet 00 \\
-1.3462 E \bullet 00 \\
2.0000 E+00 \\
-3.0000 E \bullet 00\end{array}$ & $\begin{array}{l}3.0769 E-01 \\
1.3385 E-01\end{array}$ & $: x$ & & \\
\hline $0(635) \cdot 0(115)=$ & $\begin{array}{l}+0(526): \\
: 0(546) \\
+10(625) \\
+10(645)\end{array}$ & $\begin{array}{r}-8.0764 E+00 \\
-2.0192 E-00 \\
7.5000 E-01 \\
-7.5000 E-01\end{array}$ & $\begin{array}{l}9.2300 E=01 \\
2.30 ? T E=01\end{array}$ & $: x$ & & \\
\hline $0(645) \cdot 0(115)=$ & $\begin{array}{l}+0(536): \\
+0(556): \\
+j 0(635): \\
+10(655):\end{array}$ & $\begin{array}{r}-3.3654 E+00 \\
-6.7308 E=01 \\
2.3000 E+00 \\
-5.5000 E+00\end{array}$ & $\begin{array}{l}3.8162 E-01 \\
7.6923 E-02\end{array}$ & $x_{x}^{x}$ & & \\
\hline $0(655) \cdot 0(115)=$ & $\begin{array}{l}+0(540): \\
+10(645) \\
+10(665)\end{array}$ & $\begin{array}{r}-3,3654 E+00 \\
2,3000 E=01 \\
-2.3000 E-01\end{array}$ & $3.0482 E-01$ & - $x$ & & \\
\hline $0(665) \cdot 0(115)=$ & $\begin{array}{l}+0(55 c) \\
+10(855):\end{array}$ & $\begin{array}{r}-4.0305 E+00 \\
3.0000 E+00\end{array}$ & $4.6254 E-01$ & $\cdot x$ & & \\
\hline $0(615)=0(215)=$ & $\begin{array}{l}+o(406): \\
+0(426): \\
+10(525): \\
+0(606): \\
+0(626) .\end{array}$ & $\begin{array}{r}1.8357 E+00 \\
3.6713 E+00 \\
3.02808+01 \\
-9.3182 E-02 \\
1.3977 E+00\end{array}$ & $\begin{array}{r}-5.1573 E-01 \\
-1.031 S E \rightarrow 00 \\
-3.4613 E+00 \\
3.0303 E-03 \\
-4.3455 E-02\end{array}$ & $\begin{array}{l}: x \\
: x \\
: x \\
: x \\
: x\end{array}$ & $\begin{array}{l}3.4965 E=02 \\
6.9930 E=02\end{array}$ & $\begin{array}{l}\times 2 \\
\times 2\end{array}$ \\
\hline $0(615) \div 0(235)=$ & 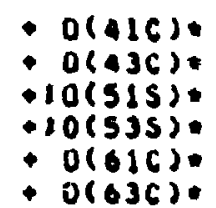 & $\begin{array}{l}-7.3427 E+00 \\
-7.3427 E+00 \\
-1.0096 E+01 \\
-6.7308 E+00 \\
-7.0273 E+00 \\
-1.1102 E+01\end{array}$ & $\begin{array}{l}2.0629 E+00 \\
2.0629 E+00 \\
1.1539 E+00 \\
7.6023 E=01 \\
2.5135 E=01 \\
3.6364 E \cdots 01\end{array}$ & $\begin{array}{l}: x \\
: x \\
: x \\
x \\
: x \\
x \\
x\end{array}$ & $\begin{array}{l}-1.3986 E=01 \\
-1.3980 E=01\end{array}$ & $\begin{array}{l}\times 2 \\
1 \times 2\end{array}$ \\
\hline
\end{tabular}




\begin{tabular}{|c|c|c|c|c|c|c|}
\hline $0(625) \div 0(215)=$ & $\begin{array}{l}+O(416) \\
+0(43 C) \\
+\quad D(515) \\
+O(53 S) \\
+0(61 C) \\
+O(63 C)\end{array}$ & $\begin{array}{r}1.1748 E+01 \\
1.1740 E+01 \\
-1.0305 E+00 \\
7.4038 E+00 \\
-2.2364 E+00 \\
5.5909 E+00\end{array}$ & $\begin{array}{r}-3.3007 E+00 \\
-3.3007 E+00 \\
4.6154 E-01 \\
-0.4615 E-01 \\
7.2727 E-02 \\
-1.0182 E-01\end{array}$ & $\begin{array}{l}: x \\
: x \\
: x \\
: x \\
: x \\
x\end{array}$ & $\begin{array}{l}2.2378 E-01 \\
2.2370 E=01\end{array}$ & $\begin{array}{l}: \times 2 \\
: \times 2\end{array}$ \\
\hline $0(625) * 0(225)=$ & $\begin{array}{l}O(\triangle O C): \\
+O(4 A C): \\
+O(54 S)= \\
+O(60 C): \\
+O(64 C)\end{array}$ & $\begin{array}{r}2.9371 E+00 \\
-1.4605 E+00 \\
-2.4231 E+01 \\
1.4909 E+00 \\
-6.7091 E+00\end{array}$ & $\begin{array}{r}-8.2517 E-01 \\
1.1259 E-01 \\
2.7692 E+00 \\
-4.8485 E-02 \\
2.1818 E-01\end{array}$ & $\begin{array}{l}: x \\
: x \\
: x \\
x \\
x\end{array}$ & $\begin{array}{r}5.5944 E-02 \\
-2.7972 E-02\end{array}$ & $\begin{array}{l}* \times 2 \\
+\times 2\end{array}$ \\
\hline $0(635) * 0(215)=$ & $\begin{array}{l}+0(42 C): \\
+0(44 C) \\
+10(525) \\
+10(545) \\
+0(62 C) \\
+0(64 C) .\end{array}$ & $\begin{array}{r}4.4056 E+00 \\
1.1014 E+00 \\
-4.0305 E+00 \\
1.3125 E+01 \\
-1.3977 E+00 \\
1.9568 E+00\end{array}$ & $\begin{array}{r}-1.2378 E+00 \\
-3.0944 E-01 \\
4.6154 E-01 \\
-1.5000 E+00 \\
4.5455 E=02 \\
-6.3636 E-02\end{array}$ & $\begin{array}{l}* x \\
* x \\
: x \\
: x \\
: x \\
* x\end{array}$ & $\begin{array}{l}0.3916 E-02 \\
2.0979 E-02\end{array}$ & $\begin{array}{l}\times \times 2 \\
\times \times 2\end{array}$ \\
\hline $0(635)=0(225)=$ & $\begin{array}{l}+0(41 C): \\
+10(515): \\
+10(555) \\
+0(61 C) \\
+0(65 C) .\end{array}$ & $\begin{array}{r}8.8112 E+00 \\
-8.0769 E+00 \\
-2.0192 E+00 \\
4.4727 E+00 \\
-1.2300 E+01\end{array}$ & $\begin{array}{r}-2.4755 E+00 \\
9.2308 E-01 \\
2.3077 E-01 \\
-1.4545 E-01 \\
4.0000 E-01\end{array}$ & $\begin{array}{l}: x \\
: x \\
: x \\
: x \\
: x \\
x\end{array}$ & $1.6783 E=01$ & $\times 2$ \\
\hline $0(645) \oplus 0(215)=$ & $\begin{array}{l}+0(436) \\
+10(535) \\
+10(555) \\
+0(636) \\
+0(65 C) .\end{array}$ & $\begin{array}{r}2.9371 E+01 \\
1.6827 E+00 \\
5.0481 E+00 \\
-6.5227 E+00 \\
1.8450 E+01\end{array}$ & $\begin{array}{r}-0.2517 E+00 \\
-1.9231 E-01 \\
-5.7692 E-01 \\
2.1212 E-01 \\
-6.0000 E-01\end{array}$ & $\begin{array}{l}: x \\
: x \\
: x \\
: x \\
x \\
x\end{array}$ & $5.5944 E-01$ & $+\times 2$ \\
\hline $0(645)=0(225)=$ & $\begin{array}{l}+0(42 C) * \\
+10(525) \\
+\quad 0(62 C) * \\
+0(66 C) *\end{array}$ & $\begin{array}{r}1.4605 E+01 \\
-8.0769 E+01 \\
5.5909 E+00 \\
-4.1000 E+00\end{array}$ & $\begin{array}{r}-4.1259 E+00 \\
9.2300 E+00 \\
-1.8162 E-01 \\
1.3333 E-01\end{array}$ & $\begin{array}{l}\cdot x \\
0 x \\
0 x \\
x\end{array}$ & $2.7972 E-01$ & $\cdot \times 2$ \\
\hline $0(655) * 0(215)=$ & $\begin{array}{l}+0(44 C) \\
+10(545) \\
+0(64 C) \\
+0(66 C)\end{array}$ & $\begin{array}{r}1.0357 E+00 \\
5.0401 E+00 \\
-8.3064 E-01 \\
1.0250 E+00\end{array}$ & $\begin{array}{r}-5.1573 E-01 \\
-5.7692 E-01 \\
2.7273 E-02 \\
-3.3333 E-02\end{array}$ & $\begin{array}{l}: x \\
: x \\
: x \\
: x\end{array}$ & $3.4965 E-02$ & $+\times 2$ \\
\hline $0(655)+0(225)=$ & $\begin{array}{l}+D(43 C) \\
+\quad 0(535) \\
+0(636)\end{array}$ & $\begin{array}{r}1.4685 E+01 \\
-3.3654 E+00 \\
1.8636 E+00\end{array}$ & $\begin{array}{r}-4.1239 E+00 \\
3.8462 E-01 \\
-6.0606 E-02\end{array}$ & $\begin{array}{l}-x \\
: x \\
0 x\end{array}$ & $2.79726=02$ & $* \times 2$ \\
\hline
\end{tabular}




\begin{tabular}{|c|c|c|c|c|c|c|c|c|}
\hline $0(665) * 0(215)=$ & $\begin{array}{l}+10(555) \\
+0(65 C)\end{array}$ & $\begin{array}{r}1.0096 E+01 \\
-1.2300 E+01\end{array}$ & $\begin{array}{r}-1.1538 E+00 \\
.0000 E=01\end{array}$ & $\begin{array}{l}x \\
x\end{array}$ & & & & \\
\hline $0(665) \times 0(225)=$ & $\begin{array}{l}+0(446): \\
+80(545): \\
+0(646):\end{array}$ & $\begin{array}{r}2.2028 E+01 \\
-4.0385 E+01 \\
2.2364 E+00\end{array}$ & $\begin{array}{r}-6.1888 E+00 \\
-16154 E+00 \\
-7.2727 E-02\end{array}$ & $\begin{array}{l}: x \\
: x \\
: x\end{array}$ & 4.19585001 & $1 \times 2$ & & \\
\hline $0(615) \times 0(315)=$ & $\begin{array}{l}+0(306) \\
+0(326) \\
+\quad 0(425) \\
+0(506) \\
+0(526) \\
+0(625)\end{array}$ & $\begin{array}{r}-6.11096+01 \\
-9.1703 E+01 \\
-9.1783 E+01 \\
1.2564 E+01 \\
-1.0046 E+02 \\
2.3102 E+01\end{array}$ & $\begin{array}{r}3.3506 E+01 \\
3.0262 E+01 \\
2.5787 E+01 \\
-1.7330 E+00 \\
2.6026 E+01 \\
-1.3636 E+00\end{array}$ & $\begin{array}{l}* x \\
: x \\
: x \\
: x \\
: x \\
\cdot x\end{array}$ & $\begin{array}{r}-5.7498 E+00 \\
-6.6247 E+00 \\
-1.7403 E+00 \\
3.4100 E=02 \\
-5.1282 E-01\end{array}$ & $\begin{array}{l}: \times 2 \\
0 \times 2 \\
0 \times 2 \\
\times \times 2 \\
\times 2\end{array}$ & $\begin{array}{l}3.1000 E=01 \\
4.6620 E=01\end{array}$ & $\begin{array}{l}x=3 \\
03 \\
x\end{array}$ \\
\hline $0(615)+0(325)=$ & $\begin{array}{r}+0(316): \\
+0(336) \\
+10(415) \\
+0(435) \\
+0(516) \\
+0(536) \\
+10(635)\end{array}$ & $\begin{array}{l}9.1783 E+00 \\
3.0594 E+00 \\
1.0305 E+01 \\
6.2413 E \circ 01 \\
1.8646 E+01 \\
2.5126 E+01 \\
1.8545 E+01\end{array}$ & $\begin{array}{l}-5.0262 E+00 \\
-1.6754 E+00 \\
-1.1346 E+01 \\
-1.7535 E+01 \\
-2.6026 E+00 \\
-3.4701 E+00 \\
-1.0909 E+00\end{array}$ & $\begin{array}{l}* x \\
: x \\
: x \\
: x \\
: x \\
: x \\
: x \\
: x\end{array}$ & $\begin{array}{l}0.0247 E-01 \\
2.0749 E-01 \\
7.6923 E-01 \\
1.1886 E+00 \\
5.1282 E-02 \\
6.8376 E-02\end{array}$ & $\begin{array}{l}\times 2 \\
: \times 2 \\
: \times 2 \\
: \times 2 \\
\times 2 \\
\times 2 \\
\times 2\end{array}$ & $\begin{array}{l}-4.6620 E=02 \\
-1.3540 E=02\end{array}$ & $\begin{array}{l}0 \times 3 \\
0 \times 3\end{array}$ \\
\hline $0(615) \oplus 0(335)=$ & $\begin{array}{r}+0(326): \\
+10(425): \\
+10(445): \\
+0(526): \\
+0(546) \\
+10(625): \\
+10(645)\end{array}$ & $\begin{array}{l}-.0357 E+01 \\
-3.3042 E+01 \\
-1.1014 E+01 \\
-2.6385 E+02 \\
-1.1308 E+02 \\
-3.2455 E+01 \\
-2.7810 E+01\end{array}$ & $\begin{array}{l}1.0052 E+01 \\
9.2032 E+00 \\
3.0944 E+00 \\
3.6436 E+01 \\
1.5615 E+01 \\
1.0091 E+00 \\
1.6364 E+00\end{array}$ & $\begin{array}{l}: x \\
: x \\
: x \\
: x \\
: x \\
: x \\
x \\
x\end{array}$ & $\begin{array}{l}-1.7249 E+00 \\
-6.29376-01 \\
-2.09798-01 \\
-7.17956-01 \\
-3.07690-01\end{array}$ & $\begin{array}{l}: \times 2 \\
: \times 2 \\
: \times 2 \\
: \times 2 \\
x 2\end{array}$ & $9.3240 E=02$ & $\cdot \times 3$ \\
\hline $0(625) \neq 0(315)=$ & $\begin{aligned} & 0(316) \\
+ & 0(336) \\
+ & 10(415) \\
+ & 10(35) \\
+ & 0(516) \\
+ & 0(536) \\
+ & 10(615) \\
+ & 10(635)\end{aligned}$ & $\begin{array}{r}-7.3427 E+01 \\
-2.4476 E+01 \\
2.9371 E+01 \\
-3.8102 E+02 \\
7.5385 E+01 \\
-6.7949 E+01 \\
-3.7091 E+01 \\
1.8545 E+01\end{array}$ & $\begin{array}{r}4.0210 E * 01 \\
1.3403 E+01 \\
-8.2517 E+00 \\
1.0727 E+02 \\
-1.0410 E+01 \\
1.2145 E+01 \\
2.1818 E+00 \\
-1.0909 E+00\end{array}$ & $\begin{array}{l}: x \\
: x \\
: x \\
: x \\
: x \\
: x \\
: x \\
* x \\
x \\
x\end{array}$ & $\begin{array}{r}-6.0998 E+00 \\
-2.2999 E+00 \\
5.5944 E-01 \\
-7.2727 E+00 \\
2.0513 E=01 \\
-2.3932 E=01\end{array}$ & 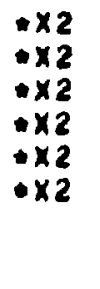 & $\begin{array}{l}3.7296 E-01 \\
1.2432 E-01\end{array}$ & 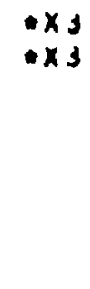 \\
\hline $0(625)=0(325)=$ & $\begin{array}{l}+0(306) \\
+10(445) \\
+0(506) \\
+0(546) \\
+10(645)\end{array}$ & $\begin{array}{r}-1.9580 E+01 \\
1.4605 E+01 \\
-5.0256 E+00 \\
1.1308 E+02 \\
1.6691 E+01\end{array}$ & $\begin{array}{r}1.0723 E+01 \\
-4.1259 E+00 \\
6.9402 E-01 \\
-1.5615 E+01 \\
-9.0182 E-01\end{array}$ & $\begin{array}{l}: x \\
: x \\
: x \\
: x \\
x\end{array}$ & $\begin{array}{r}-1.03946+00 \\
2.19725-01 \\
-1.2675 E-02 \\
3.07696-01\end{array}$ & $\begin{array}{l}+\times 2 \\
\times 2 \\
\times 2 \\
\times 2 \\
\times 2\end{array}$ & $9.9456 E-02$ &.$\times 3$ \\
\hline
\end{tabular}




\begin{tabular}{|c|c|c|c|c|c|c|c|c|}
\hline $0(625)+0(335)=$ & 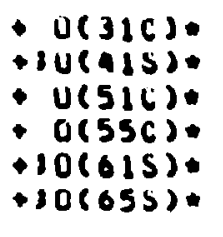 & $\begin{array}{r}1.4685 E+01 \\
6.0112 E+01 \\
7.5 j 45 E+01 \\
-2.2615 E+01 \\
5.1927 E+01 \\
-1.2240 E+02\end{array}$ & $\begin{array}{r}-0.0420 E+00 \\
-2.0755 E+01 \\
-1.0410 E+01 \\
3.12316+00 \\
-3.0545 E+00 \\
7.2000 E+00\end{array}$ & $\begin{array}{l}x \\
: x \\
: x \\
: x \\
x \\
x \\
x\end{array}$ & $\begin{array}{r}1.3000 E+00 \\
1.6703 E+00 \\
2.0513 E-01 \\
-6.1538 E-02\end{array}$ & $\begin{array}{r}\times 2 \\
-\times 2 \\
: \times 2 \\
-\times 2 \\
-\times 2\end{array}$ & $-7.45928=02$ & 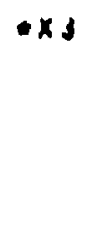 \\
\hline $0(635)+0(315)=$ & 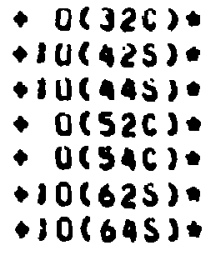 & $\begin{array}{r}-1.1014 E+02 \\
-2.2028 E+01 \\
-4.4056 E+01 \\
2.2615 E+02 \\
-2.2615 E+02 \\
-4.6364 E+00 \\
-9.2727 E+00\end{array}$ & $\begin{array}{r}6.03156+01 \\
6.10896+00 \\
1.23786+01 \\
-3.12316+01 \\
3.12316+01 \\
2.72738-01 \\
5.45456-01\end{array}$ & 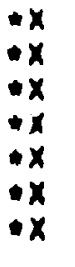 & $\begin{array}{r}-1.035 U E+01 \\
-4.1958 E-01 \\
-8.3916 E-01 \\
6.1538 E-01 \\
-6.1536 E-01\end{array}$ & $\begin{array}{l}-\times 2 \\
\times \times 2 \\
\times \times 2 \\
\times \times 2 \\
\times \times 2\end{array}$ & $5.5944 E-01$ & 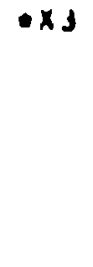 \\
\hline $0(635)+U(325)=$ & $\begin{array}{l}-O(31(6): \\
+10(415): \\
00(556): \\
+10(615): \\
+10(055)\end{array}$ & $\begin{array}{r}-1.1014 E+01 \\
2.2020 E+01 \\
1.1300 E+01 \\
-7.1182 E+00 \\
4.0000 E+01\end{array}$ & $\begin{array}{r}6.03156+00 \\
-6.18886+00 \\
-1.5615 E+00 \\
.1 .3636 E-01 \\
-2.40006+00\end{array}$ & $\begin{array}{l}* x \\
0 x \\
0 x \\
* x \\
x \\
x\end{array}$ & $\begin{array}{r}-1.03506 \bullet 00 \\
1.19546 \bullet 01 \\
3.07696-02\end{array}$ & $\begin{array}{l}: \times 2 \\
: \times 2 \\
\times \times 2\end{array}$ & $5.5944 E=02$ & $\cdot x J$ \\
\hline $0(635)+0(335)=$ & $\begin{array}{l}+O(30 C): \\
-O(506): \\
+10(665)=\end{array}$ & $\begin{array}{l}-1.4685 E+01 \\
-1.5077 E+01 \\
-1.0200 E+01\end{array}$ & $\begin{array}{l}8.0420 E+00 \\
2.0021 E+00 \\
6.0000 E=01\end{array}$ & $\begin{array}{l}: x \\
: x \\
: x\end{array}$ & $\begin{array}{l}-1.3800 E+00 \\
-4.1026 E-02\end{array}$ & $\begin{array}{l}: \times 2 \\
-\times 2\end{array}$ & $7.45925 \cdot 02$ & $\cdot \times 3$ \\
\hline $0(645)+0(325)=$ & $\begin{array}{l}+0(336): \\
+10(435): \\
+0(536) \\
+0(556) \\
+10(635) \\
+10(655)\end{array}$ & $\begin{array}{r}-6.1109 E+02 \\
-3.6719 E+02 \\
6.2021 E+01 \\
-1.1308 E+02 \\
3.0904 E+02 \\
-2.0400 E+02\end{array}$ & $\begin{array}{r}3.3508 E+01 \\
1.0315 E+02 \\
-8.6752 E+00 \\
1.5615 E+01 \\
-1.61025+00 \\
1.2000 E+01\end{array}$ & $\begin{array}{l}: x \\
: x \\
: x \\
: x \\
: x \\
: x\end{array}$ & $\begin{array}{r}-5.74906+00 \\
-6.89306+00 \\
1.70946-01 \\
-3.07646-01\end{array}$ & $\begin{array}{l}-\times 2 \\
: \times 2 \\
1 \times 2 \\
1 \times 2\end{array}$ & $3.1080 E=01$ & $\cdot x s$ \\
\hline $0(645) * 0(325)=$ & $\begin{array}{r}0(326): \\
+10(425): \\
+0(526) \\
+10(625): \\
10(665)\end{array}$ & $\begin{array}{r}-7.3427 E+01 \\
1.3605 E+01 \\
7.5305 E+01 \\
-1.3909 E+01 \\
1.7000 E+01\end{array}$ & $\begin{array}{r}4.0210 E+01 \\
-4.1259 E+00 \\
-1.0410 E+01 \\
8.1010 E-01 \\
-1.0000 E+00\end{array}$ & $\begin{array}{l}: x \\
: x \\
: x \\
: x \\
x \\
x\end{array}$ & $\begin{array}{r}-6.0990 E+00 \\
2.7972 E \cdot 01 \\
2.0513 E \cdot 01\end{array}$ & $\begin{array}{l}: \times 2 \\
1 \times 2 \\
1 \times 2\end{array}$ & $3.7296 E-01$ & $\cdot \times 3$ \\
\hline $0(645) \bullet 0(335)=$ & $\begin{array}{l}+3(316): \\
+10(415) \\
+0(516): \\
+10(615) .\end{array}$ & $\begin{array}{r}-3.6713 E+01 \\
1.3217 E+02 \\
-7.5355 E+01 \\
3.7091 E+01\end{array}$ & $\begin{array}{r}2.01055+01 \\
-3.71335 * 01 \\
1.0410 E+01 \\
-2.18106+00\end{array}$ & $\begin{array}{l}: x \\
: x \\
: x \\
: x\end{array}$ & $\begin{array}{r}-3.44995+00 \\
2.5175 E+00 \\
-2.0513 E \cdot 01\end{array}$ & $\begin{array}{l}: \times 2 \\
: \times 2 \\
\times \times 2\end{array}$ & $1.06408-01$ & $\times x_{3}$ \\
\hline $0(65 s)+0(315)=$ & $\begin{array}{l}+10(445)= \\
+10(645)= \\
+10(665)=\end{array}$ & $\begin{array}{r}-3.6713 E+01 \\
9.82 .27 E+00 \\
-1.7000 E \bullet 01\end{array}$ & $\begin{array}{r}1.0315 E+01 \\
-5.4545 E=01 \\
1.0000 E+00\end{array}$ & $\begin{array}{l}: x \\
: x \\
: x\end{array}$ & $-6.99308 \cdot 01$ & $\times 2$ & & \\
\hline
\end{tabular}




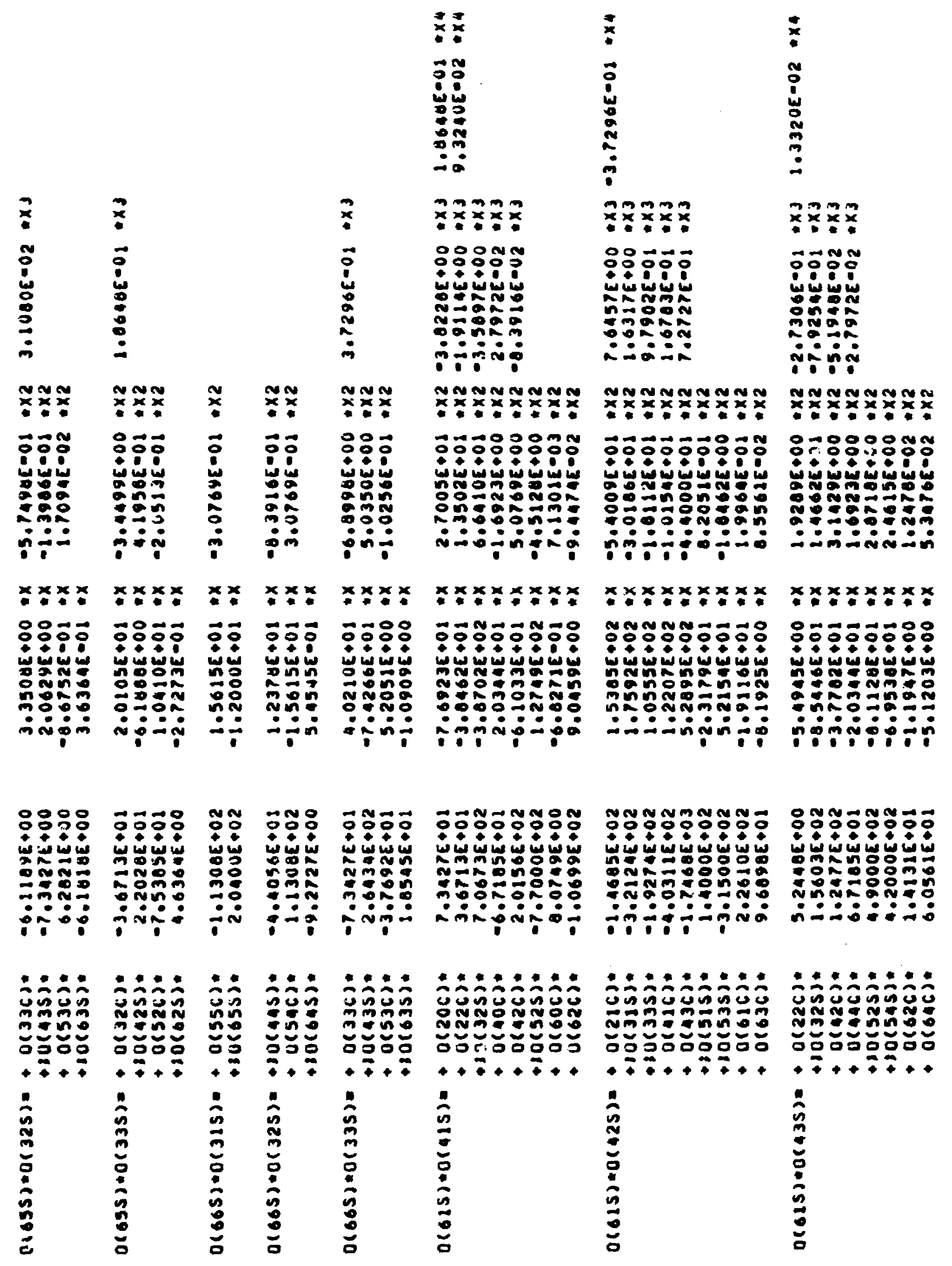




\begin{tabular}{|c|c|c|c|c|c|c|c|c|c|c|}
\hline $0(615)+0(445)=$ & $\begin{array}{l}+10(335): \\
+0(43 C): \\
+10(535): \\
+0(555) \\
+0(63 C) \\
+0(65 C)\end{array}$ & $\begin{array}{l}-3.6713 E+01 \\
-3.3700 E+02 \\
-2.4500 E+02 \\
-1.0500 E+02 \\
-3.3914 E+02 \\
-5.3294 E+02\end{array}$ & $\begin{array}{l}2.0105 E+02 \\
1.6276 E+02 \\
1.0564 E+01 \\
1.7385 E+01 \\
2.8674 E+01 \\
1.5059 E+01\end{array}$ & $\begin{array}{l}x \\
: x \\
: x \\
: x \\
: x \\
: x\end{array}$ & $\begin{array}{l}-3.0499 E+00 \\
-1.3530 E+01 \\
-1.4350 E+00 \\
-6.1530 E=01 \\
-2.9947=01 \\
-4.7059 E-01\end{array}$ & 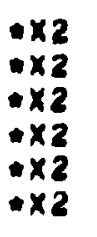 & $\begin{array}{l}1.06485-01 \\
2.2378 E-01\end{array}$ & $: \times 3$ & & \\
\hline $0(625) \cdot 0(415)=$ & 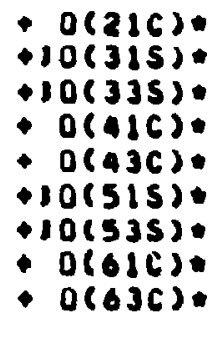 & $\begin{array}{r}2.3497 E+02 \\
5.1399 E+01 \\
2.5699 E+02 \\
-5.3798 E+02 \\
1.6124 E+03 \\
7.0000 E+01 \\
3.5000 E+01 \\
1.7119 E+02 \\
-3.1492 E+02\end{array}$ & 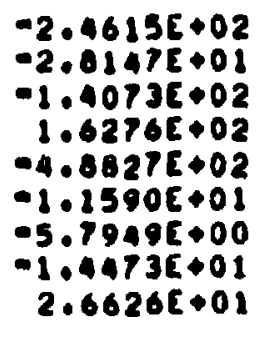 & $\begin{array}{l}* x \\
* x \\
* x \\
* x \\
* x \\
: x \\
* x \\
* x \\
x\end{array}$ & $\begin{array}{r}8.6415 E+01 \\
4.0290 E+00 \\
2.41496+01 \\
-1.3530 E+01 \\
4.0615 E+01 \\
1.1026 E=01 \\
2.0513 E-01 \\
1.51166-01 \\
-2.7007 E-01\end{array}$ & 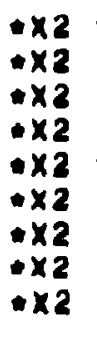 & $\begin{array}{r}-1.2233 E+01 \\
-2.6107 E-01 \\
-1.3054 E+09 \\
2.2378 E-01 \\
-6.7133 E-01\end{array}$ & $\begin{array}{l}: \times 3 \\
0 \times 3 \\
0 \times 3 \\
0 \times 3 \\
0 \times 3 \\
03\end{array}$ & $5.9674 E-01$ & $\cdot \times 4$ \\
\hline $0(625) \cdot 0(425)=$ & 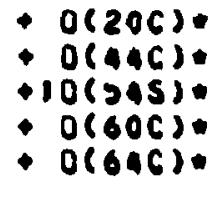 & $\begin{array}{r}1.1748 E+02 \\
-5.3748 E+02 \\
-2.3100 E+03 \\
-3.5529 E+01 \\
-1.1143 E+02\end{array}$ & $\begin{array}{r}-1.2300 E+02 \\
1.6276 E+02 \\
3.0246 E+02 \\
3.0039 E+00 \\
9.4214 E+00\end{array}$ & $\begin{array}{l}: x \\
: x \\
: x \\
x \\
x \\
x\end{array}$ & $\begin{array}{r}1.3207 E+01 \\
-1.3538 E+01 \\
-1.3538 E+01 \\
-3.1373 E-02 \\
-9.8396 E-02\end{array}$ & $\begin{array}{l}\times 2 \\
1 \times 2 \\
1 \times 2 \\
1 \times 2 \\
\times 2\end{array}$ & $\begin{array}{r}-6.1166 E \div 00 \\
2.2370 E-01\end{array}$ & $\begin{array}{l}\times 3 \\
\times 3 \\
\times 3\end{array}$ & $2.9037[001$ & $1 \times 4$ \\
\hline $0(625) \cdot 0(435)=$ & 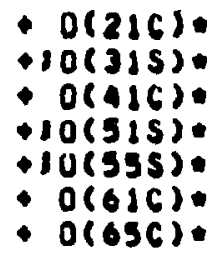 & $\begin{array}{r}-3.3566 E+01 \\
-9.5455 E+01 \\
-2.30355+02 \\
-7.00000+01 \\
1.0500 E+02 \\
2.26100+01 \\
3.7306 E+02\end{array}$ & $\begin{array}{r}3.5165 E+01 \\
3.22735+01 \\
6.97525+01 \\
1.1590 E+01 \\
-1.73 A 5 E+01 \\
-1.9116 E+00 \\
-3.1541 E+01\end{array}$ & $\begin{array}{l}: x \\
x \\
x \\
x \\
x \\
x \\
x \\
x\end{array}$ & $\begin{array}{r}-1.23456+01 \\
-8.9697 E+00 \\
-5.0022 E+00 \\
-9.1026 E-01 \\
6.1536 E-01 \\
1.9964 E-02 \\
3.2941 E-01\end{array}$ & 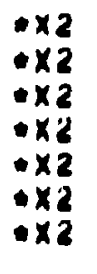 & $\begin{array}{l}1.7476 E+00 \\
1.8485 E=01 \\
9.5904 E=02\end{array}$ & $\begin{array}{l}-\times 3 \\
0 \times 3 \\
0 \times 3\end{array}$ & $-0.5240 E-02$ & $1 \times 4$ \\
\hline $0(625) \cdot 0(445)=$ & $\begin{array}{l}0(226) \\
+10(325) \\
+\quad 0(42 C) \\
+0(525) \\
+0(62 C) \\
+0(66 C)\end{array}$ & $\begin{array}{r}0.3916 E+00 \\
2.9371 E+02 \\
3.0713 E+02 \\
1.9600 E+03 \\
2.2610 E+02 \\
-1.0659 E+02\end{array}$ & $\begin{array}{l}-4.1912 E+00 \\
-1.6004 E+0 ? \\
-9.3003 E+01 \\
-3.2451 E+02 \\
-1.91165+01 \\
9.0118 E+00\end{array}$ & $\begin{array}{l}x \\
x \\
x \\
x \\
x \\
x \\
x \\
x\end{array}$ & $\begin{array}{r}3.08626 \cdot 00 \\
2.75096 \bullet 01 \\
1.73630000 \\
1.14676001 \\
1.99646 \cdot 01 \\
-9.41105 \cdot 02\end{array}$ & $\begin{array}{l}-\times 2 \\
* \times 2 \\
-\times 2 \\
-\times 2 \\
-\times 2 \\
-\times 2\end{array}$ & $\begin{array}{l}-4.3690 E-01 \\
-1.09188 .00 \\
-1.2787 E-01\end{array}$ & $\begin{array}{l}x_{3} 3 \\
x y \\
03\end{array}$ & $2.13126 \cdot 02$ & $\cdot \times 4$ \\
\hline $0(435) \cdot 0(415) 0$ & 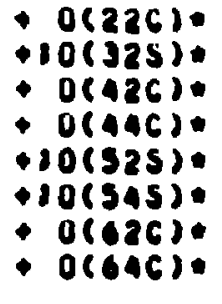 & $\begin{array}{r}4.40566+01 \\
3.85496+02 \\
-1.34376+02 \\
2.60746+02 \\
-4.20006+02 \\
6.30006+02 \\
7.07305+01 \\
-5.0072 E \bullet 01\end{array}$ & $\begin{array}{r}-4.015 A E+01 \\
-2.1110 E+02 \\
4.0682+01 \\
-8.1379 E+01 \\
6.9538 E+01 \\
-1.0431 E+02 \\
-6.05646+00 \\
. .3011 E+00\end{array}$ & $\begin{array}{l}: x \\
: x \\
0 x \\
: x \\
: x \\
: x \\
: x \\
x\end{array}$ & 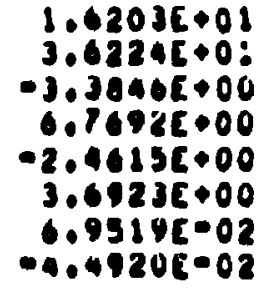 & 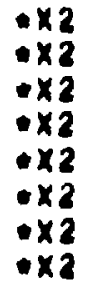 & $\begin{array}{r}-2.29315+00 \\
-1.9580 E \cdot 00 \\
5.34446002 \\
-1.1189 E-01\end{array}$ & $\begin{array}{l}0 \times 3 \\
03 \\
0 \times 3 \\
03 \\
03\end{array}$ & $1.1184[006$ & $\cdot x_{4}$ \\
\hline $0(635) \cdot 0(425) 0$ & 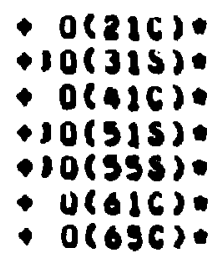 & $\begin{array}{r}1.76226+02 \\
-7.70096+01 \\
-2.68786+02 \\
2.10005+22 \\
-3.15005+02 \\
-3.07595+01 \\
-6.39535+02\end{array}$ & 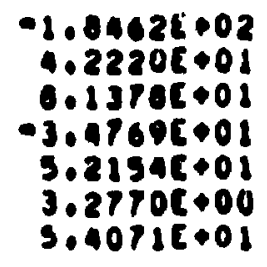 & $\begin{array}{l}: x \\
\bullet x \\
0 x \\
0 x \\
0 x \\
0 x \\
0 x \\
0 x\end{array}$ & $\begin{array}{r}0.40116+01 \\
-7.2940 E+00 \\
-6.7692 E+00 \\
1.2300 E+00 \\
-1.0442 E+00 \\
-3.4225 E-02 \\
-5.6471 E-01\end{array}$ & $\begin{array}{l}1 \times 2 \\
0 \times 2 \\
0 \times 2 \\
1 \times 2 \\
0 \times 2 \\
0 \times 2 \\
02 \\
02\end{array}$ & $\begin{array}{r}-4.17006+00 \\
3.91616-01 \\
1.1180 E-01\end{array}$ & $\begin{array}{l}0 x y \\
0 x \\
0 x \\
0 x\end{array}$ & 4.47556001 & $\times 4$ \\
\hline
\end{tabular}


$2 x \cdot 20030<28 \cdot 1-x+00+32461 \cdot 1$ $c x=50-30260^{\circ}, 2 x+10-31502 \cdot 8 \quad x=10+3611 x^{\circ}: 20$

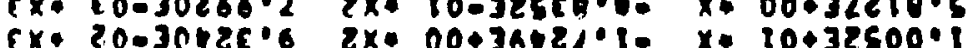

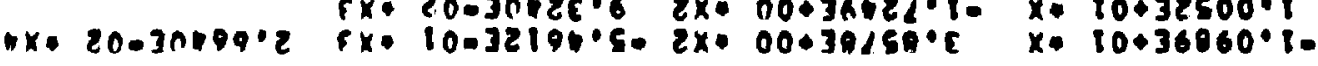

$2 x+20-31955 \cdot 0 \quad x \cdot 00+35261000$ $2 x+10=36150^{2} z-x+00+36462^{\circ} \cdot 5$

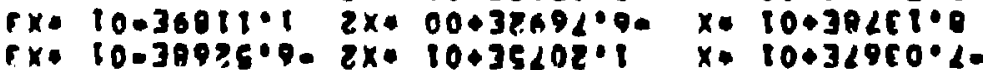

$2 x \cdot 20-36200 \cdot 5 \quad x=00+36259.5$. $2 x \cdot 20.35690 .10 x+00+31620 \cdot 1$

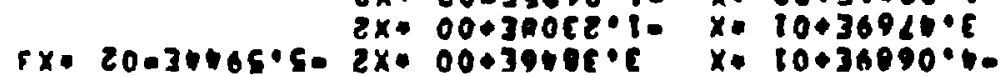

\section{$3 x \cdot 20-30250^{\circ} 8 \quad x=00+30062.80$}

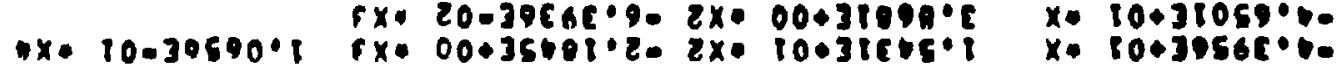

\section{$2 x+20.31061 .20 x+00+31280 \cdot 9$}

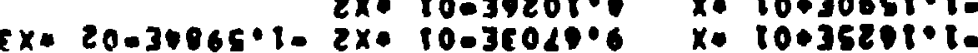
列

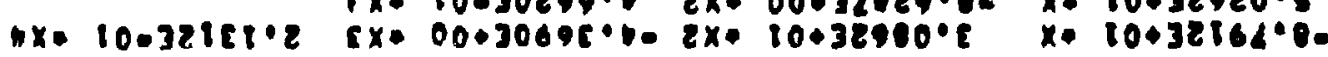

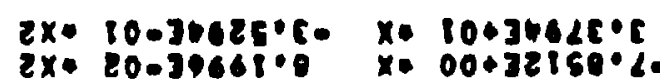

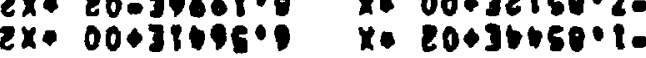

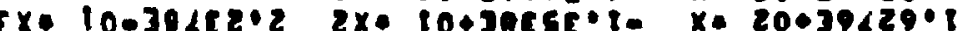

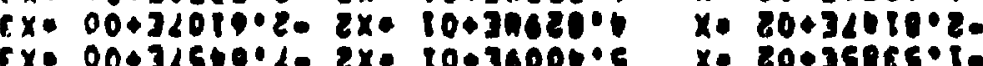

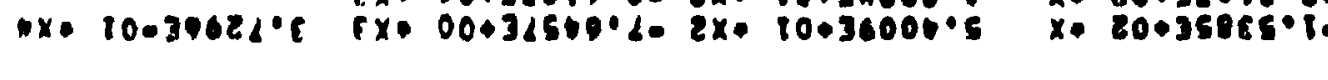

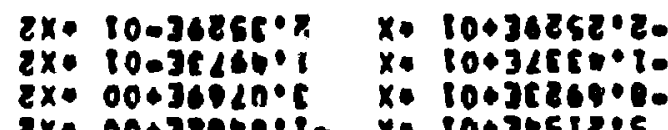

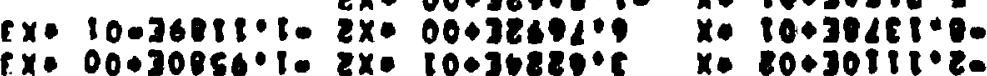

$2 x \cdot 10-36261 \cdot 1 \cdot x+10+30<61 \cdot 1$

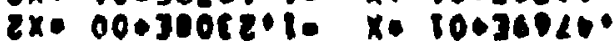

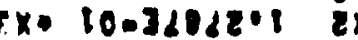

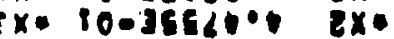

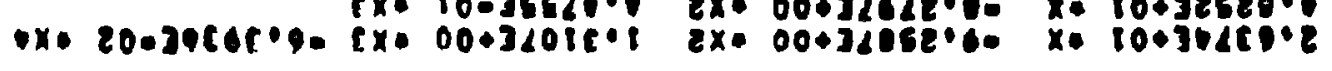

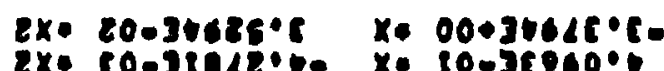

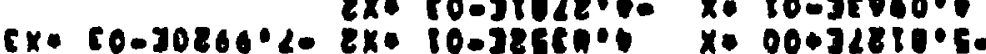

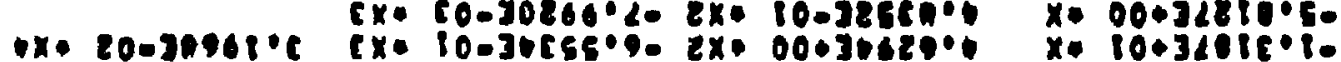

\author{
$10+31610 \cdot 1-$ \\ $20+30000 \cdot 1$ \\ $10+39616 \cdot 1-$ \\ $10+306+0.1$
$10+315600$ \\ $-(029) 0+$ \\ $-(\cos ) 014$ \\ 0.02050 .

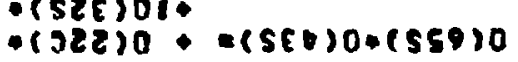 \\ $10+39609 \cdot 6$ \\ (50.300000 \\ $20+30502.10(5 c 5) 01+0(520) 0=(559) 0$ \\ $10+39290 \cdot 9$ \\ $10+3814.10$ \\ $20030001 \cdot 20$ (5+5)014 \\ sn+37ene. 1 \\ $1565) 08$. \\ $10+366220 \mathrm{C} \quad(909) 0$ \\ $20+34565 \cdot 1$
$10+385610$ \\ $10+360<000-\quad 191930$. \\ $10+30000 \% 2 \quad+(515) 010$

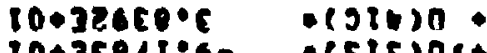 \\ 10+3625\%- isteror: \\ $20+31<60 \cdot 5-\quad *(909) 0$ - \\ ises)o: \\ 20+300, \\ 200306ios \\ $80+35090 \cdot 1$ \\ $-(928) 0 \cdot=(286) 0 \cdot(209) 0$ \\ $20+36099 \cdot 2$ \\ $80+325690$ \\ $20+30052 \cdot 5$ \\ $20+30051 \cdot 6 \cdot$ \\ $80+30489 \cdot 2$ \\ $+1258)$ \\ - cescios: \\ - $\csc (2)$ or. \\ - $(j e \cos 0$ \\ $20+39955 \cdot 10$ \\ - (see)oet $=(816) 0 \cdot(504) 0$ \\ $2003000 t \cdot 2$. \\ $20+361100.6$ \\ $10+38110.06$
$10+36280$ \\ $-(915) 0$. \\ - isterora \\ istion:

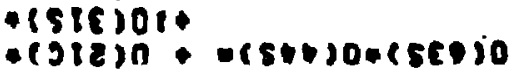 \\ $10+31260.6 \quad 0(29810$. \\ $00036+10 \%$ - $(209) 0$.

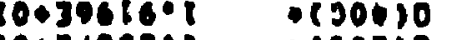 \\ - $(502) 0$ : e(sen)onecseno
}




\begin{tabular}{|c|c|c|}
\hline $0(655) * 0(445)=$ & $\begin{array}{l}0(216): \\
+80(315): \\
+0(416): \\
+10(515): \\
+0(616) *\end{array}$ & $\begin{array}{r}4.1958 E+01 \\
-7.3427 E+01 \\
1.5337 E+02 \\
-7.0000 E+01 \\
3.2294 E+01\end{array}$ \\
\hline $0(665) \cdot 0(415)=$ & $\begin{array}{l}+10(555) . \\
+0(650)\end{array}$ & $\begin{array}{r}3.1500 E+02 \\
-7.9941 E+02\end{array}$ \\
\hline $0(665) \cdot 0(425)=$ & $\begin{array}{l}+O(44 C) \\
+20(54 S) \\
+\quad O(64 C)\end{array}$ & $\begin{array}{r}5.3748 E+02 \\
-1.8900 E+03 \\
2.1802 E+02\end{array}$ \\
\hline $0(665) \cdot 0(435)=$ & $\begin{array}{l}+80(335)= \\
+0(43 C) \\
+20(535) \\
+0(63 C)\end{array}$ & $\begin{array}{r}1.1014 E+02 \\
-5.374 d E+02 \\
1.0500 E+02 \\
-7.2674 E+01\end{array}$ \\
\hline $0(665)=0(44 S)=$ & $\begin{array}{l}+0(226): \\
+10(325) \\
+\quad U(426) \\
+30(525) \\
+\quad 0(62 C)\end{array}$ & $\begin{array}{r}1.2587 E+02 \\
-0.6112 E+02 \\
3.0713 E+02 \\
-8.4000 E+02 \\
4.8449 E+01\end{array}$ \\
\hline $0(615)+0(515)=$ & $\begin{array}{r}+0(10 C): \\
+10(22 S) \\
+0(30 C) \\
+0(32 C) \\
+10(425) \\
+0(50 c) \\
+0(52 C) \\
+10(625)\end{array}$ & $\begin{array}{r}-1.3217 E+02 \\
-6.6084 E+02 \\
1.4392 E+03 \\
-4.3175 E+03 \\
9.1636 E+02 \\
-3.6552 E+02 \\
8.7125 E+03 \\
-2.6037 E+02\end{array}$ \\
\hline $0(615)+0(525)=$ & 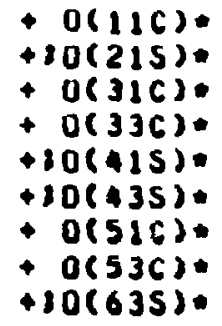 & $\begin{array}{r}9.4406 E+00 \\
2.8322 E+02 \\
1.5420 E+02 \\
3.5979 E+02 \\
-3.6000 E+02 \\
1.6036 E+03 \\
-3.1330 E+02 \\
-4.5690 E+01 \\
-3.9056 E+02\end{array}$ \\
\hline
\end{tabular}

$-4.3956 E+01 \times x \quad 1.5431 E+01 * \times 2-2.1845 E+00 \times x 3 \quad 1.0656 E-01 * \times 4$

$4.0210 E+01 * x-6.0990 E+00 \times \times 2 \quad 3,7296 E-01 * x 3$

$0 x \quad 3.0682 E+00: \times 2-6.3936 E-02 * x]$

$1.1590 E+01 * x \quad-1.10261001: \times 2$
$-2.7308 E+00 * x \quad 2.8520 E=02 \div \times 2$

$-5.2154 E+01 * x \quad 1.8462 E+00 * \times 2$

$6.7580 E+01 * x-7.0580 E=01 * x 2$

$-1.6276 E+02 \div x \quad 1.3530 E+01 * x 2-2.2378 E-01 * x J$

$3.1292 E+02 * x-1.1077 E+01 * x 2$

$1.84336+01$

$-6.0315 E+01 * x \quad 1.0350 E+01 * \times 2-5.5944 E=01 * x 3$

$1.6276 E+02 * x-1.3530 E+01 * \times 2 \quad 2.2378 E-01 * x J$

$1.7365 E+01 * x \quad 0.1534 E=01 * \times 2$
$6.1444 E+00 * x \quad-6.4171 E-02 * x 2$

$-1.3107 E+02 * x \quad 4.0294 E+01 * \times 2-6.5534 E+00 * x 3 \quad 3.1960 E-01 * \times 4$

$4.0252 E+02 * x-8.2797 E+01 \times x 2 \times 0.455 E+00 \times x$

$-9.3003 E+01 * x \quad 1.7363 E+00: \times 2-1.2787 E-01: \times 1$

$1.3908 E+02 * x \quad-4.9231 E+00 * x 2$

$963 E \bullet 00: x$

$1.27021-02 * \times 2$

$3.1469 E+02 * x-2.3322 E+02 * \times 2 \quad 7.1692 E+01 * x 3-9.5105 E+00 * \times 4 \quad 4.4755 E-01 * \times 3$

$6.9231 E+02 * x-2.4304 E+02 * \times 2 \quad 3.4406 E+01 * \times 3-1.6703 E+00 * \times 4$

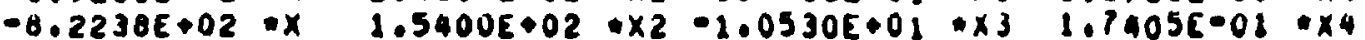

$2.4671 E+03+x-4.6200 E+02 * x 2-3.1590 E+01 * x 3-5.2214 E-01 * x 4$

$-3.0445 E+02: x \quad 3.0657 E+01 * x 2-8.0510 E-01 * x_{3}$

$7.1125 E+01 * x-3.6360 E+00 \times 2 \quad 3.2177 E-02: x 3$

$-1.7070 E+03 * x \quad 0.7264 E+01 * x 2-7.7225 E-01 * x y$

$2.8717 E+01 * x-6.4171 E-01 . \times 2$

$-2.2478 E+01 * x \quad 1.6659 E+01 * \times 2-5.1209 E+00 * x_{3} \quad 6.79325-01 * \times 4-3.1968 E-02 * \times 5$

$-2.9670 E+02 * x \quad 1.0416 E+02 \times x^{2}-1.1745 E+01 * x_{3} \quad 7.2920 E-08 * x_{4}$

$1.6500 E+01 * \times 2-1.1282 E+00 * \times 3 \quad 1.13640 E-02 * \times 4$

$-2.0559 E+02 * x \quad 3.8500 E+01 * x 2-2.6325 E+00 * x 3 \quad 4.3512 E-02 \times 44$

$1.1960 E+02 \times x-1.2044 E+01+x 2-3.5165 E-01: x J$

$-5.3278 E+02 * X \quad 5.3650 E+01 * \times 2-1.5664 E+00 * X 3$

$6.0960 E+01 * x-3.1166 E+00 * \times 2 \quad 2.7580 E=02 * X J$

$0.0906 E+00 * x-9.5450 E-01 * x 24.0221 E=03 * x 3$

$4.3075 E+01 * x \quad-9.6257 E-01 * \times 2$

$+0(536)$
$+10(635)$ 


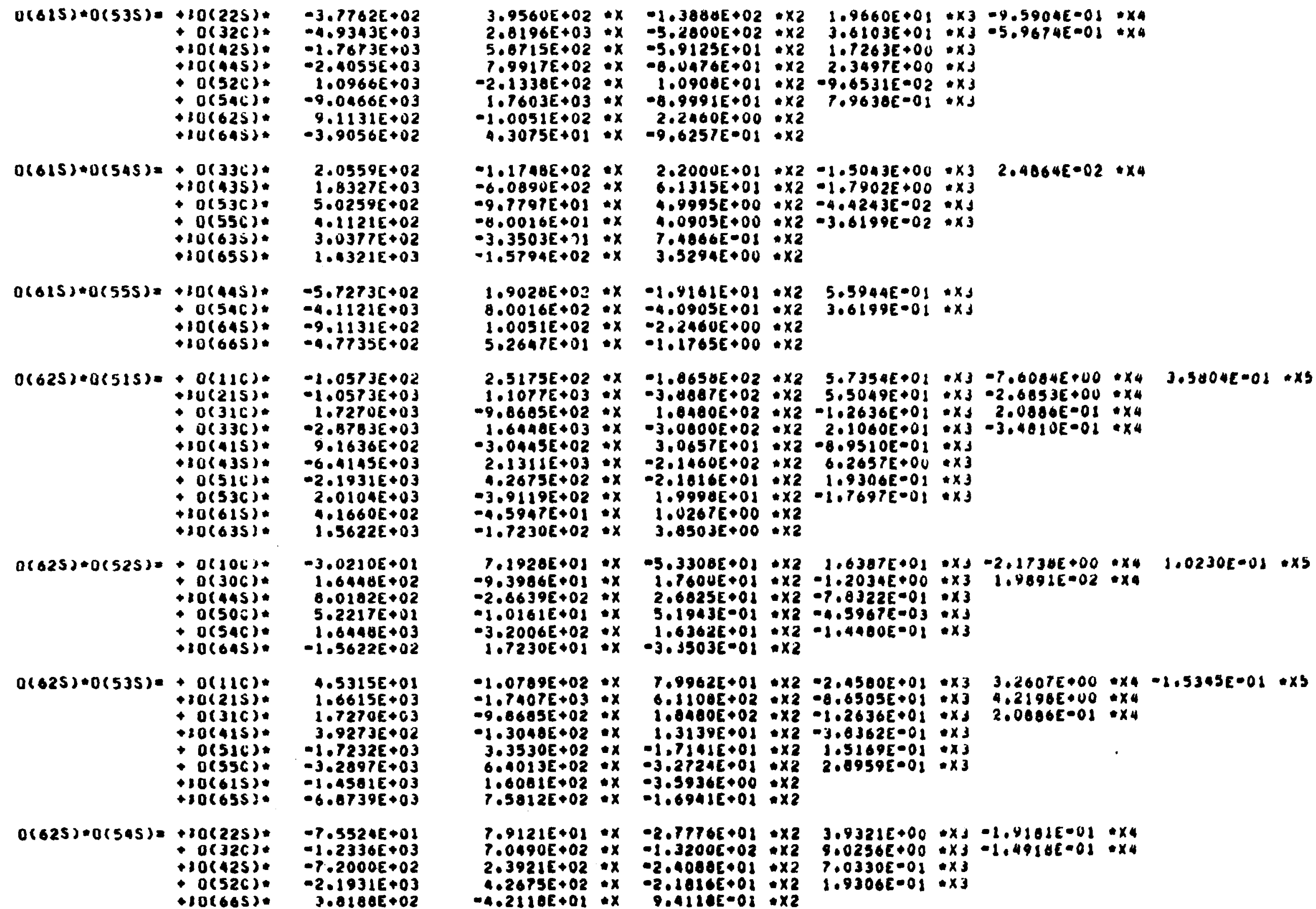

- $0(116)$.

$\rightarrow 30(215)$

- O(33C).

$+10(415)$.

$+30(435)$

$0(510)$.

- $0(536)$.

$+10(615)$.

$-1.0573 E+02$

$-1.05735+03$

$-1.7270 E+03$
$-1.0503 E+03$

$-2 . B 7 B J E+03$

$9.1636 E+02$

$-6.4145 E+03$

$-2.1931 E+03$

$2.0104 E+03$

$4.1660 E+02$

$1.5622 E+03$

$0(825)+0(525)=0(100)$

$0(306)$

$-3.0210 E+02$

$1.6448 E+02$

$0.0182 E+02$

$1.6446 E+03$

$+10(045)$.

$-1.5622 E+02$

$0(625) * 0(535)=+0(116)$

$4.5315 E+01$

$10(215)$ 1.6615E+0

$+0(31 C) \quad 1.7270 E+C 3$

$20(415)$

$3.9273 E+02$

$-1.7232 E+03$

$-1.205015+03$

+306655 : $-6.0739 E+03$

$0(625) \cdot 0(545)=+10(225)$

$0(32()$.

$-7.5524 E+01$

10(42s).

$-1.2336 E+03$

- Ocsecs.

$-7.2000 E+02$
$-2.1931 E+03$

$3.0180 E+02$

$3.9560 E+02 * x-1.3804 E+02 * x 2 \quad 1.9660 E+01 * x 3=9.5904 E=01 * x$ $2.8196 E+03 * x-5.2800 E+02 * \times 2 \quad 3.6103 E+02 * x 3-5.9674 E-01 * x$ $5.6715 E+02 * x-5.9125 E+01 * x 21.7263 E+00 * x 3$ $7.9917 E+02$ *X $-6.0476 E+01$ *X2 $2.3497 E+00 * x J$ $1.7603 E+03 * x \quad-8.9991 E+01: x 2 \quad 7.9638 E-01: x J$ $1.0051 E+02: x \quad 2.2060 E+00 * x 2$
$4.3075 E+01: x \quad-9.6257 E+01 * x 2$

$-1.1748 \varepsilon+02 \div x$ $-6.0890 E+02+x$ $-9.7797 E+01 * x$
$-8.0016 E+01 * x$ $-8.0016 E+01: x$ $-3 \cdot 3503 E+71: x$
$-1.5794 E+02$

$2.2000 E+01 \times 2-1.5043 E+00 \div \times 3$ $6.13155+0\}$ : $x 2-1.7902 E+00: x 3$ $4.9995 E+00$ : $x 2$ - 40.42438002 axj $1.0905 E+00=\times 2-3.6199 E-02+x 3$ $3.5294 E+00 \times 2$

$-1.9161 E+01+\times 2 \quad 5.5944 E=01 * x J$

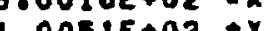
$5.2647 E+01 \div x-1.1765 E+00 \times x 2$

$2.5175 E+02 * x$

$-1.0656 E+02 * \times 2 \quad 5.7354 E+02 \times x_{3}-7.0084 E+00 * x_{4} \quad 3.5804 E-01 * \times 5$

$1.1077 E+03 * x-3.4087 E+02 * \times 2 \quad 5.5049 E+01 \times x-2.6853 E+00 * \times 4$

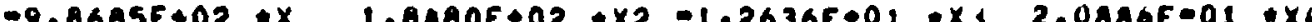

$1.6440 E+03=x-3000005002=x 2=2010005+01: x 3-3.40105001 \times x^{4}$

$-3.0445 E+02 * x \quad 3.0657 E+01 * x 2-8.9510 E=01 * x$

$2.131 \delta E+03: x-2.1460 E+02 * x 26.2657 E+00 * x 3$

(1.

$0.9119 E+02 * x \quad 1.9990 E+01 * x 2-1.76972=01 * x 3$

$\begin{array}{ll}-1.5947 E+01 * x \quad 1.0267 E * 00 & * \times 2 \\ -1.7230 E+02 * x \quad 3.8503 E+00 * \times 2 & \end{array}$

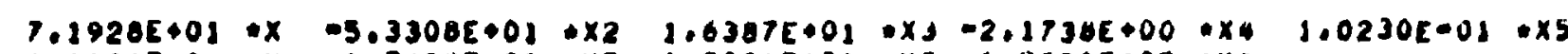
$-9.3906 E+01 * x \quad 1.7600 E+01 * x 2-1.2034 E+00 * x 3$

$-1.01815+01$ : $x$

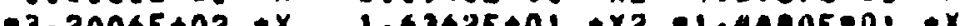
$1.7230 E+01: x-3.3503 E=01: \times 2$.

$-1.07895+02+x$

$.0789 E+02 * x$

$7.9962 E+01 * \times 2-2.4500 E+01 \div \times 3$

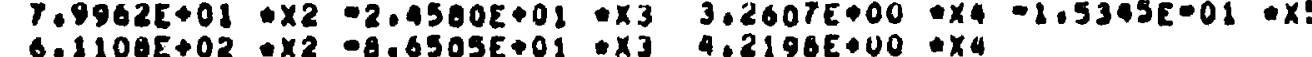
$1.0480 E+02 \times x=1.2636 E+01$ *x $2.0086 E-01 * x 4$

$3.3530 E+02 * x-1.7101 E+01 * x 2 \quad 1.5169 E-01 * x 3$

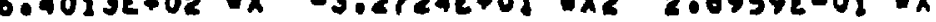

$\begin{array}{lll}1.6001 E+02: x & -3.5936 E+00 & \times x 2 \\ 7.5812 E+02 * x & -1.6941 E+01: \times 2\end{array}$

$7.9121 E+01 \div x-2.7176 E+01+x 2$

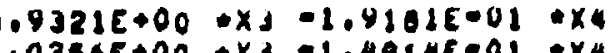

$9.0250 E+00 \times x 3-1.49100$

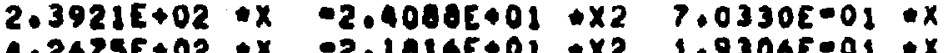

$-4.211 \theta E+02$ \& $x$ Q.4110E-01 $0 \times 2$ 


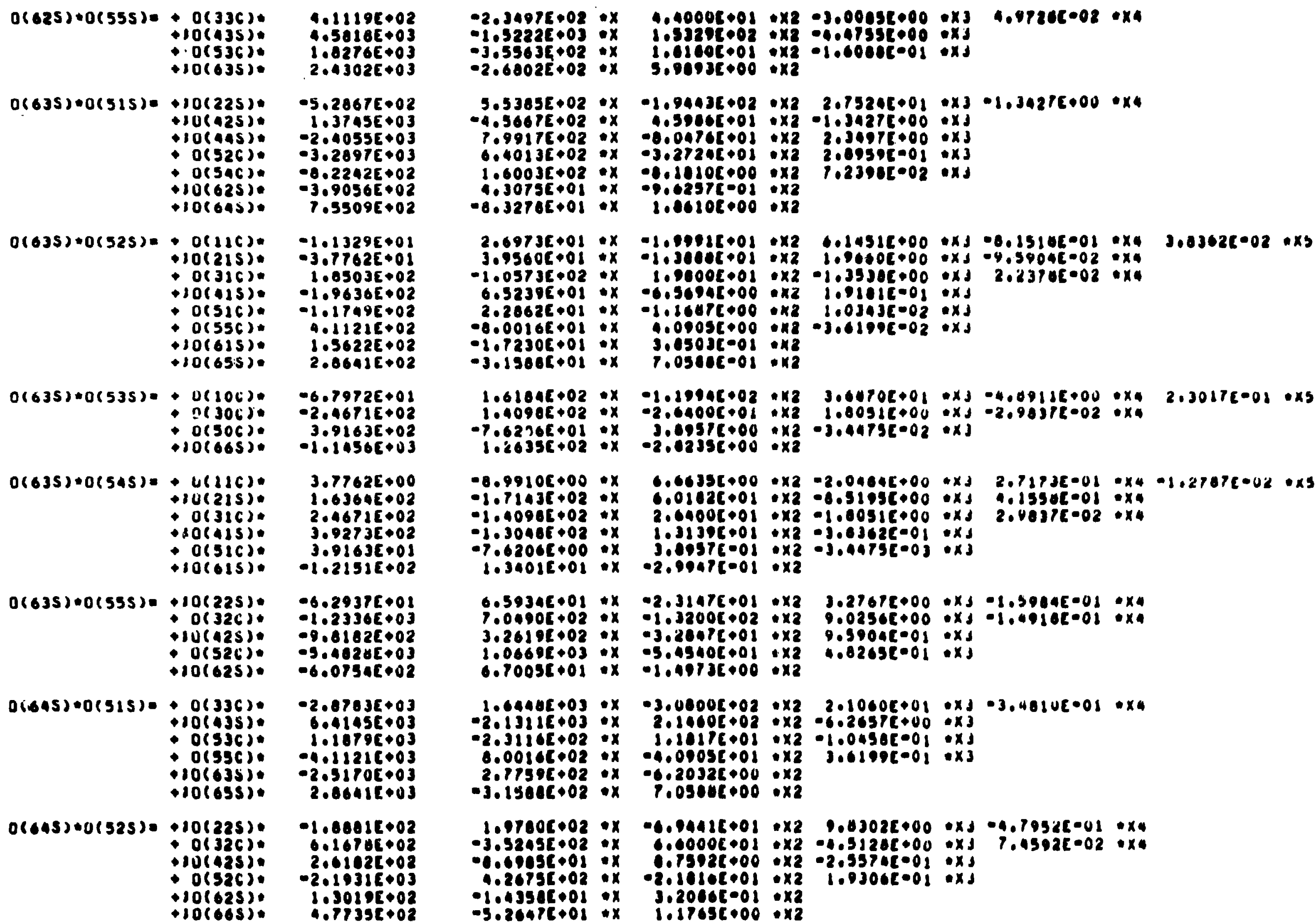

$0(625)+0(555)=0(336) 0$ $10(435)$. $+10(635)$

1.11196002 $4.5816 E+03$ $2.4302 E+03$

$0(635) \cdot 0(515)=+10(225)$ $+10(425)$. - io(atas).

- $0(526)$.

- O(sac).

$+10(645)$.

$O(635) * 0(325)=\quad O(11 C)$

$10(215)$.

- O(316)

$+10(115)$.

$+0(516)$

O $0(556)$.

$+10(655)$

$0(635)+0(535)=+U(10()$

- n(30c)

- O(506).

$-10(665)$

$0(635)+0(545)=+u(116)$.

$+i 0(215)$.

o(3ic).

- $0(415)$.

$+10(015)$.

$-5.2867 E+02$

1.37ASE+03

$-2.4055 E+03$

$-3.2897 E+03$

$-8.2242 E+02$

$-3.9056 E+02$
$7.5509 E+02$

$-1 \cdot 1329 E+01$

$-3.77625+01$

$1.0503 E+02$

$-1.9636 E+02$

$1.1749 E+02$

$0.11216+02$

$1.5622 E+02$

$2.0641 E+02$

$-6.79726+02$

$-2.4671 E+02$

$3.9163 E+02$

$-1.10565+03$

3.7762E+00

$1.6364 E+02$

$2.1671 E+02$

3.92732002

$3.9163 E+01$
$-1.2151 E+02$

$0(635) \cdot 0(555)=+10(225) \cdot$

O O(326).

1uchess.

- Orses.

$-6.29375001$

$-1.23365+03$ 0

- 961026002

-

$-2.0703 E+03$

$0(645) \div 0(315)=+0(336)=$

$+10(435)$

- O(s)

$+10(635)$

$6.41456+03$

1.18rectos

- ilizictos

a.siroctos

$+10(655)$.

2.8641[00]

$0(445) \cdot 0(525)=\cdot 10(225)$ O(32) o(52C). orsas. $+10(625):$

$1.0801 E \bullet 02$ $0.1670 E+02$ $2.61025+02$ $-2.19316003$ $1.30192 \bullet 02$
$4.7735 t \mapsto 02$

$-2.34975+02$ :x $-1.52225003 \bullet x$ $-3.59635+02: x$

5.5305E.02 $0 x$

$5.5365 E+02$
$-4.5667 E+02: x$ 7.99176002 : $x$ $6.40135+02$ : $x$ $1.60036+02$ :x $1.30756+01: x$
$-0.32785+01: x$

2.69735 .001 : $x$ $3.95606+01 * x$ -1.05736 .02 \&x

$6.52396+01$ ix

2.26625001 : $x$

$-8000165+01$ ix

$-1.72306+01$ : $x$
$-3.15806+01 * x$

$1.61846+02 * x$ $-1.40906+02$ :x $-7.62366+01$
$1.26356+02$$* x$

$1.26356+02$ : $x$

$-8.99106+00$ *x $-1 \cdot 71436+02$ : $x$ - $100905+02$ :x $1.30405+02$ :x

$-7.62068+00$ : $x$ $103401 E+01$ : $x$

$6.59346+01 * x$ $8.00906+02$ : $x$ $1.0669 E+03$ ix $6.7005 e+01 \cdot x$

$1.0440[+0]=x$ $-2.13116+03 \cdot x$ $2.31162+02$ : $x$ $2.17506+02$ :x $-3.158 c c+02$ ox

$1.0780 c \cdot 02 * x$ $-3.52455002: x$ - $0.60056+02$ : $x$ 0.26755002 :x $-1.0350[001$ ax -5.2047601 ix

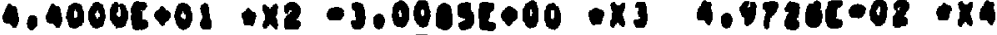

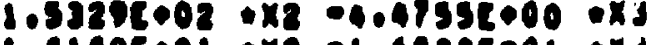

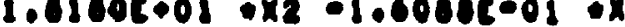

$5,9083 E \bullet 00 \times \times 2$

$-1.94435002 \cdot \times 2 \quad 2.75245001 * x 3 \cdot 1.34275000 \cdot x$

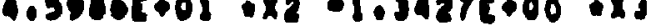

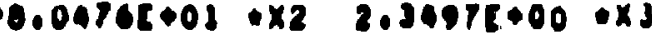
-

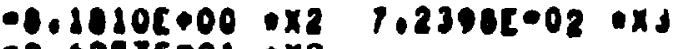

$\begin{array}{lll}1.06106+00 & 0 \times 2\end{array}$

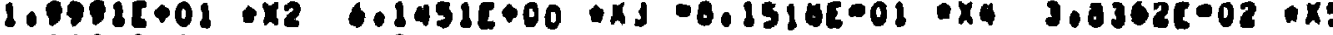

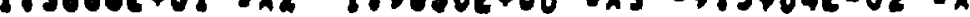

$1.90002001 \cdot \times 2-1.3530[+00 * x] 2.2370[-02 * x$

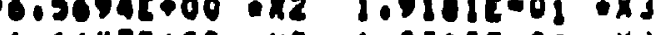

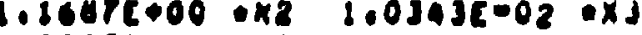

$4.09056+00$-12 $-3.41996-02$ ०xJ

$3,05036-01$ ine

r.0580e-01 : Ka

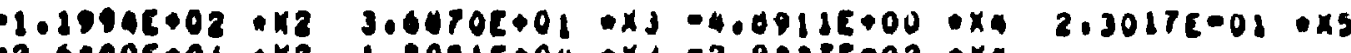

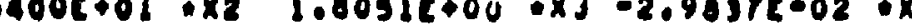

$3.0957(+00, \times 2-3.4475[-02 * x]$

$6.66355+00 \cdot \times 2-2.04046+00$ *x $2.7173 E-01 \quad \times 4-1.27076-02$ ox

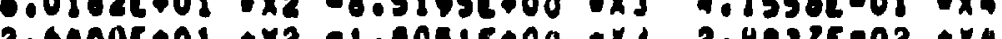

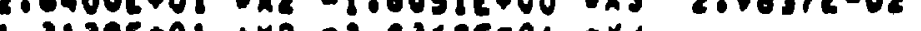

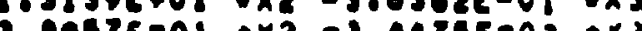

$2.09475010 \times 2$

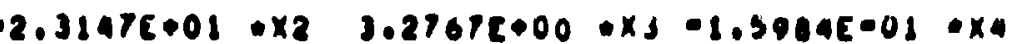

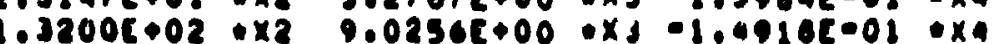

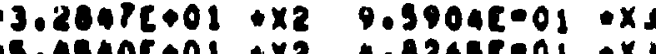

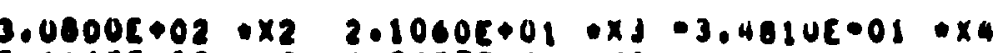

$2.1060[002 \div \times 2-6.2658[+00 \div x]$

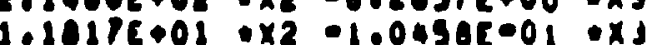

$0.0905[001: x 2 \quad 3.6190[-01: x 3$

$0.20325000 \cdot x 2$

$-0.04416+02 \times x_{2} \quad 9.03025000$ ox $-4.79525-01 * x_{4}$

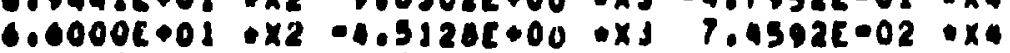

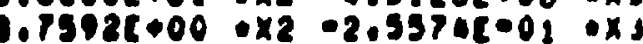

0.10102001 : $\times 2 \quad 1.030050010 x d$

$3.20605001 \times \times 2$ 


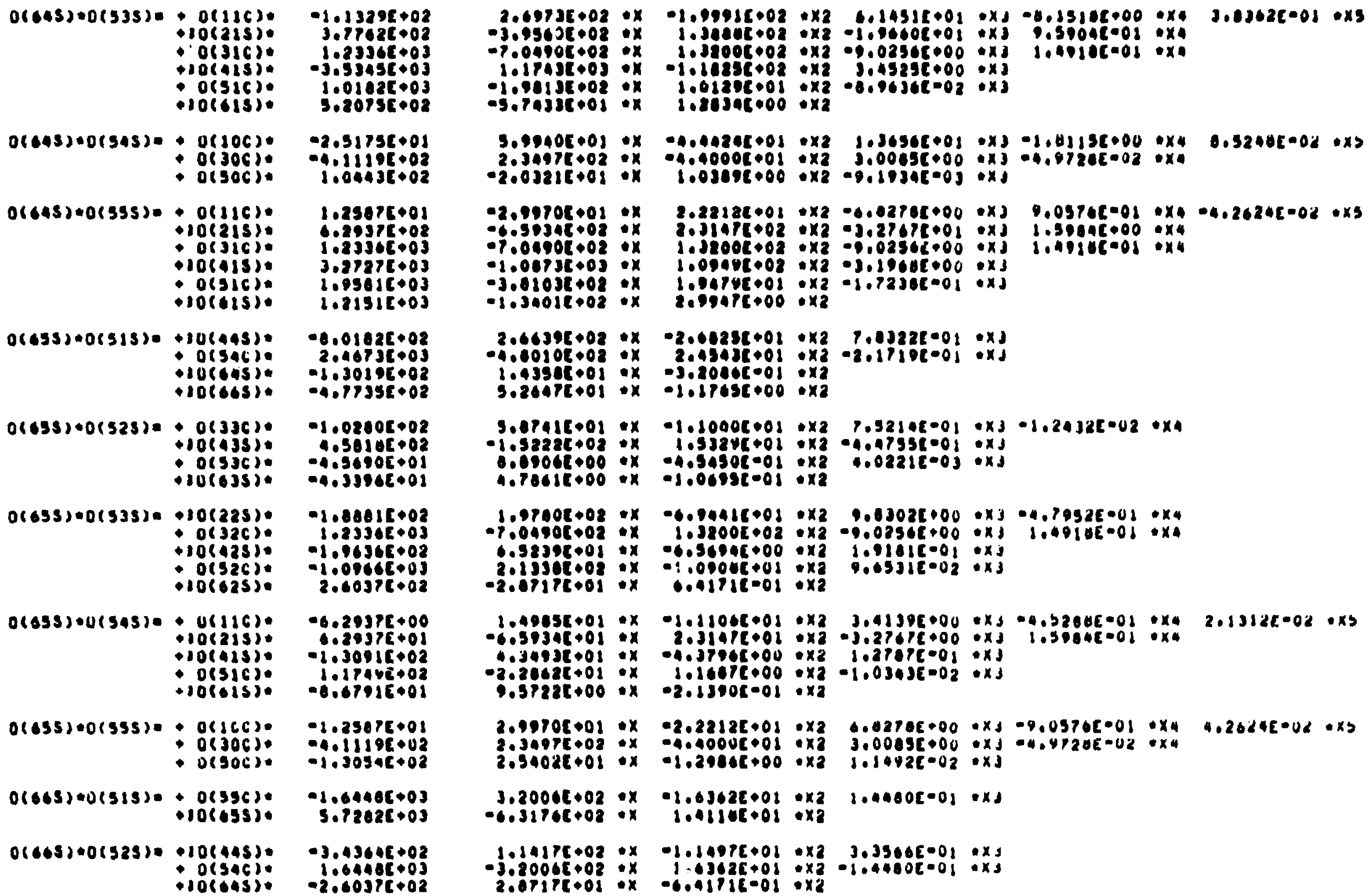

$0(653)+0(535)=+10(225)$ O $0(32 \mathrm{C})$ - o(saci. - idocass.

$-1.0001[+02$

$1.23365+03$

$-1.96365 .02$ $-1.0966003$

$0(453) \cdot 0(545)=0(116)$ $+10(213)$ $-10(425)$ + D(SIC):

$-6.29375+00$ $0.2837 E+01$ $-1.30916 \cdot 02$ $1.17408+02$ $-0.67918001$

$0(035) \cdot 0(355)+0(10)+0$ 0rsocs.

$-1.25075001$ $-4.11196+02$

1.0408003 $10(558)$ $3.72028 \div 03$

$0(665)+0(313)=$

$0(605) 00(523) \cdot 0 \cdot 10(445)$. io(bas):

$-3.43645+02$ 1.64406003
$-2.00370+02$

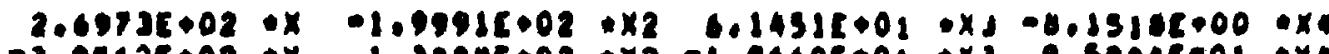
$-3.256 x+02$ :x

1.38005

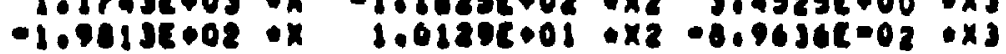

$-5.703 a c+01$ ox

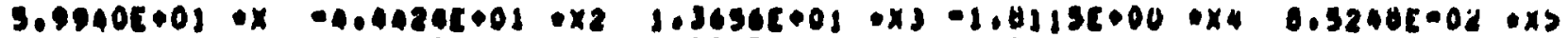

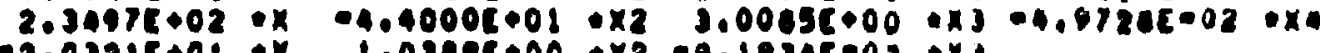

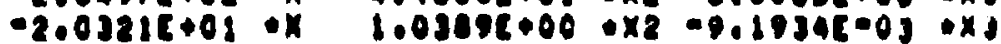

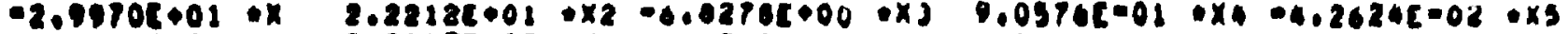

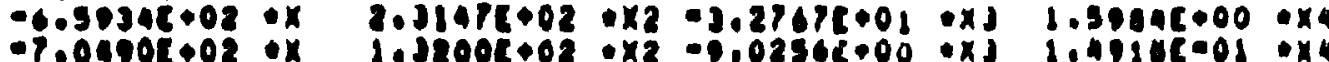

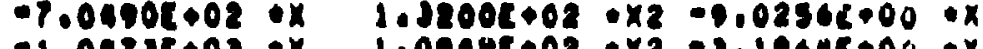

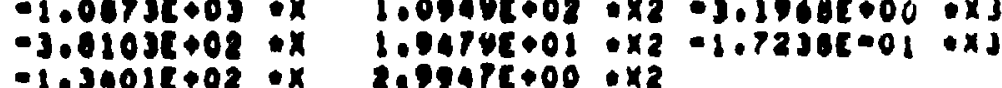

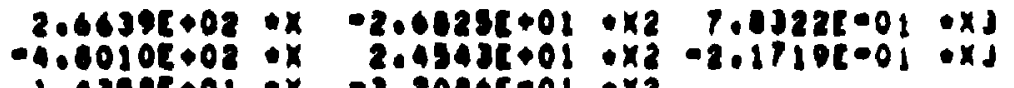

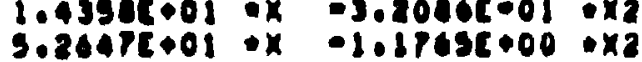

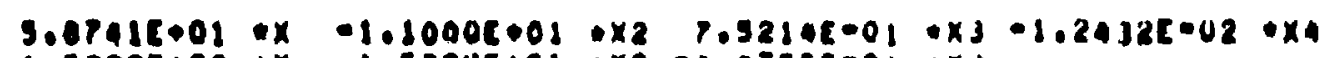

i.

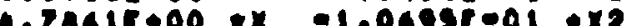

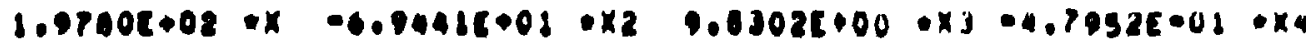

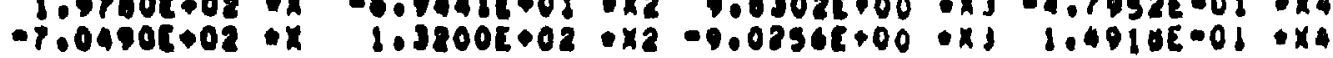

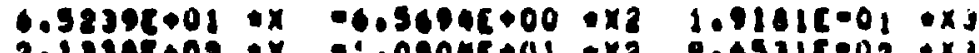

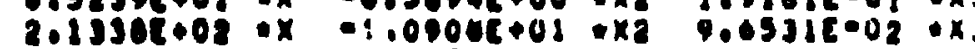

$-2.07170+01$ ix

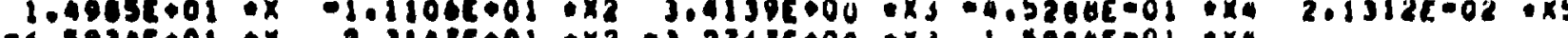

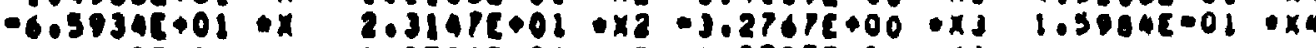

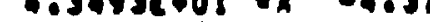

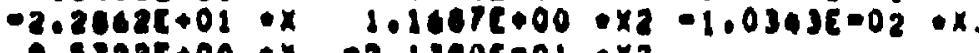

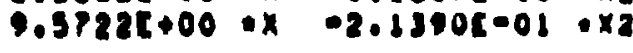

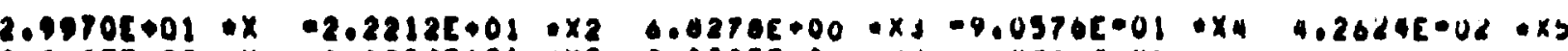

$1.14428002 \cdot x J$

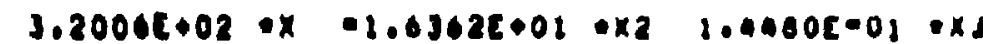
$-6.31766+02$ ix 1.41146001 ix

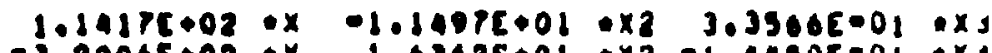

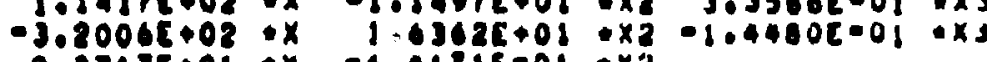

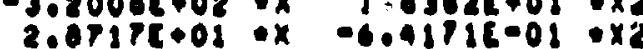




\begin{tabular}{|c|c|c|c|c|c|c|c|c|c|c|c|c|c|}
\hline$(665) * 0(535)=$ & $\begin{array}{l}+0(336) * \\
+10(435) . \\
+0(536) \\
+80(635) .\end{array}$ & $\begin{array}{r}-1.2336 E+03 \\
8.2473 E+03 \\
-2.1931 E+03 \\
2.0830 E+03\end{array}$ & & $\begin{array}{r}7.0490 E+02 \\
-2.7400 E+03 \\
4.2675 E+02 \\
-2.2973 E+02\end{array}$ & $\begin{array}{l}x \\
-x \\
0 x \\
0 x\end{array}$ & $\begin{array}{r}-1.3200 E \bullet 02 \\
2.73925 \bullet 02 \\
-2.16165 * 01 \\
5.1331 E \bullet 00\end{array}$ & $\begin{array}{l}* \times 2 \\
: \times 2 \\
: \times 2 \\
\times 2\end{array}$ & $\begin{array}{r}9.0256 E+00 \\
-8.0559 E+00 \\
1.9306 E=01\end{array}$ & $\begin{array}{l}03 \\
0 \times 3 \\
0 \times 3 \\
03\end{array}$ & -1.491 UE -01 & $\times 4$ & & \\
\hline $0(665) * 0(545)=$ & $\begin{array}{l}+10(225) * \\
+0(32 C) \\
+10(425) \\
+0(52 C) \\
+10(625)\end{array}$ & $\begin{array}{r}-1.2587 E+02 \\
1.2336 E+03 \\
-5.8909 E+02 \\
2.1931 E+03 \\
-1.7358 E+02\end{array}$ & & $\begin{array}{r}1.3187 E+02 \\
-7.0490 E+02 \\
1.9572 E+02 \\
-4.2675 E+02 \\
1.9144 E+01\end{array}$ & $\begin{array}{l}: x \\
: x \\
: x \\
: x \\
: x\end{array}$ & $\begin{array}{r}-4.6294 E+01 \\
1.3200 E+02 \\
-1.9708 E+01 \\
2.1816 E+01 \\
-4.2781 E-01\end{array}$ & $\begin{array}{l}+\times 2 \\
\times \times 2 \\
0 \times 2 \\
\times \times 2 \\
\times \times 2\end{array}$ & $\begin{array}{r}6.5534 E+00 \\
-9.0256 E+00 \\
5.7542 E=01 \\
-1.9306 E-01\end{array}$ & $\begin{array}{l}\times 3 \\
\times 1 \times 3 \\
1 \times 3 \\
\times 3\end{array}$ & $\begin{array}{r}-3.19606=01 \\
1.49185 .-01\end{array}$ & $: \times 4$ & & \\
\hline $0(665) \cdot 0(555)=$ & 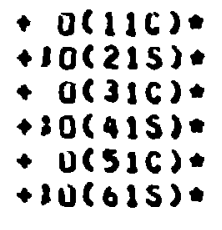 & $\begin{array}{r}-7.5524 E+01 \\
1.25 A 7 E+03 \\
-1.2326 E+03 \\
1.9636 E+03 \\
-7.8326 E+02 \\
3.4717 E+02\end{array}$ & & $\begin{array}{r}1.7982 E+02 \\
-1.3187 E+03 \\
7.0490 E+02 \\
-6.5239 E+02 \\
1.5241 E+02 \\
-3.8289 E+01\end{array}$ & $\begin{array}{l}x_{x} \\
-x \\
-x \\
-x \\
0 x \\
0 x\end{array}$ & $\begin{array}{r}-1.3327 E \bullet 02 \\
0.6294 E+02 \\
-1.3200 E+02 \\
6.5694 E \bullet 01 \\
-7.7914 E \bullet 00 \\
8.5561 E-01\end{array}$ & $\begin{array}{l}\times 2 \\
\times \times 2 \\
\times \times 2 \\
\times 2 \\
\times 2 \\
\times 2 \\
\times 2\end{array}$ & $\begin{array}{r}4.0967 E+01 \\
-6.5 j 34 E+01 \\
9.0250 E+00 \\
-1.9111 E+00 \\
6.8951 E=02\end{array}$ & $\begin{array}{l}: \times 3 \\
: \times 3 \\
: \times 3 \\
: \times 3 \\
\times 13 \\
\times 3\end{array}$ & $\begin{array}{r}-5.4346 E+00 \\
3.1964 E+00 \\
-1.4918 E-01\end{array}$ & $\begin{array}{l}\times 4 \\
\times 4 \\
\times 4 \\
\times 4\end{array}$ & $2.5574 E-01$ & $\cdot \times 5$ \\
\hline $0(1,15) \cdot 0(615)=$ & 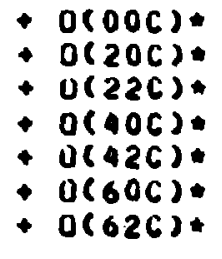 & $\begin{array}{r}-6.9231 E+01 \\
-1.6773 E+03 \\
2.7094 E+03 \\
1.7889 E+03 \\
-7.8264 E+03 \\
-3.9588 E+02 \\
4.1567 E+03\end{array}$ & $\bullet x$ & $\begin{array}{r}1.6484 E+02 \\
1.8117 E+03 \\
-2.9266 E+03 \\
-6.5384 E+02 \\
2.8606 E+03 \\
5.3928 E+01 \\
-5.6623 E \div 02\end{array}$ & $\begin{array}{l}n: x \cdot 2 \\
: x \\
: x \\
: x \\
: x \\
: x \\
: x \\
0 x\end{array}$ & $\begin{array}{r}-1.2210 E+02 \\
-6.7401 E+02 \\
1.0686 E+03 \\
7.0147 E+01 \\
-3.4109 E+02 \\
-1.8071 E+00 \\
1.9014 E+01\end{array}$ & $\begin{array}{l}* 3 \\
\times 23 \\
\times 2 \\
\times 2 \\
\times 2 \\
\times 2 \\
\times 22 \\
\times 2 \\
\times 2\end{array}$ & $\begin{array}{r}3.7553 E+01 \\
1.0739 E+02 \\
-1.7347 E+02 \\
-3.3247 E+00 \\
1.4545 E+01 \\
1.0722 E-02 \\
-1.1250 E-01\end{array}$ & 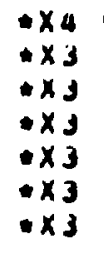 & $\begin{array}{r}-4.9817 E+00 \\
-7.0990 E+00 \\
1.1469 E+01 \\
3.0080 E-02 \\
-1.3163 E-01\end{array}$ & $\begin{array}{l}1 \times 5 \\
\times 4 \\
\times 44 \\
\times 44 \\
\times 4\end{array}$ & $\begin{array}{r}2.3443 E-01 \\
1.3853 E-01 \\
-2.237 \forall E-01\end{array}$ & $\begin{array}{l}* x 6 \\
\times 55 \\
\times 5\end{array}$ \\
\hline $0(625) \cdot 0(615)=$ & 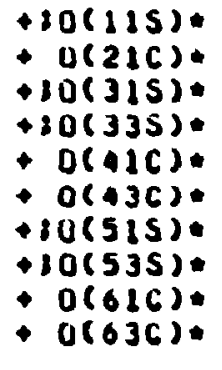 & $\begin{array}{r}4.1530 E+02 \\
-3.0965 E+03 \\
-4.4937 E+03 \\
1.0405 E+04 \\
1.1851 E+04 \\
-1.0957 E+04 \\
2.2032 E+03 \\
-8.9963 E+03 \\
-6.6507 E+03 \\
4.9000 E+03\end{array}$ & & $\begin{array}{r}-9.8901 E+02 \\
3.3447 E+03 \\
2.7252 E+03 \\
-6.3587 E+03 \\
-4.3317 E+03 \\
4.0048 E+03 \\
-4.9478 E+02 \\
2.0203 E+03 \\
9.0599 E+02 \\
-6.7950 E+02\end{array}$ & $\begin{array}{l}* x \\
: x \\
: x \\
* x \\
* x \\
* x \\
: x \\
: x \\
: x \\
: x \\
: x\end{array}$ & $\begin{array}{r}7.3299 E+02 \\
-1.2443 E+03 \\
-5.6702 E+02 \\
1.3230 E+03 \\
5.1772 E+02 \\
-4.7865 E+02 \\
3.3200 E+01 \\
-1.3557 E+02 \\
-3.1703 E+01 \\
2.3777 E+01\end{array}$ & $\begin{array}{l}\times 2 \\
\times 22 \\
\times 2 \\
\times 2 \\
\times 2 \\
\times 2 \\
\times 2 \\
\times 2 \\
\times 2 \\
\times 2 \\
\times 2 \\
\times 2 \\
\times 2\end{array}$ & $\begin{array}{r}-2.2532 E+02 \\
1.9025 E+02 \\
4.7664 E+01 \\
-1.1122 E+02 \\
-2.2026 E+01 \\
2.0364 E+01 \\
-6.2056 E=01 \\
2.5339 E+00 \\
1.0013 E-01 \\
-1.3510 E=01\end{array}$ & 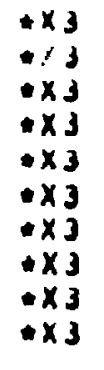 & $\begin{array}{r}2.4890 E+01 \\
-1.3107 E+01 \\
-1.3427 E+00 \\
3.1324 E+00 \\
1.9933 E-01 \\
-1.6429 E-01\end{array}$ & 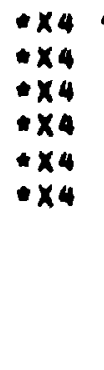 & $\begin{array}{r}-1.4066 E+00 \\
2.5574 E=01\end{array}$ & $\begin{array}{l}\text { *xs } \\
\text { :x5 }\end{array}$ \\
\hline $0(625) \cdot 0(625)=$ & 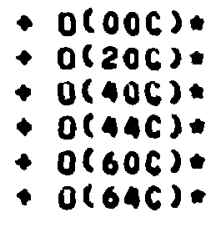 & $\begin{array}{r}-1.1077 E+02 \\
-2.0643 E+03 \\
1.9195 E+02 \\
-2.1914 E+04 \\
6.9674 E+02 \\
7.9009 E+03\end{array}$ & $\star x$ & $\begin{array}{r}2.6374 E+02 \\
2.2298 E+03 \\
-1.0981 E+02 \\
6.0095 E+03 \\
-9.4914 E+01 \\
-1.0072 E+03\end{array}$ & $\begin{array}{l}\cdot x 2 \\
: x \\
* x \\
* x \\
* x \\
* x\end{array}$ & $\begin{array}{r}-1.9546 E \bullet 02 \\
-0.2955 E \bullet 02 \\
2.1490 E+01 \\
-9.5730 E \bullet 02 \\
3.32125+00 \\
3.0043 E \bullet 01\end{array}$ & $\begin{array}{l}: \times 3 \\
: \times 2 \\
\times 2 \\
\times 2 \\
\times 2 \\
* 2 \\
\times 2 \\
\times 2\end{array}$ & $\begin{array}{r}6.0085 E+01 \\
1.3217 E+02 \\
-9.1429 E-01 \\
1.0727 E+01 \\
-1.0871 E-02 \\
-2.1616 E-01\end{array}$ & $\begin{array}{l}: \times 4 \\
1 \times 3 \\
1 \times 3 \\
0 \times 3 \\
1 \times 3 \\
03\end{array}$ & $\begin{array}{r}-7.9707 E+00 \\
-0.7374 E+00 \\
0.2741 E=03 \\
-3.6857 E-01\end{array}$ & $\begin{array}{l}x 5 \\
: \times 4 \\
04 \\
x 4 \\
x 4\end{array}$ & $\begin{array}{l}3.7509 E=08 \\
1.7050 E-08\end{array}$ & $\begin{array}{l}\times 66 \\
\times 55\end{array}$ \\
\hline $0(635)+0(615)=$ & 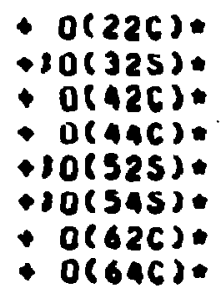 & $\begin{array}{r}1.5483 E+03 \\
-8.9874 E+03 \\
-1.3417 E+03 \\
6.2177 E+03 \\
2.3133 E+04 \\
-1.3494 E+04 \\
-1.2470 E+03 \\
-2.0784 E+03\end{array}$ & & $\begin{array}{r}-1.6723 E+03 \\
5.4503 E+03 \\
4.9030 E+02 \\
-3.0036 E+03 \\
-5.1952 E+03 \\
3.0305 E+03 \\
1.6987 E+02 \\
2.8312 E+02\end{array}$ & 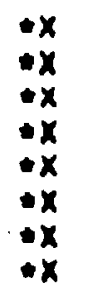 & $\begin{array}{r}6.2216 E+02 \\
-1.1340 E+03 \\
-5.8610 E+01 \\
3.5090 E+02 \\
3.4060 E+02 \\
-2.0335 E+02 \\
-5.9443 E+00 \\
-9.9071 E+00\end{array}$ & $\begin{array}{l}: \times 2 \\
: \times 2 \\
: \times 2 \\
: \times 2 \\
: \times 2 \\
: \times 2 \\
: \times 2 \\
x \times 2 \\
x 2\end{array}$ & $\begin{array}{r}-9.9125 E+01 \\
9.5329 E * 01 \\
2.4935+00 \\
-1.5273 E * 01 \\
-6.5158 E * 00 \\
3.0009 E+00 \\
3.3774 E-02 \\
5.6290 E-02\end{array}$ & $\begin{array}{l}+x 3 \\
0 \times 3 \\
0 \times 3 \\
0 \times 3 \\
0 \times 3 \\
0 \times 3 \\
0 \times 3 \\
0 \times 3 \\
03\end{array}$ & $\begin{array}{r}6.5534 E+00 \\
-2.0853 E+00 \\
-2.2566 E=02 \\
1.3021 E-01\end{array}$ & $\begin{array}{l}0 \times 4 \\
: \times 4 \\
04 \\
04 \\
+x 4\end{array}$ & $-1.2767 E-01$ &.$\times 5$ \\
\hline
\end{tabular}




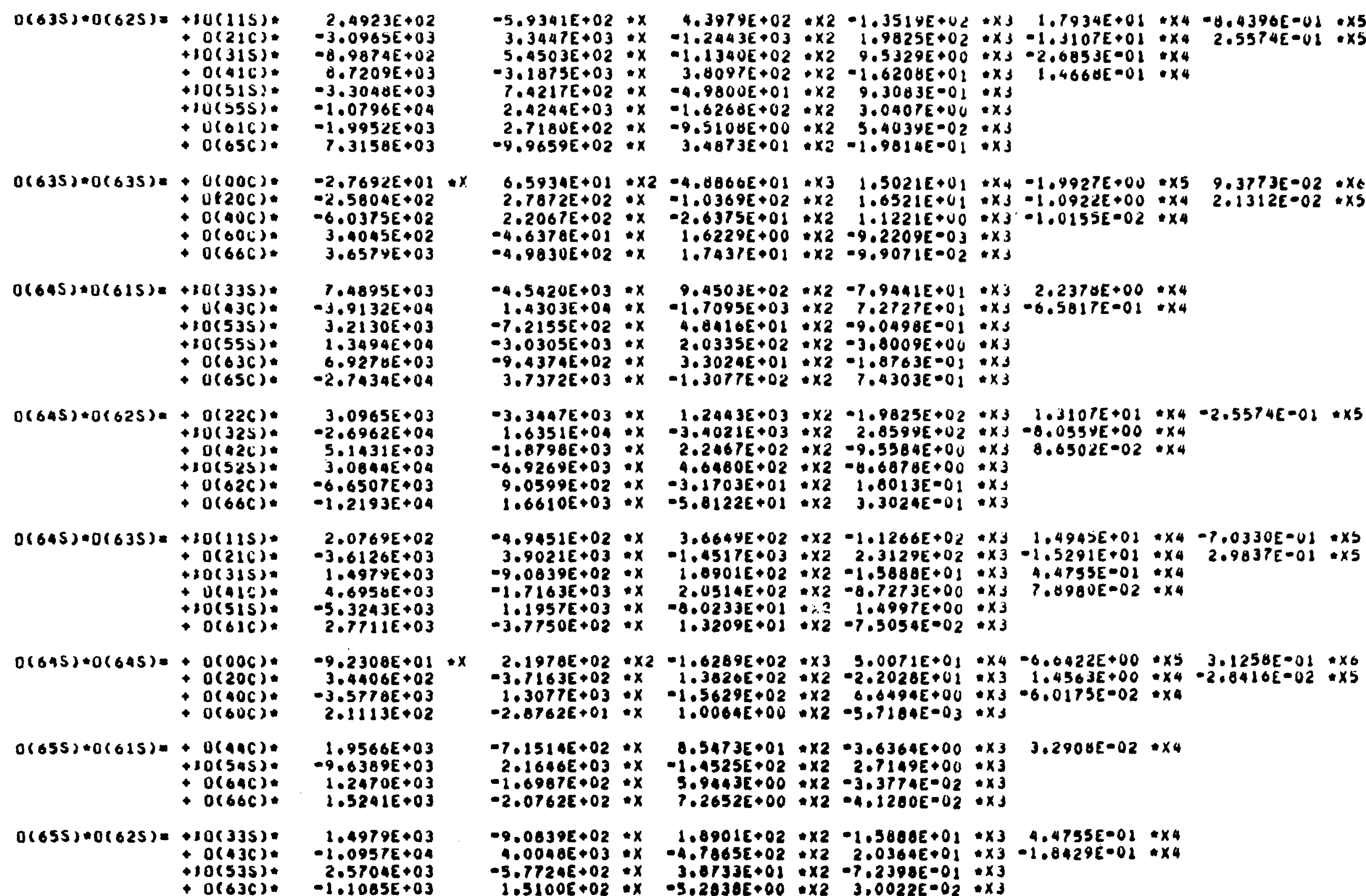

$0(635) \cdot 0(625)=+10(115) * \quad 2.4923 E+02$ $+0(216)=-3.0965 E+03$ $-8.9874 E+02$

$0.1209 E+0$

$+10(515):$

$-1.0796 E+04$

$+v(016)=-1.9952 E+03$

$7.3158 E+03$

$0(635)=0(635)=0.0(00 c$ $0(206)$ O(40) - $0(000)$

$-2.7692 E+01$ $-2.5804 E+02$ $-6.0375 E+02$ $3.0045 \varepsilon+02$

$0.0955 \cdot 03$

$0(645)+0(615)=+10(335)$. $0.0(436)$. $+10(555)$.

- o(b3c).

- $0(65 C)$.

$0(645)+0(625)=+0(22 C) *$ $+30(325)$. $0(426):$

$+10(620)$. $+0(66 C)$.

$0(645)+0(635)=+30(115)=$ $+0(216)$. $+30(315)$. $+20(515)$. + o(b)

$0(645)+0(645)=+0(00 C)$ $+0(20 C)$ o( $0(600):$

$0(655)+0(615)=+0(410)=$ $+10(545)=$ $+0(64 C):$
$+0(66 C)$.

$0(655) * 0(625)=+10(335)=$ $+0(43 C)$. $+10(535)$ :

$-5.9341 E+02 * x$ $3.3447 E+03: x$ $5.4503 E+02 * x$ $-3.1875 E+03 * x$ $7.4217 E+02: x$ $2.4244 E+03: x$ $2.7180 E+02: x$
$-9.9659 E+02: x$

$4.3979 E+02 \times \times 2-1.3519 E+02 * x 3 \quad 1.7934 E+01+\times 4=8.4396 E-01+\times 5$ $-1.2443 E+03 * \times 2 \quad 1.9825 E+02 * x 3-1.1107 E+01 * \times 42.5574 E-01 * \times 5$

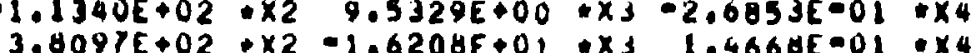

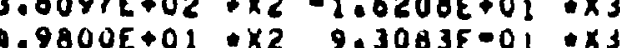

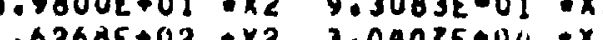

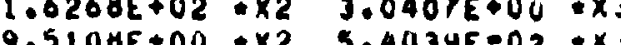

$9.5108 E+00: \times 2$
$3.4873 E+01: \times 2-1.9814 E=01: x 3$

$6.5934 E+01 * \times 2-4.0066 E+01 * \times 3 \quad 1.5021 E+01+x 4-1.9927 E+00=\times 5 \quad 9.3773 E=02 * x_{E}$ $2.7872 E+02 * x-1.0369 E+02 * \times 2 \quad 1.6521 E+U_{1} * X_{3}-1.0922 E+00 * x_{4} \quad 2.1312 E-02 * x 5$ $2.2067 E+02 * x-2.6375 E+01 * \times 2 \quad 1.1221 E+00 * x 3-1.0255 E-02+x 4$

$-4.637 a E+01 * x \quad 1.6229 E+00 * x 2-9.2209 E-03 * x 3$

$0.5420 E+03 * x \quad 9.4503 E+02 * \times 2-7.9441 E+01 * x 3 \quad 2.2370 E+00 * x 4$

$1.4303 E+04: x-1.7095 E+03 * x 27.2727 E+01 * x 3-6.5817 E=01 \times \times 4$

$-3.9132 E+04$

(1)

$6.92765+03$

$-2.7434 E+04$

$3.0965 E+03$ $-2.6962 E+04$ $5.1431 E+03$ $3.0844 E+04$ $-6.6507 E+03$ $-1.2193 E+04$

$2.0769 E+02$ $3.6126 E+03$ $1.4979 E+03$ $4.6958 \varepsilon+03$ $-5.3243 E+03$
$2.7711 E+03$

$+02 * x$

$-3.0305 E+03 * x$

$x \quad 2.0335 E+02: x 2-3.0009 E+00: x$

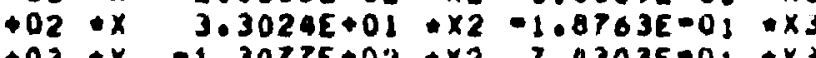

$-1.3077 E+02+\times 2 \quad 7.4303 E=01 \times 3$

$-3.3447 E+03 * x \quad 1.2443 E+03 * x 2-1.9825 E+02 * x 3 \quad 1.3107 E+02 * x 4-2.5574 E-01 * \times 5$ $1.6351 E+04 * x-3.4021 E+03 * x 2 \quad 2.8599 E+02 * x 3-8.0554 E+00 * x 4$

$-1.0798 E+03 * x \quad 2.2467 E+02 * x 2-9.5384 E+0 u * x_{3} \quad 8.6502 E-02 * x$

$-6.9269 E+03 * x \quad 4.0460 E+02 \times x 2-0.6078 E+00 * x 3$

$1.6610 E+03 * x \quad-5.0122 E+01 * x 2 \quad 3.3024 E-01: x 3$

$04.9451 E+02 * x \quad 3.6649 E+02 * x 2-1.1266 E+02 * x J \quad 1.4945 E+01 * x 4-1.0330 E=01 * x b$ $3.9021 E+03 * x-1.4517 E+03 * \times 2 \quad 2.3129 E+02 * \times 3-1.5291 E+01 * \times 4209837 E-01 * \times 5$ $-9.0839 E+02 * x \quad 1.0901 E+02 * x 2-1.5888 E+01 * x 3 \quad 4.4755 E-01 * x$

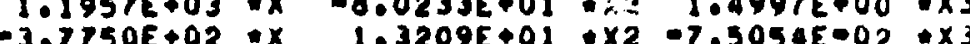

$-9.2308 E+01 . x$ $3.4406 E+02$ $3.5778 E+03$
$2.1113 E+02$

$2.1978 E+02 * \times 2-1.6209 E+02 \times 3 \quad 5.0071 E+01 \times \times 4-6.0422 E+00 * \times 5 \quad 3.1258 E-01 * \times 6$ $-3.1163 E+02 * x \quad 1.3820 E+02 * \times 2-2.2028 E+01 * \times 3 \quad 1.4563 E+00 * x 4-2.8410 E-02 * \times 5$ $1.3077 E+03 * x-1.5629 E+02 * x 20.6494 E+00 * x 3-6.0175 E=02 * x 4$ $-2.8762 E+01 \cdot x \quad 1.0064 E+00 * x 2-5.7184 E-03 * x$

$1.9566 \varepsilon+03$

$-9.6389 E+03$

$1.3241 E+03$
1.2410

$1.49796 \bullet 03$

$-1.0957 E+04$

$-1.57046+03$

$-7.1514 E+02 * x \quad 0.5473 E+01 * \times 2=3.6364 E+00 * \times 3 \quad 3.2908 E-02 * \times 4$

$2.1646 \varepsilon+03 * x-1.4525 E+02 * x 2 \quad 2.7149 E+00 * x 3$

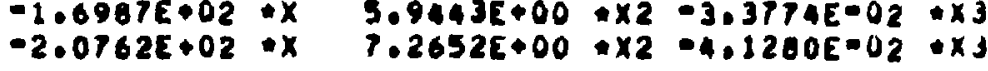

$-9,0039 E+02 * x \quad 1.8901 E+02 * \times 2-1.5800 E+01 * \times 3 \quad 4.4755 E-02 * \times 4$

$4.0048 E+03 * x-4.1065 E+02 \times x \quad 2.0364 E+01 \times 3=1.8429 E-02$ a $x$

$-5.7724 E+02 * x \quad 3.8733 E+01: x_{2}-7.2398 E-01: x_{3}$ 


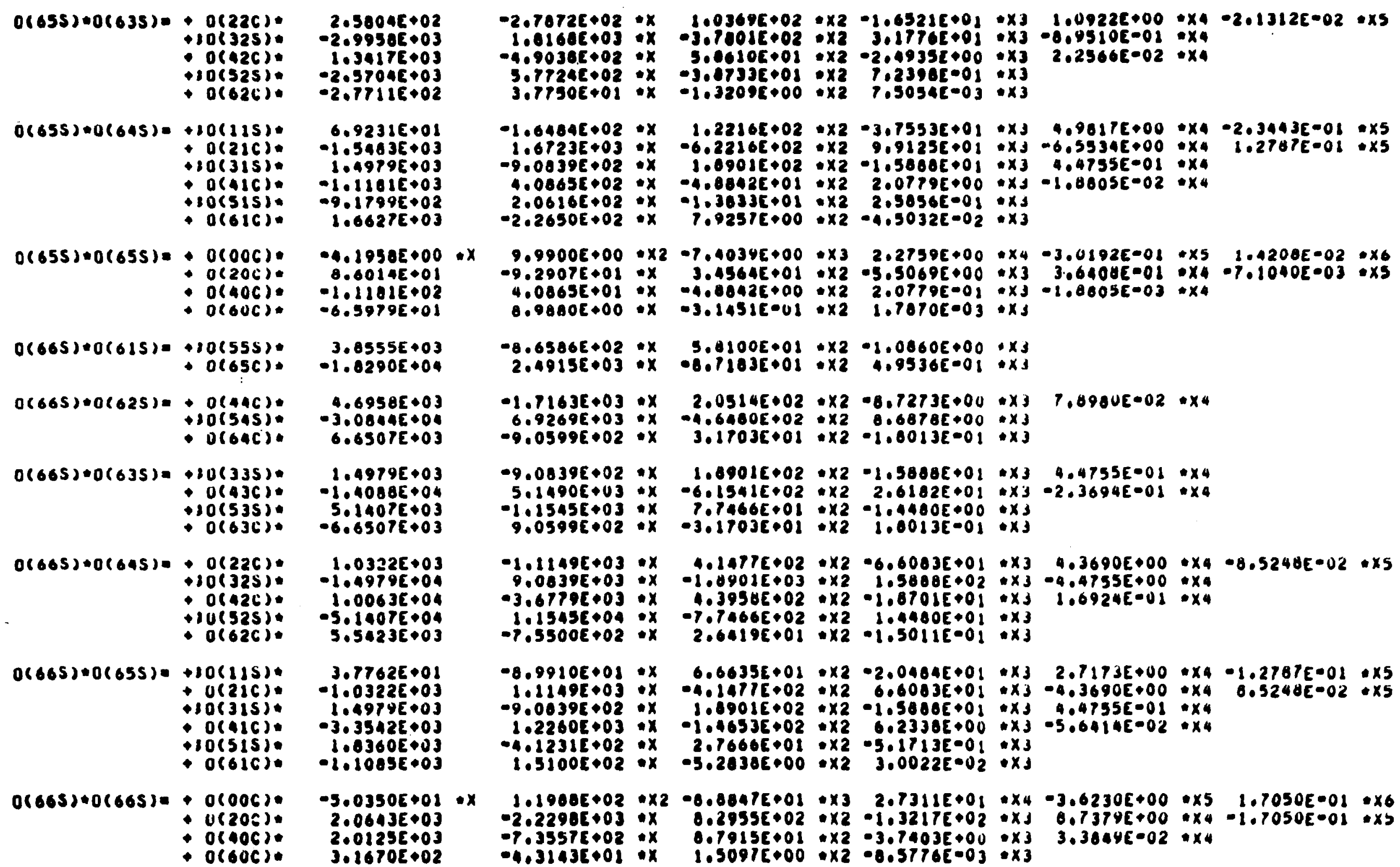

$0(655) \cdot 0(635)=-O(226)=$ $10(325)$ - $0(426)$. $+0(525):$
$+0(626)$. 2.58046+02 $-2.99585+03$ i. 3417 itos $-2.57046+0$ $-2,77116+02$

$0(655)=0(645)=+10(115)=$ +o(2ic). $+20(315)$. $\rightarrow$ o(aic). $+10(515)$.

$6.9231 E+01$ $-1.54635+03$ $1.49795+03$ $-1.11016+03$ $-9.1799 E+02$ $1.6627 E+03$

$0(655) \cdot 0(655)=+0(006)$. o(20c). $\because$ O(boc).

$-4.1958 E \bullet 00$ $8.6014 E+01$ $-1.11215+02$
$-6.59795+01$

$0(665)+0(615)=+10(555)=$ - $0(656)$.

$\begin{aligned} 0(665) \cdot 0(625)= & +0(44 C) \\ + & 0(545)\end{aligned}$ o(bac).

$3.0555 E \div 03$ $-1.0200 E+0$ $4.69585+03$ $-3.0814 E 004$

$0(665) \cdot 0(635)=+80(335)=$ $+20(535)$.

$1.4979 E+03$ $-1.4088 E+0$ $-6.65078003$

$1.0322 E+03$

$0(665)=0(645)=0(226)$ $\rightarrow 0(425)$. $+10(325)$. $1.0063 E+04$ $-5.1107 E+04$ $5.5423 E \bullet 03$

$0(665)=0(655)=+10(115)+$ $+10(315)$. - o(taic). $+10(515)$. $3.7762 E \bullet 01$
$-1.0322 E \bullet 03$ $1.4974 E \bullet 03$ $-3.3542 E+03$ $1.03605 \bullet 03$ $-i .1085 E \bullet 03$

$0(665) \times 0(665)=+0(006)$ - $u(205)$. - o( 900$).$

$-2.70726+02 \cdot x \quad 1003696+02 \times x 2-1.6521 E+01 \cdot x 3 \quad 1.0922 E+00 \cdot x 4-201312 E-02 \cdot x 5$

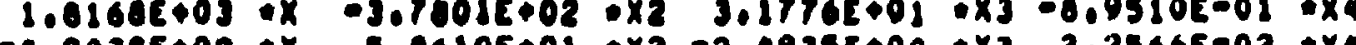
$-4.9036 E+02 * x \quad 50610 E+02 * x 2-2.4935 E+00 * x] 2.2566 E-02 * x 4$

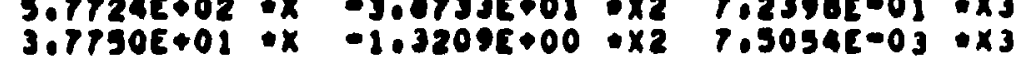

$-1.6484[002 * x \quad 1.2216 E+02 * \times 2-3.7533 E+01 * x] \quad 4.9017 E+00 * \times 4-2.3443 E-01 * x 5$ $1.6723 E+03 * x-6.2216 E+02 * x 2 \quad 9.9125 E+01 * x J-6.5534 E+00 * x 4 \quad 1.2747 E-01 * x 5$ $-9.0039 E+02 * x \quad 1.0901 E+02 * x_{2}-1.5800 E+01 * x_{3} \quad 404755 E-01 * x_{4}$

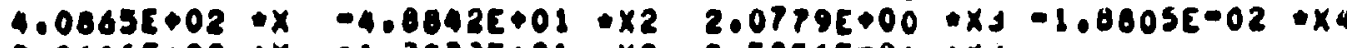

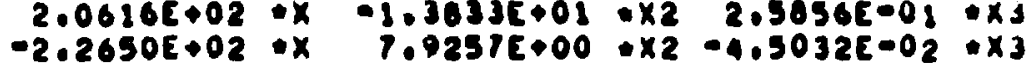

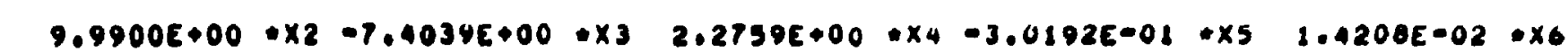
$-9.2907 E+01 * x \quad 3.4564 E+01 \times \times 2-5.5060 E+00 * x 3 \quad 3.6404 E-01 * x 4-7.10405-03: \times 5$

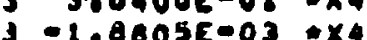

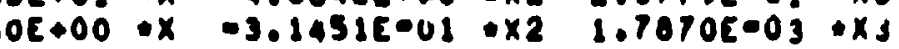

$-8.6586 E+02 * x \quad 5.0100 E+01 * \times 2 \cdot 1.0060 E+00 * x s$

$2.4915 E+03 * x-8.1103 E+01 * \times 2 \quad 4.9536 E-01 * x 3$

$-1.7163 E+03 * x \quad 2.0514 E+02 * \times 2-8.7273 E+00 * x 3 \quad 7.8900 E-02 * x 4$ $6.9269 E+03 * x-1.6460 E+02+x 2-0.6878 E+00 * x 3$

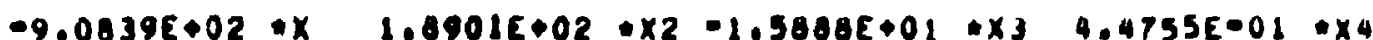
$5.1490 E+03: x-6.1541 E+02: x 22.6182 E+02 \times x-2.3694 E-01: x 4$

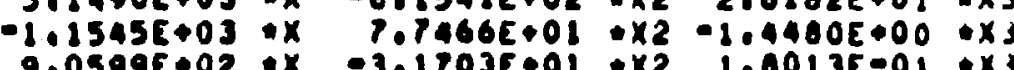

$-1.1149 E+03 * x \quad 4.14 T 7 E+02 * \times 2 \cdot 0.6003 E+01 * \times 3 \quad 4.3690 E+00 * \times 4 \cdot 8.5248 E-02 * \times 5$ $9.0839 E+03 * x-1.0901 E+03 * x_{2} \quad 1.5808 E+02 * x_{3}-4.4755 E+00 * x_{4}$

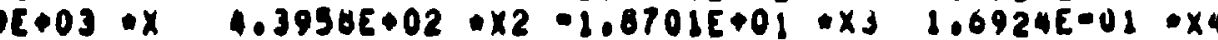

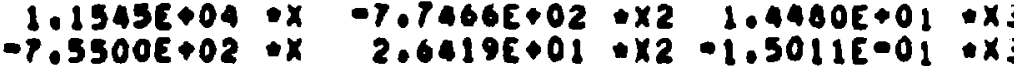

$-8.9910 E+01 \cdot \times \quad 6.6635 E+01 \cdot \times 2 \cdot 2.0404 E+01 * \times 3 \quad 2.7173 E+00 * \times 4-1.2767 E-01 * \times 5$ $1.12149 E+03 * x-4.1477 E+02 * x 2 \quad 6.6003 E+01 * x_{3}-4.3690 E+00 * x_{4} \quad 6.5248 E-02+x 5$

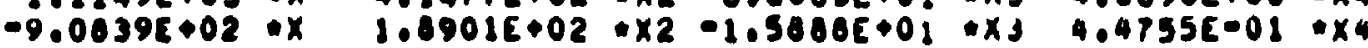
$1.2260 E+03 * x-1.4653 E+02 * \times 2 \quad 6.2338 E+00 * x_{3}-5.6414 E-02 * x_{4}$

$-4.1231 E+02: x \quad 2.76665+01 \div x^{2}-5.1713 E-01: x_{3}$

$-5.03505+01 \cdot x$ $2.0643 E+03$ $3.16705 \bullet 02$

$1.1900 E+02 * \times 2-0.0847 E+01 * \times 3 \quad 2.7311 E+01 * \times 4-3.6230 E+00 * \times 5 \quad 1.7050 E-01 * \times 6$

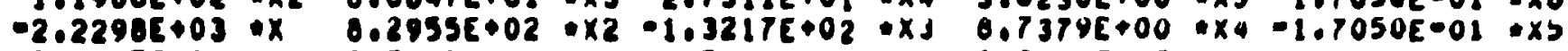
$-7.3557 E+02 * x \quad 0.7915 E+01 * \times 2-3.7403 E \div 00 * x 3 \quad 3.38446-02 \times \times 4$ 


\section{Table 3}

Cotfficients relating Stevens operators to Racah operaturs

\begin{tabular}{|c|c|c|c|}
\hline 1 & $m$ & $k_{1}^{m}$ & $\frac{\sqrt{\frac{21+1}{k_{2}}}}{k_{1}^{m}}$ \\
\hline 1 & 0 & $\frac{1}{2} \sqrt{\frac{3}{x}}$ & 1 \\
\hline 1 & 1 & $\frac{1}{2} \sqrt{\frac{3}{x}}$ & 1 \\
\hline 2 & 0 & $\frac{1}{3 / \sqrt{3}}$ & 2 \\
\hline 2 & 1 & $\frac{1}{2} \sqrt{\frac{13}{2}}$ & $\frac{1}{13}$ \\
\hline 2 & 2 & $\sqrt[1]{\frac{15}{2}}$ & $\frac{2}{13}$ \\
\hline 3 & 0 & $9 \sqrt{7}$ & 2 \\
\hline 3 & 1 & $\sqrt[1]{\sqrt{\frac{42}{x}}}$ & $\frac{1}{16}$ \\
\hline 3 & 2 & $\sqrt[1]{\frac{105}{1}}$ & $\frac{2}{113}$ \\
\hline 3 & 3 & $\sqrt[3]{\frac{70}{n}}$ & ivo \\
\hline 4 & 0 & ret & 1 \\
\hline 4 & 1 & $\sqrt[3]{10}$ & ito \\
\hline 4 & 2 & $3 \sqrt{\frac{5}{1}}$ & $\frac{4}{5}$ \\
\hline 4 & 3 & $3 \cdot \sqrt{\frac{10}{10}}$ & $\frac{1}{10}$ \\
\hline 4 & 4 & $\sqrt[3]{\frac{35}{\pi}}$ & $\frac{8}{1+3}$ \\
\hline 3 & - & $\frac{1}{11}$ & 8 \\
\hline 5 & 1 & $\frac{1}{1 \frac{16 B}{a}}$ & $\frac{1}{13}$ \\
\hline 5 & 2 & $1 \sqrt{\frac{3155}{2}}$ & ines \\
\hline 3 & 3 & $\frac{1}{3 r} \sqrt{\frac{770}{x}}$ & $\frac{16}{100}$ \\
\hline 3 & 4 & $\sqrt[3]{\frac{305}{x}}$ & $\frac{8}{325}$ \\
\hline 3 & 5 & $\frac{3}{32} \sqrt{\frac{134}{4}}$ & $\frac{11}{\sin }$ \\
\hline
\end{tabular}

\begin{tabular}{|c|c|c|c|}
\hline 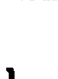 & \multirow{2}{*}{$m$} & \multirow{2}{*}{$\kappa_{1}^{m}$} & \multirow{2}{*}{$\frac{\sqrt{\frac{21+1}{k_{2}}}}{k_{1}^{m}}$} \\
\hline 8 & & & \\
\hline 6 & 0 & $\frac{1}{32} \sqrt{\frac{13}{3}}$ & 16 \\
\hline 6 & 1 & $10 \sqrt{\frac{273}{x}}$ & $\frac{8}{\sqrt{22}}$ \\
\hline 6 & 2 & 614 $\sqrt{\frac{2730}{x}}$ & $\frac{32}{3210}$ \\
\hline 6 & 3 & $\frac{1}{33} \sqrt{\frac{\sqrt{2730}}{x}}$ & $\frac{16}{1210}$ \\
\hline 6 & 1 & $\frac{3}{32} \sqrt{\frac{91}{\pi}}$ & $\frac{16}{317}$ \\
\hline 6 & 5 & $\frac{3}{32} \sqrt{\frac{2002}{1}}$ & $\frac{16}{315 x}$ \\
\hline 6 & 6 & $\frac{1}{6006}$ & $\frac{32}{k 12}$ \\
\hline 7 & 0 & $\frac{1}{32} \sqrt{\frac{15}{n}}$ & 16 \\
\hline 7 & 1 & $\frac{1}{258} \sqrt{\frac{105}{x}}$ & $\frac{120}{11}$ \\
\hline 7 & 2 & $\frac{3}{6 x} \sqrt{\frac{10}{x}}$ & $\frac{32}{12}$ \\
\hline 7 & 3 & $\frac{3}{126} \sqrt{\frac{35}{2}}$ & $\frac{G}{|z|}$ \\
\hline 7 & 4 & $\frac{3}{256} \sqrt{\frac{35}{n}}$ & $\frac{128}{121}$ \\
\hline 7 & 5 & $\sqrt[3]{21} \sqrt{\frac{770}{4}}$ & $\frac{6}{1612}$ \\
\hline 7 & 6 & $34 \sqrt{10.010}$ & 32 \\
\hline 7 & 7 & $\frac{21}{51} \sqrt{\frac{715}{1}}$ & $\frac{32}{\pi \sqrt{12 t}}$ \\
\hline 8 & 0 & $\frac{1}{235} \sqrt{\frac{17}{x}}$ & 120 \\
\hline 1 & 1 & $34 \sqrt{\frac{\pi}{\pi}}$ & $\frac{32}{3}$ \\
\hline 8 & 2 & $\frac{3}{\sqrt{2}} \sqrt{\frac{\pi n}{n}}$ & $\frac{40}{300}$ \\
\hline$\bullet$ & 3 & $6 \pi \sqrt{\frac{1205}{n}}$ & $\frac{32}{1+135}$ \\
\hline 8 & 4 & $\frac{3}{2 x} \sqrt{\frac{1300}{x}}$ & $\frac{4}{319}$ \\
\hline- & 3 & $\frac{3}{17.017}$ & 32 \\
\hline 8 & 6 & $\frac{1}{\sqrt{2 u} \sqrt{\frac{11.3 \mathrm{C}}{n}}}$ & $=\frac{4}{u x}$ \\
\hline - & 7 & $\frac{3}{61} \sqrt{\frac{12.138}{n}}$ & $\frac{32}{3 x+15}$ \\
\hline - & 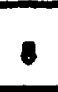 & $\frac{3}{250} \sqrt{\frac{\sqrt{2.13 E}}{1}}$ & $\frac{120}{2121}$ \\
\hline
\end{tabular}


ISUN 875500281 